\title{
Real-time quality control using interactive vision : a theoretical and practical approach to interactive sensing and classification
}

Citation for published version (APA):

Westra, R. L. (2001). Real-time quality control using interactive vision : a theoretical and practical approach to interactive sensing and classification. [Doctoral Thesis, Maastricht University]. Datawyse / Universitaire Pers Maastricht. https://doi.org/10.26481/dis.20011129rw

Document status and date:

Published: 01/01/2001

DOI:

10.26481/dis.20011129rw

Document Version:

Publisher's PDF, also known as Version of record

Please check the document version of this publication:

- A submitted manuscript is the version of the article upon submission and before peer-review. There can be important differences between the submitted version and the official published version of record.

People interested in the research are advised to contact the author for the final version of the publication, or visit the DOI to the publisher's website.

- The final author version and the galley proof are versions of the publication after peer review.

- The final published version features the final layout of the paper including the volume, issue and page numbers.

Link to publication

\footnotetext{
General rights rights.

- You may freely distribute the URL identifying the publication in the public portal. please follow below link for the End User Agreement:

www.umlib.nl/taverne-license

Take down policy

If you believe that this document breaches copyright please contact us at:

repository@maastrichtuniversity.nl

providing details and we will investigate your claim.
}

Copyright and moral rights for the publications made accessible in the public portal are retained by the authors and/or other copyright owners and it is a condition of accessing publications that users recognise and abide by the legal requirements associated with these

- Users may download and print one copy of any publication from the public portal for the purpose of private study or research.

- You may not further distribute the material or use it for any profit-making activity or commercial gain

If the publication is distributed under the terms of Article $25 \mathrm{fa}$ of the Dutch Copyright Act, indicated by the "Taverne" license above, 


\section{Real-time Quality Control using Interactive Vision}




\title{
Real-time Quality Control using Interactive Vision
}

\author{
A theoretical and practical approach
} to interactive sensing and classification

\section{PROEFSCHRIFT}

ter verkrijging van de graad van doctor aan de Universiteit Maastricht, op gezag van de Rector Magnificus,

Prof. Dr. A.C. Nieuwenhuijzen Kruseman, volgens het besluit van het College van Decanen, in het openbaar te verdedigen op donderdag 29 november 2001 om 14.00 uur

door

\section{Ronald Leonard Westra}

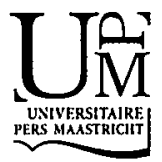


Promotor: $\quad$ Prof. dr. ir. drs. O.J. Vrieze

Co-promotor: Dr. ir. R.L.M. Peeters

Leden van de beoordelingscommissic:

Prof. dr. ir. K. L. Boon (voorzitter)

Prof. dr. ir. A. Hasman

Prof. dr. H. Th. Jongen (RWTH Aachen, ALMA)

Dr. E. O. Postma

The support by the Commission of the European Communion, DG XII, under grant BE3180, is gratefully acknowledged.

Philips Netherlands is acknowledged for their permission to use CCD-images containing their logo.

ISBN $90-5278-326-8$

Print: Datawyse | Universitaire Pers Maastricht

Cover: Peter Stroo

Pictures 7.15 - 7.20 courtesy Andreas Reiss, TampoPrint GmbH.

(C) 2001 by R.L. Westra, Maastricht, The Netherlands

All rights reserved. No part of this publication may be reproduced, stored in a retrieval system, or transmitted, in any form or by any means, electronically, mechanically, phofocopying, recording or otherwise, without prior permission of the author. 


\section{Die Sinne trïgen nicht,}

aber das Urteil trügt

Goethe

Aan mijn ouders, Marijke, Janneke, en Annelies 


\section{Preface}

This work is largely based on the European research project 'IntelPadPrint'. In its course, a tight schedule commanded the swift and dead-line constrained development of prototypes in demanding real-world environments, largely aimed at demonstrating a 'proof of principle'. When I started writing down this thesis in the fall of 1999, I quickly found that most of the work lacked a proper scientific and methodological basis. Therefore, more profound research was required. In the succeeding period, research and writing intertwined into one activity. In the course of writing this thesis, I found that the popular dictum that a good' book writes itself, contains a high degree of truth. The genesis of this work had its own dynamics, and during its conception, I often felt its obedient servant; in quiet moments an addict obsessed with unremitting mathematical brainteasers, and during night a restless zombie.

This work could not have been completed if not so many people had assisted me in so many ways. In the first place, I would like to thank my promotor, Koos Vrieze, for his infallible patience with my inborn Frisian-Groningian stubbornness, especially in those cases where I proofed demonstrably wrong. He more than once showed me a direct way to a result that I had obtained only in zigzag movement. Second, I thank my co-promotor, Ralf Peeters. Among the many things I learned from him is not becoming tired of being critical to your own ideas. Similarly, I greatly appreciate the effort of the assessment-team in reading the sizeable manuscript.

I thank my former colleagues, Bernhard Hahn from RIKS for initiating me in Mathematica, and Piet Boekhoudt and Rob Luesink from the mathematics department for their fruitful discussions. From the department of computer science I thank Eric Postma for inspiring me to the field of active vision, and Nico Roos for his introduction to Bayesian Belief Networks. I thank Emile Aarts from the Technical University Eindhoven/Philips Research for his help in letting me participate in the IntelPadPrint project, and Kasper Boon for his encouragement to pursue a Ph.D. I thank all my colleagues from the IntelPadPrint-team, especially Peter Legierse, project coordinator, Frits Wittgreve, Loes Bouwkamp-Wijnoltz, and Johan Vanhauwe (all from Philips), Calle Schmidt (LEGO), Renate Bultemeier-Bäuerle (Marabu), and Andreas Reiß (TampoPrint). In particular I would like to thank James Shippen and Nick Freear from Birmingham University for the very pleasant, intensive, and scientifically inspiring cooperation during the project. Jean Derks is acknowledged a.o. for his help during a fatal hard disk error. In the same spirit, I thank Coen Dekker for his inventiveness and dexterity. Thanks also to everybody who assisted me in various different ways, in particular all my colleagues from dep. mathematics, notably Marijke Verheij and Dianne Crook. The same for Jan Paredis and Jacques Lenting from the faculty of General Sciences. Many thanks to Peter Stroo for his advice during the printing, and the fabrication of the cover.

Last, but not least, I thank my wife Marijke and daughters Janneke and Annelies for enduring my absence in body and mind.

The main text start of this thesis starts with a famous classical Chinese proverb. To paraphrase another Chinese dictum we can conclude: 'even a journey of a thousand miles ends with one single step'.

Maastricht, September 2001

'without making any claim to the quality of this work. 


\section{Contents}

Preface

\section{Introduction}

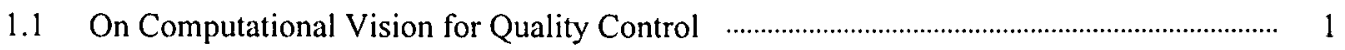

1.2 Computational Vision Control in Industrial Processes ……………………………………..... 4

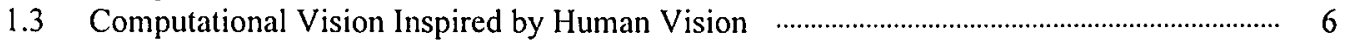

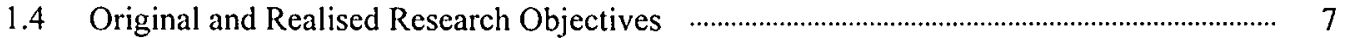

1.5 Innovative Aspects of the Research ……………………....................................................... 8

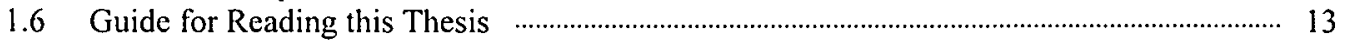

2. The Analysis of Printed Images

2.1 Introduction: Defining Structure in Printed Images ………......................................... 15

2.2 Structure and Characteristics of Printed Images ………................................................ 17

2.3 Image Analysis of Printed Images ................................................................................. 22

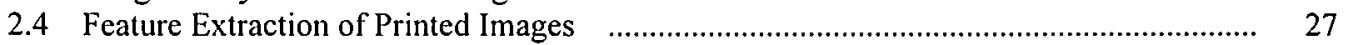

2.5 The Euclidean Distance Transform for Printed Images ……………………………....... 29

2.6 1-D Greyscale Transformations on Printed Images ………………………………….... 44

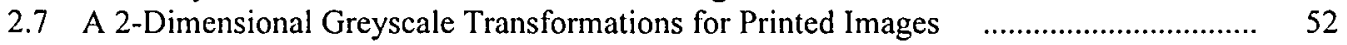

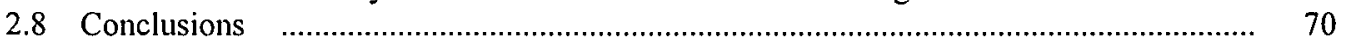

3. The Classification of Textures and Scan-profiles of Printing Defects using 71 Fractal Wavelet Analysis

3.1 Introduction ......................................................................................... 71

3.2 Applicability of Fourier Analysis for Defect Classification ………………………….... 74

3.3 Wavelets, Multi Resolution Analysis and Continuous Wavelet Transform ……........ 85

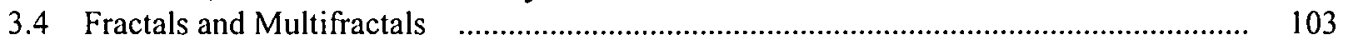

3.5 Wavelet Analysis of 1D-Fractal Functions and MultiFractals …………………....... 111

3.6 Application of Fractal Wavelet Analysis to Defect Identification and Classification $\quad \ldots \quad 116$

3.7 Wavelet Analysis of N-Dimensional Multifractal Sets f............................................... 123

3.8 Perspective on Strong Template-free Defect Classification with Fractal Wavelet
Analysis ................................................................................................ 132

3.9 Conclusions ………………………………………......................................... 134

4. Clustering Methods for Weakly-Separated Many-Parameter Classes 135

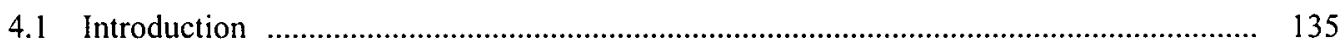

4.2 Representation of Weakly Separated Many-Parameter Classes …………………….... 139

4.3 Survey of Major Approaches to Clustering ………................................................. 140

4.4. Supervised Clustering with Artificial Neural Networks ………………………........... 148

4.5 Partitional Clustering with Fuzzy Class-Membership Functions ................................. 155

4.6 Exploring Data-Sets with Automatic Clustering Techniques ………….......................... 159

4.7 Applications of Clustering Methods in Computational Vision ………………………... 174

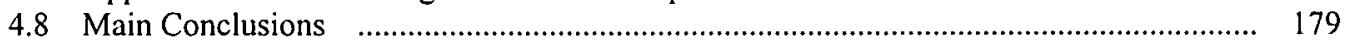




\section{Interactive Classification of Numerous Weakly Separated Many- Parameter Prototypes with Noise}

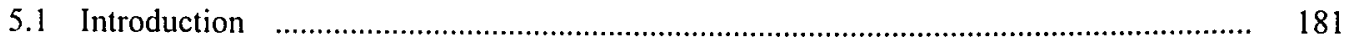

5.2 Parameter Selection by Functional Optimisation …………………………………….... 187

5.3. The Fusion of Heterogeneous Information ……...................................................... 201

5.4. Control of the Reasoning Process ………………..................................................... 206

5.5. IASC: InterActive Sensing and Classification Algorithm .......................................... 209

5.6. Convergence and Stability of the IASC Algorithm ……........................................... 215

5.7. Dynamic Properties of the IASC Algorithm …….................................................... 239

5.8 Main Conclusions regarding Interactive Classification ……..................................... 260

6. Numerical Simulations with the IASC Algorithm 261

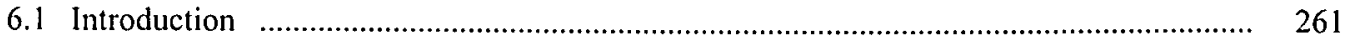

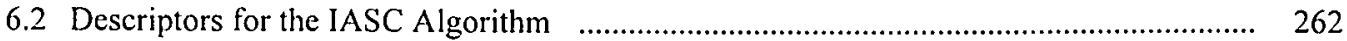

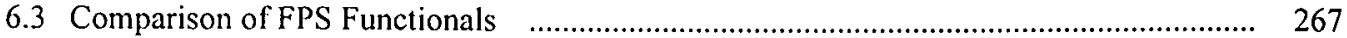

6.4 Comparison of Alpha-Increment Schemes ………...................................................... 277

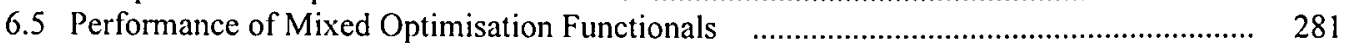

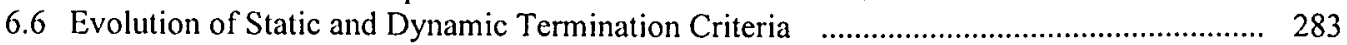

6.7 General Conclusions ....................................................................................... 293

7. On-line Control of Printing Quality Using Computational Vision 295

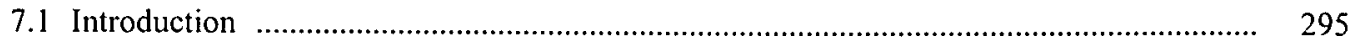

7.2 Intelligent Control of Padprinting Processes ………….................................................... 297

7.3 Defining Features of Padprinting Defects …………............................................... 304

7.4 The IPP-PT1 Approach: Fuzzy Correlations between Scan-features and Defect

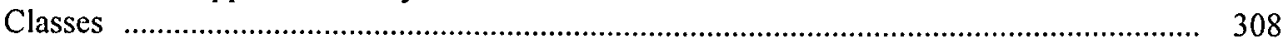

7.5 The IPP-PT2 Approach: Interactive Sensing and Classification ...................................... 317

7.6 General Conclusions regarding the Integrated Approach for Interactive Vision and
Control in the 'IntelPadPrint' Project ….................................................................. 327

\section{Evaluation and Conclusions}

Appendix A. Extracting Features from Printing Defects

A.1 Features Defined by Basic Image Characteristics ........................................................... 339

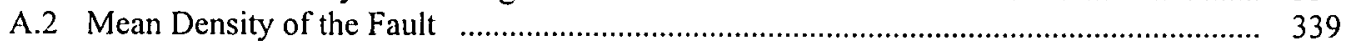

A.3 Feature parameters for Scan Profiles ………….......................................................... 340

A.4 Detecting Oscillatory Signals with 'hairity' ………….............................................. 343

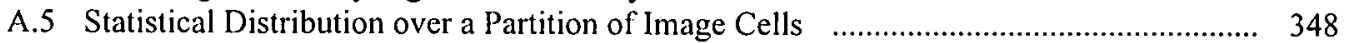

A.6. Identification of Spherical versus Irregular Shapes ……………………………........ 353

A.7 Identification of Rasters versus Irregular Patterns ……………………………......... 361

A.8 Identification of Streaks, Lines and Scratches …….................................................. 364

A.9 Identification of Dirt Particles ................................................................................... 366

Appendix B - Greyscale Transformations $\quad 369$

B.1 CID Transforms Of 18 Printing Defects …………..................................................... 369

B.2 2DI Transforms of 18 Printing Defects …………................................................. 370 
Appendix C - Dynamic Properties of IASC 375

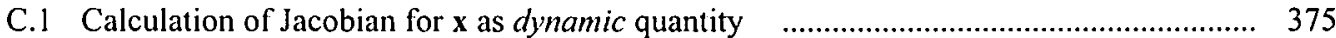

C.2 Simplifications of Jacobian lead to conflicting results for convergence $\quad$....................... 376

Appendix D -Example of Session with IPP-prototypes 377

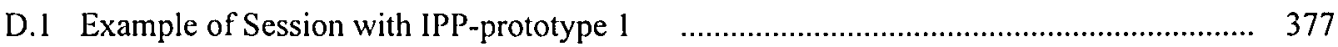

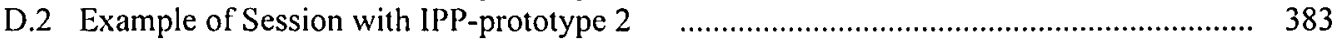

General References $\quad 391$

$\begin{array}{ll}\text { Author Index } & 405\end{array}$

$\begin{array}{ll}\text { Subject Index } & 407\end{array}$

$\begin{array}{ll}\text { Summary } & 413\end{array}$

$\begin{array}{ll}\text { Samenvatting } & 421\end{array}$

$\begin{array}{lr}\text { Curriculum Vitae } & 429\end{array}$ 


\section{Chapter 1}

\section{Introduction}

\subsection{On Computational Vision for Quality Control}

'One picture is worth more than a thousand words". This popular yet classical Chinese proverb vividly expresses human attitude towards the visual sense and its role in cognition. The parallel nature of visual information allows fast and basic processing, as opposed to the sequential and symbolic processing in spoken language. The great ease and skill of human vision creates disbelief when humans are confronted with the difficulties computer vision encounters even in elementary visual tasks like object recognition. Computers, whose computing powers are freely acknowledged to outcompete human capabilities, are not expected to yield to a task that is considered by humans as absolutely elementary. This misunderstanding is primarily caused by a lack of understanding of human vision and the mental processing and interpretation of visual information. Historically, computer vision has pursued an amalgam of distinct approaches towards visual analysis, resulting in a different performance and outcome than human vision.

This thesis is situated in the field of 'computational vision', also called 'machine vision' or 'computer vision'. This is a relatively new field. The first vision systems presented stem from the mid sixties of the previous century [Roberts, 1965], [Guzman, 1968], see [Petrou,2001]. At present, progress in this area seems to be exponential, both on the scientific/technical level as in the number of realised applications. The reasons for this interest are varied. On the one hand prices for hardware are constantly dropping, while simultaneously the performance and speed are continuously increasing, roughly following Moore's Law ${ }^{2}$. Likewise, good general image processing toolboxes are now available that allow efficient software development. On the other hand, there exists a large interest from industrial and governmental institutions. These include high-tech industries like aerospace and military manufacturers, but also lowtech industries like automatic inspection and surveillance. Nonetheless, computational vision has not yet fully matured as a solid scientific area. This expresses itself in the fact that many if not all - applications have an improvised character, and most fully exploit domain-specific

\footnotetext{
'Here 'thousand' represents a number beyond counting, see: Theodora Lau, 'Chinese Proverbs', Harperperennial Library, Boston, 1995.

2 Moore's Law states that the number of transistors on a chip doubles every 18 months as manufacturers find ways to make components ever smaller. It has long been realised that this process can not continue on forever.
} 
characteristics. This last property inhibits the spread of ideas to other areas of application, and the growth of a general theory of machine vision. Or, as one expert in the field once whimsically remarked" : 'The state-of-the-art of machine vision is the subtraction of digital images, and the rest is ingenuity and good luck'. There are numerous developments in machine vision that are difficult to combine under one denominator. On the other hand, there is no progress towards general incorporating theories or methodologies. At present, it looks like there is no need for a 'grand unified theory' of computer vision, and it may as well be non-existent [Haralick, 1986].

The current wave of interest for computational vision fits in well with the contemporary trend in informatics to focus on integrated and embedded sensor-actuator systems of low cognitive complexity. Such machines mimic primary unconsciousness processes, like animal reflexes, rather than higher mental processes, like symbolic reasoning. In a historic perspective, this trend may be a reaction to earlier developments in Artificial Intelligence that fully concentrated on higher cognitive symbolic processing, culminating in the well-known 'expert systems' in the early 1980's'. This field resulted in a surge of interest that, when it eventually eased down, left many of its initial aspirations unfulfilled. The present approach is more modest in its aims, but perhaps more pragmatic in its objectives. In concentrating on feasible systems, it focuses on multifarious, detailed bottom-up processes, rather than the former general top-down approach. In this view, machine vision represents a basic automatic process, in which a (digital) image of a scene is analysed and processed into some sort of diagnosis. Though the interest for the bottom-up approach can be seen as a reaction to earlier developments, the associated field itself is of roughly the same age, and developed alongside its rule-based counterpart. It started in the 1940's with work from McCulloch and Pitts [McCulloch,Pitts, 1943], and later resulted in Rosenblatt's famous 'perceptrons', It further developed in the 'Multilayer Neuronal Networks', Kohonen's 'Self-Organising Feature Maps' and 'Genetic Algorithms'. At present it may be victim to the same wave of enthusiasm that eventually abashed the field of expert systems.

An essential challenge for computer vision concerns its computational complexity. All approaches in computational vision systems suffer from a condition called 'the conundrum of combinatorial complexity', as defined by Perlovsky in [Perlovsky, 1998]. This means that in one stage or another, all these systems experience a combinatorially complex phase. For systems based on a priory knowledge, like Rule Based Systems, this phase expresses itself by an exponential explosion of the complexity of the rule-systems used. For systems based on adaptivity alone, like Artificial Neural Networks, this phase is manifest by the exponential training requirements. Model-based techniques, combining adaptivity and a priority, lead to a combinatorial explosion of the computational complexity. Perlovsky argued that fuzzy and adaptive parametric approaches, like for instance in Gaussian Mixture Decomposition (see section 4.3.g), exhibit the right balance between a-priory form and adaptivity. On the other hand, human intelligence seems to effortless combine these two factors 'automatically'.

The subject of this thesis is the automatic interpretation of printed objects based on visual information. We can and will study such an extensive topic only in a limited and well-defined domain. In our case the main context is the deduction of the physical and chemical causes of a printing defect, based on an appropriate digital image. To reach even this restricted objective requires the development of algorithmic tools from several scientific disciplines. At the basis, it demands an adequate understanding of the physical and chemical principles that govern the printing process. This requires the systematic classification by human domain experts of all

\footnotetext{
${ }^{3}$ Tim King of Birmingham University, personal communication. 1997

"For a contemporary chronology and a feeling of the enthusiasm of that period sec: [Buchanan, 1985]

S See [Pfeifer et al, 1989b]
} 
printing quality and defect classes, together with their visual characteristics and their physical-chemical origins. On the other hand, this knowledge can be used directly for the control of the printing process.

Similarly fundamental, it assumes a thorough comprehension of the structure of printed images. Based on this general structure, suitable methods for image analysis utilise specific characteristics of printed images in order to focus on relevant features defining the quality of a printed object. These methods include transformations of printed images into one standardised geometric form. Quantifying the identified features in an image is the main objective of feature extraction algorithms. These algorithms are domain-dependent in the sense that they have a meaning only within our specific context. Their number relates to the collection of all relevant features discernible in a printed image - literally - in the eyes of human experts. The collection of all specified features together compound the 'feature-space'. Specific areas in feature-space correspond with specific printing defects discernible in an image, because the features were constructed specifically to represent specific defect-properties. An important research topic of this thesis concerns the construction of features that stem from the fractal wavelet analysis of the image. Collectively, image processing and feature extraction can transform a sizeable digital image of a printed object into a considerably smaller subset in a well-defined and structured feature-space. This results into a significant dimension-reduction. The next aspect that demands our attention is the topology of the printing-defect classes in feature-space. This demands for the discovery of significant structures in feature-space. This phrasing is the literal definition of 'Pattern Recognition' by Bezdek and Pal [Bezdek,Pal, 1992]. Important characteristics of the feature-space in our context are its high dimensionality and the high degree of overlap of the clusters representing the different defects. This is an impediment shared by most computational vision applications. As an absolute prerequisite, however, for high-dimensional feature spaces, is the availability of ample empirical data to calibrate the system. Due to the 'curse of dimensionality' (see Section 2.4.2) the number of required empirical data increases exponentially with the number of features. From the pattern analysis it may turn out that some of the printing defects - as defined by human experts - are more similar than others. In other words, hierarchies may become visible in feature-space, and another organisation of printing defects may appear than provided by human experts. To this end suitable automatic clustering algorithms are developed.

Application of our methods to image processing, and the resulting ordering in feature-space, permits a simple method for interpreting a new digital image. First, the image is transformed to a vector in feature-space, and second, this feature-vector is matched to all defect clusters in feature-space. The defect cluster with the best match is then interpreted as representing the most likely printing defect. This process is most efficient when the complete feature-vector is available. In many cases, however, it is not necessary to extract all features from an image, as for most defect-classes there exist idiosyncratic correlations between the feature-parameters. Moreover, significant computation-time can be gained by extracting only the most significant features. This poses the problem of determining the most important feature-parameter that can be extracted under the current circumstances, or terminating the process altogether. The problem of concurrent and interactive classification poses an important research topic in this thesis. Finally, the success of this approach must be evaluated on a real-world problem.

Much of the research in this thesis is performed as part of an international research project, aimed at the development of autonomous, flexible, and 'intelligent' printing robots. The project was called 'IntelPadPrint', and funded under a European Union scientific programme. It was a cooperation between six industrial and scientific partners from four European nations, performed in the course from late 1996 to early 2000. After the end of the project one additional year was devoted to lay the theoretical foundation for this thesis. 
Before discussing the structure of this thesis, we will, for a better understanding, put our research in a wider perspective. First, we discuss the fast-developing area of industrial computational vision. Second, we briefly compare human vision with computational vision. Next, we summarise the main innovative aspects of our research. Finally then, we address the composition of this thesis.

\subsection{Computational Vision Control in Industrial Processes}

The last decade has witnessed an unprecedented explosion of patents for industrial applications of computer vision. Figure 1.1 shows the increase in awarded European patents in the last 15 years. The reasons for this increase were mentioned before; more powerful, efficient, and cheaper hardware, more general applicable software, and increasing competitive pressure. Most of the developed systems, however, remain close to the level of 'image processing'. 'Image processing' is aimed predominantly at converting an image for later human inspection. 'Computer vision', on the other hand, refers to the automated extraction of information from an image. At present, most systems explicitly compare an observed image with an image of the required situation. Additionally, they extensively exploit domaindependent characteristics. A typical example is the automatic detection of missing parts in assembly packages ${ }^{6}$. Moreover, computer vision is becoming increasingly important for the automatic assessment of product quality in industrial manufacturing. It offers a reliable approach for visual inspection with constant performance under varying circumstances.

Given the enormous size of the literature in this area, it is extremely difficult to obtain an objective view on the state-of-the-art of the industrial applications (See for instance: [Christensen et al,1997], [Courtney et al,1997], [Firschein et al,1993], [Ho et al,1997], [Price, 1986]). This would require a set of objective evaluation criteria for such applications. Various authors have proposed methods that would allow a comparative assessment of computer vision algorithms [Haralick,1986], [Pavlidis, 1992]. According to Bowyer and Philips [Bowyer et al,1998], [Philips,Bowyer,1999], the benefits of such methods would include (1) placing computer vision on a solid experimental and scientific ground, (2) assisting in the development of engineering solutions to practical problems, (3) allowing for accurate assessment of the state of the art, and (4) providing convincing evidence to potential users that computer vision research has indeed found a practical solution to their problems.

\footnotetext{
${ }^{6}$ Another example is military object recognition, see for instance: [Ratches et al.,1997].
} 


\begin{tabular}{|c|c|}
\hline year & $\begin{array}{c}\text { number } \\
\text { of patents }\end{array}$ \\
\hline 1985 & 5 \\
\hline 1986 & 6 \\
\hline 1987 & 16 \\
\hline 1988 & 19 \\
\hline 1989 & 29 \\
\hline 1990 & 26 \\
\hline 1991 & 26 \\
\hline 1992 & 33 \\
\hline 1993 & 40 \\
\hline 1994 & 39 \\
\hline 1995 & 43 \\
\hline 1996 & 63 \\
\hline 1997 & 70 \\
\hline 1998 & 108 \\
\hline 1999 & $87^{*}$ \\
\hline 2000 & $87^{*}$ \\
\hline Total & $697^{*}$ \\
\hline
\end{tabular}

*) not all submissions processed

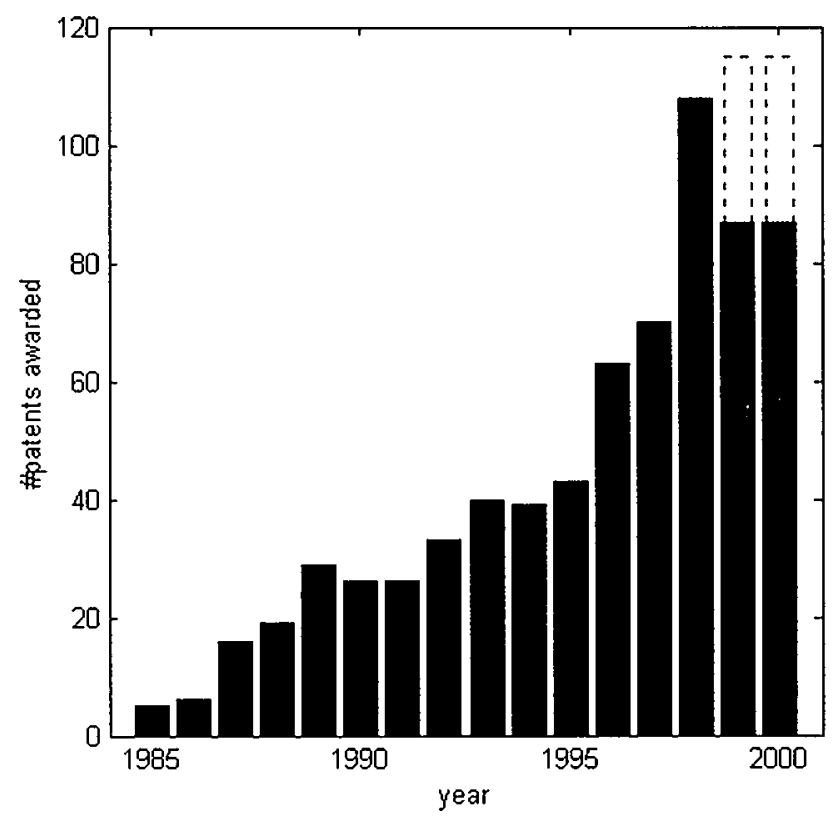

Fig. 1.1. Growth of awarded European patents tor computer/machine vision since 1985. Source: Ihe European Patent Office, 2001

In this thesis we study the application of computational vision to quality control of industrially printed decorations. Quality control is at present mostly performed by human visual inspection, sometimes with the aid of some automated tools. This work is typically performed at a conveyor belt, and has a temporarily and hired character. However, this type of visual inspection is subject to large variations in skill from person to person, between age and gender groups, or for one person throughout a working day, and throughout the seasons of a year. In some industrial areas, like for instance in steel and paper industries, visual inspection predominantly relates to the high-resolution analysis of textures and their statistical properties. The resulting quantities are used to compute physical and chemical characteristics that define the quality of the product. Such an approach is called 'Statistical Pattern Recognition', see: [Jain et al.,2000]. This work often takes place in a laboratory setting and has a specialised scientific character. However, also in this case there is a lack of standardisation and automation.

From the industrial point of view there is a strong interest in the application of automated, reliable, and low-cost systems for visual inspection. At present, such systems are costintensive, rather unstable and low-performance. Skilled humans easily outperform such automated systems. Another major problem of present automated visual inspection systems is their absolute lack of flexibility, which causes large investments for relative little changes. For each new variety of product these systems require elaborate reconfiguration. In that case the system has to be fed with a large number of elaborate descriptions, such as CAD/CAM designs, and a mass of details relating to the product, and the production setting. This fact seriously counters attempts to improve production efficiency by making manufacturing more flexible. However, industries remain largely interested in the further development of computer vision because automated or computer aided visual inspection has the potential for a more stable and robust performance. In that respect it would be more uniform and eventually also more reliable than a human operator. 


\subsection{Computational Vision Inspired by Human Vision}

It is illustrative to compare the current state of the art of computational vision with the visualcognitive abilities of human beings. For outsiders, the first and most striking impression created by machine vision is its weak performance in comparison with human achievement. Humans are visual experts equipped with excellent physiological sensors and a complex, hitherto not fully understood cognitive apparatus to process this information. For instance, humans readily acquire the skills required to identify and classify slight variations and deviations of a given image, and easily outperform current computer vision algorithms. Despite certain limitations, such as 'change-blindness' - the incapability to detect slow or minute changes in a scene - human vision is extremely agile at invariant recognition tasks. However, the human recognition process strongly relies on context, knowledge, and experience [Jain et $a l ., 2000$ ]. The ability to exploit contextual information in resolving ambiguity and recognising complex patterns is amongst the major distinctions between human and machine vision.

Three aspects of human vision are specifically relevant for our context of print defect classification:

\section{Active Vision}

Human vision is understood as an active, dynamic and parallel process [Hildreth, 1998]. Images are processed in parallel as complexes of characteristic features, the 'Gestalts' [Pelah,1997]. Visual attention is the process of confining high-level neuronal processing to a mere part of the entire visual field, called the Focus-of-Attention (FOA). The FOA directs the search for specific features, based on current beliefs and expectations. E.O. Postma [Postma, 1994] constructed a model for covert attention, based on ART-neural networks, that emulates this behaviour of the FOA. Palmer [Palmer,1997] has suggested that visual attention is the process of locating the object of interest, and positioning it in an object-centred reference frame, suitable for recognition by template matching. $B$. Moghaddam and A. Pentland [Moghaddam,Pentland,1997] have developed an efficient computational technique for automatic object recognition, in accordance with Palmer's model of visual attention. In a similar fashion, H.D. Tagare et al. [Tagare et al.,2001] were inspired by the FOA in devising a maximum likelihood strategy for directing attention during visual search. The results of the FOA search iteratively generate new beliefs, creating a process that quickly converges to a result: the interpretation of the image. Therefore, human vision is an interactive and parallel process between sensing and (unconscious) cognition.

\section{Invariant Recognition}

Another notable capacity of human vision lies in its ability of invariant recognition. In the foremost place, this refers to the ability to recognise objects, irrespective of their orientation and perspective. Moreover, invariant recognition refers to the identification of implicitly defined patterns, such as artificial and natural textures. The basis for this ability lies in deep, fundamental processing of the visual information, rather than in specific cognitive processes. Just like in the interactive vision mentioned above, humans readily outcompete computer vision systems in invariant recognition. Pioneering work in this respect has been done by A.P. Pentland [Pentland,1986] in providing a model for the perceptual organisation and representation of natural forms. 


\section{Autonomous Sensing}

In human vision, the eye acts as an active and autonomous sensor rather than the passive registration-devices, like CCD-cameras, used in computational vision. Much of the visual preprocesing occurs directly in the eye itself, such as accommodation and autofocussing. These abilities inspired several publications. M. Subbarao and J.Q. Tyan [Subbarao,Tyan, 1998] compute the optimal value for autofocussing and depth-from-focus from a biologically inspired model. Leung et al. [Leung et al.,2000] obtain a robust hierarchical clustering algorithm by modelling the scale-space blurring effect of the lateral retinal interconnections. Using a different biological metaphor, S. Hati and S. Sengupta [Hati,Sengupta,2001], estimate camera parameters using a genetic algorithm.

Computational vision algorithms, on the other hand, mostly engage in a series of distinct techniques like template matching, optimisation, feature extraction, Fourier or wavelet analysis, and correlation functions. Information is processed sequentially as a fixed inputoutput system, for normally there are no interactions or feedback loops between the output and the input. This combination yields static systems, complex to maintain and develop, which cannot compete with human vision. Numerous approaches in computational vision have been directly inspired by the efficiency of human vision, like the examples mentioned before: [Pentland, 1986], [Postma, 1994], [Moghaddam,Pentland, 1997], [Subbarao,Tyan, 1998], [Leung et al.,2000], [Tagare et al.,2001].

In our research, the three aspects of human vision described above inspired the following developments:

1. the modelling of print defect identification as an interactive vision process.

2. mimicking the human ability for invariant texture identification by applying multifractal wavelet analysis.

3. the development and application of an intelligent camera system, that can automatically focus and zoom-in on specified parts of the object, and automatically perform basic image processing tasks, like finding the orientation and adjusting the illumination of the image. This work is performed by our partner in the research, the department of Electrical and Mechanical Engineering of the University of Birmingham, and is not further described in this thesis, see: [Shippen et al.,1999] and [Freear et al., 1999].

\subsection{Original and Realised Research Objectives}

The research in this thesis is performed as part of an international research project. The prime objective of this project concerned the realisation of a fast, flexible, autonomous, and robust real-time system for the control of print quality in industrial mass-production. This objective demanded the integration of varied information, stemming from numerous distinct sensors, such as measurements on viscosities, temperatures, mechanical pressures, as well as entire images from a digital still-camera. Using process knowledge, this information should somehow be translated into a proper control action.

However, two additional insights notably redefine the original objective. First, since print quality is defined by human visual evaluation [Chan,1998], [Kaplanova,Fribert, 1998], the most relevant sensor for establishing post-printing quality is a digital camera. Second, as an overwhelming knowledge of the underlying physical-chemical processes became available, it became possible to infer the physical-chemical cause of a printing defect directly from its 
correct classification. This cause directly defines the most suited control-action. Thus, it is possible to directly utilise the proper classification of the actual realised printing quality for the control of the printing process. This means that we can formulate the main objective of our research as follows.

The development of a framework for real-time classification of print quality, based on the analysis of images of the printed objects, integrated with other multi-sensor information.

In practice, this goal had to be disseminated in various sub-goals. These sub-goals correspond to the topics described in this thesis. Three relevant sub-goals can be distinguished.

1. Provide a suitable framework for the image analysis of printed objects, that makes it independent of the template applied ${ }^{7}$. This topic is covered by Chapters 2 and 3 .

2. Find a representation of print quality and printing defects that allows for wellseparated partitioning. This topic is covered by Chapter 4 .

3. Develop a framework for fast real-time classification of print quality, utilising all available sensor information. This topic is covered by Chapters 5 and 6 .

4. Construct prototypes based on these frameworks, and test them in realistic conditions. This topic is described in Chapter 7.

\subsection{Innovative Aspects of the Research}

We will now describe the original and innovative aspects of our research..

\section{Interactive Vision}

An important inspiration for our research is the interactive vision process exhibited by human vision. Here, 'interactive' refers to the interaction between basic perceptual tasks and cerebral processing and control, displayed by the brain in controlling the focus-of-attention during visual attention. By this motivation, in our research printing defect identification is modelled as an interactive vision process. This means that our system principally operates on two levels.

1. low-level perceptual tasks, like automatically concentrating on specified parts of the observed object, and basic image processing tasks such as extracting textures or intensity profiles from suitable segments and scan-lines on the object.

2. high-level processing, like computing the most-likely hypotheses regarding the state of the object (e.g. its quality class) from the available sensor-information. From these hypotheses to construct expectations, and subsequently to define the most relevant locations on the object, and the most informative perceptual tasks to be performed, such as generating suitable scan-lines or segments on the object, and managing the global exploration of the object.

The interaction between these two levels thus consists of where to search on the object, and what feature to look for, based on the current hypotheses and expectations. This interaction gives rise to a computational implementation of the focus-of-attention. The high-level

\footnotetext{
${ }^{7}$ In the context of printed images a 'template' is the image intended for printing.
} 
processing can be interpreted as a reasoning process. This reasoning process is depicted in Figure 1.2. The 'interactive sensing' in this scheme corresponds to the low-level perceptual tasks, and is depicted in detail in Figure 1.3. Notice that the 'reasoning process' depicted in Figure 1.2 is more general than the 'interactive sensing process' shown in Figure 1.3. This form of reasoning process can also be applied to medical diagnosis or chemical analysis. In that sense, the 'interactive image analysis' depicted in Figure 1.3, is merely a particular instance of the general 'interactive sensing' in Figure 1.2.

To implement real-time print quality classification as an interactive vision process constitutes a new and original approach to on-line image analysis. This approach is worked out in detail in Chapter 5, and its evaluation in Chapters 6 and 7.

Our research focussed on this interaction, and the eventual quality diagnosis and subsequent printer control. Similarly important is the role of the camera. As mentioned before, our partner in the research, the department of Electrical and Mechanical Engineering of the University of Birmingham, devised an intelligent camera system.

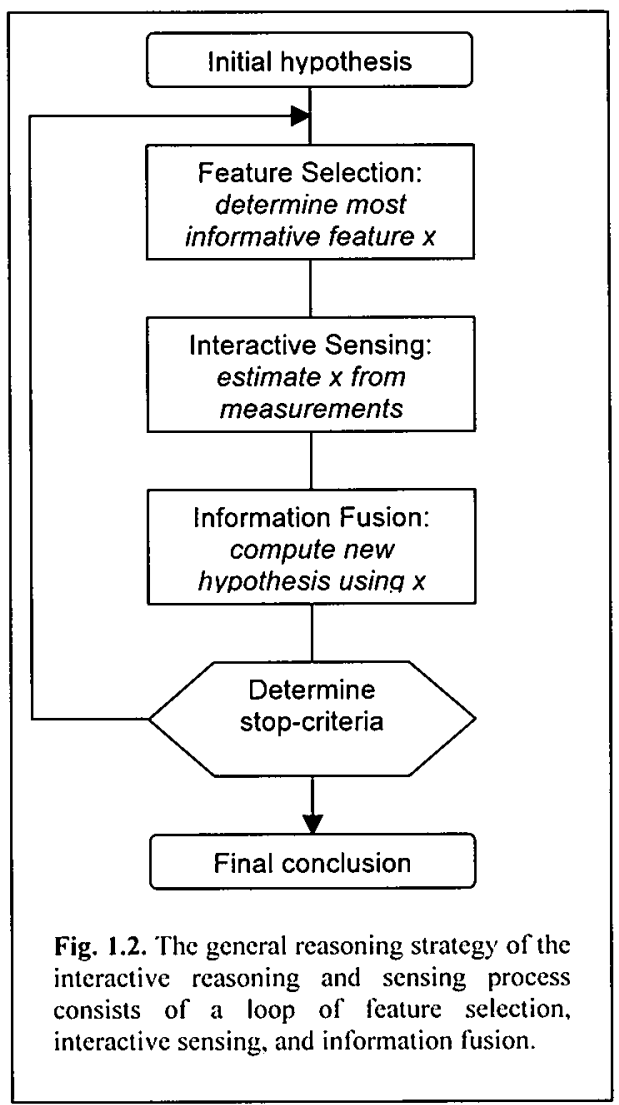




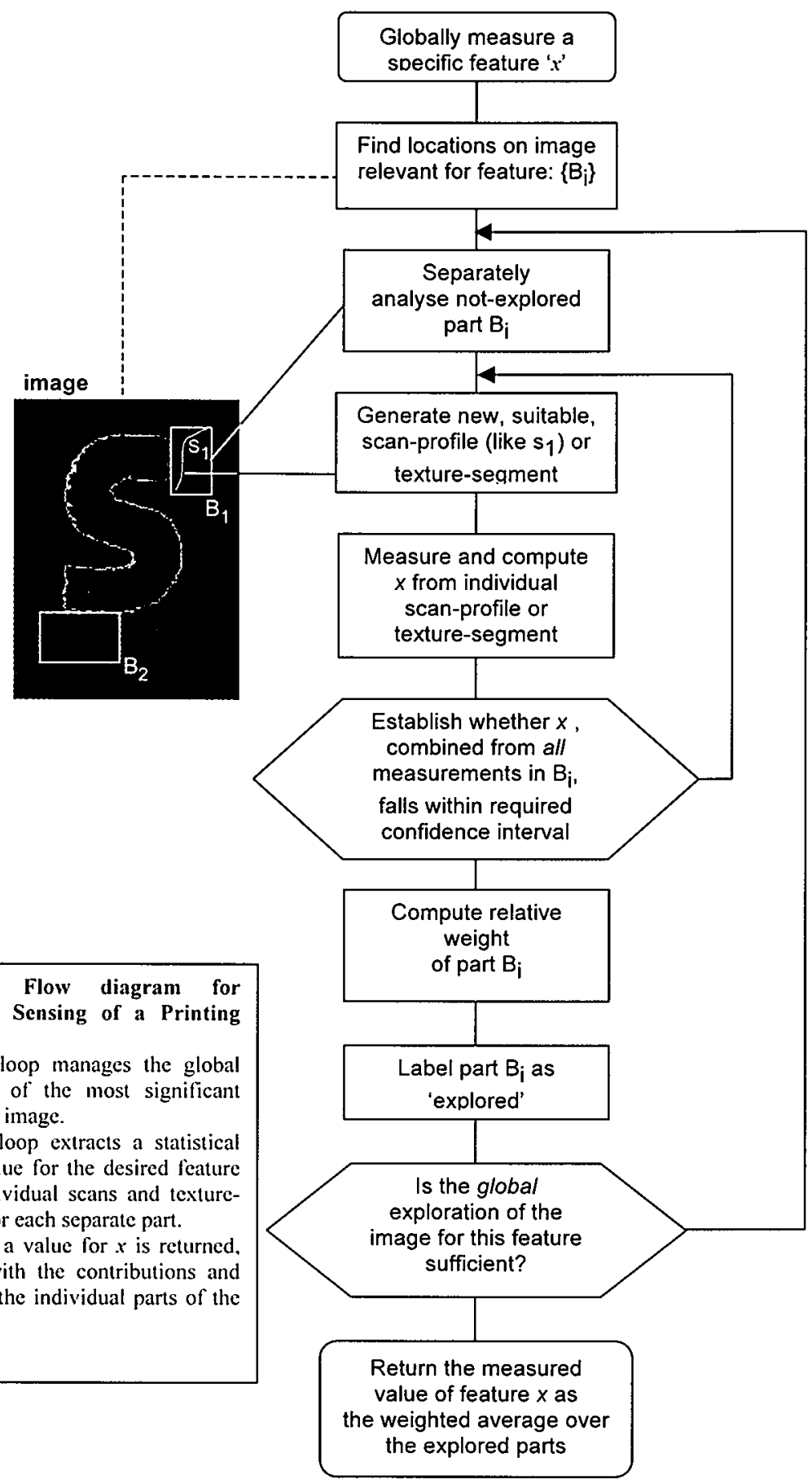

Fig. 1.3. Flow diagram for Interactive Sensing of a Printing Defect.

The outer loop manages the global exploration of the most significant parts on the image.

The inner loop extracts a statistical relevant value for the desired feature $x$ from individual scans and texturesegments for each separate part.

Ultimately, a value for $x$ is returned. averaged with the contributions and weights of the individual parts of the image.
Return the measured

value of feature $x$ as

the explored parts 


\section{Geometrical Intensity Transformations}

Printing defect analysis appears to depend heavily on the template applied in the printing process. This would prevent a more general approach. For this reason, we concentrate on representations that eliminate the influence of the template as much as possible. This leads to the construction of geometrical transformations that map any arbitrary printed image into a uniform 1D- or 2D-representation. This representation can be used for further templateindependent processing. Figure 1.4 shows an example. This method is described in Chapter 2.
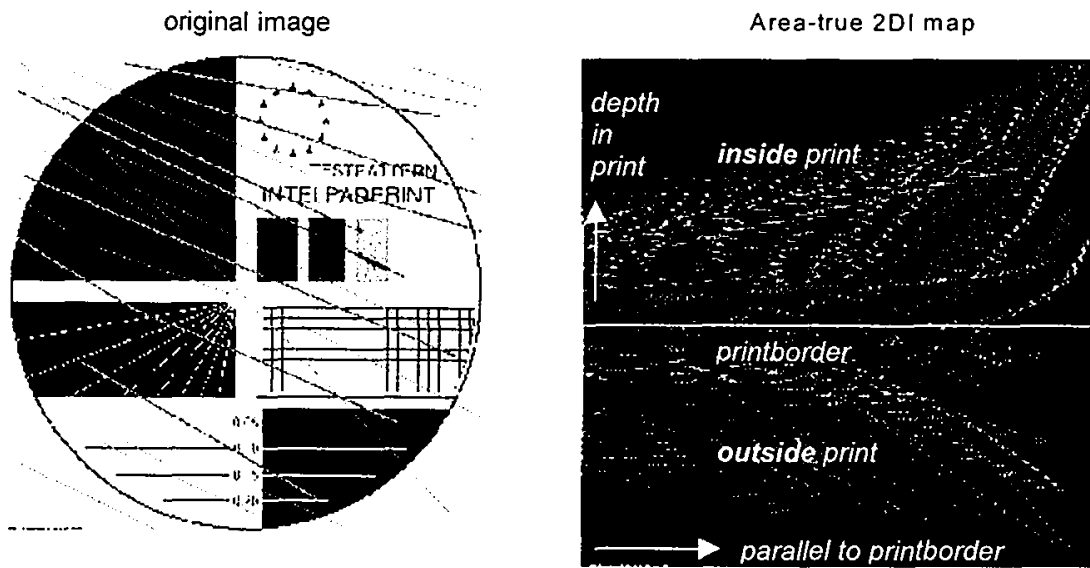

Fig. 1.4. Left: digital image of defect called: streaks of lines, right: congruent 2Dl mapping of the faults visible in the image.

\section{Multifractal Wavelet Analysis for Print Defect Classification}

In the last five years wavelet analysis has been applied for the analysis of multifractal sets. Recently, this formalism has also been successfully utilised for the classification of artificial
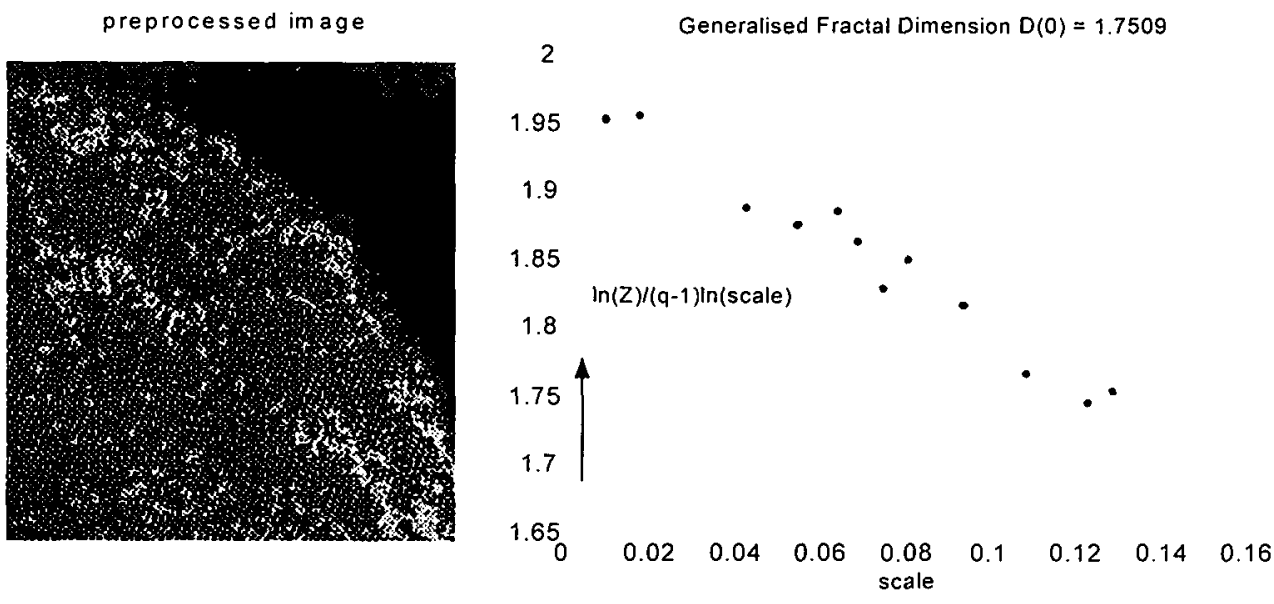

Fig. 1.5 Calculation of the Multifractal properties of a printing defect using the Continwous Wavelet Transform. Left: pre-processed image of a printing defect, right: dependence of the macro-canonical ensemble function with the scale, based upon the so-called Wavelet Transform Modulus-Maxima Method. determining the principal generalised multi-fractal dimensions of this image. From: [Westra,2000a]. 
and real textures. In our approach, we consider printing defects as textures, and we apply a method called the Wavelet-Transform Modulus-Maxima Method for their analysis and classification. This approach allows for a strong method for printing defect identification and classification, without any prior knowledge of the template of the print. The attractive side of this new approach is that it is successful in invariant recognition, and moreover, with the help of clustering and classification techniques, is capable to efficiently 'learn' new texture patterns - ergo, new types of printing defects. It also opens a perspective for estimating the parameters from the underlying physical-chemical processes, that ultimately determine the print quality.

\section{Automatic Fuzzy Cluster Analysis using Gaussian Membership Functions}

A new method for automatic fuzzy cluster analysis, called iterated $F M V$, based on multivariate Gaussian membership functions. With this method faster, yet equally reliable clustering results can be obtained, compared with conventional clustering methods like Fuzzy $C$-means $(F C M)$. Consequently, $F M V$ enables automatic and unsupervised clustering of the large data-sets of multi-dimensional, weakly separated clusters, that are typical for our context.
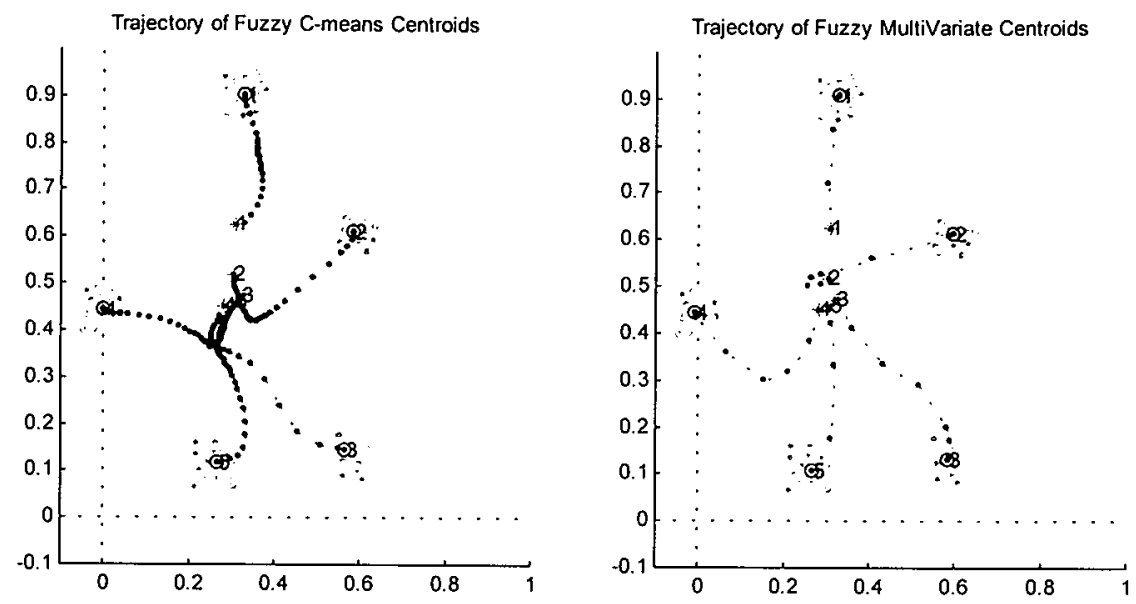

Fig. 1.6. Typical FMV and FCM sessions for the same data set consisting of 5 separated clusters, showing the smaller number of iterations and the comparable end result of $F M V$. Left: trajectories of the FCM-prototypes, right: likewise for the $F M V$-prototypes. 


\subsection{Guide for Reading this Thesis}

As it is our intent in this thesis to describe a current state-of-the-art automatic visual inspection system, we shall highlight all relevant contributions from the scientific fields mentioned in Section 1.1 in more detail. This inevitably implicates that several different disciplines are addressed, whose relations only follow from their eventual integration in our context. This non-relatedness is reflected in the composition of this thesis.

Chapter 2 is devoted to the analysis of printed images. Here, we focus on the object of our analysis; digital images of printed objects. We restrict the infinite range of textures and patterns that can be printed by providing a pragmatic definition of 'printed image', as treated in this thesis. We discuss the relevant characteristics and structures defining such printed images. With an eye to the later integration in an interactive classification system, we discuss the analysis of printed images using scanning in some detail. Finally, much attention is devoted to original work [Westra,1999d] on geometrical transformations that map arbitrary printed images into a explicit ID- or 2D-representation. This leads to an explicit representation of any printed image that can be used for template-independent processing.

In Chapter 3 printing defects are characterised as multifractal textures. Next, a method, based on the analysis of multifractals using the continuous wavelet transform, is applied. Thus, a representation of the defect in terms of its fractal texture properties is obtained. This is valid both for the fractal properties of $1 \mathrm{D}$-scans as of 2D-textures. This chapter describes original work in the application of fractal wavelet analysis to defect classification [Westra,2000a].

In Chapter 4 fuzzy clustering is applied to reveal structure in the representation space, and thus reduce its dimensionality. In this chapter original work is presented in an algorithm for automatic fuzzy cluster analysis, based on Gaussian membership functions [Westra,2000b]. This approach is especially applicable to high-dimensional feature spaces with weakly separated clusters. This part can be read independent of the rest of the thesis. In this chapter we also describe the application of computational neuronal networks for mapping the obtained representation to a fuzzy membership function of the defect. Finally, we sketch how the identification of a printing defect can be used for the direct control of the printing process.

Chapter 5 represents the core of the thesis. It concerns interactive classification and sensing, given a high-dimensional feature space with numerous weakly separated clusters. The central question in this chapter is whether a new feature needs to be extracted, and if so, which feature-parameter would be most proficient given the currently acquired information. To this end, first a suitable symmetric normalised metric is proposed that complements the nonsymmetric Mahalanobis-distance. Second, utilising this metric, a dynamic algorithm is proposed for Functional Parameter Selection, called FPS, in which the topology of featurespace is combined with the current set of hypothesis to generate the best feature-parameter to be examined next. Third, the fusion with other kinds of (sensor) information is considered. Next, the FPS-approach is integrated in a formalism for InterActive Sensing and Classification, called IASC. Finally, much effort is devoted to the convergence, stability, and dynamic properties of the IASC-algorithm. The whole approach can be considered as a reasoning process for interactive vision, mimicking to some extent the focus of attention in human vision. Using IASC, the image analysis approach becomes active vision. This chapter is based entirely on original work [Westra,2000b]. It is also independent of the rest of the thesis, as it is likewise applicable for on-line diagnosis in chemical analysis or medical examination. 
In Chapter 6 we present a number of numerical experiments with the IASC-approach on theoretical problems. This provides a deeper understanding of the IASC-algorithm, and its dependence on noise in the input, and the degree of cluster-overlap in feature-space. Also, the effect of the termination-conditions is studied, leading to the assessment of optimal values for the IASC-parameters for a given noise-level and cluster-overlap.

In Chapter 7 we discuss the application of IASC-approach on a real industrial problem. The area of this application is on-line visual quality control of industrial padprinting processes. The application serves as a test-bed for the consistency of the IASC-method, and its evaluation in an intricate and demanding environment. In this chapter our main results from the European research project 'IntelPadPrint' are presented. In this course two prototype computational vision control systems are discussed that show the results of the IASCapproach in a demanding real-world setting.

We conclude this thesis with a separate section on the major conclusions, some final remarks, and an evaluation of the possible output of this study.

Appendix $A$ is devoted to the subject of feature extraction in the specific field of padprintingdefects. In this appendix, special-purpose algorithms are described, that were specifically constructed for the quantification of padprinting-defect features in the form of fuzzy membership-functions. This information is not included in the main text, as it would by its sheer size divert too much from the main subject. However, for a thorough understanding of the integrative approach it is inevitable, because the quality of feature extraction determines the efficiency of the subsequent clustering and classification of an image. 


\section{Chapter 2}

\section{The Analysis of Printed Images}

\subsection{Introduction: Defining Structure in Printed Images}

In this chapter we provide the basic terminology for the specification of printing defects. In order to do so, we concentrate on the primary object under study: digital images of printed elements.

First, we need to emphasise the practical distinction between the actual printed element and the digital image thereof - in our case stemming from a CCD-camera system. Trivial as this seems, the resulting digital image differs so much in quality from the original, that even trained domain experts have problems in identifying the defining - but subtle - traits of printing defects, so well visible on the original object for the naked eye. During the imaging and subsequent processing, a significant amount of the original information can disappear, and other undesired effects can be superimposed on the signal. Information can be lost due to optical processes like absorption in the lens, insufficient photon energy for triggering the photo-electric effect on the CCD-cell, or because it is thresholded away in the subsequent hardware or software processing. Unwanted effects can be added in the form of noise, ambient light, other lighting effects, optical effects in the lens like spherical aberration, electrokinetic effects of the $C C D$, effects in the frame grabber, or effects in the image preprocessing software (rotation, scaling, translation). We represent a digital image as a finite collection of greyscale values, defined on a compact domain, with a calibrated axial position, direction, and scaling relative to the printed element. In the following section, we provide a more precise definition of 'image'. Moreover, in order to compare colours and greyscales, we fixate the digital output intensity relative to the desired output. For this reason we make another basic observation: the distinction between the desired output and the actual output. The desired output is defined by the pattern intended for printing on the element. In current decoration practices, this pattern is mostly designed as a bitmap by a design-engineer using some suitable computer program. Next, this bitmap is etched automatically on a cliché. This cliché is used in the printing process. This basic image on the cliché containing the pattern to be printed (in negative form) is called the template. It is this template that is the gauge for the actually printed image. Print quality is defined as the relative and absolute 
difference between the digital image of the actual print and its associated template. This definition enables a workable approach for automatic vision quality control. Let the absolute cumulative difference be bound by a threshold, defined in advance by the product designer. If this threshold is exceeded, the print quality of the element is regarded as insufficient and the element is rejected.

In order to use the information contained in the relative distribution of the difference over the image, it is important to classify the set of possible locations on an image. Consequently, we identify four basic PrintContexts. In order to be general applicable, the analysis of a digital image must be performed in a manner that does not rely on the template. We call such an approach template-free. We make a distinction between a strong and a weak template-free approach. A strong template-free approach does not require any information from the template at all. In the weak template-free approach at least some information of the template is required - mostly during its preprocessing. An example of a weak template-free approach is the subtraction of an image with its template. The resulting difference highlights the actual printing defect. On the other hand, human operators are - to a large extent - capable of performing strong template-free defect identification: Most domain experts are able to identify the correct printing defect class, without knowing the template used.

The most central observation in our context is that a printing defect can be classified into a limited number of printing defect classes, according to distinctive characteristic variations between the image and its template. Essential therein is the spatial distribution of the defect over the image and the PrintContexts. An analysis of this distribution enables a template-independent representation of the printing defect. By its nature, the collection of printing defects are fuzzy sets. Therefore, we introduce membership functions that define to what degree an observed defect corresponds to a specific printing defect class.

An important element in the image analysis is the scanning of the image at some characteristic points. Scanning here is defined as the variation in greyscale, according to a curve drawn at a certain location on the image. Therefore, besides the four PrintContexts previously defined, a set of five characteristic types of curves is defined, called scan curve topologies. However, in scanning the image into a collection of 1D-scanprofiles, much of the original 2D-information is lost. Therefore, at its best, it can only be a part of the entire image analysis strategy. Examples of the complete analysis of an image in a way that focuses on faulty parts of the image are provided in Chapter 7 in the context of real-world applications of our approach.

Next, we must identify, and consequently quantify and qualify, the characteristic features that define the printing defect classes. This is an activity closely connected to the area of application. For this reason, this subject is treated separately in a separate appendix, appendix A, where we treat this subject in extenso in a real practical application.

In the final sections of this chapter, we extent the notion of the qualitative PrintContext to a more general and quantitative gauge on printed images. This gauge can be consequently employed to transform any digital image of a print into a explicit $1 \mathrm{D}$ - or 2D-representation. Such a representation allows a uniform and templateindependent approach to printing defect classification.

Thanks to the general and template-independent description of printing defects in later chapters we can concentrate on clustering and classification techniques without being concerned by the original context of printing defects, and thus be independent of this specific application. 


\subsection{Structure and Characteristics of Printed Images}

\subsubsection{Digital Images and Templates}

First, we define the basic concept of an image.

\section{Digital Images}

With a digital image we mean the discrete representation of a printed surface produced by a digital still-camera'. In our context, the colour in an image can be decomposed in three basic greyscale values Red, Green and Blue - the RGB values. Therefore, coloured images can be considered as composed of three separate greyscale images. For that reason, in the remainder we need only to consider greyscale images. Therefore, we define a digital image of size $N_{1} \times N_{2}$ as a spatial greyscale pattern, represented by a $N_{1} \times N_{2}$ matrix $P$. The coordinates $\left(x_{1}, x_{2}\right) \in\left\{1, \ldots, N_{1}\right\} \times$ $\left\{1, \ldots, N_{2}\right\}$ of $P$ correspond to the so-called pixels of the image. The value of matrix $P\left[x_{1}, x_{2}\right]$ lies in the interval $[0,1]$, and corresponds to the greyscale of the associated CCD-cell on the camera. Normally, there are 256 greyscales defined.

\section{Original Decorations and Continuous Images}

In many of the situations that we encounter in this thesis, it is more practical to employ a continuous representation of an image. This is for instance the case when we can apply differential and integral operators later in this chapter, or in the framework of 'Multi Resolution Analysis' and fractal decomposition (Chapter 3). Moreover, we are ultimately only interested in the real, continuous decoration on the printed object, and the CCD-image is merely a discrete approximation of this condition. In the continuous case, images are defined as mappings $f: \mathbb{I}^{2} \rightarrow \mathbb{R}$ with continuous $\operatorname{domain}(f) \equiv \Omega=\left[1, N_{1}\right] \times\left[1, N_{2}\right]$. For all $\mathbf{x}=\left(x_{1}, x_{2}\right) \in \Omega: f(\mathbf{x}) \in[0,1]$, corresponding to the local greyscale of the decoration. Here we assume that $f$ is a faithful representation of the printed element, and that the scale-difference between the image and the actual print can be represented by an isotropic scaling matrix $\sigma \mathrm{I}$, where $\sigma$ is the scaling in $\mathrm{mm} / \mathrm{pixel}$, and I the identity matrix as: $\mathbf{x}^{\prime}=\sigma \mathrm{I} \mathbf{x}$. In the rest we assume $\sigma=1$, due to good calibration. In the remainder, we always use the continuous representation, even in the case when we speak of a 'digital' image. The 'translation' of the results thus obtained to a discrete framework is straightforward, for instance by averaging over the lattice-cells, corresponding to the CCD-cells of the camera. In this way integrals become summations, and so forth.

\section{The Template of the Print}

A special case is the pattern designed for the print. This pattern is called the template and is present as negative image on the cliche of the printing device. The pattern associated with the template is denoted by $T$. The template $T$ defines an oriented coordinate system and gauge on the image, and therefore on the element. This coordinate system is called the principal template gauge (PTG). In the remainder of the text it is always implicitly assumed that actual digital images are gauged and oriented according to this primary coordinate system PTG. Situations, where the alignment of the CCD-camera-framegrabber-system fails to align with the PTG are

\footnotetext{
' In our context that will always be a CCD digital-camera.
} 
called mismatches ${ }^{2}$. This can be caused by a translation, rotation, incorrect scaling, or by a tilt of the focal plane of the camera relative to the surface of the element.

\section{The Print-Border}

In the remainder of this thesis we only consider greyscale maps where a definite border can be identified. This border separates the printed area from not-printed area, and therefore we call this the print-border.

\subsubsection{PrintContext: A Classification of Areas on an Printed Element}

Above we assumed that images can be represented as continuous mappings $f$. Now we look how we can describe and analyse printed areas. For the sake of simplicity, we assume in the following that not-printed areas have intensity zero, and printed areas have a non-zero intensity. In other words, we assume the print to be white-on-black. However, in the illustrations we show examples of both black/white and white/black. Of course, black/white printing can be mapped to this state by a simple linear transformation: $f \leftarrow 1-f$.

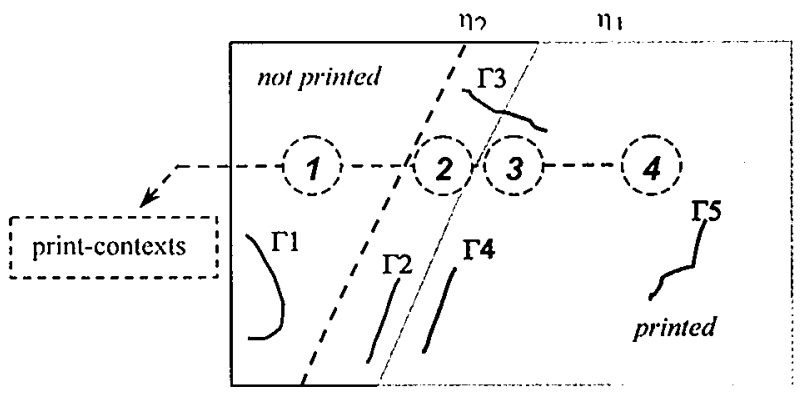

Fig. 2.1 Typology of PrintContexts and scan curves.

Consider a compact area $A \subset \Omega$. Let $f$ be the continuous representation of an image with domain $\Omega$. Principally, we distinguish only two different types of area; printed areas, with $f(\mathbf{x})>0$, and not-printed areas, with $f(\mathbf{x})=0^{3}$. This allows us to transform all possible greyscale-images to black-and-white images, an operation called thresholding. Area $A$ represents the 'shape' of the print if for all $\mathbf{x} \in \mathrm{A}: f(\mathbf{x})>0$, and all $\mathbf{x} \notin \mathrm{A}: f(\mathbf{x})=0$. The print-border is the border of the printed area $A$, denoted $\partial A$. We employ standard mathematical notation, such as $\bar{A}$ for the closure of $A$, and $A^{*}=$ $A \backslash \partial A$ for the interior of a $A$. The exterior of $A$ is the set of points $\Omega \backslash \bar{A}$. Partitioning algorithms are transformations that decompose a disconnected compact area $A$ into a union: $A=\cup_{k} A_{k}$, where $A_{k}$ is a disjoined connected compact area.

In many situations, printing defects are characterised by phenomena occurring near the print border. The problem is what near in our context means. The concept near derives from the average boundary-distance of known near-border phenomena. The value for the distinction near versus far can be determined empirically to be about 0.5

\footnotetext{
' This should be distinguished from a printing defect class called 'mismatch' where the print is placed on a wrong location on the element.

'In practice there is a lower limit $\varepsilon$ to the greyscale due to discretisation, and the definition of a printed area can be reformulated as: $\{x \in \Omega \mid f(x) \geq \varepsilon\}$.
} 
$\mathrm{mm}$. Here, we assume that there are two such values; one inside the print and one outside the print. Thus, we come to four essential types of location on an area. We call these types the PrintContexts. The four PrintContexts are depicted in Figure 2.1. Table 2.1 provides a definition in terms of two parameters $\eta_{1}$ and $\eta_{2}$.

\begin{tabular}{|c|l|c|}
\hline PC & Description & Distance to print border \\
\hline 1 & far outside the printed area & $\geq \eta_{2}$ \\
\hline 2 & outside the printed area, but near to its border & $<\eta_{2}$ \\
\hline 3 & inside the printed area, and near to its border & $<\eta_{1}$ \\
\hline 4 & deep within the printed area & $\geq \eta_{1}$ \\
\hline
\end{tabular}

Table 2.1. PrintContexts (PC)

In practice, the division between the PrintContexts is not sharp. Especially within the pairs $\{1,2\}$ and $\{3,4\}$ it is arbitrary which to call near or far. Moreover, in practice even the distinction between inside $\{1,2\}$ and outside $\{3,4\}$ is vague, due to slight deficiencies in the image processing. Therefore, it is more appropriate to define a 'fuzzy' generalisation of PrintContext. Let the 4-dimensional fuzzy membershipfunction $\pi(x)$ for a point $x$ on the image be defined as:

$$
\pi(\mathbf{x})=\left(\pi_{1}(\mathbf{x}), \pi_{2}(\mathbf{x}), \pi_{3}(\mathbf{x}), \pi_{4}(\mathbf{x})\right)
$$

Where: $\pi_{k}(\mathbf{x}) \in[0,1]$ represents the extent to which $\mathbf{x}$ can be considered as belonging to PrintContext ' $k$ '. Here, we assume function $\pi(x)$ to be normalised: $\Sigma_{\mathrm{k}} \pi_{\mathrm{k}}(\mathbf{x})=1$. Our choice for the fuzzy membership-functions $\pi_{k}$ is shown in Figure 2.2 below. The membership functions are defined in terms of the distance $D$ to the print-border. Function $D(\mathbf{x})$ is defined more accurately in Section 2.5. The membership function of an area $S$ is defined as a Sugeno-integral [Sugeno, 1974]:

$$
\pi(S)=\int_{S} \pi(D(\mathbf{x})) f(\mathbf{x}) d_{2} \mathbf{x} / \int_{S} f(\mathbf{x}) d_{2} \mathbf{x}
$$

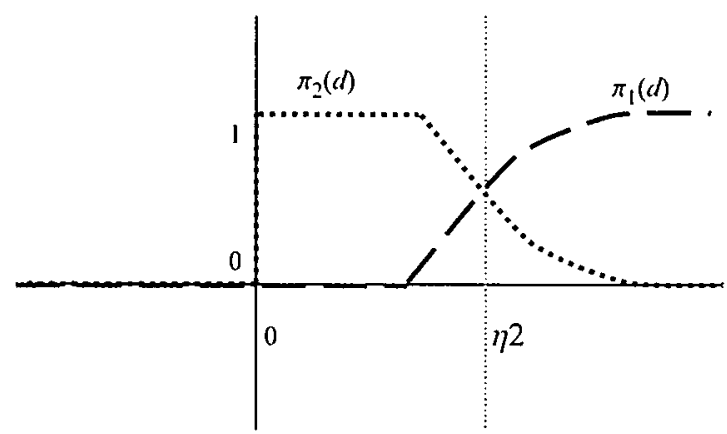

Fig. 2.2 Functions for fuzzy membership of a point at distance ' $d$ ' from the printborder to a given PrintContext. Shown are the functions $\pi_{1}$ and $\pi_{2}$. The representations for $\pi_{3}$ and $\pi_{4}$ are identical, when $d$ is changed into $-d$, and $\eta_{2}$ into $\eta_{1}$.

\subsubsection{The $Z$-representation of an Image}

An obvious way of comparing a digital image $f$ with its template $T$, is to directly subtract them point-wise. A pre-condition for such an operation is that the image is gauged and oriented according to the primary template co-ordinate system PTG, as defined in Section 2.2.1. Under the assumption of white-on-black print, the value of this difference directly relates to a specific set of defects. A negative difference implies locally ink missing, because the ink-intensity of the image is lower than the intended template intensity. Similarly, a positive difference implies that ink is smeared outside the print, because non-negative intensity is observed outside the 
template. There are two problems with a quantity based on a direct subtraction $f-T$. First, it depends on ink colour and background colour, i.e. whether the print is blackon-white or white-on-black. Second, the information it contains depends on the PrintContext, e.g. 'ink missing' always occurs in PrintContexts 3 and 4. For a true (weak) template-free approach, it is therefore better to take the absolute value of this difference. Thus, we define the absolute difference, denoted $Z$ :

$$
Z(\mathbf{x})=|f(\mathbf{x})-T(\mathbf{x})|
$$

We call this the 'Z-representation' of an image. $Z$ has non-zero values wherever the image differs from the template. Thus, a perfect image obeys: $Z(\mathbf{x}) \equiv 0$. In the entity $Z$ the true spatial extent of the defect is represented, rather than in the original image itself. Therefore it offers the possibility to concentrate on the defect, independent of the specific details of the template. Thus, the $Z$-representation highlights the defect and makes it independent of the template. However, because of the operation: $|f-T|$ the procedure itself relies on a detailed knowledge of the template. An example of this method is shown in Figure 2.3.

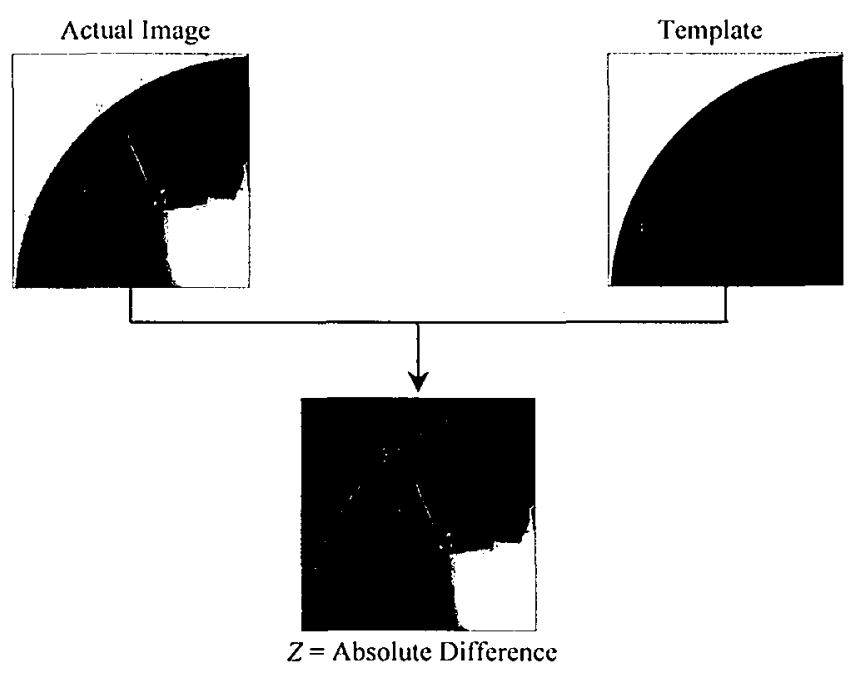

Fig. 2.3. $Z$, the absolute difference between actual image and template The residual border of the template, visible below, is called 'ghost-image'.

Though subtraction is a primitive action, the quality of this step has a large impact on the efficiency of the resulting image analysis. The reason is that in practice, due to round-off errors, even in the most ideal case, there is a slight difference between the image and its template. Other problems concern: [1] the lighting; i.e. no ambient light, reflections, and a light level that matches the template. [2] Defects in the optical and electronic parts of the CCD-system. [3] Errors due to pre-matching software, such as translation, rotation, and scaling of the image to the template $P T G$. In practice, there is always a residual image after subtraction, called the ghost-image. The ghost-image generally outlines the borders of the print. The direct dependency of the subtraction on the template means that this approach is strong template-dependent in the sense of Section 2.1.1. An example of a ghost-image is shown in the lower image in Figure 2.3. In later sections we shall see that the entity $Z$ forms the general starting-point for a weak template-free representation. 


\subsubsection{Print Quality as Function of $Z$}

Above definitions allow for a simple formulation of the print quality of an element. Let the defect density $\rho$ of an image $f$ be defined as the sum of $Z$ divided by its size:

$$
\rho(f)=|\Omega|^{-1} \int_{\Omega} Z(x) d_{2} x
$$

where $\Omega$ is the domain of the image, and $|\Omega|$ its size: $|\Omega|=\int_{\Omega} \mathrm{d}_{2} \mathbf{x}$. The quality threshold is defined as a critical value of $\rho$, denoted as $\rho_{\text {crit }}$. Above this threshold a printed element is said to have insufficient print quality and it is rejected. Below the threshold the element is accepted. This can be represented as an if-then rule:

$$
\text { if }\left(\rho(f) \geq \rho_{\text {crit }}\right) \text { then 'print quality is insufficient' }
$$

Implicitly, it is assumed that the default value for print quality is 'sufficient'. This rule is used as a primary tool for quality control, called the 'red bulb' approach: if this rule fires (the condition is $/$ rue), the machine is stopped and a human operator is called. The value for $\rho_{\text {crit }}$ must be set before the production process starts by the design engineer based on the desired printing quality of the product. Note that in order to be gauge independent, $\rho$ has to have the dimension of $\mathrm{mm}^{-2}$.

More elaborate definitions of print quality incorporate the detailed spatial distribution of $Z$. This can avoid situations of an intense, but highly localised defect with $\rho(f)<\rho_{\text {crit, }}$ but otherwise unacceptable print-quality. An example is a small scratch on the print.

\subsubsection{Print Defect Classes: Characteristic Patterns in Variations of $T$}

Now that we have defined the basic concept of $a$ printing defect, we can utilise the following observation, central to our context. Consider a print with template $T$. All possible printing defects of $T$ can be arranged in a limited set of similarity classes. These printing-defect classes are defined as specific patterns of variations of template $T$ with identifiable characteristics. This formulation leads to a partition based on a 'look-alike' principle, and thus highly vulnerable to subjective interpretation. A straight-forward way to make such a partition is by offering a large number of printing defects to a human expert and letting him decide to which defect class $k$ - if any - it belongs. Later, especially in Chapter 4, we describe methods that can automatically generate clusters for classification. Thus, these classes are based on visual characteristics, and most certainly they differ from a classification based on the underlying physical and chemical causes of the failure. Because of its subjective nature, we shall here regard defect-classes as a fuzzy partitioning. Let $u_{k}$ indicate the membership to defect class $k$. For a certain image $f$ that is a variation of template $T$, the quantity $u_{k}(f \mid T)$ expresses the extent in which $f$ can be considered as a failure in defect class $k$, given its template $T . u_{k}(f \mid \mathrm{T})=0$ implies that $f$ can certainly not be considered as a failure of type $k$. Thus, a class $V_{k}$ of defect type ' $k$ ' is defined as the set of images: $V_{k}=\left\{f \mid u_{k}(f \mid \mathrm{T})>0\right\}$. 


\subsection{Image Analysis of Printed Images}

The definitions from the previous sections provide us with a framework for the systematic analysis of printed images. This analysis is aimed at the identification of the 'interesting' parts of the image - i.e. the areas where the faults are situated - and their subsequent analysis. For the identification of these interesting parts we can best employ the Z-representation of the image, because this directly shows the faulty parts. Thus, the most straight-forward approach for image analysis is to obtain the parts of the image, and in combination with their PrintContext compare the area with the corresponding area of the template. Another approach for the image analysis is by scanning the image. A scan is the measurement of the intensity - i.e. the greyscale over a one-dimensional curve drawn on the image. In our context we can make a simple topological classification of relevant types of curves that we can use for scanning. Likewise, an even more simple classification can be constructed for the possible intensity-profiles resulting from the scanning.

\subsubsection{Intelligent Binary Search for Relevant Areas on the Image}

The absolute difference $Z$ of an image $f$ allows for a focussed analysis of an image. First, isolated areas of the image are selected where $Z(\mathbf{x})$ is above a predefined value. The collection of these areas is called a SearchMap. The image then is decomposed into a union of disjunct compact areas called searchboxes, where the 'energy' of the image is sufficiently high. The energy of a searchbox $B$ is defined as:

$$
E_{B}=\int_{B}|f(\mathbf{x})-T(\mathbf{x})| \mathrm{d}_{2} \mathbf{x} .
$$

For this decomposition an algorithm has been developed that iteratively covers the SearchMap with a set of searchboxes. This algorithm involves binary search on the absolute difference $Z$. Figure $2.4 a$ shows the iterative search of the algorithm to a specific area on the image. In Figure $2.4 b, 75$ of these searches are performed, resulting in a covering of the 'interesting' parts of the image. The algorithm must balance between finding the smallest significant areas, and not cutting relevant areas in multiple fragments. Attempts to add some intelligence to the algorithm improved its performance, but also significantly decreased its speed. Therefore, empirically a balance was sought between 'SearchBox-quality' and 'speed'. This resulted in an 'Intelligent Binary Search' algorithm called FindAreas.

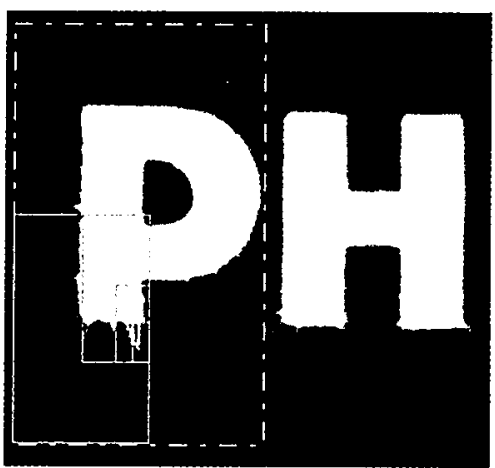

Fig 2.4a Iterative computation of one SearchBox

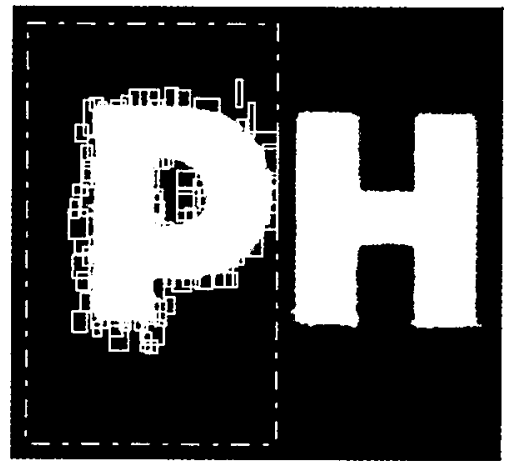

Fig 2.4b Covering with 75 Searchboxes 
The basic trick of FindAreas is to iteratively dispose the $k$-th found SearchBox $S_{k}$ from the actual SearchMap $Z_{k}: Z_{k+1} \leftarrow Z_{k} S_{k}$. Therefore, the algorithm exhaustively covers the SearchMap $Z$ with a set of searchboxes $\left\{S_{k}\right\}$, until the remaining cumulative energy of $Z_{k}$ falls below a certain pre-set threshold ' $\epsilon$ ':

$$
\int_{Z[k]}|/(\mathbf{x})-T(\mathbf{x})| \mathrm{d}_{2} \mathbf{x}<\epsilon \cdot \int_{Z[k]} \mathrm{d}_{2} \mathbf{x} .
$$

Or simply: $\rho_{k}(f)<\epsilon$, with $\rho_{k}$ the local defect density in SearchMap $Z_{k}$, as defined in equation 2.2. A partitioning algorithm based on finding the location of the global maximum discrepancy on the SearchMap, rather than binary search did not yield better results. Another interesting alternative is by employing Multi Resolution Analysis (MRA). MRA generates wavelets with translations $\left(k_{1}, k_{2}\right)$ and dilatations $\left(\lambda_{1}, \lambda_{2}\right)$ from a mother wavelet. So box ' $i$ ' is defined by corners: $\left(k_{1} / \lambda_{1}, k_{2} / \lambda_{2}\right)$ and sides: $\left(\lambda_{1}, \lambda_{2}\right)$. In this way a covering of boxes of SearchMap $Z$ is generated. This, however, did not necessarily improve the covering of the FindAreas algorithm. The further analysis of the SearchBox in relation with its PrintContext is the subject of Chapter 3 and appendix $\mathrm{A}$.

\subsubsection{Image Analysis Based on Scan-curves and Scan-profiles}

Another approach for image analysis is to make a number of scans on the actual image on locations that convey relevant information for the identification of the defect class. In this section we define such characteristic locations for scanning and compare the possible results of a scan with the predicted results in the case of no defect, i.e. with the scan-result of an identical scan curve on the template. Relative to the PrintContexts defined in table 2.1, we can define five classes of curves on an image. We can group these curves according to two criteria:

criterion 1: 'curves that fall entirely inside the printed area'versus 'curves that fall entirely outside the printed area'

criterion 2: 'curves that are parallel to the border of the print' versus 'curves that are perpendicular to the border of the print'

Combining these criteria we can define five relevant scan curve types:

\begin{tabular}{|c|c|}
\hline $\begin{array}{l}\text { Curve } \\
\text { Type }\end{array}$ & Definition \\
\hline$\Gamma 1$ & Far outside the printed area [i.e. in PrintContext l] \\
\hline$\Gamma 2$ & near outside the printed area and parallel to the print border [PrintContext 2] \\
\hline$\Gamma 3$ & perpendicular to, and crossing, the print border [PrintContexts 2 and 3] \\
\hline$\Gamma 4$ & near inside the printed area and parallel to the print border [PrintContext 3] \\
\hline$\Gamma 5$ & deep inside the interior of the printed area $[$ PrintContext $A]$ \\
\hline
\end{tabular}

Table 2.2 Scan-curve Typology

These curve types and their relation with the PrintContexts are depicted in Figure 2.1. In this way curves are defined as continuous topological mappings of an interval $[0$, $\left.\Lambda_{\max }\right] \rightarrow \mathbb{I R}^{2}$, where $\Lambda_{\max }$ is the length of the curve in $\mathrm{mm}$. Next, a scan-profile is defined as the intensity of an image measured along this scan curve. This means that if a scan-curve $\gamma$ on image $f$ is parameterised with a parameter $\lambda \in[0, \Lambda$ max $]$ as $\mathbf{x}(\lambda)$, then the resulting scan-profile $I$ is expressed by: $\mathrm{I}(\lambda)=f(\mathbf{x}(\lambda))$. The parameterisation of the scan-curve $\gamma$ is performed such that the parameterisation is isometric: 
$\|\partial \mathrm{x} / \partial \lambda\|=1$, in order to conserve the gauge dependence of the curve. For each position on a print an appropriate scan-curve can now be defined:

\begin{tabular}{|l|l|}
\hline Situation of printed area & Possible ScanCurves \\
\hline Totally outside printed area & $\Gamma 1, \Gamma 2, \Gamma 3$ \\
\hline Totally within printed area & $\Gamma 3, \Gamma 4, \Gamma 5$ \\
\hline Over several printed areas & $\Gamma 1, \Gamma 2, \Gamma 3, \Gamma 4, \Gamma 5$ \\
\hline
\end{tabular}

Table 2.3. Relation between PrintContext and ScanCurve Type

In Figure 2.5 an example is shown of a scanning operation over a defect in the 'deep' interior of the print - i.e. PrintContext 4 - over a scan-curve of type $\Gamma 5$.

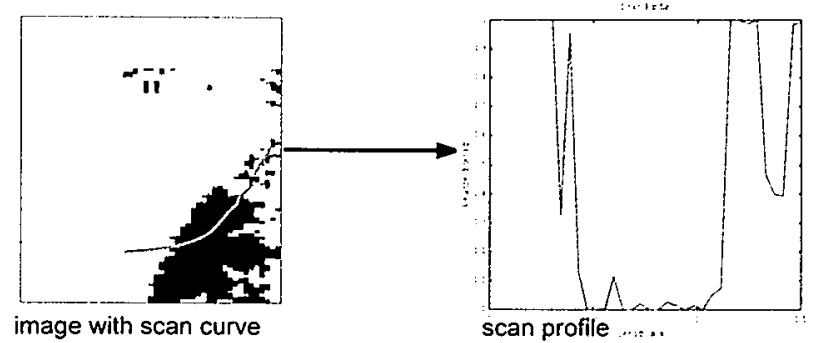

Fig. 2.5 Scan profile of defect 'ink missing', taken over a scancurve of type $\Gamma 5$ : 'deep inside the print'.

\section{Expected Scanning Results}

Let us assume that the template $T$ consists of a set of compact areas with constant greyscale. Now, suppose that we make a scan on template T. For each of the five topology types defined above there are but a limited number of possible outcomes. These are described in table 2.4. We can cluster the curve-types according to these outcomes and thus obtain three possibilities: $\{\Gamma 3\},\{\Gamma 1, \Gamma 2\}$, and $\{\Gamma 3, \Gamma 4\}$. These can be reduced to two classes because the last two are topologically equivalent: $\{\Gamma 3\}$ and $\{\Gamma 1, \Gamma 2, \Gamma 4, \Gamma 5\}$. Therefore, we define two expected types: E1: a constant representing $\{\Gamma 1, \Gamma 2, \Gamma 4, \Gamma 5\}$, and E2: a step-function representing $\{\Gamma 3\}$. We shall here adopt the convention to parameterise the curve $\Gamma$ in such a way that it starts at high intensity and subsequently drops to the lower intensity level. A step-function is consequently always of the kind 'Hi-Lo step' - a 'Lo-Hi step' can never occur. The small number of possible outcomes is caused by the restriction of the position of the curve to one (or in case of $\Gamma 3$ two) area(s) - otherwise more discrete steps would be observed. The two possible outcomes are depicted below in Figure 2.6.

\begin{tabular}{|c|l|}
\hline \multicolumn{1}{|c|}{ Curve Type } & Expected Scan Result on Template $\boldsymbol{T}$ \\
\hline$\Gamma 1$ & only 1 possibility: a constant equal to the background greyscale \\
\hline$\Gamma 2$ & only 1 possibility: a constant equal to the background greyscale \\
\hline$\Gamma 3$ & only 1 possibility: a step-function Hi-Lo or Lo-Hi, depending where the curve starts \\
\hline$\Gamma 4$ & only 1 possibility: a constant equal to the printcolour greyscale \\
\hline$\Gamma 5$ & only 1 possibility: a constant equal to the printcolour greyscale \\
\hline
\end{tabular}

Table 2.4. Expected results from scanprofiles on template. 
The expected results for a scan on the absolute difference $Z$ rather than the image $f$ itself are even more facile. Since in the case of a template $Z=|f-T|=0$, the only expected result is a constant zero.

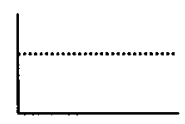

$\mathrm{E}_{1}$ : Constant

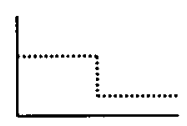

$\mathrm{E}_{2}$ : ‘Hi-Lo'-step

Figure 2.6. The possible outcomes of scans of a template.

\section{Observed Scanning Results}

We must make a clear distinction between expected scan-profiles and the actually observed scan-profile. Comparison of these two, in combination with additional information like PrintContext and the scan-curve-topology can provide us with important information about the printing defect class $k$. Such an approach must be built on a learning-phase, where a large collection of known defect-types are studied, and the for all possible PrintContexts and the scan-curve-types the relation between the expected and observed scan-profiles are identified. The results of this phase can be utilised in on-line classification of a new scan-profile in searching for and recognising the characteristic traits of the defect-classes.

However, in scanning an image into a collection of one-dimensional scan-profiles much of the original two-dimensional information is lost. Therefore, at its best, it can only be a part of the entire strategy for image analysis. An approach that relies to a large extent on scan-analysis in is presented in Chapter 7 in the context of a real-world application.

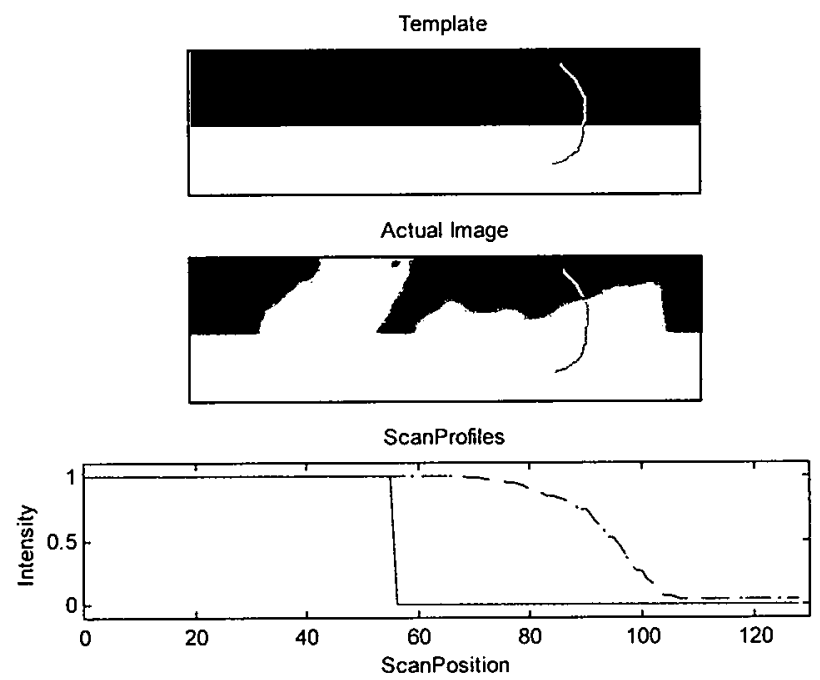

Fig. 2.7 Example of expected and observed scanprofiles. Above: Image of Template, lower half is printed. Middle: Actual Image in same frame. In both images the same scanline that crosses the printborder. Below: Expected scanprofile (solid) and Observed scanprofile (dashed) of the scanline. The line is scanned in upward direction. 


\section{Multi Scan-line Complexes}

One single scan-line does in general not provide sufficient information to decide on the nature of a defect, if any. For this reason, it seems attractive to analyse a collection of scan-lines. This can be done by simultaneously generating a complex of scan-lines, relevant to the current PrintContext. In this approach the entire complex is evaluated as a whole. This enables the analysis of correlations between the resulting profiles. In the case of defects emerging perpendicular to the print-border the correlation between the two scan-profiles from parallel scan-curves is high, as visible in Figure 2.8.

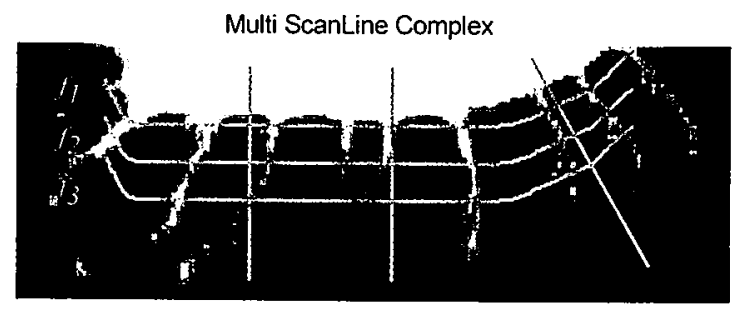

Fig. 2.8 Multi ScanLine Complex, consisting of three parallel (Г2) and three perpendicular (ГЗ) ScanLines on a print with defect class 'hairs'.

In this case the individual filaments are dissimilarly curved. This results in a relatively low average correlation between the scan-profiles, that makes it difficult to discern from random variations. Another disadvantage of this approach is that the conclusion may already be clear after the first scans, while this approach persists in analysing the scans in the complex. Therefore, in our context the multi-scanline approach is considerably better than single scan-curves.

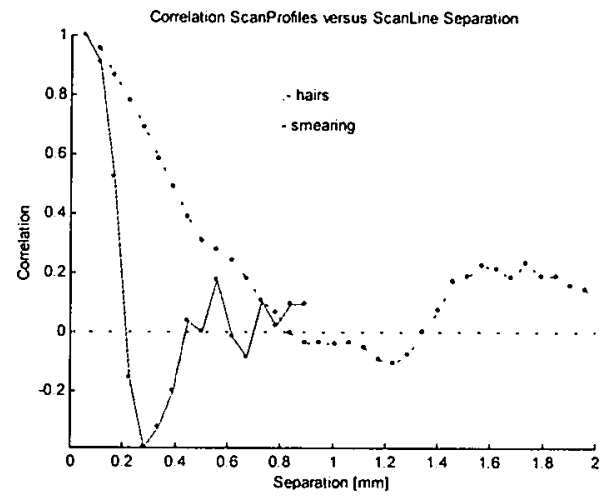

Fig. 2.9 Correlation between Parallel ScanLines. The correlation between parallel ScanLines against their distance. Solid: 'smearing', Dotted: 'hairs'.

In Chapter 7 we present a reasoning process that combines information of PrintContext and resulting scans and selects the best type and position for a new scanline. In that way the same information is aggregated dynamically and iteratively, rather than static and simultaneously like in the multi-scan line approach. 


\subsubsection{Conclusions}

An approach based on search-boxes and scan-profiles offers a solid framework for a focused analysis of printed images. The relation between PrintContext, scancurvetopology, and expected and observed outcomes offers the possibility for an empirical measurement of a correlation with a printing defect classes. In order to do so, it is important to quantify or qualify the relevant features of the printing defect classes in the search-box or scan-profile.

\subsection{Feature Extraction of Printed Images}

\subsubsection{Defining Features for Printing Defects}

The ultimate goal of the image analysis, as for instance with scanning, is the identification of the differences between the digital image of the actual printed element and its associated template. In order to utilise these differences in determining the printing defect class, we employ our earlier and most essential observation; i.e. that printing defect classes are all defined by their own specific collection of visual deviations from the associated printing-template. It is the primary objective of the feature-extraction to qualify or quantify these deviations. Each specific feature is thus represented by a feature-parameter, denoting the degree to which this feature is present in the image. In this way, an image can be translated from a matrix of colour values into a string of feature-parameters, called the feature-vector. The collection of all possible values of the feature-vector is the feature-space. The approach that we describe in the following chapters is applicable only on subsets of $\mathbb{R}^{\prime \prime}$, therefore we define features solely as continuous parameters, preferably in the domain $[0,1]$. This definition enables the interpretation of the feature-parameter as the fuzzy membership function of the associated feature.

From its very definition, a feature is often strongly related to the domain of its application. This means that the construction of feature-parameters heavily utilises domain-specific elements and frequently has an improvised and detailed character. The quality of this exercise, however, strongly determines the success of all subsequent feature-processing. In practice, it is extremely difficult to construct a consistent set of features, especially in the context of image analysis. Though of utmost importance for our approach, we do not describe the feature-extraction of printed images at this place. This subject is treated extensively in the separate appendix $A$, where it is presented in every detail in the context of a specific real-world printing application. This information is not included in the main text, as it would by its sheer size divert too much from our main line. However, for a good understanding of the integrative approach it is inevitable, because the quality of feature extraction determines the efficiency of the subsequent clustering and classification of an image. For this reason, this appendix is frequently referred to in the succeeding text.

However, a different and more general approach to feature extraction is possible, and is sketched in Chapter 3. There, fractal wavelet analysis is applied to obtain the 'generalised fractal dimension' of an image. This is an - in principle infinite - string of numbers, representing multifractal properties of the defect interpreted as a texture. This string can be directly used as a feature-vector representing the image. From a pragmatic point of view, though, both approaches for feature-extraction are combined, 
because some constructed feature-parameters exhibit stronger correlations with the printing defect classes than the generalised fractal dimension.

A general observation is that in our context the correlation between either type of the feature-parameter and defect class is small. This leads to the introduction of large quantities of feature parameters in order to combine and amplify the subtle correlations. However, due to the 'curse of dimensionality' (Section 2.4.2) the number of required empirical data increases exponentially with the number of features. Managing high-dimensional feature-spaces with numerous weakly-separated defectclasses introduces new kinds of problems which are discussed extensively in Chapters 4 and 5.

\subsubsection{On the ideal number of feature parameters}

The number of feature parameters together with the number of measurements determine the performance of a classifier. A straightforward classifier, such as a cellpartitioning of feature space, requires an exponential number of measurements as function of the feature dimension [Bishop, 1995]. This predicament is called the 'curse of dimensionality' [Bellman,1961]. Therefore, for a fixed number of measurements there exists an optimal number of features to represent the data. A lower number would not utilise all available information, and a higher number would introduce too much uncertainty or feature-redundancy. This observation is called the "peaking phenomenon' [Raudys,Pikelis, 1980] ${ }^{4}$. For this reason, for a limited set of measurements only the most expressive features should be used. The curse of dimensionality applies to all types of classifiers, including multi-layer feed-forward neural networks. Though, there exists no exact relationship between the number of features, the number of measurements, and the probability of misclassification, it is generally accepted that the number of measurements is at least ten times as large as the number of features used [Jain,Chandrasekaran,1982]. Equally important is the expressivity of the features. Watanabe [Watanabe,1985] noted that two different patterns, however complex, can be made arbitrary similar by encoding them with a large number of meaningless or redundant features (the so-called 'ugly duckling theorem').

These phenomena imply that for a given, but limited, set of observations, we must be moderate in composing the number of features that we employ in our pattern recognition system. For this reason, a number of methodologies have been developed to construct the smallest, yet most meaningful, subset from a larger pool of potential features. In literature they are called principal component analysis, feature extraction, and feature selection. Below, we discuss these formalisms in the sense of our context.

\section{Identifying the most salien features with Principal Component Analysis}

In Principal Component Analysis (PCA), also called Karhunen-Loève expansion, [Bow,1992] the largest $m$ eigen-values of the covariance-matrix of the zero-mean normalised pattern-matrix $M$ are used to determine the $m$ most salient features. This works as follows. The zero-mean normalised pattern-matrix $M$ is defined as: $\left(\widetilde{\mathbf{m}}_{1}, \ldots, \widetilde{\mathbf{m}}_{K}\right)$, with: $\widetilde{\mathbf{m}}_{k}=\mathbf{m}_{k}-\mathbf{m}$, and: $\mathbf{m}=\sum_{k=1}^{K} \mathbf{m}_{k} / K$. The covariance-matrix of $\mathrm{M}$ is defined as: $\mathrm{C}=\mathrm{M} \cdot \mathrm{M}^{\top}$. Next, the $m$ largest eigen-values $\lambda_{k}$ of $\mathrm{C}$ are determined,

\footnotetext{
${ }^{4}$ For a recent survey and discussion on the peaking phenomenon see: [Jain et al.,2000]
} 
and their associated eigen-vectors $\mathbf{h}_{k}$. The $N \times m$ matrix $\mathrm{H}=\left(\mathbf{h}_{1}, . ., \mathbf{h}_{m}\right)$ is used to construct a new pattern-matrix $\mathrm{M}^{\prime}=\mathrm{M}$.H. These $m$ largest eigen-values correspond to the $m$ expressive features. As PCA uses the most expressive features, it effectively approximates the data by a linear subspace using a mean squared error criterion.

\section{Feature extraction}

With feature extraction we mean the process of obtaining the value $x_{n}$ of a given feature-parameter with index $n$ from a given object by measuring or computation ${ }^{5}$.

\section{Feature parameter selection}

In current literature feature parameter selection indicates the process of selecting the 'best' subset of the feature parameter set [Jain et al.,2000]. In our context we can refine this definition to:

selecting the specific feature parameter, most relevant to the current hypothesis in the present sensing process,

i.e. the feature parameter that is expected, once measured, to provide the most accurate hypothesis regarding the state of the object.

In literature feature selection is commonly regarded as an exhaustive process following feature extraction. After the extraction from the sensed data, the features with low discrimination ability (e.g. based on principal component or discriminant analysis) are discarded [Jain et al.,2000]. In this way feature selection leads to saving in computational effort, as the final hypothesis is based only the most expressive features.

\subsection{The Euclidean Distance Transform for Printed Images}

The introduction of the PrintContext in Section 2.2 enabled a qualitative positioning on an image. We now extend and quantify the PrintContext into a generalised gauge for printed images. We use this gauge to construct a map of the distances of all imagepoints to the print-border, called the 'distancemap' of the print. We provide algorithms for calculating distancemaps of discrete images, but also present a generalised approach for continuous images in terms of the Helmholtz-equation.

\subsubsection{The Euclidean Distance Transform and the Distance Map}

The Euclidean distance transform is a mathematical transformation that, for each point $\mathbf{x}$ in a given set $\Omega \subset \mathbb{I R}^{N}$, defines the Euclidean distance to a given subset $S \subset \Omega$. The entire collection of Euclidean distances, defined on $\Omega$, is called the distance map $D$. The Euclidean distance transform is an important technique in image analysis and pattern recognition. It can be applied for specifying the 'context' or relative position

\footnotetext{
sIn literature feature extraction is often defined as determining the most approximate subspace of feature space, i.c. removing the feature(s) containing the least significant information, see: [Jain $e t$ al.,2000].
} 
in an image, for image processing - like 'thinning' or 'erosion', or as a basis for specific image transformations. Several efficient algorithms for computing discrete distance maps of two-dimensional binary images have been described, see: [Shih,Mitchell,1992], [Paglieroni,1992a], [Paglieroni,1992b], [Ragnehalm,1993], [Breu et al.,1995]. The algorithm of Breu et al. is in linear time, and hence asymptotically optimal. The problem of continuous distance maps is not addressed in image analysis and pattern recognition literature. The reason for this omission is that these algorithms are specifically conceived for digital images. Preferably, therefore, Euclidean metrics are approximated by digital distances [Mukherjee et al.,2000], or straightforwardly defined for the matrix of image pixels. The Euclidean distance transform is closely related to the Voronoi diagram of the set $\Omega$, which assigns to each point in $\Omega$ the 'label' of the closest point in subset $S$. For this reason, the Voronoitransform of a map is frequently expressed in terms of the Euclidean distance transform, see: [Guan,Ma,1998].

\subsubsection{The Distancemap of a Printed Image}

The entity 'PrintContext', defined in Section 2.2.2, provides a useful definition for the qualitative and relative position on an image. However, it conveys only information concerning whether a point is inside or outside the print, and whether it is near the print-border, or not. In several circumstances, however, it is necessary to know the absolute position of a point on the image. This is the case, for instance, if for the purpose of scanning we want to draw lines perpendicular or parallel to the printborder. The Euclidean Distance Transform (EDT) provides a useful starting point for the definition of the concept distance on a printed image. In the EDT, the distance $d_{E}$ to a set $A \subset \Omega$ is defined for each point $\mathbf{x} \in \Omega$ as the minimal Euclidean distance of $\mathbf{x}$ to any point $a$ in $A: d_{E}(\mathbf{x}, A)=\min a \in A\|\mathbf{x}-a\|$. In order to emphasise the difference between printed and not-printed areas, we make the following addition to the EDT. Let $D$ denote the concept distance in a printed image. We define the value of $D$ for points $\mathbf{x}$ outside a printed area $A$ as equal to the EDT:

$$
\mathbf{x} \notin A \Rightarrow D(\mathbf{x})=d_{E}(\mathbf{x}, A) .
$$

However, if $\mathbf{x}$ is inside a printed area $A$, then $D(\mathbf{x})$ is defined as a negative value, and in size equals the minimum distance to the exterior of $A$ :

$$
\mathbf{x} \in A \Rightarrow D(\mathbf{x})=-\min a \notin A\|\mathbf{x}-\boldsymbol{a}\| .
$$

The collection of the distances of all points of the image to the printed shape is called the distancemap. Definitions 2.1 and 2.2 provide a more precise description.

\section{Definition 2.1 Membership to Print}

Let the mapping $f: \Omega \subset \mathbb{I R}^{2} \rightarrow[0,1]$ define an image of a printed object, and let $A=$ $\{\mathbf{x} \in \Omega \mid f(\mathbf{x})>0\}$ be the support of $f$, indicating the print-shape. Now, let function $s$ be defined as: $s: \Omega \rightarrow\{-1,1\}: \mathbf{p} \in A \Leftrightarrow s(\mathbf{p})=1$ and $\mathbf{p} \notin A \Leftrightarrow s(\mathbf{p})=-1$. Thus, $s$ represents the crisp membership-function to the printed area $A$, and directly depends on the characteristic function $\chi_{A}$ on the printed shape $A: s(\mathbf{x})=2 \chi_{A}(\mathbf{x})-1$. 


\section{Definition 2.2 Distancemap for Printed Images}

Let $f, A$, and $s$ be defined as in definition 2.1. Let $\partial A$ denote the border of $A$. Then the distancemap $D: \Omega \rightarrow$ IR is defined as a function for all $\mathbf{p} \in \Omega$ as:

$$
D(\mathbf{p})=s(\mathbf{p}) \min \mathbf{x} \in \partial \mathrm{A}\|\mathbf{x}-\mathbf{p}\| .
$$

Notice that for any printed shape $S$, and all $\mathbf{p} \in \Omega S$, the value: $s(\mathbf{p}) \cdot \min \mathbf{x} \in S\|\mathbf{x}-\mathbf{p}\|$ is always assumed on its border ${ }^{6} \partial S$. This restriction from $S$ to $\partial S$ causes a considerable reduction in the search-space for computing $D$. In calculating the smallest $\|\mathbf{x}-\mathbf{p}\|$, we need now only to search set $\partial S$, instead of the entire set $S$. The distance to the border is expressed in metric units. This value can easily be transformed to a distance in pixels with the use of a scaling-factor, for instance in $\mathrm{mm} /$ pixel. An example of a distancemap is shown in Figure 2.10. On the left side in that figure the image of a template is shown, and also two points with their appropriate distances. On the right side of the figure, a contour plot of the distancemap is shown, as sets of curves connecting all points $x$ with the same distance $D(x)$. With this gauge it is possible to define neighbourhoods of borders, for instance all points $x$ in the image with $D(x)$ between -0.3 and +0.3 . Notice that this definition of distance allows a simple redefinition of the PrintContexts as defined in Figure 2.1 of Section 2.2.1.

\section{Definition 2.3 PrintContexts}

$$
\begin{aligned}
& \text { PrintContext 1: }\left\{\mathbf{x} \in \Omega \mid D(\mathbf{x})>\eta_{2}\right\}, \text { PrintContext } 3:\left\{\mathbf{x} \in \Omega \mid 0 \geq D(\mathbf{x})>-\eta_{1}\right\} \\
& \text { PrintContext 2: }\left\{\mathbf{x} \in \Omega \mid \eta_{2} \geq D(\mathbf{x})>0\right\}, \text { PrintContext } 4:\left\{\mathbf{x} \in \Omega \mid D(\mathbf{x}) \leq-\eta_{1}\right\}
\end{aligned}
$$

Another useful application of the distance map is for the definition of special curves on the image. A curve $\gamma_{/ /}$with length $L$ through a given point $\mathbf{p}$ parallel to the printborder of an image with distancemap $D$ can be expressed as:

$$
\gamma_{/ /}(\mathbf{p})=\{\mathbf{x} \in \Omega \mid D(\mathbf{x})=D(\mathbf{p}) \wedge\|\mathbf{x}-\mathbf{p}\|<L / 2\} .
$$

Where $\|\bullet\|$ is the Euclidean distance on $\Omega$. Likewise, a curve through a point $p$, perpendicular to the print-border in an image with distancemap $D$ is given by ${ }^{7}$ :

$\gamma_{\perp}(\mathbf{p})=\left\{\mathbf{x} \in \Omega \mid \partial \mathbf{x}(t) / \partial t=-s(\mathbf{p}) \nabla D(\mathbf{x}(t)) \wedge \mathbf{x}(0)=\mathbf{p} \wedge t>0 \wedge \sup _{t, t^{\prime}}\left\|\mathbf{x}(t)-\mathbf{x}\left(t^{\prime}\right)\right\|<L\right\}$.

From these examples it is clear that the applications of the distancemap are extremely sensitive to the quality of the distancemap. If the obtained distancemap $D$ differs in any respect from the true distancemap $D^{*}$, the curves and areas defined above accumulates these errors. Many of the entities that are defined in later sections are based on the distancemap. Hence, these applications also rely sensitively on the correctness of the distancemap. Therefore it is important to define a good computation scheme for the distancemap for a given image. The distancemap can be calculated by an algorithm that is comparable to the diffusion equation applied on the border of the

\footnotetext{
"Proof: Let $\mathrm{p} \notin S$, and let $\mathrm{x} \in S$ be the closest point to $\mathrm{p}$. Now, suppose $\mathrm{x} \notin \partial S$. Then there exists a neighbourhood $B(\mathbf{x}, \varepsilon)$ of $\mathbf{x}$, which lies entirely within $S$. But then, also, exists an $\mathbf{y} \in B$ closer to $\mathbf{p}$ than $\mathbf{x}$, such as: $\mathbf{y}=\mathbf{x}+\varepsilon(\mathbf{p}-\mathbf{x}) /(2 \|(\mathbf{p}-\mathbf{x} \|) \neq \mathbf{x}$. This violates our original assumption that $\mathbf{x}$ was the closest point to $p$, so this assumption must be false.

${ }^{7}$ This definition will only applicable to continuous diffeomorphic distancemaps $D$.
} 
image. In the next sections we discuss the computation of the distancemap of both discrete and continuous templates.

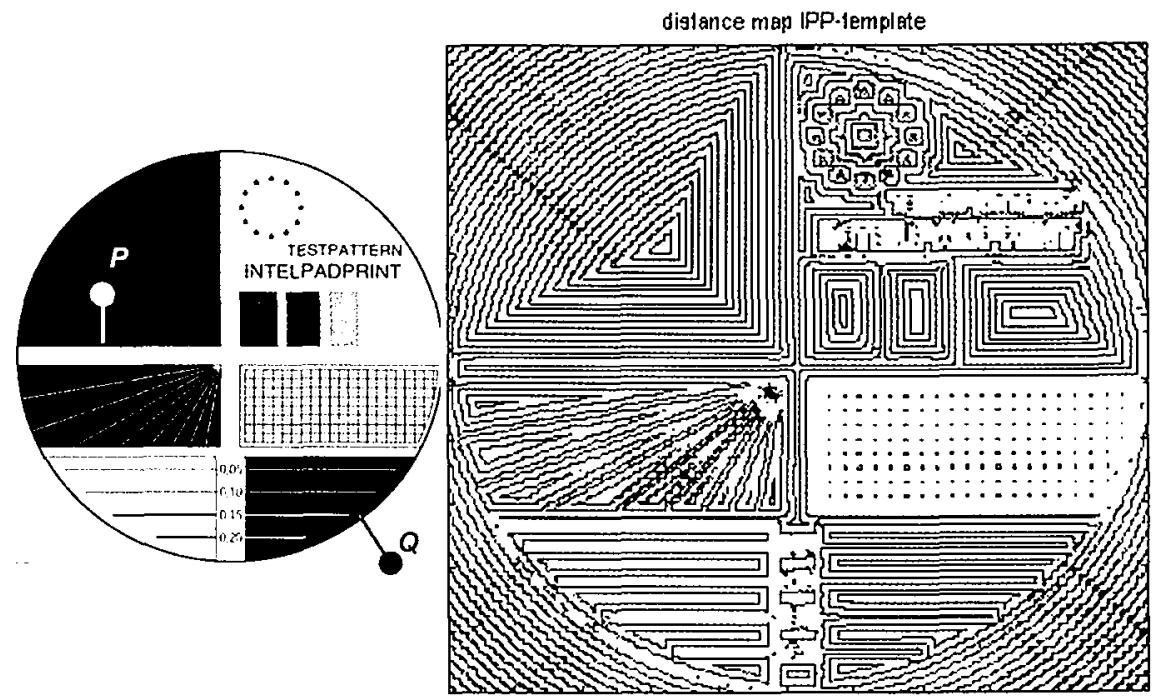

Fig. 2.10 (a): Examples of distances in a printed image. $P$ inside the print has negative value, whereas $Q$ outside the print has positive value. (b): Contour-plot of distance map of the same image; inside the print negative, outside positive. For this image a lower-threshold of 0.05 for the greyscale was employed, resulting in fusion of the two rightmost rastered blocks.

\subsubsection{Efficient Algorithms for Discrete Distancemaps}

Let us first assume that the image is discrete, so that $\mathbf{x}$ and $\mathbf{y}$ are pixels on the image, and all their components are integer. In that case, the distancemap $D$ can be easily computed from its definition by calculating the minimum distance to the print-border for each pixel $\mathrm{x}$ on the image $\Omega$.

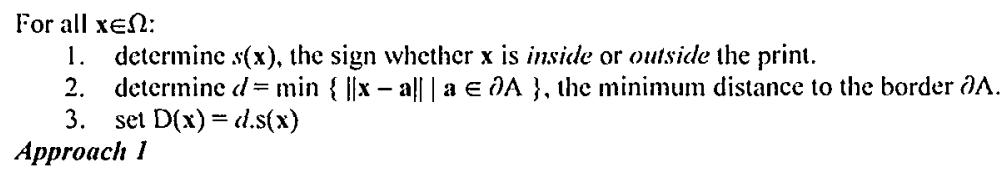

1. determine $s(\mathbf{x})$, the sign whether $\mathbf{x}$ is inside or outside the print.

2. determine $d=\min \{\|\mathbf{x}-\mathbf{a}\| \mid \mathbf{a} \in \partial \mathrm{A}\}$, the minimum distance to the border $\partial \Lambda$.

3. $\operatorname{set} \mathrm{D}(\mathrm{x})=d . \mathrm{s}(\mathrm{x})$

Approach I

However, this approach requires substantial computation time. If $N_{A}$ is the measure of $\partial A$ and $N_{\Omega}$ the measure of $\Omega$, then above computation would require $N_{A} \cdot\left(N_{\Omega}-N_{A}\right)$ computations $^{8}$. Another approach is to utilise some prime characteristics of the distancemap:

1. $D \mid \partial A=0$

2. if $\mathbf{a}$ is outside the isodistance-contour ${ }^{9} S=\{\mathbf{x} \in \Omega \mid D(\mathbf{x})=d\}$, then:

$$
D(\mathbf{a})=d+\min _{\mathbf{x} \in \mathrm{S}}\|\mathbf{x}-\mathbf{a}\|
$$

3. if $\mathbf{a}$ is inside the isodistance-contour ${ }^{10} S$, then: $D(\mathbf{a})=d-\min _{\mathbf{x} \in \mathrm{S}}\|\mathbf{x}-\mathbf{a}\|$

\footnotetext{
${ }^{8}$ For a typical map $N_{\Omega} \approx 10^{5}$, and $N_{\Lambda} \approx 10^{3}$, so algorithm 1 requires many millions of computations.

"So $D(\mathrm{a})>d$.
} 
This can be used for the following discrete algorithm:

Let $S_{1}$ be the set of unexplored points, and $S_{2}$ the set of explored points on image $\Omega$. Note that: $S_{1} \cup S_{2}=\Omega$. Initially, set: $S_{1}=\Omega \backslash \partial A, S_{2}=\partial A$, and for all $\mathbf{x} \in S_{2}$ set: $D(\mathbf{x})=0$. Discretise the image $\Omega$ with minimum grid size $\varepsilon>0$. Then perform the following iteration until $S_{1}$ is empty:

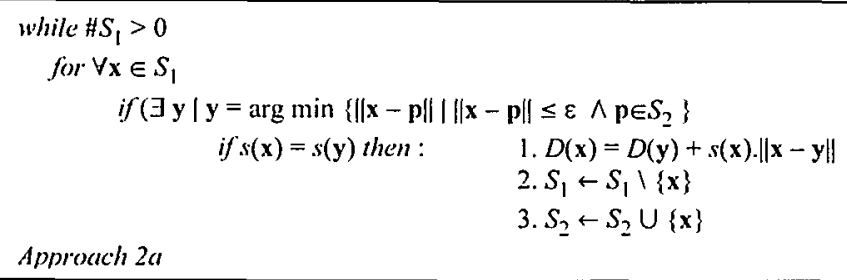

This algorithm converges towards a fair discrete approximation of $D(\mathbf{x})$. This algorithm still has the disadvantage that all points of $S_{1}$ have to be compared with all points of $S_{2}$. Set $S_{1}$ shrinks, but set $S_{2}$ grows, so the number of checks a $\# S_{1}$.\# $S_{2}$ is on average high. The following algorithm performs exactly the same actions, but checks only on points $\mathbf{x}$ on the border $\partial S_{2}$ of set $S_{2}$, rather than the entire set itself.

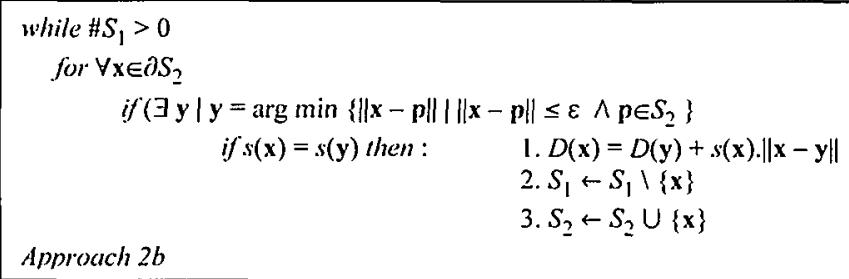

This algorithm exclusively operates on a small strip of diameter $\varepsilon$ around the border $\partial S_{2}$ of the explored set. During the iteration $\partial S_{2}$ increases weakly and $S_{1}$ decreases substantially. So, the number of computations $\propto \# \partial S_{2} . \# S_{1}$ is significantly lower than for approach $2 a$.

\subsubsection{Joining Distancemaps of Disconnected Parts}

Disconnected parts of an image can be efficiently joined by the following observation:

- The distancemap $D$ is non-additive with regard to composition of parts: if image $H$ consists of a union of disconnected parts $H_{i}$, each with associated distancemap $D_{i}$, then the distancemap of $H$ is:

$D(\mathbf{x})=\min _{i} D_{i}(\mathbf{x})$

Let for instance a map $H$ consist of two disconnected parts $H_{1}$ and $H_{2}$, as in Figure 2.11. Let $D_{1}$ be the map relative to part $H_{1}$ and equally $D_{2}$ for $H_{2}$. Let $H_{1} \cap H_{2}=\varnothing$, then the map $H$ consisting of $H_{1} \cup H_{2}$ has a distancemap: $D(\mathbf{x})=\min \left\{D_{1}(\mathbf{x}), D_{2}(\mathbf{x})\right\}$. So, $D\left(H_{1} \cup H_{2}\right)=\min \left\{D\left(H_{1}\right), D\left(H_{2}\right)\right\}$.

Notice that equation [2.4] also holds for the interior of a part. In the interior of part ' $k$ ' only term $D_{k}$ is negative, because this area belongs to the exterior of all others parts. 
The separatrices, i.e. lines that separate the sectors of the disconnected parts are:

$$
\gamma_{\mathrm{ij}}=\left\{\mathbf{x} \in \Omega \mid D_{i}(\mathbf{x})=D_{j}(\mathbf{x}) \wedge D_{i}(\mathbf{x})=\min _{k} D_{k}(\mathbf{x})\right\} .
$$

This is a generalisation of a Voronoi partitioning ${ }^{\prime \prime}$. If each part $B_{i}$ consists of just one single point $\mathbf{p}_{i}$, then $\gamma$ is the standard Voronoi partitioning of $\cup_{i} B_{i}$ on $\Omega$.

The disadvantage of this approach is that it is elaborate, and introduces the extra obstacle of first determining the disconnected parts. An example of this approach is depicted in Figure 2.11.

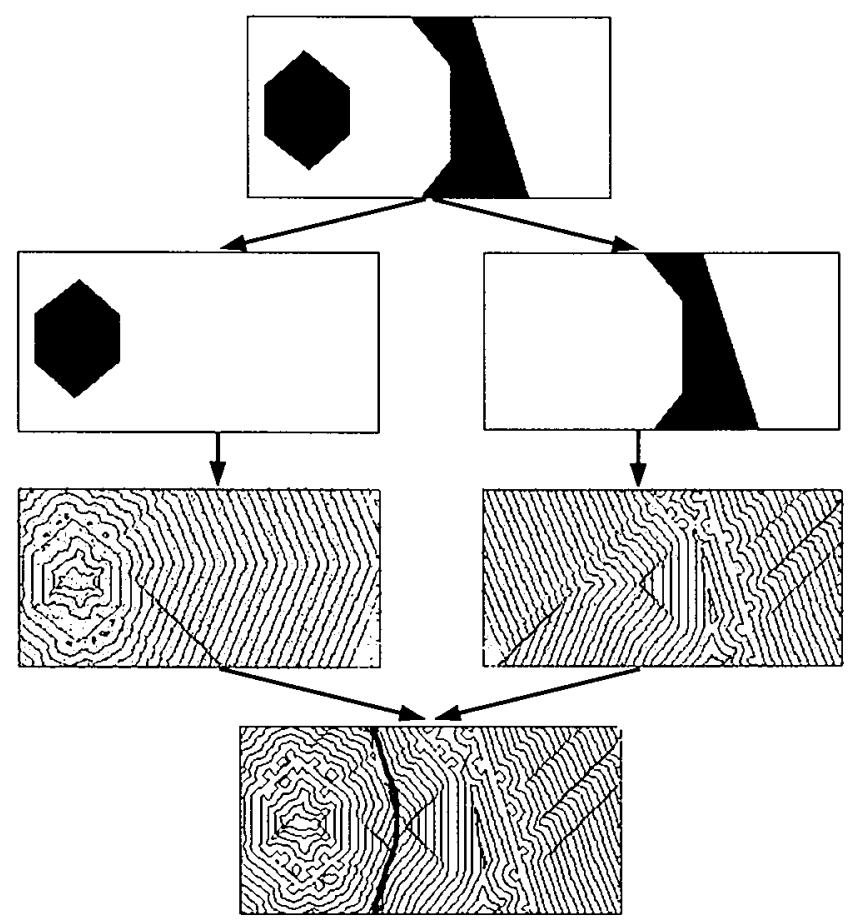

Fig. 2.11. Utilising the non-additiveness property of the distancemap. Separate the image in disconnected parts, solve the equations for each individual part, and find the global distancemap by combining the individual parts by determining $\min _{k} D_{k}(x)$. In the lower picture the separatrix between the two parts is indicated as a fat line. Distortions in the lines are caused by the discrete nature of the digital image.

\footnotetext{
"A Voronoi partitioning for a given set of discrete points $P \subset \mathbb{R}$ ", is a collection of convex subsets in $\mathbb{R}^{n}$, such that each subset consists of the points in $\mathbb{R}^{n}$ that are closest to a specific member of $P$.
} 


\subsubsection{Computation of Continuous Distancemaps}

Now, let us assume that the domain of the image is a compact subset of $\mathbb{I R}^{2}$, so all points on the image have real coordinates. In order to design a general approach for the calculation of continuous distancemaps, we first provide some useful definitions and assertions. Building on these, we observe that the basic expression for the distance-map in definition 2.2 is equivalent Huygens' principle of wave-propagation. This interpretation allows for a simple expression for the continuous distance-map for a given print-shape. First, let us define the following basic entities.

Definition 2.4: Consider an image with domain $\Omega \subset \mathrm{IR}^{2}$, containing a printed shape $A$. An isodistance-contour (IDC) is the collection of all points on the image with the same distance to the print-border $\partial A$. So, the isodistancecontour for distance $d$ is:

$$
H(d)=\{\mathbf{x} \in \Omega \mid D(\mathbf{x})=d\} \text {. }
$$

For $d=0$ we again obtain the original print-border. Likewise, we can define 'rays' emanating from the border.

Definition 2.5: Let $a$ be an arbitrary point on the print-border $\partial A$. The ray ' $\rho$ ' is then defined as the line that connects all points $\mathbf{x} \in \Omega$ for which $a$ is the closest point in the printed area $A$ :

$$
\rho(\boldsymbol{a})=\{\mathbf{x} \in \Omega \mid D(\mathbf{x})=\|\mathbf{x}-a\| s(\mathbf{x})\}=\left\{\mathbf{x} \in \Omega \mid a \in \arg \min _{\mathbf{u} \in \partial S}\|\mathbf{x}-\mathbf{u}\| s(\mathbf{x})\right\}
$$

Moreover, we distinguish between the outward ray ' $\rho_{+}$', as the part of the ray in the not-printed part of the image. Similarly, the inward ray ' $\rho$.' is the part of the ray inside the print. Furthermore, we define the subset of $A$, that is closest to a given point $P$.

Definition 2.6: Let $A$ be a printed area, and let $P$ be an arbitrary point in $\Omega$. The collection of points in $A$ that are closest to $P$ is called: $\operatorname{Cset}(P, A)$.

So, $C$ set defines a transformation $\Omega \rightarrow A$. Notice that $\operatorname{Cset}(P, A)$ can be a single point, a collection of points on a circle, or a continuous arc of a circle. Based on these definitions, we can now formulate the following Lemma.

Lemma 2.1 Let $A$ be a convex printed area with border $\partial A$. Let $P$ be a point outside $A$. Let $Q$ be the closest point on $\partial A$ to $P: Q \in \operatorname{Cset}(P, \partial A)$.

Then:

either [1]: $\partial A$ is differentiable ${ }^{12}$ in $Q$, and $P Q$ is perpendicular to $\partial A$,

or [2]: $\partial A$ is not-differentiable in $Q$, and $Q$ is the centre of a sheaf of emanating rays $Q R$ defining the points $R \notin A$ for which $Q$ is the closest point of $A$.

Proof: [1] Let $\partial A$ be a differentiable curve near $Q$, and let $\mathbf{a}(\lambda)$ for $\lambda \in[0,1]$ be a suitable continuous differentiable parameterisation of $\partial A$ near $Q$, and let $\lambda^{*}$ be the parameter value of $Q$, the closest point on $\partial A$ to $P$, so: $\lambda^{*}=\arg \min _{\lambda \in[0,1]}\|\mathbf{p}-\mathbf{a}(\lambda)\|$.

Since $\mathbf{a}$ is differentiable, this implies: $\frac{\partial}{\partial \lambda}\|\mathbf{p}-\mathbf{a}(\lambda)\|=0$ for $\lambda=\lambda^{*}$. Performing the

\footnotetext{
${ }^{12}$ i.e. that the derivative of $\partial A$ is a continuous curve.
} 
differentiation gives: $\left(\mathbf{p}-\mathbf{a}\left(\lambda^{*}\right)\right) \cdot \frac{\partial \mathbf{a}\left(\lambda^{*}\right)}{\partial \lambda}=0$. As $\frac{\partial \mathbf{a}\left(\lambda^{*}\right)}{\partial \lambda}$ is the tangent vector to $\partial A$ in $Q$, and $\left(\mathbf{p}-\mathbf{a}\left(\lambda^{*}\right)\right)$ is the vector $\overrightarrow{P Q}$, we find that: $\overrightarrow{P Q} \perp \frac{\partial \mathbf{a}\left(\lambda^{*}\right)}{\partial \lambda}$

[2] Let $\mathbf{a}(\lambda)$ and $\lambda^{*}$ be defined as above. Let: $\hat{\mathbf{n}}(\lambda)$ be the outward directed normal vector to $\partial A$ at $\mathbf{a}(\lambda)$. Let: $\hat{\mathbf{n}}_{1}=\lim _{\lambda \uparrow \lambda^{*}} \hat{\mathbf{n}}(\lambda)$, and: $\hat{\mathbf{n}}_{2}=\lim _{\lambda \downarrow \lambda^{*}} \hat{\mathbf{n}}(\lambda)$. As $\partial A$ is not differentiable at $Q$, this means that: $\hat{\mathbf{n}}_{1} \neq \hat{\mathbf{n}}_{2}$. Moreover, as $A$ is convex, the collections of normal vectors left and right of $Q$ do not overlap. Thus, at $Q$ there is an open edge between $\hat{\mathbf{n}}_{1}$ and $\hat{\mathbf{n}}_{2}$. Therefore, all lines emanating outward from $Q$ within this edge define points $R \notin A$ for which $Q$ is their closest point on $\partial A, Q \in \operatorname{Cset}(R, A) \square$

Lemma 2.2 Let $A$ be a printed area with border $\partial A$. Let $Q$ be a point on $\partial A$. Now, let $A$ be locally non-convex at $Q$.

Then:

either [1]: $\partial A$ is differentiable in $Q$, and all rays are perpendicular to $\partial A$, or [2]: $\partial A$ is not differentiable in $Q$, and there are no points outside $A$ for which $Q$ is the closest point of $A$.

Proof: [1] The proof is identical to proof [1] of Lemma 2.1.

Proof: [2] Let $\hat{\mathbf{n}}_{1}$ and $\hat{\mathbf{n}}_{2}$ be defined as in proof [2] of Lemma 2.1. Let $N_{l e f i}$ be the collection of normal vectors left of $Q$, and $N_{\text {righ }}$ the ditto collection right of $Q$. As $A$ is non-convex at $Q$, this time the collections $N_{\text {lcf }}$ and $N_{\text {righ }}$ do overlap. Therefore, all points outside $A$ near $Q$ lie on rays defined by both $N_{l e f i}$ or $N_{\text {right }}$. Let $S$ be the separatrix, consisting of intersections of left- and right-rays with identical distancevalues. Let $W_{l y f t}$ and $W_{\text {right }}$ be the areas outside the print respectively left, and right of $S$. Now, $Q$ is the rightmost extreme of the line $\overline{W_{l e f l}} \cap \partial A$, and leftmost extreme of $\overline{W_{\text {right }}} \cap \partial A$. Application of Lemma 2.1 means that the closest point on $\partial A$ for all points $\mathbf{w}$ in either $W_{l e f t}$ or $W_{\text {right }}$ is defined by the intersection of the ray $\rho$ through $\mathbf{w}$ with $\partial A$. As $\partial A$ is not differentiable in $Q$, however, there is no ray emanating from $Q$, and so $Q$ is not the closest point to $P \square$

Lemma 2.3 Let $A$ be a printed area, with border $\partial A$. Let $P$ be a point outside $A$. Let $Q$ be the closest point to $P$ on $\partial A: Q \in \operatorname{Cset}(P, \partial A)$. Let $R$ be a point on the ray $Q P$. Let $H_{1}$ be the IDC passing through $R$, and $H_{2}$ be the IDC passing through $P$.

\section{Then:}

[1]: $Q$ belongs to the subset of $\partial A$ of closest points to $R: Q \in \operatorname{Cset}(R, \partial A)$,

[2]: $R$ belongs to the subset of $H_{1}$ of closest points to $P: R \in \operatorname{Cset}\left(P, H_{1}\right)$.

[3]: the distance between $H_{1}$ and $H_{2}$ is: $\mathrm{d}\left(H_{1}, H_{2}\right)=\left|\mathrm{d}\left(H_{1}, \partial A\right)-\mathrm{d}\left(H_{2}, \partial A\right)\right|$ 


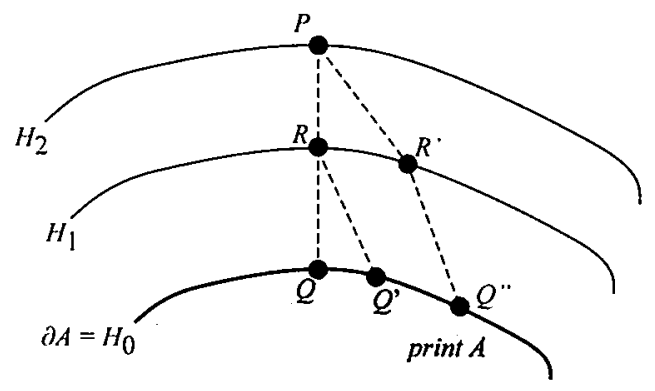

Fig. 2.12.

Proof: [1] Suppose there was another point $Q^{\prime} \neq Q$ on $\partial A$ with distance $R Q^{\prime}<R Q$. Then the piecewise linear path $P R Q^{\prime}$ passing through $R$ has length: $P R Q^{\prime}=P R+R Q$ ' $<P R+R Q=P Q$, which is impossible, because $Q$ is the closest point on $\partial A$ to $P \square$

[2]: Suppose there was another point $R^{\prime} \neq R$ on $H_{1}$ with distance $P R^{\prime}<P R$. Let $Q^{\prime \prime}$ be the closest point on $\partial A$ to $R^{\prime}$. Then the piecewise linear path $P R$ ' $Q^{\prime \prime}$ passing through $R^{\prime}$ has length: $P R^{\prime} Q^{\prime \prime}=P R^{\prime}+R^{\prime} Q^{\prime}$. But $R^{\prime} Q^{\prime \prime}=R Q$, for both $R$ and $R$ ' lie on the same isodistance-contour, and both $Q^{\prime \prime}$ and $Q$ lie on $\partial A$. So: $P R{ }^{\prime} Q^{\prime \prime}=P R^{\prime}+R Q<P R$ $+R Q=P Q$, which is impossible, because $Q$ is the closest point on $\partial A$ to $P \square$

[3]: Let IDC $H_{2}$ have a distance $d_{2}$ to the print-border $H_{0} . H_{1}$ is an IDC inbetween $H_{0}$ $=\partial A$ and $H_{2}$, on a distance $d_{1}$ from $H_{0}$. Let $d_{12}$ be the distance between $H_{1}$ and $H_{2}$.

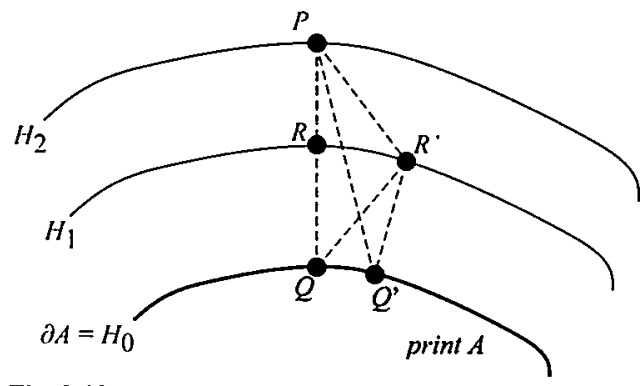

Fig. 2.13.

Now, $R$ is the intersection of $H_{1}$ and line $P Q$. Let us suppose that $R^{\prime}$ is the closest point on $H_{1}$ to $P$, and that $Q^{\prime}$ is the closest point of $H_{0}$ to $R$ '. Since $R^{\prime}$ is the closest point on $H_{1}$ to $P$, it follows directly that: $P R \geq P R$ '. Similarly, one can deduce that: $R Q \geq R^{\prime} Q^{\prime}$. Since $P Q$ is a straight line, it follows that:

$$
P Q=P R+R Q \geq P R^{\prime}+R^{\prime} Q^{\prime}=d_{12}+d_{1} \text {. }
$$

Since $P Q$ is by definition equal to $d_{2}$, it follows:

$$
d_{2} \geq d_{12}+d_{1}
$$

Otherwise, from triangle $\triangle P Q^{\prime} R^{\prime}$ the triangular inequality dictates that:

$$
P Q^{\prime} \leq P R^{\prime}+R^{\prime} Q^{\prime}=d_{12}+d_{1} \text {. }
$$

Similarly, since $P$ is on $H_{2}$, and $Q^{\prime}$ is on $H_{0}$, it follows that: $P Q^{\prime} \geq d_{2}$. Therefore:

$$
d_{2} \leq P Q^{\prime} \leq d_{12}+d_{1}
$$

From $[\alpha]$ and $[\beta]$ it can be concluded that:

$$
d_{12}=d_{2}-d_{1}
$$


This means that the print-depth from an IDC has the folowing additive property:

$\mathrm{d}\left(H\left(d_{1}\right), H\left(d_{2}\right)\right)=\left|d_{1}-d_{2}\right| \square$

Lemma 2.4 Let $A$ be a printed area with border $\partial A$. Let $P$ be an arbitrary point outside $A$. Let $Q$ be the closest point on $\partial A$ to $P$. Let $R$ be an arbitrary point on ray $Q P$.

\section{Then:}

[1]: the IDC $H_{1}$ passing through $R$ is locally differentiable at $R$,

[2]: ray $Q P$ is perpendicular to IDC $H_{1}$ passing through $R$.

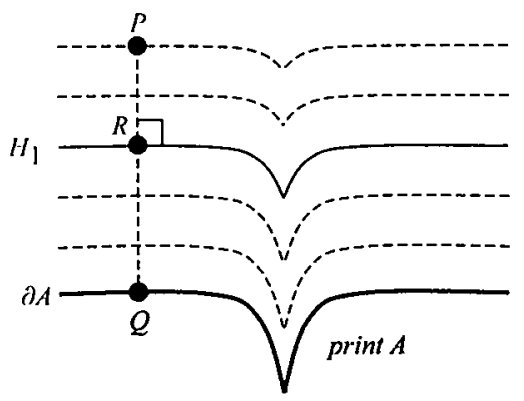

Fig. 2.14.

Proof: [1]: According to Lemma 2.1, there are two possibilities for $Q$ : Either: i. $\partial A$ is differentiable at $Q$. Then IDC $H_{1}$ is a projection-like transformation of print-border $\partial A$, and thus differentiable at $R . O r:$ ii. $\partial A$ is not-differentiable at $Q$. Then there are two possibilities. $\alpha$ ) If $A$ is locally convex at $Q$, according to Lemma $2.1-2, Q$ acts as the centre of a sheaf of emanating lines. Therefore, locally the IDCs form concentric arcs with centre $Q$. Therefore, IDC $H_{1}$ at $R$ is such an arc, and therefore differentiable at $R . \beta$ ) If $A$ is locally non-convex at $Q$. Then, according to Lemma $2.2-2$, there are no points outside $A$ for which $Q$ is the closest point of $A$. Therefore, $Q$ can not be the source of the ray $Q P \square$

[2]: First, we prove that $R Q \perp H_{1}$. From Lemma 2.4 .1 we know that near $R$ there exists a local differentiable parameterisation $\mathbf{a}(\lambda)$ of $H_{1}$. Let $\mathbf{r}=\mathbf{a}\left(\lambda^{*}\right)$ be the coordinate-vector representing $R$, and similarly $\mathbf{q}$ the vector of $Q$. From Lemma 2.3.1 we know that $Q$ is the closest point on $\partial A$ to $R$. Therefore, $\lambda^{*}$ minimises: $\|\mathbf{q}-\mathbf{a}(\lambda)\|$. Then, similar to Lemma $2.1-1$, the proof follows from differentiating $\|\mathbf{q}-\mathbf{a}(\lambda)\|$ to $\lambda$ at $\lambda=\lambda^{*}$, and obtaining: $Q R \perp H_{1}$. We also know that path $P R Q$ is a straight line, so if: $Q R \perp H_{1}$, then also: $Q P \perp H_{1}$ 口

This Lemma does not mean that all points on a IDC $H_{1}$ are differentiable. Points on $H_{1}$ that are equally close to multiple disconnected points on $\partial A$, can be nondifferentiable. An example is shown in figure 2.X. Here, all IDCs reproduce the central 'dint' of the print-border, where they are locally not differentiable. Lemma 2.4 merely dictates that emanating rays will never intersect non-differentiable points on intermediate IDCs. Whenever a ray hits a non-differentiable point on an IDC, it will end there. For the determination of the not-differentiable points on IDCs, the radius of curvature of the print-border plays an important role. 
Finally, we can make a statement on the gradient of the associated distancemap $D$.

Lemma 2.5 Let $A$ be a printed shape with distancemap $D$. Let $P$ be a point outside $A$, and let $H$ be the isodistance-contour passing through $P$. If $H$ is differentiable at $P$ then $\nabla D$ is perpendicular to $H$ at $P$.

Proof: This follows directly from Lemma 2.4-2.

With these five Lemmas, we can now formulate two Theorems on the propagation of a continuous distancemap $D$.

Theorem 2.1 Let $A$ be a printed area with distancemap $D$. Let $H$ be an isodistancecontour of $A$. Let $\mathbf{p}$ be a point outside $A: D(\mathbf{p})>D(H)$. Let $\mathbf{q} \in \operatorname{Cset}(\mathbf{p}, H)$. Then:

$$
D(\mathbf{p})=D(H)+\|\mathbf{p}-\mathbf{q}\|
$$

Proof: This follows directly from Lemma 2.3-3.

Theorem 2.2 Let $A$ be a printed area with distancemap $D$. Let $D$ be differentiable at $\mathbf{x}$, then it obeys:

$$
\|\nabla D(\mathbf{x})\|=1
$$

Proof: From Lemma 2.5 we know that: $\nabla D \perp H$, and from Lemma 2.3-3 that: $d\left(H_{1}, H_{2}\right)=\left|d_{2}-d_{1}\right|$. So, for two adjacent isodistance-contours $H_{1}$ and $H_{2}$, separated by a distance $h$, we have that: $\left|D\left(H_{2}\right)-D\left(H_{1}\right)\right|=h$. For $h \rightarrow 0$ we find:

$$
\|\nabla D\|=\lim _{h \rightarrow 0} \frac{\left|D\left(H_{2}\right)-D\left(H_{1}\right)\right|}{h}=1
$$

Theorem 2.2 determines the length of the gradient $\nabla D$, but leaves its direction totally free. Consequently, $\nabla D(\mathrm{x})$ can be written as: $\nabla D(\mathbf{x})=\hat{\mathbf{u}}(\phi(\mathbf{x}))$, where $\hat{\mathbf{u}}$ is the unit vector directed in angle $\phi(\mathbf{x}): \hat{\mathbf{u}}(\phi)=(\cos \phi, \sin \phi)^{\top}$. The same considerations are valid for IDCs inside a print, if the sign-change of $s(\mathrm{x})$ is included.

Above assertions, especially theorem 2.1 for the distancemap, can now be interpreted as follows. Any point $\mathbf{x}$ on an isodistance-contour $H_{2}$, lying some distance farther away from the print-border, obeys:

$$
D\left(H_{2}\right)=D(\mathbf{x})=D\left(H_{1}\right)+s\left(H_{1}\right) \min \left\{\|\mathbf{x}-\mathbf{y}\| \mid \mathbf{y} \in H_{l}\right\}
$$

Thus, the new isodistance-contour $\mathrm{H}_{2}$ can be constructed directly from the old isodistance-contour $H_{1}$, by letting 'rays' of equal length emanate from all points y on $H_{1}$, and determining the outer envelope. A similar expression holds for isodistancecontours in the interior of the print. This formulation for the construction of the distance-map is equivalent to Christiaan Huygens' famous principle for the propagation of light-waves:

"...every point on a primary wavefront serves as the source of secondary wavelets, such that the primary wavefrom at some later time is the envelope of these wavelets..." [Huygens, 1678] 
It becomes identical when the term 'wavefront' is replaced by the term 'isodistancecontour'. This principle is shown in Figure 2.15.

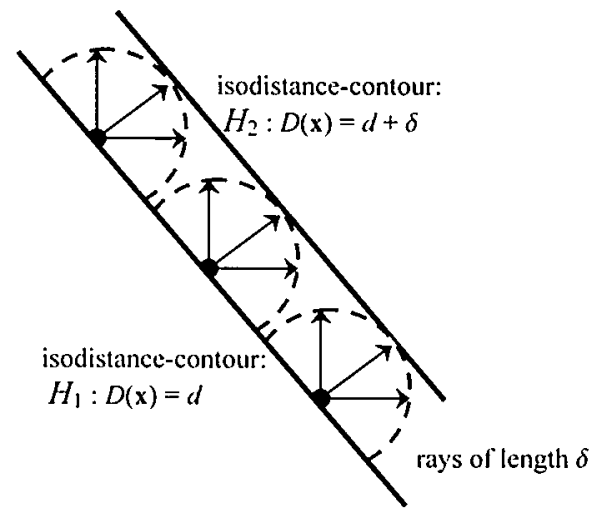

Fig. 2.15. Huygens' principle for wave propagation.

The mathematical consequences of this qualitative principle involves the solution of the wave propagation of a scalar optical field $\Psi(\mathbf{x}, t)$. The exact derivation of this expression from Huygens' principle can be found in textbooks on geometrical optics, such as: [Hecht, 1987].

$$
\nabla^{2} \Psi-\Psi_{t} / c^{2}=0
$$

where $c$ is the velocity of the wave front. This is a homogeneous expression in $\Psi$. This has as serious consequence that we can not impose the primary boundary-condition for a distance-map $D$ of a shape $S: D \mid \partial S=0$, because $\Psi \mid \partial S=0$ would directly imply $\Psi(\mathbf{x}, t) \equiv 0$. Therefore, we impose a boundary-condition on the print-shape $S$ as:

$$
\Psi \mid \partial S=\text { constant }>0
$$

For convenience we set this constant to 1 . Moreover, in line with Lemma 2.5, we demand that $\Psi$ emanates perpendicular from the print-border $\partial S$, as far as it is differentiable, so:

$$
\nabla \Psi \perp \partial S
$$

In our case, we are not so much interested in the actual manner in which an optical field propagates from one surface to another. We are interested in stationary solutions. Therefore, we separate the scalar field $\Psi(\mathbf{x}, t)$ in a spatial and a temporal part: $\Psi(\mathbf{x}, t)=$ $\psi(\mathbf{x}) \tau(t)$. Substitution in expression [2.5] gives for the spatial part $\psi(\mathbf{x})$ :

$$
\nabla^{2} \psi-K \psi=0
$$

with separation-constant $K$. In our case, the distance is a monotonous increasing function, and not an oscillating function. Therefore, we only allow non-negative numbers for $K$. For simplicity, we set $K=1$. The same boundary conditions as above apply for $\psi$ : 


$$
\psi \mid \partial S=1, \nabla \psi \perp \partial S
$$

Equation [2.8] is the well-known Helmholtz equation, where $\psi(\mathbf{x})$ represents the amplitude of the field ${ }^{13}$. The solution to the Helmholtz equation is the mathematical description of the isopotential lines of the scalar field. Green's theorem can be employed to rigorously solve equation [2.8].

Thus, the solution to expressions [2.8], [2.9] provides us with the shape and location of the isodistance-contours in our distance-map. However, we have not yet asserted the value of the distance-map $D$ at that location. Since the equipotential lines: $V=\{\mathbf{x} \mid$ $\psi(\mathbf{x})=v\}$, and isodistance-contours $H=\{\mathbf{x} \mid D(\mathbf{x})=d\}$ have the same morphology, we can locally define a simple scalar transformation $h: I R \rightarrow$ IR:D $\rightarrow \psi$, such that:

$$
D(\mathbf{x})=h(\psi(\mathbf{x}))
$$

Now, the problem has become to determine $h$. Mapping $h$ can be defined implicitly, by determining its relation on a distinctive subset of $\Omega$.

For a connected shape $S$, the scalar transformation $h$ can now be determined as follows. Select an arbitrary point a on $\partial S$, and determine its entire ray $\rho(\mathbf{a})$ - inward and outward. If $\mathbf{a}$ is a differentiable point, then there is exactly one unique ray. This ray follows from solving $\mathbf{p}(t)$ from: $\dot{\mathbf{p}}=\nabla \psi(\mathbf{p})$, with: $\mathbf{p}(0)=\mathbf{a}$. If $\mathbf{a}$ is notdifferentiable point, there is a continuum of rays. In that case, select one arbitrary ray ${ }^{14}$. Next, for the entire ray: $\rho(\mathbf{a})=\left\{\mathbf{p}(t) \mid t \in\left[0, t_{\operatorname{mon}}\right]\right\}$ determine the values for distancemap $D$ directly from its basic expression in definition $2.2: D(\mathbf{p}(t))=\| \mathbf{p}(t)-$ $\left.\mathbf{a} \|, t \in\left[0, t_{\max }\right]\right\}$. The values of $\psi$ on ray $\rho(\mathbf{a})$ are known from the solution of [2.8] and [2.9]: $\psi(\mathbf{p}(t))$. Together, this directly defines the mapping $h$ defined in [2.10]. In case of a connected print-shape $S$, the mapping $h$ can consequently be employed for all points $\mathbf{x} \in \Omega$. A point with field $\psi(\mathbf{x})$ thus has a distancemap-value: $D(\mathbf{x})=h(\psi(\mathbf{x}))$. It is possible that the ray $\rho(\mathbf{a})$ does not contain all possible - positive and negative values of $\psi$ in $\Omega$. In that case, the same procedure must be repeated for other $a \in \partial S$, until the mapping $h$ holds for all observed values of $\psi$.

For disconnected print-shapes $S$ the scalar transformation $h$ must be determined separately for each connected part $S_{j}$ as a local mapping $h_{j}$. Let $S$ consist of a disconnected collection of $J$ connected parts $S_{j}$ as: $S=\cup_{j} S_{j}$, with: $S_{i} \cap S_{j}=\varnothing$. Now, let $D_{j}$ be the distance-map of part $S_{j}$ if it were in isolation of all other parts: $D_{j}(\mathbf{x})=s_{j}(\mathbf{x})$. $\min \left\{\|\mathbf{x}-\mathbf{y}\| \mid \mathbf{y} \in \partial S_{j}\right\}$, where $s_{j}$ provides the sign as in definition 2.1. Now, define the cell $\omega_{j}$ of $S_{j}$ as the set of points in $\Omega$ closer to part $S_{j}$ than to any other part: $\omega_{j}=\{\mathbf{x} \in \Omega$ $\left.\mid D_{j}(\mathbf{x})=\inf _{j=1 . . J} D_{i}(\mathbf{x})\right\}$. The borders $\partial \omega_{j}$ of these cells are visible as ascending 'ridges' in the global surface $\psi(\mathrm{x}, \mathrm{y})$. These ridges can be determined directly from the

\footnotetext{
${ }^{13}$ Incidentally, this is the same equation as the stationary diffusion equation: $\nabla \Phi=-\Phi_{l}$, with separation: $\Phi(\mathbf{x}, t)=\phi(\mathbf{x}) \cdot T(t)$ we obtain [2.8] for the spatial part, and $T_{t}=-K T$ for the temporal part. Thus the same line of reasoning can be given for an interpretation like heat-transfer, where isodistance-contours correspond to isotemperature-curves. ${ }^{14}$ For instance: determine whether there exists a $\mathbf{b} \in \rho(\mathbf{a})$ such that $\psi$ is continuous and differentiable in a small neighbourhood $B(\mathbf{b}, \epsilon)$ of $\mathbf{b}$. Then solve $\mathbf{p}(t)$ from: $\dot{\mathbf{p}}=\nabla \psi(\mathbf{p})$, with: $\mathbf{p}(0)=\mathbf{b}$.
} 
field $\psi(\mathbf{x})$ using differential geometry. Now, for each cell $\partial \omega_{j}$ in $\Omega$ we can apply the method for a single connected shape $S_{j}$, described above, and thus find the local mapping $D_{j}(\mathbf{x})=h_{j}(\psi(\mathbf{x}))$.

The entire approach for calculating the continuous distancemap can now be represented in the following scheme.

Let $S$ be a printed shape on an image with domain $\Omega$.

i. Solve Helmholtz's equation: $\nabla^{2} \psi-\psi=0$ for all $\mathrm{x} \in \Omega$ with: $\psi \mid \partial S=1$, and: $\nabla \psi \perp \partial S$

ii. Partition $S$ in a set of disconnected parts $U_{j} S_{j}$ as follows. Determine the collection ' $Y$ ' of ridges in the surface of $\psi$. Together with the image border $\partial \Omega$ this defines the borders of the separate cells $\omega_{j}: \curlyvee \cup \partial \Omega \equiv \bigcup_{j} \partial \omega_{j}$.

iii. For each cell $\omega_{j}$ :

1. Determine the points with the highest, respectively lowest, local value of $\psi$ : $\mathbf{c}_{j}^{+}=\arg \max \left\{\psi(\mathbf{x}) \mid \mathbf{x} \in \omega_{j}\right\}$, and $\mathbf{c}_{j}^{-}=\arg \min \left\{\psi(\mathbf{x}) \mid \mathbf{x} \in \omega_{j}\right\}$, the associated border-points: $\mathbf{a}^{+/-}{ }_{j}=\arg \min \left\{\left\|\mathbf{x}-\mathrm{c}^{+/-}{ }_{j}\right\| \mid \mathbf{x} \in \partial S\right\}$, and the associated rays: $\rho_{j}^{+}=\rho\left(\mathbf{a}_{j}^{+}\right)$, and $\rho_{j}^{-}=\rho\left(\mathbf{a}_{j}^{-}\right)$.

2. For both rays determine $D$ directly from definition 2.2 : $D(\mathbf{x})=\min \{\|\mathbf{x}-\mathbf{u}\| \mid \mathbf{u} \in \partial S\}$, and construct the local mapping $h_{j}: \psi \rightarrow D$.

3. Apply mapping $h_{j}$ on all values of $\psi(\mathbf{x})$ in cell $\omega_{j}$, and thus obtain the full local distance-map $D \mid \omega_{j}$.

Finally, notice that because: $\|\nabla D\|=1$, the distancemap $D$ can also be derived from field $\psi$ using: $\nabla D(\mathbf{x})=\nabla \psi(\mathbf{x}) /\|\nabla \psi(\mathbf{x})\|$. Below are a few examples of this calculation.

Example 1. As an example we calculate the distancemap $D$ of a printed circle with radius ' $R$ '. First, we transform equation [2.8] to polar coordinates with the origin situated in the centre of the circle. Let: $F(r, \varphi)=\psi(x)$, with: $\mathrm{x}=(r \cos \varphi, r \sin \varphi)$. Then equation [2.8] can be rewritten as:

$$
F_{r r}+\frac{1}{r} F_{r}+\frac{1}{r^{2}} F_{\varphi \varphi}=F
$$

with boundary condition: $F(R, \varphi)=1$ for all $\varphi$. Next, we separate $F(r, \varphi)$ in a radial part $X(r)$ and an angular part $\Phi(\varphi): F(r, \varphi)=X(r) \Phi(\varphi)$, and thus obtain:

$$
\frac{r^{2} X_{r}}{X}+\frac{r X_{r}}{X}=1-\frac{\Phi_{\varphi \varphi}}{\Phi}
$$

Because of our special choice of the coordinate system we note that in this case: $\Phi(\varphi)$ $=$ constant $>0$, and thus obtain:

$$
r^{2} X_{r r}+r X_{r}-X=0
$$

The (real part of the) solution is: $X(r)=A r+B r^{-1}$, with constants $A$ and $B$. We dismiss the singular part, so $B=0$. The boundary-conditions for $F$ imply that for $X$ : 
$A R=1 \Rightarrow A=1 / R$, and for $\Phi: \Phi(\varphi)=1$. So: $F(r, \varphi)=r / R$, and: $\psi(\mathbf{x})=R^{-1} \sqrt{x^{2}+y^{2}}$, with: $\mathbf{x}=(x, y)$. For determining the global scalar mapping $h$, we compute the distancemap $D$ directly for the ray: $\mathbf{x}(\lambda)=\lambda(1,0)^{\top}, \lambda \geq 0$, and obtain: $D(\mathbf{x}(\lambda))=\lambda-R$. Using the expression for field $\psi$ we find for the same ray: $\psi(\mathbf{x}(\lambda))=\lambda / R$. Therefore, we find: $D=h(\psi)=R \psi-R$. Application of $h$ on the entire field $\psi$ gives the global expression for the distancemap: $D(\mathbf{x})=\sqrt{x^{2}+y^{2}}-R$.

Example 2. Consider the print-shape defined by the half-plane: $x \leq 0$. In that case the isodistance-contours are vertical lines, so the scalar field $\psi$ is only a function of $x$, and equation [2.8] becomes: $\psi_{x x}=\psi$, with solution: $\psi(x)=A e^{x}+B e^{-x}$. Application of the boundary-conditions at $x=0$ gives: $B=1-A$, and $A>1 / 2$. So: $\psi(x)=A e^{x}+(1-A) e^{-x}$. For the ray defined by the $x$-axis: $\mathbf{x}(\lambda)=\lambda(1,0) \mathrm{T}, \lambda \in \mathbb{R}$, we can compute the distancemap $D$ directly and obtain: $D(\mathbf{x}(\lambda))=\lambda$. Application of $\psi$ on the same ray gives: $\psi(\mathbf{x}(\lambda))=A e^{\lambda}+(1-A) e^{-\lambda}$. Therefore, $h$ is implicitly determined by: $\psi=A \exp (h(\psi))+(1-A) \exp (-h(\psi))$. Global substitution of $h$ in field $\psi$ gives the global distancemap: $D(\mathbf{x})=D(x, y)=x$.

Example 3. As an example of combining two disconnected parts, we now consider a print with a circle and a half-plane. Let $C$ be the circle with radius $R$, and centre: $\mathbf{m}=$ $(-m, 0)$, and $V$ the half-plane: $x \geq L$, let: $R-m<L$. The separatrix $S$ of $C$ and $V$ is: $S=$ $\left\{\mathbf{x} \mid D_{C}(\mathbf{x})=D_{V}(\mathbf{x})\right\}=\left\{(x, y) \mid \sqrt{(x+m)^{2}+y^{2}}-R=L-x\right\}$. This is a parabola with focal point $\mathbf{m}$ and as axis the negative $x$-axis. The separatrix is visible as a ridge in the map; on lines perpendicular crossing $S, D(\mathbf{x})$ assumes a maximum exactly on $S$. Tracking this optimum $\left(s_{1}, s_{2}\right)$ over $S$, it increases as: $D\left(s_{1}, s_{2}\right)=L-s_{1}$. The joint solution for the distancemap is found by separating left from right of the separatrix $S$. On the left-side $D(\mathbf{x})$ is defined by $D_{C}(\mathbf{x})=\|\mathbf{x}-\mathbf{m}\|-R$, and on the other side by: $D_{V}(\mathbf{x})=L-x_{1}$.

In most cases, however, this approach is too complex for an exact solution, and numerical approximations, for instance using Kirchhoff's integral formulation [Kirchhoff,1883] must be employed. We now have algorithmic (approaches 1,2a, 2b) and non-algorithmic (definition 2.2, equations [2.8]\&[2.9]) representations for computing the distancemap. Algorithmic approaches are valid for discrete, and nonalgorithmic for continuous problems. In real-world applications the image is discrete, such as a CCD-image, so the approach for the discrete distance-map is used. With that approach it was possible to efficiently compute complex distancemaps, as for instance in Figure 2.10, but at the lowest level round-off errors could create inhomogenities and oscillations, as for instance visible in Figure 2.11. 


\subsection{1-D Greyscale Transformations on Printed Images}

The introduction of a generalised gauge for printed images can be used to construct uniform transformations that transform printed images into a standardised onedimensional or two-dimensional representation. This representation can be used for dimension reduction, or to construct other, more powerful representations of printed images [Westra,1999d].

\subsubsection{The 1-Dimensional Cumulative Intensity Distribution Transform}

The distancemap $D$ provides an explicit distance for each point $\mathbf{x}$ on an image. This opens the possibility to represent some of the characteristics of an image as a function of the scalar $d=D(\mathbf{x})$, rather than of the position $\mathbf{x}$ on the image itself. Below, we describe a transformation that represents an image as a function of distance $d$ and constitutes in this way a template invariant representation of the image.
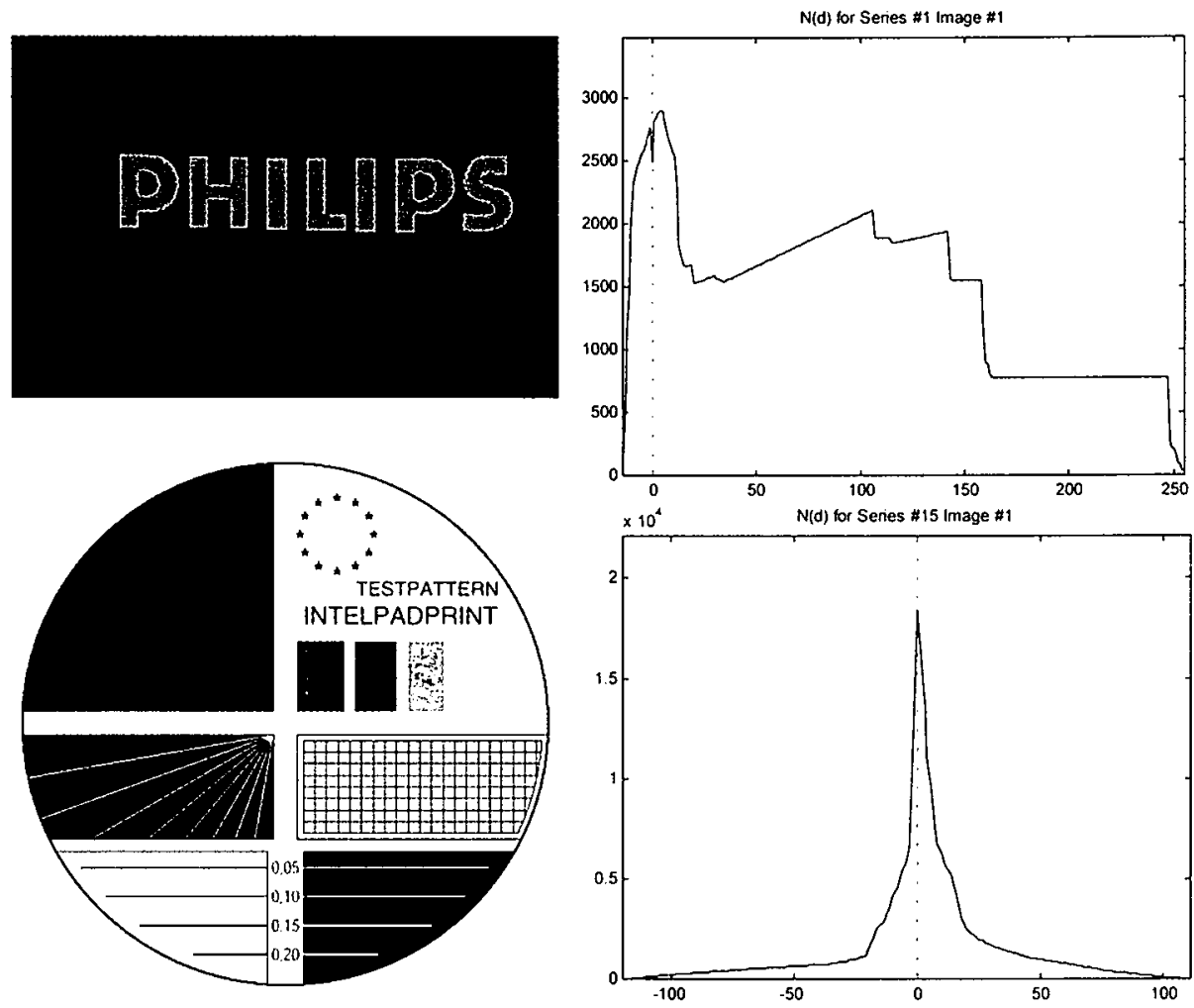

Fig. 2.16. Dependency of the number $N(d)$ of points in a template with distancemap-value $z$, for two different templates. This examples demonstrate that $N(d)$ depends on specific characteristics from the template.

We can now employ the distancemap to transform the absolute difference $Z(\mathbf{x})$ between an image $f(\mathbf{x})$ and its corresponding template $T(\mathbf{x})$ into a representation in printdepth $d$. Let $H_{d}$ be the isodistance-contour defined in the previous section: 


$$
H_{d}=\{\mathbf{x} \in \Omega \mid D(\mathbf{x})=d\}
$$

Then we can compute the integral sum of the absolute difference $Z(\mathbf{x})$ over $H_{d}$. This sum is called the Cumulative Intensity Distribution of the image, denoted $C(d)$ :

$$
C(d)=\int_{\mathbf{x} \in H_{d}} Z(\mathbf{x}) \mathrm{d}_{2} \mathbf{x}
$$

$\mathrm{C}(d)$ represents the cumulative intensity o a distance $d$ to the print-border. Using the distancemap $D$ and the Dirac $\delta$-function, this expression can also be written as:

$$
C(z)=\int_{\Omega} Z(\mathbf{x}) \cdot \delta(D(\mathbf{x})-z) \mathrm{d}_{2} \mathbf{x}
$$

For a given template, the transformation $C$ indicates the aggregated intensity as function as distance. The precise shape of $C$ as function of $z$ for a given defect, however, depends on the specific characteristics of the template.
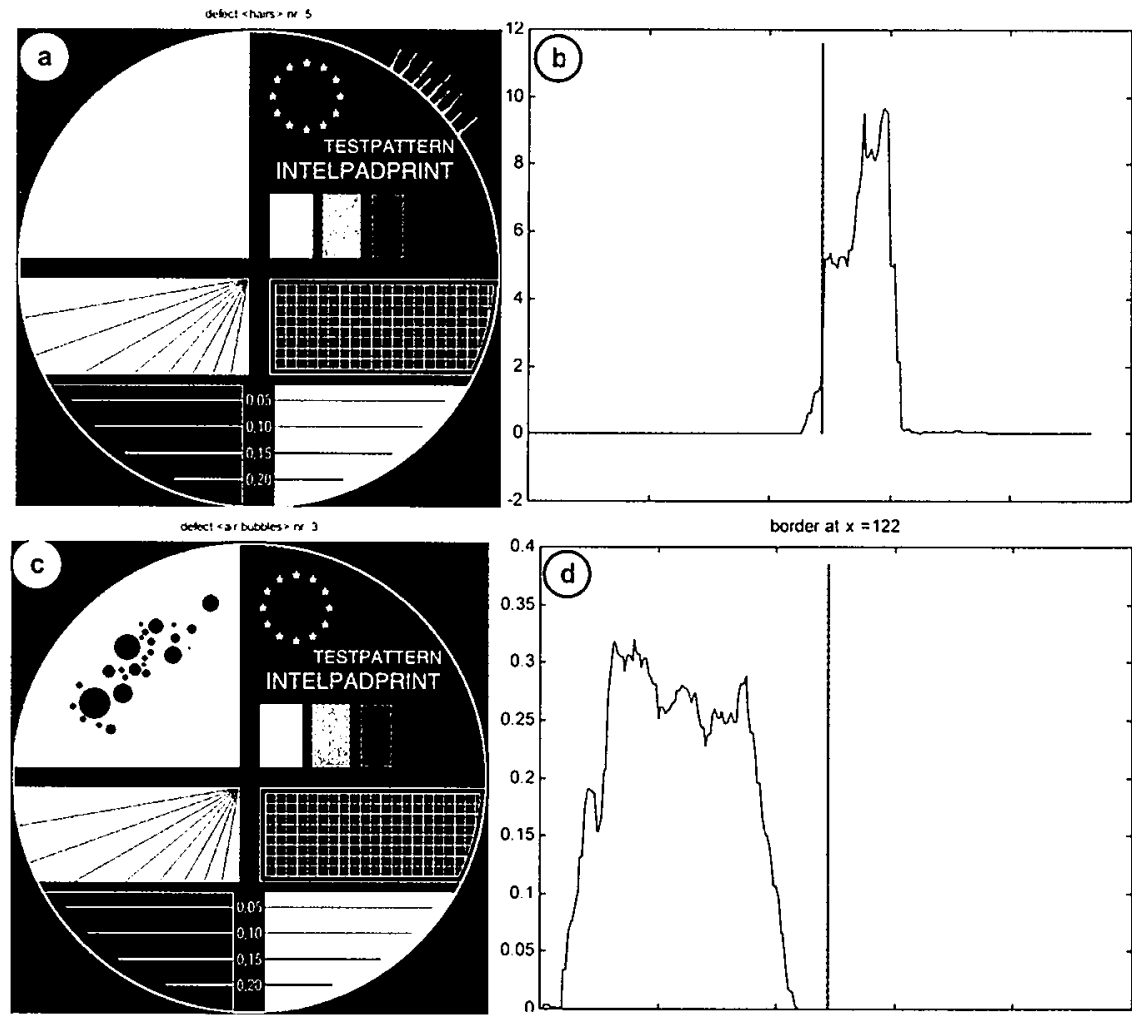

Fig. 2.17 (a): Print defect 'hairs', (c): Print defect 'air bubbles'. (b) and (d): Plot of intensity of difference as function of distance to printed area for respectively (a) and (c).

If we want to construct a template-free entity from $C$, we need to divide $C(z)$ by the measure of the set on the template with a given distance $z$, denoted by $N(z)^{15}$ :

\footnotetext{
Is In the discrete case this is the number of points with the given distance, in the continuous case it is the length of the curve on the distancemap that connects all points with the given distance.
} 


$$
N(z)=\int_{\mathbf{x} \in H_{d}} \mathrm{~d}_{2} \mathbf{x}=\int_{\Omega} \delta(D(\mathbf{x})-z) \mathrm{d}_{2} \mathbf{x}
$$

That the number $N(z)$ of points with given distance $z$ can differ tremendously amongst different templates is demonstrated by an example in Figure 2.16. The first template has much space around the print, so there are many points with large distance. The second template has much print-borders, so there are many points with small distances. For this reason we shall refer to $N(z)$ as the characteristic of the template. Using above definitions, we can write the expression for a Template-free or Relative Cumulative Intensity Distribution of the Difference - or short CID - denoted as $\Gamma(z)$ :

$$
\Gamma(z)=C(z) / N(z)
$$

The form $\Gamma(z)$ relates to the proportion of points on an image with print-depth $z$ that differ from the template, hence relative CID. It is clear that this approach is very sensitive to defects in the distancemap. In fact, the CID-profile needs to be corrected for the before-mentioned 'ghost'-image. Empirically, each template has one specific ghost-image $Z^{\text {ghrost }}(x)$. Therefore, the correction needs to be calculated only once. This correction, called the zero-artefact, is computed as:

$$
\Gamma{ }^{g h o s t}(z)=\int_{\Omega} Z^{g h o s t}(\mathbf{x}) \cdot \delta(D(\mathbf{x})-z) \mathrm{d}_{2} \mathbf{x} / N(z)
$$

and the correct CID is defined by:

$$
\Gamma^{*}(z)=\Gamma(z)-\Gamma^{g h o s t}(z)
$$

Henceforth, we always use this corrected form, and drop the asterisk. On first sight, it may appear possible to decrease the computation time of the CID by only considering those areas in the image $\Omega$ where the difference $Z$ with the template is above a lower threshold:

$$
W(\theta)=\{\mathbf{x} \in \Omega \mid Z(\mathbf{x})>\theta\}
$$

For some suitable, empirically determined threshold $\theta$. We can obtain a 'reduced' CID by applying the integrations in [2.12] on this sub-area $W$ rather than on the entire image $\Omega$. First, this actually causes the number of computations in equation [2.13] to drop as $\# W<\# \Omega$. Thereafter, however, both the reduced characteristic [2.14] and the reduced ghost-image [2.16] have to be calculated for each new image. This disadvantage effaces any benefit from the lessened summation.

In Figure 2.17 two examples of CIDs are shown. On the left side the defect is depicted $^{16}$, on the right side the cumulative intensity $\Gamma$ is plotted versus the distance $D$. The relation between the image of the defect and the resulting cumulative intensity plot is clear. For instance, with the defect 'hairs' most of the intensity is just outside the print (i.e. just right of the line demarcating the print border), whereas in defect 'air bubbles' the defect is totally located inside the printed area. In appendix B typical examples of more printing defects are shown. In all cases the plots are very distinct and characteristic.

With the CID-transform we have obtained a uniform and template-free representation of a defect. It represents the location of a defect as compatible with the PrintContexts; inside, near or outside the print border. Because of the independence of the CIDtransform of the characteristics of the image it offers the possibility to determine absolute characteristics of defects, independent of specific peculiarities of the image. This in turn offers the possibility to determine the CID-transform of a new image, and find out with which of the characteristic defects it shows the greatest degree of similarity.

\footnotetext{
16 The image, not the difference $Z$.
} 
We conclude that with the gauge $D$ we can obtain a useful one-dimensionalrepresentation of a print. The cumulative intensity distribution function $\Gamma(d)$ offers interesting possibilities for image processing.

Possibility 1: Filtering and Convolutions: $G(x)=\int \Gamma(y) \cdot K(x, y) \mathrm{d} y$

Possibility 2: Moments: $\omega_{n}=\int \Gamma(x) \cdot x^{n} \mathrm{~d} x$

Possibility 3: Transforms (Fourier and Wavelets): $a_{m n}=\int \Gamma(x) \cdot \psi_{m n}(x) \mathrm{d} x$

In the next sections we investigate two examples to demonstrate the use of this representation. First, we present a simple form of filtering. 


\subsubsection{Examples of Representations based on the 1D-CID-transform}

\subsubsection{A Tetrahedral Representation of Defects Based on the CID- transform}

The one-dimensional cumulative intensity representation described in the previous section allows some interesting possibilities for image processing. In this section we focus on a simple form of filtering.

Based on the distancemap-gauge we define a ordinary 4-band 'filter' that operates on the four PrintContexts defined in Section 2.2. First we define the total energy ' $E_{\Gamma}$ ' of the cumulative intensity distribution as:

$$
E_{\Gamma}=\int \Gamma(z) \cdot \mathrm{d} z
$$

In general this energy is not equal to the energy $E$ of the absolute difference ' $Z$ ',

$$
E=\int Z(\mathbf{x}) \cdot \mathrm{d}_{2} \mathbf{x}
$$

due to the division by $N(z)$ as in equation [2.14] In the first approach the cumulative intensity $E_{\Gamma}$ is subsequently 'filtered' in each of the four PrintContexts. Let ' $\xi_{k}$ ' denote the fraction of the energy $E_{\Gamma}$ in PrintContext ' $k$ ' - apart from a constant discussed below:

$$
\xi_{k} \propto \int \pi_{k}(z) \Gamma(z) . \mathrm{d} z
$$

Here $\pi_{k}$ denotes the fuzzy PrintContext -membership function as defined in Section 2.2.2. In this way we obtain a vector $\xi$ with four components $\left(\xi_{1}, \xi_{2}, \xi_{3}, \xi_{4}\right)$, where $\xi_{k}$ is proportional to the sum of the cumulative intensity $E$ in PrintContext $k$. A typical example of this approach is shown in Figure 2.18. Next, the proportionality factor implicit in equation [2.20] is chosen such that $\xi$ is normalised:

$$
\xi_{1}+\xi_{2}+\xi_{3}+\xi_{4}=1
$$

This very simple filter can be regarded as sampling in the four PrintContexts rather than a total measurement of the integral cumulative intensity.

Because the $\xi_{k}$ 's are normalised according to equation [2.21], and because $0 \leq \xi_{k} \leq 1$, the $4 \mathrm{D}$-vector $\xi$ can be represented in a 3D-pyramid as shown in Figure 2.18. This representation is called the $3 D$ Pyramidal Representation. Thus each point can be represented by 3 indices indicated as $\xi$.

For a set of more than 500 example images containing 18 printing-defects the four band filtered values $\xi$ ' were calculated and plotted in the pyramidal representation. These calculations clearly show that the 18 printing-defects indeed inhabit characteristic parts of the pyramid, so that we can conclude that specific regions of the pyramid correspond to specific defects.

This data-segmentation is used in section 4.7.1 to model some defects.

\footnotetext{
${ }^{17}$ But equal to the energy $\mathrm{E}_{\mathrm{C}}$ of the absolute $\operatorname{CID} \mathrm{C}(\mathrm{z})$, as follows directly from equation [2.13] and the basic properties of $\delta$-function: $\int \delta(\mathrm{z}) \mathrm{dz}=1$ (normalization), and $\delta(-\mathrm{x})=\delta(\mathrm{x})$ (symmetry). In practice, due to round-off errors during the calculation of the $\mathrm{C}(\mathrm{z}), \mathrm{E}_{\mathrm{C}}$ may slightly differ from $\mathrm{E}$.
} 


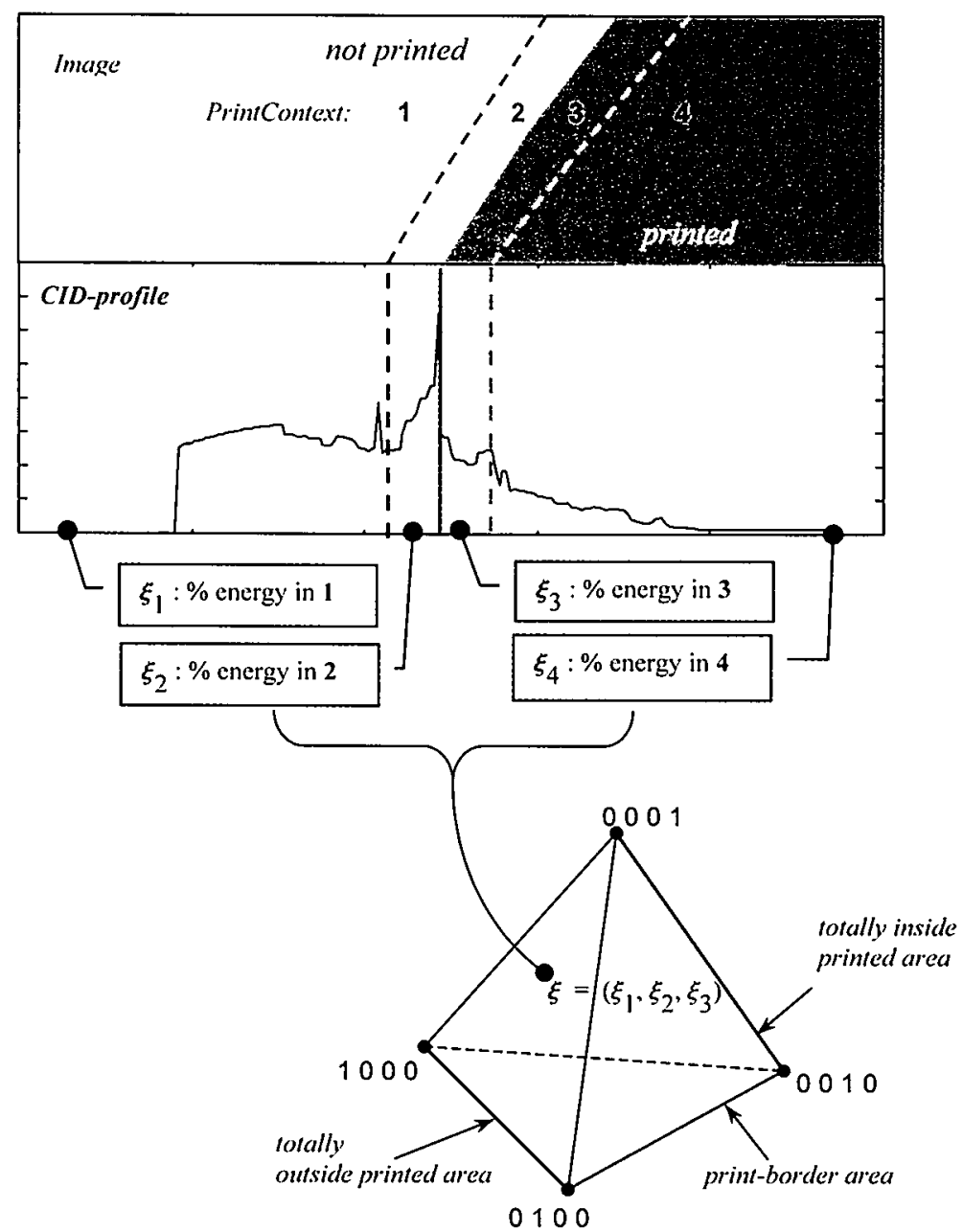

Fig. 2.18. Transformation of the image of a printed object into a point $\xi$ in the $3 D$-pyramidal representation. This transformation proceeds in four stages. [1] Above an image of a print, with the printed part in grey. The dashed lines indicate the four PrintContexts. [2] Second, the cumulative intensity plot, with vertically the integrated intensity, and horizontally the distance $d$. Horizontally, four bands corresponding with the four PrintContexts are indicated. [3] In each band the cumulative intensity can be integrated to obtain the cnergy in printcontext ' $i$ ' called $\xi_{i}$. [4] Below, the 3D-pyramidal representation of the 4D-vector $\left(\xi_{1}, \xi_{2}, \xi_{3}, \xi_{4}\right)^{\top}$. The corners are marked by their 4D-coordinates. The edge between (1000) and (0100) represents defects that occur totally outside of the print, similar for the other edges. The representation of the image is indicated as a point $\xi$ in the pyramid. 


\subsubsection{A Planar Representation of Defects Using the CID-transform}

We can also apply a straight-forward transformation on the CID-representation of an image in order to obtain a template-free characterisation of defects. We do this by determining the optimal fit of the 1D-CID-function $\Gamma(x)$ with a simple block-function. This block is defined by a function $B(x)$ with three variables $\left(L_{1}, L_{2}, h\right)$ as follows:

$$
\begin{aligned}
& x \in\left[L_{1}, L_{2}\right] \Leftrightarrow B\left(x, L_{1}, L_{2}, h\right)=h \\
& x \notin\left[L_{1}, L_{2}\right] \Leftrightarrow B\left(x, L_{1}, L_{2}, h\right)=0
\end{aligned}
$$

Next we define an algorithm $O p t B l o c k F i t$ that determines the block $B^{*}$ that minimises the quadratic criterion:

$$
Q\left(L_{1}, L_{2}, h\right)=\int\left(\Gamma(z)-B\left(z, L_{1}, L_{2}, h\right)\right)^{2} d z
$$

So:

$$
\left(\mathrm{L}_{1} *, \mathrm{~L}_{2} *, \mathrm{~h}^{*}\right)=\arg \min _{\mathrm{L} 1, \mathrm{~L}, \mathrm{~h}} \mathrm{Q}\left(\mathrm{L}_{1}, \mathrm{~L}_{2}, \mathrm{~h}\right)
$$

and: $B^{*}(x)=B\left(x, L_{1}, L_{2}, h\right)$, is the 'best' block in the sense of $Q$. The 'fit' of the best match $B^{*}$ is defined as:

$$
\mathrm{Q}^{*}=\mathrm{Q}\left(\mathrm{L}_{1}{ }^{*}, \mathrm{~L}_{2} *, \mathrm{~h}^{*}\right)
$$

The algorithm OptBlockFit establishes the solution to equation [2.25] by simply applying LSQ with criterion $Q$ from equation [2.23]:

$$
\partial \mathrm{Q} / \partial \mathrm{L}_{1}=0 \wedge \partial \mathrm{Q} / \partial \mathrm{L}_{2}=0 \wedge \partial \mathrm{Q} / \partial \mathrm{h}=0
$$

Application of this approach therefore produces four numbers $\left(\mathrm{L}_{1}^{*}, \mathrm{~L}_{2}{ }^{*}, \mathrm{~h}^{*}, \mathrm{Q}^{*}\right)$ that characterise the image. The algorithm OptBlockFit thus induces a transformation $B^{*}$ from the set of possible CID-functions to $I^{4}$. In Figure 2.19 a typical example of this approach is shown. From a clustering of a large number of printing defects in this representation it will later in Chapter 4 become clear that the features $L_{1}$ and $L_{2}$ contain significant information about the defect class. Consequently, we restrict our representation initially to $\left(\mathrm{L}_{1}{ }^{*}, \mathrm{~L}_{2}{ }^{*}\right)$, and investigate the distribution of the defects in the $L_{1} L_{2}$-plane. In combination with the CID-transform $\Gamma$, and the Z-transform ${ }^{18}$, the composition $\mathrm{B}^{*} \circ \mathrm{\Gamma} \circ \mathrm{Z}$ is a transformation from the set of possible images $F f: \Omega \rightarrow[0,1]$ of a given template $T$ to $\mathrm{IR}^{2}$ as:

$$
\mathrm{B}^{*} \circ \Gamma \circ \mathrm{Z}: F \rightarrow \mathrm{IR}^{2}: f \rightarrow\left(\mathrm{L}_{1}{ }^{*}, \mathrm{~L}_{2}{ }^{*}\right) \text {. }
$$

This means that this approach generates a significant dimension-reduction from $F$ to $I^{2}$. The $L_{1} L_{2}$-plane can be divided in several areas of interest. Note first that, by definition, $\mathrm{L}_{2} \geqslant \mathrm{~L}_{1}$. Therefore, we only need to be concerned with the half -plane above $\mathrm{L}_{1}=\mathrm{L}_{2}$. In this upper half-plane there are three main areas of interest. Defects that lie totally outside the print have $L_{1}>0$. Likewise, defects that lie totally inside the print have $L_{2}<0$. Defects that overlap printed and not-printed parts of an image are found in the area $\left\{L_{1}<0\right\} \cap\left\{L_{2}>0\right\}$. Defects that lie just outside the printed area, like 'hairs', can be found in the area: $\left\{L_{1} \geq 0\right\} \cap\left\{L_{2}<\varepsilon\right\}$ for some small $\varepsilon$. Similarly, defects just inside the print, like 'edge roughness', can be found in the area: $\left\{L_{1} \geq\right.$ $\varepsilon\} \cap\left\{\mathrm{L}_{2} \leq 0\right\}$.

\footnotetext{
${ }^{18}$ The calculation of the absolute difference $Z(x)$ can be regarded as a transformation on the image ' $\mathrm{f}$ ' and template ' $T$ ', defined as: $Z(f, T)=|\mathrm{f}-\mathrm{T}|$
} 
Based on these considerations we conclude that, a priori, it is to be expected that specific defects inhabit specific parts of the $\mathrm{L}_{1} \mathrm{~L}_{2}$-plane. The distribution of printingdefects in the $\mathrm{L}_{1} \mathrm{~L}_{2}$-plane, and suitable techniques for structuring them are studied in more detail in Section 4.7.2.

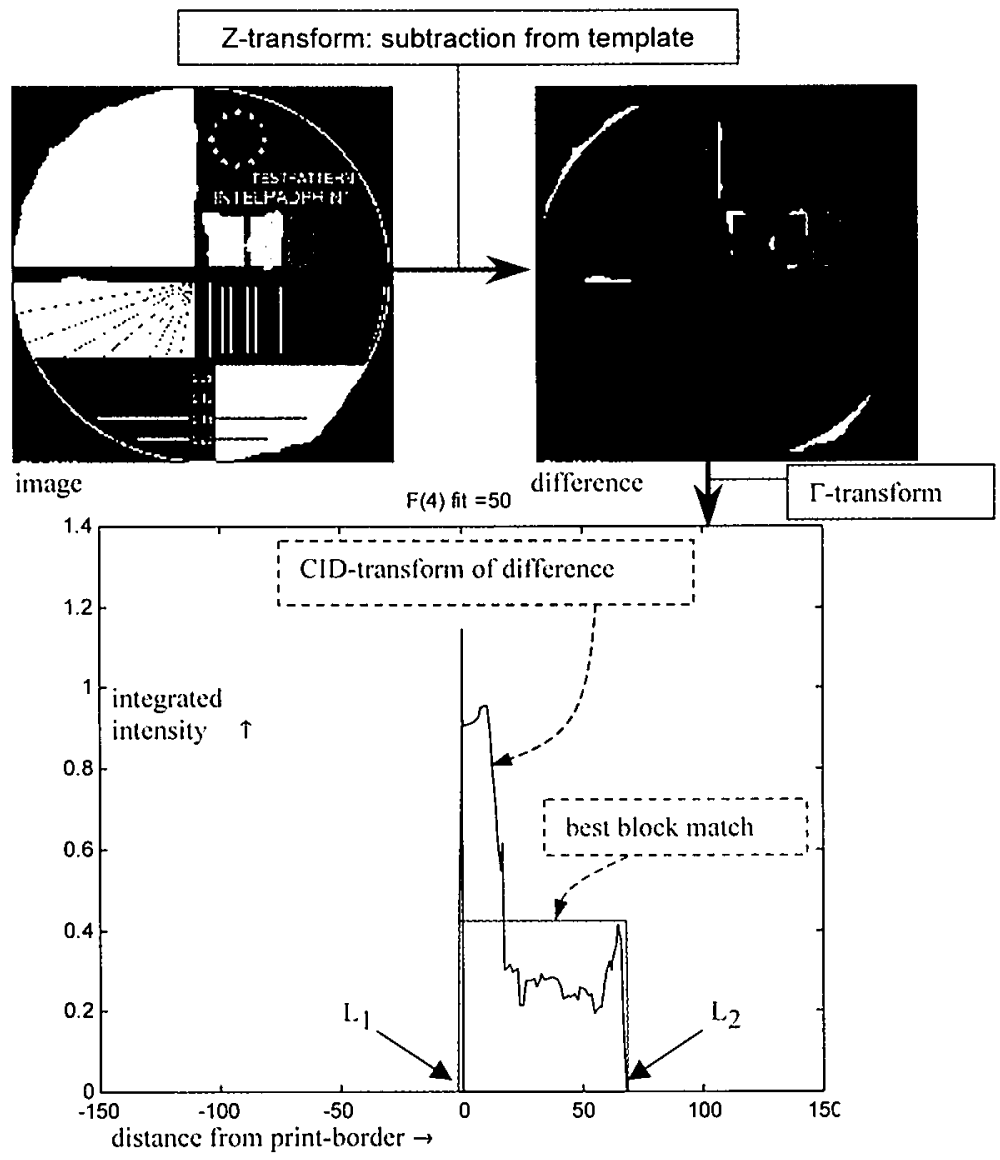

Fig. 2.19. The composed 1 'ansformation of $Z, \Gamma$ and $B^{*}$. After subtraction from the template, the absolute difference ' $Z$ ' is transformed using the CID-transform $\Gamma$. The resulting CIDprofile is matched with a block $\mathrm{B}^{*}$. 


\subsection{A 2-Dimensional Greyscale Transformations for Printed Images.}

Now that we can successfully transform an image into a one-dimensional weak template-independent representation using the CID-transform, we investigate whether it is possible to construct a comparable two-dimensional transformation. Such a transformation would open a perspective on approaches that could focus on this twodimensional representation, irrespective of all characteristics and details of the template. Constructing a two-dimensional analogon of the CID-transformation, however, is mathematically complex. The complications include distortions and cuts in the image, the treatment of local optima in the distancemap, the treatment of disconnected parts of the image, smoothly connecting the interior and exterior mapping at the print-border, the calibration of the origin of the mapping, and excessive computing times. These problems make it difficult to provide a general applicable and practical usable approach for all possible templates. We propose a partial continuous transformation from a template to a uniform 2D-representation, which we call the 2D-Intensity Map, short 2DI-map. However, we show that when there are closed isodistance-contours (IDCs) in the distancemap, this mapping will always be discontinuous. Similarly, the mapping is discontinuous for certain assemblies of multiple disconnected parts. Moreover, near local optima in the distancemap, image distortions always occur, even when isometric scaling is applied. These conditions severely limit the practical applicability of a 2DI-approach.

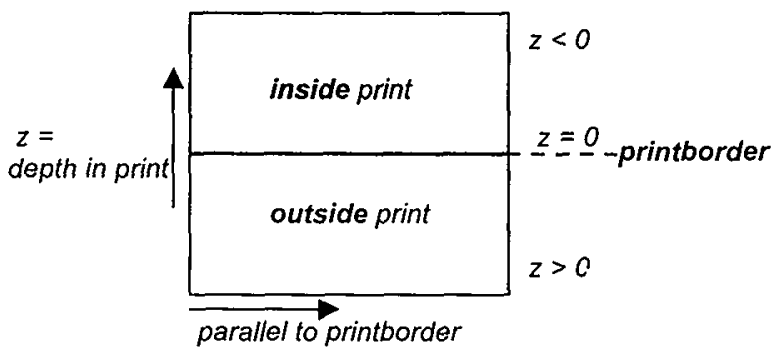

Fig. 2.20. Template-free 2D-representation of a print, denoted as the $2 D / M a p$.

\subsubsection{Mathematical Complications of a 2D-Parameterisation of an Image}

Let us now attempt to construct a two-dimensional generalisation from the onedimensional cumulative intensity transform. Such a 2D-transform can be based on a suitable general 2D-parameterisation of an image. Starting point is the following couple of observations: (i) the 'print-depth' $z$ on distancemap $D$ can be considered as a dimension perpendicular to the print-border. The local direction of that axis is directed along the 'rays' emanating from the print-border. In differentiable points these rays are directed along $\nabla D$. Rays were defined in definition 2.5 in Section 2.5.5 for points a on the print-border. For points $\mathbf{a} \in \Omega$ where $D$ is differentiable, and which do not lie on the print-border, we can compute the rays $\rho(\mathbf{a})$ passing through them as parameterisations $\mathbf{x}(t)$ with: 


$$
\dot{\mathbf{x}}=\nabla D(\mathbf{x}), \mathbf{x}(0)=\mathbf{a},
$$

with a time-interval $T=\left[t_{1}, t_{2}\right]$ with $t_{1}<0, t_{2}>0$, such that $D$ is differentiable on $T$. Then, according to Theorem 2.2:

$$
\forall(t \in T)\|\nabla D(\mathbf{x}(t))\|=1 .
$$

The second observation is that: (ii) the isodistance-contours (IDCs) at distance $z$ to the print-border can be considered as a generalised parallel dimension. In definition 2.4 in Section 2.5.5 these contours were defined by:

$$
H_{z}=\{\mathbf{x} \in \Omega \mid D(\mathbf{x})=z\} .
$$

A 2-dimensional representation is attractive, because it would depict all defects in a uniform, template-free way on a well-defined 2-dimensional map, as depicted in Figure 2.20. This would open the way for a general approach to defect classification, that would focus solely on the 2DI representation, and would search for discriminating characteristics of defect classes. However, there are some formidable obstacles that complicate such a 2D-approach.

[1] The first problem concerns the calibration of the parallel axis. There is freedom in selecting the location of the origin on the parallel axis on a closed print-border. This can be avoided by adopting a decision criterion. An example is the following procedure. Let $\mathbf{a}(\lambda)$ be a suitable continuous parameterisation of print-border $\partial A$ for $\lambda \in \Lambda \subset \mathbb{I R}$. Define a quadratic criterion:

$$
Q_{N}(\lambda)=\sum_{n=1}^{N}\left(\frac{\partial^{n} \mathbf{a}(\lambda)}{\partial \lambda^{n}}\right)^{2}
$$

Now, select as parallel origin the point $\mathbf{a}(\lambda)$ on $\partial A$ with the parameter-value $\lambda_{0}$ :

$$
\lambda_{0}=\inf \arg \inf Q_{N}(\lambda)
$$

[2] The second problem occurs when a printed shape $S$ contains multiple disconnected parts. Then the possibility arises that the 2DI-map becomes discontinuous. Close outside the border $\partial S$, there will exist disconnected IDCs associated with the different disconnected parts of $S$. Therefore, just outside the print-border, the parallel axis in the 2DI-map is partitioned in non-overlapping intervals $V_{i}$, where $V_{i}$ is the interval associated to the ' $i$-th' disconnected part of $S$. This partitioning is such that the union of all intervals constitutes the entire parallel axis, and the intervals do not mutually intersect. Farther away from the print-border, the IDCs will merge ${ }^{19}$. The desired 2DI transform must be such, that this merger will not cause any reshuffling of the lateral axis. This means that if the IDCs of two parts $i$ and $j$ merge, their corresponding intervals must merge also. Hence, these two intervals must be neighbouring, and either: $\sup V_{i}=\inf V_{j}$, or: $\sup V_{j}=\inf V_{i}$. For this reason, the aforementioned freedom of selecting the origin on the lateral axis does not apply for each of these disconnected parts. However, when more than two IDCs connect simultaneously, i.e. for the same print-depth $D$, this argument fails. This is because the associated intervals can not be placed in a linear sequence such that their union corresponds to a continuous parameterisation of the joined IDC. For this reason it is not possible to provide a unique indexing of simultaneously-splitting disconnected parts.

${ }^{19}$ If they do so within the frame of the image. 
[3] The third problem stems from discontinuities caused by splitting of the IDCs near local optima in the distancemap. Let $V$ represent the collection of local optima of $D$, including boundary-extremes. Then, somewhere in-between the print-border and these optima the IDCs will split-up, as they converge towards the individual optima in $V$. This can cause discontinuities in the intended 2DI-mapping. In order to be continuous at the point where two interior IDCs split, the two segments on the parallel axis of the 2DI-map, representing the IDCs in the image, must join smoothly. This is similar to the joining of two intervals in the previous case. Therefore, the same problem regarding the unique indexing applies, as multiple IDCs cannot be joined by a continuous 2DI-mapping. In fact, these two problems are identical, for both deal with local disconnected optima. We deal with this problem later in more detail. We already remark that in areas where the IDCs are closed curves the 2DI-mapping are always discontinuous.

[4] The number of interior and exterior local optima, i.e. the number of maxima and minima of distancemap $D$, in general differ. Segments of the print-border that border multiple exterior and interior optima must therefore be cautiously joined smoothly on the 2DI-map. This is especially true for disconnected parts of the print.

[5] The fifth problem concerns the connection of the positive with the negative perpendicular axis. This may cause a discontinuity in the 2DI-mapping. Problems [4] and [5] also cause that the indices ' $k$ ' for interior or exterior rays $\rho^{+\gamma-}$, from Definition 2.5 , and scaling-functions $N_{k}(z)$, defined in expression [2.14], are totally unrelated. This in its turn means that, generally, inner and outer rays, meeting on the same local print-boundary have different indices. Again, we deal with this problem later in more detail.

[6] The last problem is of a practical nature. It concerns whether the generated 2DItransformation is congruent or non-congruent. Non-congruent transformations distort the image, possibly beyond recognition. A true template-free analysis of an image requires a congruent transformation. Let $\phi(\mathbf{x})$ be the sought $2 \mathrm{DI}$ transform and let $J(\mathbf{x})$ denote its Jacobian. An isometric transformation obeys: $\forall \mathbf{x} \in \Omega \mid$ det $J(\mathbf{x}) \mid=1$. A congruent transformation is isometric, but the opposite need not be the case. The difference can be caused by inhomogeneous scaling-factors $N(z)$. A transformation realised by scaling the IDC at distance ' $z$ ' with the characteristic ' $N(z)$ ' - i.e. the length of the IDC - is isometric but not necessarily congruent. For this reason, we therefore always use the interval $[0, N(z)]$ to map the IDC on the 2DI-map. The overall scaling-factor $N(z)$ entirely depends on properties of the template, and as such lessens the desired template-free nature of the transform. In Figures 2.23-2.26 examples of congruent and non-congruent transformations of the same image are shown. The starting-point of the parameterisation has been fixed by remarks [1] and [2] above. 


\subsubsection{Relation between the 2DI Transform and the 2D- Parameterisation}

The distancemap $D$ can be considered as a kind of geometry introduced on the image space $\Omega$. It creates a field of IDCs and orthogonal gradients on the image. Our approach for constructing a 2DI-map is based on the 2D-parameterisation of this field. Let $\mathbf{X}(\lambda, z)$, with $\lambda \in[0, N(z)]$, represent the general parameterisation of a IDC on printdepth $z$. This means that $\mathbf{X}$ maps the interval $[0, N(z)]$ to the entire IDC $H_{z}$. Now, let $Z(\mathbf{x})$ represent the absolute difference in greyscale between image $f$ and its template $T$ in point $x$ on the image: $Z(x)=|\ell(x)-T(x)|$. Then the transformation: $\phi(\lambda, z)=$ $Z(\mathbf{X}(\lambda, z))$ represents a valid $2 D I$ transform from the image to the map: $\left[0, N_{\max }\right] \times$ $\left[d_{\min }, d_{\max }\right]$. This transformation is isometric, but in general not congruent. The vertical axis is directed perpendicular to the IDC $H_{z}$ defined by the distancemap $D$. Notice that by theorem 2.2 the gradient $\partial_{z} \mathrm{X}=1$, so the vertical component always contributes to isometric transformations. Therefore, in the case of above parameterisation $\mathbf{X}$, the local Jacobean is: $J(\lambda, z)=\left|\partial_{\lambda} \mathbf{X}\right|=1$.

Like in the 1D-case, in all practical situations the map must be corrected for ghostimages. This is performed analogous to equation [2.17]. Let $Z$ ghost $(x)$ be the ghostimage, and $\phi{ }^{g h o s t}(z)$ its $2 \mathrm{DI}$ transform. Then the corrected 2DI transform is:

$$
\phi^{*}(\mathbf{z})=\phi(\mathbf{z})-\phi \text { ghost }^{(\mathbf{z})}
$$

Again, in the remainder we drop the asterisk, and always assume $\phi$ is subtracted.

\subsubsection{Definitions for an Algorithmic Approach for the 2DI-Mapping}

In the next sections we construct an algorithmic approach for computing the 2DItransform. We start with a few useful definitions and propositions.

Definition 2.7: The 'interior' $H^{*}$ of a closed IDC $H$ is the connected set with boundary $H: \partial H^{*}=H$. If $w(\mathbf{x}, H)$ represents the winding-number of $\mathbf{x}$ relative to $H$, then: $H^{*} \equiv\{\mathbf{x} \in \Omega \mid w(\mathbf{x}, H) \neq 0\}$.

Definition 2.8: Let $\mathrm{x}_{1}, \mathrm{x}_{2}, . ., \mathrm{x}_{N}$ be a collection of points on distancemap $D$. Then curve $H\left(\mathbf{x}_{1}, \mathbf{x}_{2}, . ., \mathbf{x}_{N}\right)$ is the IDC with smallest possible distancemap-value, such that $H^{*}$ contains all points: $H\left(\mathbf{x}_{1}, \mathbf{x}_{2}, . ., \mathbf{x}_{N}\right) \equiv\left\{\mathbf{x} \in \Omega \mid D(\mathbf{x})=d^{*}\right\}$, with: $d^{*}=\sup \left\{D\left(\mathbf{x}_{1}\right), D\left(\mathbf{x}_{2}\right), \ldots, D\left(\mathbf{x}_{N}\right)\right\}$.

Definition 2.9: Let $\mu$ be a local optimum of a distancemap $D$, including boundary extremes. Then the 'basin' $\beta$ of $\mu$ is the set of image-points for which $\mu$ is the global optimum inside its interior, such that both $\mathrm{x}$ and $\mu$ lie on the same side of the print-border: $\beta(\mu)=\left\{\mathbf{x} \in \Omega \mid \mu \subset H^{*}(\mathbf{x}) \wedge s(\mu)=s(\mathbf{x}) \wedge D(\mu) s(\mu)=\sup D\left(H^{*}(\mathbf{x})\right) s(\mathbf{x})\right\}$ with $s$ the print-membership function, defined in definition 2.1. Notice that a basin can be completely enclosed in some other basin, containing a superior optimum.

Equipped with these definitions, we can proof the following two theorems. 
Theorem 2.3: Suppose, $x_{1}, x_{2}$, and $x_{3}$ are points on a distancemap $D$, such that:

$$
\begin{aligned}
& D\left(\mathbf{x}_{1}\right) \leq D\left(\mathbf{x}_{2}\right) \leq D\left(\mathbf{x}_{3}\right) . \\
& \text { Then either: [1] } H\left(\mathbf{x}_{1}, \mathbf{x}_{2}\right) \subset H^{*}\left(\mathbf{x}_{3}\right), \\
& \text { or: [2] } H\left(\mathbf{x}_{1}, \mathbf{x}_{2}, \mathbf{x}_{3}\right)=H\left(\mathbf{x}_{1}, \mathbf{x}_{2}\right) .
\end{aligned}
$$

Proof:

[1] Suppose: $D\left(\mathbf{x}_{2}\right)<D\left(\mathbf{x}_{3}\right)$. Then by definition $2.4 H\left(\mathbf{x}_{1}, \mathbf{x}_{2}\right)$ is an IDC with distancemap-value $D\left(\mathbf{x}_{2}\right)$, so: $H\left(\mathbf{x}_{1}, \mathbf{x}_{2}\right)=H\left(\mathbf{x}_{2}\right)$. Let: $d=\left(D\left(\mathbf{x}_{2}\right)+D\left(\mathbf{x}_{3}\right)\right) / 2$, and: $r=D\left(\mathbf{x}_{3}\right)$. As: $D\left(\mathbf{x}_{1}\right)<D\left(\mathbf{x}_{2}\right) \leq D\left(\mathbf{x}_{3}\right)$, it is clear that: $H\left(\mathbf{x}_{1}, \mathbf{x}_{2}\right) \subset H_{d}{ }^{*}$, and that: $H_{d} \subset H_{r}{ }^{*}$. Therefore: $H\left(\mathrm{x}_{1}, \mathrm{x}_{2}\right) \subset H_{r}{ }^{*}=H\left(\mathrm{x}_{3}\right)^{*} \square$

[2] Suppose: $D\left(\mathbf{x}_{2}\right)=D\left(\mathbf{x}_{3}\right)$. Since: $D\left(\mathbf{x}_{1}\right) \leq D\left(\mathbf{x}_{2}\right)$, we know that: $H\left(\mathbf{x}_{1}, \mathbf{x}_{2}\right)=H\left(\mathbf{x}_{2}\right)$. Since: $D\left(\mathbf{x}_{2}\right)=D\left(\mathbf{x}_{3}\right)$, we know that: $H\left(\mathbf{x}_{2}\right)=H\left(\mathbf{x}_{3}\right)=H\left(\mathbf{x}_{1}, \mathbf{x}_{2}, \mathbf{x}_{3}\right)$. So: $H\left(\mathbf{x}_{1}, \mathbf{x}_{2}\right)=$ $H\left(\mathbf{x}_{1}, \mathbf{x}_{2}, \mathbf{x}_{3}\right) \square$

Theorem 2.4: In areas where the IDCs are closed curves the 2DI-mapping is discontinuous.

\section{Proof:}

Suppose that $H$ is a closed IDC. Then $H^{*}$ is a compact area containing local optima of the distancemap $D$, for the negative rays extending inside $H^{*}$ will have to converge locally. Let $M$ be the absolute optimum inside $H^{*}$. Then some ray ' $\rho$ ' originating c.q. ending in $M$, will act as starting-point for the parameterisation of all IDCs crossing $\rho$. Let IDC $h$ correspond to a parameterisation: $\mathbf{x}(\tau)$, with: $\tau \in\left[t_{1}, t_{2}>\right.$, and: $t_{2}-t_{1}=$ length $(h)$. Let $\mathbf{a} \equiv h \cap \rho \neq \emptyset$. Then both:

$$
\lim _{\tau \downarrow_{1}} \mathbf{x}(\tau)=\mathbf{a} \text {, and: } \lim _{\tau \uparrow t_{2}} \mathbf{x}(\tau)=\mathbf{a} \text {. }
$$

Let $B(\mathbf{a}, \epsilon)$ be a $\epsilon$-neighbourhood of a, with $\epsilon \ll$ length $(h)$. Then the intersection $h \cap B$ is mapped on the two disconnected intervals: $\left[t_{1}, t_{1}+\epsilon_{1}\right\rangle U\left\langle t_{2}-\epsilon_{2}, t_{2}\right\rangle$ which suffice: $0<\epsilon_{1}, \epsilon_{2} \ll$ length $(h)$. Consequently, the entire neighbourhood is mapped in two disconnected parts in the 2DI-map $\square$

\subsubsection{Four Different Scenarios for Computing the 2DI-Map- Coordinates}

One of the complexities involved in computing the 2DI-mapping of an image point is that we can distinguish different conditions for the calculation. These conditions are composed of the two opposing pairs: insideloutside the print, and single connected/multiple disconnected parts. For each of the four possible combinations, we point out the major associated problems, and define a suitable approach. In all cases, the perpendicular coordinate of a point simply equals the value of its distancemap.

\section{A. Determining the 2DI-Coordinates in the Interior of a Single Connected Part.}

In this case the parallel coordinate depends on two factors.

[1] The first factor concerns the relative position of the point on its resident IDC. This again depends on the location of the local starting-point of the parameterisation on its IDC, which is determined by the intersection of the main-ray and the IDC. Then, the 
relative position is determined by clockwise measuring the distance to the intersection. In the case of a not-closed IDC, an additional scalar transformation is applied on this distance in order to avoid negative values.

[2] The second factor concerns the order of the resident IDC in the ensemble of all IDCs. The IDCs converge towards the collection of local minima inside the printshape, including the border extremes. Notice that a minimum can be a single point or a continuous segment of a $1 \mathrm{D}$-curve. The minima inside a print-shape can be envisaged as a branching tree, stemming from a single root at depth zero at the printborder, and branches ultimately extending to the deepest minima, see Figures 2.21 and

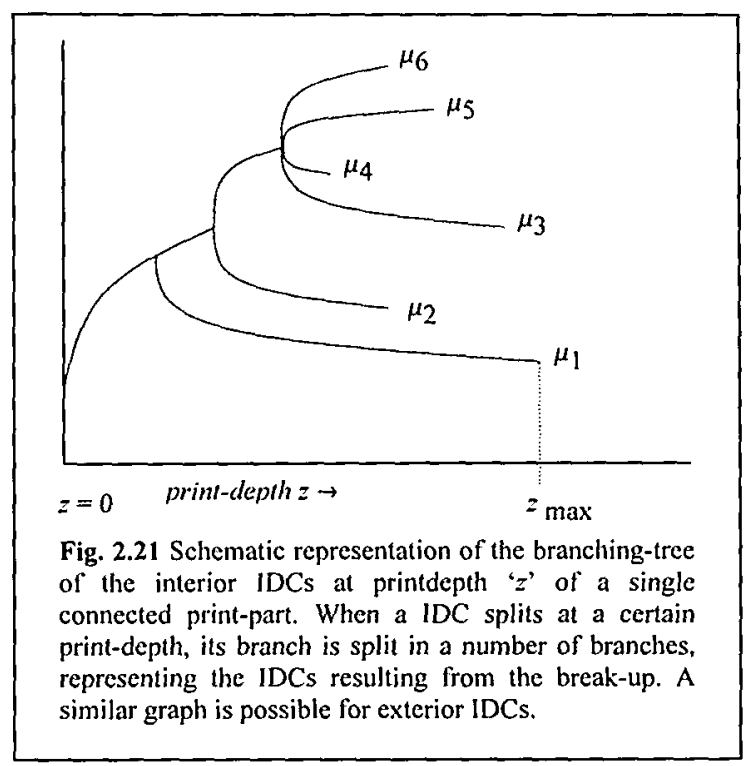
2.22. If we want to map the IDCs to intervals of the parallel axis, we need merging branches to be neighbouring intervals. Even though we know that closed IDCs cause discontinuities by the cutting effect of their main-ray, this coupling increases the local continuity of the mapping. For this reason we must compute the branching-tree of the local minima. This branching-tree concerns the intervals mapped from the IDCs. It is a diagram in the plane of print-depth $z=D(\mathbf{x})$, versus the lower value of the interval representing the associated local minimum.

So, it is a diagram in the 2DI-plane. The length of an interval is the length of the associated IDC. If two IDCs merge, the two corresponding intervals merge. Therefore, local continuity is promoted if the upper-value of the interval coincides with the lower part from the next. The branching-tree is computed with an algorithm specified below. First, the branching-tree is constructed in the 2DI-plane, such that different branches do not intersect. Next, the branches are numbered, starting from below. Thus, we have implicitly assigned a rank to the entire collection of IDCs inside a connected shape, by indicating the order of its associated local minimum in the branching-tree.

Finally, if both factors are known, the absolute parallel position of a point follows from the sum of its relative position on its resident IDC, increased with the cumulative sum of the lengths of all IDCs with lower rank..

\section{B. Determining the 2DI-Coordinates in the Interior of a collection of Disconnected Parts.}

If a print-shape consists of a collection of non-overlapping print-shapes, the approach sketched above can be applied to each individual connected part in the collection. This requires that first the collection is indexed. Subsequently, for each part the 
scaling-function must be determined, i.e. the cumulative length of all its IDCs at printdepth $z$. The 2DI-coordinates are then calculated as follows.

The parallel coordinate depends on two factors. First, the order of the connected part in the entire collection making op the total, disconnected, print-shape. This order determines the cumulative length of IDCs at print-depth $z$ of the parts with lower index than the present part. Second, this cumulative length is increased with the outcome of approach $\mathrm{A}$, applied on the part containing the point.

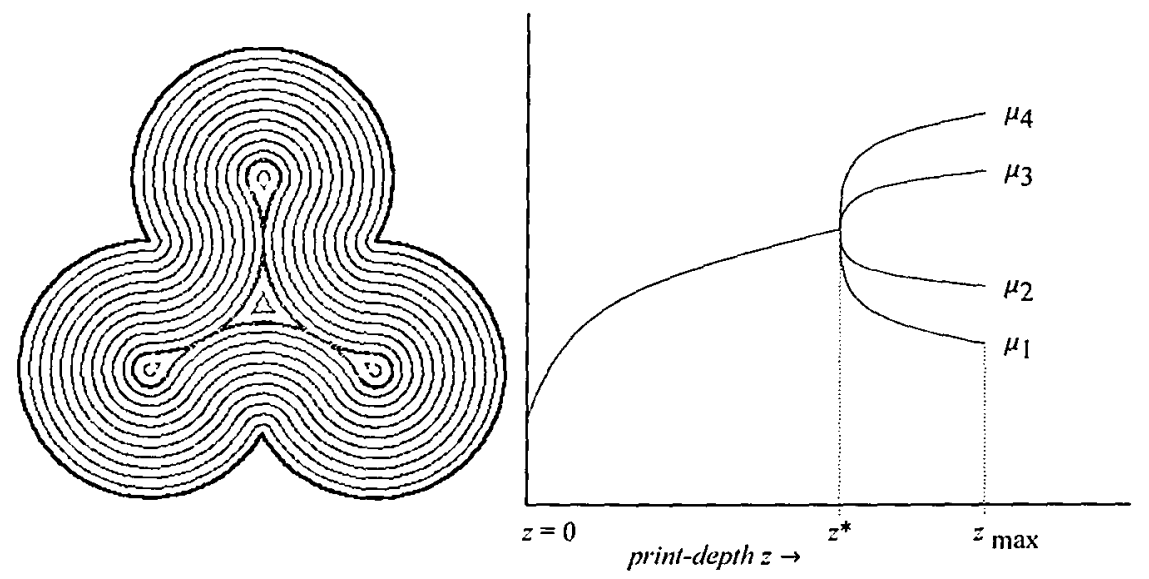

Fig. 2.22 Left: IDCs inside a three-lobed print-shape. The border of the printshape is outlined by the bold outer curve. From the border up to a certain print-depth $z^{*}$ the IDCs are closed curves. At print-depth $z^{*}$ (indicated by fat curve) the IDC splits up in four disconnected curves that further converge to the four local minima. Right: Graph of the interior IDCs at printdepth ' $z$ ' of the three-lobed print-shape. When the IDC splits at print-depth $z^{*}$, the single branch splits in four pieces, representing the four IDCs resulting from the break-up. At the maximum print-depth $z_{\max }$ the IDCs have converged to their minima $\mu_{1}, \mu_{2}, \mu_{3}$, and $\mu_{4}$.

\section{Determining the 2DI-Coordinates in a Single Connected Exterior Region.}

The approach for calculating the 2DI-map for a point outside the print is identical to approach $A$, provided we exchange in for out, minimum for maximum, and $+D(\mathbf{x})$ for $-D(\mathbf{x})$. Local maxima can originate from non-printed areas enclosed inside a printed area, as for example in the numeral ' 8 '. Connecting the positive with the negative perpendicular axis, however, poses a new and severe problem. This problem concerns a possible discontinuity in the 2DI-mapping. It can assume two forms.

i. If both the interior and exterior of a print-shape are single connected areas, then the problem is how to connect the axes on the print-border. Both axes in fact are rays, stemming from global optima connected to the print-border. However, the intersection of inner and outer ray with the print-border will, in general, differ. Since these intersections determine the origin on the 2DI-map, the upper and lower limit for approaching the origin via the perpendicular axis will differ. A solution can be found in determining the value of the exterior perpendicular axis on the boundary, measured over the print-border. Let a be the intersection of the interior main-ray with the print-border: let $\mathbf{m}$ be the global minimum in the interior of the print-shape $S$, and let ray $\rho^{-}$be 
determined by: $\rho^{-}=\rho(\mathbf{m})$. Now, let a be the intersection of $\rho^{-}$with the printborder: $\mathbf{a}=\rho^{-} \cap \partial S$. Then, print-border $\partial S$ can be parameterised measuring from a as: $V(s \in \partial S) \exists !\left(\lambda \in \mathrm{IR}^{+}\right) \mathbf{s}=\mathbf{x}(\lambda)$. Similarly, let $\mathbf{M}$ be the global maximum in the exterior of $S$, and let ray $\rho^{+}$be: $\rho^{+}=\rho(\mathbf{M})$. Define the intersection of $\rho^{+}$with the print-border as: $\mathbf{b}=\rho^{+} \cap \partial S$. Let $\lambda^{*}$ be the parameter such that: $\mathbf{b}=\mathbf{x}\left(\lambda^{*}\right)$. Since: $\mathbf{a}=\mathbf{x}(0), \lambda^{*}$ represents the parallel coordinate of the entire ray $\rho^{+}$. Therefore, the exterior IDCs $H$ are parameterised clockwise from their intersection with main-ray $\rho^{+}$, such that the parameter on $\rho^{+} \cap H$. assumes the value $\lambda^{*}$. This guarantees continuity at the print-border $\partial S$.

ii. If the interior and/or exterior of a print-shape consists of a collection of disconnected areas, then above approach can be applied to lock the axes of each of the local objects, be it that the intersections are not determined by the perpendicular axis itself, but by a suitable ray, representing a line in the 2DImap parallel to the perpendicular axis, that intersects the object. A problem is that the rays $\rho^{-}=\rho(\mathbf{m})$ and $\rho^{+}=\rho(\mathbf{M})$, from the global minimum $\mathbf{m}$ and global maximum $\mathbf{M}$ need not meet on the same local segment of $\partial S$. Therefore, we have to make a choice for the origin of the 2DI-map. We thus define the origin a of the 2DI-map as the intersection of the interior ray $\rho^{-}=\rho(\mathbf{m})$, stemming from the global minimum $\mathbf{m}$, with the exterior ray $\rho^{+}$with highest possible length. Let $R$ denote the border of the part containing the global minimum $\mathbf{m}$. The exterior point $\mathrm{y}_{0}$ most distant to $R$ is: $\mathrm{y}_{0}=\arg ! \max \{D(\mathrm{y}) \mid \mathrm{y} \in \Omega \wedge \mathrm{s} \in R \wedge$ $\left.\mathbf{s} \in \rho^{+}(\mathbf{y})\right\}$. Then we define: $\mathbf{a} \equiv \rho^{+}\left(\mathbf{y}_{0}\right) \cap R$, where $\rho^{+}\left(\mathbf{y}_{0}\right)$ is a ray connecting $\mathbf{y}_{0}$ with $R$.

\section{Determining the 2DI-Coordinates in a Collection of Disconnected Exterior Regions.}

In a similar fashion this approach is identical to approach $B$, i.e. when we exchange in approach $\mathrm{B}$ in for out, minimum for maximum, the sign of the distancemap $D$, and connect the interior and exterior axis with the recipe described above in approach $\mathrm{C}$. 


\subsubsection{Algorithmic Formulation of the General 2DI-Mapping}

We can now formulise an approach for 2DI-mapping. We do this in an algorithmic way, expressed in natural language. Suppose, we wish to determine the 2DI-mapping $\phi$ of a point $\mathbf{x} \in \Omega$ to its $2 \mathrm{DI}$-coordinates $\mathbf{z}=\left(z_{1}, z_{2}\right)$ as: $\mathbf{z}=\phi(\mathbf{x})$.

\section{Partition and Index all Disconnected Parts:}

Identify the disconnected parts $D_{n}$ in the map $D$, and partition the map in cells $\omega_{n}$ for which these parts are the most proximate part: $\omega_{n}=\left\{\mathbf{x} \in \Omega \mid D(\mathbf{x})=\inf _{k} D_{k}(\mathbf{x})\right\}$. Now, index all cells (ergo parts), i.e. provide all cells $\omega_{n}$ with a unique $n$. This can not be done in a uniform way if more than two cells join 'simultaneously' (i.e. at same printdepth $\left.z_{2}\right)$. In such cases an arbitrary decision criterion must be designed. Let $N_{L}\left(z_{2}\right)$ denote the cumulative length of the IDCs at printdepth $z_{2}$ of cell $\omega_{L}$ with index ' $L$ '.

\section{For each disconnected cell with index $L$ :}

\section{1a. Partition the Local Minima}

Determine all local minima - including boundary extremes: $M=\left\{\mu_{1}, \mu_{2}, \ldots, \mu_{N}\right\}$, ordered such that $D$ forms a monotonous non-decreasing sequence: $D\left(\mu_{1}\right) \leq D\left(\mu_{2}\right) \leq \ldots \leq D\left(\mu_{N}\right)<0$.

Determine the intradistance-matrix $d: d_{i j}=\min \left\{\|\mathbf{x}-\mathbf{y}\| \mid \mathbf{x} \in \mu_{i} \wedge \mathbf{y} \in \mu_{j}\right\}$

For each minimum $\mu_{k} \in M$ :

- determine the centre-of-mass:

$\mathbf{m}_{k}=\int_{\mu[k]} \mathbf{x} d_{2} \mathbf{x} / \int_{\mu[k]} d_{2} \mathbf{x}$

- determine a suitable ${ }^{20}$ vector $\mathbf{v}_{k}$ that can function as initial direction for the main-ray $\rho_{k}$ starting at $\mathbf{m}_{k}$.

- determine basin $\beta_{k}$ of $\mu_{k}$, as defined in definition 2.9 .

- determine the local scaling-function $n_{k}\left(z_{2}\right)$ that gives the length of the ICD at depth $z_{2}$ inside the basin $\beta_{k}$. The domain of this function assumes values only for values of $z_{2}$ that fall outside the basin.

\section{1b. Partition the Local Maxima}

Partition in a similar way the local maxima.

\section{2a Determine the Branching Tree of the Local Minima}

As discussed above, it is not possible to uniquely index the local optima inside the cell of a part. This is only possible for binary splitting IDCs. Let $\# M=N$ be the number of local minima inside a part, and let $\operatorname{rank}(n)$ indicate the index of the $n$th minimum. Then the algorithm below computes the unique fusion for binary-splitting IDCs.

\footnotetext{
${ }^{20}$ For a line segment this may be a normal vector, for a point we may define some rule, as for instance a vector pointing away from the centre of the image.
} 


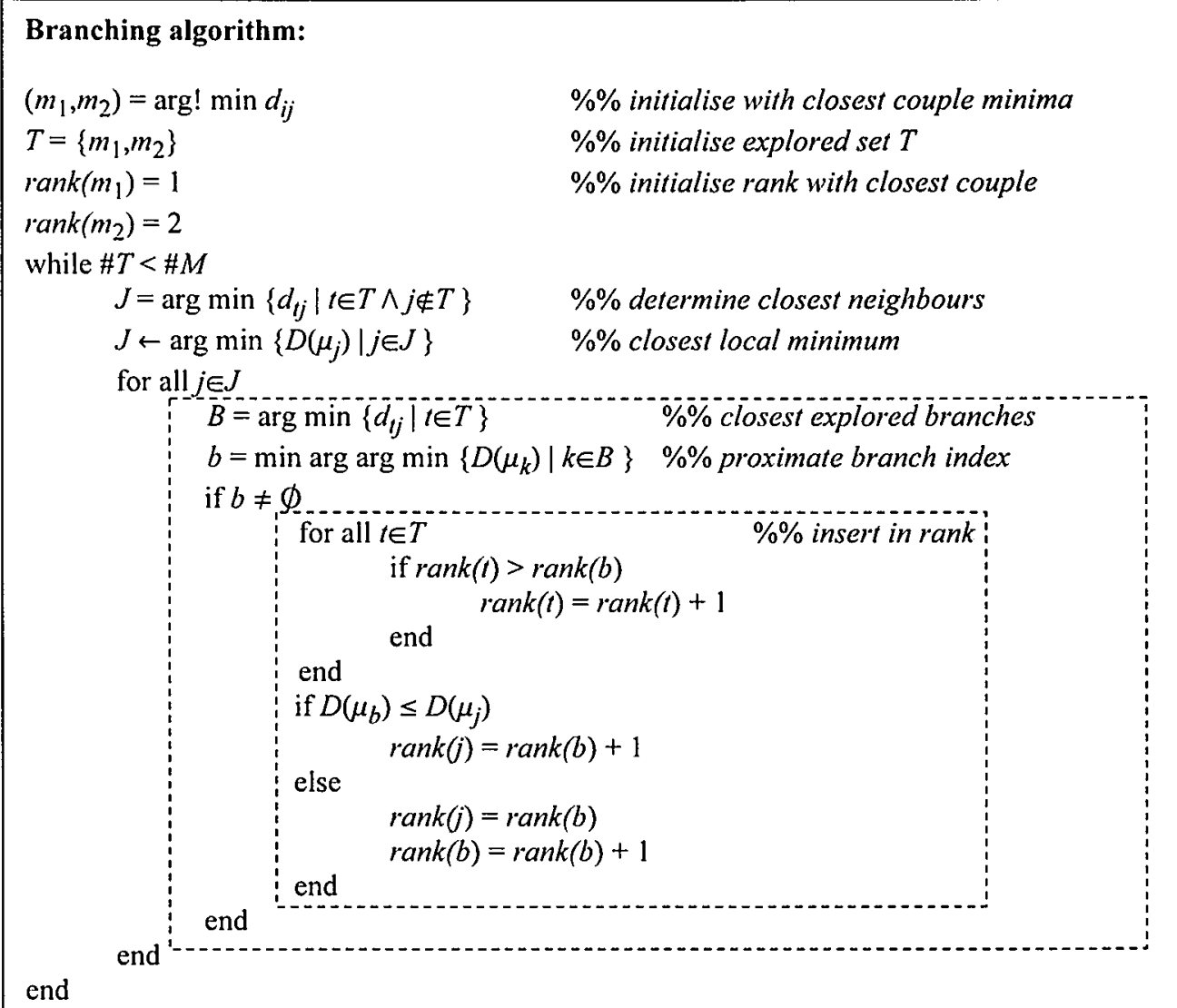

\section{2. $2 b$ Determine in a similar way the Branching Tree of the Local Maxima}

2.3 Determine the Relative Position of $\mathrm{x}$ on its Resident IDC

The relative position of $\mathbf{x}$ on its IDC follows from determining:

- the perpendicular coordinate: $z_{2}=D(\mathbf{x})$,

- the index ' $L$ ' of the disconnected part in the ensemble of print-parts,

- the resident IDC of $\mathbf{x}: H(\mathbf{x})$,

- the optima inside $H(\mathbf{x}): M \cap H^{*}(\mathbf{x})$,

- the index $K$ of the global optimum in $H(\mathbf{x}): K=\arg \arg \min D\left(M \cap H^{*}(\mathbf{x})\right)$,

- the main-ray emerging from the minimum $\mu_{K}: \rho_{K}$,

- the intersection of the main-ray $\rho_{K}$ and the local IDC: $\mathbf{a}=\rho_{K} \cap H(\mathbf{x})$,

- parameterisation of the local IDC $H(\mathbf{x}): \frac{\partial \mathbf{u}}{\partial \lambda}=\left(\begin{array}{cc}0 & -1 \\ 1 & 0\end{array}\right) \nabla D(\mathbf{u})$ with: $\mathbf{u}(0)=\mathbf{a}$,

- the value of $\lambda$ of the IDC in $\mathbf{x}: \lambda(\mathbf{x})$, this is the relative position of $\mathbf{x}$.

\subsection{Determine Global Parallel Position of $\mathrm{x}$ :}

Finally, the parallel coordinate of $\mathbf{x}$ is: $z_{1}=\lambda(\mathbf{x})+\sum_{k=1}^{K-1} n_{\operatorname{rank}(k)}\left(z_{2}\right)+\sum_{l=1}^{L-1} N_{l}\left(z_{2}\right)$ 


\subsubsection{Evaluation of the 2DI-Mapping}

At first sight the 2DI-map appears attractive and concise, mainly because the representation - not the transform - is completely template-free. However, this approach suffers from serious mathematical complexities, and exhibits inherent discontinuities, distortions and segmentations. This distortion and cutting especially affects those defects that have a well-defined and characteristic shape, like the typical spherical 'air bubbles'. Even the isometric mapping that we have constructed can considerably distort an image, as demonstrated in Figure 2.23. The largest distortions and discontinuities are found near local optima of the distancemap $D$. This is caused by the decrease in length of the IDCs as they approach a local optimum, and by the free calibration of the parallel axis. An awkward additional problem is the 'slicing' of digital images in horizontal segments, clearly visible in Figure 2.24. In many cases this practical problem overshadows the negative effects of the distortion and cutting, which we so meticulously tried to relegate in the sections above.

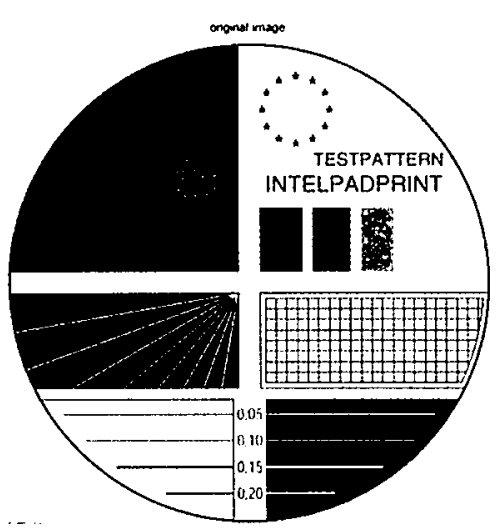

Area-false $2 D \mid$ map

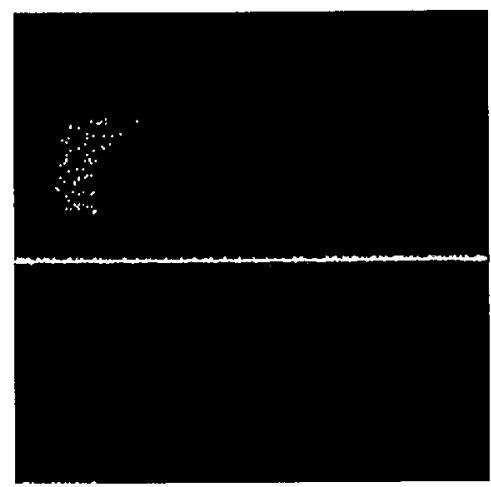

Area-true 2DI map

Fig. 2.23. Distortions due to decreasing length of interior IDC contours.

An image of the printing defect 'dirt particles', i.e. the collection of dots in the upper left quarter of the image. Clockwise from above left:

(i) the original image.

(ii) A non-isometric representation, where no scaling with the 'characteristic' $N(z)$ is applied.

(iii) An isometric 2DI-transform with scaling with $N(z)$.

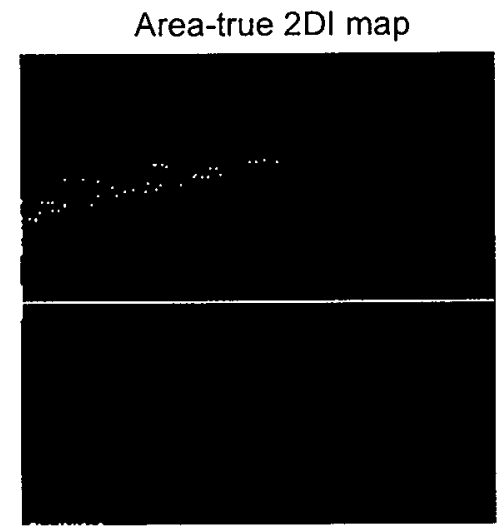

These defects stem from the limited resolution of discretised images, but also to even the weakest of the imperfections present in the distancemap. Also small variations in the ghost-image confuse the mapping. A general disadvantage of the 2DI-approach is the massive computation times it requires. This is especially true for the, in principle off-line, construction of the geometrical transform, $\phi(\lambda, z)$, defined in Section 2.7.5. 
The 2DI-mapping can be regarded as a global map of an image. An attractive alternative is to instead consider a collection of local maps [Milnor, 1969]. These maps can for instance be constructed from the individual cells of the disconnected print-parts, or the basins of the individual optima. The individual 2DI-maps of this 'quasi-Voronoi' partitioning of the print can be analysed separately. Each of these maps can be constructed to be continuous, besides a cut for closed IDCs. The problem then becomes to combine the analysis of all these parts into one synthesis.

In the Figures 2.24 to 2.26 some examples of the $2 \mathrm{DI}$ transforms for three printing defects are depicted. These figures show that, despite the problems of the 2DImapping, the algorithm was in general reasonable accurate.

Finally, notice that there is a simple relation between the $1 D$ - transform $\Gamma(z)$ and the 2Dl- transform $\phi(\lambda, z)$ :

$$
\Gamma(z)=\int_{0}^{N(z)} \phi(\lambda, z) d \lambda
$$

For all these reasons the $1 D$-CID appears more practicable, even as it lacks the attractive 'parallel' dimension of the 2DI-map. 
original image

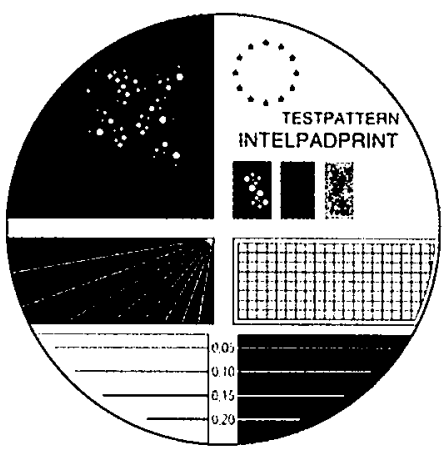

Area-true 2DI map

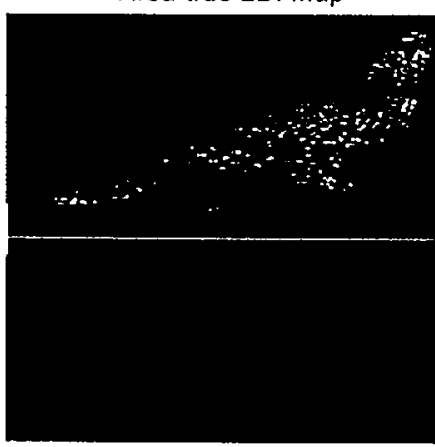

Area-false 2DI map

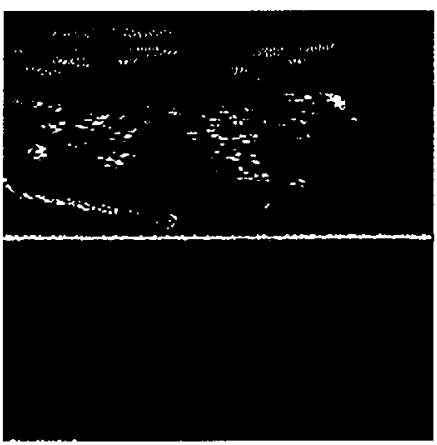

Fig 2.24. An example of a manufactured image of defect air bubbles with its associated 2DI transforms, above 'congruent', below 'non-congruent.

original image
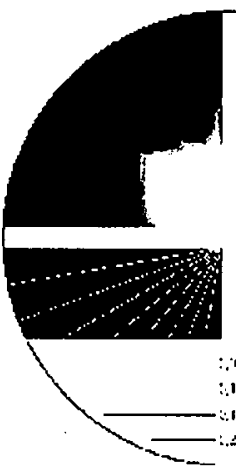
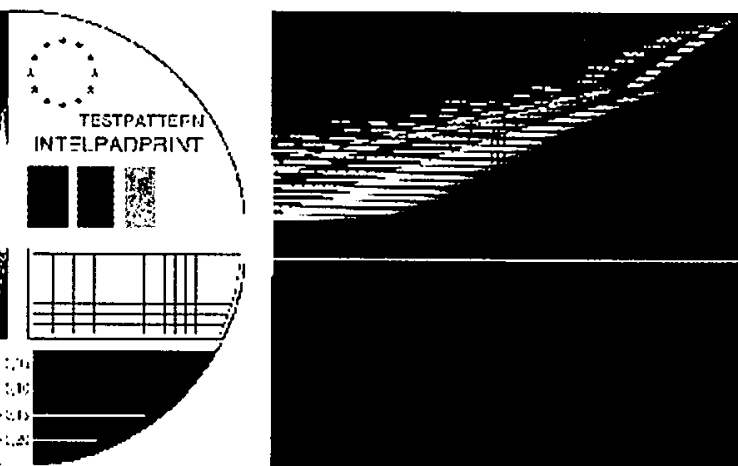

Area-true 2DI map

Fig. 2.25. This real image of defect 'ink missing' clearly shows effects of segmentation and deformation, especially in the deepest (upper) part of the map. Left: a congruent transformation, right: a non-congruent transformation.

orig in a 1 im age

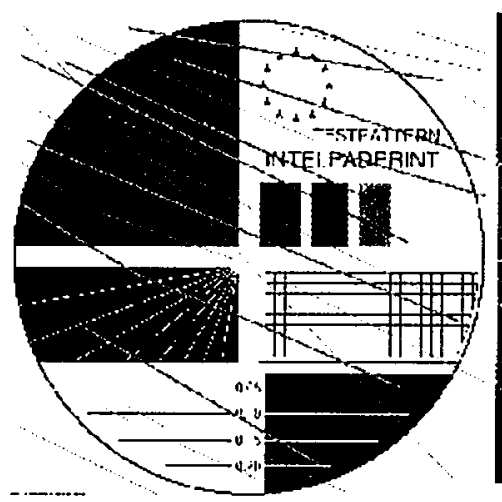

Area-true 201 map

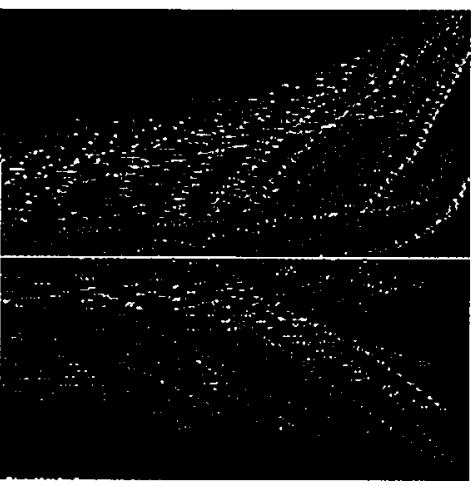

Area-false 2DI map

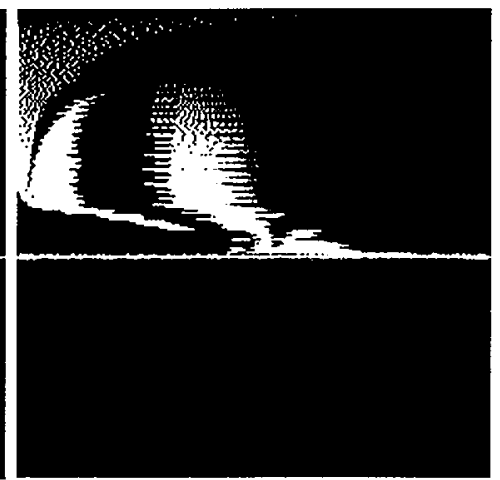

Fig. 2.26. Image of defect streaks of lines. From left to right: [1] adapted digital image of defect, [2] congruent 2DI map, [3] non-congruent 2DI mapping. The 2DI map clearly shows the effects of segmentation and deformation, especially in the deepest (upper) part of the map. Segmentation is strongest in congruent map, and deformations is strongest in the noncongruent map. 


\subsubsection{Potential Applications of the 2DI Map}

The 2DI-transform can potentially be employed in searching the characteristic features of specific defects. Two of such applications are discussed in this section.

\subsubsection{Fourier and Wavelet Transformations on the 2DI Map}

First, we apply the 2D-Fourier transform to the 2DI representation of a defect. Let $\phi(\mathbf{z})$ represent the 2DI-map at point $\mathbf{z}$. Then the Fourier-transform $f$ of $\phi$ is:

$$
f(\mathbf{k})=(2 \pi)^{-1} \int_{\Omega} \phi(\mathbf{z}) \mathrm{e}^{-i k . z} \mathrm{~d}_{2} \mathbf{z}
$$

In Figures 2.27-2.28 some examples are shown. From these images it is hard to find any conspicuous information. The quality of the Fourier-transform is seriously impaired by imperfections in the 2DI map, which in its turn are largely caused by small defects in the distancemap. Another approach based on Fourier-transform is to define a cut-off frequency $k_{\mathrm{min}}$, and ignore all higher - or lower - frequencies, and subsequently apply the inverse Fourier-transform. In this way higher - or lower frequencies are filtered from the image. Again, this approach did not give convincing results, due to imperfections in the map. Results are shown in Figures 2.29.

\subsubsection{Special Purpose Filters on the 2DI Map}

In this approach special filters were designed to detect specific defects, based on the integration or convolution of the 2DI map with this filter. In many cases it is interesting to decompose the analysis of an image in a horizontal and a vertical direction. Therefore, a particular suitable form for special purpose filters is:

$$
\psi_{k}(\omega)=\int_{\Omega} \phi(\mathbf{z}) \pi_{k}(z) b(\omega, \lambda) \mathrm{d}_{2} \mathbf{z}
$$

where $\mathbf{z}$ is a point on the 2DI-map with coordinates: $(z, \lambda)$, with $z$ the print-depth, and $\lambda$ the parallel coordinate. $\pi_{k}(z)$ is the 'fuzzy' membership-function for PrintContext $k$, defined in Section 2.2.2. Function $b$ relates to the parallel effect of the 2DI map. This filter is suitable for defects situated in PrintContext $k$, with a specific parallel characteristics that can be captured in function $b(\omega, \lambda)$. An example of a such a special purpose filter is given in equation [2.30]. This filter is specially designed for the detection of a printing defect called 'hairs'.

$$
h(\omega)=(2 \pi)^{-1} \int_{\Omega} \phi(\mathbf{z}) \pi_{2}(z) \mathrm{e}^{-i \omega \lambda} \mathrm{d}_{2} \mathbf{z}
$$

'Hairs' occur just outside the print, and are characterised by their quasi-periodic behaviour parallel to the print-border with relative high frequency. Therefore the first term in the filter is $\pi_{2}(z)$, the 'fuzzy' membership-function for 'just outside the print', as depicted in Figure 2.2. The second term $\mathrm{e}^{-i \omega \lambda}$ focuses on periodic signals with frequency $\omega$. This filter therefore is sensitive to periodic signals parallel to the nearoutside of the print. Function $h$ is the 'spectrum' obtained from filtering the 2DI transform of the image. Therefore, $h$ is characterised by clear localisation in the higher frequency-domain when compared with other characteristic defects. Figure 2.29 shows two characteristic examples of 'hairs'. These images clearly show the distortions and segmentations of the 2DI transform. Fore these reasons this filter did not give good results. Also other filters based on equation [2.29], specially designed for 'bubbles' or 'lines' did not give noteworthy results. In general, this approach suffered from the many defects in 2DI algorithm, especially the 'slicing' and inhomogeneous deformation. 
original image

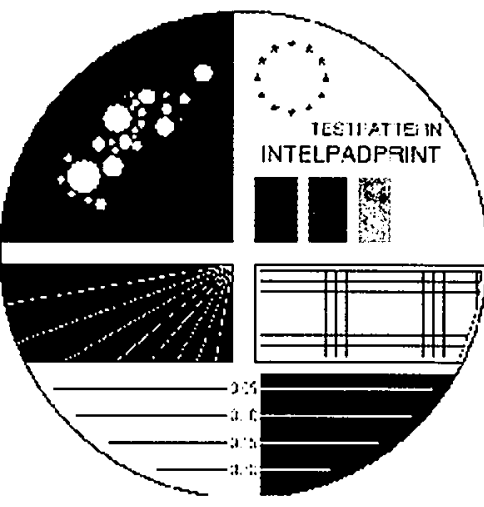

original image
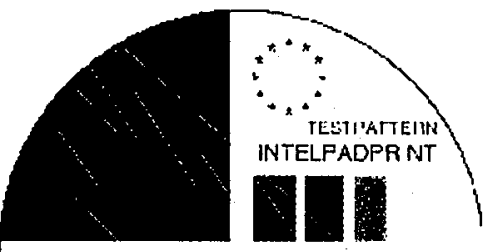

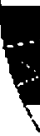

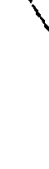

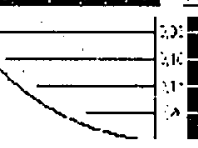

origin a I im age
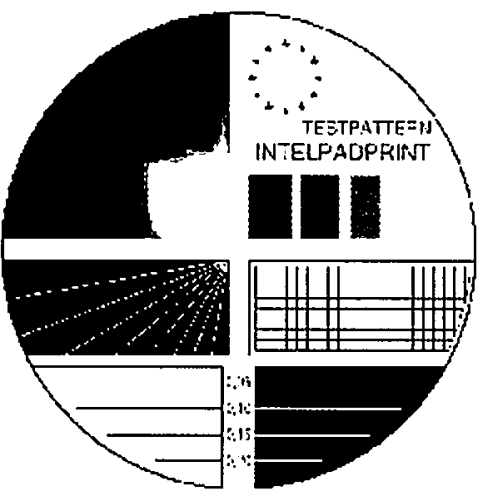

Area-true 2DI map

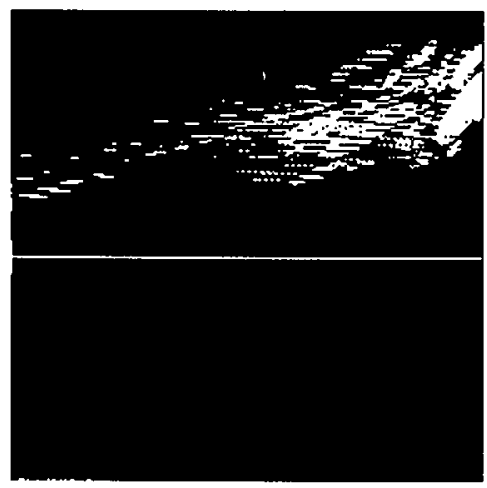

Area-true 2DI map

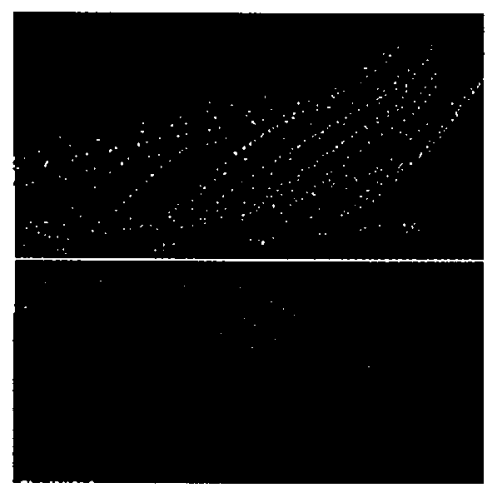

Area-true 2DI map

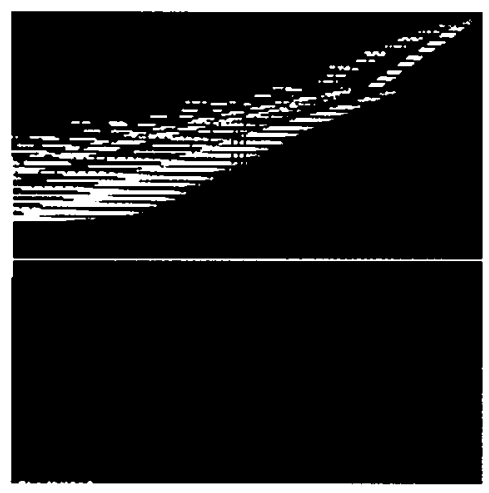

Fast Fourier Transform

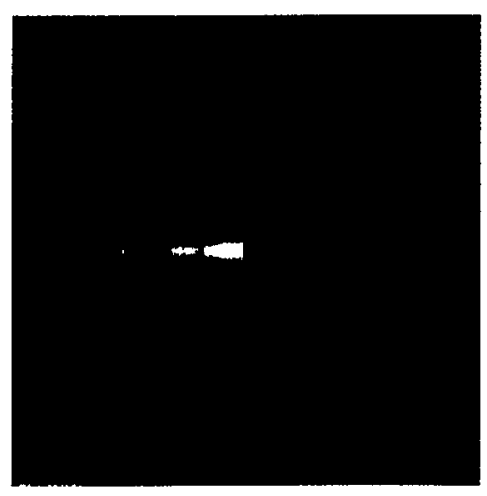

Fast Fourier Transform

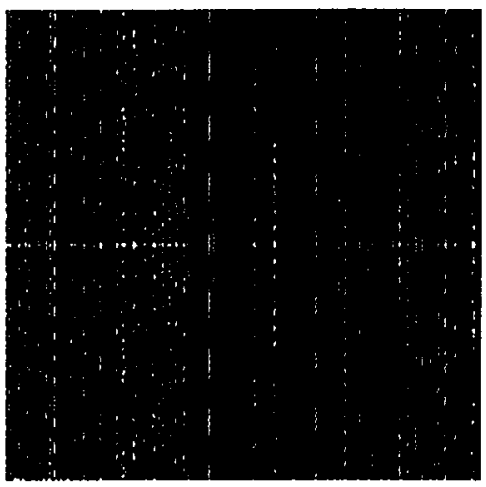

Fast Fourier Transform

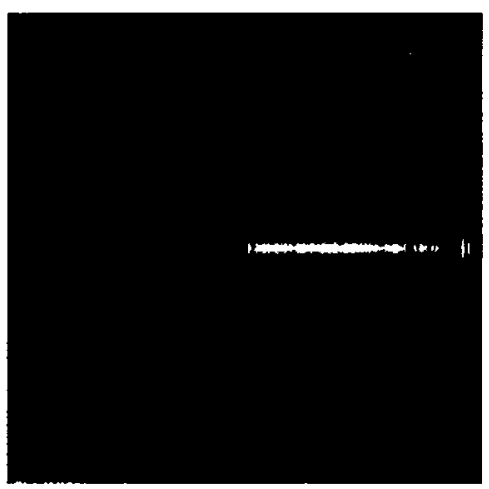

Fig. 2.27. Three examples of images of defects (left), their $2 \mathrm{DI}$ map (middle), and their fast Fourier transform (right). 


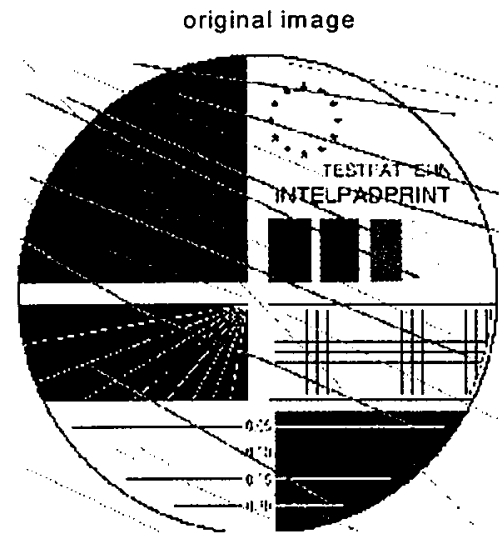

2DI map

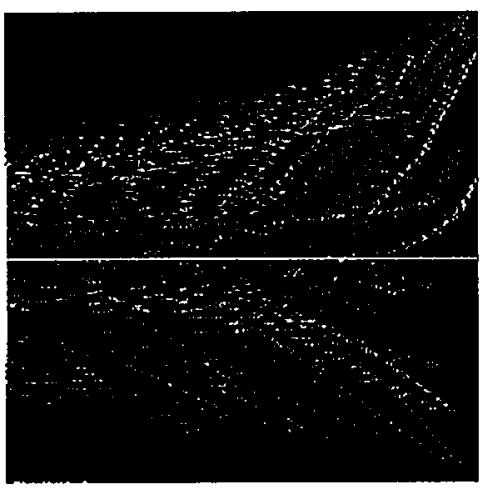

Fourier Highpass filter

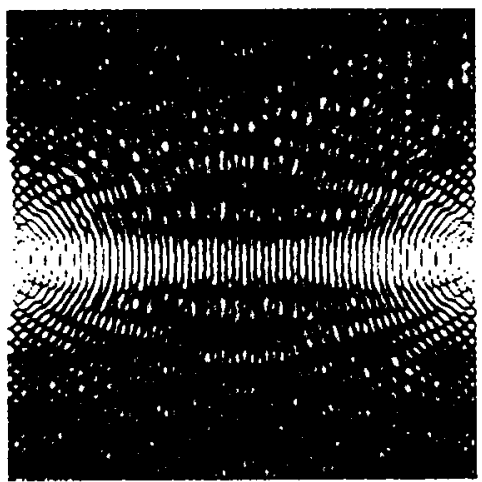

Fast Fourier Transform

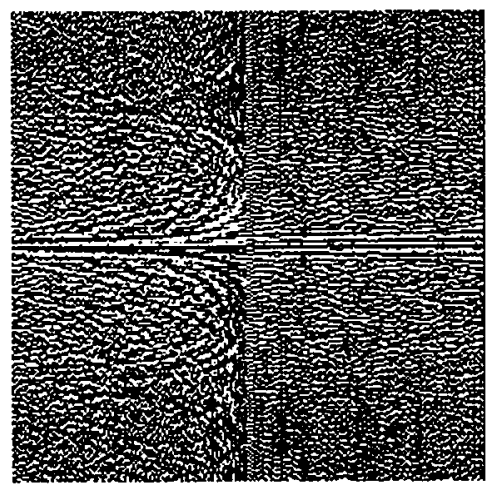

Fourier Lowpass filter

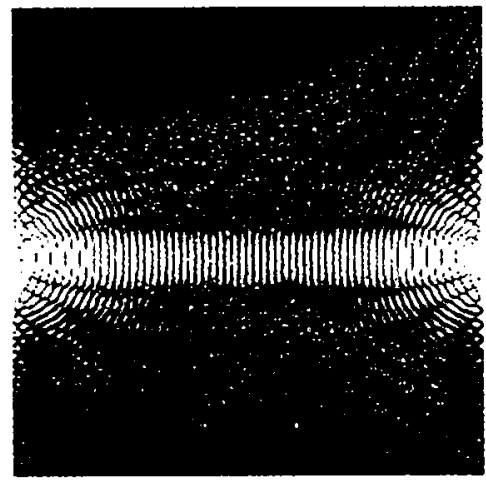

Fig. 2.28. Image of defect 'stripes' (above), 2DI map of the image (middle left), Fast Fourier transform of the 2DI map (middle right), Fourier Highpass filter of the 2DI map (lower left), Fourier Lowpass filter of the 2DI map (lower right). 

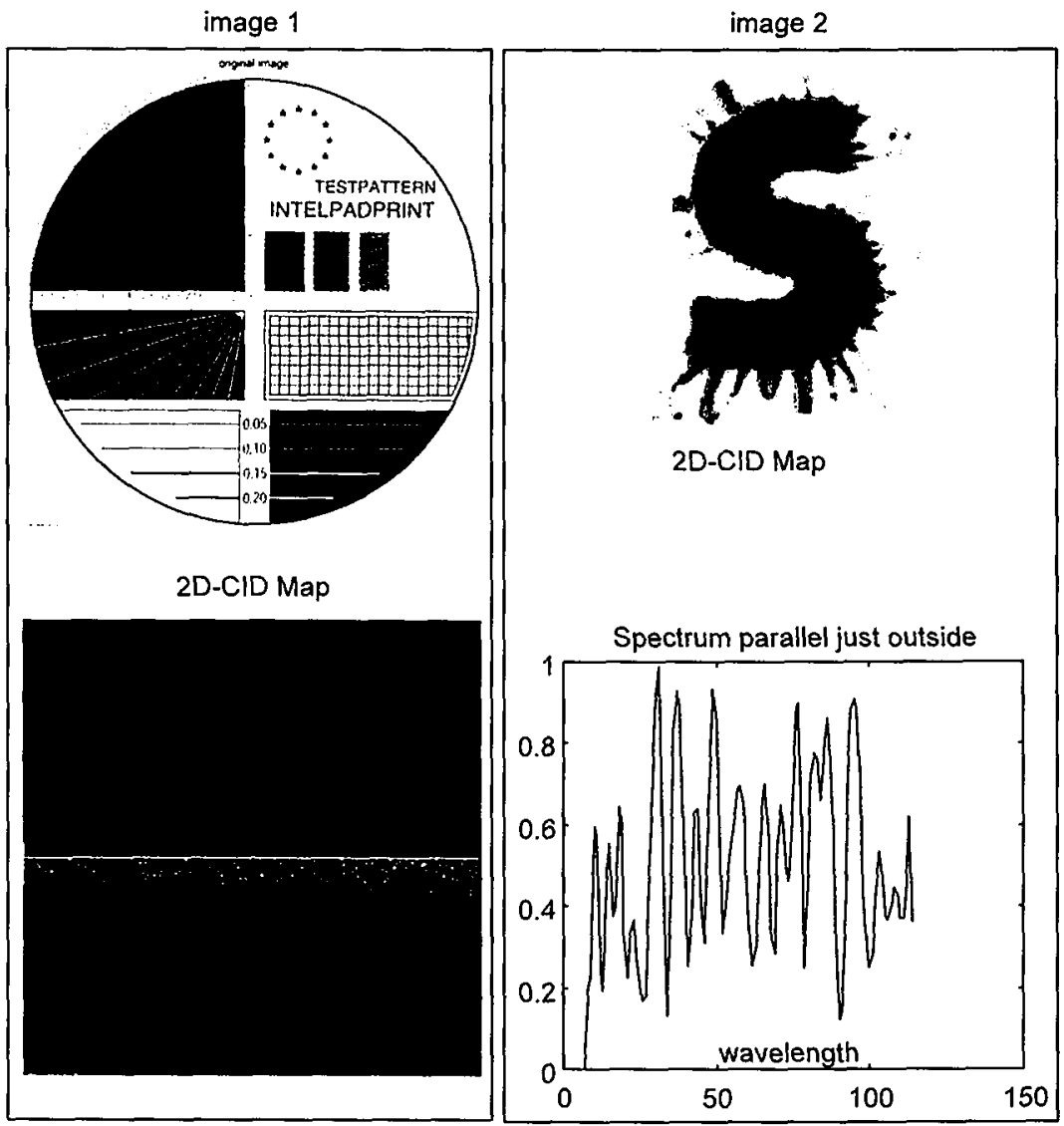

Fig. 2.29. Two examples of images with defect 'hairs'. Left-above: a test-pattern with right-above 'hairs' protruding from the ink. Left-below: the associated 2DI-map, showing the hairs protruding outside the print (the lower part of the rectangle). . Because of the 'slicing' of the transformation, the hairs are not good visible.

Right-above: a CCD-image of the character ' $S$ ', with hairs. Right-middle: the associated 2DI-map, again with slicing. Right-below: the Fourier transform of the strip parallel to and just outside the print, defined by the special fitter in equation [2.30]. 


\subsubsection{Correction of Small Translations Using the CID-Transform}

The following application does not explicitly use the 2DI-map, but it does depend on the parameterisation of the print-border. With that, we employ the CID-transform of an image to estimate the combined strength and direction of a small translation.

Even in the case of a perfect printing, the observed image may differ from the associated template due to small changes in the alignment of the camera with the printed object, within the coordinate-plane of the template, the PTG defined in Section 2.2.1. A small spatial shift parallel to the PTG causes a translation relative to the template. A shift in the vertical distance of the camera to the coordinate-plane causes a scaling, a rotation of the camera causes a rotation, and a shift from the vertical axis causes a projection of the $P T G$ to a tilted plane relative to the camera. The composition of all such deficiencies leads to an affine transform to new coordinates as: $\mathbf{u}=A \mathbf{x}+\mathbf{t}$, with $\mathbf{x}$ is in the $P T G$, and $\mathbf{u}$ in the coordinate system on the focal plane of the camera. $t$ is the translation-vector and $A$ is a $2 \times 2$ matrix that can be composed by projection, scaling, and rotation. In the non-perturbed case, the CIDfunction $\Gamma$ is defined by equation [2.15]:

$$
\Gamma(z)=\int_{\Omega} Z(\mathbf{x}) \cdot \delta(D(\mathbf{x})-z) \mathrm{d}_{2} \mathbf{x} / N(z)
$$

With $D$ the distance-map on the template, $z$ the print-depth, $N$ the characteristic i.e. the cumulative length of IDCs at depth $z, \delta$ the Dirac-delta function, and $Z$ the absolute difference between image $f$ and template $T: Z(\mathbf{x})=|f(\mathbf{x})-T(\mathbf{x})|$. Let us now only consider small translations of the template relative to itself, i.e.: $f(\mathbf{x})=T(\mathbf{x}+\mathbf{t})$. In that case the difference $Z$ can be approximated as:

$$
Z(\mathbf{x})=|T(\mathbf{x}+\mathbf{t})-T(\mathbf{x})| \approx|\nabla T(\mathbf{x}) \cdot \mathbf{t}|
$$

Insertion in above expression for $\Gamma$ gives:

$$
\Gamma(z) \approx \int_{\Omega}|\nabla T(\mathbf{x}) \cdot \mathbf{t}| \delta(D(\mathbf{x})-z) \mathrm{d}_{2} \mathbf{x} / N(z)
$$

Let us assume that the translation vector $\mathbf{t}$ can be written as: $\mathbf{t}=t \hat{\mathbf{u}}(\varphi)$, where $\hat{\mathbf{u}}(\varphi)$ denotes the unit vector with angle $\varphi$ with the positive $x$-axis. Then, the expression for $\Gamma$ becomes:

$$
\Gamma(z)=t \int_{\Omega}|\nabla T(\mathbf{x}) \cdot \hat{\mathbf{u}}(\varphi)| \delta(D(\mathbf{x})-z) \mathrm{d}_{2} \mathbf{x} / N(z) \equiv t w(\varphi, z)
$$

Now consider the set $C$ of images without greyscale-gradients, i.e. images that consists only of parts with constant grey-level. For these images $\nabla T$ differs only from zero near the borders of the print, i.e. where $D(\mathbf{x}) \approx 0$. Therefore $\Gamma$ is mainly concentrated near the print-border zone $z=0$. In practice $\Gamma$ is found to be approximated by a block-function centred around $z=0$. Suppose that the actual template $T$ belongs to this set: $T \in C$. In other words, $T$ consists of a collection of one or more parts $A_{k}$, each with constant greyscale $g_{k}$ :

$$
T(\mathbf{x})=\sum_{k=1}^{K} g_{k} \theta_{k}(\mathbf{x})
$$

where $\theta_{k}(\mathbf{x})$ is the characteristic function on part $A_{k}$. Then $\nabla T(\mathbf{x})$ is everywhere zero, except on the border: $\partial T=U_{k} \partial A_{k}$. There, it is perpendicular to the border, and directed outward. From Lemma 2.5 we know that the normal is directed according to $\nabla D$, so: 


$$
\nabla T(\mathbf{x})=\sum_{k=1}^{K} g_{k} \nabla D(\mathbf{x}) \delta_{k}(\mathbf{x})
$$

where $\delta_{k}(\mathbf{x})$ is the Dirac-delta function on boundary $\partial A_{k}$. Now let, similar to the 2DImap, $\hat{\mathbf{n}}_{k}(\lambda)$ represent a valid parameterisation of the outward directed normal on the print-border of part $A_{k}$, for parameter $\lambda \in\left[0, N_{k}(0)>\right.$, i.e. the length of border $\partial A_{k}$. Then on this border we have:

$$
\nabla T_{k}(\lambda)=g_{k} \hat{\mathbf{n}}_{k}(\lambda)
$$

and the expression [2.35] for $w(\varphi, z)$ becomes:

$$
\begin{aligned}
& w(\varphi, z)=\sum_{k=1}^{K} g_{k} \oint_{\lambda_{k}}\left|\hat{\mathbf{n}}_{k}(\lambda) \cdot \hat{\mathbf{u}}(\varphi)\right| d \lambda \delta(z) \\
& =\sum_{k=1}^{K} g_{k} \oint_{A_{k}}\left|n_{1 k}(\lambda) \cos (\varphi)+n_{2 k}(\lambda) \sin (\varphi)\right| d \lambda \delta(z)
\end{aligned}
$$

We can write this last term briefly as: $w(\varphi) \delta(z)$. Now we employ our theoretical predication, in line with empirical observation, that for misregistration $\Gamma(z)$ can be fairly approximated by a block-function from some $z_{1}<0$ to some $z_{2}>0$. This blockfunction corresponds to a 'smeared-out' variation of the above theoretically predicted $\delta$-peak. Replacing the $\delta$-peak above by a block-function, we may approximate the energy, defined in equation [2.18] as:

$$
E_{\Gamma} \equiv \int_{z_{1}}^{z_{2}} \Gamma(z) d z=\int_{z_{1}}^{z_{2}} t \cdot w(\varphi, z) d z \approx\left(z_{2}-z_{1}\right) t \cdot w(\varphi) \equiv t \cdot w(\varphi) \Delta z
$$

This leads to the following estimation:

$$
w(\varphi) t=E_{\Gamma} / \Delta z
$$

$E_{\Gamma}$ and $\Delta z$ can be directly computed from the CID-transform $\Gamma(z)$, and function $w$ can be calculated off-line from the known template $T(\mathbf{x})$. Though this expression combines the angle $\varphi$ and the strength $t$ of the translation, we can use it for iteratively adjusting the planar position of the print-head, in a way similar to the popular 'highlow game', provided we register the sequence of positions and corresponding energy $E_{\Gamma}$ and shift $\Delta z$. Numerical simulations show that this approach is able to significantly reduce the mismatch in a few steps.

\subsection{Conclusions}

In this chapter we have provided a general framework for the description of images of printed decorations. In this framework we are now better equipped to qualify and quantify structure, position, and form of defects occurring in the image. Because of this general and template-independent description in later chapters we need not be concerned by the original context of printing defects, and thus we are able to concentrate on clustering and classification techniques, independent of our specific application. 


\section{Chapter 3}

\section{The Classification of Textures and Scan-profiles of Printing Defects using Fractal Wavelet Analysis}

\subsection{Introduction.}

\subsubsection{Composition and Objectives: Identifying Printing Defects from their Textural Properties}

While defect classes have their own distinctive appearance, so easy to recognise for a human observer, it is hard to express their characteristics in a limited set of feature parameters based on the extraction of geometrical attributes. Likewise, it is not possible to simply match a printing defect with a fixed and empirically observed defect class prototype-pattern, for the defining element of a defect class lies in its variation of the applied template, rather than being a specific pattern. This variation in itself has some archetypical features that set it apart from other defect classes, and it are those features that must be identified. A 'typical' defect class prototype-pattern, based on the average over a large set of examples, mostly has lost all detail and thus lacks the major distinctive characteristics of an authentic specimen. In practice, it is difficult to find a specimen that in effect fully matches this prototype, for real specimen are both highly variable and at the same time very recognisable.

In this chapter we consider a number of related issues regarding the scaling structure of defects. First, we investigate whether we can utilise the scaling properties of the contour and texture of a typical fault in order to define an archetype of that defect class. Second, we ask whether we can perhaps model this condition by a multifractal formalism. Subsequently, we consider images of printing defects as geometric fractal objects. In that light we investigate whether its characteristic features can be expressed as fractal properties that set it apart from the other defect classes.

This chapter has four objectives; 1 . to study the spectral and spatial decomposition of an image or scan profile, rather than the image itself, 2. to interpret defects as fractal structures and to study the relation between fractal properties of the image of a defect and its defect class, 3 . to calculate fractal properties based on spectral and spatial 
analysis, and 4. to contemplate on a method for template-free defect identification based on fractal and spectral properties of defects.

First, we discuss the importance of fractal analysis for texture characterisation relevant for the identification and classification of defects. In the following section, we concentrate on the decomposition of signals stemming from 1D-scan profiles and 2D-textures. For this purpose we employ techniques that analyse the spectral composition of the signal, Fourier and wavelet analysis. Fourier analysis is able to elucidate the spectral composition of a signal, which can be more characteristic than the original scan profile. We apply Fourier analysis on typical 1D- and 2D-signals, and investigate whether we can find correlates with the defect class.

Next, we concentrate on wavelet analysis. Wavelets can be considered as an extrapolation of spectral decomposition, that can also represent spatial variations in the spectrum. Wavelet analysis is capable of more efficient approximation of especially discontinuous and discrete signals than Fourier analysis. Whereas Fourier analysis averages signals spatially, wavelet analysis can 'zoom-in' on local phenomena of the signal. Multi Resolution Analysis (MRA) is an efficient iterative approach for computing the constituting wavelet coefficients. The Continuous Wavelet Transform (CWT) is useful to understand the degree of detail and characteristic locations at each scale. Here, we also introduce the Global Wavelet Decomposition Approach (GWDA), that results in a more generic formulation than MRA, but does not share its advantages of orthogonal function decomposition. The wavelets obtained with MRA can be considered as a special case of GWDA. The ultimate objective of the analysis of the signals and images is to obtain a representation that contains a stronger correlation with the defect classes than the original signal. Put in an other way, the approach must average out the individual portion of the signal, and extract the defect-class characteristic part. This task of clustering and classification is treated separately in Chapter 4, but in some cases we already discuss examples of classification.

Next, we briefly review the main concepts of the multifractal formalism as relevant for our context. We describe the original technique for computation of multifractal properties based on box-coverings, and a recent method based on the continuous wavelet transform. Both methods are applied on 1D-scanprofiles and parts of 2Dimages of real digital images, and the results are compared. The wavelet-based analysis of fractal sets is called: (Multi)Fractal Wavelet Analysis'.

We end this chapter with indicating how this approach can be used for the construction of a method for true template-free identification and classification of a printing defect.

\subsubsection{Motivation: The Significance of Fractal Texture Analysis for Printing Defect Classification}

The principal information humans obtain from visual analysis concern the shape, colour and texture of an object. This information is somehow integrated and used to decide on the nature and status of the object. While shapes and colour gradients can be modelled sufficiently accurately, textural analysis is far from simple. Natural textures are highly irregular and cannot be modelled precisely with present mathematical techniques. There is no general accepted definition of the concept texture. Most publications provide a definition suited to the specific context or method presented. A reason for this is the strong intuitive notion of texture, which is indeed

\footnotetext{
I This does not indicate that the employed wavelets are necessarily fractals themselves.
} 
hard to fit in one definition. This diversity can be demonstrated by a brief review of some definitions collected from major handbooks and papers:

- A region in an image has a constant texture if a set of local statistics or other local properties of the picture function are constant, slowly varying, or approximately periodic. [Sklansky, 1978)]

- texture is an atribute representing the spatial arrangement of the grey levels of the pixels in a region.[IEEE Standard 610.4-1990, 1990]

- texture is a [...] descriptor as providing a measure of properties such as smoothness, coarseness, and regularity. [Gonzalez and Woods, 1992]

- patterns which characterise objects are called textures in image processing. [Jahne, 1997]

Although there exists no formal definition of the concept 'texture', it provides an intuitive appealing qualification of a surface. This qualification does not depend on form or colour, but describes a surface in terms of regular versus irregular patterns, smoothness versus coarseness, and colour gradients.

The first step to characterise textures is by analysing their spectral properties. In [Azencott, 1997] textures are represented in a metric space. The distance between textures is defined using windowed Fourier filters. This distance relates to the spectral density relative to a Gaussian random field. An experimental analysis and comparison with Mahalanobis distances shows the advantage of their method. Similarly, the Fourier-based characteristic scale approach presented in Section 3.2.3 is found to characterise and distinguish defects.

The observation that texture has a high degree of self-similarity under scaletransformations, appeals to the mathematical fields of fractals and multi resolution analysis. In recent years therefore, research in the fractal properties of textures is enjoying much attention. Pentland [Pentland,1994] showed that a fractal dimension based on box counting could distinguish textures of natural versus artificial objects. Likewise, attempts have been made to use fractal properties to define standard texture classes. However, such texture characterisation is phenomenological, since it lacks a precise mathematical basis. Another quantity used for texture classification other than the fractal dimension is the Lipschitz exponent, that has been related directly to the fractal dimension. The Lipschitz exponent, however, is difficult to calculate experimentally since it defines whole sets in an image rather than individual points. To characterise individual singularity points in a texture the Hölder exponent is of more practical use. The Hölder exponent for two dimensional images can be computed by a method similar to the WTMM-method, described in Section 3.5.4. Based on such methods, it is found that Hölder exponents with negative values correspond to sharp edges such as contours, whereas positive Hölder exponents correspond to naturally occurring phenomena like vegetation, or cloud formation [Mascle et al,1994], [Rioul,1993]. Recently this analysis has also been applied to characterise texture classes [Leblanc et al,1997].

Fractal and multifractal concepts [Mandelbrot,1975] are now widely used to characterise multiscale phenomena that occur in a variety of physical situations [Pietronero,1986]. In its present form, the multifractal approach is basically adapted to describe statistically the scaling properties of singular measures. Many notable examples of growth phenomena that obey fractal scaling laws are known from physics, biology, economy, geology, and urban development [White,Engelen, 1994]. 
The wavelet transform has been recently advocated as a highly efficient technique to collect microscopic information about the scaling properties of multifractal measures [Arneodo,1996]. Extensive applications to various multifractal measures, including the invariant measures of some well-known discrete dynamical systems, have clearly demonstrated the fascinating ability of this mathematical telescope to reveal the underlying hierarchy that governs the spatial distribution of the singularities. What makes the wavelet transform such an attractive tool is that its singularity scanning ability applies to any distribution, including singular functions [Malat, Hwang, 1992].

\subsubsection{Computational Models for Invariant Visual Recognition}

At present, several computational models for invariant visual recognition have been reported. These reports concern models that can recognise specific geometrical shapes, independent of affine transformations and small deformations. Park and Yang [Park, Yang,2001] describe invariant object detection, based on evidence accumulation using the Gabor transform. López-Rubio et al. [López-Rubio et al.,2001] describe a method for invariant identification of shapes and patterns recognition by selforganising feature maps, based on a robust deformation index. Khalil and Bayoumi [Khalil,Bayoumi,2000] apply the Wavelet Transform Modulus Maxima method for the recognition of two-dimensional objects, invariant to affine transformations. Similar to our approach, described below, their study is inspired by the work of Arneodo ${ }^{2}$. This approach applies a wavelet-formalism to the analysis of fractal objects. In Section 3.8 is discussed how, applied to our context, such an approach allows the template-free recognition of printing-defects.

\subsection{Applicability of Fourier Analysis for Defect Classification}

First, we investigate the applicability of Fourier Analysis to the characterisation of printing defects. For a good introduction on Fourier Analysis see for instance: [Priestley, 1981].

Fourier Analysis is defined on the set of Lebesgue measurable functions $L^{2}[0,2 \pi]-$ and thus correspondingly on all compact intervals $[a, a+\lambda]$. Therefore, Fourier Analysis is also applicable on all functions $f$ in $L^{2}(\mathbb{R})$ that are periodic extensions of $[a, a+\lambda]: f(x+\mathrm{k} . \lambda)=f(x)$ for all $x \in[a, a+\lambda]$ and $k \in \mathbb{Z}$. In Fourier Analysis all functions in $\mathrm{L}^{2}[0,2 \pi]$ are constructed by an infinite series, generated by one single basic function, $\psi(x)=\mathrm{e}^{\mathrm{i} x}$. This sinusoidal wave is the only function that is required to generate the entire set of functions $\mathrm{L}^{2}[0,2 \pi]$.

Generally, in $n$ dimensions, Fourier Analysis generates the spectral decomposition of functions: $f: \mathbb{I R}^{n} \rightarrow \operatorname{IR}$ The spectrum $\mathrm{F}[f]$ of $f$ is a function $\mathrm{F}[f]: \mathbb{C}^{n} \rightarrow \mathbb{C}$ according to the continuous Fourier transform:

$$
F\left[f(k)=(2 \pi)^{-1 / 2 n} \int f(x) e^{i k \cdot x} d_{n} x\right.
$$

\footnotetext{
2 A thorough review of the 'wavelet transform modulus maxima method' is given by Arneodo in [Erlebacher et al.,eds.,1996].
} 
In our case, we are primarily interested in $n=1$ for scan-profiles, and $n=2$ for images. The set: $\left\{\psi_{\mathrm{k}}(x)=\mathrm{e}^{\mathrm{ikx}} \mid x \in \mathbb{R}, k \in \mathbb{Z}\right\}$ forms an orthonormal basis of the Lebesguespace of periodic functions $\mathrm{L}^{2}\left(\mathrm{IR}^{n}\right)$. Based on this set for $n=2$, any function $f$ in $\mathrm{L}^{2}\left(\mathbb{R}^{2}\right)$ that is defined on a compact domain $D_{f} \subset \mathbb{R}^{2}$, can be uniquely decomposed into an infinite sum:

$$
f=\Sigma_{\mathrm{k} 1, k 2 \in \mathbb{Z}} a_{\mathrm{k} 1, \mathrm{k} 2} \psi_{\mathrm{k} 1, \mathrm{k} 2}
$$

with coefficients $a_{\mathrm{k} 1, \mathrm{k} 2}$ defined as:

$$
\begin{aligned}
& a_{\mathrm{k} 1, \mathrm{k} 2}=\left\langle\psi_{\mathrm{k} 1, \mathrm{k} 2} \mid f\right\rangle=\mathrm{F}\left[f\left(2 \pi \mathrm{k}_{1} / \lambda_{1}, 2 \pi \mathrm{k}_{2} / \lambda_{2}\right)\right. \\
& =(2 \pi)^{-1} \int f\left(x_{1}, x_{2}\right) \mathrm{e}^{\mathrm{i} 2 \pi \mathrm{k}_{1} x_{1} / \lambda_{1}} \mathrm{e}^{\mathrm{i} 2 \pi \mathrm{k}_{2} x_{2} / \lambda_{2} \mathrm{~d} x_{1} \mathrm{~d} x_{2}}
\end{aligned}
$$

where $\langle\cdot \mid \cdot\rangle$ is the inner product in $\mathrm{L}^{2}\left(\mathrm{IR}^{2}\right)$, and $\lambda_{1}, \lambda_{2}$ are the respective lengths of the intervals in its both dimensions. For this reason, all functions that are periodic extensions of $\mathrm{D}_{f}: f\left(x_{1}, x_{2}\right)=f\left(x_{1}+l_{1} \lambda_{1}, x_{2}+l_{2} \lambda_{2}\right), l_{1}, l_{2} \in \mathbb{Z}$, with wavelengths $\lambda_{1}, \lambda_{2}$, have the same spectral decomposition. This approach works similarly for $n=1$. The transformation: $f \rightarrow\left\{a_{\mathrm{k}}\right\}$ is called the Discrete Fourier Transform.

There exists an extensive literature on the application of Fourier Analysis to the identification of $1 \mathrm{D}$ signals and 2D images, and the subsequent classification of the resulting Fourier coefficients. See for instance: [Matsuyama et al., 1983], [Lin,Chellappa,1987], [Hsu et al.,1993], and [Azencott et al.,1997]. For this classification often fuzzy or neural clustering is employed. Typical area's of application are the interpretation of seismic signals, EEG's and ECG's, radar and sonar reflections. Most of these applications use adaptations of the standard Fourier Transform or targeted post-processing aimed to identify a certain feature of a defect in the profile. The reason for an incidental use rather than structural approach based on Fourier Analysis is twofold.

First, the Fourier Transform of a scanprofile contains information on the spectral composition of the signal, so the question shifts from characterising spatial features of the scanprofile to space-invariant spectral properties of the Fourier transformed signal. Not all defects, however, exhibit spectral features so characteristic that they may serve to define them. The problem is to correlate elements of the Fourier spectrum to the defect classes in such a way that a 1-1 correspondence emerges. Once a scanprofile has been obtained the definitions of the PrintContext and CurveType provide an essential framework for further interpretation. Such a framework is lacking for the Fourier spectrum.

The second reason lies in the spatial details and scaling characteristics of the scanprofile. The Fourier Transform smears out all spatial details and gives the contribution of the spectrum at scale ' $\lambda$ ' (the wavelength in the Fourier space). Because of this averaged information it is badly suited to reconstruct local scalinglaws and self-similarity. For that reason the Fourier Analysis is ideal in recognising periodic properties on the one hand, but practically incapable of describing fractal scaling laws on the other. To resolve this inability we will later apply a recent refinement of Fourier Analysis; the Wavelet Analysis.

Other basic disadvantages to the application of Fourier Analysis are:

- As it is only applicable on periodic functions, or on relatively small intervals, it is not valid for realistic intervals. 
- It is incapable of efficiently describing localised discontinuities because of spatial averaging of local effects. Since the basis function $\mathrm{e}^{\mathrm{i} \omega \mathrm{x}}$ operates on the entire $\mathbb{R}$, a localised effect affects the entire $F[f(\omega)$.

- Its inability to efficiently describe time dependent frequency effects $\omega(t)$.

- A more basic problem is the semantics of the spectrum, i.e. the interpretation of the Fourier transform and its correlations with meaningful entities, like in our context the printing defect classes.

In the following sections we discuss general advantages and disadvantages of Fourier Analysis of scanprofiles and textures, and shortly review the value of the subsequent clustering of the defect classes.

\subsubsection{Fourier Analysis of ScanProfiles}

As mentioned above, the main problem in applying Fourier Analysis lies in the interpretation of the Fourier Transform of the scanprofile and the subsequent mapping of its features to the defect classes. Since the interpretation is solely aimed at the recognition of features characteristic to print defects, both items can be handled with techniques that combine pattern recognition and clustering/classification tasks like Neural Networks, Genetic Algorithms and Fuzzy Clustering. As demonstration of this principle two of such techniques were applied for the detection of 'hairs' in an original scanprofile and its Fourier Transform. In both approaches the signal is considered as a large vector over space or frequency. These examples are described in detail later in Chapters 4 and 5 that deal with clustering and classification techniques. Here we use the resulting quality of the mapping to show that these techniques are in fact capable to discriminate the defect classes from each other. The first technique is based on a simple feed-forward neural network and is aimed at the identification of a Г3-curve scanprofile (see Section 2.3.2) for a limited set of defect classes. The quality of the mapping of the Fourier Transformed spectrum of the scanprofile is represented by the fraction of positive identification, versus false positives and false negatives. The second example uses Kohonen's Self Organising Map to cluster the defects into the space of the Fourier spectrum. Figure 3.1 shows the results of this mapping. This last example demonstrates the difficulty of obtaining meaningful correlates between the Fourier Transform of the scan and the defect classes. The techniques succeeds in identifying 'hairs', but the two other defect clusters are not fully separated relative to their error-size. The same holds for a similar representation of the first technique. Therefore the predictive capabilities of these techniques are weak. In Chapter 4 we will see that other representations can give better cluster separation, and in Chapter 5 we will discuss how to handle poor cluster separation. 


\begin{tabular}{|c|c|c|c|}
\hline $\mathrm{nr}$ & Defect & Pos & False pos \\
\hline 1 & 'hairs' & 0.95 & 0.27 \\
\hline 4 & 'recess' & 0.52 & 0.42 \\
\hline 10 & 'smearing' & 0.46 & 0.38 \\
\hline
\end{tabular}

Table 3.1. Fraction of positives and false positives for the classification of the Fourier zone-vector by an artificial neuronal network.

Kohonen Map of Wave Groups
\begin{tabular}{|c|c|c|c|}
\hline$R S$ & $S$ & $S$ & $R R$ \\
\hline & $R S$ & $\begin{array}{c}R R S \\
S\end{array}$ & \\
\hline $\begin{array}{c}R R R \\
S S\end{array}$ & & & $H H H$ \\
\hline$R S S$ & & $\begin{array}{c}H H H \\
H\end{array}$ & $H H H$ \\
\hline
\end{tabular}

Fig. 3.1. Kohonen map of wavenumber-zone vectors, $H=$ 'hairs', $R=$ 'recess', $S=$ 'smearing'.

\subsubsection{Shape Analysis with Fourier Descriptors}

Two dimensional objects can be characterised by their shape, texture and colour distribution. Analysis of the colour distribution presents no serious problem. Problems concerning texture is discussed in Sections 3.2.3 and 3.7.4. In many applications the shape of an object can be represented by its contour. This is for instance the case in vehicle recognition, chromosome classification, and the identification of elementary particles [Altmann et al,1996]. The shape of 2D-contours can be efficiently characterised with the use of Fourier Analysis [Gonzalez, Woods, 1992]. The basic idea behind the analysis is to parameterise the contour of the object as a vector $\mathbf{x}(\lambda) \in \Omega$ of a single parameter $\lambda$, then to expand this function in its Fourier series: $a_{n}=$ $F[\mathbf{x}](2 \pi n / \lambda)$, and finally use the coefficients $\left\{a_{n}\right\}$ of these series as the so-called Fourier Descriptors, see: [Bow,1992]. Figure 3.2 illustrates one of the main difficulties of obtaining significant correlates between the Fourier Descriptors of the shape of the print, and the defect class. This relation is found to be weak. The application of this method to the analysis of digital images of defects meets three major computational difficulties. First, the defects are typically 'fuzzy' in the sense that most are not defined by definite and sharp contours. Their intensity can be seen as continuously varying from 1 to 0 . In this respect they exhibit an appearance characteristic to fractal geometry. Therefore it is difficult to parameterise their shape by one parameter. Even artificially 'sharpened' borders will not resolve this problem. Moreover, even in the case of definable shapes like 'air bubbles' or 'ink missing', it is not trivial to design efficient algorithms to parameterise the contours. Second, the Fourier analysis generates a large, in principle unlimited, number of coefficients, and it is a matter of taste how many of them are involved as input for classification, just as in the 1D-Fourier Analysis. Of course, one guideline would be to explore the 
dependence of the average cluster segregation on the number of Fourier Descriptors involved. This, however, is related to the third problem; the prototypes of the defect classes are not well separated, even when many Fourier coefficients are involved.

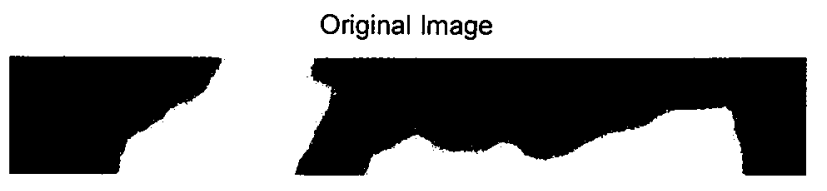

Edge for threshold $=0.95$

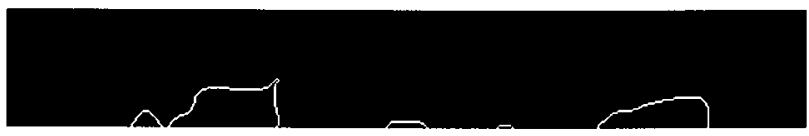

Edge for threshold $=0.8$

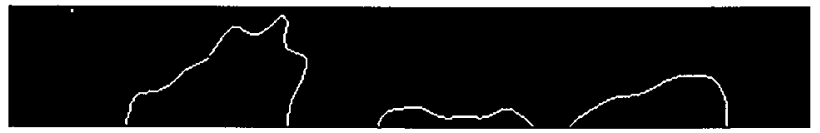

Fig. 3.2 Problems concerning the 'shape' from a defect. Shown above is an image of defect 'recess' outside the print, the print is white-on-black. Middle: Contour of defect for an intensity-threshold of 0.95. Below: Similar for threshold 0.8

Finally, some of the shapes may have more than one appearance. For instance 'air bubbles' may be recognisable as circles of various sizes, but also as set of 'crescent moons', depending on the lighting conditions.
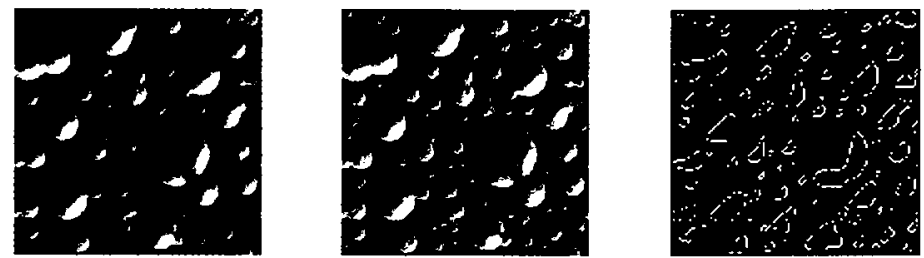

Fig. 3.3. Fundamental problem of shape-analysis. Due to the lighting conditions, the air bubbles are illuminated from one side. In the original image this hardly creates a problem, but after thresholding with 0.35 the typical shape is a crescent rather than a circle. After edge extraction little of the supposed archetypal circular shapes is visible.

Summarising, shape analysis in general and Fourier Descriptors in particular, are not very useful in the context of printing defect characterisation. Given the continuous and fractal geometry of the defect, we must look for other tools suitable to represent these features of the defect. 


\subsubsection{Fourier Analysis of Textures}

The analysis of textures can be based on statistical analysis using auto-correlation functions [Sanna et al.,1999], distance-matching functions [Oh et al.,1999], and multidimensional co-occurrence histograms [Valkealahti,1998],[Gimelfarb, 1999]. However, it is more useful to look for some periodic structure in the texture. In that respect, Fourier analysis is an important tool for texture classification. This approach similarly offers the possibility for a statistical analysis, as employed by Sziranyi and Hanis [Sziranyi,Hannis, 1999]. Azencott et al. [Azencott et al., 1997] treat textures as Gaussian random fields, and use windowed Fourier filters to compute their spectral density. Thus, a metric texture space is constructed, where the distance between two textures is expressed in these attributes.

Here, we propose another and original approach, based on Fourier analysis, by quantifying a 'typical periodic scale' in the texture. First, we compute the contribution of wavelength ' $\lambda$ ', as present in the texture, to the entire Fourier spectrum. Since the Fourier Transform is expressed in terms of a 'wave number' $k=2 \pi / \lambda$ rather than the wavelength $\lambda$, we construct the following transformation, called the Fourier scale representation, using the Dirac $\delta$-function:

$$
\phi(\kappa)=\int \mathrm{F}[f](\mathbf{k}) \delta(|\mathbf{k}|-\kappa) \mathrm{d}_{2} \mathbf{k}
$$

In order to avoid a disproportional high contribution of a uniform background, we omit the zero-term and set: $\phi(0) \equiv 0$. Next, we construct a normalised Power spectral density function $P$ :

$$
\mathrm{P}(\kappa)=|\phi(\kappa)|^{2} / \int|\phi(\mathrm{x})|^{2} \mathrm{dx}
$$

Here $\kappa$ represents the wavenumber in $\mathrm{mm}^{-1}$. This representation 'counts' the number of structures in the texture with a typical length $1 / \kappa$. Images with a well-defined length $\kappa_{0}$ reveal a peak near $\kappa=\kappa_{0}$. The characteristic scale $\lambda$ for the texture is then defined by the wavelength associated to wavenumber $k$ :

$$
2 \pi \lambda^{-1}=<k>=\int k \mathrm{P}(k) \mathrm{d} k
$$

(in $\mathrm{mm}$ ). This entity, together with its standard deviation $\delta \lambda$, is expected to show some correlation with the defect class. The white noise in $P(\kappa)$ is expected to follow a $\chi^{2-}$ distribution. Therefore, $P$ can be de-noised by subtraction with the variance $\operatorname{Var}(P)$ of the observed $P$ in areas where $\langle\phi\rangle$ is expected to be zero. In the following we show some examples where the characteristic scale can be calculated exactly. We finish this section with the application of this method to some real examples of defects.

Furthermore, we remark that this method was recently successfully applied for estimating paper roughness in high-quality paper production [Peeters, Westra,2000].

\section{Examples of Fourier Power spectral density functions}

\section{Example 1. Typical Scale of a Single Square}

As an example of the application of the Fourier Power spectral density function we consider the image of one single square represented by:

$$
\mathrm{b}_{\mathrm{L}}\left(\mathrm{x}_{1}, \mathrm{x}_{2}\right)=h \chi_{[-\mathrm{L}, \mathrm{L}]}\left(\mathrm{x}_{1}\right) \chi_{[-\mathrm{L}, \mathrm{L}]}\left(\mathrm{x}_{2}\right)
$$

where $\chi_{[\mathrm{a}, \mathrm{b}]}(\mathrm{x})$ is the characteristic function on interval $[\mathrm{a}, \mathrm{b}]: \chi=1$ if $\mathrm{x} \in[\mathrm{a}, \mathrm{b}]$ and $\chi=$ 0 if $\mathrm{x} \notin[\mathrm{a}, \mathrm{b}]$, the side of the square is $2 \mathrm{~L}$, and $h \in[0,1]$ is the greylevel of the square, see Figure 3.4. For this function the Fourier transform is: 


$$
\mathrm{F}\left[\mathrm{b}_{\mathrm{L}}\right]\left(\mathrm{k}_{1}, \mathrm{k}_{2}\right)=A \mathrm{u}\left(\mathrm{k}_{1} \mathrm{~L}\right) \mathrm{u}\left(\mathrm{k}_{2} \mathrm{~L}\right)
$$

with $\mathrm{u}(\mathrm{x})=\sin (\mathrm{x}) / \mathrm{x}$, and $A=2 h \mathrm{~L}^{2} / \pi$.
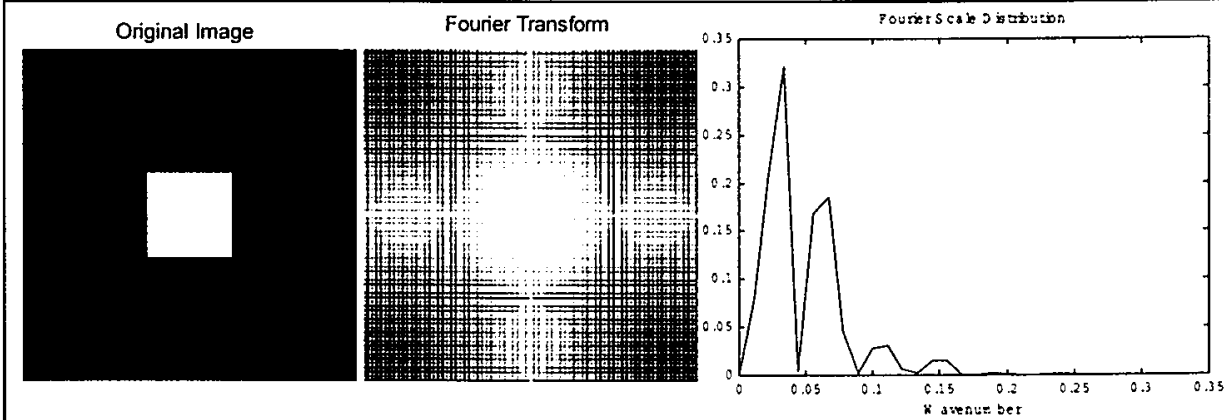

Fig. 3.4. Left: Image of a white square, with suitable coordinate system. Middle: Fourier transform. Right: Fourier power spectral density.

Therefore function $\phi$ can be written as:

$$
\phi(k)=A k \mathrm{~g}(k \mathrm{~L})
$$

with:

$$
\mathrm{g}(\mathrm{x})=\int_{[0,2 \pi]} \mathrm{u}(\mathrm{x} \cos \varphi) \mathrm{u}(\mathrm{x} \sin \varphi) \mathrm{d} \varphi
$$

Therefore: $\mathrm{P}(k)=C k^{2} \mathrm{~g}(k \mathrm{~L})^{2}$, where $C$ is a constant such that $\int \mathrm{P}(k) \mathrm{d} k=1$, so: $C^{-1}=$ $L^{-3} M_{2}$. Here we have used the definition of the moments $M_{n}$ of $g^{2}$ :

$$
\mathrm{M}_{n}=\int \mathrm{g}(k)^{2} k^{\mathrm{n}} \mathrm{d} k .
$$

The typical size ' $\lambda$ ' of the single square follows from equation [3.3]:

$$
2 \pi \lambda^{-1}=\int k \mathrm{P}(k) \mathrm{d} k=C \int k^{3} \mathrm{~g}(k \mathrm{~L})^{2} \mathrm{~d} k=\mathrm{L}^{-1} \mathrm{M}_{3} / \mathrm{M}_{2} \text {. }
$$

So in this example the characteristic scale is directly proportional with the side $\mathrm{d}=2 \mathrm{~L}$ of the square: $\lambda=c \mathrm{~L}$, with $c=\mathrm{M}_{2} / 2 \pi \mathrm{M}_{3}=\int x^{2} \mathrm{~g}(x)^{2} \mathrm{~d} x / 2 \pi \int x^{3} \mathrm{~g}(x)^{2} \mathrm{~d} x \approx 2.02$ So in this case $\lambda \approx 1.01 \mathrm{~d}$.

\section{Example 2. Typical Scale of a single Sphere}

As an example of the application of the Fourier Power spectral density function we consider the image of a sphere with radius $R$ and centre at the origin. This sphere is represented by:

$$
\mathrm{s}_{\mathrm{R}}(\mathrm{r}, \varphi)=h \chi_{[0, \mathrm{R}]}(\mathrm{r})
$$

where $(r, \varphi)$ are polar coordinates, $\chi$ is the characteristic function, $2 \mathrm{R}$ is the diameter of the sphere, and $h \in[0,1]$ is the greylevel. For this sphere the Fourier scale representation $\phi$ follows from:

$$
\begin{aligned}
& \phi(k)=h(2 \pi)^{-1} \int_{[0, \mathrm{R}]} \mathrm{dr} \int_{[0,2 \pi]} \mathrm{rd} \varphi \mathrm{e}^{\mathrm{i} k \mathrm{r} \cos \varphi} \\
& =h(2 \pi)^{-1} k^{-2} \int_{[0,2 \pi]} \mathrm{d} \varphi\left\{\mathrm{e}^{\mathrm{i} k \mathrm{R} \cos \varphi(1-\mathrm{i} k \mathrm{R} \cos \varphi)-1\}}\right.
\end{aligned}
$$

Define $\mathrm{x}$ and $\mathrm{z}$ as: $\mathrm{x}=k \mathrm{R}$ and $\mathrm{z}=x \cos \varphi$, then $\phi$ can be written as:

$$
\phi(k)=h(2 \pi)^{-1} k^{-2} \int_{[0,2 \pi]} \mathrm{d} \varphi \mathrm{e}^{\mathrm{i} x z}(1-\mathrm{i} x z)-h k^{-2} \equiv h(2 \pi)^{-1} k^{-2} A(k \mathrm{R})-h k^{-2}
$$

with complex function $A$ defined as an integral: 


$$
A(x)=\int_{[-1,1]} \mathrm{e}^{2 \mathrm{ix} z}(\mathrm{i} x z-1)\left(1-z^{2}\right)^{-1 / 2} \mathrm{~d} z
$$

Therefore we can write the power density $\mathrm{P}(k)$ as:

$$
\mathrm{P}(k)=C h^{2} k^{4}\left|(2 \pi)^{-1} A(k \mathrm{R})-1\right|^{2}
$$

where constant $C$ follows from $\int \mathrm{P}(k) \mathrm{d} k=1$, so:

$$
C^{-1}=h^{2} \mathrm{R}^{3} \int x^{-4}\left|(2 \pi)^{-1} A(x)-1\right|^{2} \mathrm{~d} x=h^{2} \mathrm{R}^{3} \mathrm{Q}_{4}
$$

with generalised moments: $\mathrm{Q}_{\mathrm{n}}=\int x^{-\mathrm{n}}\left|(2 \pi)^{-1} A(x)-1\right|^{2} \mathrm{~d} x$. The typical size ' $\lambda$ ' of a sphere follows from:

$$
2 \pi \lambda^{-1}=\int k \mathrm{P}(k) \mathrm{d} k=C h^{2} \int k^{-3}\left|(2 \pi)^{-1} A(k \mathrm{R})-1\right|^{2} \mathrm{~d} k=C h^{2} \mathrm{R}^{2} \mathrm{Q}_{3}
$$

Combining with the expression for $C$ we find:

$$
2 \pi \lambda^{-1}=h^{2} \mathrm{R}^{2} \mathrm{Q}_{3} / h^{2} \mathrm{R}^{3} \mathrm{Q}_{4}=\mathrm{R}^{-1} \mathrm{Q}_{3} / \mathrm{Q}_{4}
$$

Like in the case of a square, the characteristic scale of a sphere is directly proportional to its diameter $\mathrm{D}=2 \mathrm{R}: \lambda=2 \pi \mathrm{Q}_{4} \mathrm{R} / \mathrm{Q}_{3}=\pi \mathrm{Q}_{4} \mathrm{D} / \mathrm{Q}_{3}$. Numerical approximation of the constant gives $\lambda_{\text {sphere }} \approx 0.8209 \mathrm{D}$.

\section{Example 3. Typical Scale of a Lattice of Squares}

Consider a lattice of $(2 \mathrm{~N}+1) \times(2 \mathrm{~N}+1)$ squares, centred at the origin. In this case we can write the intensity distribution on the image as:

$$
f(\mathbf{x})=\Sigma_{\mathrm{n} 1, \mathrm{n} 2=-\mathrm{N}: \mathrm{N}} h(\mathbf{n}) \mathrm{b}_{\mathrm{L}}(\mathbf{x}-\boldsymbol{a}(\mathbf{n}))
$$

where $b_{L}$ is the block-function from the single square example, $\mathbf{n}=\left(\mathrm{n}_{1}, \mathrm{n}_{2}\right), \boldsymbol{a}(\mathbf{n})=$ $\left(\mathrm{Dn}_{1}, \mathrm{Dn}_{2}\right)$ is the translation along the lattice, with $D$ the grid-size of the lattice. The Fourier transform of this function is:

$\mathbf{F}[f](\mathbf{k})=\Sigma_{\mathrm{n} 1, \mathrm{n} 2} h(\mathbf{n}) \mathrm{F}\left[\mathrm{b}_{\mathrm{L}}\right](\mathbf{k}) \mathrm{e}^{-\mathrm{i} \mathrm{k}_{1} D \mathrm{n}_{1} \mathrm{e}^{-\mathrm{ik}} \mathrm{k}_{2} D \mathrm{n}_{2}}$

$=-2\left(\mathrm{~L}^{2} / \pi\right) \mathrm{u}\left(\mathrm{k}_{1} \mathrm{~L}\right) \mathrm{u}\left(\mathrm{k}_{2} \mathrm{~L}\right) \Sigma_{\mathrm{n} 1, \mathrm{n} 2} h(\mathbf{n}) \mathrm{e}^{-\mathrm{ik}} \mathrm{k}_{1} \mathrm{Dn}_{1} \mathrm{e}^{-\mathrm{i} \mathrm{k}_{2} \mathrm{Dn}} \mathrm{n}_{2}$

with $\mathrm{u}(\mathrm{x})=\sin (\mathrm{x}) / \mathrm{x}$. Now let us assume that all squares have the same greylevel $h$ : $h(\mathbf{n})=h$ (constant), and define $\mathrm{v}_{\mathrm{N}}(\mathrm{x})$ as:

$$
\mathrm{v}_{N}(\mathrm{x})=\sum_{\mathrm{n}=-N: N} \mathrm{e}^{-i n \mathrm{x}}=-1+2 \cos (1 / 2 N \mathrm{x}) \sin (1 / 2(N+1) \mathrm{x}) / \sin (1 / 2 \mathrm{x})
$$

Then we can write $\mathrm{F}[\mathrm{f}]$ as:

$$
\mathrm{F}\left[f(\mathrm{k})=-2 h\left(\mathrm{~L}^{2} / \pi\right) \mathrm{u}\left(\mathrm{k}_{1} \mathrm{~L}\right) \mathrm{u}\left(\mathrm{k}_{2} \mathrm{~L}\right) \mathrm{v}_{M}\left(\mathrm{k}_{1} \mathrm{D}\right) \mathrm{v}_{M}\left(\mathrm{k}_{2} \mathrm{D}\right)\right.
$$

So the function $\phi$ from equation [3.1] can be written as:

$$
\phi(\kappa)=-2 h\left(\mathrm{~L}^{2} / \pi\right) \kappa \mathrm{G}_{N}(\kappa \mathrm{L}, \kappa \mathrm{D})
$$

with:

$$
\mathrm{G}_{N}(\mathrm{x}, \mathrm{y})=\int_{[0,2 \pi]} \mathrm{u}(\mathrm{x} \cos \phi) \mathrm{u}(\mathrm{x} \sin \phi) \mathrm{v}_{N}(\mathrm{y} \cos \phi) \mathrm{v}_{N}(\mathrm{y} \sin \phi) \mathrm{d} \phi
$$



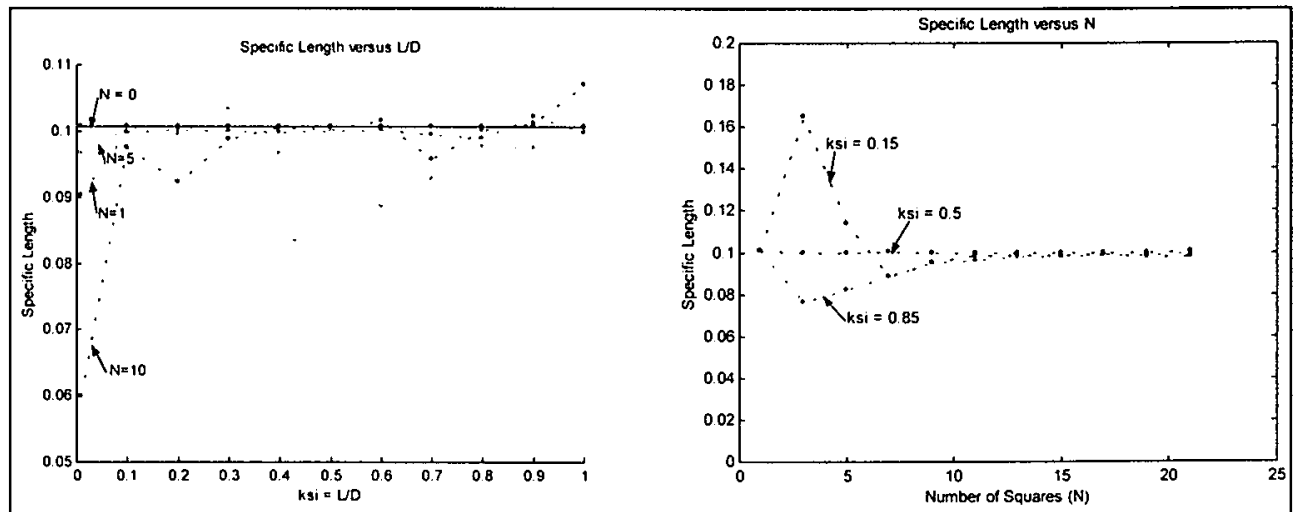

Fig. 3.5 Specific length 1 versus $x=L / D$ for $L=10$, and several numbers of squares $(2 N+1)$

So:

$$
\mathrm{P}(\kappa)=\kappa^{2} \mathrm{G}_{N}(\kappa \mathrm{L}, \kappa \mathrm{D})^{2} / \mathrm{M}_{2}(\mathrm{~L}, \mathrm{D}, \mathrm{N})
$$

Where we have used the generalised $n$-moments of $\mathrm{G}^{2}$ as:

$$
M_{n}(L, D, N)=\int G_{N}(L x, D x)^{2} x^{n} d x .
$$

which constraints $\int P(x) d x$ to 1 . The typical size ' $\lambda$ ' of the lattice can now be written according to equation [3.3]:

$2 \pi \lambda^{-1}=\int k \mathrm{P}(k) \mathrm{d} k=\int k^{3} \mathrm{G}_{\mathrm{N}}(\mathrm{L} k, \mathrm{D} k)^{2} \mathrm{~d} k / \mathrm{M}_{2}(\mathrm{~L}, \mathrm{D}, \mathrm{N})=\mathrm{M}_{3}(\mathrm{~L}, \mathrm{D}, \mathrm{N}) / \mathrm{M}_{2}(\mathrm{~L}, \mathrm{D}, \mathrm{N})$

To investigate the relation with the side $d=2 \mathrm{~L}$ of the square, and grid-size $\mathrm{D}$, define: $\xi=L / D \in[0,1]$. Then we can rewrite the expression for $M_{n}$ as:

$$
M_{n}(\xi, L, N)=\xi^{1+n} L^{-1-n} \int G_{N}(\xi x, x)^{2} x^{n} d x
$$

and rewrite the expression for $\lambda$ as:

$$
\lambda(\xi)=L \xi^{-1} \int G_{N}(\xi x, x)^{2} x^{2} d x / \int G_{N}(\xi x, x)^{2} x^{3} d x
$$

The dependence of $\lambda$ versus $L$ and $\xi$, hence $\mathrm{D}=\mathrm{L} / \xi$, is represented in Figure 3.6. For $N=0$ we find $\mathrm{v}_{0}(\mathrm{x})=1$, so there it coincides - as to be expected - with the expression for one single square. For a lattice-like configuration of comparable, square-like objects of size $D$ we therefore expect a typical size of $\lambda \approx D$. And so obtain a size comparable with the object. 


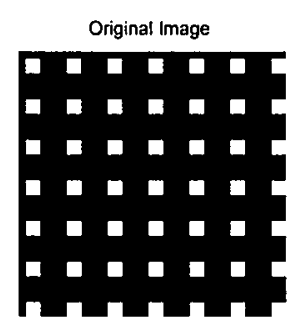

Typical scale $=5.127+-3.354 \mathrm{~mm}$ $\mathbf{k s i}=0.333$
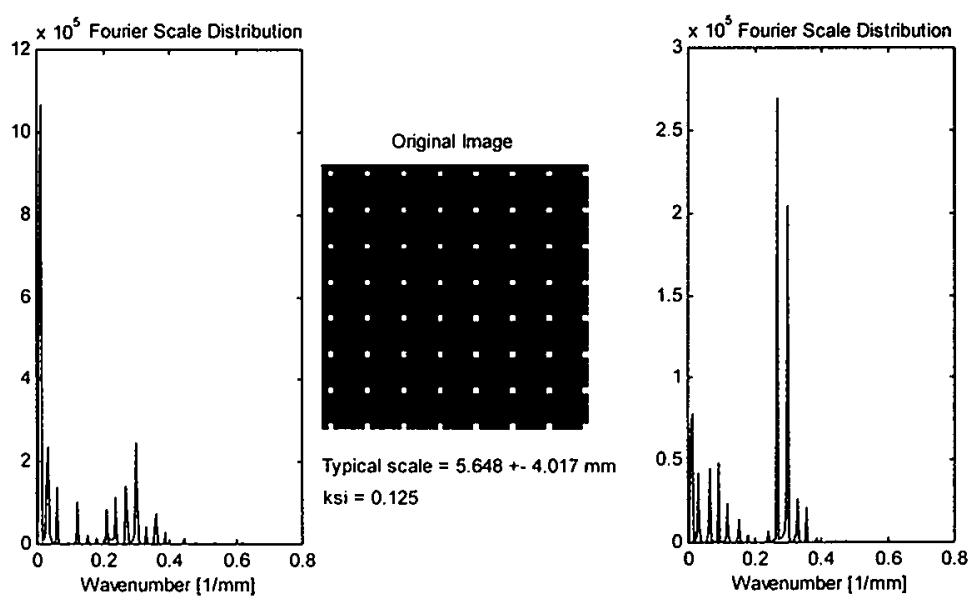

Fig. 3.6. Lattice of $(2 N+1)^{2}$ squares, for different $L$ and $D$.

\section{Example 4. Typical Scales of some Defects}

The figures below show some typical defects with their Fourier Power spectral density function (PSD) and their typical scale $\lambda$. It is found that for typical 'small' defects the characteristic scale $\lambda$ is relatively small, and that for somewhat 'larger' defects $\lambda$ is likewise larger. In Table 3.2 below some defects and their rescaled characteristic length $\lambda$ are listed.

\begin{tabular}{|c|c|}
\hline Defect Name & Typical Scale ' $\lambda_{\mathbf{r}}$ ' $[\mathbf{m m}]$ \\
\hline 'ink missing' & $9.9 \pm 6.0$ \\
\hline 'air bubbles' & $1.5 \pm 0.9$ \\
\hline 'dust particles' & $0.11 \pm 0.07$ \\
\hline
\end{tabular}

Table 3.2. Typical Fourier scales for some defects 


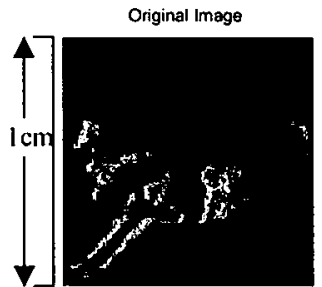

Typical scale $=9.866+-6.030 \mathrm{~mm}$

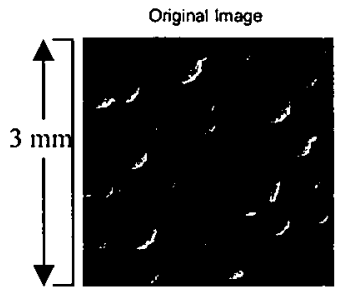

Typical scale $=1.474+-0.851 \mathrm{~mm}$

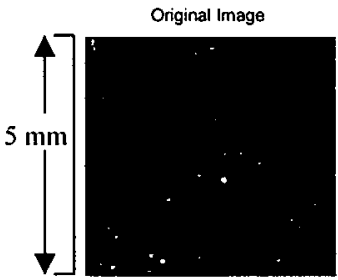

Typical scale $=0.112+.0 .068 \mathrm{~mm}$
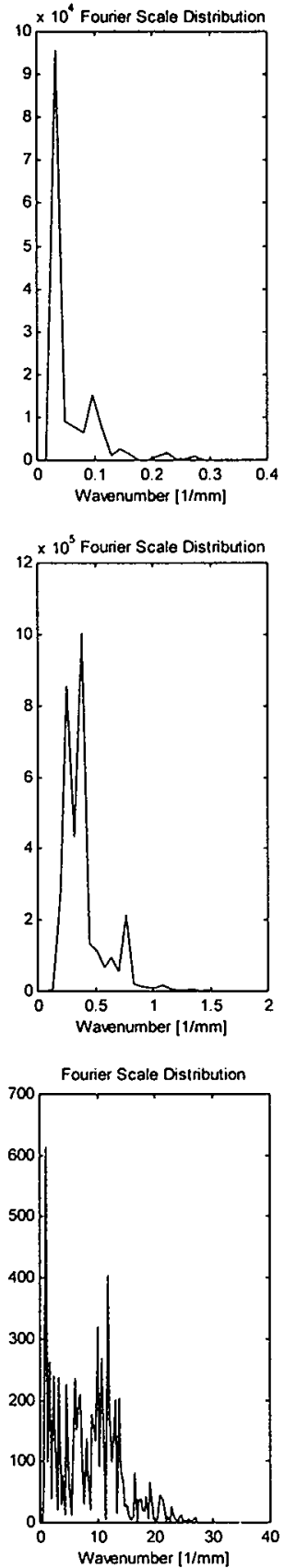

Fig. 3.7. Texture analysis with Fourier Transform. Above: left image of defect 'Ink missing' and right its Fourier Power spectral density function. This gives a characteristic length $\lambda_{\mathrm{r}}=9.9 \pm$ $6.0 \mathrm{~mm}$. Middle: image and PSD of defect 'Air Bubbles', with $\lambda_{\mathrm{r}}=1.5 \pm 0.9 \mathrm{~mm}$. Below: same pictures for defect 'Dust Particles', with characteristic length $\lambda_{r}$ of $0.11 \pm 0.07 \mathrm{~mm}$. 


\subsection{Wavelets, Multi Resolution Analysis and Continuous Wavelet Transform}

\subsubsection{Short Review of Wavelets}

Fourier analysis is an adequate tool for the spectral decomposition of signals. If there is relevant information contained in the spectrum of a signal or an image, then this technique is capable to identify and adequately represent it. All $\mathrm{N}$-dimensional signals that are periodic, or contained in a small window, can be efficiently analysed and represented with its Fourier decomposition. In practice, there are some important limitations to the method. If the signal essentially is non-periodic or not contained in a small window, the Fourier technique needs a substantial number of coefficients to adequately represent it. Likewise, if the signals vary in time, the coefficients must be made time-dependent.

More problematic is the fact that Fourier analysis practically fails to represent sharp discontinuities, like edges in images. In principle, given sufficient coefficients, the Fourier decomposition can approximate each discontinuity to any desired accuracy. In reality, however, the technique causes oscillations near sharp edges. This is of essential importance for signals that convey an infinite regression of detail like the famous Mandelbrot-set. Even with the limited resolution of digital images, this is also an important aspect of the texture of some printing defects. To cope with this limitations a number of adaptations to the Fourier technique were proposed, as for instance the windowed Fourier analysis. A successful adaptation of the Fourier technique developed in the last two decades is the wavelet analysis. Wavelets were introduced by J. Morlet [Morlet, 1983] to characterise specific seismic signals. Whereas the Fourier analysis relies on sinusoidal functions to decompose the signal, the wavelet analysis is based on the analysis with oscillating functions - hence wave with a relative small domain - hence wavelet. Because of their limited extension they are also capable of a spatial decomposition of the signal. While there is one generating function for the Fourier analysis : $\mathrm{e}^{\mathrm{ix}}$, there are an infinite number of functions that can be used for wavelet analysis. In Figure 3.8 two important wavelets; the Daubechies wavelet [Daubechies, 1990] and the Coiflet, are shown.
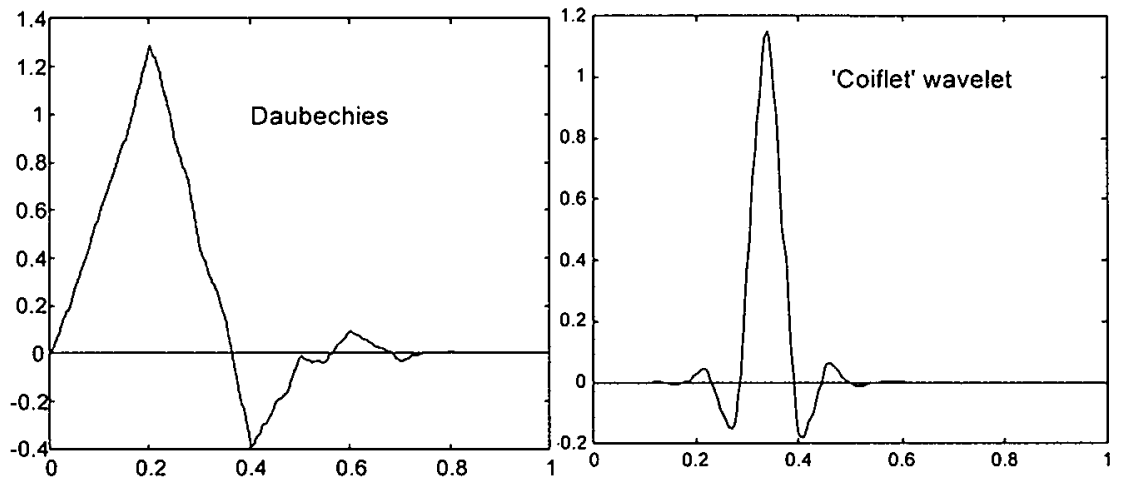

Fig. 3.8 Examples of two widespread wavelets, the Daubechies wavelet and the 'Coiflet'. 
For many applications it is important that the set of translations of the wavelet are orthogonal. Consider translations by $b$ and dilatations by $a$ of wavelet $\psi(\mathrm{x})$, called the 'mother' wavelet function, and define:

$$
\mathrm{T}_{\mathrm{ab}} \psi(\mathrm{x}) \equiv a^{1 / 2} \psi((\mathrm{x}-\mathrm{b}) / a)
$$

where $T$ is an affine transformation with translation $b$ and dilatation $a$. This function has a $L^{2}(\mathrm{IR})$-norm of $1 ;\left\|\mathrm{T}_{\mathrm{ab}} \psi\right\|^{2}=\left\langle\left.\mathrm{T}_{\mathrm{ab}} \psi\left|\mathrm{T}_{\mathrm{ab}} \psi>=\int\right| \mathrm{T}_{\mathrm{ab}} \psi\right|^{2} \mathrm{dx}=1\right.$, with $\left.<\bullet\right| \cdot>$ the inner product in $\mathrm{L}^{2}(\mathrm{IR})$, and where we have required that $\|\psi\|=1$. In order to generate an orthonormal basis we restrict $a$ and $b$ to a set of discrete values; the so-called binary dilations $a=2^{-\mathrm{n}}$, and dyadic translations $b=\mathrm{k} 2^{-\mathrm{n}}$, with $\mathrm{k}, \mathrm{n} \in \mathrm{Z}$. Now we define the basis functions $\psi_{\mathrm{nk}}$ as:

$$
\psi_{\mathrm{nk}}(\mathrm{x})=\mathrm{T}_{\left(2^{\mathrm{n}}, \mathrm{k}\right)} \psi(\mathrm{x}) \equiv 2^{1 / 2 \mathrm{n}} \psi\left(2^{\mathrm{n}_{\mathrm{x}}}-\mathrm{k}\right)
$$

Then for an orthonormal basis of wavelets we demand that:

$$
<\psi_{\mathrm{nk}}\left|\psi_{\mathrm{m} l}\right\rangle=\delta_{\mathrm{nm}} \delta_{\mathrm{kl}}
$$

where $\delta_{\mathrm{ij}}$ is the Kronecker-delta: $\delta_{\mathrm{ij}}=1$ if $\mathrm{i}=\mathrm{j}$, and else zero. With such basis any signal $f(x)$ in $L^{2}(I R)$ can be decomposed as a sum of wavelets:

$$
f(\mathrm{x})=\Sigma_{\mathrm{ml}} a_{\mathrm{ml}} \psi_{\mathrm{ml}}(\mathrm{x})
$$

with coefficients $a_{\mathrm{mn}}$ defined as:

$$
a_{\mathrm{ml}}=\left\langle\psi_{\mathrm{m} l}\right| f>=2^{1 / 2 \mathrm{~m}} \int \psi\left(2^{\mathrm{m}} \mathrm{x}-\mathrm{l}\right) f(\mathrm{x}) \mathrm{dx}
$$

The transformation: $f \rightarrow a_{\mathrm{ml}}$ is called the Discrete Wavelet Transform (DWT). Another important characteristic of wavelets is the number of vanishing moments, $n_{\psi}$. This means that all the moments of the wavelet $\psi(x)$ up to $n_{\psi}$ are equal to zero:

$$
0 \leq \mathrm{n} \leq \mathrm{n}_{\psi} \Rightarrow\left\langle\psi \mid \mathrm{x}^{\mathrm{n}}\right\rangle=\int \psi(\mathrm{x}) \mathrm{x}^{\mathrm{n}} \mathrm{dx}=0
$$

For a thorough and comprehensive introduction to wavelets see [Chui,1992].

\subsubsection{The Continuous Wavelet Transform}

The wavelet analogon to the Fourier transform can easily be defined. The continuous wavelet transformation (CWT) of a function $f(x)$ with regard to basis wavelet $\psi$ for translation $b$ and dilatation $a$ is defined as:

$$
\mathrm{W}_{\psi}[f](\mathrm{b}, a)=\left\langle\mathrm{T}_{\mathrm{ab}} \psi \mid f\right\rangle=a^{1 / 2} \int \psi((\mathrm{x}-\mathrm{b}) / a) f(\mathrm{x}) \mathrm{dx}
$$

The amplitude of the wavelet transform at position $\mathrm{x}=\mathrm{b}$ and scale ' $\mathrm{a}$ ', $\left|\mathrm{W}_{\psi}[f](\mathrm{b}, a)\right|$, is a measure for the characteristic details at scale $a$.

\subsubsection{The Multi Resolution Approach to Wavelet Analysis}

In Multi Resolution Analysis (MRA) (also multiresolution approximation) a signal is decomposed both in scale as spatially. The scale decomposition can be considered analogous to the frequency domain of the Fourier-decomposition. In MRA the wavelets are a basis to represent the details gained between a resolution at one scale of magnification, and the next finer scale. MRA is based on consecutive approximations at finer and finer resolutions. The concept is formalised by the following definition, [Malat, 1989]: 
Definition 3.1 A sequence $\left\{V_{n} \mid n \in \mathbb{Z}\right\}$ of closed sub-spaces of $L^{2}(\mathbb{R})$ is a Multi Resolution Analysis if and only if the following six following conditions are satisfied:

1. $\forall(\mathrm{n}, \mathrm{k} \in \mathbb{Z}) f(\mathrm{x}) \in \mathrm{V}_{\mathrm{n}} \Leftrightarrow f\left(\mathrm{x}-2^{\mathrm{n}} \mathrm{k}\right) \in \mathrm{V}_{\mathrm{n}}$

2. $\forall(\mathrm{n} \in \mathbb{Z}) \quad \mathrm{V}_{\mathrm{n}+1} \subset \mathrm{V}_{\mathrm{n}}$

3. $\forall(n \in \mathbb{Z}) f(x) \in V_{n} \Leftrightarrow f(x / 2) \in V_{n+1}$

4. $\lim _{n \rightarrow \infty} V_{n}=\cap_{n=-\infty}+\infty V_{n}=\{0\}$

5. $\lim _{n \rightarrow-\infty} V_{n}=$ closure $U_{n=-\infty}:+\infty V_{n}=L^{2}(I R)$

6. $\exists\left(\varphi \in \mathrm{L}^{2}(\mathbb{R})\right) \quad\{\varphi(\mathrm{x}-k) \mid k \in \mathbb{Z}\}$ is an orthonormal basis of $\mathrm{V}_{0}$

So, the MRA generates a decomposition of the Hilbert space $L^{2}(I R)$ into a chain of closed subspaces: ... $\subset \mathrm{V}_{2} \subset \mathrm{V}_{1} \subset \mathrm{V}_{0} \subset \mathrm{V}_{-1} \subset \mathrm{V}_{-2} \subset \ldots$. Furthermore, we can define subspaces $W_{j}$ as an orthogonal complement of $V_{j}$ in $V_{j-1}: V_{j-1}=V_{j} \oplus W_{j}$, so that: $L^{2}(I R)=\oplus_{j \in Z} W_{j}$. If there exists a coarsest scale $n$, as indeed it does for digital images, we can write the chain of spaces as: $V_{n} \subset \ldots \subset V_{1} \subset V_{0} \subset V_{-1} \subset \ldots$ and obtain:

$$
\mathrm{L}^{2}(\mathbb{R})=\mathrm{V}_{\mathrm{n}} \oplus_{\mathrm{j}} \leq \mathrm{n} \mathrm{W}_{\mathrm{j}}
$$

The spaces $\mathrm{W}$ are called detail spaces, for they contain the new details in the function revealed by increasing scale $m$ to scale $m+1$. Let us assume that the reference subspace $\mathrm{V}_{0}$ is generated by a single function $\phi \in \mathrm{L}^{2}(\mathrm{IR})$, like stated in condition 6 in definition 3.1. So $\{\phi(\mathrm{x}-k) \mid k \in Z\}$ is an orthonormal basis of $\mathrm{V}_{0}$. If $\phi$ generates a MRA then $\phi$ is called a scaling function, also known as 'mother' scaling function.

\subsubsection{Wavelet Packages}

The decomposition of a signal proceeds according to fixed relations between the wavelet coefficients at different scales. Such scale relations can be regarded as 'zooming' independently into both the high and low frequency bands of the signal. Such related packages of coefficients are known as wavelet packages (sometimes called wavelet packets). Starting point for the wavelet packages is the mother scaling function $\phi$, defined above, and ditto mother wavelet $\psi$, defined in equation [3.4]. The equation that pair-wise relates scales is known as the dilation equation, also called a two-scale equation:

$$
\begin{aligned}
& \phi(\mathrm{x})=\Sigma_{\mathrm{k}} h_{0}(\mathrm{k}) \phi(2 \mathrm{x}-\mathrm{k}) \\
& \psi(\mathrm{x})=\Sigma_{\mathrm{k}} h_{1}(\mathrm{k}) \phi(2 \mathrm{x}-\mathrm{k})
\end{aligned}
$$

with certain coefficients $h_{0}$ and $h_{1}$. This is a consequence from the fact that $\phi, \psi \in V_{0}$, and $V_{0} \subset V_{1}$, and the space $V_{1}$ is generated by $\{\phi(2 x-k) \mid k \in Z\}$. More specific, this defines the relation between the mother wavelet function $\psi$ to the scaling function $\phi$. Based on the two-scale decomposition, it is possible to construct a hierarchical decomposition, both upward as downward in scale. This creates a mechanism of a filter bank, the signal is rewritten in terms of a new basis in the new scale-regime. Substitution of $x^{\prime}=2^{n} x$ gives the dilation equation for higher scales: 


$$
\begin{aligned}
& \phi\left(2^{\mathrm{n}} \mathrm{x}\right)=2 \sum_{\mathrm{k}} h_{0}(\mathrm{k}) \phi\left(2^{\mathrm{n}+1} \mathrm{x}-\mathrm{k}\right) \\
& \psi\left(2^{\mathrm{n}} \mathrm{x}\right)=2 \sum_{\mathrm{k}} h_{1}(\mathrm{k}) \phi\left(2^{\mathrm{n}+1} \mathrm{x}-\mathrm{k}\right)
\end{aligned}
$$

These hierarchies can be used for an efficient decomposition and reconstruction algorithms, see [Chui, 1996].

\subsubsection{Application of Wavelets for Feature Extraction and Shape Analysis}

Similarly to Fourier analysis, wavelet analysis lends itself for the description of geometric objects in terms of their spectral composition. Because of its local character, opposed to the global nature of Fourier analysis, wavelets are better suited to represent local discontinuities, such as sharp edges in an image, whereas Fourier decomposition will generally smear out such borders. Mallet et al. [Mallet et al.,1997] utilise the discrete wavelet-transform to produce wavelet coefficients which are used for classification. Rather than using one of the standard wavelet bases, they compute the wavelet which optimises some specified discriminant criteria, relevant to the specific context. Pittner and Kamarthi [Pittner,Kamarthi,1999] propose a comprehensive method for feature extraction, based on the discrete wavelet coefficient matrix. Substantial clusters in this matrix represent the most relevant frequencies for the object. Next, they represent the (norm of) these clusters as Euclidean vectors, and consider them as the most salient features. Drolon et al. [Drolon et al.,2000] use the continuous wavelet transform to analyse the multiscale shape of sedimentary particles. By applying Sammon-mapping to this representation, they are able to fully separate relevant sedimentary classes Other examples using wavelets for feature extraction are [Khalil,Bayoumi,2000] and [Tao et al.,2001]. These approaches are discussed in section 3.5.

In the next two sections, we propose two applications of the wavelet formalism in our context. First, as an alternative to the 'FindAreas'-algorithm (Section 2.3.1) for searching an image for relevant parts. Second, we propose an algorithm for wavelet decomposition, that in contrast to MRA does not generate equidistant separated wavelets at a discrete dilatation, but the collection of multiscale wavelets that minimises the cumulative squared difference between a function and its approximation. Finally, we consider the wavelet analysis of textures by computing a 'typical' length, similarly to the Fourier approach in Section 3.2.3.

\subsubsection{The Wavelet Transform as an Alternative for the FindAreas Algorithm}

As the 2-dimensional continuous wavelet transform CWT generates a complete covering of an object with wavelets, this algorithm can be used as an alternative to the FindAreas algorithm, discussed in Section 2.3.1. This algorithm generates a set of SearchBoxes that collectively cover the Z-representation of an image. 

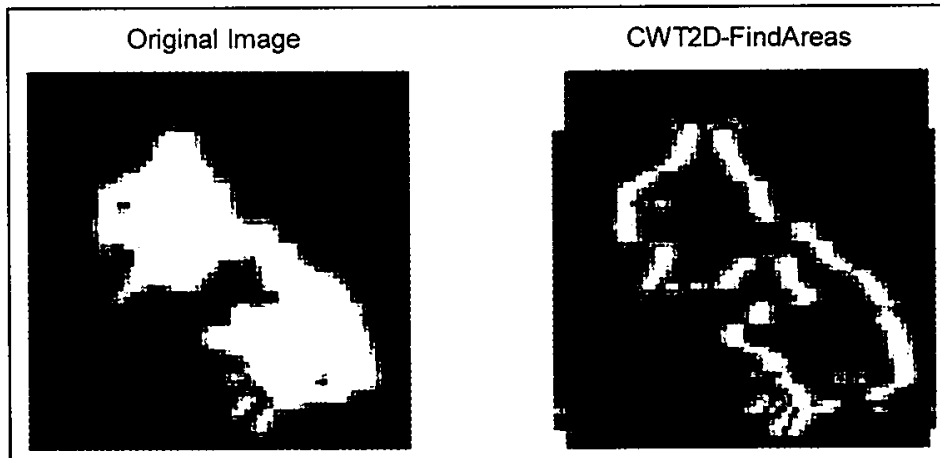

Fig 3.9 Left: Z-representation of an image with defect 'Ink Missing'. The intensity acts as a trigger for the FindAreas algorithm. Right: Alternative algorithm based on the absolute value of the CWT2D-transform for fixed scale $a=2$. A Gaussian[1] function was used, having 1 vanishing moment. This triggers the function to be relatively high near the border of the area.

As a measure for the intensity of the difference between image and template, FindAreas uses the intensity, i.e. the greyscale, of $Z$. In terms of the CWT the intensity of the difference is reflected in the value of $\left|\mathrm{W}_{\psi}[f]\right|$ at $\mathbf{x}$ for scale $a$. therefore, we can relate the area of interest with a scale $a$. Since each defect can have a different set of relevant scales, we can 'zoom-in' interactively, based on the actual most likely hypothesis. For the present most likely defect class we select the most relevant scale a. Iterative application of the exclusion of investigated points generated with this algorithm will lead to an 'erosion' of the area; it will shrink from the outside to the inside. This is also visible in Figure 3.9.

This approach is in general, however, not similar to the greyscales of $Z$. If the applied wavelet has $n_{\psi}$ vanishing moments, the $2 \mathrm{D}-\mathrm{CWT}$ intensity $\left|\mathrm{W}_{\psi}[f]\right|$ generates a set of points $\{\mathbf{x}\}$ where at scale $a$ the $n_{\psi}$-spatial derivative tensor has the highest values. So for $\mathrm{n}_{\psi}=1$ this indicates the borders of the areas, see Figure 3.9. For this reason the approach works best if $n_{\psi}$ is chosen to be zero. This means that we apply a onehumped scaling function $\phi$ rather than the wavelet. But in that case the CWT $\left|W_{\psi}[f]\right|$ recreates the original image $f(\mathbf{x})$, and the optima are represented by the highest greyscale values of $f$, just as the FindAreas-algorithm. Another problem with this approach is that the CWT is the high computational load, which makes this algorithm substantially slower compared with FindAreas.

\subsubsection{Global Wavelet Decomposition}

\section{a Non-orthogonal Function Space Alternative to Multi Resolution Analysis}

MRA and wavelet packages appear as attractive formalisms for the analysis of irregular signals. In the case of the analysis of a scanprofile of the defect 'hairs' the signal consists of a quasi-periodic collection of highly irregular, but on its own nearsimilar peaks. The disadvantage of MRA in the analysis of such functions, consisting of non-equidistant separated identical peaks, is that they are analysed at an infinite regression of higher resolutions. Thus, a function consisting of two identical peaks: $f(x)=\phi\left(x-b_{1}\right)+\phi\left(x-b_{2}\right)$ normally leads to an infinite sum of higher order wavelets, whereas a sum of two correctly constructed scaling functions $\phi$ should suffice. This is 
caused by the periodic dyadic spacing $x_{n}=2^{n} x-k, k=0,1, \ldots, 2^{n}-1$ at resolution $n$. If we relax this condition, we can write $x_{k}=a_{k} x-b_{k}$. More in general, we propose to write a signal as the following decomposition:

$$
\mathrm{H}_{\phi}[f](\mathrm{x})=\Sigma_{\mathrm{k}=1}^{\mathrm{n}_{\mathrm{k}}} \mathrm{c}_{\mathrm{k}} \phi\left(\mathrm{a}_{\mathrm{k}} \mathrm{x}-\mathrm{b}_{\mathrm{k}}\right)
$$

with 'mother' scaling function $\phi$. We call this approach the Global Wovelet Decomposition Approach (GWDA). From this representation it is clear that MRA is a special case of GWDA such that $a_{m}$ and $b_{m}$ are respectively a dilation and a translation, and $c_{m}$ is the corresponding wavelet coefficient:

$$
\forall_{\mathrm{n}, \mathrm{k} \in Z} \exists_{\mathrm{m} \in Z} \mathrm{a}_{\mathrm{m}}=2^{\mathrm{n}}, \mathrm{b}_{\mathrm{m}}=\mathrm{k} 2^{-\mathrm{n}}, \mathrm{c}_{\mathrm{m}}=\left\langle\psi_{\mathrm{nk}} \mid f\right\rangle
$$

First, we try to generate a computation scheme for the GWDA-coefficients $\left\{n, a_{k}, b_{k}, c_{k}\right\}$, representing the entire set of solutions. Within this set we can consequently look or the solution with the smallest number of terms. Next, we discuss one global search technique and an approximation scheme.

\section{Approach 1: Global Least Square Approximation}

In the Global Least Squares Approximation (GLSQ) the signal is approximated globally with series $\mathrm{H}_{\phi}$ in the mother scaling function $\phi$. Here $G L S Q$ is used with a quadratic criterion $V$ in terms of the series $\mathbf{a}, \mathbf{b}$, and $\mathbf{c}$ :

$$
V(\mathbf{a}, \mathbf{b}, \mathbf{c})=\left\|f-\mathrm{H}_{\phi}[f]\right\|^{2}
$$

Here, $\|$.$\| is the \mathrm{L}^{2}$-norm. The minima of $V$ are defined by:

$$
\partial V / \partial \mathrm{a}_{\mathrm{k}}=0 \wedge \partial \mathrm{V} / \partial \mathrm{b}_{\mathrm{k}}=0 \wedge \partial \mathrm{V} / \partial \mathrm{c}_{\mathrm{k}} \wedge \partial \mathrm{V} / \partial \mathrm{n}=0
$$

This can be written as a series of complexly entangled equations.

$$
\begin{array}{lll}
\left\langle f \mid \phi\left(\lambda_{\mathrm{k}} \mathrm{x}-\beta_{\mathrm{k}}\right)\right\rangle & = & \Sigma_{\mathrm{k}=1: \mathrm{N}} \alpha_{\mathrm{n}} \mathrm{P}\left(\lambda_{\mathrm{k}}, \beta_{\mathrm{k}}, \lambda_{\mathrm{n}}, \beta_{\mathrm{n}}\right) \\
\left\langle f \mid \phi^{\prime}\left(\lambda_{\mathrm{k}} \mathrm{x}-\beta_{\mathrm{k}}\right)\right\rangle & = & \Sigma_{\mathrm{k}=1: \mathrm{N}} \alpha_{\mathrm{n}} \mathrm{Q}\left(\lambda_{\mathrm{k}}, \beta_{\mathrm{k}}, \lambda_{\mathrm{n}}, \beta_{\mathrm{n}}\right) \\
\left\langle f \mid \mathrm{x} \phi^{\prime}\left(\lambda_{\mathrm{k}} \mathrm{x}-\beta_{\mathrm{k}}\right)\right\rangle= & \Sigma_{\mathrm{k}=1: \mathrm{N}} \alpha_{\mathrm{n}} \mathrm{R}\left(\lambda_{\mathrm{k}}, \beta_{\mathrm{k}}, \lambda_{\mathrm{n}}, \beta_{\mathrm{n}}\right)
\end{array}
$$

with constants $P, Q$, and $R$ depending on $\left(\lambda_{\mathrm{k}}, \beta_{\mathrm{k}}, \lambda_{\mathrm{n}}, \beta_{\mathrm{n}}\right)$ and defined by:

$$
\begin{array}{lll}
\mathrm{P}\left(\lambda_{\mathrm{k}}, \beta_{\mathrm{k}}, \lambda_{\mathrm{n}}, \beta_{\mathrm{n}}\right) & = & \left\langle\phi\left(\lambda_{\mathrm{k}} \mathrm{x}-\beta_{\mathrm{k}}\right) \mid \phi\left(\lambda_{\mathrm{n}} \mathrm{x}-\beta_{\mathrm{n}}\right)\right\rangle \\
\mathrm{Q}\left(\lambda_{\mathrm{k}}, \beta_{\mathrm{k}}, \lambda_{\mathrm{n}}, \beta_{\mathrm{n}}\right) & = & \left\langle\phi^{\prime}\left(\lambda_{\mathrm{k}} \mathrm{x}-\beta_{\mathrm{k}}\right) \mid \phi\left(\lambda_{\mathrm{n}} \mathrm{x}-\beta_{\mathrm{n}}\right)\right\rangle \\
\mathrm{R}\left(\lambda_{\mathrm{k}}, \beta_{\mathrm{k}}, \lambda_{\mathrm{n}}, \beta_{\mathrm{n}}\right)= & <\mathrm{x} \phi^{\prime}\left(\lambda_{\mathrm{k}} \mathrm{x}-\beta_{\mathrm{k}}\right)\left|\phi\left(\lambda_{\mathrm{n}} \mathrm{x}-\beta_{\mathrm{n}}\right)\right\rangle
\end{array}
$$

This, in principle infinite, set of coupled equations can not be solved in general. Therefore we must look to efficient search techniques.

\section{Approach 2: A Genetic Algorithm for GWDA}

In this approach we apply a simple Genetic Algorithm (GA) for determining the parameters in the Global Wavelet Decomposition Approach to approximate a given function $f(\mathrm{x})$. In this way, the problem is defined as a distributed local search in the GWDA parameter-space. Genetic Algorithms (GAs) [Holland,1975], [Goldberg,1989] are computational schemes, based on the principle of survival of the fittest by means of natural selection, as originally postulated by Charles Darwin ${ }^{3}$ in

\footnotetext{
${ }^{3}$ Actually, the addition 'survival of the fittest' was advocated by the co-discoverer of natural selection, Alfred Russel Wallace, while Darwin himself favoured a restriction to: 'The principle of natural selection'. Ultimately, Darwin regretted this addition, but did not change it in later editions [Gould,1991].
} 
his celebrated 'On the origin of species' in 1859 [Darwin, 1859]. Whereas in the original context the term 'fittest' is tautological, because the 'fittest' are defined as those individuals that somehow manage to survive, in GAs the 'fittest' is accurately defined in terms of an optimisation process. In general, in the context of GAs 'fitness' denotes the ability of an 'individual' to solve a given problem. Compared to natural selection, GAs are greatly simplified abstractions, and the entire process could be termed 'artificial selection', were it not that this term is reserved for agricultural breeding. A GA consists of four basic ingredients. In the first place stands the specified problem that must be solved. This can concern an optimisation of a closed, well-defined, and parameterised function, or some implicit multi-agent interaction. Second, a GA operates on a population of agents, which interact through their fitness. Third, the fitness measures the efficiency of an agent to solve the problem. Fourth, each agent in the population contains a set of parameters that in some way determine its modus operandi, and thus its efficiency to solve the problem. This set is comparable to the genotype in natural selection, while the modus operandi for solving the problem corresponds to the phenotype of the individual.

Applied to the context of finding the best GWDA-approximation to a given function, the problem can be stated as a simple optimisation problem. For this reason, it suffices to employ a simple genetic representation for an agent: a linear string of the parameters, belonging to the GWDA-parameters that collectively define the $H$ approximation of function $f$ through equation [3.15]. Thus, each agent contains a full set of parameters: $\left\{\mathrm{n}, a_{k}, b_{k}, c_{k} \mid k=1 . \mathrm{n}\right\}$. These values are encoded directly as real numbers. Furthermore, the parameters in the initial population are in selected ranges; $\{1, . ., 128\}$ for $n,[1 / 16,16]$ for $a$, domain $(f)$ for $b$, and $[0, \max (f)]$ for $c$. The fitness $F$ of an agent is determined by the quadratic $L S Q$-criterion $V$ from equation [3.17] applied on its parameter set $(\mathbf{n}, \mathbf{a}, \mathbf{b}, \mathbf{c})$. The lower this criterion, the higher its fitness. More specific: $F=-V(\mathbf{a}, \mathbf{b}, \mathbf{c})$. If: $V(\mathbf{a}, \mathbf{b}, \mathbf{c})=0$ the iteration ends. The entire GA consists of an initiation and an (infinite) iteration cycle. First, the initial population is created. Next, the following basic cycle is repeated, theoretically until $V(\mathbf{a}, \mathbf{b}, \mathbf{c})$ becomes zero:

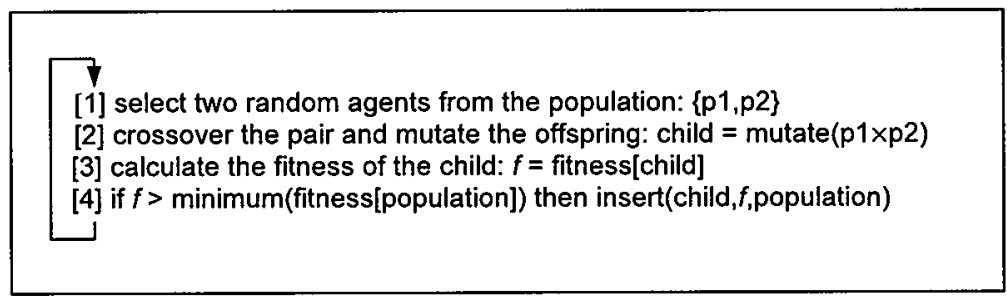

This iteration cycle is based on a steady-state optimisation GA called Genitor [Whitley,1989], [Paredis, Westra,1997]. In this cycle, first two agents are selected in order to reproduce, using standard two-point crossover and adaptive mutation. Subsequently, the fitness of the 'offspring' is computed according to: $F=-V(\mathbf{a}, \mathbf{b}, \mathbf{c})$. If this fitness exceeds the minimum fitness in the agent population, the offspring is inserted at the appropriate rank position. In order to keep the population constant, the least fit individual is removed.

Several numerical experiments were performed with diverse scanprofiles. These experiments illustrate the advantage of the GA approach. In Figure 3.10 the result of an approximation of a 'hairy' scanprofile after $10 \mathrm{~K}$ generations is shown. The left side shows the change of the quadratic criterion $V$ as function of the generations, the 
right side shows the best agent after $10 \mathrm{~K}$ generations, i.e. the function $\mathrm{H}_{\phi}[f](\mathrm{x})$ with the parameters $\left\{n, a_{k}, b_{k}, c_{k}\right\}$ of the best agent. The general conclusions after these experiments is that the GA-approach is capable of efficiently approximating a given signal using the GWDA-expansion $\left.\mathrm{H}_{\phi}[f](\mathbf{x}) \mathbf{a}, \mathbf{b}, \mathbf{c}, n\right)$, at a large computational cost. This approach suffers from the same problem as similar local search algorithms, the fact that it can end in a local optimum. The quadratic criterion function $V(\mathbf{a}, \mathbf{b}, \mathbf{c}, n)$ defined above can be used to measure the degree of 'hairiness' of a given scanprofile. This approach is therefore employed in appendix A.4 in discussing specialised techniques for detecting the defect 'hairs'.
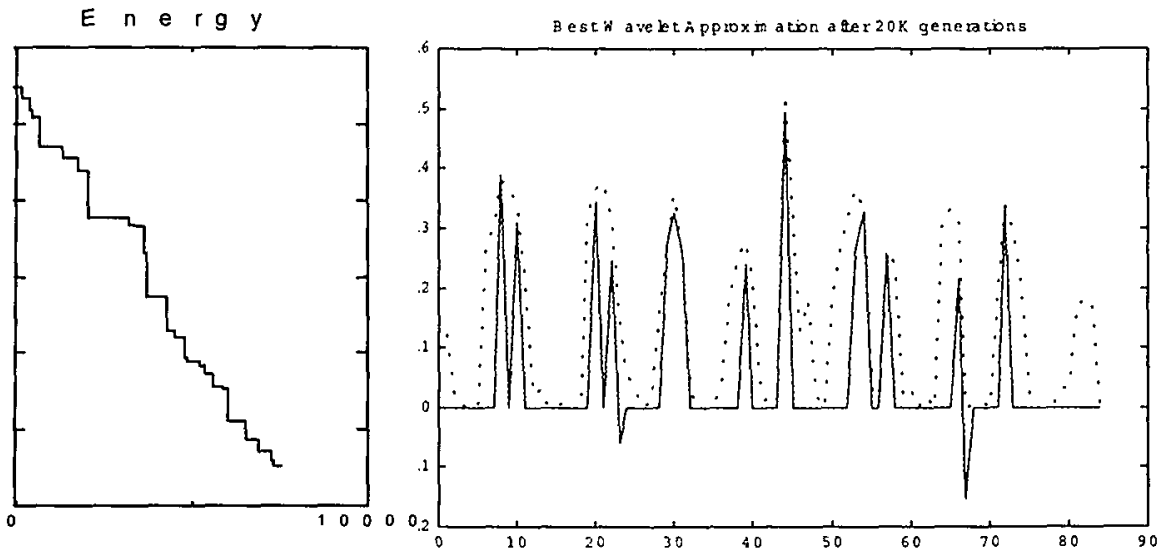

Fig. 3.10 Results of the approximation of a 'hairy' profile after $10 \mathrm{~K}$ generations. Left: criterion $V$ as function of the generation, Right: the best approximation (dashed) after $10 \mathrm{~K}$ generations.

\section{Approach 3: Local Least Square Approximation}

The third approach to determine the GWDA-decomposition of a given scanprofile is based on an algorithm called Iterative Local Least Squares Approximation (ILLSQ). In ILLSQ the signal is optimally approximated locally with the 'mother scaling function' $\phi$. In this approach the scaling function of the $2^{\text {nd }}$ degree B-splines were used. The algorithm is as follows.

[1] determine the intervals $x_{k}=\left[x_{1}^{(k)}, x_{2}^{(k)}\right]$ where the scan is above the noise-level:

$f(x)>v$ for all $x_{1}{ }^{(k)}<x<x_{2}{ }^{(k)}$

[2] for each interval $X_{k}$ with $\operatorname{size}\left(X_{k}\right)>\epsilon$ :

- approximate the scan with a mother function $\phi(x)$ using LSQ on $X_{k}$

- calculate 'fit' and the coefficients $\left\{a_{k}, b_{k}, c_{k}\right\}$ of the approximation $c_{k} \phi\left(a_{k} x-b_{k}\right)$

In the ILLSQ algorithm, first the domain of $f$ is partitioned in a collection of closed intervals $X_{k}=\left[x_{1}(k), X_{2}(k)\right]$, such that: (i) $\forall\left(x \in X_{k}\right) f(x)>v$, and (ii) $U_{k} X_{k} \subseteq$ domain $(f)$, where $v$ is a given threshold related to the actual noise level. Next, for each interval $X_{k}$ with minimal length $\epsilon$ (to avoid noisy peaks), the best fit with one single peak $c \phi(a \mathrm{x}+b)$ is determined, using LSQ with a criterion $\mathrm{v}(a, b, c)=\|c \phi(a \mathrm{x}+b)-f\|^{2}$ for $\mathrm{x} \in \mathrm{X}_{\mathrm{k}}$. In the case of our mother function $\phi(\mathrm{x})=\gamma \mathrm{x}(1-\mathrm{x})$ this LSQ-problem can be 
solved exactly. This results in a set $\left(a_{\mathrm{k}}, b_{\mathrm{k}}, c_{\mathrm{k}}, n\right)=\mathrm{LSQ}_{\phi}(f, \mathrm{X}, v)$. The total error $E$ is defined as:

$$
E=\Sigma_{\mathrm{k}=1: \mathrm{n}} \mathrm{v}\left(a_{\mathrm{k}}, b_{\mathrm{k}}, c_{\mathrm{k}}\right)=\Sigma_{\mathrm{k}=1: \mathrm{n}} \int_{\mathrm{X}[\mathrm{k}]}\left|c_{\mathrm{k}} \phi\left(a_{\mathrm{k}} \mathrm{x}+b_{\mathrm{k}}\right)-f(\mathrm{x})\right|^{2} \mathrm{dx}
$$

Figure 3.11 shows the demonstration of ILLSQ to a hairy signal. The original signal is indicated by a solid curve. The 'ground-level' of the approximation $H$ is determined by the noise level $\nu$. Next, all peaks with minimum width above the noise level are approximated with the scaling function $\phi(x)$. This gives a good approximation of the original scanprofile. The coefficients $\left(a_{\mathrm{k}}, b_{\mathrm{k}}, c_{k}, n\right)$ from the $H$-decomposition of $f$ can subsequently be used to obtain a match with the profile of a standard defect type. For instance, based on a large set of empirical data, typical coefficients for all defect classes can be calculated. For a given scanprofile the $H$-coefficients can be calculated and the normalised metric distance - called "Mahalanobis-distance" and discussed in Chapter 5 - in the parameters space to all defect classes can be compared. The best match is the closest defect class. This procedure was found the best way to detect scanprofiles of hairs. An example is shown in Figure 3.11.

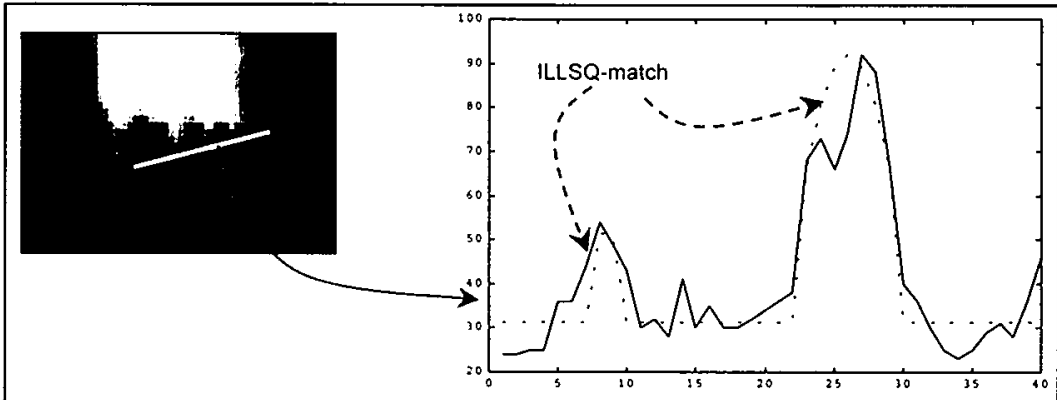

Fig 3.11 Left: example of an image with 'hairs', and a $\Gamma 2$-scanline. Right: resulting scanprofile, and the approximation of the signal generated by the ILLSQ-algorithm. This algorithm only operates on: [1] peaks above the noise level $v$, indicated by the base of the approximation, and [2] peaks wider than 4 units. The resulting coefficients can be matched with a reference set, and the best match can be obtained. 


\subsubsection{The Wavelet Analysis of Textures}

In this section we present an analogon for the Fourier-based computation of a 'typical length', as presented in Section 3.2.3, in terms of wavelets. Starting point is the 2Dcontinuous wavelet transform (2D-CWT) for translation vector $\mathbf{x}$ and dilation $a$; $\mathrm{W}_{\psi}[f](\mathbf{x}, a)$. In this method we search for the maximal absolute difference between the 2D-CWT $\mathrm{W}_{\psi}[f]$ and the original image $f$. The basic idea behind this approach is that the 2D-CWT represents features near location $\mathrm{x}$ relevant at scale $a$. Therefore, the difference between image and the 2D-CWT should be maximal at scales typical for the object. Let for $a>0 \mathrm{U}(a)$ be equal to the absolute difference:

$$
\mathrm{U}(a)=\int_{\Omega}\left|\mathrm{W}_{\psi}[f](\mathbf{x}, a)-f(\mathbf{x})\right| \mathrm{d}_{2} \mathbf{x}
$$

where $\Omega$ is the domain of the image. Let $A$ contain the values of $a$ for which the derivative of $U$ with respect to $a$ is maximal.

$$
A=\arg \sup _{a} \frac{\partial U(a)}{\partial a}
$$

This set $A$ contains sizes typical for the image. In reality noise has a detrimental effect on this approach. For that reason we average $\mathrm{U}$ in an neighbourhood $\mathrm{B}_{a}$ around $a$. This will not affect $A$. Best results were obtained by:

$$
\begin{aligned}
& \mathrm{u}(a)=a^{-1} \int_{[0, a]} \mathrm{U}\left(a^{\prime}\right) \mathrm{d} a^{\prime} \\
& A^{\prime}=\arg \sup _{a} \frac{\partial u(a)}{\partial a}
\end{aligned}
$$

In order to construct one value for the typical size we can take the average over $A$ '. The resulting entity, denoted $a_{0}$, shows a relation with the defect class. In order to investigate the validity of this approach a number of test images was produced consisting of identical spheres with diameter $D$. In Figure 3.12 the values of $a_{0}$ is plotted versus sphere diameters $D$. The correlation in this figure is $98 \%$. Figure 3.13 shows some typical examples of such images, together with two 2D-CWTs of the image at different scales. In the same figure $\partial_{a} \mathrm{u}$ is plotted versus $a$. We finish this section with the application of this method to some real examples of defects. Figures 3.14 and 3.15 show images of defects, with a bar indicating length. In the figure the typical size is denoted. 


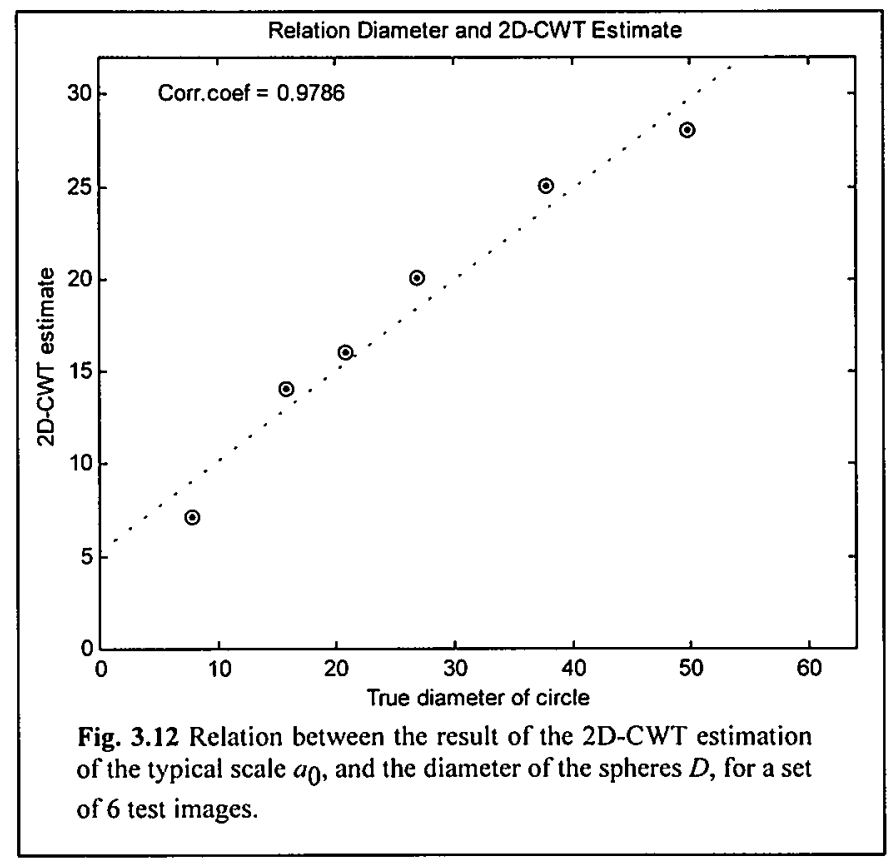


Original Image

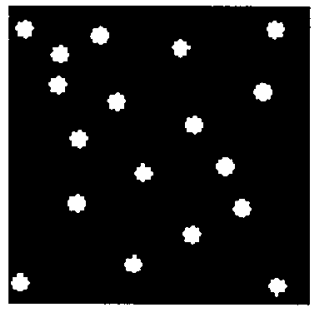

Wavelet Transform at scale 43

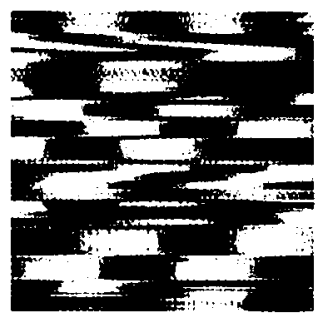

Original

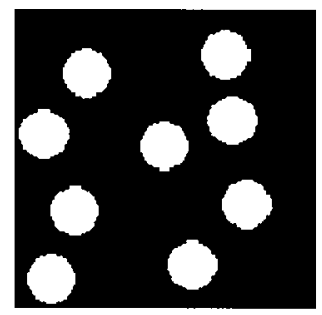

Wavelet Transform at scale 43

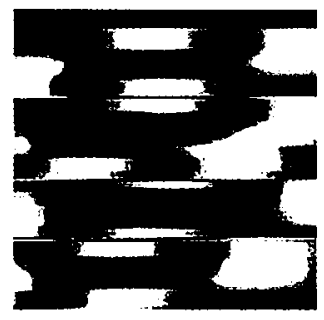

Wavelet Transform at scale 21

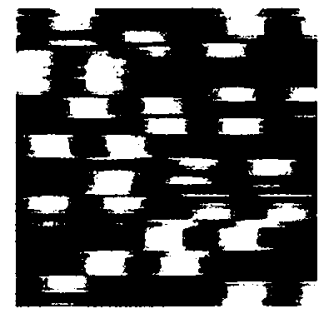

Increase of Difference versus Scale

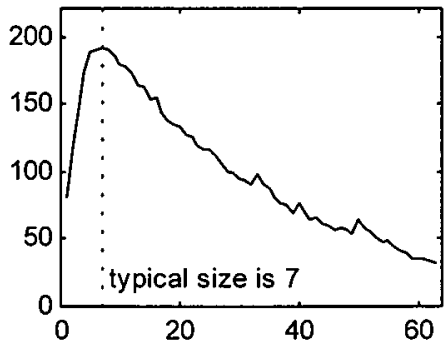

Wavelet Transform at scale 21

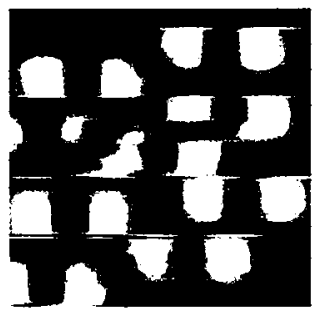

Increase of Difference versus Scale

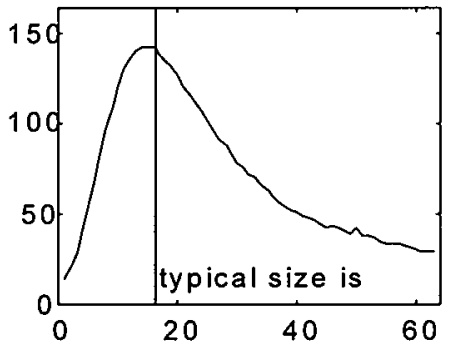

Fig. 3.13 Examples of test images of spheres. Above: four figures with the original spheres of size 8. Two CWT transforms are shown for scale 21 and 43 respectively. Also shown is the dependence of $\partial_{a} \mathrm{u}$ versus $a$. The maximal value is attained for $a$ is 7 . Below: similar for spheres of diameter 21 . Here the maximal value for $\partial_{a} \mathrm{u}$ is attained for $a$ is 16 . 
Original Image

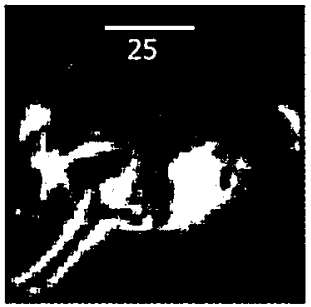

Wavelet Transform at scale 43

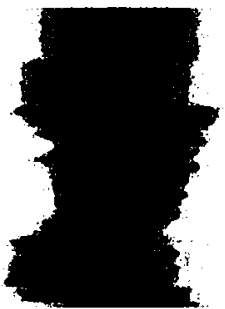

Original Image

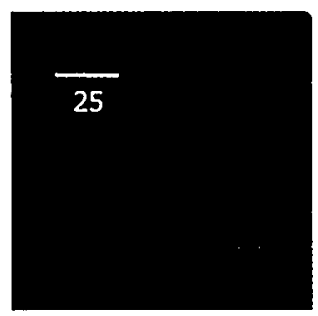

Wavelet Transform at scale 43

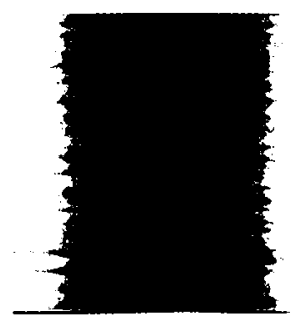

Wavelet Transform at scale 21

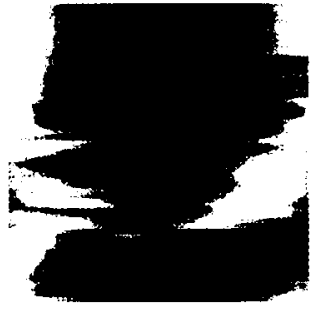

Increase of Difference versus Scale

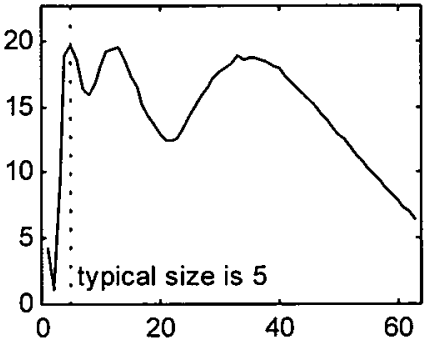

Wavelet Transform at scale 21

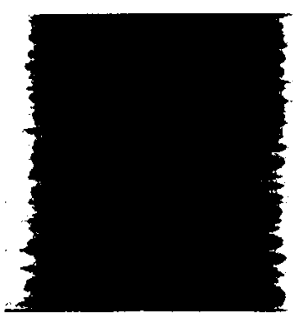

Increase of Difference versus Sce

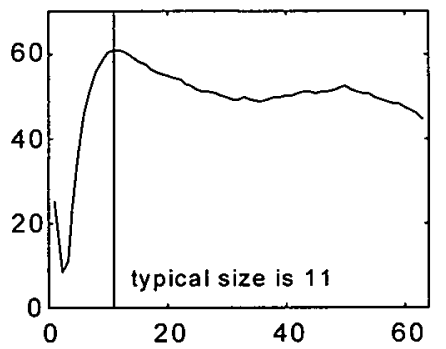

Fig. 3.14 Examples of real images of defects. Above: defect 'ink missing'. Shown is the original defect and two CWT transforms for scales 21 and 43. Also shown is the dependence of $\partial_{a} \mathrm{u}$ versus $a$. These reveal three characteristic sizes, the lowest being 5 . Below: similar for defect 'air bubbles - foam'. Here the maximal value for $\partial_{a} \mathrm{u}$ is attained for $a$ is 11 , though other peaks are visible for $a \approx 1$, and $a \approx 50$. In the original images a bar of length 25 is indicated. 
Original Image

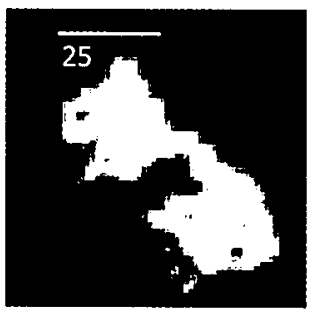

Wavelet Transform at scale 43

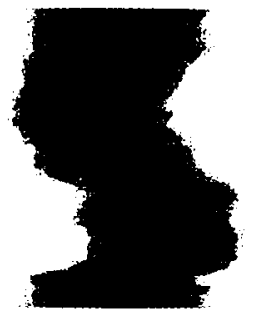

Original Image

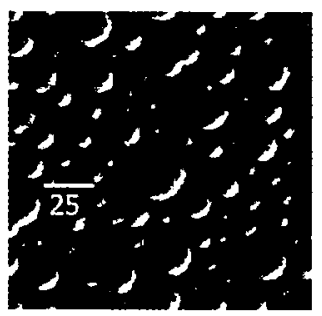

Wavelet Transform at scale 43

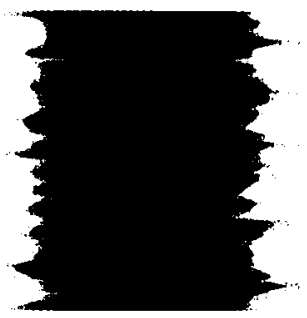

Wavelet Transform at scale 21

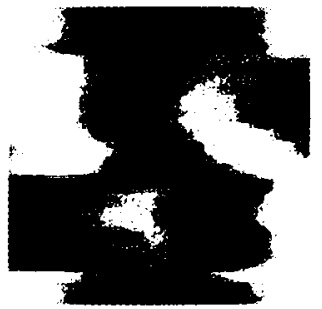

Increase of Difference versus Scale

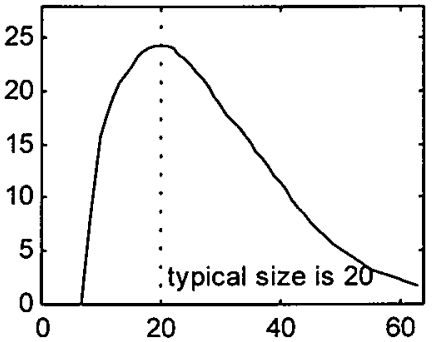

Wavelet Transform at scale 21

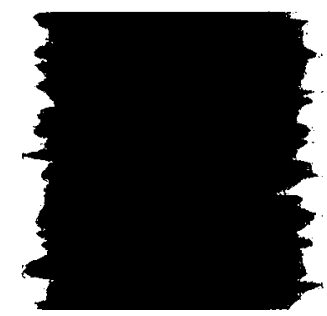

Increase of Difference versus Sca

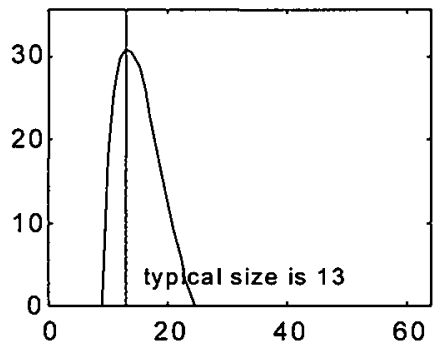

Fig. 3.15 Examples of real images of defects. Above: defect 'ink missing'. Shown is the original defect and two CWT transforms for scales 21 and 43. Also shown is the dependence of $\partial_{a^{\mathrm{u}}}$ versus $a$. This lot shows a definite peak for $a \approx 20$. Below: similar for defect 'air bubbles'. Here the maximal value for $\partial_{a} \mathrm{u}$ is attained at a sharp peak for $a \approx 13$. In the original images a bar of length 25 is indicated. 


\section{Application of Typical Wavelet Length on a ID-Block-function}

As a simple example of this approach, we study its application on a 1D-blockfunction: $f(\mathrm{x})=h \chi_{[-\mathrm{L}, \mathrm{L}]}(\mathrm{x})$, where $\chi_{\mathrm{V}}$ is the characteristic function on set $\mathrm{V}$, and $h$ is the greyscale of the block. For the CWT we use a localised wavelet such that for $\mathrm{x} \notin[-$ $1,1] \Rightarrow \psi(\mathrm{x})=0$. For this wavelet $\psi$ the continuous wavelet transform of $f$ is:

$$
\mathrm{W}_{\psi}[f](a, \mathrm{~b})=h \cdot \sigma^{-1 / 2} \int_{[-\mathrm{L}, \mathrm{L}]} \psi(\mathrm{x}-\mathrm{b} / a) \mathrm{dx}
$$

In three cases this transform $W$ is equal to zero; if the wavelet lies left or right outside the interval $[-L, L]-$ because then $\psi$ is zero, and if the wavelet lies entirely inside the interval $[-L, L]-$ because by definition $\int \psi(x) d x=0$. So the only non-zero values are assumed if the domain of the wavelet overlaps with $[-\mathrm{L}, \mathrm{L}]$, which is the case when:

$$
\text { i. }(\mathrm{b}+a>-\mathrm{L}) \wedge(\mathrm{b}-a<-\mathrm{L}) \text { or ii. }(\mathrm{b}+a>\mathrm{L}) \wedge(\mathrm{b}-a<\mathrm{L})
$$

These conditions are visible in the CWT of a block, depicted in Figure 3.16. If we restrict wavelet $\psi$ to a Haar-wavelet, defined on $[-1,1]$ :

$$
\mathrm{H}(\mathrm{x})=\left(\chi_{[-1,0]}(\mathrm{x})-\chi_{[0,1]}(\mathrm{x})\right) / \sqrt{ } 2
$$

we can for each given scale $a$ distinguish the different topological possibilities of the relative position of the wavelet and the block-function. Figure 3.17 shows an example. The defining points of the wavelet are left-side: $x_{1}=b-a$, middle: $x_{2}=b$, and right-side: $\mathrm{x}_{3}=\mathrm{b}+a$. Each of these three points can be in one of the three possible areas of the block-function: left: $\left.A_{1}=<-\infty,-L\right\rangle$, inside: $A_{2}=[-L, L]$, and right: $A_{3}=\langle L, \infty\rangle$. So, a priori there are $3^{3}=27$ possible combinations of $x_{k}$ and $A_{j}$. These combinations can be encoded as a triplet $\left\{\alpha_{1}, \alpha_{2}, \alpha_{3}\right\}$, with $\alpha_{\mathrm{k}}$ representing the relative position $x_{k}$. Let $\alpha_{k}$ is equal to: -1 for $x_{k} \in A_{1}, 0$ for $x_{k} \in A_{2}$, and +1 for $x_{k} \in A_{3}$ s. However, there is redundancy in these triplets due to the constraint: $x_{1}<x_{2}<x_{3}$. Therefore all states $\left\{{ }^{*}, *,-1\right\}$ reduce to one case; the wavelet is left of the block. Likewise all $\{1, *, *\}$ reduce to 'wavelet right of the block'. In the same manner $\{-$ $1, *, 1\}$ implies $*=0$ and represents one rather than three cases. In this way only 10 possibilities remain. These ten cases are listed in the Table below.

\begin{tabular}{|l|l|l|l|}
\hline $\mathbf{n r}$ & \multicolumn{1}{l}{ code } & \multicolumn{1}{l|}{ condition } & wavelet transform $W(a \mathrm{~b}) \sqrt{(2 \mathrm{a}) / \mathbf{h}}$ \\
\hline 1 & $\{* *-1\}$ & $\mathrm{b}+\mathrm{a}<-\mathrm{L}$ & 0 \\
\hline 2 & $\{-1-10\}$ & $(\mathrm{b}>-\mathrm{L}-\mathrm{a}),(\mathrm{b} \leq-\mathrm{L})$ & $-(\mathrm{b}+\mathrm{a}+\mathrm{L})$ \\
\hline 3 & $\{-1-11\}$ & $(\mathrm{b}<-\mathrm{L}),(\mathrm{b}>\mathrm{L}-\mathrm{a})$ & $-2 \mathrm{~L}$ \\
\hline 4 & $\{-100\}$ & $(\mathrm{b}>-\mathrm{L}),(\mathrm{b}<-\mathrm{L}+\mathrm{a}),(\mathrm{b}<\mathrm{L}-\mathrm{a})$ & $(\mathrm{b}-\mathrm{a}+\mathrm{L})$ \\
\hline 5 & $\{-101\}$ & $(\mathrm{b}<\mathrm{a}-\mathrm{L}),(\mathrm{b}>\mathrm{L}-\mathrm{a}),(\mathrm{b}<\mathrm{L}),(\mathrm{b}>-\mathrm{L})$ & $2 \mathrm{~b}$ \\
\hline 6 & $\{-111\}$ & $(\mathrm{b}>\mathrm{L}),(\mathrm{b}<\mathrm{a}-\mathrm{L})$ & $2 \mathrm{~L}$ \\
\hline 7 & $\{000\}$ & $(\mathrm{b}>-\mathrm{L}+\mathrm{a}),(\mathrm{b}<\mathrm{L}-\mathrm{a})$ & 0 \\
\hline 8 & $\{001\}$ & $(\mathrm{b}>-\mathrm{L}+\mathrm{a}),(\mathrm{b}<\mathrm{L}),(\mathrm{b}>\mathrm{L}-\mathrm{a})$ & $\mathrm{a}+\mathrm{b}-\mathrm{L}$ \\
\hline 9 & $\{011\}$ & $(\mathrm{b}>-\mathrm{L}+\mathrm{a}),(\mathrm{b}>\mathrm{L})$ & $\mathrm{a}-\mathrm{b}+\mathrm{L}$ \\
\hline 10 & $\{1 * *\}$ & $\mathrm{b}>\mathrm{L}+\mathrm{a}$ & 0 \\
\hline
\end{tabular}

Table 3.3 Ten possible conditions for wavelet-transform of $1 D$-block function.

So, for the absolute difference between the CWT and the block-function we can write in this case:

\footnotetext{
${ }^{4}$ We can write an expression for $\alpha_{k}$ as: $\operatorname{sign}\left(\operatorname{sign}\left(x_{k}-L\right)+\operatorname{sign}\left(x_{k}+L\right)\right)$, with sign: $x<0 \rightarrow-1, \operatorname{sign}(0)=0$, and sign: $x>0 \rightarrow 1$.
} 


\begin{tabular}{|c|c|c|c|}
\hline nr & coule & condition & Difference: $W(\mathrm{a} b)-h .\left.\mathrm{Blok}\right|_{-L}, \mathrm{~L}$ \\
\hline 1 & $\{* *-1\}$ & $b+a<-L$ & 0 \\
\hline 2 & $\{-1-10\}$ & $(b>-L-a),(b \leq-L)$ & $-(b+a+L) \cdot h / \sqrt{ }(2 a)$ \\
\hline 3 & $\{-1-11\}$ & $(b<-L),(b>L-a)$ & $-2 \mathrm{~L} \cdot \mathrm{h} / \sqrt{ }(2 \mathrm{a})$ \\
\hline 4 & $\{-100\}$ & $(b>-L),(b<-L+a),(b<L-a)$ & $(b-a+L) \cdot h / \sqrt{(2 a)-h}$ \\
\hline 5 & $\{-101\}$ & $(b<a-L),(b>L-a),(b<L),(b>-L)$ & $2 b \cdot h / \sqrt{(2 a)-h}$ \\
\hline 6 & $\{-111\}$ & $(b>L),(b<a-L)$ & $2 \mathrm{~L} . \mathrm{h} / \sqrt{(2 a)}$ \\
\hline 7 & $\{000\}$ & $(b>-L+a),(b<L-a)$ & $-\mathrm{h}$ \\
\hline 8 & $\{001\}$ & $(b>-L+a),(b<L),(b>L-a)$ & $(a+b-L) \cdot h / \sqrt{ }(2 a)-h$ \\
\hline 9 & $\{011\}$ & $(b>-L+a),(b>L)$ & $(a-b+L) \cdot h / \sqrt{(2 a)}$ \\
\hline 10 & $\{1 * *\}$ & $b>L+a$ & 0 \\
\hline
\end{tabular}

Table 3.4 Ten possibilities for difference: wavelet-transform - ID-block function.

The function $U$ from equation [3.18] thus follows from: $\mathrm{U}(a)=\int|W \backslash f(a, b)-f(b)| \mathrm{d} b$. In Figure 3.18 function $U$ is plotted for some typical cases. Figure 3.19 shows $U$ for one specific block, and Figure 3.20 shows the relation between estimated size and true diameter for some blocks. For this specific example a clear relation could be established between diameter $2 \mathrm{~L}$ and the output $A$ of this method.

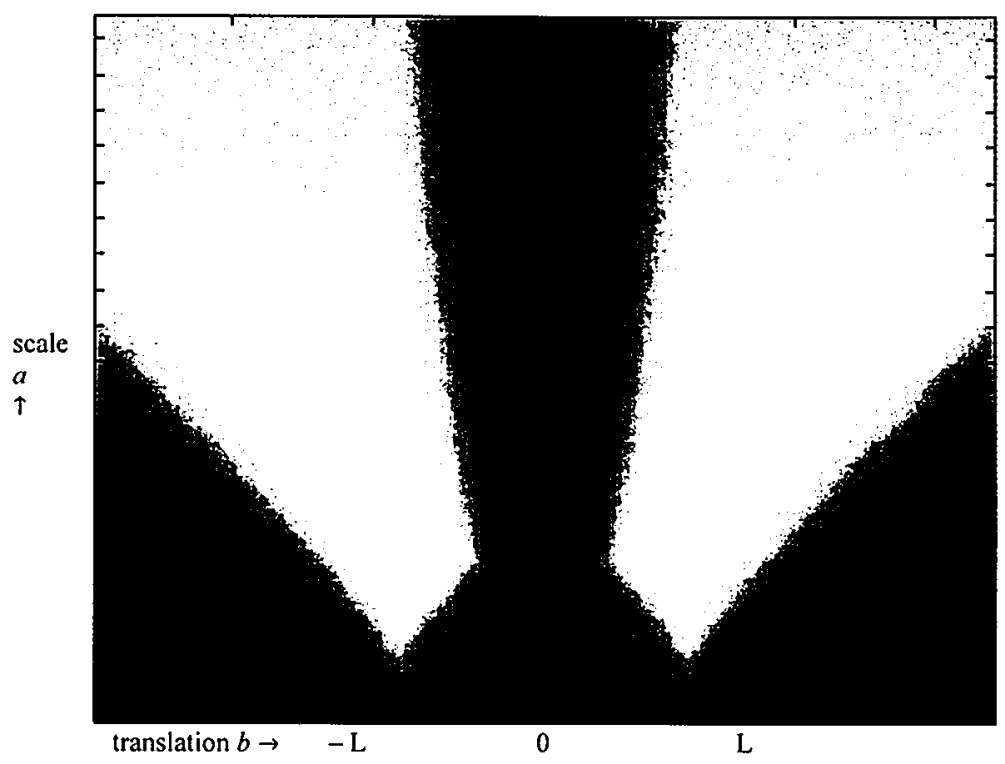

Fig. 3.16 CWT for a block-function. The non-zero area is bounded by: $(a>-\mathrm{b}-\mathrm{L}) \wedge(a>\mathrm{L}+\mathrm{b})$ or $(a>\mathrm{L}-\mathrm{b}) \wedge(a>\mathrm{b}-\mathrm{L})$ 


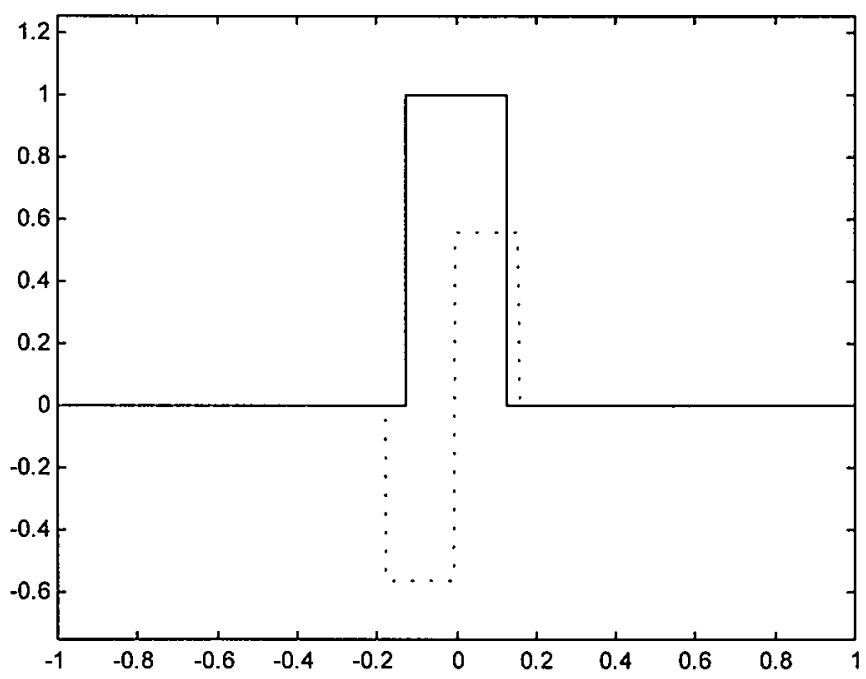

Fig. 3.17 Example of the relative position of a Haar-wavelet to the block-function.

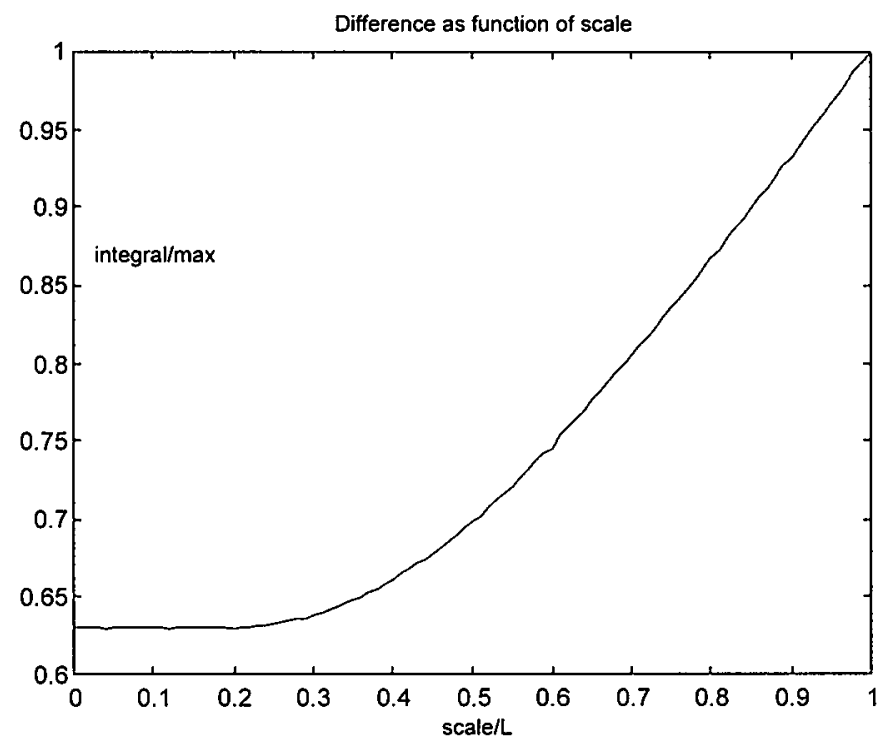

Fig 3.18 Relation between the integral $\mathrm{U}(a)$ and the scale $a$, for $a<\mathrm{L}$. 


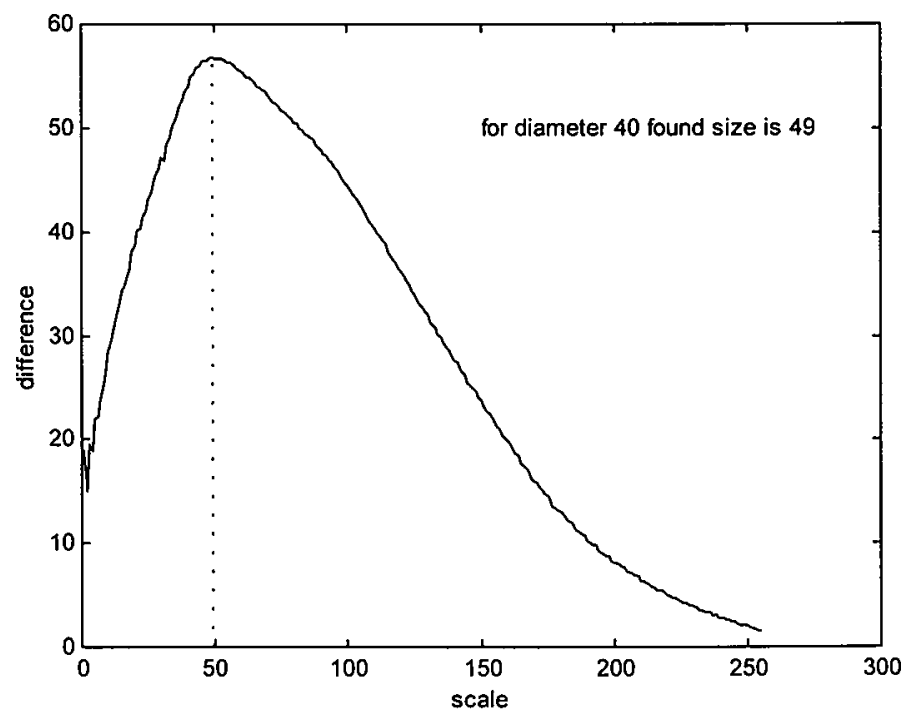

Fig. 3.19 Function $\mathrm{U}(a)$ for one specific block-function with diameter 40.

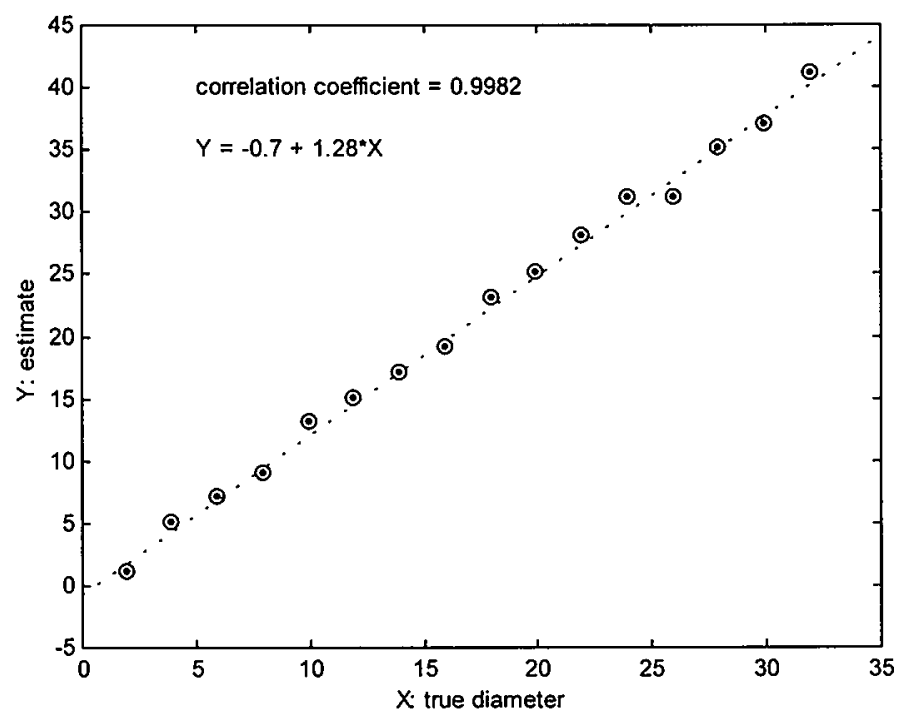

Fig. 3.20 The relation between estimated size and true diameter for some blocks. 


\subsection{Fractals and Multifractals}

In this section we introduce the necessary tools and definitions required to characterise fractal sets relevant to our context. More profound and extensive treatment of this subject can be found in various standard textbooks on the subject, see for instance [Mandelbrot,1999].

\subsubsection{Fractals and Fractal Dimensions}

For traditional geometric sets, such as squares, pyramids and hyper-spheres, the notion of dimension is associated with the minimum number of parameters required to uniquely describe the position of its points. This degree of freedom is called the topological dimension $\mathrm{d}_{\mathrm{T}}$. This is always an integer number. This definition is applicable for all geometric sets, even for 'pathological' objects such as the Peanocurve and the Cantor-set. However, this concept fails to represent the obvious differences of such sets with the conventional geometric objects. First, we generalise the definition of dimension on the basis of a scaling law.

Consider a geometric set $S$, with $\mathrm{d}_{\mathrm{T}}(S)=\mathrm{n}$, revealing new detail at each scale of magnification. Let us consider an adequate covering of this set with $N(\epsilon)$ (hyper)blocs of size $\epsilon$. Now let us study how $N$ varies with $\epsilon$ in the limit $\epsilon \downarrow 0$. For a traditional geometric set of topological dimension $n$, the number $N$ varies like: $\mathrm{N}(\epsilon) \propto \epsilon^{-\mathrm{n}}$. In 1919 Hausdorff [Hausdorff,1919] introduced a new definition for dimension, that is now called a fractal dimension. He first defined a measure $\mu_{\mathrm{H}}(S, \mathrm{~d})$ of $S$ in a generalised dimension $d$. He showed that there exists one unique real number $\mathrm{d}_{\mathrm{H}}$, such that: $\mu_{\mathrm{H}}(S, \mathrm{~d})=\infty$ if $\mathrm{d}_{\mathrm{H}}<\mathrm{d}_{\mathrm{H}}$, and $\mu_{\mathrm{H}}(S, \mathrm{~d})=0$ if $\mathrm{d}>\mathrm{d}_{\mathrm{H}}$. The boundary value $\mathrm{d}_{\mathrm{H}}$ is called the Hausdorff dimension, and the associate measure of the set is $\mu_{\mathrm{H}}\left(S, \mathrm{~d}_{\mathrm{H}}\right) \in<0, \infty>$ indicates the size of the set. In general this construction is not practical for direct computation for a given set. In practice it is more simple to study the scaling power laws exhibited by $N(\epsilon)$. In 1958 Kolmogorov introduced a quantity to specify the scaling behaviour which he called capacity. Because of its ease of computation this is a suitable parameter to characterise such sets ${ }^{5}$ :

$$
\mathrm{d}_{\mathrm{K}}(\mathrm{S})=\limsup _{\epsilon \downarrow 0} \ln \mathrm{N}(\epsilon) / \ln (1 / \epsilon)
$$

This quantity is also called the box-dimension. In 1978 Mandelbrot [Mandelbrot, 1978] introduced the term fractals for sets that exhibit a non-integer fractal dimension. A fractal is an irregular geometric object with an infinite nesting of structure at all scales. The importance of fractals is that most natural objects are fractals. Even all real dynamics in nature exhibit chaotic trajectories (called strange attractors) that are fractals. Assessing the fractal properties of an object is informative, as it can be used to characterise and thus identify an object. For this reason we study the fractal properties of the printing defects and investigate whether their fractal properties can be used to identify them.

Using the Triadic Cantor set (TCS) as an example, we demonstrate the principle of the calculation of a fractal dimension. The Triadic Cantor set is a collection of points on a line segment that is the result of an infinite number of iterations, in which each step consists of removing the middle-third of the segment(s) remaining from the previous step. Figure 3.21 shows the construction process of the Triadic Cantor set, starting above with a line segment that is iteratively dissected in downward direction.

\footnotetext{
${ }^{3}$ Sometimes this is confused with the Hausdorff-Besicovitch dimension.
} 
As the construction process starts at iteration 0 with 1 interval of length 1 , in iteration $n$ there are $2^{n}$ segments of length $3^{-n}$. So, we can cover the $n$-th stage of construction process with $N=2^{n}$ boxes of size $\epsilon=3^{-n}$, so $N(\epsilon)=\epsilon^{-\ln (2) / \ln (3)}$. Therefore the fractal dimension of the TCS is: $d_{K}($ TCS $)=\limsup _{\epsilon \downarrow 0} \ln N(\epsilon) / \ln (1 / \epsilon)=3 \log 2$. There are many definitions of fractal dimensions, which are partly related, see [Westra, 1982] and [Farmer et al. 1983].

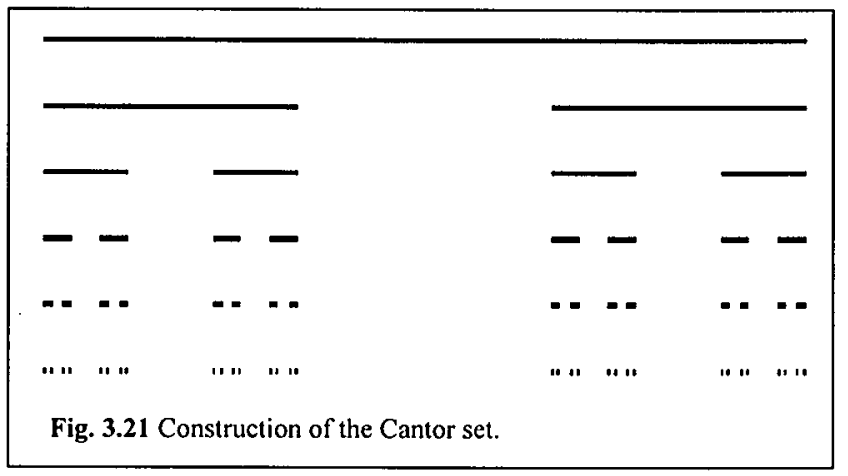

This method for obtaining the fractal dimension from a covering with finite intervals can readily be generalised to higher dimensions. The covering of a set with hypercubes, or 'boxes', with an upper-bound size, is called the 'box-counting method', or the 'grid dimension method'. The box-counting method is demonstrable not wellsuited for the estimation of fractal dimension [Theiler,1990]. This method underestimates the true fractal dimension above the topological dimension 1, mainly due to discretisation in pixels and grey-scales. Since discrete images have a finite resolution, there exists a lower bound for the box-size for the box-counting method. Bisoi and Mishra [Bisoi,Mishra,2001] found that the box-size for an image of $N$ pixels should vary roughly in the range $\left[N^{1 / 6}, N^{1 / 2} / 2\right]$. Because of the lower bound, Termonia and Alexandrowicz [Termonia,Alexandrowicz, 1983] scaled the boxes such, that they contain the same number of points. This, so called, fixed-mass method resulted in a simple, and fast algorithm, yet providing very accurate estimates. Asvestas et al. [Asvestas et al.,1999] used this approach to estimate the fractal dimension of several discrete images. In this thesis we study another suitable alternative to the box-counting method for accurately estimating the fractal dimension, called the multifractal formalism.

\subsubsection{Synopsis of the Multifractal Formalism}

Most fractals found in nature do not exhibit the simple power scaling laws found in conventional geometric objects and in self-similar fractals like the Cantor set. These sets can not be represented by either a continuous density function or as a finite sum of Dirac delta-functions. An example is the Hausdorff measure of the Triadic Cantor set, discussed before. Such measures are called singular. Sets of this kind can not be specified by merely one single fractal dimension. For their characterisation it is required to define a whole spectrum of dimensions called the generalised dimensions $D_{\mathrm{q}}$. The traditional fractal dimension is but one element in this continuous spectrum. 
To distinguish such multi-scale fractal sets from traditional one-scale self-similar sets, they are called multifractals. For this reason we propose to call the traditional selfsimilar fractals unifractals. The essential difference between them is that for unifractals there exists some affine transformation that translates each part of the set into identically but smaller sub-parts. This downward self-similarly is repeated ad infinitum. The formalism of multifractal objects is described in terms of the microcanonical ensemble theory, stemming from statistical physics. This describes the dependence of some meaningful statistical quantities, like entropy and free energy, as function of the box-covering of the set, and noise considered as 'temperature'. Let the multifractal set $S$ be covered with an ensemble $C$ of hyperboxes $B_{i}$ of size $\epsilon_{i} \leq \epsilon$, and centres $\mathbf{x}_{\mathbf{i}}$.

$$
C(\epsilon)=\left\{B_{i} \mid B_{i}=B_{\epsilon_{i}}\left(x_{i}\right) \wedge \epsilon_{i} \leq \epsilon \wedge \exists_{n \in I N} i=1 \ldots n\right\}
$$

$\mathrm{C}(\epsilon)$ is any collection of hyperboxes that may cover $S$. The minimal number of hyperboxes, needed to cover the set entirely, so $S \subseteq \mathrm{C}(\epsilon)$, is defined as $\mathrm{N}(\epsilon)$ :

$$
\mathrm{N}(\epsilon)=\inf \#\{\mathrm{C}(\epsilon) \mid S \subseteq \mathrm{C}(\epsilon)\}
$$

The a-priori spatial probability to encounter box $i$ in the multifractal, denoted as $\mathrm{p}_{\mathrm{i}}$, is equal to the number of points in the hyperbox, denoted as $n_{i}$, divided by the total number of points in the complete set, i.e., its total normalised mass, $M=\Sigma_{i} n_{i}$. In ensemble theory we consider the ensemble of all possible coverings - ergo partitions - of $S$. The partition function ${ }^{6} Z$ for this ensemble is expressed in terms of the local probabilities and masses, the box-sizes, and the total mass ${ }^{7}$.

$$
Z(a, q)=\sum_{i=1: N(\epsilon)} p_{i}^{q} / \epsilon_{i}^{a}=M^{-q} \epsilon^{-a} \sum_{i=1: N(\epsilon)} n_{i} q
$$

where $a$ is called the mass exponent, $q$ the moment order, and $\epsilon_{\mathrm{i}}$ is the length of the hyperbox $i$. In the last step of equation [3.26] we assumed that all hyperboxes have the same size $\epsilon$. Similar to the Hausdorff measure there are three possible outcomes for $\mathrm{Z}$, depending on $a$ and $\mathrm{q}$. If for fixed $\mathrm{q}, a$ is greater than some relation $\tau(\mathrm{q})$ the partition sum diverges to infinity. On the other hand, if $a$ is less than $\tau(q)$ the sum converges to zero. Only if $a$ is exactly equal to $\tau(q)$ the sum approaches a finite value different from zero. Thus, by requiring that $Z$ is equal to some non-zero constant, we can obtain the relationship between $a$ and $q$. If we normalise the probabilities $p_{i}$ we obtain $Z=1$. In analogy with ensemble theory the function $\tau(q)$ is called the free energy. This is given by:

$$
\tau(\mathrm{q})=\lim _{\epsilon \downarrow 0} \ln \left(\sum_{\mathrm{i}=1: \mathrm{N}(\epsilon)} \mathrm{p}_{\mathrm{i}} \mathrm{q}\right) / \ln \epsilon
$$

The generalised dimension $\mathrm{D}_{\mathfrak{q}}$ [Hentschel,Procaccia,1983] is then defined as:

$$
\mathrm{D}_{\mathrm{q}}=\tau(\mathrm{q})(\mathrm{q}-1)^{-1}
$$

This equation defines a whole family of fractal dimensions. For certain values of $q$ a special meaning can be attributed to $D_{q}$. For instance, for $q=0$ we obtain the original expression for the fractal Kolmogorov dimension: $D_{0}=\lim _{\epsilon \downarrow 0} \ln N(\epsilon) / \ln 1 / \epsilon \equiv d_{K}$. For a uniform fractal all $p_{i}$ are equal. Therefore the general dimension $D_{q}$ does not vary with $q$ and the set is not a multifractal. For a non-uniform fractal, the variation of $D_{q}$ quantifies the non-uniformity and the set is multifractal. For multifractals the generalised dimensions are positive and decrease monotonically with $q$, while for

\footnotetext{
${ }^{6}$ We use the standard notation of ensemble theory, ' $Z$ ' for German 'Zustand'. Of course, there is no relation to the absolute difference $Z$, defined earlier.

${ }^{7}$ For a detailed discussion see [Roman, 1995]
} 
simple fractals all the dimensions coincide. Now, let the probability of box $i$ assume the following scaling relation for $\epsilon \downarrow 0$ :

$$
\mathrm{p}_{\mathrm{i}} \propto \epsilon^{-h_{\mathrm{i}}}
$$

where $h_{i}$ is position independent. The scaling index $h_{i}$ is called the Hölder exponent and is discussed later in more detail. Since $h$ controls the singularity of the density, it is sometimes called the crowding index or the exponent of the singularity. In general, let the fractal set $S$ be represented by a density function $\mu$, which is a measure on $\mathrm{IR}^{\mathrm{n}}$. The singularity exponent $\alpha$ at point $\mathbf{x} \in \mathbb{R}^{\mathrm{n}}$ is then defined as:

$$
\alpha(\mathbf{x})=\limsup _{\epsilon \downarrow 0} \ln \mu\left(\mathrm{B}_{\epsilon}(\mathrm{x})\right) / \ln \epsilon
$$

with hyperboxes B of size $\epsilon$ and centres $\mathbf{x}$. The $\sigma$-singularity spectrum of $\mu$ associates to any given singularity exponent $\alpha$ the fractal dimension of the set of all the points $x$ with singularity exponent $\alpha$ :

$$
\sigma(\alpha)=\operatorname{dim}(\{\mathbf{x} \mid \alpha(\mathbf{x})=\alpha\})
$$

For a multifractal where equation [3.29] holds, the same scaling relation can be found in many boxes for small $\epsilon$, and all boxes with the same scaling index are said to be a sub-fractal with a local dimension $h_{i}$. This sub-fractal is said to have a dimension equal to the singularity spectrum $\sigma\left(h_{i}\right)$. In other words, the function $\sigma(h)$ can be interpreted as the fractal dimension of the set of points with the same local dimension $h$. For a simple fractal like the triadic Cantor set the function $\sigma(h)$ is only defined in a single point $(h, \sigma)=\left(\mathrm{d}_{\mathrm{K}}, \mathrm{d}_{\mathrm{K}}\right)$. Such a fractal is not a multifractal. In contrast, for multifractals $h$ is no longer unique but may assume values in a finite range $\left[h_{\mathrm{min}}\right.$, $h_{\max }$, while $\sigma(h)$ turns out to be, in general, a single humped function with $\mathrm{d}_{\mathrm{K}}$ as its maximum. In order to find a relation between $\sigma(h)$ and $\tau(q)$, the partition sum $Z$ is redefined in terms of measure $\mu$ and local exponent $h$ [Halsey,1986].

$$
\mathrm{Z}(a, \mathrm{q})=\int \mathrm{d} h \mu(h) \epsilon(\mathrm{q} h-\sigma(h))
$$

The measure $\mu(h) \mathrm{d} h$ represents the number of times $h$ assumes a value in the interval $[h, h+\mathrm{d} h]$. For $\in \downarrow 0$, the main contribution to $Z$ stems from the exponent when it is close to its minimum value. Therefore, we perform a saddle-point approximation:

$$
\frac{\partial}{\partial h}(q h-\sigma(h))=0
$$

This leads to a Legendre transformation (see [Frisch, Parisi,1985]), which can be used to determine the $\sigma(h)$ spectrum. From this Legendre transformation we note that the function $\sigma(h)$ is a convex function with a slope $q$ in each dense point. As $q \rightarrow \infty$ the largest $p_{i}-$ representing the most concentrated part of the multifractal at that scale dominates the partition function. This corresponds to the point where the $\sigma(h)$ curve vanishes with an infinite slope, which is at the leftmost part for the minimum h. As $\mathrm{q} \rightarrow \infty$ the smallest $\mathrm{p}_{\mathrm{i}}$ dominates - representing the least concentrated part - and the corresponding $h$-value, the rightmost part of the $\sigma(h)$ curve, vanishes with negative infinite slope. By increasing (decreasing) the exponent $q$, boxes with higher (lower) probabilities - i.e. fractal regions with denser (more ramified) occupation - are selected. We shall use this characteristic later to determine the free energy.

\subsubsection{A Thermodynamical Interpretation of Multifractal Measures}

Arneodo [Arneodo,1996] proposed a useful thermodynamical interpretation to the multifractal formalism. In this analogy the variable $q$ corresponds to the inverse temperature $T^{-1}$, function $\tau$, as mentioned before, acts as free energy, and the Hölder 
exponent $h$ corresponds to energy. In this context a thermodynamic function is a statistical average that provides macroscopic - i.e. global - information about the scaling properties of singular measures that are self-similar. The thermodynamic analogy offers an attractive explanation to phase transition phenomena occurring in the scaling properties of fractal measures. Of special importance is the case $q=1$, when the generalised dimension $D_{1}$ is defined by:

$$
D_{1}=\lim _{\epsilon \downarrow 0} \Sigma_{i=1: N(a)} p_{i} \ln p_{i} / \ln \epsilon \equiv \lim _{\epsilon \downarrow 0}-S(\epsilon) / \ln \epsilon
$$

The function $S(\epsilon)$ is called the entropy of the fractal, and is defined similar to information entropy [Shannon,1948]. In statistical thermodynamics, entropy represents unavailable energy or molecular disorder. According its definition, analogue to equation [3.30], entropy is maximal when particles in a medium have equal energy levels. Entropy should not be confused with uncertainty. Uncertainty is minimal when all elements are in the same category [Umpleby,1977]. The quantity $\mathrm{D}_{\text {, }}$ thus measures how information scales ${ }^{8}$ with scale $\epsilon$. Therefore $D_{1}$ is called the information dimension [Amitrano et al.,1986]. These concepts have been used to give a more precise definition of a multifractal [Farmer,1982]. A distribution is defined as a multifractal measure if its fractal dimension $\mathrm{D}_{0}$ exceeds its information dimension $D_{1}$. In a similar way one can show that the dimension $D_{2}$ corresponds to the correlation between the probabilities $\mathrm{p}_{\mathrm{i}}$. Therefore it is called the correlation dimension of the set.

\subsubsection{The Application of Fractal and Multifractal Formalism to Printing Defects}

Images of print defects belonging to one defect class are found to have a characteristic appearance that is difficult fully to encompass in one set of parameters, as those presented in appendix $A$. In this section we analyse such images in the light of the multifractal formalism developed before.

A. Pentland [Pentland,1984] [Pentland,1986] formulated the first principles for the application of a fractal formulism to image analysis. He remarked that fractal dimension correlates well with the human perception of 'smoothness' versus 'roughness' of surfaces. In his view, fractal dimensions near 2 correspond to smooth surfaces, and near 3 correspond to a maximum rough "salt-and-pepper"-like surface. The analysis of geometrical objects in a fractal framework can furnish relevant information, even for non-fractal sets. For this purpose M. Carlin [Carlin,2000] used the traditional Hausdorff measure to quantify the complexity of non-fractal objects. Asvestas et al. [Asvestas et al.,1999] used a fixed mass approach to estimate the fractal dimension of discrete images. Here, we apply two straightforward methods.

As a first approach, we directly apply the box-counting method. A given image of the z-representation of an image is covered with boxes of size $\epsilon$, and the scaling of $N(\epsilon)$ with $\epsilon$ is analysed. A typical example is shown in Figure 3.22. If we regard the object as a unifractal, we can calculate $d_{K}=$ limsup $_{\epsilon \downarrow 0} \ln N(\epsilon) / \ln (1 / \epsilon)$. In the second approach we consider the object as a multifractal object. The results are labelled in Table 3.5.

\footnotetext{
${ }^{8}$ or more exactly: $\ln \epsilon$
} 
In order that we can successfully apply the multifractal formalism on CCD-images of printing defects we make a few simplifications and approximations.

1. We study the set created by the absolute difference between image and template, which we call $z$ here to avoid confusion with the partition-sum $Z$.

2. We assume all boxes of equal size, such that the partition-sum $Z$ does not depend on the infimum size as in equation [3.25].

3. In fact, we lay a grid on the image with gridsize $\epsilon$, and will only include the number of grid-cells that have integral value of intensity above a given threshold to avoid noise.

4. The ensemble probability for cell $\omega_{\mathrm{i}}$ is given by: $\mathrm{p}_{\mathrm{i}}=\int_{\omega_{\mathrm{i}}} \mathrm{z}(\mathbf{x}) \mathrm{d}_{2} \mathbf{x} / \int_{\Omega} \mathrm{z}(\mathbf{x}) \mathrm{d}_{2} \mathbf{x}$, where $\Omega=\cup_{\mathrm{i}} \omega_{\mathrm{i}}$ is the entire covering of the image $z$.

Equipped with these definitions the generalised fractal dimension $D(q)$ is calculated for $q=0$ (traditional fractal dimension), $q=1$ (information Entropy in the image), and $q$ $=2$ (correlation between the points). The free energy is estimated from the slope of In $Z(a, q)$ versus $\ln \epsilon$, as is clear from equation [3.27]. The entropy is calculated from equation [3.34]. The results are listed in Table 3.5 and plotted in Figure 3.23. From the results it is clear that the most 'point-like' objects are 'dirt particles' with $\mathrm{d} \approx$ 0.57 , the most 'line-like' are 'streaks of lines' with $\mathrm{d} \approx 1.08$, and several defects that are semi-planar like 'haze' with $\mathrm{d} \approx 1.85$.

In our context the spatial variation of the fractal dimension over the image was considerable, indicating that the defects are genuine multifractals. The figures in Table 3.5 are averaged over the image, weighted with the local intensity on the image. From Figure 3.234 it is clear that there is a considerable overlap of the defect classes. The defects, however, largely obey $D_{0} \geq D_{1} \geq D_{2}$. The multifractal formalism is able to separate 'point'-like (as e.g. nr. 7 'dust particles'), 'line'-like (as e.g. nr. 15 'streaks of lines'), and 'area'-like objects (as e.g. nr. 5 'ink missing'). Typical examples of printing defects can be found in appendix B2. 

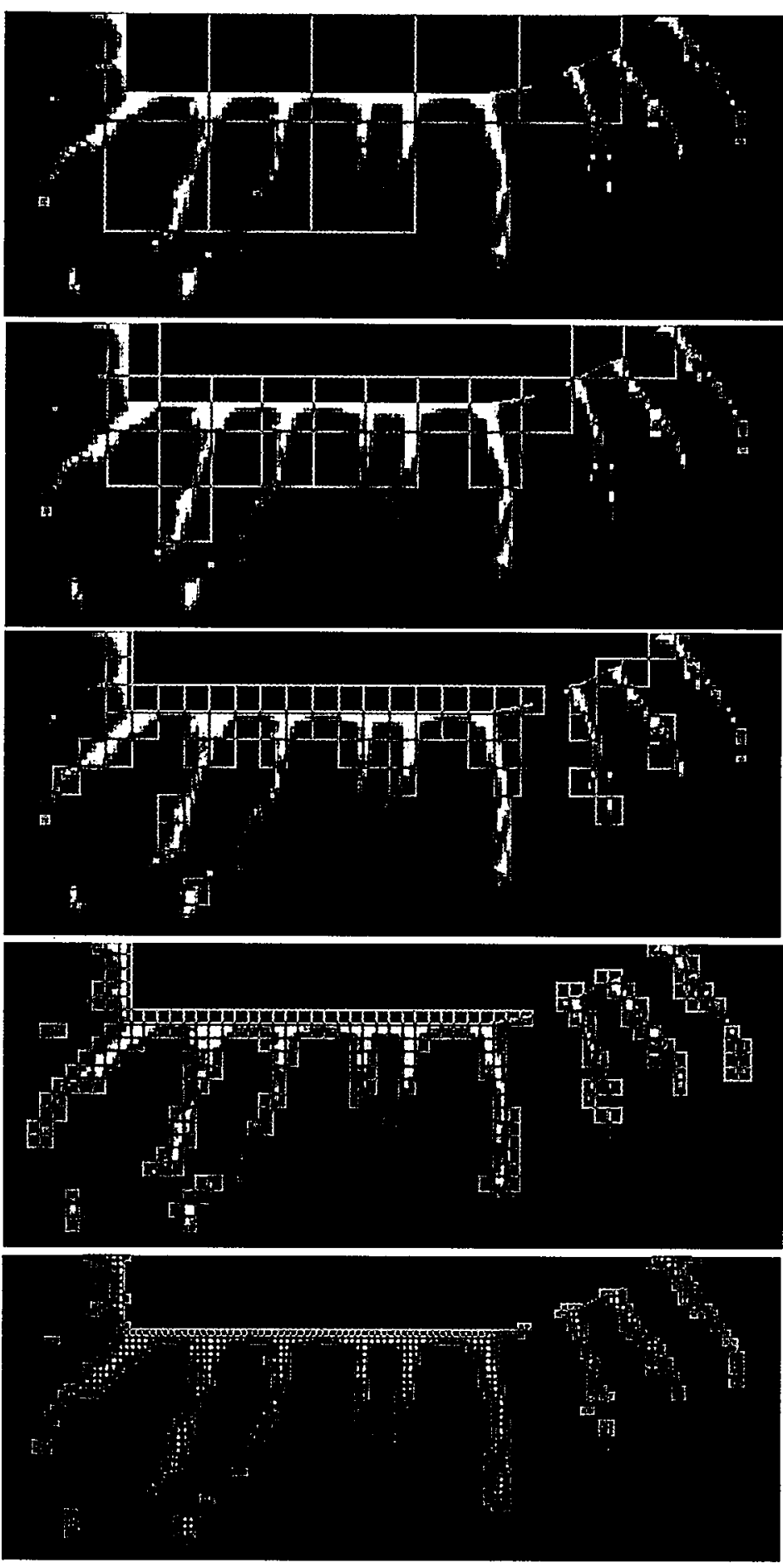

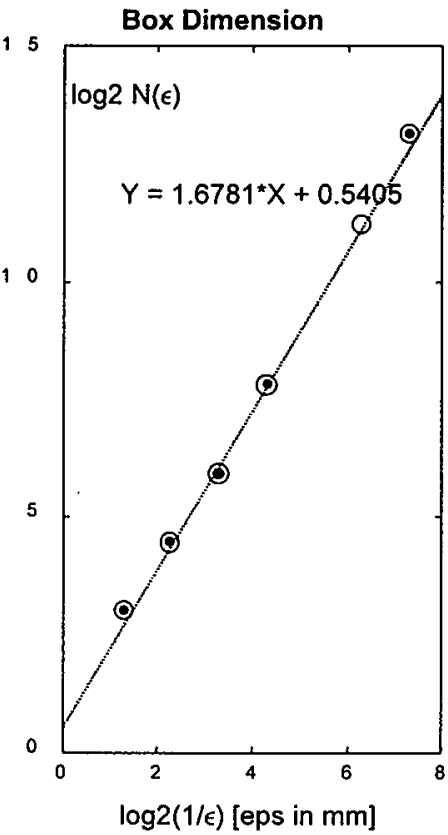

Fig. 3.22 Box covering of an image with defect 'hairs'. Left: covering for exponentially increasing number of boxes. Right: Relation between number of boxes and box-size, plotted as ${ }^{2} \log (\mathrm{N}(\epsilon))$ versus ${ }^{2} \log (1 / \epsilon)$. The box-covering leads to a fractal dimension: 1.6781 for hairs. The number and size of boxes in the pictures are:

\begin{tabular}{|c|c|c|}
\hline Pic\# & $\boldsymbol{\epsilon}(\mathbf{m m})$ & \#Boxes \\
\hline 1 & 0.400 & 8 \\
\hline 2 & 0.200 & 20 \\
\hline 3 & 0.100 & 59 \\
\hline 4 & 0.050 & 231 \\
\hline 5 & 0.025 & 583 \\
\hline
\end{tabular}




\begin{tabular}{|c|c|c|c|}
\hline Defect & Fractal dim D0 & Entropy D1 & Correlation D2 \\
\hline hairs & $1.514 \pm 0.241$ & $1.314 \pm 0.132$ & $1.001 \pm 0.273$ \\
\hline air bubbles & $1.946 \pm 0.139$ & $1.143 \pm 0.170$ & $0.932 \pm 0.082$ \\
\hline air bubbles (foam) & $1.273 \pm 0.199$ & $1.267 \pm 0.247$ & $0.956 \pm 0.118$ \\
\hline constriction & $1.459 \pm 0.126$ & $0.787 \pm 0.089$ & $0.769 \pm 0.181$ \\
\hline recess & $1.607 \pm 0.181$ & $0.991 \pm 0.094$ & $0.778 \pm 0.223$ \\
\hline ink missing & $1.829 \pm 0.196$ & $1.499 \pm 0.127$ & $0.892 \pm 0.210$ \\
\hline misregistration or double print & $1.607 \pm 0.195$ & $1.033 \pm 0.256$ & $0.797 \pm 0.330$ \\
\hline dirt particles & $0.566 \pm 0.147$ & $0.290 \pm 0.114$ & $0.238 \pm 0.091$ \\
\hline edge roughness & $1.200 \pm 0.156$ & $0.822 \pm 0.201$ & $0.310 \pm 0.163$ \\
\hline ink flow & $1.764 \pm 0.298$ & $1.747 \pm 0.309$ & $1.317 \pm 0.163$ \\
\hline smearing & $1.819 \pm 0.279$ & $1.039 \pm 0.418$ & $0.899 \pm 0.321$ \\
\hline fine lines flow together & $1.705 \pm 0.239$ & $1.646 \pm 0.106$ & $1.217 \pm 0.228$ \\
\hline colour differences & $1.865 \pm 0.327$ & $1.151 \pm 0.077$ & $1.068 \pm 0.386$ \\
\hline raster point visible & $1.592 \pm 0.062$ & $1.474 \pm 0.120$ & $1.203 \pm 0.280$ \\
\hline colour or mis registration & $1.841 \pm 0.353$ & $1.826 \pm 0.302$ & $1.451 \pm 0.197$ \\
\hline streaks of lines and droplets of ink & $1.077 \pm 0.037$ & $0.895 \pm 0.067$ & $0.718 \pm 0.089$ \\
\hline haze & $1.846 \pm 0.355$ & $1.656 \pm 0.283$ & $1.488 \pm 0.180$ \\
\hline orange peel & $1.644 \pm 0.172$ & $1.557 \pm 0.161$ & $1.240 \pm 0.081$ \\
\hline colour match wrong & $1.847 \pm 0.318$ & $1.766 \pm 0.271$ & $1.509 \pm 0.110$ \\
\hline
\end{tabular}

Table 3.5 Generalised dimensions based on a set of 476 examples of 18 typical printing defects.
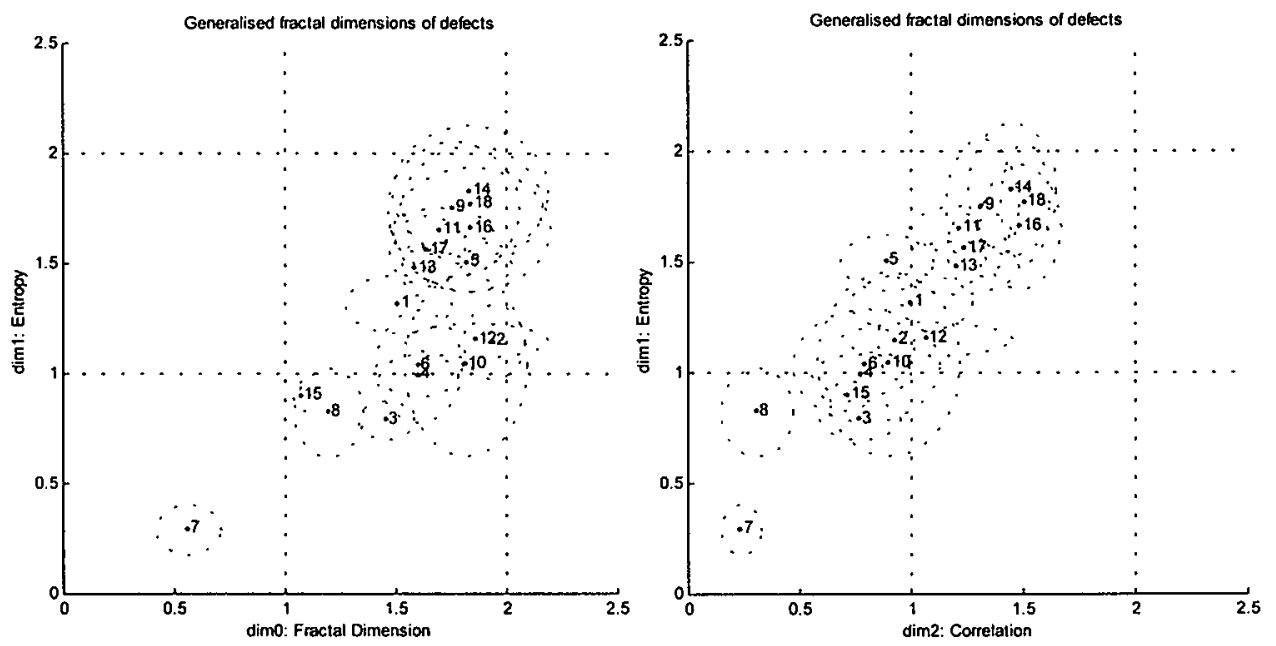

Fig. 3.23 Printing defects mapped in the plane of two generalised dimensions, left: fractal dimension and entropy, right: entropy and correlation. 


\subsection{Wavelet Analysis of 1D-Fractal Functions and MultiFractals}

In the previous section we defined fractal dimensions in terms of box-coverings. In this section we define these properties in terms of the continuous wavelet transform (CWT). The CWT can be considered as generating a covering of the relevant features of the set at scale $a$ with a collection of wavelets with lower dilatations. First, we formalise singular behaviour in 1D-functions more accurately. We define the concept singularity, describe its 'strength', and approximate the behaviour of the function near the singularity. Finally, we define free energy and generalised dimensions in terms of the CWT. We do this in some length, for the CWT provides an automatic and more reliable method to obtain the $D(q)$-spectrum.

Several methods have been published regarding the application of a waveletformalism for the analysis of fractal objects. Khalil and Bayoumi [Khalil,Bayoumi,2000] show how the Wavelet Transform modulus maxima can be used for invariant two-dimensional object recognition. For this, they use a parameterisation of the edge of an object. For this edge, they plot the tangent angle $\Theta$ versus the length $S$ of the edge traversed. With the Wavelet Transform modulus maxima they determine the Lipschitz exponents of the function $Q(S)$, which indicate the singular points on the edge. Thus, they compose a feature vector consisting of pairs $\left(\alpha_{i}, d_{i}\right)$, that for the $i$-th singular point denote its Lipschitz exponent and its distance to the succeeding singular point $i+1$. They show that this feature vector is invariant for affine transformations. Tao et al. [Tao et al.,2001] describe a more general applicable methodology for feature extraction using wavelets and fractals. First, they obtain the wavelet transform of the Central Projection Transformation of a geometric set. Next, they compute a modified Hausdorff dimension for three different scales. These three numbers are used as a feature-vector for clustering and classification. This approach appeared satisfactory for the recognition of Chinese characters. Other examples of fractal wavelet-analysis concern texture analysis. These are discussed later in Section 3.7.3

\subsubsection{Fractal Analysis with Wavelet Transform using Hölder Exponents}

We now construct suitable indicators for discontinuities and regions where a 1dimensional function reveals infinite deep non-convergent structures, i.e. where its fractal dimension is not-integer. This allows us to formulate a symbolic representation for characterising fractal $1 \mathrm{D}$-functions $f(\mathrm{x})$. This condition can be specified by the use of a generalised fractal dimension. Up to now, we have defined fractal dimension in terms of the box covering dimension. We now look for a more precise mathematical method for characterising fractal dimension. Therefore, we define a generalisation of the derivative $f^{\prime}(\mathrm{x})$. First, we extent the definition of the concept continuity. A function $f: \mathbb{R}^{\mathrm{n}} \rightarrow \mathbb{R}^{\mathrm{m}}$ is said to be Lipschitz continuous with constant $\gamma$ in an open neighbourhood $D \subset \mathbb{R}^{\mathrm{n}}$, written $\operatorname{Lipschitz}(f, \gamma)$, if for all $\mathbf{x}, \mathbf{y} \in D\|f(\mathbf{x})-f(\mathbf{y})\| \leq \boldsymbol{\gamma} \| \mathbf{x}-\mathbf{y}$ $\|$. Here $\|\cdot \cdot\|$ and $\|\cdot\|$ are appropriate norms in spaces $\operatorname{IR}^{n}$ and $\mathbb{I R}^{m}$. The Lipschitz $\gamma$ has been directly related to the fractal dimension in literature [Arneodo,1996]. However, the Lipschitz $\gamma$ is difficult to obtain experimentally, since it does not characterise individual points in an image, but sets of points instead. To characterise individual 
singularity points in an image, we turn instead to the Hölder exponent, mentioned before. The Hölder exponent $h(x)$ of a distribution $f$ at point $\mathrm{x}_{0}$ is determined as the greatest $h$, such that $f$ is $\operatorname{Lipschitz}(f, \gamma)$ at $\mathrm{x}_{0}$. Moreover, there must exist a constant $C$ and a polynomial $\mathrm{P}$ of order $\mathrm{n}$, such that for all $\mathrm{x}$ in a neighbourhood of $\mathrm{x}_{0}, f(\mathrm{x})$ does not differ more than $\mathrm{C}\left|\mathrm{x}-\mathrm{x}_{0}\right|^{h}$ from $\mathrm{P}(\mathrm{x})$.

Definition 3.2 Let $f$ be a function $\mathbb{R} \rightarrow \mathbb{R}$. Let $P_{n}(x)$ be a polynomial of order $n \leq \infty$, such that in a $\varepsilon$-neighborhood of a point $x_{0}-$ defined as: $B_{\varepsilon}\left(x_{0}\right)=\left\{x \mid x_{0}-\varepsilon<x<x_{0}+\varepsilon\right\}-$ holds:

$$
\mathrm{x} \in \mathrm{B}_{\varepsilon}\left(\mathrm{x}_{0}\right) \Rightarrow\left|f(\mathrm{x})-\mathrm{P}_{\mathrm{n}}(\mathrm{x})\right|<=\mathrm{C}\left|\mathrm{x}-\mathrm{x}_{0}\right|^{\alpha}
$$

for some $\alpha$ and $C$. The Hölder exponent $h$ of $f$ is the largest exponent $\alpha$ such that $f$ is Lipschitz $(f, \alpha)$ at $x_{0}$ :

$$
\mathrm{h}=\sup \left\{\alpha\left|\exists_{\alpha, \mathrm{C}}\right| f(\mathrm{x})-\mathrm{P}_{\mathrm{n}}(\mathrm{x})|<=\mathrm{C}| \mathrm{x}-\left.\mathrm{x}_{0}\right|^{\alpha} \wedge \operatorname{Lipschitz}\left(f\left(\mathrm{x}_{0}\right), \alpha\right)\right\}
$$

In this way, the strength of a singularity in a function $f$ can be described with the Hölder exponent. The wavelet transform can be used to calculate the Hölder exponent in a function. Different wavelet basis sets have different ranges of permissible Hölder exponents which they can reveal. To study this phenomenon Arneodo [Arneodo, 1996] slightly redefined the original wavelet transform from equation [3.4] as:

$$
\psi_{\mathrm{ab}}(\mathrm{x})=a^{1 / 2} \psi((\mathrm{x}-\mathrm{b}) / a)
$$

where $a$ is a scale parameter and $b$ is a real valued space parameter. Let function $f$ have a Hölder exponent $h \in<n, n+1>$ at $x=x_{0}$. Then, near $x_{0} f$ can be expanded as:

$$
f(\mathrm{x})=\mathrm{c}_{0}+\mathrm{c}_{1}\left(\mathrm{x}-\mathrm{x}_{0}\right)+\ldots+\mathrm{c}_{\mathrm{n}}\left(\mathrm{x}-\mathrm{x}_{0}\right)^{\mathrm{n}}+\mathrm{C}\left|\mathrm{x}-\mathrm{x}_{0}\right|^{h}
$$

Suppose we use an analysing wavelet $\psi$ with $n_{\psi}>n$ vanishing moments. This means that $\psi$ is orthogonal with the polynomials $P_{k}(x)$ with $k \leq n_{\psi}$. The continuous wavelet transformation of $f$ can thus be written as:

$$
\mathrm{W}_{\psi}[f](\mathrm{x}, a)=a^{\mathrm{h}+1 / 2} \mathrm{C} \mathrm{W} \psi[f]\left(\mathrm{x}_{0}, a\right)
$$

This means that for $a \downarrow 0$ the wavelet transform obeys a scaling law near $\mathrm{x}=\mathrm{x}_{0}$ as:

$$
\mathrm{W}_{\psi}\left[f\left(\mathrm{x}_{0}, a\right) \sim a \mathrm{~h}\left(\mathrm{x}_{0}\right)\right.
$$

Elaborating on this approach, Jaffard [Jaffard, 1989] obtained:

Theorem 3.1. Let $\psi$ be an analysing wavelet with $n_{\psi}$ vanishing moments and let $f$ be a bounded function.

$[\Rightarrow]$ If $f$ is Lipschitz $\gamma \leq \mathrm{n}_{\psi}$ at $\mathrm{x}_{0}$ then near $\mathrm{x}_{0}: \mid \mathrm{W}_{\psi}\left[f(\mathrm{x}, a) \mid=O\left(a^{\gamma}+\left|\mathrm{x}-\mathrm{x}_{0}\right| \eta\right)\right.$.

$[\Leftrightarrow]$ If $\gamma \leq n_{\psi}$ and

[i] $\exists_{v>0}:\left|W_{\psi}[f](x, a)\right|=O\left(a^{3}\right)$ uniformly in $x$, and :

[ii] $\left|\mathrm{W}_{\psi}[f](\mathrm{x}, a)\right|=O\left(a^{\gamma}+\left|\mathrm{x}-\mathrm{x}_{0}\right|^{\gamma / \mid}|\mathrm{in}| \mathrm{x}-\mathrm{x}_{0} \|\right)$,

then $f$ is Lipschitz $\gamma$ at $\mathrm{x}_{0}$.

So the greatest value of the Lipschitz-coefficient $\gamma$ corresponds with the Hölder exponent. For non-oscillating singularities the behaviour in the cone $\left|\mathrm{x}-\mathrm{x}_{0}\right| \leq \mathrm{Ca}$ 
suffices to compute the Hölder exponent $h\left(x_{0}\right)$, as proved by Malat and Hwang [Malat,Hwang, 1992].

$$
\sup _{|\mathrm{x}-\mathrm{x} 0|<\mathrm{C} a}\left|\mathrm{~W}_{\psi}[f]\left(\mathrm{x}_{0}, a\right)\right|=O\left(a^{\mathrm{h}\left(\mathrm{x}_{0}\right)}\right)
$$

So the Hölder exponent can be defined as the largest exponent $\gamma$, provided $h \leq \mathrm{n}_{\psi}$ :

$$
h\left(\mathrm{x}_{0}\right)=\limsup _{a \downarrow 0} \ln \left|\mathrm{W}_{\psi}[f]\left(\mathrm{x}_{0}, a\right)\right| / \ln a
$$

for $\mathrm{x}$ in a $\varepsilon$-neighbourhood of a point $\mathrm{x}_{0}: \mathrm{x} \in \mathrm{B}_{\mathcal{E}}\left(\mathrm{x}_{0}\right)$. Therefore it is sufficient to inspect at each scale $a$ the local maxima of the wavelet transform in the cone $\left|x-x_{0}\right|$ $\leq \mathrm{C} a$, and to study the behaviour of these maxima as $a \downarrow 0$. This approach was formalised by Malat and Hwang [Malat,Hwang,1992]. Here we follow their definition of maxima-lines:

\section{Definition 3.3}

- We call a modulus maximum of the wavelet transform $\mathrm{W}_{\psi}[f]$ any point $\left(\mathrm{x}_{0}, a_{0}\right)$ of the space-scale half plane which corresponds to a local maximum of the modulus of $\mathrm{W}_{\psi}[]\left(\mathrm{x}, \mathrm{a}_{0}\right)$, considered as a function of $x$, i.e. $\left|\mathrm{W}_{\psi}[]\left(\mathrm{x}_{0}, \mathrm{a}_{0}\right)\right|>\left|\mathrm{W}_{\psi}[f]\left(\mathrm{x}, \mathrm{a}_{0}\right)\right|$ for all $x$ in a neighbourhood $\mathrm{B}_{\varepsilon}\left(\mathrm{x}_{0}\right)$ of $\mathrm{x}_{0}$. Thus, $\partial \mathrm{W}_{\psi}[f] / \partial \mathrm{x}$ at $\left(\mathrm{x}_{0}, a_{0}\right)$ is zero.

- We call maxima-line any connected curve in the space-scale half plane consisting of modulus maxima.

They proved that singular behaviour near $\mathrm{x}=\mathrm{x}_{0}$ indicates the existence of maximalines converging for $a \downarrow 0$ towards $x_{0}$ on the space axis. Similarly, they proved that in the absence of such lines $f$ is uniformly $\operatorname{Lipschitz}\left(f, \mathrm{n}_{\psi}\right)$ in a neighbourhood $\mathrm{B}_{\varepsilon}\left(\mathrm{x}_{0}\right)$ of $\mathrm{x}_{0}$. Thus the modulus maxima-lines (MML) indicate the location of singularities in $f$. Moreover, the local singular behaviour can be computed with equation [3.40], provided $h \leq \mathrm{n}_{\psi}$. In the case of a fractal function, however, the singularities are not isolated but locally dense. Let $\mu$ be the measure of the set of these singularities. First we define the singularity exponent.

Definition 3.4 Let $\mu$ be a measure on IR and $\sup (\mu)$ its support.

- For all $x$ in a neighbourhood $\mathrm{B}_{\mathcal{E}}\left(\mathrm{x}_{0}\right)$ of $\mathrm{x}_{0}$ the singularity exponent at point $x_{0} \in \sup (\mu)$ is defined as:

$$
\alpha\left(\mathrm{x}_{0}\right)=\limsup _{\epsilon \downarrow 0} \ln \mu\left(\mathrm{B}_{\varepsilon}\left(\mathrm{x}_{0}\right)\right) / \ln \varepsilon
$$

- The $\Phi(\alpha)$ singularity spectrum of measure $\mu$ associates to any given $\alpha_{0}$ the Hausdorff dimension of the set of all the points $\mathrm{x}_{0}$ such that $\alpha\left(\mathrm{x}_{0}\right)=\alpha_{0}$ :

$$
\Phi\left(\alpha_{0}\right)=\mathrm{d}_{\mathrm{H}}\left(\left\{\mathrm{x}_{0} \in \sup (\mu) \mid \alpha\left(\mathrm{x}_{0}\right)=\alpha_{0}\right\}\right.
$$

For the singularity exponent $\alpha\left(\mathrm{x}_{0}\right)$ one can prove in a similar way to the Hölder exponent [Arneodo, 1996]:

$$
\alpha\left(\mathrm{x}_{0}\right)=\limsup _{a \downarrow 0} \ln \left|\mathrm{W}_{\psi}[f](\mathrm{x}, a)\right| / \ln a
$$

for $\mathrm{x} \in \mathrm{B}_{\varepsilon}\left(\mathrm{x}_{0}\right)$. 


\subsubsection{Similarity between Wavelet Analysis and Box Covering}

The characteristic function on the interval $[0,1]$ is called $\chi[0,1]$ and defined as 1 on $[0,1]$ and $O$ outside that interval. If we take the characteristic function $\chi[0,1]$ as analysing wavelet, then $\mu\left(\mathrm{B}_{\varepsilon}\left(\mathrm{x}_{0}\right)\right.$ is the wavelet transform of $\mu$ [Arneodo,1996]. With the 'box' function $\chi[0,1]$ we see that the wavelet transform generates a covering of the fractal set $\sup (\mu)$ with boxes. Therefore, this approach becomes identical to the Kolmogorov approach. In general, wavelets can be regarded as 'fuzified' boxes. The covering with wavelets by the wavelet transform of the set defined by $\mu$ thus becomes equivalent to the covering with boxes. Most boxes - so also most wavelets - are generated at locations with much detail at that scale. In fact, this fuzified covering with wavelets, rather than the box-covering itself, evades any smooth behaviour in a covering that could mask singularities, or perturbate their strength $\alpha$, and thus lead to possible errors in the singularity spectrum. Therefore, we adopt wavelets instead of boxes as the basic mechanism in the multifractal formalism.

\subsubsection{Calculation of the Partition Function $\mathrm{Z}$ for a CWT-Covering}

Now that we are equipped with definitions of maxima-lines and wavelet coverings of fractal sets, we reformulate the ensemble partition function $Z$, as defined in equation [3.26] in Section 3.4.2, in terms of the CWT. $Z$ can intuitively be understood to model the total measure at scale $a$, considered in a fractal dimension $q$ by the entire partition of wavelets. As mentioned before, the basic foundation of the application of wavelets to estimate fractal dimensions is the fact that given its localised spatial extension the wavelets can be considered as a continuous approximation of a box. Therefore, the partition function $Z$ for an ensemble of boxes with size $\epsilon$, as defined by equation [3.26]:

$$
\mathrm{Z}(\mathrm{q}, \epsilon)=\sum_{i=1: \mathrm{N}(\epsilon)} \mu_{\mathrm{i}}(\epsilon)^{\mathrm{q}}
$$

can now be approximated as an integral over the ensemble of wavelets:

$$
\mathrm{Z}(\mathrm{q}, a)=\int\left|\mathrm{W}_{\psi}[f](\mathbf{x}, a)\right|^{\mathrm{q}-1} \mathrm{~d} \mu(\mathbf{x})
$$

Here the exponent $q-l$ is caused by the vanishing moments of the CWT. In both cases the assumption is that in the thermodynamic limit $a \downarrow 0$ we can approximate $Z$ by [Arneodo,1996]:

$$
\mathrm{Z}(\mathrm{q}, a) \propto a^{(\mathrm{q}-1) \mathrm{D}(\mathrm{q})}
$$

where $\mathrm{D}(\mathrm{q})$ is the generalised fractal dimension, defined in equation [3.28]. Therefore:

$$
\mathrm{D}(\mathrm{q})=\lim _{a \downarrow 0}(\mathrm{q}-1)^{-1} \ln \mathrm{Z}(\mathrm{q}, a) / \ln a
$$

In order to avoid divergences in equation [3.42], rather than as a continuous sum, we base the definition of $Z$ on a discrete sum over the maxima of the modulus CWT function: $x \rightarrow\left|\mathrm{W}_{\psi}[f](\mathbf{x}, a)\right|$ [Andreodo, 1996].

The maxima-lines that connect the maxima for all scales $a$, exhibit a number of useful properties that make them attractive for computing fractal properties, as shown by Muzy [Muzy,1991], Malat [Malat, 1992], and Bacry [Bacry,1993]:

a. They can reproduce the full hierarchical structure of the original fractal function; 
b. Each of the maxima-lines points for $a \downarrow 0$ towards a point $\mathrm{x}_{0}$ at the scale-axis that corresponds to a singularity of $f$. For a maxima-line with index ' $l$ ' the position of the maxima at scale $a$ is indicated as $x_{l}(a)$. Let $L(a)$ be the set of all maxima-lines at scale $a$.

c. The number of maxima-lines at scale $a, \mathrm{~N}(a)$, follows the general scaling law:

$$
\mathrm{N}(a) \sim a^{-D_{0}}
$$

These conditions can be naively interpreted as follows. The nature of the proliferation of the maxima-lines as $a$ approaches zero, corresponds with the rate of multiplication of singularities. In order to avoid inflections in $\mathrm{W}_{\psi}$, caused by sign-changes of $\mathrm{q}$, Arneodo [Arneodo, 1996] further refined the partition function to:

$$
\mathrm{Z}(\mathrm{q}, a)=\Sigma_{l \in L(a)}\left(\sup _{\xi \leq a}\left|\mathrm{~W}_{\psi}[f]\left(\mathrm{x}_{l}(\xi), \xi\right)\right|\right)^{\mathrm{q}}
$$

\subsubsection{The Wavelet Transform Modulus Maxima Method}

Now we posses the necessary ingredients to redefine the generalised fractal dimensions in terms of the continuous wavelet transform CWT. For a given function $f$, first the CWT $\mathrm{W}_{\psi}[f]$ is determined. This can be represented on a 2-dimensional plane with horizontally the domain $(f)$, and vertically the scales $a$. In this plane we can draw the maxima-lines, the lines that vertically connect the maxima of the transform considered as function of $x$. Next, for each maxima-line $l$ we calculate the supremum at scale $a: \mathrm{m}_{1}(a)=\sup _{\xi \leq a}\left|\mathrm{~W}_{\psi}[f]\left(\mathrm{x}_{/}(\xi), \xi\right)\right|$. We calculate this quantity for the whole ensemble of maxima-lines at scale $a$. The sum of these quantities defines the partition function $Z$ as defined in equation $[3.46] ; \mathrm{Z}(\mathrm{q}, a)=\Sigma_{l \in L(a)} \mathrm{m}_{\mathrm{l}}(a) \mathrm{q}$.

Finally we use equation [3.44] to calculate $D(q)$. We do this by plotting $\ln Z$ versus $\ln$ $a$ and calculating the slope as $a \downarrow 0$, and then divide the result by $(q-1)$. This gives us the spectrum of generalised dimensions $D(q)$ for this image. This approach is called the Wavelet Transform Modulus Maxima method (WTMM) [Arneodo,1996]. Generally, it gives consistent results and robust estimates of the $\mathrm{D}(q)$ singularity spectrum.

In order to adapt this method to our context of discrete images with additional noise and low resolution, we can introduce two new elements. First, there is a maximum resolution represented by the lowest attainable scale. Second, noise is introduced as the equivalent of temperature in the thermodynamic analogon of the theory. Let $T$ represents the amount of noise in the image, and $\beta$ represent the inverse temperature: $\beta=1 / \mathrm{T}$. Then the partition sum can be redefined as: $\mathrm{Z}_{\beta}(\mathrm{q}, a)=\Sigma_{l \in L(a)} \mathrm{m}_{\mathrm{l}}(a) \mathrm{q} \beta$, and the WTMM approach be adapted similarly. 


\subsection{Application of Fractal Wavelet Analysis to Defect Identification and Classification}

In this section we apply the fractal wavelet analysis, as developed in the previous sections, for characterising $1 \mathrm{D}$-functions and $1 \mathrm{D}$-scanprofiles of printing defects.

\subsubsection{Application of the Wavelet Transform Modulus Maxima Method to the Cantor Set}

We illustrate the WTMM method with a simple example. The WTMM method is applied to the well-known Triadic Cantor Set (TCS) as a set for which the spectrum of dimensions can easily be obtained exactly. More precise, we look to the function defined by the density over the TCS: $\mathrm{x} \rightarrow \mu_{\mathrm{TCS}}([0, \mathrm{x}])$, called the 'Devil's staircase', shown in Figure 3.24. The TCS is defined by a self-similar scaling law that yields a fractal dimension $d_{\mathrm{TCS}}={ }^{3} \log 2 \approx 0.6309$. Therefore, the number of boxes, here intervals, of size (length) ' $\epsilon$ ', $N$, needed to cover the TCS entirely, is determined by: $\mathrm{N}(\epsilon) \sim \epsilon^{-\mathrm{D}}$, with $\mathrm{D}={ }^{3} \log 2$. Let: $a=1 / \epsilon$, so the partition function is defined by: $\mathrm{Z}(\mathrm{q}, a)$ $=\mathrm{N}(1 / a) \mathrm{q}=a \mathrm{qD}$. Now, define ' $\tau$ ' as the limit power of $\mathrm{Z}: \mathrm{Z} \sim a^{\tau(\mathrm{q})}$. From this is clear that: $\tau(q)=q D$. So, the slope of $\tau(q)$ is equal to $D$.

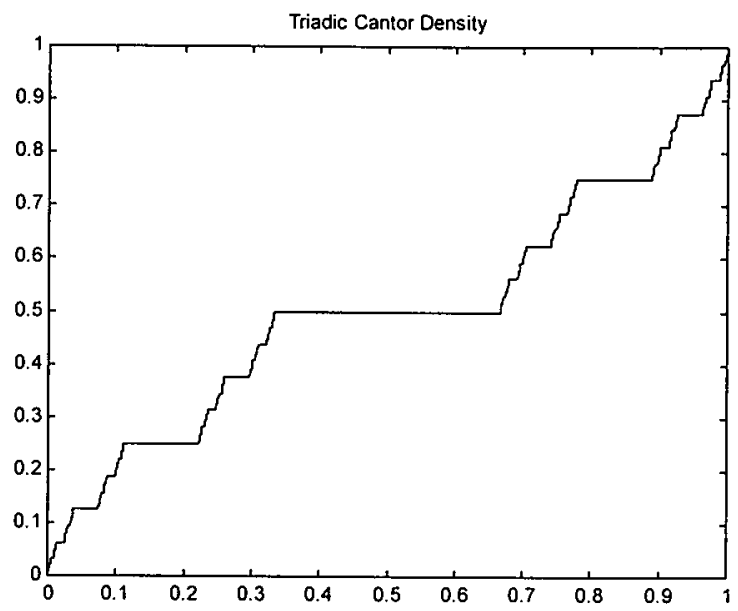

Fig. 3.24 The measure over the triadic Cantor set, called "Devil's Staircase".

In Figure 3.25 the continuous wavelet transform of the Devil's staircase is shown. In the same figure the maxima-lines are indicated, that vertically connect maxima of the transform considered as function of $x$. Figure 3.26 depicts the proliferation of the maxima-lines as the scale zooms in to zero. The slope of the line is $-0.628-$ which corresponds well with the fractal dimension of the set $\mathrm{d}_{\mathrm{TCS}}=3 \log 2 \approx 0.631$. The horizontal lines are caused by the scaling behaviour; every (1/3) of the scale the set doubles. This gives a good result. Next we apply the WTMM method and plot $\ln Z$ over the maxima-lines versus In $a$, following equation [3.43]. This is shown in Figure 3.27. Here we obtain a value for the main fractal dimension $D_{0}=0.6467$, that is also near its known value. 


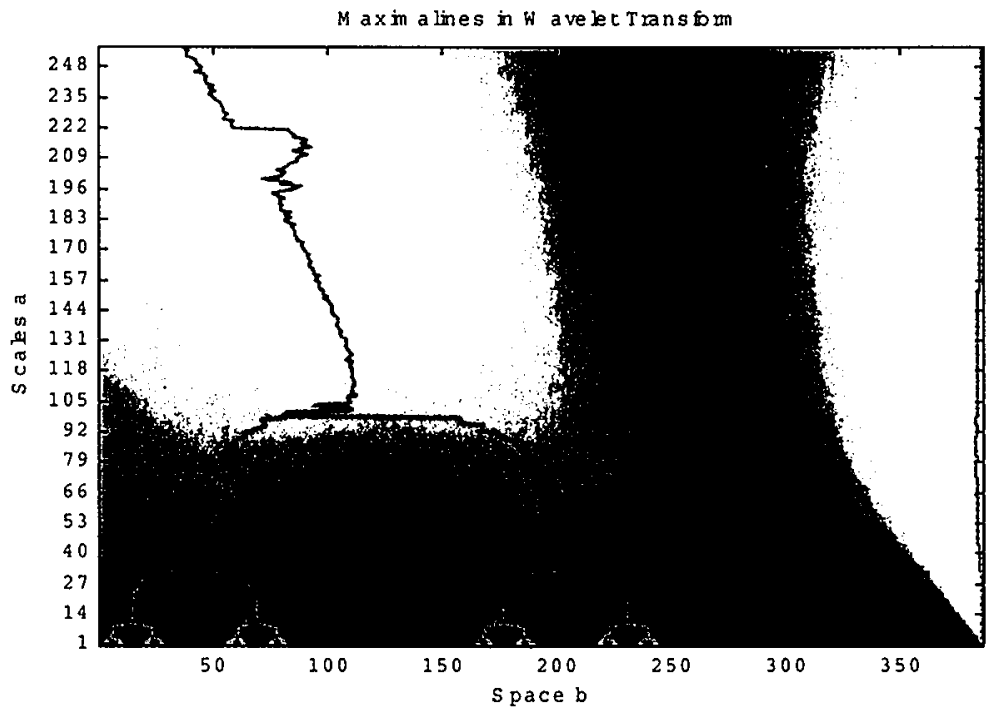

Fig. 3.25 The continuous Wavelet Transform of the Devil's staircase function, color-scaled at each scale, with the maxima-lines,

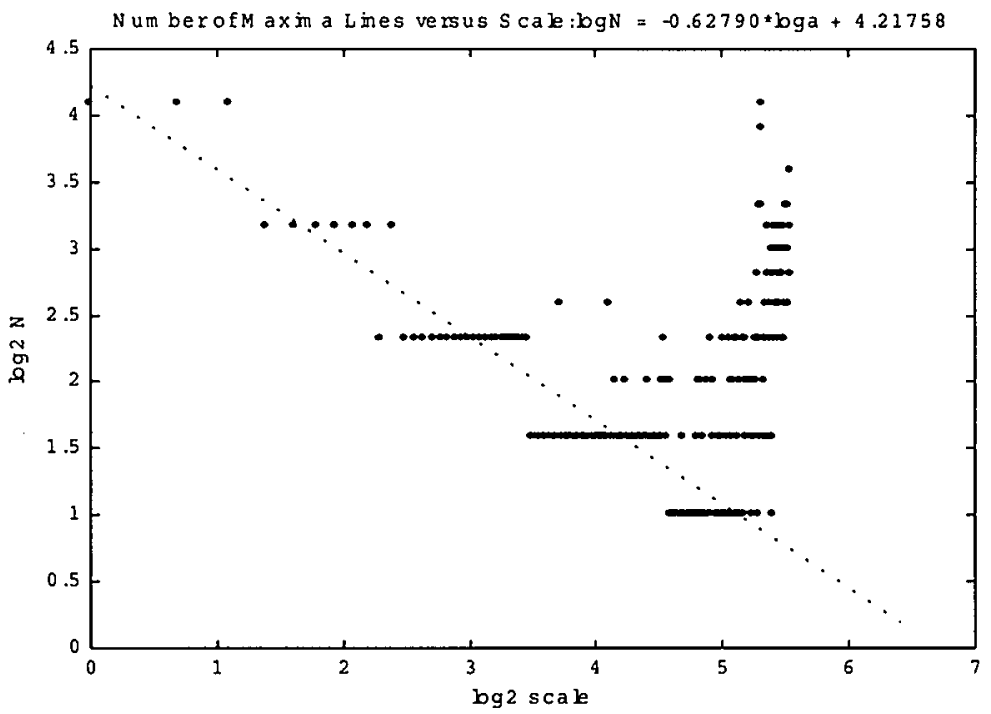

Fig. 3.26 The number of Maxima-lines versus the scale (double log-plot). The best fit is with a line with gradient close to the expected $3 \log (2)$. The horizontal lines are caused by the scaling behavior; every $(1 / 3)$ of the scale the set doubles. 
Partitionsum versus scale for TCS

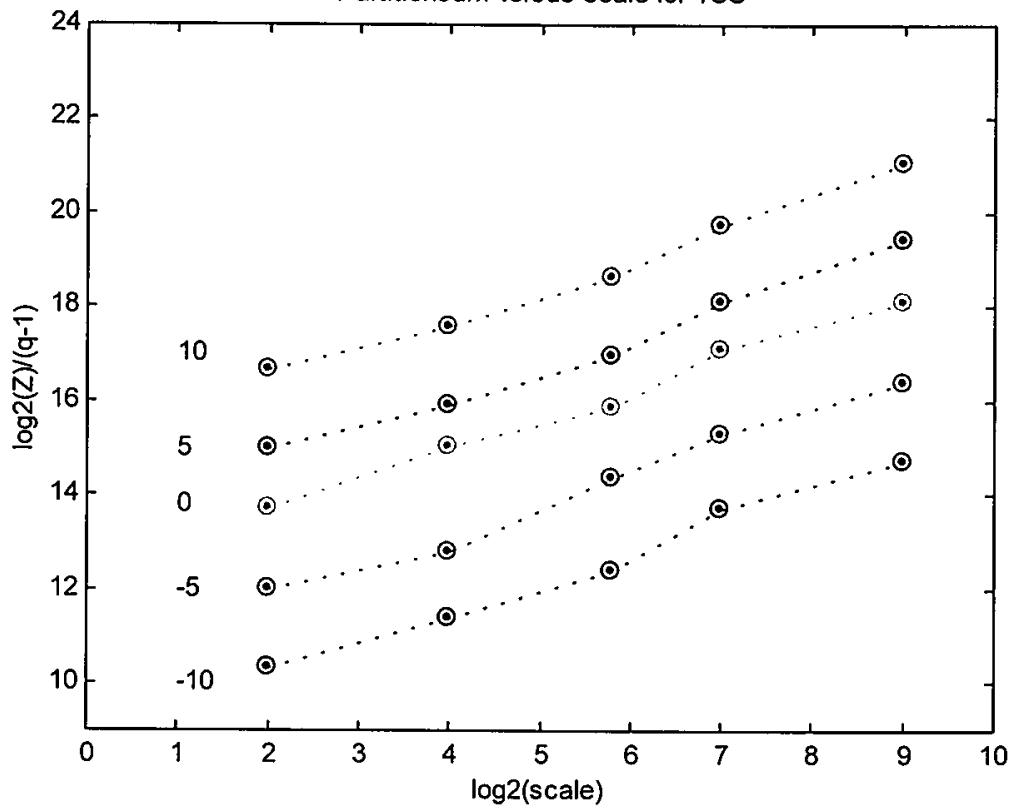

Generalised Dimension for TCS

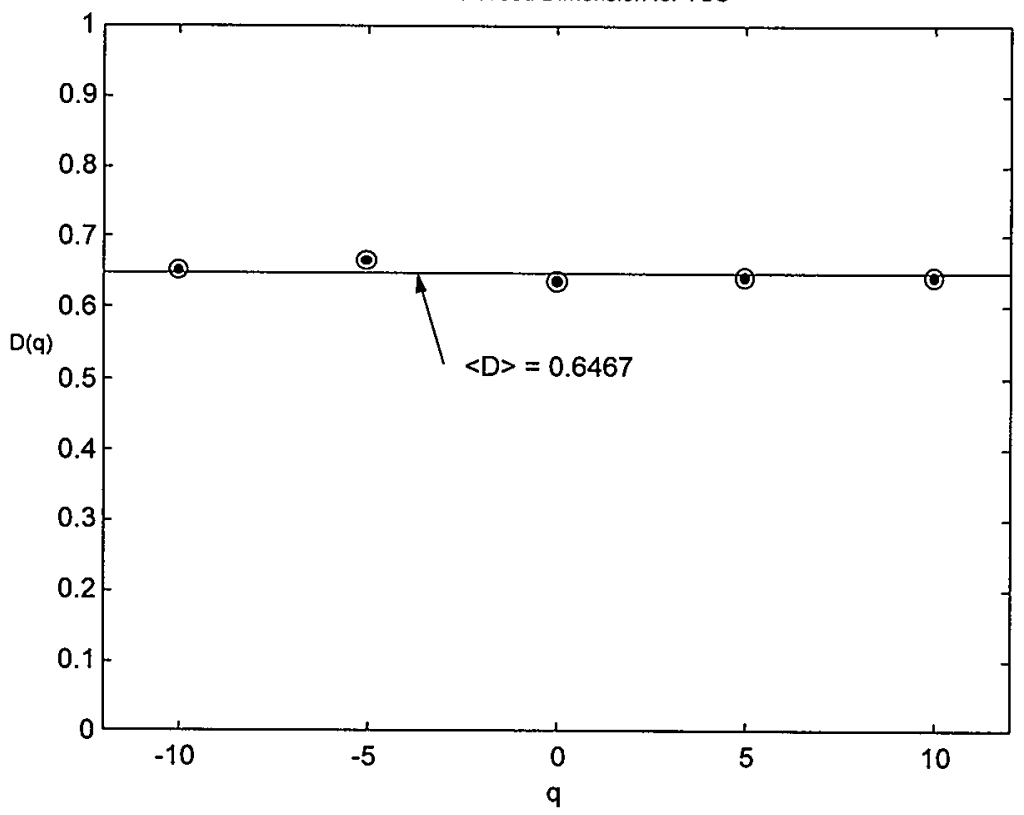

Fig. 3.27 MMWT-method for the measure over TCS. Above: the relation between the partition function $Z$ versus the scale (double log-plot). Indicated are the curves for different values of $q$. Below: the generalised dimension $\mathrm{D}(\mathrm{q})$ based on the average gradient of the $\log Z$-loga curve for $a \downarrow 0$. Within the precision the $\mathrm{D}(\mathrm{q})$ are equal, $\mathrm{D}(\mathrm{q})=0.65 \pm 0.01$. 


\subsubsection{Application to Fractal Characterisation of ScanProfiles}

As described in Section 3.4, there are a number of suitable fractal descriptors for scanprofiles. The most meaningful being the maxima-line-scaling law, and the MMWT-method. These quantities are described in equations [3.41] and [3.42] in the previous section. In this section we apply this formalism to scanprofiles. As an example we investigate the wavelet analysis of a scanprofile over a $\Gamma 2$-curve near a 'hairy' print fault. In Figure 3.28 an example of a detailed scanprofile of the defect hairs is depicted.

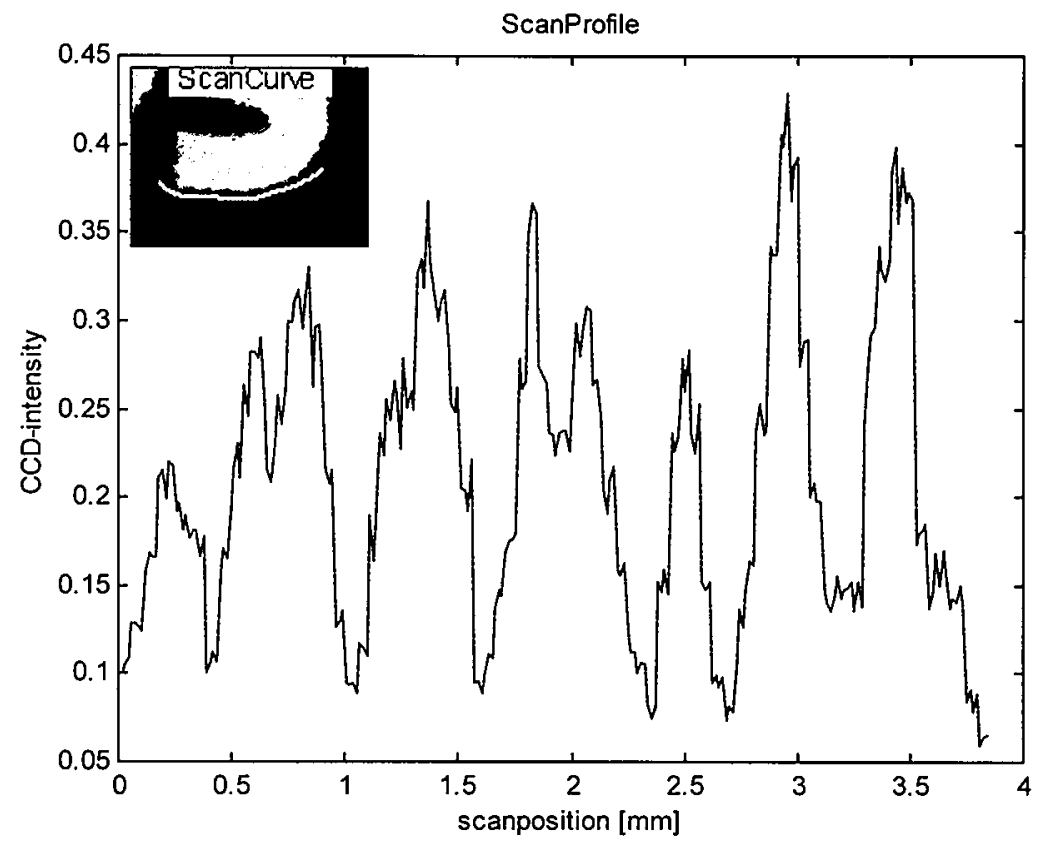

Fig. 3.28. Left above: ScanCurve on an image with a typical defect of 'hairs'. Below: Detailed ScanProfile over the ScanCurve, scanning from left to right.

From this scanprofile we first calculate the exponent ' $\alpha$ ' that drives the proliferation of maxima-lines: $\mathrm{N}_{\alpha} \propto a^{-\alpha}$. Figure 3.20b shows the relation between $\ln a$ and $\ln \mathrm{N}_{\alpha}$. The slope of this curve for $a \downarrow 0$ defines an exponent $\alpha \approx 0.76$.

Next, we determine the CWT of the profile, represented in Figure 3.29a. In the $\mathrm{x}-a$ plane we draw the maxima-lines, and calculate $Z(q, a)$ using the WTMM method. Finally, we plot $\ln Z /(q-1)$ versus $\ln a$ for different values of $q$. The slopes of these curves for $a \downarrow 0$ defines the spectrum of generalised dimensions $D(q)$. The result for this scanprofile is $\mathrm{D}(0) \approx 0.47$. The difference between $\alpha$ and $\mathrm{D}(0)$ may be explained by the difficulty of precisely determining the position of the maxima-lines due to noise, imperfections, and the digital nature of the image, and fluctuations in the calculation over one maxima-line. Table 3.6 lists the results over 7 different examples of 'hairs' scanprofiles. The values show large differences. 
Maximalines in Wavelet Transform
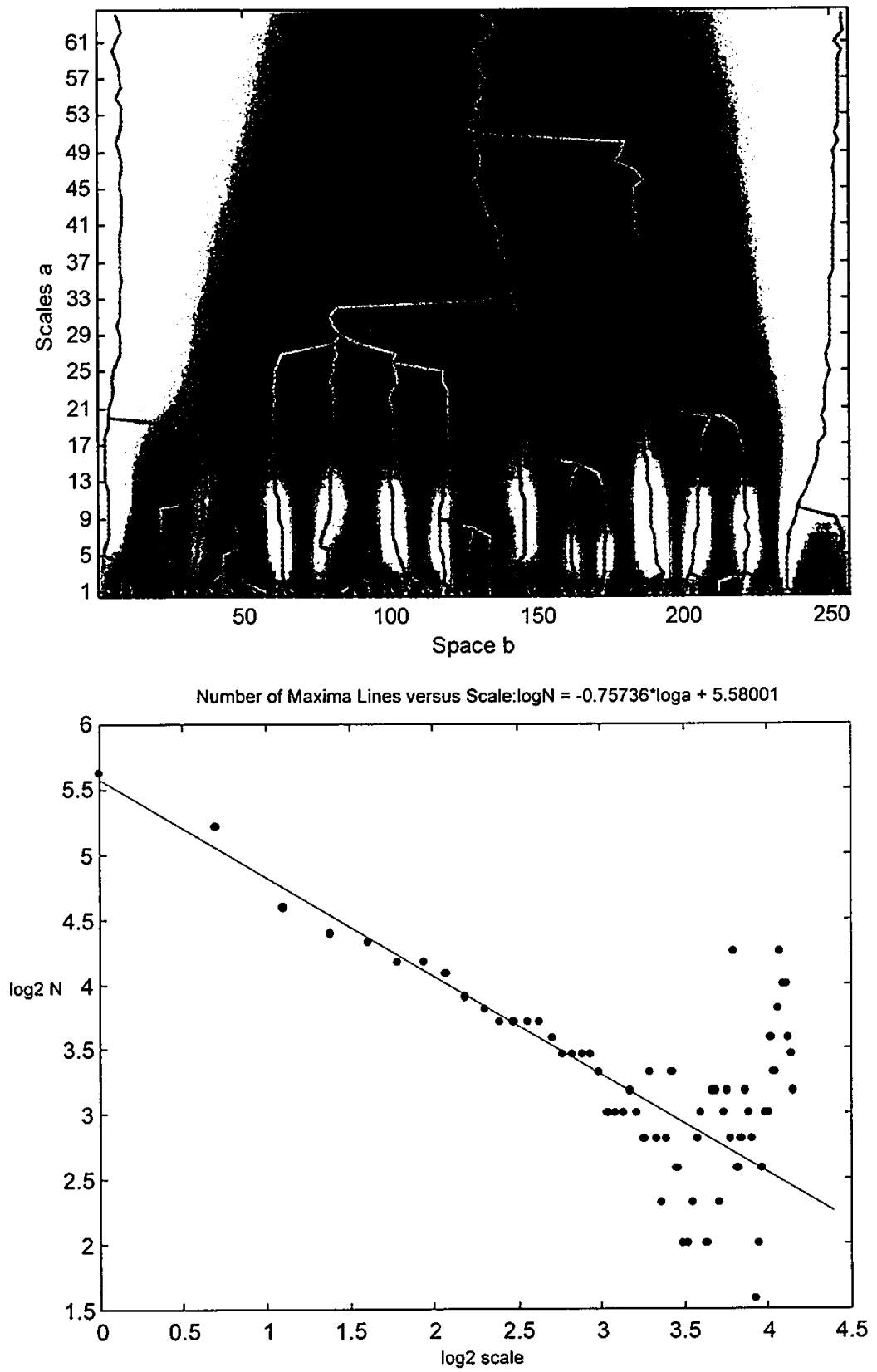

Fig. 3.29 (a) (above): the continuous wavelet transform of the 'hairs'-scanprofile of Figure 3.28, color-scaled at each scale. (b) (below): The number of Maxima-lines versus the scale (double log-plot). Indicated is the best fitting line. Its gradient defines the MM-scaling exponent of about 0.76 . 

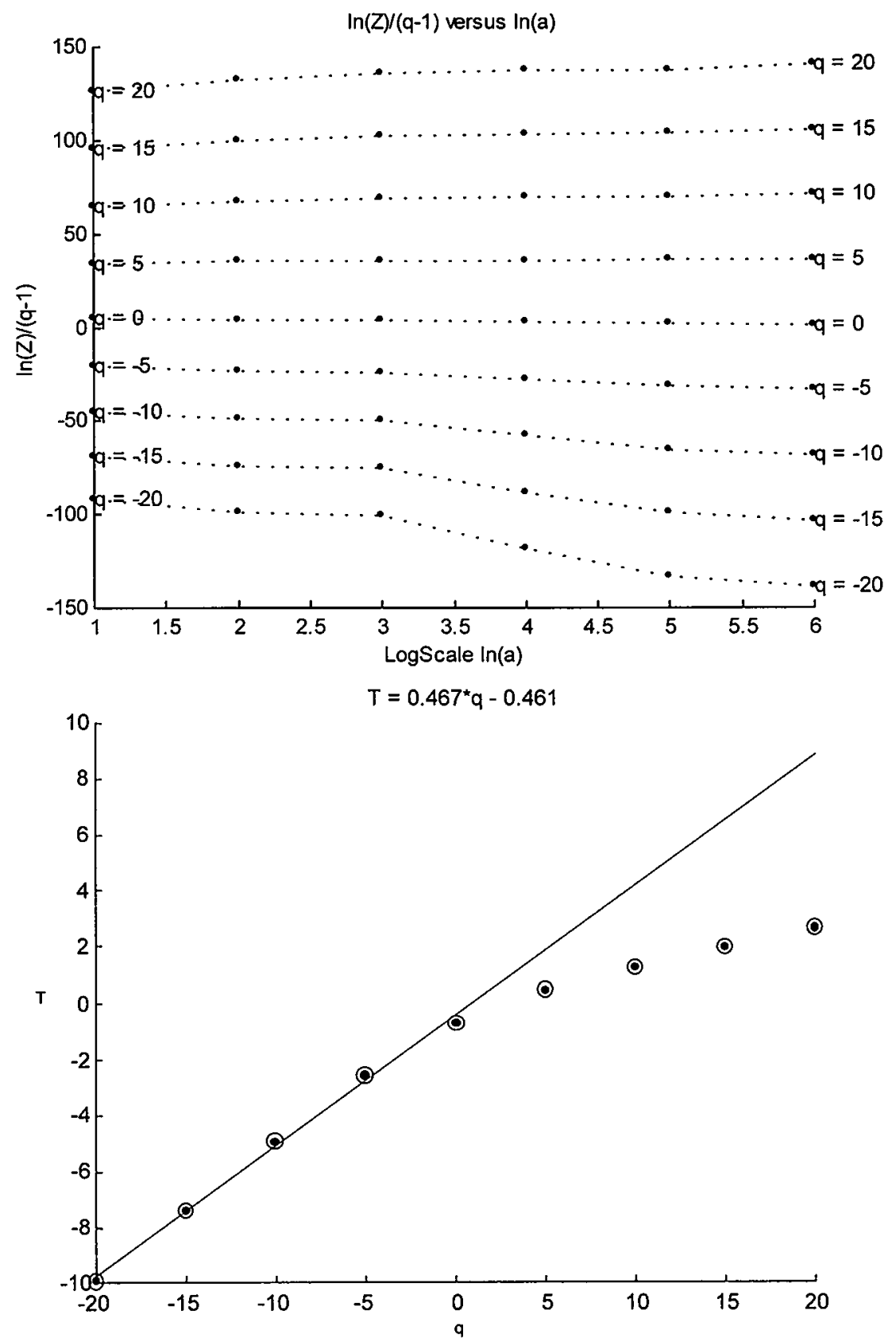

Fig. 3.30 MMWT-method belonging to the 'hairs'-scanprofile of Figure 3.28. Above: the relation between the partition function $Z$ versus the scale (double logplot). Below: the average gradient of the $\log Z$ - $\log a$ curve for $a \downarrow 0$ versus the scale $a$. Indicated is the best fit with a line. Its gradient defines the MMWT-scaling exponent of about 0.47 . 
From this example it is clear that there are a number of problems with the application of the wavelet formalism. The first problem is the amount of computing time necessary to calculate the dimensions. The computation times of the maxima-linescaling exponent $\alpha$ of about was significantly faster than the WTMM-method $D(0)^{9}$. Moreover, the two dimensions were found to differ considerably, as expressed by the example in Figure 3.31. Moreover, they were hardly correlated. Table 3.6 lists a number of observed cases of dimensions $\alpha(\operatorname{dim} 1)$ and $D(0)(\operatorname{dim} 2)$ for a printing defect called 'hairs'. The next approach is to plot a number of printing defects in the $\operatorname{dim} 1$,dim2-plane. This did not provide sufficient division between the defects. The

\begin{tabular}{|c|c|c|}
\hline Example & dim1 & dim2 \\
\hline hairs1 & 0.7574 & 0.4666 \\
\hline hairs2 & 0.9498 & 0.7055 \\
\hline hairs3 & 0.1392 & 0.9589 \\
\hline hairs4 & 0.1869 & 0.7737 \\
\hline hairs5 & 0.5065 & 1.1283 \\
\hline hairs6 & 0.2437 & 0.8693 \\
\hline hairs7 & 0.1076 & 1.3145 \\
\hline
\end{tabular}

Table 3.6. The dimensions $\alpha(\operatorname{dim} 1)$ and $D(0)$ (dim2) listed for seven examples of defect 'hairs'. problem is mainly caused by the poor quality of the maxima-lines, and low resolution of the images. Only for a set of seven images with relative high resolution meaningful results could be obtained. The exponent $\alpha$ looks somewhat more reliable than the generalised dimension $D(0)$.

Finally, we remark that in most experiments this analysis was more successfully applied to the CID-representation, rather than to the image itself.

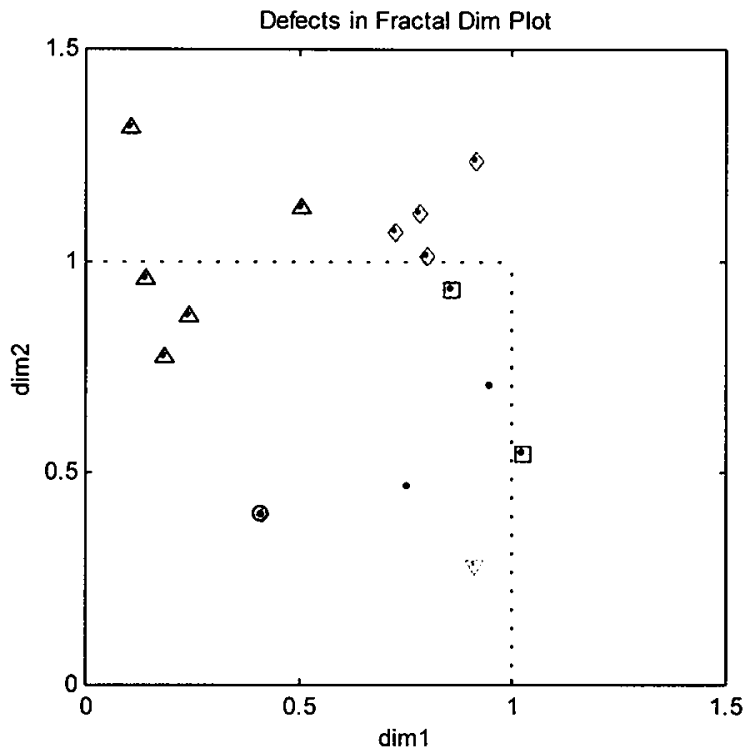

Fig. 3.31. Defects plotted in $\operatorname{dim} 1 x \operatorname{dim} 2$ plane. [triangle up]: hairs, [square:] recess, [circle:] smearing, [diamond:] air bubbles, [triangle down:] orange peel. $\operatorname{dim} 1=\operatorname{dim} M M, \operatorname{dim} 2=\operatorname{dim} M M W T$.

\footnotetext{
${ }^{9}$ On a Athlon $650 \mathrm{MHz}$ processor under Matlab: $12 \mathrm{sec}$ for the WTMM and $1 \mathrm{sec}$ for the $\alpha$ exponent.
} 


\subsection{Wavelet Analysis of N-Dimensional Multifractal Sets}

\subsubsection{N-Dimensional Wavelet Analysis}

The 1D-wavelet approach described above can be generalised to $\mathrm{n}$-dimensional fractal distributions. In this section we briefly describe the n-dimensional wavelet formalism. We can generalise the affine group of translations and dilatations: $x \rightarrow a x+b$ in IR to the $n$-dimensional Euclidean group of translations, rotations, and dilatations $E_{n}(I R)$. This is a non-unimodular locally compact group [Murenzi,1990]. The most natural unitary representation in $L^{2}\left(\mathbb{R}^{n}\right)$ that satisfies (1) square integrability, and (2) irreducibility is $\Omega(\mathbf{b}, a, \mathrm{r})$ defined for $\psi \in \mathrm{L}^{2}\left(\mathrm{IR}^{n}\right)$ by [Arneodo, 1996]:

$$
\Omega(\mathbf{b}, a, r) \psi(\mathbf{x})=a^{1 / 2} \mathrm{n} \psi\left(r^{-1}(\mathbf{x}-\mathbf{b}) / a\right)
$$

with the translation vector $b \in \mathrm{IR}^{\mathrm{n}}$, the dilation parameter $a>0$, and the $\mathrm{n}$-dimensional rotation operator $r \in \mathrm{SO}(\mathrm{n})$. In this way the wavelet transform of a function $f \in \mathrm{L}^{2}\left(\mathbb{R}^{\mathrm{n}}\right)$ is then defined as:

$$
\mathrm{W}_{\psi}[f](\mathrm{x}, a)=\langle\Omega \psi| f>
$$

where $\langle\cdot| \cdot>$ is the inner product in $L^{2}\left(\mathbb{R}^{n}\right)$. This can be written as:

$$
\mathrm{W}_{\psi}[f](\mathbf{x}, a)=a^{1 / 2 \mathrm{n}} \int \mathrm{d}^{\mathrm{n}} \mathbf{x} \psi\left(r^{-1}(\mathbf{x}-\mathbf{b}) / a\right) f(\mathbf{x})
$$

An example of an analysing wavelet in $\mathbb{R}^{2}$ is the optical wavelet [Freysz,1990].

$$
\psi(\mathrm{x})=\mathrm{c}\left(\mathrm{J}_{1}(\mathrm{x} / \mathrm{R}) /(\mathrm{x} / \mathrm{R})-\mathrm{J}_{1}(\mathrm{x} / \gamma \mathrm{R}) /(\mathrm{x} / \gamma \mathrm{R})\right)
$$

where $\mathbf{x}=\|\mathbf{x}\|$, and $\mathrm{R}, \mathrm{c}$, and $\gamma$ are suitable constants. $\mathrm{J}_{1}$ is the first Bessel-function. This wavelet is the Fourier transform of the characteristic function on a ring with inner-diameter $\mathrm{R} / \gamma$, and outer diameter $\mathrm{R}: \psi(\mathrm{x})=\mathrm{F}[\chi[\mathrm{R} / \gamma, \mathrm{R}]]$.

If we restrict the analysis to two dimensions, then there are two natural ways to construct the wavelet basis. One basis is defined by the tensor product $h_{j, j}{ }^{\prime}, k, k^{\prime}(x, y)=$ $h_{j, k}(x) h_{j^{\prime}, k^{\prime}}(y)$, so that each basis function $h_{j, j}{ }^{\prime}, k, k^{\prime}(x, y)$ is supported on a rectangle. The second basis is created by the set of three $1 \mathrm{D}$-basis functions supported on a square: $\left\{\mathrm{h}_{\mathrm{j}, \mathrm{k}}(\mathrm{x}) \mathrm{h}_{\mathrm{j}, \mathrm{k}^{\prime}}(\mathrm{y}), \mathrm{h}_{\mathrm{j}, \mathrm{k}}(\mathrm{x}) \chi_{\mathrm{j}, \mathrm{k}^{\prime}}(\mathrm{y}), \chi_{\mathrm{j}, \mathrm{k}}(\mathrm{x}) \mathrm{h}_{\mathrm{j}, \mathrm{k}^{\prime}}(\mathrm{y})\right\}$, where $\chi(\mathrm{x})$ is the characteristic function of the interval $[0,1]$, and $\chi_{\mathrm{j}, \mathrm{k}}(\mathrm{x})=2^{-\mathrm{j} / 2} \chi\left(2^{-j \mathrm{j}}-\mathrm{k}\right)$. [Andreodo, 1996].

\subsubsection{Wavelet Analysis of N-Dimensional Local Scaling Laws and Fractal Singularity Spectrum}

In a similar way as the $1 \mathrm{D}$-analysis, local self-similarity in an object near $\mathbf{x}=\mathbf{x}_{0}$ implies that the density $\mu$ obeys the local scaling law $\mu\left(\mathrm{B}_{\epsilon}\left(\mathrm{x}_{0}\right)\right) \propto \mathrm{e}^{\alpha\left(\mathrm{x}_{0}\right)}$, where $\alpha$ is the local scaling exponent. The scaling behaviour of the wavelet transform of a fractal measure is derived similarly to equation [3.48] in Section 3.5 as [Argoul et al.,1989]:

$$
\mathrm{W}_{\psi}[f]\left(\mathbf{x}_{0}, a\right) \approx a^{\alpha\left(\mathbf{x}_{0}\right)}
$$

Here, we disregard any anisotropic effects in the local scaling laws, therefore we rely on radially symmetric wavelets, like the optical wavelet. Arneodo [Arneodo, 1996] compares the continuous wavelet transform with a 'mathematical microscope', where 
b and $a$ corresponds with the position and the inverse magnification respectively. Equation [3.47] allows the efficient localisation of singularities, and the subsequent estimation of their 'strength'. In practice, the hierarchical structure of the singularities, which can accumulate locally, can lead to oscillations around this power law, and make an exact determination of $\alpha(\mathbf{x})$ somewhat uncertain.

The wavelet analysis of multifractals embedded in $\mathrm{n}$ dimensions follows the same line of thought as the 1-dimensional approach. Starting point is the partition function $Z$ for a covering of the multifractal $F$ with hyperspheres with diameter $\epsilon_{\mathrm{i}} \geq \epsilon$. Let $s$ be a specific covering of $F$ defined by: $s=U_{\mathrm{j}} \mathrm{B}_{\epsilon \mathrm{j}}\left(\mathbf{x}_{\mathrm{j}}\right)$, where $\mathrm{B}_{\epsilon}(\boldsymbol{a})$ is a hyper-sphere with radius $\epsilon$ and centre $a$. Let $S$ be the ensemble of all possible coverings $s$ of $F$ with $\epsilon_{\mathrm{i}} \geq$ $\epsilon$. Then the partition function $Z$ for this case is defined by:

$$
\mathrm{Z}(\mathrm{q}, \epsilon)=\inf _{s \in S} \Sigma_{\mathbf{j}=1: \mathrm{N}(\epsilon)} \mu\left(\mathrm{B}_{\epsilon \mathbf{j}}\left(\mathbf{x}_{\mathbf{j}}\right)\right) \mathrm{q}
$$

Like in one dimension an n-dimensional radially symmetric wavelet can been seen as smooth oscillatory variants of the characteristic function $\chi$ on a sphere. Therefore, we can rewrite equation [3.48] in terms of wavelets as (see Muzy et al [Muzy, 1992]):

$$
\mathrm{Z}(\mathrm{q}, a)=\int\left|\mathrm{W}_{\psi}[f](\mathbf{x}, a)\right|^{\mathrm{q}-1} \mathrm{~d}_{\mathrm{n}} \mathbf{x}
$$

In the limit of $a \downarrow 0$ this can be rewritten as:

$$
\mathrm{Z}(\mathrm{q}, a) \propto a^{\tau(\mathrm{q})}
$$

where $\tau(q)$ again represents the $\tau$-singularity spectrum, and:

$$
D_{\mathrm{q}}=\tau(\mathrm{q}) /(\mathrm{q}-1)
$$

is similarly the generalised fractal dimension. In the same spirit as the one dimensional case this allows the definition of an n-dimensional WTMM method: $Z$ should be limited to the modulus maxima-lines of the continuous wavelet transform [Arneodo, 1996]:

$$
\mathrm{Z}(\mathrm{q}, a)=\Sigma_{l \in L(a)}\left(\sup _{\xi \leq a}\left|\mathrm{~W}_{\psi}[f]\left(\mathbf{x}_{l}(\xi), \xi\right)\right|\right)^{\mathrm{q}}
$$

The combination of equations [3.54] and [3.55] provides a practical approach for the calculation of $\mathrm{D}_{\mathrm{q}}$ :

$$
\mathrm{D}_{\mathrm{q}}=(\mathrm{q}-1)^{-1} \limsup _{a \downarrow 0} \ln \mathrm{Z}(\mathrm{q}, a) / \ln a
$$

\subsubsection{D-Fractal Wavelet Analysis of Textures}

The multi-dimensional wavelet analysis of multifractals, described in the previous section, can be applied to the analysis of textures. In this approach we consider textures as geometric sets with characteristic fractal properties, and we apply a multiresolution approach, like the WTMM method, in order to estimate the relevant parameters. In recent years, fractal texture analysis has received much attention. Chaudhuri and Sarkar [Chaudhuri,Sarkar,1995] devise algorithms for segmentation and classification, based on six features composed from the multifractal dimension of the texture. This allowed them to segment and classify natural and artificial textures. Kaplan and Jay-Kuo [Kaplan,Jay-Kuo,1995] construct an extended self-similar measure to represent scale-dependent texture properties, and thus estimate texture roughness. An important area in texture analysis is to model textures as random fields. 
Examples are Gaussian, Gibbs and Markov random fields. The problem then is to estimate the parameters. For instance, Gimelfarb [Gimerlfarb, 1999] uses Simulated Annealing on grey-level co-occurrence histograms to obtain parameter estimates. Alternatively, MRA can be used for estimating the field parameters. Wang and Liu [Wang,Liu, 1999], [Wang,Liu,2000] model textures as Markov Random Fields (MRF) and use the Markov Chain Monte Carlo (MCMC) method for estimating the MRF parameters. However, this MRF/MCMC-method is only valid for fixed neighbourhoods. Therefore, Wang and Liu applied Multiresolution Analysis for considering texture characteristics at different scales. With this so-called MultiResolution Markov Random Fields (MRMRF) method they were able to successfully segment and classify natural and artificial textures.

Several reports describe the application of the Wavelet Transform Modulus Maxima Method. Westra [Westra,2000a] describes the adaptive control of a printing robot using the WTMM method to classify CCD-images of printing defects. Sharman et al. [Sharman et al.,2000] report on a fast and accurate method to match multimodal medical images using the WTMM method. Khalil and Bayoumi [Khalil,Bayoumi,2000] show how the WTMM method can be used for invariant twodimensional object recognition.

In the following section we describe the application of 2D-fractal wavelet analysis for the classification of printing defects, where the printing defects are interpreted as fractal textures.

\subsubsection{Application of 2D-Fractal Wavelet Analysis to Printing Defects}

The 1D-CWT of a 1D-function $f$ is a two dimensional entity. For every translation $x$ and every dilation $a$ a value $\mathrm{W}_{\psi}[f](\mathrm{x}, a)$ must be calculated. The 2D-CWT of a $2 \mathrm{D}$ function $f: \mathbf{x} \in \mathbb{R}^{2} \rightarrow \mathbb{R}$, where $f(\mathbf{x})$ denotes the greyscale at point $\mathbf{x}$, is here chosen not to be $4 \mathrm{D}$ but $3 \mathrm{D}$ by making the dilations of the $\mathrm{x}_{1}$ and $\mathrm{x}_{2}$-components dependent. Therefore, the 2D-CWT consists of 'slices' of images with the same domain as $f$. An example of such a set of images for scales 1 to 16 is visible in Figure 3.33 for an image with defect 'ink missing'.

The application of the multifractal wavelet analysis to $2 \mathrm{D}$-images proceeds similar as in the 1-dimensional case. We study two methods, the power law scaling of the CWT as function of the scale $a$, and the 2D-variant of the WTMM method. Examples of the application of these methods to the computation of fractal properties of deterministic and random snowflake fractals, and clusters formed by Diffusion Limited Aggregation $(D L A)$ can be found in [Arneodo, 1996].

\section{i. The Local Scaling Exponent}

Figure 3.32 shows the results from the local CWT analysis of the scaling properties of the depicted image with defect 'hairs'. According to equation [3.51], the local scaling exponent $\alpha(\mathbf{x})$ is estimated at different points in the image by plotting $\log _{2}$ $\left|\mathrm{W}_{\psi}[f](\mathbf{x}, a)\right|$ versus ${ }^{2} \log a$. For deterministic fractals, disregarding finite-size effects which occasionally affect the CWT at large scales, $\mathrm{W}_{\psi}[f](\mathbf{x}, a)$ provides a rather accurate estimate of the exponent $\alpha$. From the plot in Figure $3.32 b$ it is visible that there exist oscillations which are superimposed on the expected CWT power law behaviour. This behaviour is first observed by Arneodo [Arneodo, 1996]. In the case of deterministic snowflakes he could demonstrate that these oscillations are periodic, 
and that this periodicity is a consequence of the exact recursive structure of the aggregate. As far as this image of a real printing defect is concerned, the nonperiodicity of the observed oscillations indicate the non-deterministic nature of the underlying multifractal. This random nature is caused by the physical-chemical processes that create the fibrous 'hair' phenomena.

Figure 3.32 illustrates the determination of local scaling exponents at different points of the defect. In Figure 3.32a the absolute difference $z$ of the defect is shown. In the figure four separate points A,B,C and D are depicted. The four dashed lines in Figure $3.32 b$ correspond to the CWT-scaling as observed in these points. The average slope of these lines is indicated in the same figure. Averaging is performed to minimise the oscillations around the expected scaling law. For this specific image the four values vary around $D=1.68 \pm 0.15$. These large variations were observed in all real images, and may have different causes. First, this does reflect a real phenomenon of a multifractal. The fractal features vary over the object, and it are these statistical properties that ultimately characterise the object. Second, the mentioned oscillations were strong, and it is not well possible to eliminate this effect entirely, causing large variations in the observed values for $D$. Thirdly, the calculated dimensions of the observed defect relate to the fractal dimension of the template used; they mix with the dimensionality of the template. Therefore, in the example in Figure 3.32 a template with solid areas was used, so dim(template) $=2$.

Finally, there are some practical problems in calculating the fractal dimensions, mainly caused by the general poor quality of the digital CCD-image. This approach also required exceedingly high computation times. The problem of the insufficient resolution of the image can in principle be solved by an interactive magnification if the scale needed for the next analysis is fed-back to the CCD-camera. 
Original Image
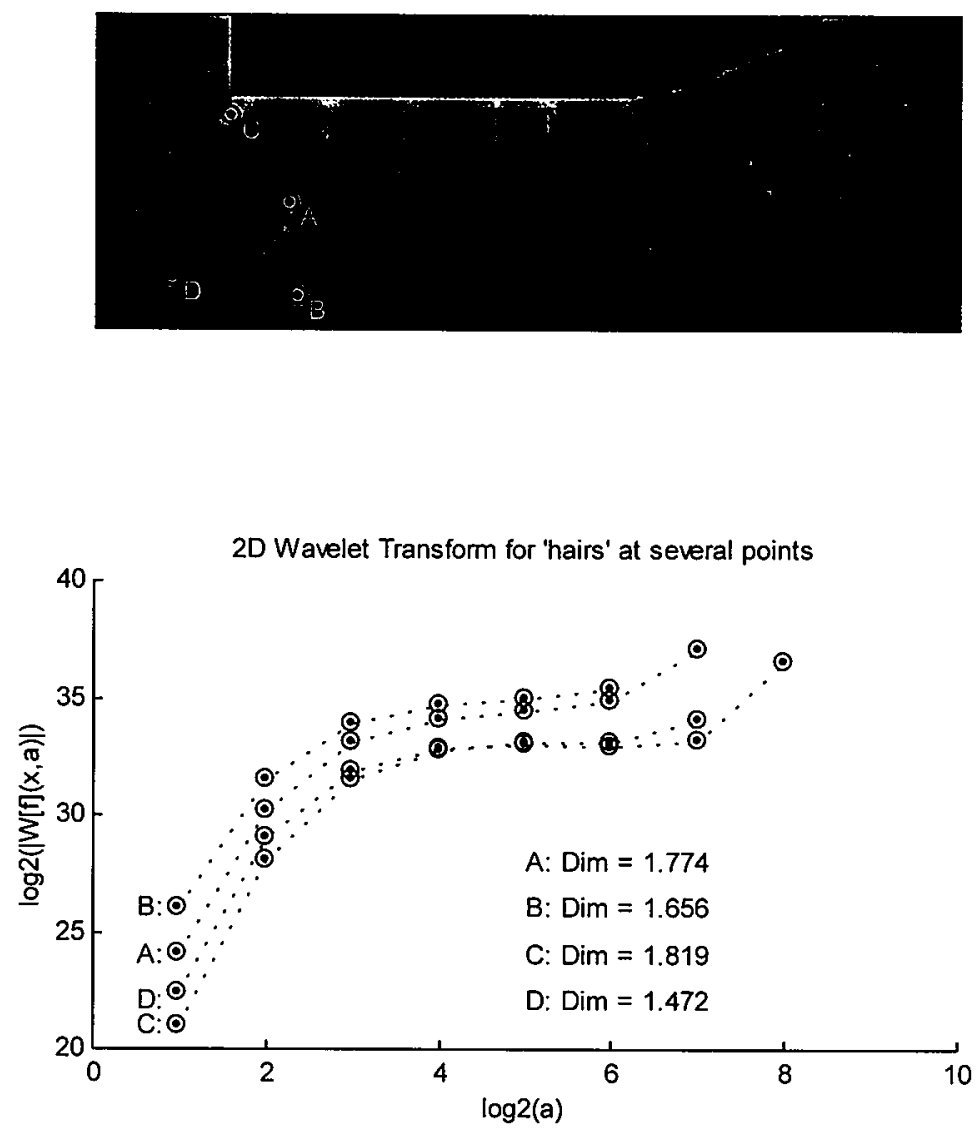

Fig. 3.32 Wavelet transform analysis of the z-representation of an image revealing the defect 'hairs'. (a) Above the original image as difference between CCD-image and template, with four points A,B,C,D. (b) Below: The determination of the local scaling exponents for these points. The average slope of the curves is listed in the figure. The large deviations for the scaling exponent are caused by local variations and oscillations around the power-law. 


\section{ii. The 2D-WTMM Method}

Figure 3.33 shows the results from the global CWT analysis of an image with defect called 'ink missing'. Besides the original $z$-representation of the image, 16 different scales of the 2D-CWT are shown. Each scale is normalised separately. In the WTMM method for each scale $a$ the maxima of the CWT are computed. Next, the maximalines, indexed ' $l$ ', are determined as the lines that connect corresponding points in the 2D-CWT slices over all scales. In this way we obtain a parameterisation for the maxima-line ' $l$ ' as: $\left(a, \mathbf{x}_{f}(a)\right)$, where $\mathbf{x}_{f}(a)$ is the 2D-position of the maximum of CWT corresponding to maxima-line ' $l$ ' at scale $a$. Using equation [3.56] the partition sum $Z$ could be computed, and with equation [3.57] the generalised dimensions $D$ could be determined. Table 3.7 shows the results of the application of this method to a number of printing defects. The main problem in the application of the 2D-WTMM method is again the poor quality of the image, both the low resolution as the noise. These made it difficult to determine high-quality maxima-lines, which in turn affected the result through equations [3.56] and [3.57]. On the other hand this method is not so sensitive for the oscillations observed in the first method.

\begin{tabular}{|c|c|c|c|c|}
\hline $\mathbf{n}$ & defect name & $\operatorname{dim}$ & std & \#samples \\
\hline 1 & hairs & 1.57 & 0.09 & 13 \\
\hline 2 & bubbles & 1.66 & 0.19 & 7 \\
\hline 2 & foam & 1.17 & 0.31 & 5 \\
\hline 3 & constriction & 1.32 & 0.28 & 5 \\
\hline 4 & recess & 1.32 & 0.10 & 5 \\
\hline 5 & ink missing & 1.73 & 0.08 & 12 \\
\hline 7 & dirt particles & 0.42 & 0.22 & 11 \\
\hline 8 & edge roughness & 1.34 & 0.07 & 5 \\
\hline 10 & smearing & 1.58 & 0.26 & 5 \\
\hline 12 & color difference & 1.71 & 0.09 & 5 \\
\hline 13 & rasterpoints & 1.20 & 0.04 & 9 \\
\hline 15 & lines & 1.15 & 0.16 & 7 \\
\hline 16 & haze & 1.53 & 0.44 & 5 \\
\hline$\overline{17}$ & orange peel & 1.89 & 0.31 & 5 \\
\hline
\end{tabular}

Table 3.7. Generalised fractal dimension $D(0)$ based on 2D-Continuous Wavelet Transform Modulus Maxima method for a large series of images. 


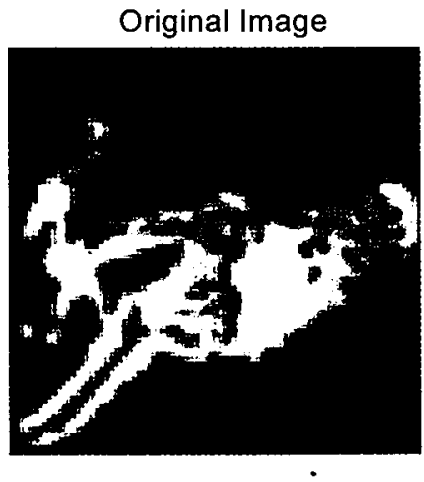

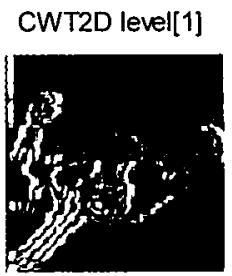

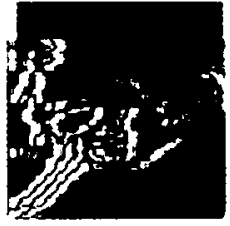

CWT2D level[5]

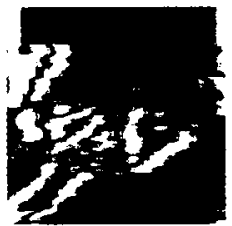

CWT2D level[9]

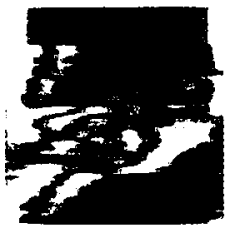

CWT2D level[13]

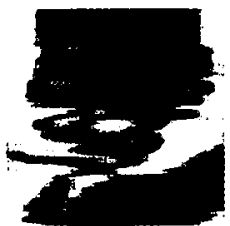

\section{CWT2D level[2]}

CWT2D level[3]

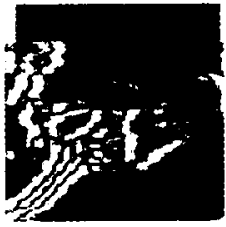

CWT2D level[7]

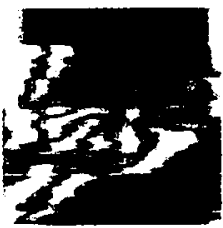

CWT2D level[11]

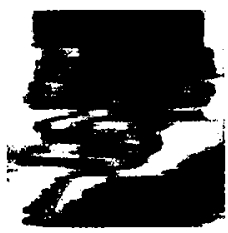

CWT2D level[15]

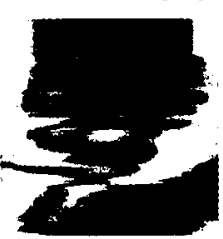

CWT2D level[4]

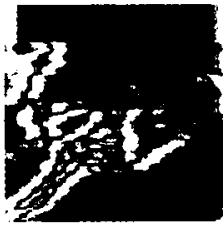

CWT2D level[8]

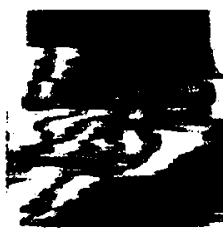

CWT2D level[12]

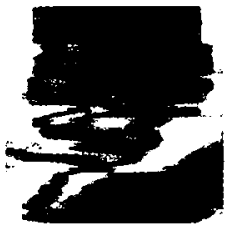

CWT2D level[16]

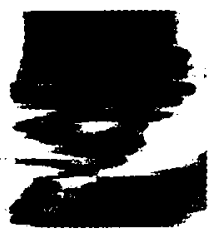

Fig. 3.33 The 2D continuous wavelet transform, $\mathrm{W}_{\psi}[f](\mathrm{x}, a)$, with equal $\mathrm{x}$ - and $\mathrm{y}$-scale ' $a$ ', with Gaussian[1] mother wavelet $\psi$. Above: original image of Z-representation of defect 'Ink Missing'. Below: images representing 16 scale levels, i.e. $\left|\mathrm{W}_{\psi}[\mathrm{f}](\mathrm{x}, a)\right|$ for $a=1 . .16$. The WTMM-method connects pixels representing the maxima of each image vertically, and so generates the maxima-lines. 


\section{iii. Comparison of the results}

The values for the 2D-WTMM method were compared with the values obtained for the 2D-multifractal formalism defined in Section 3.5. Figure 3.34 shows the relation between the 2D-box dimension and the dimension based on the 2D-CWT. Error ellipses were not indicated in order not to confuse the image. The relation between these two dimensions has a correlation coefficient of 0.87 for the whole set, and 0.70 for a reduced set without the outlier nr. 7 'dust particles'.

Table 3.8 lists the relation between the 2D-CWT and the 1D-CWT fractal dimensions. In Figure 3.35 the relation between these fractal dimensions is shown. From this figure it is clear that there is no definite relation between these two dimensions. The poor correlation of -0.33 in this figure is mainly due to poor quality of the 1D-CWT results.

\begin{tabular}{|l|l|l|l|l|}
\hline $\mathbf{n}$ & defect name & \multicolumn{1}{c|}{$\operatorname{dimCWT}$ 1D } & \multicolumn{1}{c|}{$\operatorname{dim}$ CWT 2D } \\
\hline $\mathbf{1}$ & hairs & $0.413 \pm 0.888$ & $1.570 \pm 0.090$ \\
\hline 2 & air bubbles & $0.805 \pm 1.108$ & $1.415 \pm 0.250$ \\
\hline 4 & recess & $0.940 \pm 0.738$ & $1.320 \pm 0.100$ \\
\hline 7 & dust particles & $0.912 \pm 0.280$ & $0.420 \pm 0.220$ \\
\hline 10 & smearing & $0.558 \pm 0.477$ & $1.580 \pm 0.260$ \\
\hline 17 & orange peel & $0.912 \pm 0.280$ & $1.890 \pm 0.310$ \\
\hline
\end{tabular}

Table 3.8 Relation between 2D-and ID-CWT Dimensions

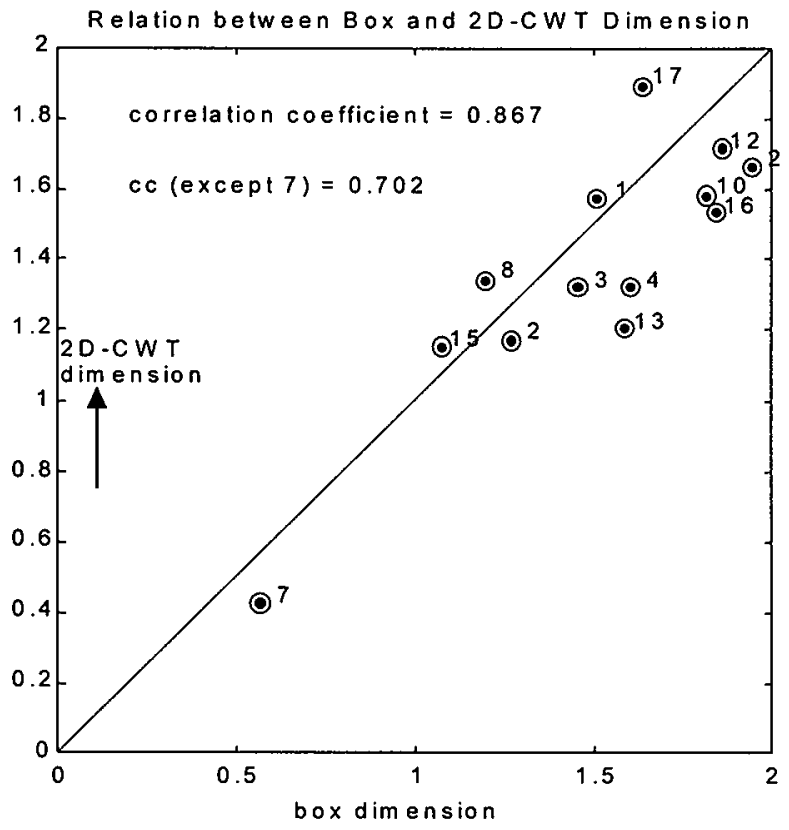

Fig. 3.34 Relation between the 2D-box dimension and 2D-CWT dimension. Numbers refer to printing defect index $n$ in Table 3.8. 


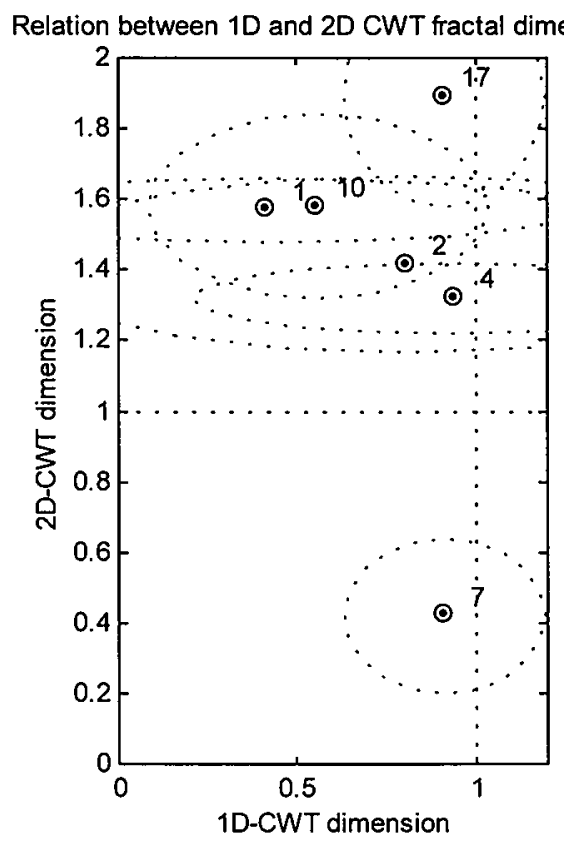

Fig. 3.35 Relation between the 2D- and 1D-CWT dimension. 


\subsection{Perspective on Strong Template-free Defect Classification with Fractal Wavelet Analysis}

Humans have a strong cultural and aesthetic intuition for fashionable and immaculate decorations, especially in the context of manufactured consumer products. Therefore, human attention is triggered when confronted with a printed decoration which in overall appearance deviates substantially from this general notion. For this reason humans are able to perform template-free defect recognition. Computers are not aware of any cultural background of a decoration, and therefore need an example in the form of the print template. In most cases, however, designed decorations have an orderly appearance and normally are non-fractals. The inspection of a decoration for areas with fractal properties may therefore reveal print faults occurring on an object. Moreover, as shown above, printing defects can have their own range of generalised fractal dimensions, somehow mixed with the dimension of the template applied. Multifractal wavelet analysis of patterns occurring in printing defects therefore offers a perspective to true template-free identification and classification. Such an approach could additionally utilise knowledge of the underlying chemical-physical processes, especially those referring to rheological properties of ink and thinner, notably the influence of turbulence and viscosity. First, this approach should exploit the knowledge of the dependencies of printing defects on the chemical-physical causes. Second, the typical patterns that characterise the defect should be understood from dynamical properties of the printing process creating it. Third, the fractal scaling properties resulting from these properties should be elucidated. Finally, the analysis of a printed surface should focus on finding structures that exhibit exactly these scaling properties, and thus indicate the most likely printing defect. Possibly, this is similar to the way how humans perform the strong template-free classification of printing defects by its power to detect order in textures. Be it, that humans do not posses any deep model based of understanding the basic chemical-physical processes, but have acquired a knowledge of admissible printing patterns and defect textures through learning.

As an example of such an approach Table 3.9 lists some observed fractal dimensions for a limited number of printing defects, all belonging to the same cluster based on a clustering of visually characteristics (as described in the next chapter). This table shows that within this cluster the visually related defects:

1. are non-integer,

2. have typical values - especially the $2 \mathrm{D}-\mathrm{CWT}$ - that sets them apart from the others.

A large practical problem is the computational load caused by this approach, and the restrictions caused by the digital nature of the CCD-images. Moreover, the quality of the images must be considerably improved before this method is applicable.

A related item is the following consideration. Suppose that we posses a deep model, existing of a set of equations describing the printed image as a result from the physical-chemical print process. Is it possible to use this deep model to make deductions about the physical-chemical process itself? Such an approach is called reverse engineering. This would allow direct process control based solely on an analysis of the printed image. The fundamental complexities associated with the construction of a deep physical-chemical-electromechanical model, if not its sheer impossibility, make this approach at the present untenable. 


\begin{tabular}{|c|c|c|c|c|}
\hline \multirow[t]{2}{*}{ DEFECT } & \multicolumn{2}{|c|}{ SCAN CURVES } & \multicolumn{2}{|c|}{ TEXTURE PATCHES } \\
\hline & $\begin{array}{l}\text { Box } \\
\text { Coumting }\end{array}$ & $\begin{array}{l}\text { ID-CWT } \\
\text { method }\end{array}$ & Bex Counting & 2D-CWT method \\
\hline hairs & 0.76 & 0.47 & $1.5 \pm 0.1$ & $1.75 \pm 0.10$ \\
\hline recess & - & 0.42 & $1.6 \pm 0.2$ & $1.32 \pm 0.10$ \\
\hline smearing & - & 1.2 & $1.82 \pm 0.28$ & $1.58 \pm 0.26$ \\
\hline
\end{tabular}

Table 3.9 Fractal dimensions for some defects with different techniques

Another way for template-free recognition to group defects is in "small scale" defects like 'dust particles', and "large scale" defects as 'wrong print-colour applied'. The scale of any aspect of an image can be estimated using the wavelet transform, this also provides the location on the image of the object with that scale.

In this case, the Fourier transform can also be applied. Using the Fourier Power spectral density function $\mathrm{P}(k)$, defined in equation [3.2] in Section 3.2.3, the parameter $a$ is represented in the same units as the original space-parameter $x$. Thus, $\mathrm{P}(k) \mathrm{d} k$ represents the energy related to the scale domain $\langle a, a+\mathrm{d} a\rangle$, with $a=k^{-1}$, and $\mathrm{d} a=k^{2} \mathrm{~d} k$. In this way, relative high contributions to the total energy can be traced. If the Fourier Power spectral density function of the template, or a relative good specimen, denoted $T$, is also available as $\mathrm{P}_{T}(a)$, then:

$$
\left|\mathrm{P}(a)-\mathrm{P}_{T}(a)\right|
$$

identifies the fault in the scale-space in a similar way as the modulus difference $Z(x)$ from section 2.2.3, identifies faults in the image-space. The latter approach also holds for the wavelet approach as:

$$
\left|\mathrm{W}_{\psi}[f](\mathbf{x}, a)-\mathrm{W}_{\psi}[T](\mathbf{x}, a)\right|
$$

The advantage of this approach, compared with the image analysis of absolute difference $Z(\mathbf{x})$ is that it not only identifies the scale of the fault, but also its precise location. 


\subsection{Conclusions}

Fourier transform is a useful representation to obtain the spectral decomposition of a scanprofile or a texture. The wavelet is furthermore capable of spatial decomposition, which is useful to specify discontinuous non-periodic signals. Whenever there is a strong spatial relation in the spectral decomposition of the signal, the wavelet transform will contain more information than the Fourier transform.

Fractal dimensions can be used to characterise printing defects. However, this characterisation suffers from the same disposition as all characterisations defined in appendix A; a weak correlation with the defect classes, and a considerable overlap of the defects relative to their error size. To establish a fractal dimension based on a digital CCD-image encounters a number of practical problems, especially related to the quality of the image. Moreover, to calculate these quantities demands a timeintensive computation. For this reason, there is no outstanding advantage to employ fractal analysis of a CCD-image at the present state of art. The formalism can be used as yet another additional tool in the toolbox of feature-extractions described in appendix $A$, to extract information for the benefit of a process reasoning about the nature of the defect.

More important is that, despite the present practical limitations, the formalism of fractal wavelet analysis bears an evident and powerful potential for strong templatefree defect recognition. In this light, the interpretation of printing defects as textures, and the characterisation of these textures is of great importance for the identification of the printing defects. Textures can be described adequately by both Fourier and Wavelet coefficients. Representation of defects in such 'texture'-spaces is advantageous in the determination of the nature of the defect.

This approach could, when combined with automatic clustering and classification methods, lead to an automatic approach to printing defect classification. In the learning mode such a combined system would extract generalised fractal dimensions of known printing defects, and cluster them in the texture space thus defined. In the operation mode, the system would obtain these generalised fractal dimensions from an actual image, and from its position in texture space deduce its most likely printing defect. 


\section{Chapter 4}

\section{Clustering Methods for Weakly- Separated Many-Parameter Classes}

\subsection{Introduction}

\section{Historical Perspective}

Not long after the introduction of high-level computer languages in the mid fifties of the previous century, their potential for automatic labelling and clustering large amounts of data was recognised. In the early days of pattern recognition, Duda and Hart [Duda,Hart,1973] attempted to define their discipline as: "the field concerned with machine recognition of meaningful regularities in noisy and complex environments". A more concise and better workable definition was provided by Bezdek and Pal in 1992 [Bezdek,Pal,1992], stating that: "pattern recognition is the search for structure in data". Today, pattern recognition has developed into an extensive field of science with many applications in the major - if not all branches of science and engineering, with its own scientific journals and branch organisations. Pattern recognition is important in identifying pathological tissues in PET scans, in monitoring agricultural exploitation and deforestation in satellite images, and for the automatic recognition of numerous elementary particles created in nuclear collision experiments, to mention but three random examples of the numerous existing applications.

We shall have little more to say about the impact of this area, except to the extent that the algorithms for pattern recognition described in this chapter have a decisive influence on the efficiency of the on-line classification process, and thereby, on the direct control of the type of vision control systems described in this thesis. As we only consider pattern recognition in relation to our main context, computational vision control, we study pattern recognition of a very specific kind of data. The main characteristic of data stemming from image analysis is the large amount of statistical and non-statistical imprecision. The statistical imprecision results from noise and processing errors in the imaging process and can be modelled by a random process, for instance with multivariate normal distributions. The concept of nonstatistical imprecision has become known as fuzziness, and forms the basis of a large collection of fields starting with 'fuzzy'. In the context of computational vision data is often of an inherently fuzzy nature. The specific field of application is the visual identification and classification of printing defects. Printing defects are defined merely on the basis of subjective 
visual criteria. Many defects, therefore, are of an ambiguous nature and can not be easily labelled as one specific type. For that reason a fuzzy modelling proved especially promising.

Another important characteristic of the representations we used to characterise imaging data is their great amount of cluster-overlap. This is an impediment shared by most computational vision applications. This predicament has as a natural consequence the definition of an awkwardly large amount of parameters. This is necessary in order to amplify the minute correlations with the defect-classes exhibited by some parameter-complexes. Related to the concept of imprecision is the uncertain exact number of classes available in the data. Since the basic defect characteristics are subjective, and their representations in parameter space hugely overlap, it is difficult to assign any definite number to their quantity.

Because of all these characteristic image data problems, we focus in this chapter on the construction of pattern recognition methods that can handle many-parameter representations with large amounts of noise and a high degree of cluster-overlap. In that framework we shall frequently employ fuzzy modelling of the underlying concepts.

The Central Role of Clustering in the Design of Pattern Recognition Systems

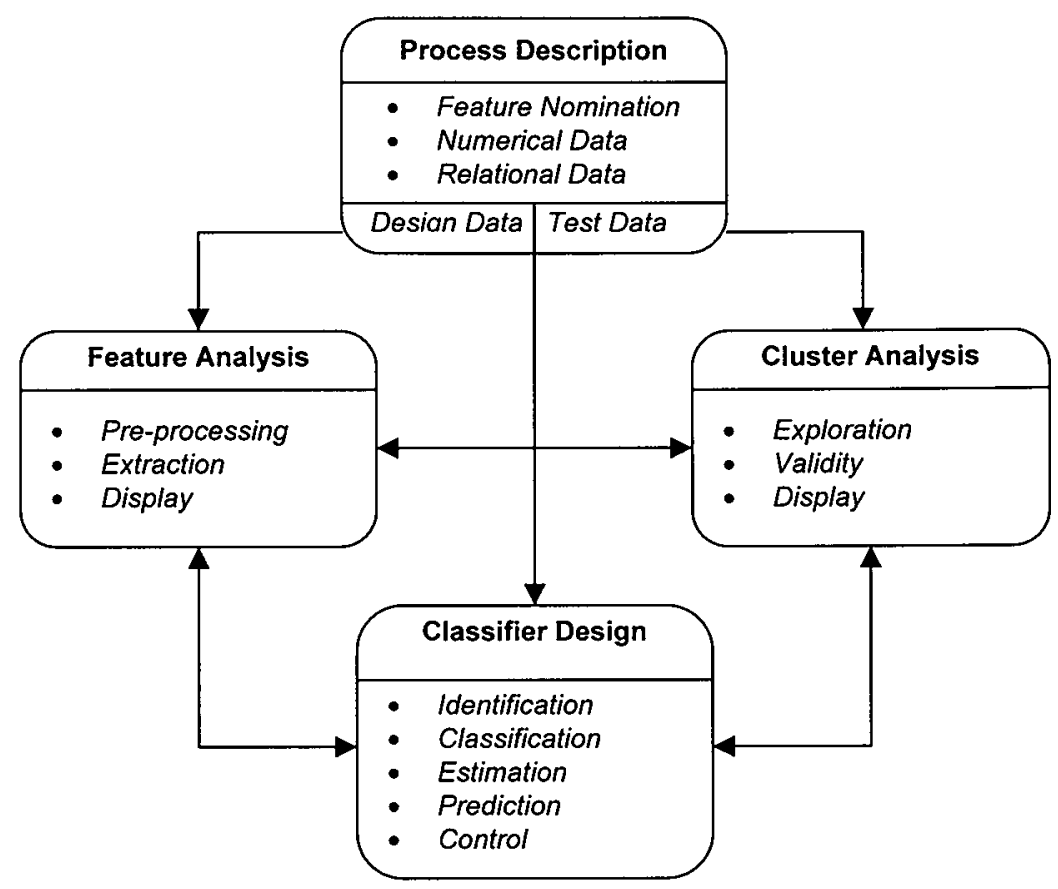

Fig. 4.1 The main activities in constructing a pattern recognition system and their relations according to Bezdek and Pal in [Bezdek,Pal,1992].

Bezdek and Pal distinguish four principal activities in the design and realisation of a pattern recognition system; Process Description, Feature Analysis, Cluster Analysis, and Classifier Design. The view of Bezdek and Pal on the relation between these activities is shown in Figure 4.1. In their view all activities are more or less connected. The four main concepts identified by Bezdek and Pal can be readily translated to our context, and we can relate the structure of this thesis directly to their layout. In our view, however, these activities are 
performed primarily sequentially, except for possible feed-back in case of redesign. This view is expressed in Figure 4.2. In our context Process Description is mainly concerned with the definition of image extraction parameters, which has largely been performed in appendix $A$.

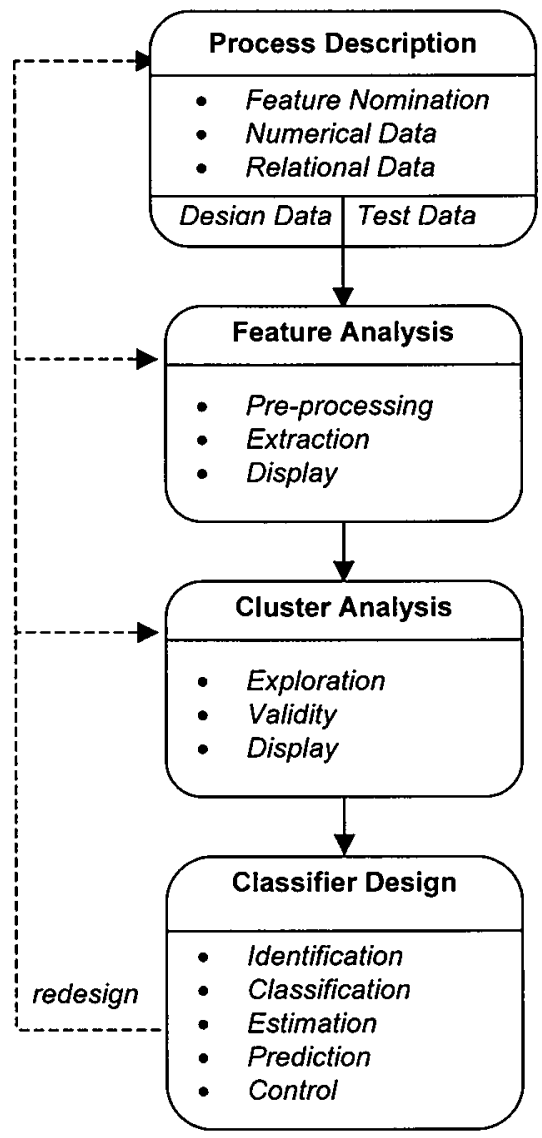

Fig. 4.2 Our vision on the interaction between the main activities.

In the following section we address another item for the description of the process, when we discuss fuzified class properties of the data. Feature Analysis in our context is also mainly addressed in Chapter 3 and appendix $A$, as most parameters are directly related to quantifying certain image and defect features. In the end of this chapter we will mathematically model some defect features to obtain insight in their distribution in parameter space for the purpose of pre-classification. Classifier Design is in our case focuses on on-line classification and direct control. These two subjects are discussed extensively in the next two chapters.

The field of Cluster Analysis lies right at the heart of pattern recognition and constitutes the main topic of the present chapter. Clustering is the partitioning of a data set in a number of distinct subsets, called classes, to which some relevant interpretation for the user can be assigned. When the data is already equipped with a label in advance this process is called 'labelled clustering', otherwise it is referred to as 'unlabeled clustering'. Clustering data can give valuable information on the structure of the data that is not directly apparent, especially 
in the case of large numbers of parameters. As such, it can form the starting point for numerical simulations, or qualitative and quantitative modelling. Since clustering empirical data results in a partitioning of a discrete number of classes, and because classification is the assignment of an actual observation to one of these classes, clustering can also be regarded as the off-line prerequisite for the - potentially on-line - process of classification. Clustering acts on a set of observations represented as a set of parameters. These parameters can be either quantitative, qualitative, or symbolic.

The result of the clustering is the assignment of a label to each observation, or the partitioning of parameter space (for instance with Voronoi-partitioning) in subsets. In addition to the traditional clustering techniques like $\mathrm{C}$-means, in recent decades many new clustering techniques have been developed. Examples are ID3 - stemming from the field of machine learning, Kohonen's self organising feature-map [Kohonen,1982,1989,1990], neural networks and genetic algorithms - all from Artificial Intelligence, and Fuzzy C-means from Fuzzy Logic. From the field of Combinatorial Optimisation stems the approach of Polyhedral Clustering [Rutten,1998]. Depending on the nature of the problem, one technique may be more applicable than an other. There does not exist a uniform approach or methodology for clustering a set of data, though some major components are recognised as described above. Most of these techniques have in common that the user must make some ad hoc assumptions about the data set, as for instance the number of relevant classes, or the size of the clusters present in the data. On the other hand, there exist some phenomenological techniques for cluster analysis that can automatically generate the number of classes, like mountain and subtractive clustering. In recent years the new fields of datamining and on-line data analysis (ODA) have emerged that aim at detecting structure in data, especially based on automatic cluster analysis and clustering techniques. Originally, unlabeled clustering techniques as Cmeans were designed for the detection of well-separated groups in the data, and the crisp assignment of a label to each of these groups, or alternatively, all relevant parts of parameter space. An important improvement to this method stemmed from the work of Zadeh [Zadeh,1965] and Bezdek [Bezdek, 1981]. In their approach they considered the assignment to a class as a fuzzy membership function. This opened the way to more complex configurations of data in parameter space, including partially overlapping classes. The development of neural networks and self-organising feature maps allowed the identification of even more complex data, like disconnected, concave, or punctured sets.

\section{Objectives of this Chapter}

The main aim of our work with regard to clustering concerns the partitioning of considerably overlapping yet labelled data sets. However, what we intend is not to reproduce the labelled sets, but to find a higher structure in parameter space. In this way some classes can be joined to one cluster. This can be used for pre-classification of the raw data.

In our context we are predominantly interested in unsupervised clustering without any prior knowledge. This may sound strange as we possess the label of each defect in our training-set. Amongst the reasons is that the experts were divided whether certain defect-classes were single or composed, i.e. whether certain features were only superficially similar, but the underlying causes are entirely different. Automatic clustering could be used to obtain more information about such questions. On the other hand, some superficially entirely different defects were thought to have almost the similar physical-chemical causes. Likewise, automatic clustering could be employed for further insight. 
This chapter can be read independent of the other chapters. As stated before, however, the techniques here described were developed primarily in the framework of computational vision control, an area notorious for its high degree of semantical and statistical cluster-overlap. For that reason we focussed on the pattern recognition of weakly-separated many-parameter defect classes.

We start this chapter in section 4.2 with a discussion of the formalism we used to represent the data and its associated partitioning.

In section 4.3 we present a concise review of the major directions in current clustering research relevant to our context, based on a survey of recent literature.

In section 4.4 we study the applicability of supervised clustering/classification techniques to our domain. This is achieved by employing artificial neural networks for pattern recognition of defects. In this section we will see that neural networks are not particularly suited for our aim of real-time classification.

Next, in section 4.5 , we consider unsupervised clustering. We describe a fuzzy clustering technique, called Fuzzy Multi-Variate clustering (FMV), that we developed for clustering particularly overlapping data, as appropriate in our domain. We compare its performance and speed to the well-known clustering algorithm 'Fuzzy C-means'.

In section 4.6 we describe an automatic clustering technique, called Iterated-FMV, that we developed for our domain. Iterated-FMV is based on cluster tree expansion, employing the FMV clustering algorithm. We compare this approach to other automatic clustering techniques like mountain and subtractive clustering.

We close this chapter in section 4.7 with two applications of clustering techniques within the context of computational vision control. First, Kohonen's self organising feature map is applied for modelling certain printing defects. Second, fuzzy clustering is used as a basis for a pre-classification method.

\subsection{Representation of Weakly Separated Many-Parameter Classes}

Our aim in this chapter is to recognise relevant features present in a given type of objects. To that extent we have possession of a sufficiently large amount of sensor data. Here, sensor data is a collection of empirical observations of the objects. Now, our intention is to quantify and qualify the required features in this collection of observations. To do so, we require a set of parameters necessary to represent the observations. Let $N$ indicate the total number of available parameters, $n$ the index of a parameter, and $x_{n}$ its value. Preferably, the parameters should be independent, i.e. the covariance for all pairs of parameters should be (close to) zero. The set of all possible values of the parameters is called parameter space. Let $\mathbf{x}$ denote an observation as a set of concurrent measurements of all $N$ parameters: $\mathbf{x}=\left(x_{1}, x_{2}, \ldots, x_{N}\right)^{\top}$. Let $X$ denote the set of empirical data consisting of $M$ observations: $X=\left\{\mathbf{x}_{1}, \mathbf{x}_{2}, . ., \mathbf{x}_{M}\right\}$. We now make the following assumption:

\section{observations that share a specific feature lie close in parameter space relative to observations that do not share that feature.}

This means that we can 'count' the relevant features in the objects by numbering the amount of isolated clusters in parameter space. This opens the concept of feature-overlap: for a certain parameter two clusters can overlap. This implies that these features are not well separated. In this way there exist 'sharp' features, corresponding to very narrow clusters with a relatively small range of parameter values, and 'broad' features, corresponding to diffuse clusters with wide ranges of parameter values. Our assumption is relative, for a specific feature may be 
present in just one single parameter, causing a well-separated clustering in that dimension, while all other parameter-values may be evenly distributed.

Besides the features, there exist classes of concepts that are composed of these features. For instance, class $A$ can be defined as a combination of features as: $F_{1} \wedge F_{2} \wedge F_{3}$, class $B$ as: $F_{1} \wedge F_{2} \wedge F_{4}$, and class $C$ as: $F_{3} \wedge F_{4}$. Classes can come in naturally, for instance for features that are sharply defined in all parameters. In that case the feature directly defines the class. Some combinations can prove to be impossible, for instance, class $B$ may be empty. In many cases, there is a human observer that provides these class definitions. An entirely different possibility, however, is that an automatic technique performs the clustering, and ends up with a specific number. Let $K$ be the number of classes that the user or the clustering technique decides to be present in the data set $X$. In the case of well-separated clusters these numbers should not differ, but for many practical cases - and also the examples that we encounter there is a certain amount of subjectivity or user intent to discriminate one rather than another number of classes.

Now we can define clustering as the act ${ }^{1}$ of partitioning set $X$ in $K$ classes, such that each measurement $\mathbf{x}_{m} \in X$ receives a label $l_{m} \in\{1,2, . ., K\}$. This is called the crisp definition of clustering. Instead of assigning a label $l_{m}$, we can define an $M \times K$ matrix $u_{m k} \in\{0,1\}$, denoting the membership of observation $m$ to class $k$. Notice that $\sum_{k} u_{m k}=1$ for all $m$, and that $\Sigma_{m} u_{m k}$ denotes the number of elements in class $k$.

The essential step of Zadeh and Bezdek [Zadeh,1965], [Bezdek,1981] was to extent the values of $u_{m k}$ to all real values in $[0,1]$. This entity $u$ is called the fuzzy partitioning matrix, and indicates the degree to which a measurement $\mathbf{x}_{m}$ belongs to a particular class $k$. The matrix is normalised with respect to the measurements, i.e. $\Sigma_{k} u_{m k}=1$ for all $m$. The summation $\Sigma_{m} u_{m k}$ now indicates the collective membership to class $k$. This process is called fuzzy clustering.

Instead of determining the partitioning matrix $u$ of the set, we can also use geometrical functions on parameter space. For each class $k$ we can thus define a distribution $\varphi_{k}(\mathbf{x})$ that gives the class membership of a measurement with parameters $\mathbf{x}$. The partitioning matrix thus equals: $u_{m k}=\varphi_{k}\left(\mathrm{x}_{m}\right)$. Methods like ID3 and artificial neural networks attempt to construct representations for such transformations. For neural networks this representation is only internal. More symmetry is introduced if all of these functions $\varphi_{k}$ can be generated by one principle generator $\Phi$ and a set of class-dependent parameters $\xi_{k}$, such that: $\varphi_{k}\left(\mathbf{x}_{m}\right)=\Phi\left(\mathbf{x}_{m} \mid \xi_{k}\right)$. Examples of class-dependent parameters of class $k$ are: (1) the prototype or centroid $\mathbf{v}_{k}$, defined as the mean value of the observations in one class weighted with the partitioning matrix $u$, and defined similarly: (2) the covariance matrix $C_{k}$. This method becomes relevant when the observations can be adequately described by a specific distribution in parameter space, as for instance a multivariate normal distribution. We encounter such examples in the next sections.

\subsection{Survey of Major Approaches to Clustering}

In this section we present a concise review of the major directions in current clustering research relevant to our context, based on a survey of recent literature. An excellent review on

\footnotetext{
' In literature the term 'clustering' is used both for the act of clustering as for the resulting set of clusters.
} 
the current state-of-the-art of clustering techniques is provided by [Jain et al.,2000]. In addition, we specify some criteria that can be used to provide a useful taxonomy of clustering techniques.

\section{a. Cluster Validity Criteria}

A Cluster Validity Criterion is a parameter that represents the degree to which an identified grouping in data-space can be regarded as a legitimate, isolated cluster. In the literature, numerous Cluster Validity Criteria have been proposed, such as the Partition Coefficient [Bezdek,1973], Partition Entropy [Bezdek,1981], Proportional Exponent [Windham,1981], Uniform Data Function [Windham,1982], Bootstrap Technique [Jain,Moreau,1987], fuzzy hypervolume and density [Gath,Geva,1989], fuzzy compactness and fuzzy separation [Pal,Bezdek,1995], [Zahid et al.,1999]. Some of these criteria are solely based on the membership-functions and do not involve the actual shape of the data-set in parameter-space. Recently, the geometric properties of the data-collection, however, receive more attention, as in the work of [Pal,Bezdek,1995], and [Zahid et al.,1999]. [Pal,Bezdek,1995] identify three groups of Cluster Validity Criteria.

1. Statistical Cluster Validity Criteria, like [Fukuyama,Sugeno, 1989], [Duda,Hart, 1973], and [Pal,Bezdek, 1995].

2. Cluster Validity Criteria based on data-membership-functions, and clusterhypervolume, like in [Gath,Geva, 1989].

3. Tests for specific distributions, like the Gaussian distribution, like provided by [Steinberg,Zeitouni,1992].

[Geva et al.,2000] compared a large number Cluster Validity Criteria for mixtures of normal distributed data (so-called 'Gaussian mixtures', see below). The clusters were obtained with the Unsupervised Optimal Fuzzy Clustering (UOFC) clustering algorithm [Gath,Geva, 1989] (discussed below). They found that no single validity criterion consistently outperformed the others under all conditions, but that group 2 and 3, of the listing above, performed somewhat better than group 1 .

\section{b. Supervised versus Unsupervised Clustering}

Consider a collection of $M$ observations in an $N$-dimensional parameter-space. If this data-set is labelled in advance, we call the clustering process supervised. When we lack prior knowledge about the data-set, we call the clustering method unsupervised. For unsupervised clustering several issues need to be clarified before or during the method is employed [Geva et al.,2000], including:

1. The data-structure(s) of the parameter space: are the parameters used to express the objects relevant enough to discern the essential differences between clusters?

2. Does there exist a 'true' number of clusters present in the data, and can the user or the method provide it?

Most prominent amongst supervised clustering/classification techniques are Artificial Neural Networks[McCulloch,1943,1965/1988], [Minsky,Papert,1969/1988] (discussed later), and Genetic Algorithms [Holland, 1975], [Goldberg, 1989].

\section{c. Parametric versus Non-Parametric Clustering}

In parametric clustering a specific distribution is assumed for the data in parameter-space, and the available observations are used to estimate the relevant parameters of the distribution. 
An example is the normal distribution $N(\mathbf{m}, C)$ with mean $\mathbf{m}$ and covariance matrix $C$. Under this assumption, the observations are used to provide the best possible estimate of $\mathbf{m}$ and $C$. In non-parametric clustering the underlying distribution is not specified. Artificial Neural Networks construct an internal representation of such an unknown distribution.

In mixture models (sometimes called model-based clustering) the probability-distribution of the observations is assumed to stem from a finite mixture of probability-distributions, such as a weighted normal distribution: $P(\mathbf{x})=\sum_{k=1}^{K} p_{k} N\left(\mathbf{x} \mid \mathbf{m}_{k}, C_{k}\right)$. In that sense, mixture-models are merely specific types of parametric models. Otherwise, mixture-models can be understood as a finite mixture of competing models $\left\{M_{1}, \ldots, M_{H}\right\}$. Then, using Bayes' theorem, the model $M_{l}$ with the highest posterior probability $P\left(M_{l} \mid \mathrm{X}\right)$, given a set of observations X, follows from:

$$
P\left(M_{l} \mid X\right)=\frac{p\left(X \mid M_{l}\right) P\left(M_{l}\right)}{\sum_{i=1}^{H} p\left(X \mid M_{i}\right) P\left(M_{i}\right)}
$$

where $P\left(M_{l}\right)$ is the a-priori probability of model $M_{l}$, and $p\left(M_{l} \mid X\right)$ is the marginal or integrated likelihood of model $M_{1}$. Instead of using this Bayesian Information Criterion, Biernacki et al. [Biernacki et al.,2000] obtain better clustering results in applying the so-called Integrated Completed Likelihood, with an additional information-entropy term, as criterion for modelselection aimed at parameter-space density estimation.

\section{d. Unsupervised Clustering Algorithms}

\section{d.1 The Fuzzy C-means algorithm}

The Fuzzy C-means algorithm (FCM) [Dunn,1973], [Bezdek,1973] is probably the most extensively studied and published clustering algorithm. It is the fuzified form of the 'crisp' Cmeans partitioning algorithm [Duda,Hart, 1973]. FCM is described in section 4.3.1.

The quality of the FCM-solution, like that of most nonlinear optimisation problems, depends strongly on the choice of initial values [Chiu,1994]. In our case that is: the number $K$ and the initial centroid-positions. Though the FCM algorithm is known for its robustness, in literature its performance is extensively reported to depend on two key factors: initial clustering and instance order [Peña et al.,1999]. This is valid for both the quality of the result, as for the required computation time. The FCM algorithm converges in finite time to a local minimum. The running of the algorithm defines a deterministic mapping from the initial centroid positions to the final solution. Moreover, FCM shares the disadvantage of most clustering algorithm in its unrealistic before-hand assumption on the number of clusters.

\section{d.2 The K-Nearest Neighbour rule}

In the $K$-Nearest Neighbour rule ( $K-\mathrm{NN}$ ) [Keller et al., 1985] an empirical set of observations $X$ is available. A new observation $\mathbf{x}$ is associated with the $K$ nearest neighbours of $\mathbf{x}$. Next, $\mathbf{x}$ is assigned to the most frequent cluster amongst these $K$ nearest neighbours.

\section{e. Automatically Finding the number of Clusters}

In supervised clustering mostly some prior knowledge about the clusters is assumed. This may involve the number, size, or shape of the clusters. In an Automatic Clustering process no assumptions whatsoever are required in advance. Regarding the resulting output-structure, 
automatic clustering algorithms can in general be classified in three types; partitional, mixture-decomposition, and hierarchical.

\section{Partitional Clustering Algorithms}

In partitional clustering algorithms ${ }^{2}$ solely a partition of the parameter space is produced. This is the most frequent employed clustering tool in pattern recognition applications. In general, all of such algorithms will find clusters in a given dataset, whether they exist or not. Moreover, literature agrees that there is no 'best' clustering algorithm [Jain et al.,2000]. Partitional clustering algorithms consist of the following three components:

1. a Search Technique in the set of possible number-of-clusters $K^{3}$.

2. a one-pass Clustering Algorithm.

3. a set of Cluster Validity Criteria.

In this approach, a large collection of alternative possibilities for $K$, generated by the Search Technique, is generated. Using a one-pass Clustering Technique, like FCM, a clustering is generated. Next, this clustering is evaluated using the Cluster Validity Criteria, like those mentioned in section 4.3.a. Finally, the number $K^{*}$ with the best evaluation is selected. An obvious disadvantage of this approach is that a large number of experiments must be performed of which ultimately only one turns out to be of use. This method is like the 'generate-and-test' procedure in automatic search programs. Even worse is that in order to obtain statistically significant decision criteria the experiments must be performed a large number of times to reduce the standard deviation. In most cases the search technique employed is exhaustive. To this category belong the methods described by Geva et al., and Zahid et al. (see section below). In [Westra et al.,1997a] we also used exhaustive search to minimise cluster energy in order to find the number of clusters. This approach is presented below in section 4.6. Approaches consisting of an iterative expansion of $K$, based on of the best results obtained during the search, are called cluster tree expansion algorithms. This approach is more comparable to (intelligent) search. The approach based on an iterated sequence of $F M V$ clustering, presented in section 4.6 , belongs to this category. More examples are discussed below.

\section{Mixture Decomposition Algorithms}

Mixture Decomposition is based on probabilistic modelling using finite mixtures [Titterington et al.,1985]. A finite mixture distribution consists of a finite collection of sources, each with its own source-conditional probability distribution. In addition, each source has an a priory probability. The entire mixture distribution now consists of the weighted sum of the sourceconditional distributions, with the a priory-probabilities as weights. Although mixtures can be built from various types of distributions, the majority of literature focuses on Gaussian Mixtures [Jain et al.,2000]. In our work we also exclusively apply Gaussian Mixtures. The central point in Mixture Decomposition is to estimate the source-dependent distribution parameters from a set of empirical observations, including the number of sources. There are two major methods with which this can be achieved; Expectation-Maximisation (EM) [Titterington et al.,1985], and the Markov Chain Monte-Carlo (MCMC) method [Richardson,Green, 1997$]^{4}$. With Mixture Decomposition it is possible to identify totally overlapping - even coinciding - classes, see for instance [Figueredo et al.,1999]. This is

\footnotetext{
${ }^{2}$ Also known as: iterative square-error partitional clustering algorithms

${ }^{3}$ Typically, for a set of $M$ observations, $K$ ranges in: $\{1,2, \ldots, \sqrt{ } M\}$.

4 This method is employed by [Wang,Liu,2000] for estimating texture properties, as discussed in section 3.7.3.
} 
because the problem is defined as a parameter-estimation task. A FCM-like algorithm will not be able to identify such overlapping classes. In our approach, however, we rely on FCM-type methods, because, citing [Jain et al.,2000]; 'the area of Mixture Decomposition, despite its formal appeal is still far too computationally demanding to be useful in pattern recognition applications'. Partitional clustering should be regarded as a specific type of Mixture decomposition.

\section{Hierarchical Clustering Algorithms}

In hierarchical clustering ${ }^{5}$ the output is a dendogram of clusters. This is a tree exhibiting the splitting/merging of related clusters, top-down from one primordial super-class corresponding to the mean of all observations, ultimately to numerous clusters corresponding to all individual observations. Such an approach is realised in: [Wilson,Hancock, 1999], who developed a Bayesian framework for matching hierarchical relational models, and applied it to clustering Synthetic Aperture Radar images. Associated with this dendogram is a corresponding sequence of relevant scales in parameter-space. Therefore, it is also called 'scale-space clustering'. This can be imagined as a discretisation of parameter space. At coarse magnifications many adjacent observations are lumped into one 'bin'. Neighbouring collection of bins, therefore, represent valid clusters at that scale. At certain scales, the merging scales, some of these collections split into multiple sub-clusters. In scale-space clustering the same three components as mentioned above for partitional clustering are present, be it that the search is performed in the space of relevant scales, rather than in the number-of-clusters. Other examples of scale-space methods are Mountain Clustering and Subtractive Clustering, and the work performed by [Kothari et al, 1999] and [Leung et al.,2000]. These approaches are discussed below in some detail.

\section{f. Partitional Clustering Algorithms for Automatic Clustering}

As mentioned above, the first set of algorithms for automatically finding the number of clusters is called partitional clustering, and consists of: 1. a search technique, 2. a clustering algorithm, and: 3. a cluster validity method. Relevant and recent developments in this field include the following methods.

\section{f.1. The Unsupervised Optimal Fuzzy Clustering Algorithm}

The Unsupervised Optimal Fuzzy Clustering (UOFC) clustering algorithm [Gath,Geva, 1989] is a combination of a precursor of the FCM-algorithm [Bezdek,Castelaz,1971] and maximum likelihood estimation [Peters, Walkers, 1978]. It consists of a number of steps from the FCMalgorithm in calculating the fuzzy memberships-functions and the cluster-means. In addition a Gaussian distribution based on these memberships-functions is constructed to estimate the maximum likelihood-distance from each of the $M$ observations to each of the $K$ clusters. This procedure requires a value for the number of clusters $K$ in the data. Next, this procedure is employed for all values of $K$ from 1 to $\sqrt{M}$. In this sense, UOFC simply exhaustively examines all feasible number of clusters, using maximum likelihood-distance as an integrated Cluster Validity Parameter.

\section{f.2 Iterated K-Nearest Neighbour Iterated Fuzzy C-Means Algorithm}

Zahid et al. [Zahid et al.,1999] propose a heuristic algorithm for fuzzy clustering without initial assumptions on the number of clusters, or the type of distribution in parameter-space.

\footnotetext{
${ }^{5}$ Also known as: agglomerative hierarchical clustering.
} 
The algorithm is called Iterated K-Nearest Neighbour Iterated Fuzzy C-Means Algorithm (KNN-IFCMA), and consists of iteratively determining the $K$ Nearest Neighbours ( $K-\mathrm{NN})$ of the observations $\mathbf{x}$, and subsequently calculating the fuzzy partition-matrix $U$ and centroids $\mathbf{m}$ using FCM, with a given number of clusters $c$. Next, a performance measure $S$, composed of two Cluster Validity Criteria - i.e. fuzzy set compactness and fuzzy set separation, is defined. Ultimately, this $S$ is calculated for all $c$ from 1 to $\sqrt{ } M$, where $M$ is the total number of available observations. Finally, the algorithm assigns a quantity $K^{*}$ to the number of clusters equal to: $K^{*}=\arg \max \{S(c) \mid c \in\{1, \ldots, \sqrt{M}\}\}$. Therefore, $K$-NN-IFCMA is an exhaustive search using a modified FCM-algorithm and a resourceful, compound fuzzy Cluster Validity Criterion. The advantage is that a fair clustering is obtained - as shown by their experiments, but at the cost of a considerable number of computations for realistic sets of observations.

\section{g. Clustering using Gaussian Mixture Decomposition}

A Gaussian mixture is a weighted sum of multivariate normal distributions (see for instance: [Schalkhoff,1992]). Thus, it is a Finite Mixture Model, defined above, with exclusively (multivariate) Gaussian distributions. Numerous publications employ this representation, for its practical relevance and its ease of computation. Also in our context we represent defectclass-conditional probability distributions as a Gaussian mixture. Gaussian Mixture Decomposition is a powerful method to find relevant clusters and class-conditional probability-distributions. [Hofbeck,Landgrebe,1996] showed that this approach works satisfactory even for limited number of training data.

As mentioned above, there are two methods for clustering. The EM-algorithm, which under mild conditions, converges to the maximum likelihood estimation of the mixture parameters. Duda and Hart [Duda,Hart,1973] provide an algorithm for computing the maximum likelihood estimates for the mean and covariance of Gaussian mixtures, based on a collection of empirical observations. This algorithm is effectively equivalent to FCM. Most approaches perform exhaustive search in the set of possible cluster-numbers, and select the number that minimises a cost-function. Mostly, this cost-function consists of the maximum likelihood estimates with an additional term to penalise high cluster-numbers. [Windham,Cutler, 1992] use this approach, and use the Akaike information criterion as additional term. More recently, [Hwang et al.,2000] use Bayes' theorem to directly infer the most likely defect class for Gaussian mixtures.

\section{h. Hierarchical and Scale-Space Clustering Algorithms for Automatic Clustering}

As mentioned above, another set of algorithms for automatically finding the number of clusters are based on hierarchical clustering. Of great importance in deciding on the number of clusters available in a data-collection is the scale-perspective in parameter-space that we adopt in observing the collection of observations. At the highest scale, the entire collection is represented by the mean of all observations, so here we find just one cluster: $K=1$. At the other extreme, each observation is seen as a separate class, and we find a number of clusters equal to the number of observations: $K=M$. Thus, if we slowly zoom-in from the highest to the lowest appropriate scale, we obtain a graph, the dendogram, in which the number of clusters develops from 1 to $M$. This idea may be utilised for finding the most significant number of clusters. That can be accomplished in two ways:

i. determine the scale where the clustering is most profound (e.g. using cluster validity criteria),

ii. determine the widest range of scales where a certain number of clusters persists. 
Both approaches are computationally intensive, as clusterings must be obtained for a relevant range of scales. The latter approach is adopted by [Kothari,Pitts,1999]. They present a functional optimisation approach to clustering, equivalent to the (conventional crisp) C-means functional $J C M$ (this means that the membership-function $u$ is either 0 or 1 ). To this functional they add a second term, representing the squared distances of cluster-centroids if they fall within a certain distance $\eta$. This $\eta$ acts as the scale-parameter. The cluster-centroids are updated by gradient-descent. The coefficients of the two terms are chosen thereby such, that the average centroid movement of the two terms cancel exactly. This defines an iteration where the centroids gradually move towards their final positions. At the end of this iteration, clusters within the distance $\eta$ are coalesced, while near-empty clusters are omitted. Finally, the number of clusters is selected that shows the widest range of scales. In ambiguous cases a cluster validity criterion, representing internal cohesion and external isolation, is employed. For this they use the Dunn index [Bezdek,Pal,1998]. The computational price paid for this approach is that, for a range of 20 scales, it is 40 times more expensive as the traditional Cmeans algorithm ${ }^{6}$.

Another example of Scale-Space Clustering is: [Leung et al.,2000]. They model the blurring effect of the lateral retinal interconnections. With this approach they blur the 'image' of the training data-set in parameter space. By increasingly blurring the image, smaller clusters fuse into larger ones, until ultimately the whole image is one connected set at sufficient low resolution. This blurring-process generates a family of clusterings along the hierarchy. Though this approach is computational intensive, it has a number of attractive features, such as: 1 . independence of initial conditions, 2 . high degree of robustness, 3 . low sensitivity to noise.

Next, we describe two closely related, simple, yet very effective, one-pass scale-space clustering techniques in more detail; Mountain Clustering and Subtractive Clustering.

\section{h.l. Mountain Clustering}

Yager and Filev [Yager,Filev, 1994] introduced a simple and efficient clustering technique, called 'Mountain Clustering', for estimating the number and initial positions of the clustercentroids. Consider a set of $M$ independent observations in a $N$-dimensional parameter-space: $X=\left\{\mathbf{x}_{m} \mid m=1 . . M, \mathbf{x}_{m} \in \mathrm{IR}^{N}\right\}$. The Mountain Clustering method is based on a discretisation of space, according to a lattice $S=\left\{s_{n i} \mid n=1 . . N, i=1 . . I\right\}$, in which each of the $N$ dimensions is partitioned in $I+1$ parts. Next, an $I^{N}$ matrix $V$ is defined, representing the 'influence' of the data-points on lattice-point $\mathbf{s}[\mathbf{i}] \equiv \mathbf{s}\left[i_{1}, i_{2}, . ., i_{N}\right]$. This influence is achieved by a potentialfunction that decays exponentially with the distance of the observation $\mathbf{x}_{m}$ to the lattice-point $\mathbf{s}[\mathbf{i}]$. The entire potential at a lattice-point $\mathbf{s}[\mathbf{i}]$ is thus defined as:

$$
V[\mathbf{i}]=\sum_{m=1}^{M} \exp \left(-\alpha\left\|\mathbf{x}_{m}-\mathbf{s}[\mathbf{i}]\right\|\right)
$$

where $\alpha$ is a chosen parameter. This potential-matrix is called the 'mountain-function'. After the construction of this mountain-function, iteratively the following three steps are performed:

i. $\quad$ the maximum of $V$ is selected as: $V^{*}=\max _{\mathbf{j}} V[\mathbf{i}]$, at position: $\mathbf{i}^{*}=\arg \max _{\mathbf{i}} V[\mathbf{i}]$.

ii. a centroid is assigned to the corresponding lattice-position: $\mathbf{m}_{k}=\mathbf{s}\left[\mathbf{i}^{*}\right]$, starting with $k=1$.

\footnotetext{
${ }^{6}$ Initialising the centroids to the convergence values obtained in the iteration-cycle of the previous scale could significantly reduce the computation time.
} 
iii. the mountain-function $V$ is destructed as: $V[\mathbf{i}]=V[\mathbf{i}]-V^{*} \exp \left(-\beta\left\|\mathbf{m}_{\mathbf{l}}-\mathrm{s}[\mathbf{i}]\right\|\right)$, where $\beta$ is a chosen parameter; $\beta>\alpha$.

The iteration is terminated when $V^{*}$ falls below a predefined parameter $\delta: V^{*}<\delta$. The generated sequence of centroids $\left\{\mathbf{m}_{1}, . ., \mathbf{m}_{K}\right\}$ represent the most concentrated parts of the distribution of observations in parameter-space. In due course, the number of clusters $K$ is automatically determined by the algorithm. However, there are some problems with this approach.

1. The number of clusters $K$ sensitively depends on the pre-defined lattice $S$ used to discretise parameter-space. The range of sizes of the intervals $\Delta_{n i}=\left[s_{n i}, s_{n, i+1}\right]$ define the range of scales with which the data-set in parameter-space is observed. In this sense, mountain-clustering is a scale-space approach.

2. $K$ and the resulting centroids strongly depend on the values of the iteration-parameters $\alpha$, $\beta$, and $\delta$.

3. parts of space deleted from $A$ during the destruction-phase, may form artificial optima that induce false centroids and, consequently, an inappropriate value for $K$. Again, this depends on the parameters $S, \alpha, \beta$, and $\delta$.

In most applications, a typical scale $\lambda$ per parameter is demanded, which implicitly defines $I$. In this way, however, again a pre-assumption on the clustering becomes required.

\section{h.2. Subtractive Clustering}

Chiu [Chiu, 1994] presented an inventive improvement to Mountain Clustering, called the Subtractive Clustering method. The approach is principally the same, but rather than constructing an entire lattice in data-space, which is considered as the collection of potential centroids, Chiu only considers the dataset $X$ itself. Therefore, the computational complexity of this method is much lower than in Mountain Clustering, and independent of the dimension of the problem. The number of steps of Subtractive Clustering is approximately proportional to the number of data-points $M$ rather than the number of lattice-points $I^{N}$. Moreover, instead of the Yager-Filev choice of an exponentially decaying potential-function, Chiu employs a Gaussian form. This leads to a faster and more robust algorithm. Another advantage of this method is that it eliminates the need to specify a lattice resolution, which normally is a compromise between desired accuracy and available computational power. However, the problem of tuning the iteration-parameters $\alpha, \beta$, and $\delta$ remains. In these parameters, too, the scale-problem returns in an other guise. The potentials for constructing c.q. destructing the mountain function have typical lengths $\alpha^{-1}$ and $\beta^{-1}$. In specifying these lengths a domain of scales is assumed for the set. Therefore, this approach too is a scale-space clustering approach.

The best results are obtained when the Mountain Clustering and Subtractive Clustering methods are combined with conventional clustering algorithms like FCM. In this combination, Mountain or Subtractive Clustering is used to estimate the number of clusters $K$ and their positions $\left\{\mathbf{m}_{k} \mid k=1 \ldots K\right\}$. This information is then used to initialise, for instance, a FCM-iteration. 


\subsection{Supervised Clustering with Artificial Neural Networks}

In this section we briefly explore the applicability of artificial neural networks for the automatic clustering and classification of scanprofiles and images of printing defects.

Artificial neural networks are networks of identical entities, called artificial neurones, linked by weighted connections. Neurones have a 'state' that is constantly updated by input signals arriving via the connections. The output of a neurone is generated by a thresholding function that acts on its current state. This output is transmitted through the out-going connections to other neurones. The strength of the transmitted signal thereby depends on both the strength of the generated output as on the weight of the connection. Numerous publications describe the application of neural networks to pattern recognition. In order to perform pattern recognition, neural networks are trained on large sets of labelled examples. During this training they build up an internal representation of the training set. This internal representation is an implicit clustering of the training set. The process of pattern recognition itself then, is an act of classification of the input-signal with respect to this implicit clustering.

In this section we study the potential of neural networks to discriminate between several typical signals stemming from the image analysis of printing defects discussed in Chapter 2. Three different applications of these techniques are described below. The objective in all cases is to obtain a 'proof-of-principle' that this approach works sufficiently well within our context. Therefore, in the last two examples we explicitly compare the performance of the neural network to a linear technique.

\subsubsection{A Neural Network for matching scanprofiles with matchprofiles}

The first example that we discuss of a neural network for pattern recognition in printing defect analysis concerns the classification of the current scanprofile of an image [Westra, 1999c]. Scanprofiles and their relation to image analysis of prints are described in Section 2.3.

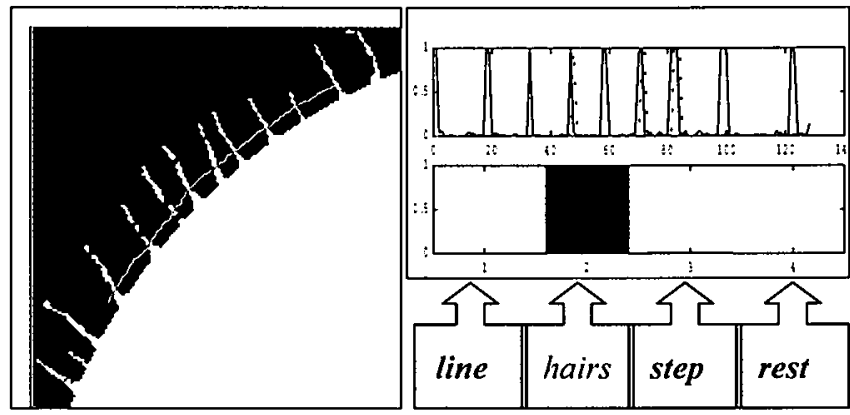

Fig. 4.3. Performance a neural network in classifying a scanprofile as either [1] 'line', [2] 'hairs', [3] 'step' or [4] 'rest'. In this example a scan on an image with defect 'hairs' is obtained (left). The resulting intensity-profile over the scan is obtained (left, above), and offered to $N N 1$. The histogram (left, below) shows the output of $N N 1$ as a probability distribution over the four defect types.

In order to somewhat restrict the problem, the set of printing defects was restricted to four elements: $\{(1)$ straight lines ; (2) step functions ; (3) oscillatory patterns ; (4) rest $\}$, where 'rest' stands for "not 1,2, or 3". A large set of empirical scanprofiles of these defects was available as training set of the neural network. The domains of the scanprofiles were scaled to the same length (64 steps), while the range - representing the intensity on the CCD-image - 
varied between zero and one. For the pattern recognition we constructed a simple 3-layer feed-forward artificial neural network, called NNI. The input-layer consisted of 16 nodes, each node representing the average value of the scanprofile over 1/16-th part of the horizontal domain of the scaled scanprofile - so the average over 4 successive points. The hidden layer consisted of 24 nodes, and the output layer contained 4 nodes, each node representing one of the four defined defect types. These four outputs can be interpreted as a not-normalised probability distribution over the set of known defects. The network was trained with the backpropagation-algorithm on the training set. The training set of $N N 1$ consisted of a set of original scanprofiles: 432 examples of defect (1) (also dubbed: 'line'), 210 examples of defect (2) (dubbed: 'hairs'), 334 examples of defect (3) (dubbed: 'step'), and 307 examples of defect (4) 'rest'. The cross-validation of the network was performed on an independent validation set containing a total of 276 separate examples. The highest output node is interpreted as the most likely defect, called the principle of 'winner takes all'. Table 4.1 shows the performance of the network on the validation set.

\begin{tabular}{|l|l|l|l|l|}
\hline defect & [1] line & [2] hairs & [3] step & [4] rest \\
\hline size of trainset & 432 & 210 & 334 & 307 \\
\hline recognised percentage & $97 \pm 3$ & $95 \pm 3$ & $95 \pm 3$ & $55 \pm 5$ \\
\hline
\end{tabular}

Table 4.1 Performance of $N N 1$, indicated is the percentage that the technique identifies correctly.

Indicated in this table is the percentage of the examples per defect type in the validationset that is classified correctly by this technique. The total size of the training set is 1283 and the number of adjustable weights in $N N I$ is 480 . Therefore, the quotient $n_{\text {train }} / n_{\text {weights }}$ is about 2.7 , which is sufficient to obtain a realistic performance ${ }^{7}$. The conclusion is that network NNI is successful in recognising $1 \mathrm{D}$-scanprofiles of real defects, but does not particularly well identify members of the rest-group.

\subsubsection{Defect Classification of Image Patches with a Neural Network}

Humans are visual experts equipped with excellent physiological sensors and a complex cognitive apparatus to process this information. In human vision images are processed in parallel as complexes of characteristic features. In most computer vision algorithms, on the other hand, information is processed sequentially as a fixed input-output system, for normally there are no interactions or feedback loops between the output and the input. This combination yields static, complex to maintain and develop systems, which cannot compete with human vision. The high performance of human vision inspired the design of a parallel (but sequentially implemented) method that utilises the entire 2D information of an image. The logical choice for such an approach is a neural network. Thus, a simple three-layer feedforward back-propagation network, called $N N 2$, was designed and trained to perform printingdefect classification [Westra,1999b]. In order to apply a neural network on this task, however, a number of typical neural network development problems must be addressed.

\section{i.The design problem}

The size of a entire CCD-image is typically $10^{5}-10^{6}$ pixels, and as such impracticably large as input-space for a neural network. Moreover, in order to be practically applicable the approach should be template-invariant ${ }^{8}$. Therefore, only small images are suitable as input. These images must represent characteristic parts of the entire image and must be large enough

\footnotetext{
${ }^{7}$ A quotient of at least 1 is desired. The problem is to obtain large representative training sets.

${ }^{8}$ For the definition of template-invariant see Chapter 2.
} 
to indicate the defect class, but small enough in order not to contain any specific information of the template. In the course of the 1D scanning approach discussed in Chapter 2, searchboxes, i.e. patches of the image, are automatically generated on locations of the largest dissension between image and its template. The searchbox-algorithm was tuned in such a way that it generated patches of $16 \times 16$ pixels. In order to compare the observed patch with the template, an identical square with the coordinates of the searchbox was placed on the template, and its contents as an image of $16 \times 16$ pixels formed the second set for the inputspace, see Figure 4.4. The input of the neural network therefore consisted of $2 \times 2^{4} \times 2^{4}=2^{9}$ inputs - for each pixel one input. Each pixel can have $2^{8}$ possible greyscale values. The best results were obtained with a hidden layer consisting of 20 neurones [Barron, 1984]. The output layer consisted of 5 neurones, each representing a specific printing defect for which the system would be trained.

\section{ii. The training problem}

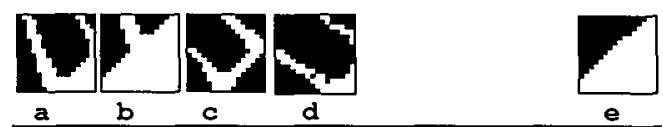

Fig. 4.4. Some examples of $16 \times 16$ searchboxes on identical spot in different test-images. (a-d): test-images with a defect called 'hairs', (e): searchbox on identical spot in the adequate template.

Due to the large size of the network a large training set was necessary. The training of the network was limited to 5 defect classes. A collection of $712 \mathrm{CCD}$-images containing those 5 defects was available. The searchbox-algorithm, discussed in Section 2.3, automatically generated relevant patches on the image of $16 \times 16$ pixels. In this way, each image generated large numbers of patches. This led to a training set of more than $25 \mathrm{~K}$ automatically generated examples. The test set contained another set of 76 experimentally obtained images. From each image large supplies of patches could be obtained using the searchbox-algorithm. While the design and realisation of this approach was extremely straightforward and fast, the training of the network, however, suffered from long training times and lack of convergence.

Because of the black-box character of neural networks, it was not clear whether backpropagation had guided the system to a local or a global optimum. The result could be improved by altering the design of the network or changing some of the many design and training parameters. This complexity constitutes another well-known disadvantage of neural nets. Though after some of such adjustments the performance of the system reached a satisfactory $80 \%$. Thereby, however, it was not able to surpass the performance of the linear scan approach discussed in Chapter 2, and later in Chapter 7, see Figure 4.6. 


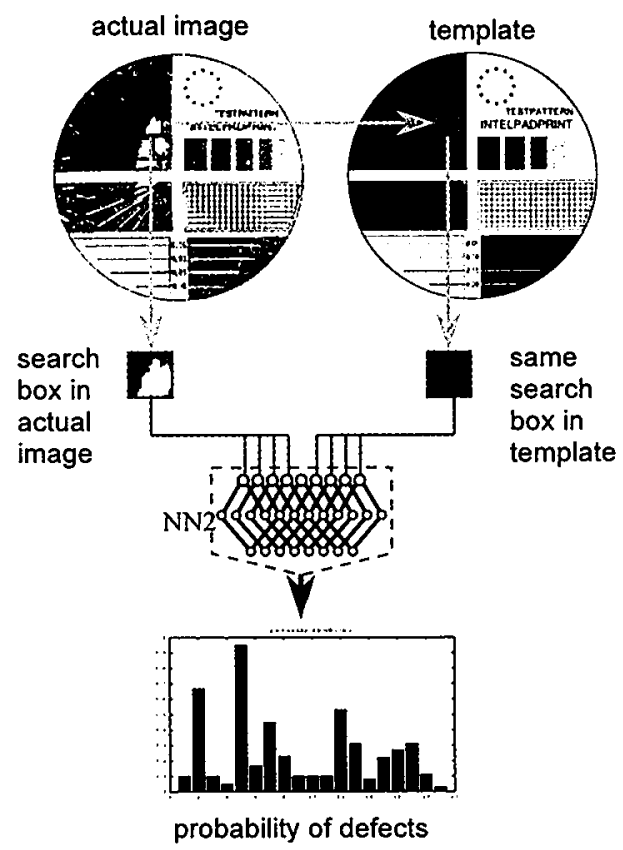

Fig. 4.5. A neural network is trained to compare a $16 \times 16$ pixel searchbox automatically generated on a relevant spot on a defect image with a searchbox on the template on the identical location.

\section{iii. Interpretation of the NN2 Output}

Rather than a probability distribution over the defect classes, the output of the neural network should be regarded as a fuzzy measure that represents the correspondence of input image to a specific defect class, or alternatively the membership of the output vector to the same defect class. This is a more genuine representation of the real situation where most defects are not sharply separated, but gradual transitions exist between certain defect-classes. At a higher level all defects can be clustered within six separate clusters sharing similar physical-chemical or mechanical causes.

\section{iv. Experimental Results and Conclusions}

Our objective was to study the applicability of network $N N 2$ to process the full 2D information of (part of) a CCD-image, and its ability to correctly identify the printing defect. A network was developed and trained on a limited set of 5 defects. The results indicate that for this set the method is indeed able to identify the defect class with an average performance of $80 \%$. The cluster to which a defect belongs, mentioned above, could be identified with an even higher performance.

A second objective was the comparison of this approach with conventional 1D scanning methods. For this reason the linear scanning approach was applied on the same validationset. The results are shown in the histogram below. 


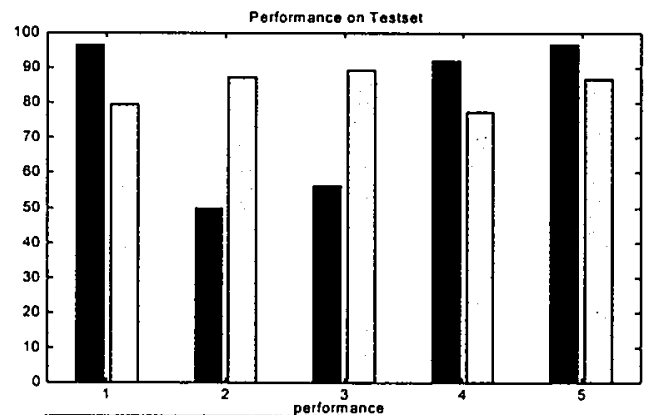

Fig. 4.6. Performance of the NN2 on an independent validationset. Horizontally the defect-nr and vertically the performance. Black $=1 \mathrm{D}$ scanning, grey $=N N 2$

Our results show that in general the performance is comparable, but the neural network is more dependent on the template than the 1D-scanning approach. On the other hand, NN2 is able to recognise certain defects that the ID-scanning approach cannot identify without employing extra tools, for instance 'air bubbles' (nr. 2 in fig. 4.6) and 'ink missing' (nr. 5 in fig. 4.6).

The design and realisation of NN2 was extremely straightforward and fast, compared with the design and development of a 1D-scanning approach, once more demonstrating the power of neural nets. On the other hand however, the training of the neural network clearly indicated one of the major drawbacks of neural nets; the long training times and the lack of convergence. A major problem was the collection of sufficient training examples. This could be achieved only by the automatic selection algorithm 'searchbox' and a large supply of appropriate CCD-images. Thus, it was possible to obtain a quotient $n_{\text {train }} / n_{\text {weights }}$ of about 2.5 , sufficient for an adequate training of the network.

The ability to adapt is shared by both approaches. The 1D-scanning method is based on statistical correlations between scans and defects, and as such can 'improve' its performance as more examples are offered to the system. This however, contrasts sharply with the ability of the NN2 to generalise and associate, as it is more 'trained' in time.

The conclusion is that the neural network offers additional information to the conventional ID scanning method. While both approaches are comparable, the neural network requires significant less development time, and has more attractive learning capabilities. Eventually, however, significantly more time was spend on collecting the test set than on its actual development time. Though larger training sets and longer training times should theoretically lead to a better performance, this approach was ultimately not implemented in the test system. The reason was the unpredictability of the neural network system for new input because of the black-box character. In the end the design was based on a perhaps potentially inferior, but better understood system with a predictable performance. 


\subsubsection{Neural Computation of the Defect from the Feature Vector}

The last 'proof-of-principle' concerns the construction of a hypothesis on the most likely

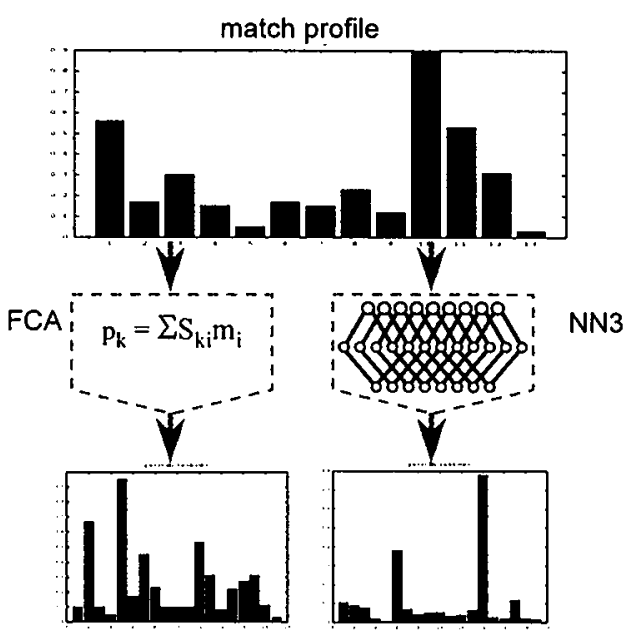

probability of defects

Fig. 4.7. Comparison of a neural network (NN3) with a linear approach in calculating the defectprobability distribution $\mathbf{p}$ from the feature-vector $\mathbf{m}$. The NN3 essentially performs a non-linear transformation on $\mathbf{m}$, while the alternative stems from a linear transformation $\mathrm{p}=S \mathrm{~m}$. Therefore, $N N 3$ should perform at least as good as FCA. defect based on the evidence in the form of a set of features extracted from the images. The hypothesis is given as a probability distribution over the defects. The most likely defect can be derived from a linear approach, where first a feature-vector $\mathbf{m}$ is extracted from the image. Second, the defect probability distribution is regarded as a vector $\mathbf{p}$. In a linear approximation $\mathbf{p}$ is calculated as a direct product of the featurevector $\mathbf{m}$ with a empirically calculated matrix $S$, called the Fuzzy Correlation Matrix (discussed later in Chapter 7 in the context of a real-world application), see Figure 4.7 below. The matrix $S$ itself can be estimated from a sufficiently large set of pairs $(\mathbf{m}, \mathbf{p})$. As an experiment a three-layer, feed-forward, back-propagation network $N N 3$ was trained on a set of 308 examples. For this experiment the size of the matchvector was restricted to 5 types: \{equal, too high, too low, step, oscillatory\}. Likewise 5 defect classes were considered: \{hairs, recess, double print, smearing, rest $\}$. The hidden layer consisted of 10 nodes. The quotient $\mathrm{n}_{\text {train }} / \mathrm{n}_{\text {weights }}$ therefore was about 3.1 , which is sufficient for a real evaluation of its performance. Not surprisingly, network $N N 3$ outperformed the linear matrix computation, as the latter can be considered as a first order approximation. The advantage of the original matrix-approach is that it is deterministic, while the $N N 3$ approach suffers from the well-established 'black-box' character inherent to neural networks, and the result may be influenced by over-training at the one hand or stuck in a local optimum on the other. Moreover, a realistic network would consist of much more feature-types as input, proportionally more hidden layer nodes, and outputs representing all defect types. This would mean a network with thousands of weights and therefore many thousands suitable training examples, creating a huge task for the data acquisition. Combined with the black-box character of neural nets, this would sufficiently favour the original linear matrix multiplication as perhaps inferior but reliable and deterministic tool.

\begin{tabular}{|l|l|l|l|l|l|}
\hline Technique & {$[1]$ hairs } & [2] recess & \multicolumn{1}{|c|}{ [3] double print } & [4 smearing & [5] rest \\
\hline matrix computation & $56 \pm 10$ & $73 \pm 6$ & $65 \pm 5$ & $48 \pm 7$ & $73 \pm 7$ \\
\hline NN3 & $76 \pm 3$ & $86 \pm 3$ & $81 \pm 3$ & $62 \pm 5$ & $82 \pm 5$ \\
\hline
\end{tabular}

Table 4.2. Comparison of the performance of the $F C A$-algorithm with $N N 3$, indicated as the percentage that the technique identifies correctly. 


\subsubsection{Conclusions on the Application of Artificial Neural Networks}

In general, neural networks have the benefit of fast design and quick implementation. The price paid for this advantage is long training times and the necessity of huge sets of examples, and problems concerning the design of the network topology and the setting of the (many) parameters. If the latter problems can be overcome neural networks will in general have a good performance. Another benefit of neural networks is that they support learning capabilities of the entire system: Neural networks have the ability to adapt to changing environments; they can 'learn' the relation between input and output, even if this relation changes in time. An important problem associated to learning systems, however, is how to maintain consistency in the training set.

A major disadvantage in our context is that they can not be used interactively. The entire input vector must be known before the network can be employed. In Chapter 5 we will concentrate on a approach in which only a part of the inputvector is required. This part, however, differs from case to case, and is therefore constructed during the sensing of the object. 


\subsection{Partitional Clustering with Fuzzy Class-Membership Functions}

\subsubsection{Clustering by Minimising Energy Functionals of Partition Matrices}

First we discuss 'fuzzy' clustering of defect classes. Consider a production process where the resulting products belong to a number of discrete defect classes, including sufficient quality. Let $X$ represent a sufficient large collection of $M$ observations of various such defect classes. Let each observation consist of $N$ independent measurements $x_{m}=\left(x_{1}, . ., x_{N}\right)^{\top}$. An observation $\mathbf{x}_{m}$ can thus be considered as one point in a $N$-dimensional parameter space. Here, we define clustering as the task of assigning values to a fuzzy matrix $u_{m k} \in[0,1]$, indicating the degree to which measurement $\mathbf{x}_{m}$ belongs to a particular member $k$ of a set of $K$ classes. Using $u_{m k}$ as weights, a class- prototype $\mathbf{v}_{k}$ is defined as the centre-of-mass of class $k$ :

$$
\mathbf{v}_{k}=\sum_{m=1}^{M} u_{m k}^{\alpha} \mathbf{x}_{m} / \sum_{m=1}^{M} u_{m k}^{\alpha}
$$

with an exponent $\alpha$, explained hereafter. For $\alpha=1, u_{m k} \in\{0,1\}$, we obtain a 'crisp' partitioning. In Fuzzy C-means (FCM) [Dunn,1973], [Bezdek, 1973] the 'energy' of a clustering is expressed in terms of the cumulative weighted distance to the class-prototypes $\mathbf{v}_{k}$ :

$$
E_{\alpha}(X, U, V)=\sum_{k=1}^{K} \sum_{m=1}^{M} u_{m k}^{\alpha} d_{m k}
$$

Here, $d_{m k}$ denotes the distance of measurement $\mathbf{x}_{m}$ to the class-prototype $\mathbf{v}_{k}$ in the overall metric in parameter space:

$$
d_{m k}^{2}=\left(\mathbf{x}_{m}-\mathbf{v}_{k}\right)^{\top} G\left(\mathbf{x}_{m}-\mathbf{v}_{k}\right)
$$

$G$ is a symmetric and semi-positive definite matrix, called the metric tensor. Now, let matrix $u_{m k}$ be based on a continuous function ' $\varphi$ ', depending solely on the relative position $\mathbf{x}_{m}$ in cluster $k$ :

$$
u_{m k}=\varphi\left(\mathbf{x}_{m}-\mathbf{v}_{k}\right)
$$

In many practical applications the probability of a defect is relatively low. Therefore, the probability of two defects occurring simultaneously can be neglected. Following this assumption, we can add an additional constraint:

$$
\forall \sum_{m=1}^{K} u_{m k}=1
$$

This constraint merely reflects the observed absence of multiple concurring defects, it does not imply that we have switched to a probabilistic framework, or that the defect classes are disjunct. Now consider the problem of finding the function $\varphi$ that minimises the energy of a given clustering. Using $M$ Lagrange multipliers $\lambda_{m}$ an optimal solution is calculated by minimising:

$$
E_{\alpha}(K, M, X)-\sum_{m=1}^{M} \lambda_{m}\left(1-\sum_{k=1}^{K} u_{m k}\right)
$$

for the $M(K+I)$ variables $\left\{\left(\lambda_{m}, u_{m k}\right) \mid m=1 . . M, k=1 . . K\right\}$. This leads to:

$$
\varphi(d)=d^{\beta}
$$

in the metric defined above, with exponent $\beta=1 /(1-\alpha)<0$. This implies that: $\alpha>1$. Expression [4.6] shows that $\alpha$ can be interpreted as the 'width' of the distribution. For $\alpha \downarrow$ l function $\varphi$ becomes a Dirac-delta function, and we again obtain the hard partitioning. Thus, in 
FCM the generating function $\varphi$ is a monotonously decreasing function in $d$. The associated partition follows from: $u_{m k}=\varphi\left(d_{m k}\right)$. This relation is a direct consequence of the choice for the FCM functional in [4.1]. Other types of functionals lead to different 'optimal' membership functions $\varphi$. The FCM definition of defect-membership function $\varphi$ in [4.6] does not take into account the specific properties of cluster $k$, nor does it reflect the specific distribution of observations within that cluster. In addition, the FCM-functional is only devised to minimise the cumulative distance to the associated prototypes. It does not tend to minimise the iteration length, the number of defect classes, or any other appropriate criterion. Instead of relying on one general tensor $G$ to represent the entire metric on parameter space, we focus on representations that can better represent the approximate shape, and the observed distribution of measurements within each defect class. Therefore, we study a generalisation of the FCMenergy of a clustering:

$$
E_{\alpha}(K, M, X)=-\sum_{k=1}^{K} \sum_{m=1}^{M} u_{m k}^{\alpha} \varphi_{k}\left(d_{m k}\right)
$$

where we require only that $\varphi_{k}$ is a monotonously increasing function. More symmetry is introduced if the dependence of $\varphi_{k}$ on $k$ can be omitted by one unique continuous function $\varphi$, and an additional class-specific parameter set $\xi_{k}$, like: $\varphi_{k}\left(\mathbf{x}_{m}\right)=\varphi\left(\mathbf{x}_{m}, \xi_{k}\right)$. In the general case that $\varphi$ is non-linear, $\varphi\left(d_{m k}\right)$ does not represent a metric on $X$, for the condition of triangular inequality does not apply. This means that, in general, the line connecting an arbitrary point with its class-prototype is curved. Again, we can write down the Lagrange equations with the additional normalisation constraint on $u_{m k}$. This optimisation leads to an optimal function for this generalised functional:

$$
u(d)=\varphi(d)^{\beta}
$$

\subsubsection{Gaussian Mixture Densities as Fuzzy Class Membership Functions}

Suppose furthermore, that all defect classes $\left\{\omega_{1}, \omega_{2}, . ., \omega_{K}\right\}$ in our context can be well approximated by multivariate normal distributions. Let, therefore, the class-membership $u$ be represented by:

$$
\varphi(\mathbf{x} \mid C)=\operatorname{det}(C)^{-1 / 2} \exp \left(-1 / 2 \mathbf{x}^{\top} C^{-1} \mathbf{x}\right)
$$

Here, $C$ is a positive semidefinite matrix. A constant term is omitted because of later renormalisation. This distribution leads to the following partition:

$$
u_{m k}=\mu_{k} \varphi\left(\mathbf{x}_{m}-\mathbf{v}_{k} \mid C_{k}\right)
$$

where $C_{k}$ represents the covariance matrix, and $\mathbf{v}_{k}$ the prototype for class $k . \mu_{k}$ is a coefficient that ensures the normalisation of [4.10]. This means that we consider our problem as a Gaussian mixture, see section 4.3.g. In that light, the coefficients $\mu_{k}$ represent the a priory probability of defect class $\omega_{k}$. and $\varphi\left(\mathbf{x} \mid \omega_{k}\right)$ represents the known class-conditional probability distribution of sampling a measurement $\mathbf{x}$, given the occurrence of defect class $\omega_{k}$. Under our assumption of a Gaussian mixture with mean $v_{k}$ and covariance $C_{k}$, we can write the entire probability distribution $P$ for sampling an observation $\mathbf{x}$ as:

$$
P(\mathbf{x})=\sum_{k=1}^{K} \mu_{k} \varphi\left(\mathbf{x}-\mathbf{v}_{k} \mid C_{k}\right)
$$

On the other hand, for a measurement $\mathbf{x}$, the most likely defect class index $k^{*}$ can be inferred with Bayes' theorem as [Hwang et al.,2000]: 
$k^{*}=\arg \max _{k} \varphi\left(\omega_{k} \mid \mathbf{x}\right)=\arg \max _{k}\left[\left(\mathbf{x}-\mathbf{v}_{k}\right)^{\mathrm{T}} C_{k}^{-1}\left(\mathbf{x}-\mathbf{v}_{k}\right)+\log \left((2 \pi)^{N / 2}\left|C_{k}\right|^{1 / 2}\right)-\log \mu_{k}\right]$

Similar to the class-prototype of equation [4.9], the class-covariance matrix $C_{k}$ can be defined as the weighted covariance for class $k$ :

$$
C_{k}=\sum_{m=1}^{M} u_{m k}^{\alpha}\left(\mathbf{x}_{m}-\mathbf{v}_{k}\right)\left(\mathbf{x}_{m}-\mathbf{v}_{k}\right)^{\top} / \sum_{m=1}^{M} u_{m k}^{\alpha}
$$

This representation for $\varphi$ decreases much faster than the FCM-membership $d^{\beta}$. Consequently, it relates to a energy functional that decreases much faster than the FCM-functional [4.2] with increasing distance to the class-prototype. This leads to equivalent prototypes as FCM, However, the steeper rate of descent of the energy-functional implies a larger gradient of $\varphi$, resulting in a larger step-size of the prototypes in the direction of their optimal position during the iteration. Because of this larger step-size, the iteration length induced by this functional is expected to be considerable shorter than in FCM, while the position of associated prototypes is hardly affected. The corresponding energy functional for this distribution $\varphi$ is:

$$
E_{\alpha}(X, U, V)=\sum_{k=1}^{K} \sum_{m=1}^{M} u_{m k}^{\alpha} \operatorname{det}\left(C_{k}\right)^{\gamma} \exp \left(\gamma d_{m k}^{2}\right)
$$

with $\gamma=(1-\alpha) / 2$.

\subsubsection{The Fuzzy Multivariate Clustering Algorithm}

Now we can define an alternative algorithm for FCM which we call Fuzzy multivariate clustering (FMV), [Westra,2000b].

Initialisation: to each measurement $m$ a random class $c$ is assigned, and $u_{m k}$ is set to one for class $c$, and zero for all other classes. These values of $u_{m k}$ are used to calculate the prototypes and covariance-matrices using [4.1] and [4.11] respectively.

Iteration Cycle: iteratively, new prototypes and covariance matrices are calculated with [4.1] and [4.11], and subsequently the new membership functions are calculated by using [4.9].

Termination: if in cycle $t$ the membership functions converge within a given precision $\epsilon$ the iteration terminates; $\max _{m k}\left\|u_{m k}(\mathrm{t})-u_{m k}(\mathrm{t}+1)\right\|<\epsilon$.

Each of these steps is open for further optimisation. For instance, Peña et al. [Peña et al.,1999] describe an empirical comparison of four different initialisation methods for the C-means algorithm.

A useful interpretation for this algorithm is to understand the covariance matrix $C_{k}$ as an approximate metric tensor on cluster $k$, and conceive the iteration as an evolution of $C_{k}$ to the 'real' metric $A_{k}$ on cluster $k$. This interpretation was introduced independently by Gustafson, Kessel, and Bock [Gustafson,Kessel,1979], [Bock, 1979] in the Adaptive Norm Algorithm, but using the FCM-membership function rather than the multivariate normal form. They also noted the correspondence between the standard FCM-membership function $d^{\beta}$ and a probabilistic maximum likelihood interpretation. 


\subsubsection{Performance of Fuzzy Multivariate Clustering}

The FMV clustering approach was tested on several sets of both artificial data, and real data stemming from selected features in CCD-images of printing defects. Figure 4.9 shows the performance of the FMV relative to the performance of FCM and the number of clusters assumed in the data. The performance is expressed in: $i$. the value of the FCM-energy of expression [4.2] of the final clustering, and ii. the number of steps in the iteration. This figure shows that FMV is faster than FCM in the sense of the number of iteration steps, and that the resulting energy of the clustering is lower. Indicated in the figure is the number $K$ of clusters in the data set. The figure shows that on average higher $K$ tend to better results for FMV. Irregularities in the distribution of $K$ over the figure are caused by the influence of the specific topological distribution of the clusters in the data set.

Figure 4.8 shows typical trajectories of the prototypes during an iteration. Left a typical FCM is shown, and right a typical FMV. This figure also shows that both result in equivalent prototypes, but that FMV proceeds faster to the final result.
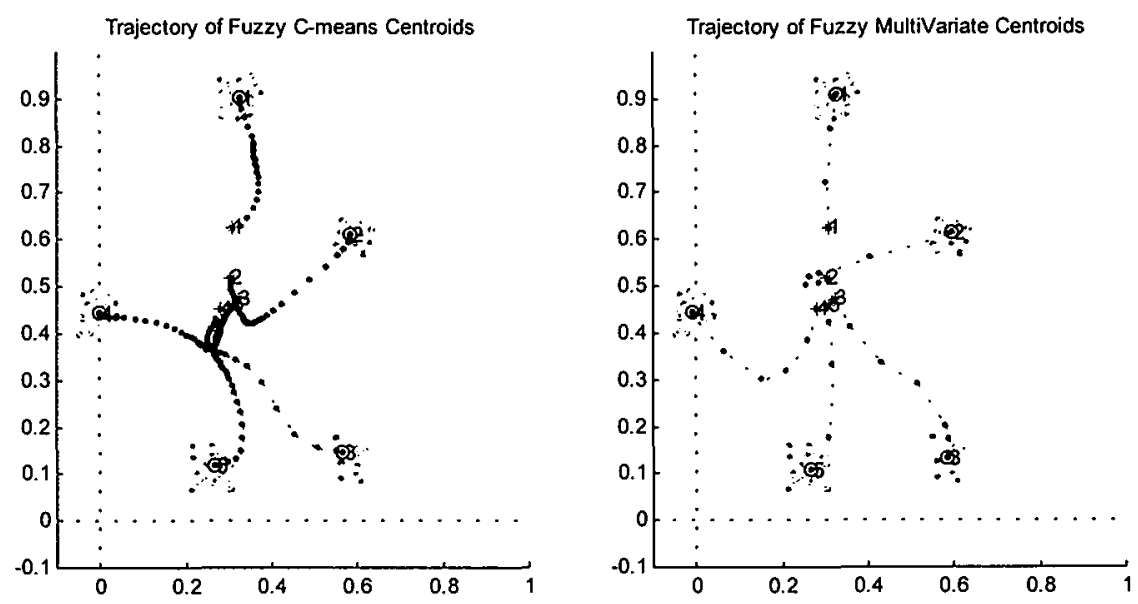

Fig. 4.8. Typical FMV and FCM sessions for the same data set, showing the smaller number of iterations and the comparability of the end result for FMV. Left: trajectories of the FCMprototypes, right: likewise for the FMV-prototypes 


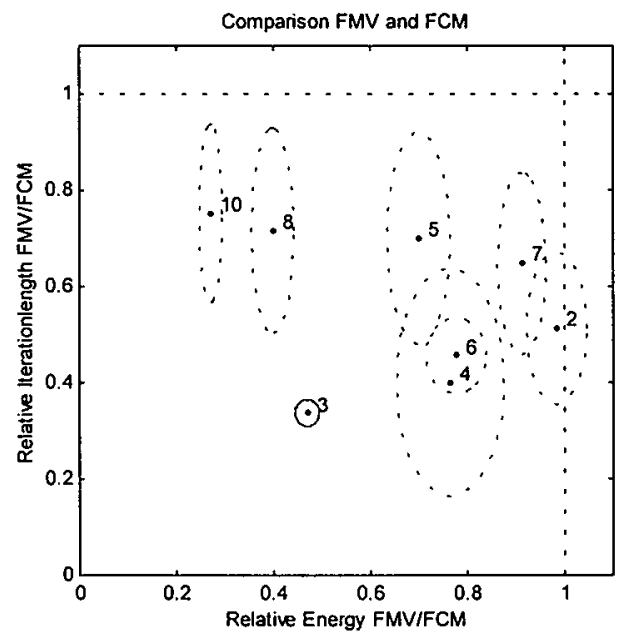

Fig. 4.9. Comparison of FMV and FCM. Numbers indicate the number of clusters $K$ present in the data. Irregularities in the distributions are caused by the specific topology of the set.

\subsection{Exploring Data-Sets with Automatic Clustering Techniques}

As described in the literature survey in section 4.3, Automatic Clustering is the unsupervised partitioning of parameter-space, without any prior knowledge on the number, size or structure of clusters available in the empirical data. This is particularly relevant for practical situations, where nothing is known of the structure aggregated experimental data. Neither its geometrical properties, nor the number of clusters it contains. However, most unsupervised clustering algorithms, like FCM and the Kohonen's Self-Organising feature Map, explicitly force the user to provide some information as input parameter. Therefore, a number of approaches have been developed, aimed to relieve the user of the burden to provide any ad-hoc assumptions on the data. From the survey in section 4.3 it is clear that in the last decade considerable progress has been achieved in this field.

In this section we describe original work in this area. First, we describe a conventional automatic clustering technique, developed for clustering power-system states in terms of their net-safety [Westra et al.,1997a]. Second, we describe an cluster tree expansion method, using the FMV algorithm described in section 4.5.

\subsubsection{Survey of Energy as function of Number of Clusters}

In [Westra et al.,1997a] we described an approach for determining the number of clusters in a large empirical data-set, containing $M$ observations. This research was performed for the Dutch Electricity Generating Boards (SEP and KEMA). The objective was to cluster netsecurity states of the Dutch power system. This method consists of three components:

1. exhaustive search in the number of clusters $K$, ranging in $\{1,2, . ., \sqrt{ } M\}$,

2. the FCM-clustering algorithm,

3. the cluster-energy as Cluster Validity Criterion. 
Principally, this method explores the cluster-energy as function of the number of clusters $K$ used in the clustering algorithm. This energy drops from a maximum value $E_{\max }$ for $K=1$ to zero for $K=M$, the number of points in the data set. More relevant, however, is the change of the energy when $K$ is increased; $\Delta E(K)=E(K)-E(K-1)$. If there is a distinct number of classes $K^{*}$ present in the data, like in the figure below with $K^{*}=8$, the energy-change $\Delta E$ decreases until $K=K^{*}$, after which it drops significantly to values near zero. Disadvantages of this approach are: 1 . the search is restricted to definition of a pre-set domain of $K$ which limits the scope of its usability, 2. the extensive number of runs needed to reduce the statistical deviations in $\Delta E$. An example of this method is depicted in figure 4.10. A similar approach is applied by [Gath,Geva, 1989] and [Zahid et al.,1999].
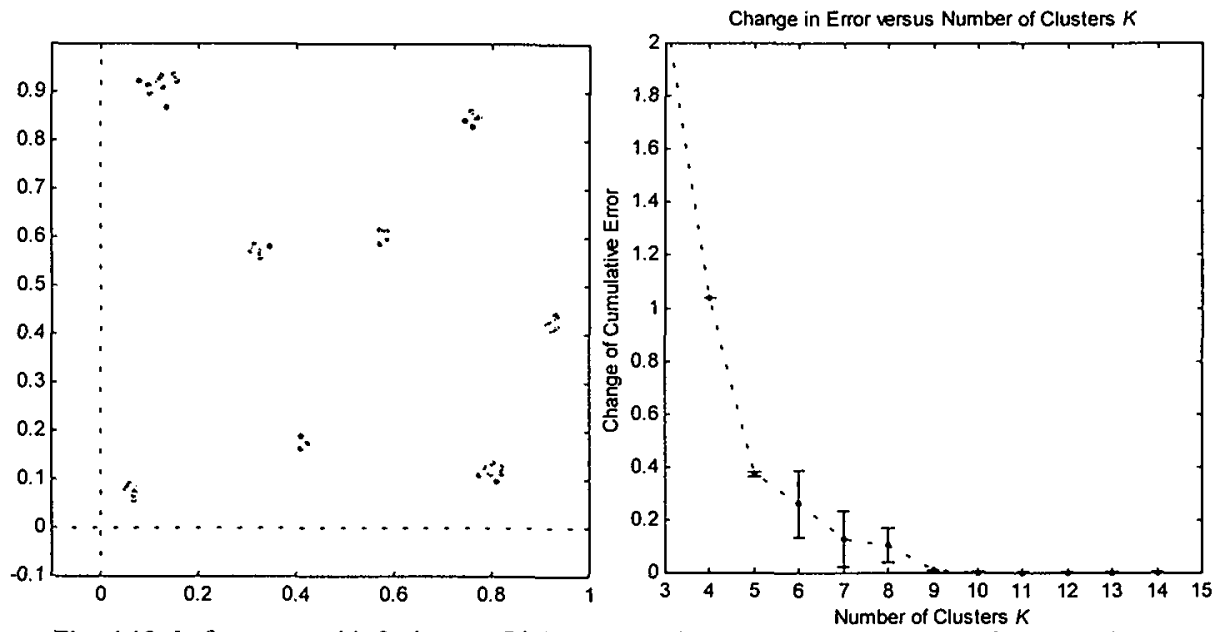

Fig. 4.10. Left: testset with 8 clusters. Right: Change in FCM-Cluster energy as function of the number of cluster $K$ used in the clustering algorithm on this test set, based on 100 different passes. 


\subsubsection{Iterative Cluster Tree Expansion}

In this section we describe a new cluster tree expansion method based on an iterated sequence of FMV clustering. Starting point of this approach is a set of cluster descriptors, including some Cluster Validity Criteria, that quantify relevant properties of clusters. Examples are average cluster separation, size, and density. These descriptors are used for two possible operations. Clusters that are found to be too close with respect to a statistically significant separation are merged. On the other hand, clusters that are found to be composed of multiple sub-unites are split. The criteria for such fusion or decomposition are expressed in terms of these descriptors. An additional constraint for these operations is an actual decrease in the cumulative cluster energy. Therefore, the progress of this process can be measured in the continuous decrease of cluster energy. The process terminates if no new candidates for fusion or decomposition are found. Finally, we evaluate and compare the performance of this algorithm on a number of test-cases.

\subsubsection{Cluster Decomposition}

If the 'number of clusters' $K$ in the clustering algorithm is set lower than the actual number of clusters $K^{*}$ present in the data, some of the resulting clusters are composed of multiple groups. In cluster decomposition the clusters are examined on the presence such sub-units. Subsequently, the sub-units are identified. This analysis is based on a number of descriptors that quantify certain relevant cluster-properties. In this section we first define a number of useful quantifiers for clusters. Next, we describe a simple model to discriminate composite from single clusters in terms of these quantifiers. Using the descriptor-representation, we construct a cluster analysis algorithm for cluster decomposition.

\section{Significant Cluster Descriptors}

Ruspini, Dunn and others [Ruspini,1969], [Dunn,1973] have proposed a number of useful parameters for describing cluster characteristics. Geva et al. [Geva et al.,2000] present a comparison of cluster validity criteria for a Gaussian mixture, which are specifically appropriate for FMV. Applied to our context we define the following cluster description parameters.

1. cluster centroid: $\mathbf{v}_{k}=\sum_{m=1}^{M} u_{m k}^{\alpha} \mathbf{x}_{m} / \sum_{m=1}^{M} u_{m k}^{\alpha}$

2. cluster radius: $R_{k}=\sum_{m} u_{m k}^{\alpha}\left\|\mathbf{x}_{m}-\mathbf{v}_{k}\right\| / \sum_{m} u_{m k}^{\alpha}$

3. intra-cluster distance: $D_{k}=\sum_{i<j} u_{i k}^{\alpha} u_{j k}^{\alpha}\left\|\mathbf{x}_{i}-\mathbf{x}_{j}\right\| / \sum_{i<j} u_{i k}^{\alpha} u_{j k}^{\alpha}$

4. inter-cluster distance: $d_{i j}=\left\|\mathbf{v}_{i}-\mathbf{v}_{j}\right\|$

5. cluster energy: $E_{k}=-\sum_{m} u_{m k}^{\alpha} \phi_{k}\left(\mathbf{x}_{m}\right) / \sum_{m} u_{m k}^{\alpha}$, with $\phi_{k}$ the class $k$ memberships function.

Moreover, we consider the averages of these descriptors: $d=\left\langle d_{i j}\right\rangle, D=\left\langle D_{k}\right\rangle, R=\left\langle R_{k}\right\rangle$, and similarly their standard deviations, like $\sigma_{R}$. The cluster energy depends on the choice for the class-potential $\phi_{k}$ acting between on measurement $\mathbf{x}_{m}$. The most straightforward choice is: $\phi_{k}(\mathbf{x})=\left\|\mathbf{x}-\mathbf{v}_{k}\right\|$. With this choice, the energy equals in size the cluster radius: $E_{k}=-R_{k}$. Notice that for sake of simplicity we use the FCM-definition of energy. 


\section{A simple model for discriminating between mono-and bi-clusters}

Consider a collection of two spheroidal sub-clusters $A_{1}$ and $A_{2}$ in an $N$-dimensional space, separated by a large distance $L$. Let, for all $i=1,2$, let set $A_{i}$ contain $N_{i}$ points, with cluster radius $R_{i}$, and intra-cluster distance $D_{i}$. Let the centroids of the sub-clusters be situated on the $x_{1}$-axis at positions: $x_{1}\left(A_{1}\right)=-L / 2$, and: $x_{1}\left(A_{2}\right)=+L / 2$, and all other coordinates are zero. Now, consider the following interpretations of the collection $A=A_{1} \cup A_{2}$.

a. collection $A$ is considered as two clusters, i.e. $A$ is a 'bi-cluster';

b. collection $A$ is considered as one cluster, i.e. $A$ is a 'mono-cluster'.

For each of these interpretations we can write down the values of the resulting descriptors, in the approximation: $L \gg R$. This means that collection $A$ is practically a straight line. The resulting descriptor values are listed in Table 4.3 below.

As a third interpretation, consider a homogeneous mono-cluster $B$, with $\left(N_{1}+N_{2}\right)$ points. Suppose that $B$ can be approximated by a straight line of length $L$, running from: $x=-L / 2$ to: $x=L / 2$. The descriptor-values for cluster $B$ are listed in the same table, where: $n_{i}=N_{i} /\left(N_{1}+N_{2}\right)$.

\begin{tabular}{|c|c|c|c|}
\hline Descriptor & $\begin{array}{c}\text { scenario }(a) \\
A \text { is bi-cluster }\end{array}$ & $\begin{array}{c}\text { scenario (b) } \\
A \text { is 'false' mono-cluster }\end{array}$ & $\begin{array}{l}\text { scenario }(c) \\
\text { cluster } B\end{array}$ \\
\hline$x_{1}$-coordinate of prototype: $\mathrm{v}$ & $\{-L / 2,+L / 2\}$ & $\left(n_{2}-n_{1}\right) L / 2$ & 0 \\
\hline mean cluster size $R$ & $\left\{R_{1}, R_{2}\right\}$ & $2 n_{1} n_{2} L$ & $L / 4$ \\
\hline variation in cluster size: $\sigma_{R}{ }^{2}$ & $\left\{\sigma_{R 1}^{2}, \sigma_{R 2}^{2}\right\}$ & $L^{2} n_{1} n_{2}$ & $L^{2} / 12$ \\
\hline mean intra-cluster distance $D$ & $\left\{D_{1}, D_{2}\right\}$ & $n_{1}^{2} D_{1}+2 n_{1} n_{2} L+n_{2}^{2} D_{2}$ & $L / 3$ \\
\hline variation in $D: \sigma_{D}^{2}$ & $\left\{\sigma_{D 1^{2}}{ }^{2}, \sigma_{D 2}{ }^{2}\right\}$ & $\begin{array}{c}n_{1} n_{2}\left(D_{1}+D_{2}\right)^{2}-4 n_{1} n_{2} L\left(n_{1} D_{1}+n_{2} D_{2}\right)+ \\
2 n_{1} n_{2} L^{2}\left(1-2 n_{1} n_{2}\right)\end{array}$ & $L^{2 / 18}$ \\
\hline mean inter-cluster distance $d$ & $L$ & - & - \\
\hline FCM-cluster-energy: $E$ & $\left\{E_{1}, E_{2}\right\}$ & $-2 n_{1} n_{2} L$ & $-L / 4$ \\
\hline
\end{tabular}

Table 4.3

Now, suppose that our clustering algorithm has generated a cluster $C$, but we are not sure whether this cluster is single or composed of two sub-clusters. This means that we have to decide between scenario (b): $C$ is composed of two sub-clusters, or scenario $(c): C$ is a homogeneous mono-cluster. In order to discriminate between these two possibilities, we can now employ the results of above table. After calculation of the cluster-descriptors, we can look for some significant quotients and compare these with the table. For instance, the quotient $\sigma_{R}{ }^{2} / R^{2}$ has a value $4 / 3$ for the pure mono-clusters of scenario (c), but only $1 / 4$ for the misinterpreted mono-cluster of scenario $(b)$. Similarly, other combinations can be employed.

\subsubsection{Iterative FMV Decomposition}

The simple model, described above, for the interpretation of clusters as mono- and bi-clusters based on their descriptors $\left\{R, \sigma_{R}, D, \sigma_{D} d, E\right\}$, can be used to design an iterative algorithm for cluster analysis. A central place in this algorithm plays a criterion $H\left(R, \sigma_{R}, D, \sigma_{D} d, E\right)$ constructed from these descriptors. If this criterion is below a certain threshold $h$, the most likely situation is that the cluster is composed of multiple sub-units, and the cluster is decomposed. If this condition is not met, the next cluster is analysed using this same procedure. The decomposition of the cluster is performed with an automatic not-labelled 
clustering algorithm, in our case Fuzzy Multivariate Clustering, with two classes - i.e. Number of Classes $=2$. The initial positions of the two new prototypes are random points near the original prototype. The result of the clustering is a new pair of prototypes that replaces the original prototype, meaning that the prototype is, so to say, split in two. As a final test the cluster-energy of the new constellation is compared with the old energy. Here, we pose the condition on the resulting pair of sub-clusters that the cumulative energy of the two subclusters lies well below the original energy of the composite cluster. If this condition is not met, the decomposition is as yet rejected, and the next cluster analysed. If this test is passed, the old cluster with all its attributes - like prototype, covariance-matrix, and membershipfunctions $u-$ is replaced by the new pair with all their attributes. The result of the iteration at this step is a new collection of prototypes and covariance-matrices, while the total number of prototypes has increased with one. The approach for cluster decomposition is sketched in Figure 4.13.

\section{Construction of Criterion $H$}

The criterion $H\left(R, \sigma_{R}, D, \sigma_{D} d, E\right)$ and the threshold $h$ can be constructed from considerations on the interpretation-model as mono- and bi-clusters, discussed in the previous section. Certainly, some conclusions can be drawn from the model, like the quotient $\sigma_{R}{ }^{2 / R^{2}}$ discussed above. The exact form of the criterion involves the dimension $N$ of the parameter space.

Instead of this analytic approach, the criterion $H$ and threshold $h$ can be estimated from sufficiently large collection of empirical data of mono- and poly-clusters in parameter space. The advantage of this approach is that instead of the oversimplified model of the previous section, we can now use all possible configurations and geometries of clusters. In this method, first, the relevant descriptor-parameters $\left(R, \sigma_{R}, D, \sigma_{D}\right)$ of the data set are calculated. Next, a labelled clustering technique - here $F M V$ - is applied on the resulting collection in the descriptor-space to find a bi-partition in of the set in mono- versus poly-clusters. In order to obtain a scale-invariant representation, we divide by the cluster-size $R$. So, define: $\xi=\left(\sigma_{R} / R\right.$, $\left.D / R, \sigma_{D} / R\right)$. For an example of 2-dimensional clusters $(N=2)$, we find the following centroids for mono- and poly-clusters in the 3 parameters $\xi$.

\begin{tabular}{|c|c|c|c|}
\hline prototypes & $\xi_{1}=\sigma_{\mathbf{R}} / \mathbf{R}$ & $\xi_{2}=\mathbf{D} / \mathbf{R}$ & $\xi_{3}=\sigma_{\mathbf{D}} / \mathbf{R}$ \\
\hline $\begin{array}{c}\text { theoretical prediction for } \\
\text { linear homogeneous mono-clusters }\end{array}$ & $\sqrt{\frac{4}{3}} \approx 1.15$ & $\frac{4}{3} \approx 1.33$ & $\sqrt{\frac{8}{9}} \approx 0.94$ \\
\hline empirical mono-clusters: $\mathbf{m}_{M}$ & $0.80 \pm 0.30$ & $1.50 \pm 0.09$ & $1.07 \pm 0.33$ \\
\hline empirical poly-clusters: $\mathrm{m}_{P}$ & $0.38 \pm 0.16$ & $1.15 \pm 0.18$ & $0.76 \pm 0.16$ \\
\hline
\end{tabular}

Table 4.4

Using this representation, the decision criterion for a cluster $C$ with parameters $\xi$ becomes:

$C$ is a mono-cluster if: $\left\|\boldsymbol{\xi}-\mathbf{m}_{M}\right\| \leq\left\|\boldsymbol{\xi}-\mathbf{m}_{P}\right\| \Rightarrow 2\left(\mathbf{m}_{P}-\mathbf{m}_{M}\right) \cdot \boldsymbol{\xi} \leq\left\|\mathbf{m}_{P}\right\|^{2}-\left\|\mathbf{m}_{M}\right\|^{2}$

So, we find a criterion:

$$
H(\xi)=2\left(\mathrm{~m}_{P}-\mathrm{m}_{M}\right) \cdot \boldsymbol{\xi}=-0.8341 . \xi_{1}-0.6964 . \xi_{2}-0.6254 . \xi_{3}
$$

and associated threshold:

$$
h=\left\|\mathbf{m}_{P}\right\|^{2}-\left\|\mathbf{m}_{M}\right\|^{2}=-1.9886
$$

Thus, a cluster with parameters $\xi$ is a mono-cluster if:

$$
H(\xi) \leq h \Rightarrow 0.8341 . \xi_{1}+0.6964 . \xi_{2}+0.6254 . \xi_{3} \geq 1.9886 \text {. }
$$


This decision criterion defines a simple linear classifier for the identification of monoclusters. The performance of this classifier is reasonable. Averaged over a large test set we obtain the following results:

$\begin{array}{ll}\text { good solution: } & 82 \% \\ \text { false positives: } & 16 \% \\ \text { false negatives: } & 2 \%\end{array}$

By constructing more elaborate classifiers than this, a better performance of the cluster analysis can be expected. In Figure 4.11 the performance of our linear classifier is visualised for a case where it no longer can discern two from one cluster in a collection of data. From this amount of overlap the algorithm considers the collection as one single cluster.
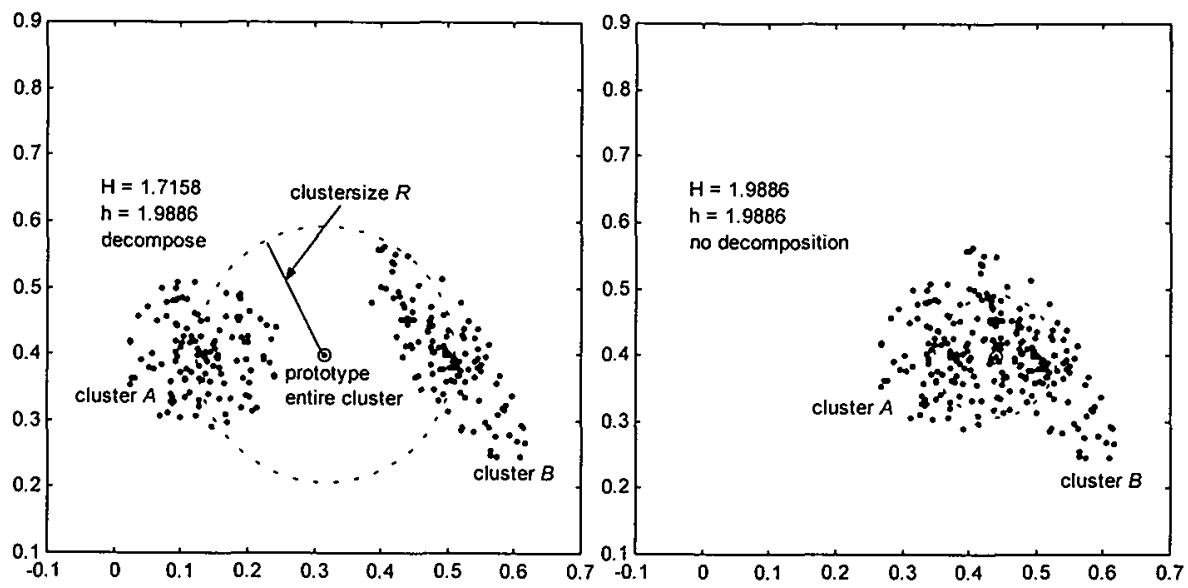

Fig. 4.11. (left): Two clusters $A$ and $B$. The criterion $H$ is calculated as if the entire set was one cluster. In this case $H$ falls below the threshold $h$, and the set is recognised as being composed.

(right): Cluster $A$ is shifted 0.245 units to the right. For the resulting cluster $A \cup B$, the criterion $H$ exactly equals the threshold $h$. Therefore, this situation represents the minimum degree of overlap for which the criterion can not distinguish the two sub-clusters.

\subsubsection{Iterative FMV Cluster Fusion}

Combining a number of clusters proceeds analogous to the decomposition of a single cluster in multiple fragments. Like in cluster decomposition, cluster fusion can be defined in terms of the cluster descriptors. In this case, a simple test is based on the inter-cluster separation of a pair of clusters. If the separation is less than statistically significant, the two clusters are merged into one cluster, and all the attributes of the new cluster are calculated. The criterion for fusion, therefore, is based on the distance between the prototypes $\left\{m_{\mathrm{A}}, \mathbf{m}_{\mathrm{B}}\right\}$ of the two clusters $A$ and $B$, and their cumulative effective width along the line joining the two prototypes. This is sketched in Figure 4.12. In this view, the shape of a cluster is approximated by its metric tensor $G$, which is the inverse of its covariance matrix; $G=C^{-1}$. This positive semi-definite matrix defines an ellipsoid in parameter-space. The effective size 
$\sigma_{\text {eff }}$ of these ellipsoids is defined by the intersection of the line through the prototypes and the ellipsoids ${ }^{9}$. This situation is depicted in Figure 4.12 below.

For that reason, the condition for fusion becomes:

$$
d_{\mathrm{AB}}<\sigma_{A, e f f}+\sigma_{B, e f f}
$$

If a certain fraction $\eta$ of cluster overlap is allowed, the right-hand side of the inequality may be reduced correspondingly; $d_{\mathrm{AB}}<(1-\eta)\left(\sigma_{A, \text { eff }}+\sigma_{B \text {, eff }}\right)$. In case of normal distributed clusters we can combine the standard deviations as: $\sigma_{e f f}{ }^{2}=\sigma_{A, e f f}+\sigma_{B \text {,eff }}{ }^{2}$. Combination of these conditions gives:

$$
d_{\mathrm{AB}}<(1-\eta) \cdot \sigma_{e f f}
$$

The Cluster Fusion Test therefore acts on all pairs of clusters. For each pair that passes the test, the number of classes $K$ is decreased by one, and a new cluster is created from the elements of the two clusters. Then, the new descriptors of the merged cluster, like prototype and covariance, are calculated. Finally, like in the case of class-decomposition, the clusterenergies of the old and the new assemblages is compared, and the new cluster must satisfy:

$$
E_{\text {new }}<E_{A}+E_{B}
$$

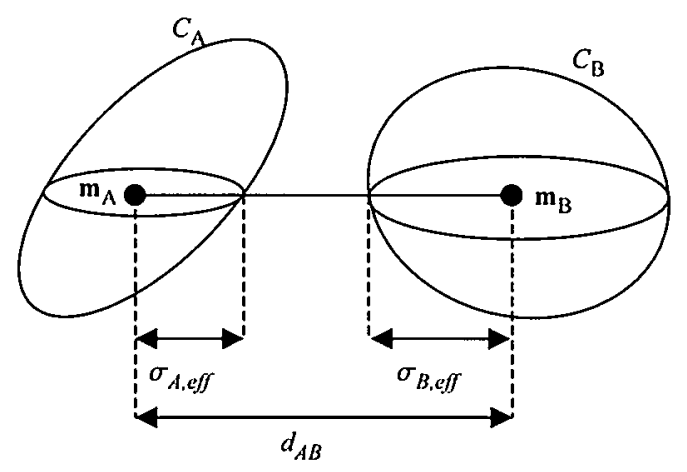

Fig. 4.12. Effective size, and inter-cluster distance of a pair of ellipsoids, representing Gaussian clusters..

\footnotetext{
${ }^{9} \sigma_{\text {A,eff }}$ follows from the following consideration. Let $M$ be the line-segment between prototype $\mathrm{m}_{A}$ of cluster $A$ and prototype $\mathbf{m}_{B}$ of cluster $B:\left\{\mathbf{x} \mid \mathbf{x}=\mathbf{m}_{A}+\alpha \mathbf{r} \wedge \alpha \in[0,1]\right\}$, with: $\mathbf{r}=\left(\mathbf{m}_{2}-\mathbf{m}_{1}\right)$. The ellipsoid $H_{1}$ : $\left(\mathbf{x}-\mathbf{m}_{1}\right)^{\top} C_{1}^{-1}\left(\mathbf{x}-\mathbf{m}_{1}\right)=1$ indicates points with equal distance to the prototype of cluster $A$. Similarly for ellipsoid $H_{2}$ of cluster $B$. The effective standard deviation in direction $\boldsymbol{r}$ follows from the intersection between $H_{1}$ or $H_{2}$ and $M$, yielding: $\sigma_{1, e f f}=\left(\mathbf{r}^{\top} C_{k}^{-1} \mathbf{r}\right)^{-1}$, and: $\sigma_{2, e f f}=\left(\mathbf{r}^{\top} C_{l}^{-1} \mathbf{r}\right)^{-1}$. Combination of the normal distributions representing of the two classes gives the effective variance of the difference: $\sigma_{e f f}^{2}=\sigma_{e f f, A^{2}}+$ $\sigma_{e f f . B^{2}}$.
} 


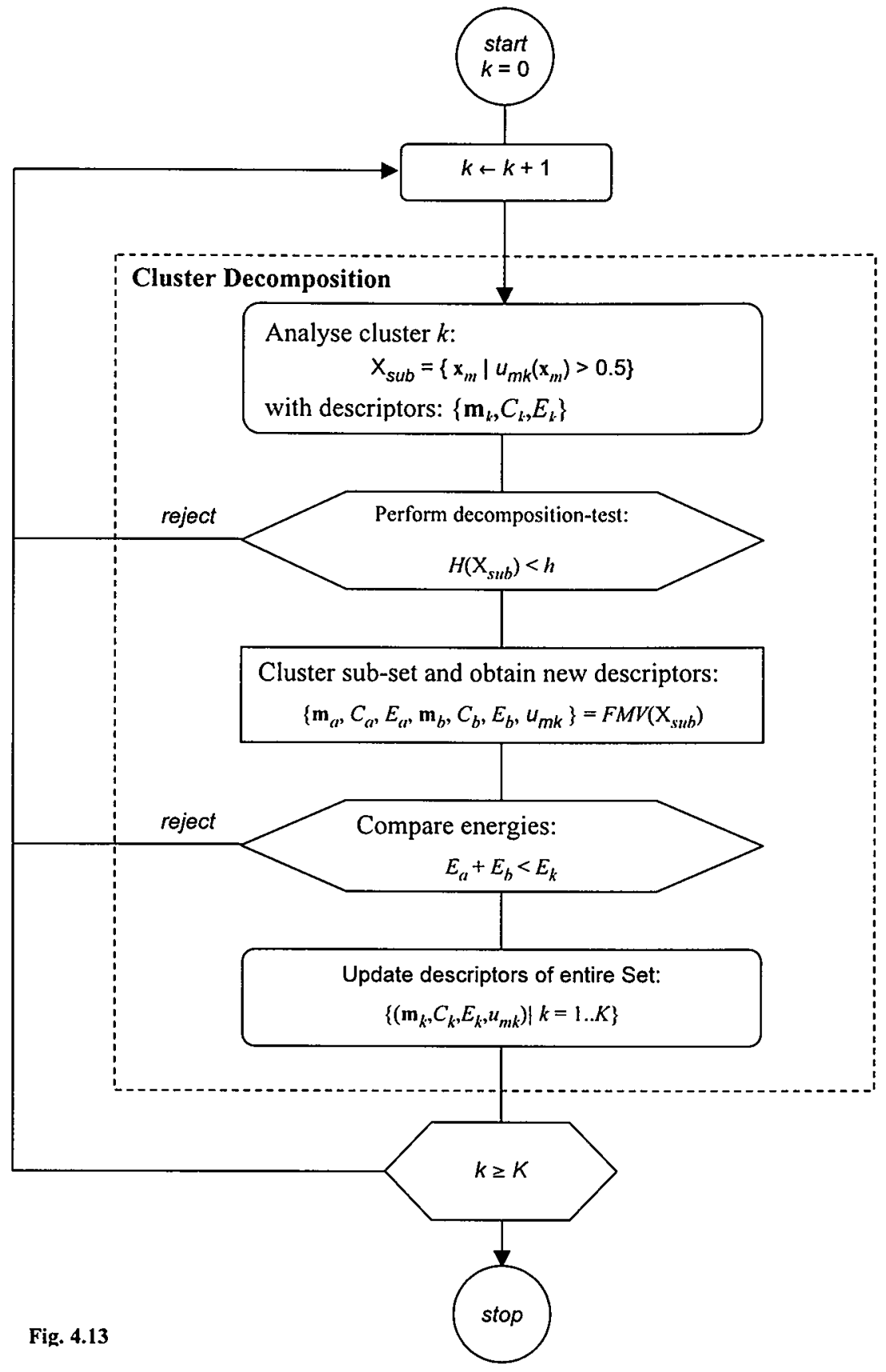




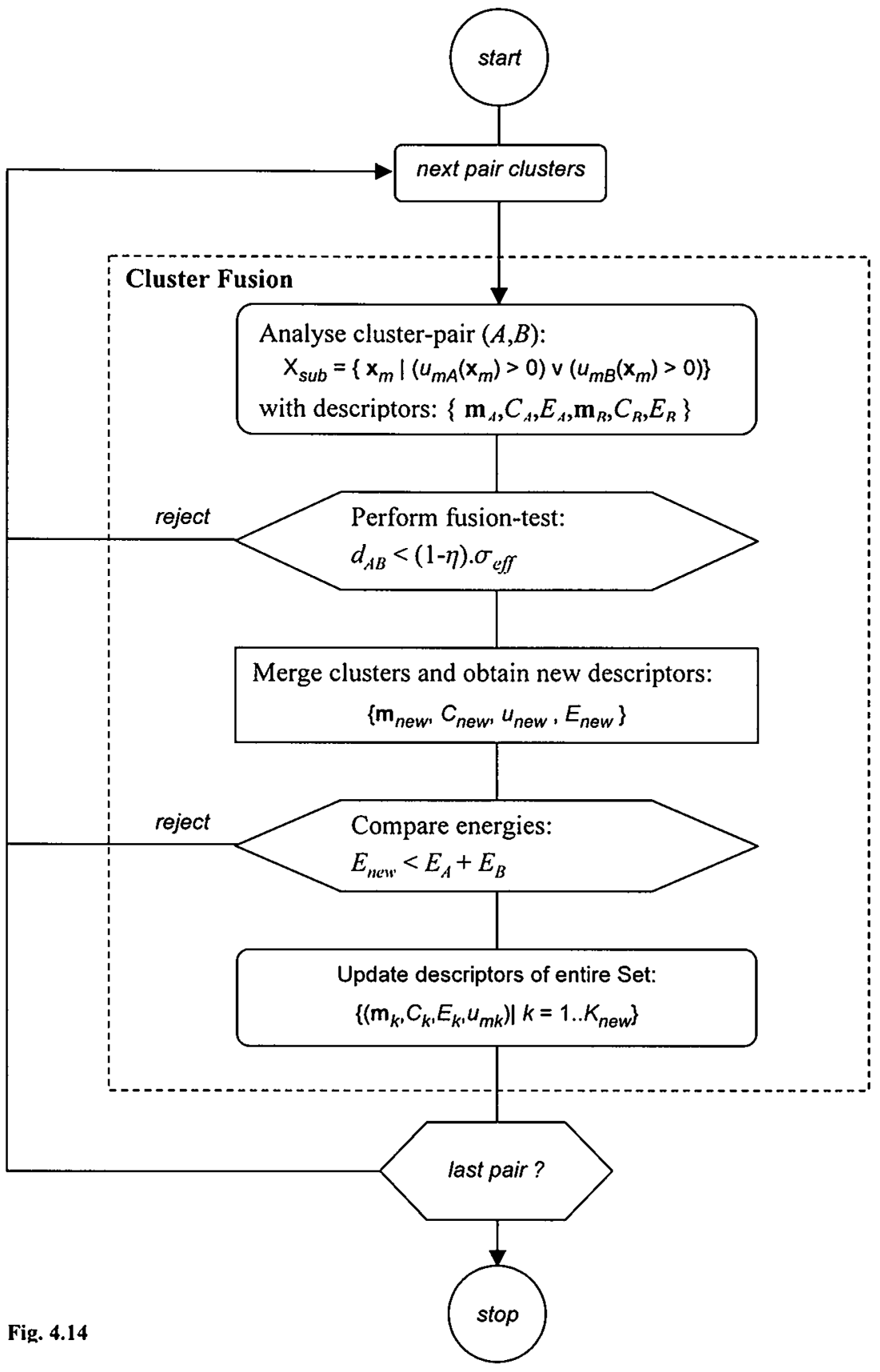


If this is not the case, the fusion is rejected as yet. The approach is sketched in Figure 4.14. The result of the entire fusion analysis is a new collection of prototypes and covariancematrices, while the total number of prototypes has decreased by the total amount of fusions.

\subsubsection{Iterative FMV Cluster Analysis}

Our approach for automatic cluster analysis is based on an iterated sequence of cluster decomposition and cluster fusion, until no modification in the prototypes and the partition matrix is observed - or, alternatively, a maximum iteration length is reached. Because of the condition that the cumulative energy effectively decreases during a fusion or decomposition, it can not happen that the system enters into infinite loops of successive fusion and later decomposition of certain subclusters.

Let $X$ represent the data set as a $M \times N$ matrix, $u$ the $M \times K$ partition matrix, and $v=\left(v_{1}{ }^{\top}, \mathbf{v}_{2}{ }^{\top}, .\right.$. , $\mathbf{v}_{K}{ }^{\top}$ ) a $K \times N$ matrix composed of the $N$ coordinates of the $K$ prototypes. Then we can write one entire run of cluster decomposition of all present classes as the application of an algorithm 'ClusterDecomposition' on the present data set, partition matrix, and set of prototypes as: $\{u, v\} \leftarrow$ ClusterDecomposition $(X, u, v)$. Similarly, we can write cluster fusion as: $\{u, v\} \leftarrow$ ClusterFusion $(X, u, v)$. The number of clusters follows from the dimensions of matrix $v$ as: $K=$ $\operatorname{dim}(v, 1)$. The entire algorithm of iterative $F M V$ cluster analysis can now be defined in pseudo-code as:

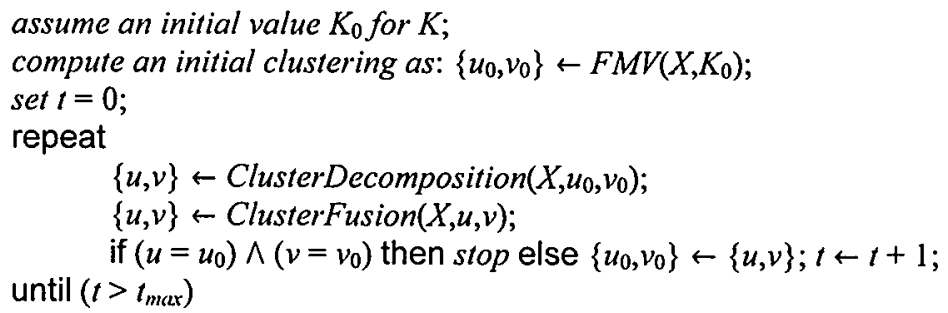

The analysis can be severely time-intensive, for in a situation with $K$ clusters, per iteration at least $K(K-1) / 2$ cluster pairs must be investigated for fusion, while $K$ clusters are checked for decomposition, and for each decomposition an $F M V$ algorithm must be applied. It is difficult to give a reasonable estimate for the entire length of the entire iteration. Because cluster fusion decreases the number of clusters $K$, while cluster decomposition on the other hand increases $K$, it may happen that during one iteration $K$ remains constant, while the position of the prototypes and the values of the partition matrix have considerably changed. Suppose that $K$ assumes an initial value $K_{0}$ and that the real number of clusters in the data is $K^{*}$. Now, let $K_{0}>K^{*}$. Then we may expect that the iteration process is initially dominated by cluster fusions. Since the minimum amount of change in $K$ per iteration is 1 , the maximum number of iterations is: $K_{0}-K^{*}$. That is if there are no non-monotonic effects that change the partition matrix but not the number of clusters, as mentioned before. A similar consideration holds for the case $K_{0}<K^{*}$, when predominantly cluster decompositions are expected. This assumption leads to a maximum of $K^{*}-K_{0}$ iterations. In general, therefore, a maximum number $\left|K^{*}-K_{0}\right|$ iterations is expected. In practice, however, the number of iterations itself is usually much less, and depends strongly on the topology of the data set.

Finally we notice our experimental observation that the algorithm for different runs with the same data set converges to approximately the same - but permutated - prototypes, and that this property is robust for small changes in the data set. 


\subsubsection{Performance of Iterative FMV Cluster Analysis}

The iterative FMV cluster analysis algorithm was tested on a large number of artificially created data sets with different topologies and data dimensions $N$. From these experiments it was found that the performance strongly depends on the topology of the data set. For the same data dimension $N$ and number of data points $M$, significant differences in the resulting number of clusters $K$ could be found. This is especially the case when clusters overlap, or are relatively near with respect to the average cluster separation. In that case prototypes are generated in between these sub-clusters. These sub-clusters act as equilibria that the algorithm can not further decompose. Another general observation was that the algorithm was found to be more efficient for cluster-fusion than for cluster-decomposition. This inequality is mainly caused by the inaccuracy in the decomposition criterion $H$. Figure 4.16 shows a typical run of the algorithm in merging and decomposing clusters. The table below shows the success rate of iterated FMV for three types of artificially generated sets. The table distinguishes between an initial guess $K_{0}$ above and below the real number of clusters $K^{*}$. In the case of sharp, wellseparated clusters the success rate was high, and the iteration length ranged in the order of 45. For partially overlapping clusters the performance dropped considerably, while the iteration length somewhat increased. As a third test set a homogeneous distributed set of 500 points in 5 dimensions was offered. In that case we would expect the algorithm to find one cluster with the mean of the set as its prototype. This was the most frequently obtained solution. In about one quarter of the runs, however, the algorithm tended to evenly split the set, with prototypes distributed around the mean of the set. If the number of points $M$ of the uniform distribution, however, was reduced, the probability for finding more prototypes increased.

\begin{tabular}{|c|c|c|l|c|c|c|c|}
\hline & \multicolumn{2}{c}{ Data set } & \multicolumn{2}{c}{ Success Rate } & \multicolumn{2}{c|}{ Iteration Length $/ \boldsymbol{K}^{*}$} \\
$K^{*}$ & $N$ & $M$ & \multicolumn{1}{c}{ type } & $K_{0}<K^{*}$ & $K_{0}>K^{*}$ & $K_{0} \leq K^{*}$ & $K_{0}>K^{*}$ \\
\hline 8 & 4 & 334 & well-separated & $85 \pm 16 \%$ & $98 \pm 6 \%$ & $1.7 \pm 0.7$ & $3.1 \pm 1.8$ \\
\hline 10 & 5 & 363 & partial overlap & $77 \pm 23 \%$ & $83 \pm 18 \%$ & $2.8 \pm 1.7$ & $2.9 \pm 1.7$ \\
\hline 1 & 5 & 500 & homogeneously & - & $65 \pm 36 \%$ & $3.2 \pm 1.8$ & $3.4 \pm 1.8$ \\
\hline
\end{tabular}

Table 4.5

Furthermore, we used two real data sets to evaluate the performance of the iterated FMV algorithm. The first data set used was collected from pre-processed data of a large series of CCD-images of a number of printing defects. The data dimension $N=32$, the number of measurements $M=531$. Figure 4.15 below shows the obtained number of clusters $K$ as function of the initial guess $K_{0}$. The actual number of clusters present in this data set is 18 , as in this case validated by domain experts. This figure clearly shows the fact that cluster fusion is more efficient than cluster decomposition.

The second test set is the Iris Plants Database, stemming from botanical taxonomy. It consists of measurements of three species of the genus Iris \{I. Setosa, I.Versicolor, I. Virginica\}, based on a classical paper of the evolutionary biologist R.A. Fisher [Fisher, 1936]. This is perhaps the best known database to be found in the pattern recognition literature and is referenced frequently to this day. This set is by far the most widely used benchmark for clustering techniques. The data set contains 3 classes of 50 instances each, where each class refers to one species of the genus Iris. The main complexity from this set lies in the fact that the first class is linearly separable from the other two; but the latter are not linearly separable from each other. For each species four numeric attributes are available \{sepal length, sepal width, petal length, petal width $\}$, and there are no missing attribute values. Though this is an exceedingly simple problem, the Iris test set facilitates comparison with other clustering techniques. Table 4.6 below lists the performance of some important clustering techniques, as 
compared to iterated FMV. This shows that most techniques have problems in distinguishing the two not linear separable clusters.

In a sense Fisher's Iris data set shows the notable development of pattern recognition. Until recently it was the essential benchmark for gauging clustering techniques to the state of the art. The typical number of clusters ranged in the order of $10 \pm 10$. Currently, clustering techniques are successfully applied on data-sets involving millions of samples, in datadimensions of several thousands, containing thousands of clusters. This is for instance the case in air and space technologies, data mining and document classification [Jain et al.,2000]. This development was partly stimulated by the advance of new clustering techniques, but the main impetus came - again - from increased processor power and speed. Handling such large numbers of prototypes requires another generation of automation in pattern analysis, in which cluster taxonomies - like in hierarchical clustering - become inevitable. In the light of these developments, there is also bound to arise a demand for automatic cluster analysis algorithms like iterated FMV.

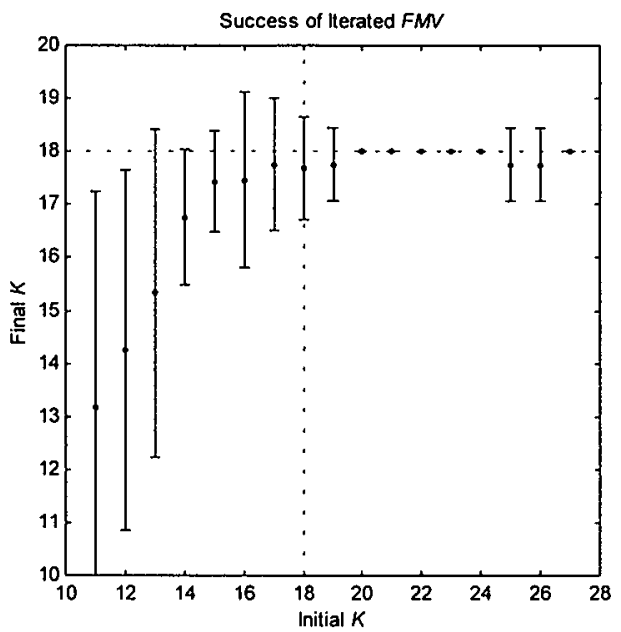

Fig. 4.15 Relation between initial guess and final result of the number of clusters $K$ of iterated $F M V$ in a experimental data set, with $M=531$, and $N=32$, stemming from CCD images.

\begin{tabular}{|l|c|c|}
\hline \multicolumn{1}{|c|}{ Technique } & Found & Iterations \\
\hline $\begin{array}{l}\text { Mountain Clustering } \\
\text { (cube length }=0.5 \text { ) }\end{array}$ & 4 & 1 \\
\hline $\begin{array}{l}\text { Subtractive Clustering } \\
\text { (cube length }=0.5 \text { ) }\end{array}$ & 4 & 1 \\
\hline $\begin{array}{l}\text { Subtractive Clustering } \\
\text { (cube length }=0.67)\end{array}$ & 3 & 1 \\
\hline Energy Survey method & $4 \pm 0$ & 6 \\
\hline $\begin{array}{l}\text { Iterated FMV } \\
\text { (100 runs) }\end{array}$ & $2.9 \pm 0.4$ & $1.3 \pm 0.4$ \\
\hline
\end{tabular}

Table 4.6. Performance on Fisher's Iris-data $\left(K^{*}=3, M=150, N=4\right)$ 

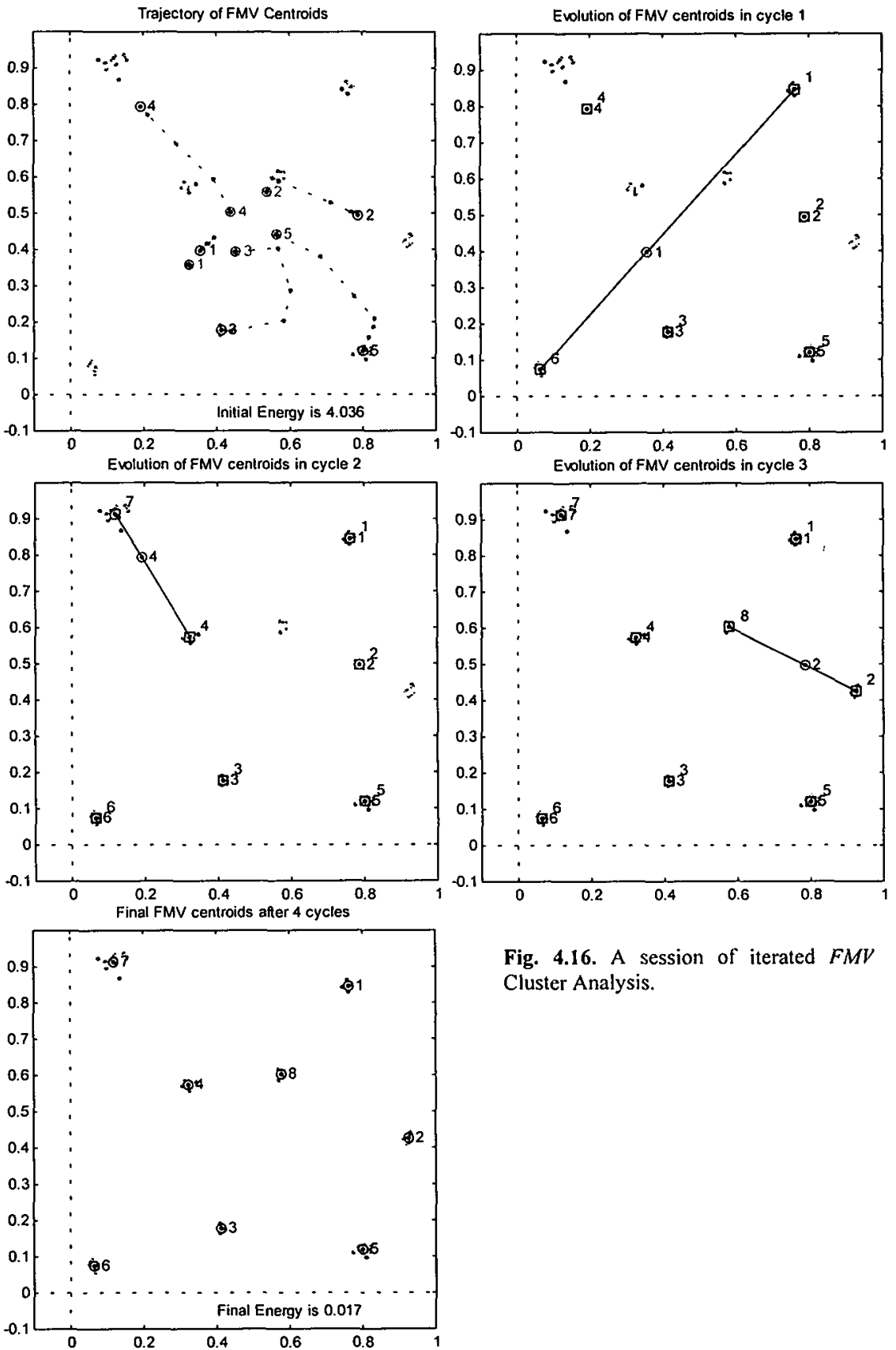

Fig. 4.16. A session of iterated $F M V$ Cluster Analysis. 


\subsubsection{Comparison with Mountain Clustering and Subtractive Analysis}

To conclude this section on automatic cluster analysis we discuss two different methods for estimating cluster numbers. These are mountain clustering and subtractive clustering, discussed in section 4.3.g. From that description we conclude that these two scale-space techniques are not as much algorithms which directly compute the optimal number of clusters from a data set. They are more like tools which provide a fast and easy way to study the number of clusters as function of the average cluster size. Under these conditions it is difficult to compare these clustering techniques with iterative FMV clustering. For each number $K$ that iterative FMV clustering finds for a data set, there exist sizes of a hyper-bloc, such that they also generate $K$ clusters. For such settings, these two scale-space techniques find almost the same prototypes with a comparable energy as iterative-FMV clustering. The figure below shows a comparison of subtractive clustering and iterated FMV clustering. On the left side it shows the relation between length $\epsilon$ of hyper-cube that is used to approximate the clusters versus the resulting number $K$ of clusters. This is performed on the data set shown right. For this data set iterated-FMV finds six clusters. This number is indicated as a horizontal line in the figure. For $\epsilon$ between 15 and 32 subtractive clustering finds six clusters. The obtained cluster-prototypes for $\epsilon=22$ are plotted right, together with the prototypes stemming from iterated-FMV. This clearly shows the normal situation that they almost coincide.
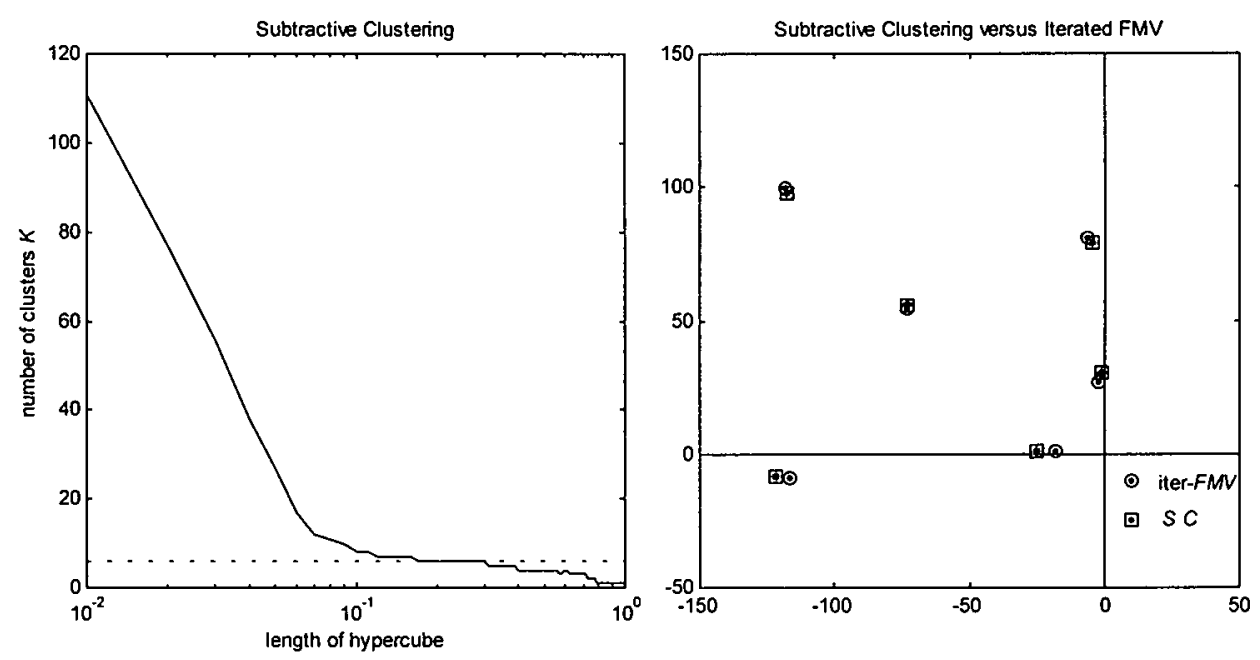

Fig. 4.17. Comparison of subtractive clustering and iterated FMV clustering. 


\subsubsection{Main Conclusions on Automatic Clustering}

Iterated FMV is an automatic cluster analysis technique that can find the relevant number of clusters in a data set. It consists of three components, a clustering technique like FMV or FCM, an algorithm for identifying and decomposing composite clusters, and an algorithm for the identification and subsequent fusion of equivalent clusters. The algorithm is driven by energy minimisation, which effectively limits the length of the iteration.

The price for this efficiency is a high computational cost, caused by the large number of clustering runs on the one hand, and the many decomposition and fusion tests of the entire collection of clusters on the other hand. Some of these calculations can be reduced. For instance, the intra-cluster distance $D$ involves $m(m-1) / 2$ operations for a specific cluster consisting of $m$ points. This can be resolved by only considering a small, but relevant, number of randomly sampled cluster points. This improves the computational effort, but also affects the success rate of the algorithm.

The IFMV algorithm exclusively uses the assumption of the underlying clustering technique on the typical shape of a cluster, i.e. spheroidal for FCM, or ellipsoidal for FMV. Since both techniques are predisposed for linear separability, IFMV shares this constitution. There are two ways FMV, and thus IFMV, can treat not linear separable clusters. First, it can assign multiple prototypes to one single cluster, thereby constructing a covering of convex Voronoicells of the principal cluster. Second, it can handle not linear separable clusters as overlapping spheroidal clusters, and assign an optimal fit to the covariance matrices of the clusters to obtain the best matching multivariate probability distributions for the measurements in the cluster.

The largest deficiency of IFMV, however, is caused by inefficiency of the decomposition criterion $H$ and its associated threshold $h$. These are caused by the simple linear model used, but also by the restriction of the considered cluster types (spheroidal, ellipsoidal) and the dimensionalities used. More complex models based on $\mathrm{N}$-dimensional ellipsoidal distributions and non-linear criteria may somewhat improve the performance.

Automatic clustering analysis leads to the number of significant clusters present in an unlabeled data set. This stands in contrast to most clustering techniques like Fuzzy C-means, Kohonen's self-organising feature map, and subtractive clustering that demand some beforehand assumptions on the topology of the data space. This requires that in the data space there indeed exists a genuine and perceptible number of clusters. In many real situations, however, data space consists of complex, not linearly separable, or overlapping distributions. In that case subjectivity or application semantics become important in interpreting the relevant regions of the data space. For this reason the identification of clusters is an activity that in practice is mostly performed in close interaction with the domain experts to find out the expected number of clusters, and to investigate whether the resulting prototypes have any practical significance. However, there are examples where clustering algorithms generated clusterings of data space that contained more attractive properties than the expert classification.

On the other hand, there is currently a tendency to apply clustering techniques on more complex and extensive fields as in the analysis of physical and astronomical experimental data, gene-protein mapping, and data mining in industrial applications. In those cases data sets count hundreds of dimensions and tens of relevant clusters, and no suitable data visualisation tools are available. Moreover, there is often no prior knowledge of the field, so in a sense there are no real domain experts. Those fields demand a new approach to clustering, where no initial assumptions on the structure of the data space are required. In such applications reliable automatic clustering techniques must lead to the automatic identification of taxonomies of the significant cluster in data space. In this development a technique like Iterated Fuzzy Multi Variate clustering can play a role. 


\subsection{Applications of Clustering Methods in Computational Vision}

\subsubsection{Simulating Parameterised Printing Defects in the SOM Map}

In Section 2.6.1 we used the one-dimensional cumulative intensity distribution (CID) to obtain a 'filtered' representation of an image. In this approach the 'energy' in the CID of the difference $Z$ between the image and the associated template in each of the four 'PrintContexts' on a printed image, were used to represent the defect as a normalised vector $\left(\xi_{1}, \xi_{2}, \xi_{3}, \xi_{4}\right)$. Because of the normalisation, and because $\xi_{k} \in[0,1]$, the $4 \mathrm{D}$-vector $\xi$ could be represented in a $3 \mathrm{D}$-pyramid. From this pyramidal representation it became clear that specific regions in the pyramid corresponded to specific printing defect-classes. Here, we further investigate the clustering of these defects and study how this observation can be utilised for the identification of the defect. As clustering technique we here employ Kohonen's Self Organising (Feature) Map (SOM) [Kohonen, 1989]. In our context it suffices to understand this approach as a two-dimensional mapping of our original 3D-pyramidal representation. This map expresses relevant topological and semantic information in the data. One such feature is that proximity on the SOM-map implies similarity of the defects.

With a simple example we now illustrate to what extent the SOM clustering-technique groups spatially separated printing defects into neighbouring locations on the map. Therefore, we

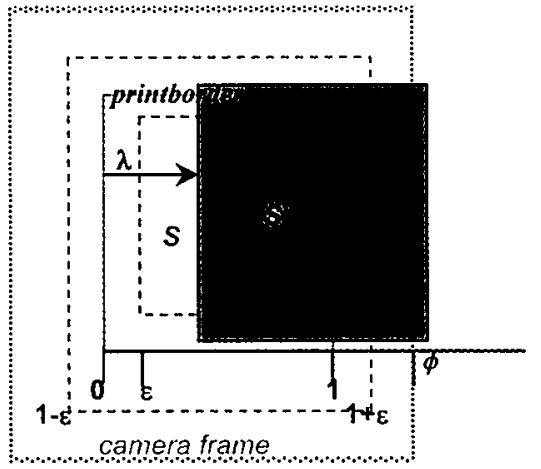

Fig. 4.18. Model for printing defects. A square $S$ is translated right by $\lambda$. The translated square $S^{\prime}$ is indicated dark grey. Also indicated is the original printborder. The dashed lines demarcate the inner and outer print-neighbourhoods. The size of the print-neighbourhoods is $\varepsilon$. The outer square represents the frame (the area observed by the camera), measured from the origin the coordinate of the frame is $\phi$. introduce a simple mathematical model to represent merely two printing defects; 'Double Print', and 'Misregistration'. 'Double print' occurs when an object is printed twice but the two printings do not fully coincide. 'Misregistration' occurs in a single printing process when the realised position of the print differs from the intended position. In Figure 4.20 the observed positions of a large set empirical data of these two defects are shown, together with the pyramid that constraints their range. In this representation the two defects are clearly separated. Kohonen Clustering can now be employed on this representation, resulting in a 2D-representation of the defects, shown in Figure 4.21. As expected, the two defect types do not overlap on the map.

The two defect types can now be modelled as following. Consider a square $S$ in a larger square $F$, see Figure 4.18. $S$ represents the template to be printed, and $F$ represents the frame of the camera - i.e. that part of the object seen by the camera. Now, let $S$ be shifted horizontally over a distance $\lambda$ and call this new square $S$ '. This generates a printing defect. In the case of 'double print' both the original $S$ and $S^{\prime}$ are printed, in the case of 'misregistration' only $S$ ' is printed. For these specific situations the representation-coordinates $\left(\xi_{1}, \xi_{2}, \xi_{3}, \xi_{4}\right)$ can be calculated exactly for each shift $\lambda^{10}$. The results are depicted in Figures 4.19 and 4.20 .

\footnotetext{
${ }^{10}$ The printcontexts depend on a parameter $\epsilon$ that defines 'neighbourhood' to the border, here: $\epsilon=0.25$.
} 
These simple models can now be plotted in the pyramidal representation, by marking the corresponding 3D-position of the parameters $\xi_{i}(\lambda)$ as the shift $\lambda$ changes. In this way we obtain 'trajectories' of the models shown in Figure 4.20. Notice that the trajectories indeed intersect the corresponding clusters of observed defects. For a given observation we could thus determine the closest point on either trajectory. The trajectory with the smallest distance identifies the most likely defect. This match provides an estimate for the best fitting parameter $\lambda$ from the model. This estimate could then be used for direct control, by adjusting the position with an amount $-\lambda$. This should of course be performed on the real template and not for this simple square. In practice this would be severely limited by problems due to the identifiability of the exact shift $\lambda$.

In a similar way the trajectories of the models can be represented on the 2D-Kohonen map, see Figure 4.21. The Kohonen-map can be divided in three exclusive parts: (1) the area where 'double print' can occur, (2) the area where 'misregistration' can occur, and (3) an area where neither (1) nor (2) can occur. In this way the Kohonen-map can be applied to classify actual printing-images like in the example discussed in the next section. Thus, this simple model demonstrates how the distribution of printing defects in output space can be modelled.
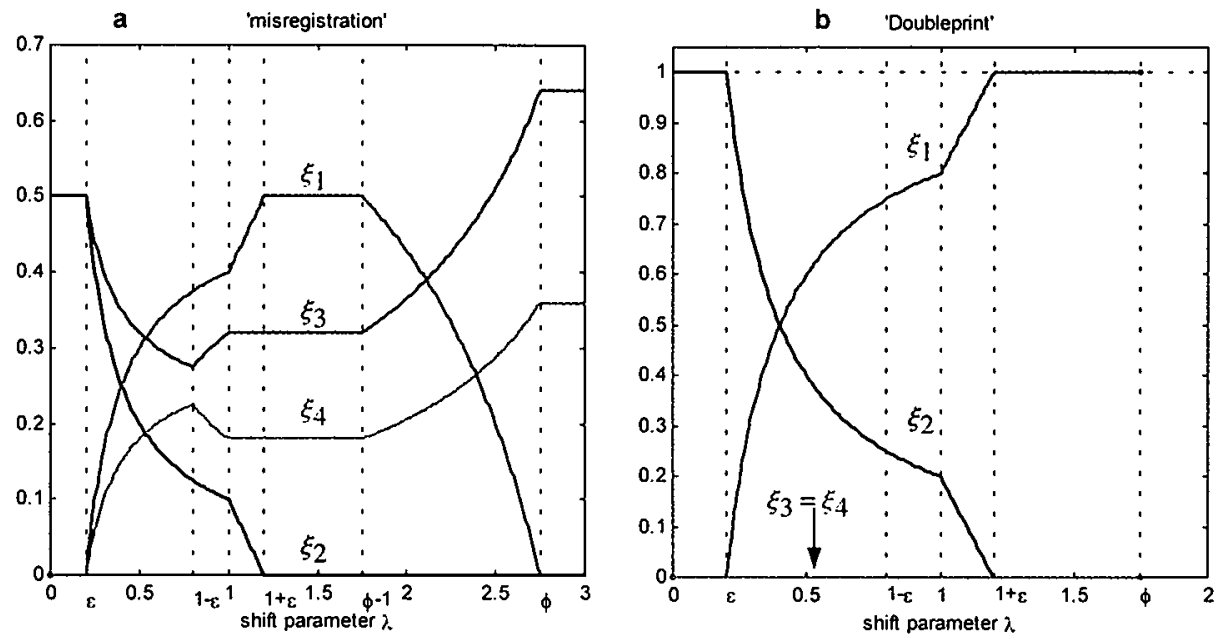

Fig. 4.19. The dependence of the normalised intensity ' $\xi$ ' over the four printcontexts as function of the translation ' $\lambda$ ' of a square. a: 'misregistration'. b: 'double print'. Printcontext-intensities refer to: $\xi_{1}=$ outside print, $\xi_{2}=$ outside print near border, $\xi_{3}=$ inside print near border, $\xi_{4}=$ inside print

Generalising this idea, the Kohonen-map can be used to classify actual printing-images, at least to their correct cluster. Moreover, a set of parameters, like $\lambda$, can be employed to model the defect and represent the 'strength of the defect'. In this way, the representation can be used to estimate the strength of an actual defect from its position in the representation space $\xi$. Extrapolation of this approach to the full set of printing defects, however, leads to a number of problems. In the first place, for this example we selected two defects that are well separated in the pyramidal representation-space. In reality, however, defects are not so well separated, and exhibit a large degree of overlap. In the real case, therefore, the nodes of the Kohonenmap corresponds to a number of classes, and many classes are clustered together in superclasses. Second, it is not always possible to propose a simple model as we did here, and even this model is for a very simple template. Realistic models should be based on the real template, and therefore severely template-dependent, meaning that for all new templates new 
models must be constructed. In the third place, even if we could surpass all these problems, the issue of the identifiability would normally impede the determination of the strength of the defect - as in our case $\lambda$.

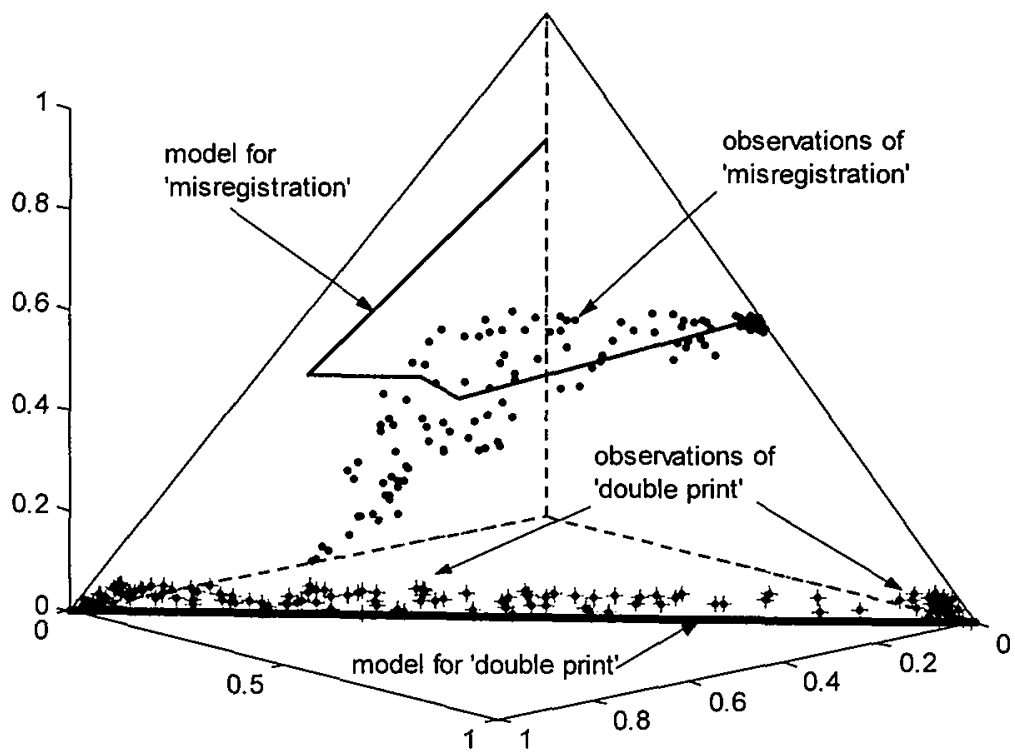

Fig. 4.20. 3D-'pyramidal' representation of parameters $\xi$. Points represent the observed positions of two types of defects, stemming from real data: 'double print' (cross), and 'misregistration' (dot). The trajectories represents the model for 'misregistration' and 'double print', with variable shift-parameter $\lambda$ and border-neighbourhood $\epsilon=0.25$.

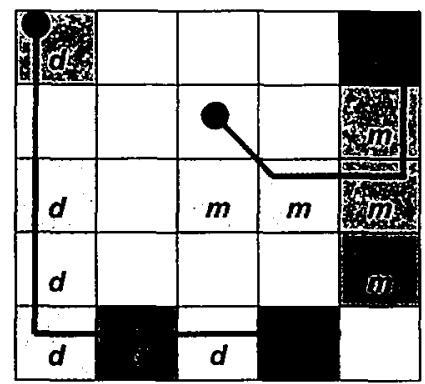

Fig. 4.21. $5 \times 5$ Kohonen-map of observed defects 'misregistration' $(m)$ and 'double print' $(d)$ in pyramidal representation. Greyscale indicates the fraction of the observations per square. The two curves represent the mapping of the models for 'misregistration' and 'double print' with varying shift-parameter $\lambda$ and border-neighbourhood $\epsilon=0.25$. 


\subsubsection{Efficient Pre-Classification based on Clustered Data}

Another dimension-reduced representation of printing-defects, described in Chapter 2, is based on the optimal fit of the 1D-cumulative intensity distribution (1D-CID) of an image with a simple block-function. This resulted in just two relevant parameters $\left(L_{l}, L_{2}\right)$, denoting the coordinates of respectively the left and right side of the best fitting block. It was found that the distribution on the $L_{1} L_{2}$-plane exhibited a meaningful partition of the printing defects. Now we apply our iterated $F M V$-cluster analysis on a large set of empirical data containing all defect classes, in order to obtain the number of meaningful clusters, their prototypes and covariance-matrices. This method straightforwardly led to six separate clusters, indicated in the figure below as $C 1, . ., C 6$. The clusters are depicted with their prototypes and metric tensors (inverse of the covariance matrix) shown as an ellipse. Subsequent Voronoipartitioning based on the cluster-prototypes divides the $L_{1} L_{2}$-plane in six different cells. These cells are visible in the figure, together with the diagonal: $L_{1}=L_{2}$, for all measurements by definition lie above the diagonal. Though the obtained clusters are well separated, most of the resulting clusters consist of multiple defect-classes, as indicated in the table below. Cluster 2 , for instance, consists of 7 totally different defect-classes. All of the defect-classes in one cluster, however, share an important semantic property. This property is also indicated in the table below. For cluster 2 this characteristic property is that the printing defect is situated totally inside the printed area. Thus, the criterion for clustering is determined by the relative position of the defect with respect to the printborders. An important consequence of this aggregation of defect classes in one cluster is that it is not possible, with this clusteringtechnique alone, to discriminate between the sub-classes of one cluster. However, because of its ease, this approach can be used as a method for pre-classification of the image. Subsequent focussed analysis must then be invoked to discriminate between the competing defect classes within one cluster. In this way, the effort of the on-line classification process, described in the next chapter, can be reduced considerably.

The pre-classification method based on this clustered data is a straightforward algorithm. As input it requires a CCD-image of the printed calibrated and scaled relative to the associate template. The algorithm itself consists of the following steps:

1. Compute the absolute difference $Z$ between the (improved) $C C D$-image and the template.

2. Obtain the 1D-CID energy distribution of $Z$ as function of the 'depth' $d$ in the print.

3. Determine the best fitting block with sides $d=L_{1}$ and $d=L_{2}$.

4. Plot the coordinates $\left(L_{1}, L_{2}\right)$ in the $L_{1}, L_{2}$-plane depicted in Figure 4.22, and determine to which Voronoi-cell it belongs.

5. In case when a multivariate normal distribution is observed: use the position $\mathbf{x}=\left(L_{1}, L_{2}\right)$ representing the defect, and the prototype $\mathbf{m}_{k}$ and covariance-matrix $C_{k}$ of the associated Voronoi cell $k$ to determine the (scaled) multivariate normal distribution for this class: $\operatorname{det}^{-1} C_{k} \exp \left(\left(\mathbf{x}-\mathbf{m}_{k}\right)^{\top} C_{k}^{-1}\left(\mathbf{x}-\mathbf{m}_{k}\right)\right)$. This can be done for all classes in the same way. Successive normalisation gives the probability-distribution of the observed image over the set of defect classes. 


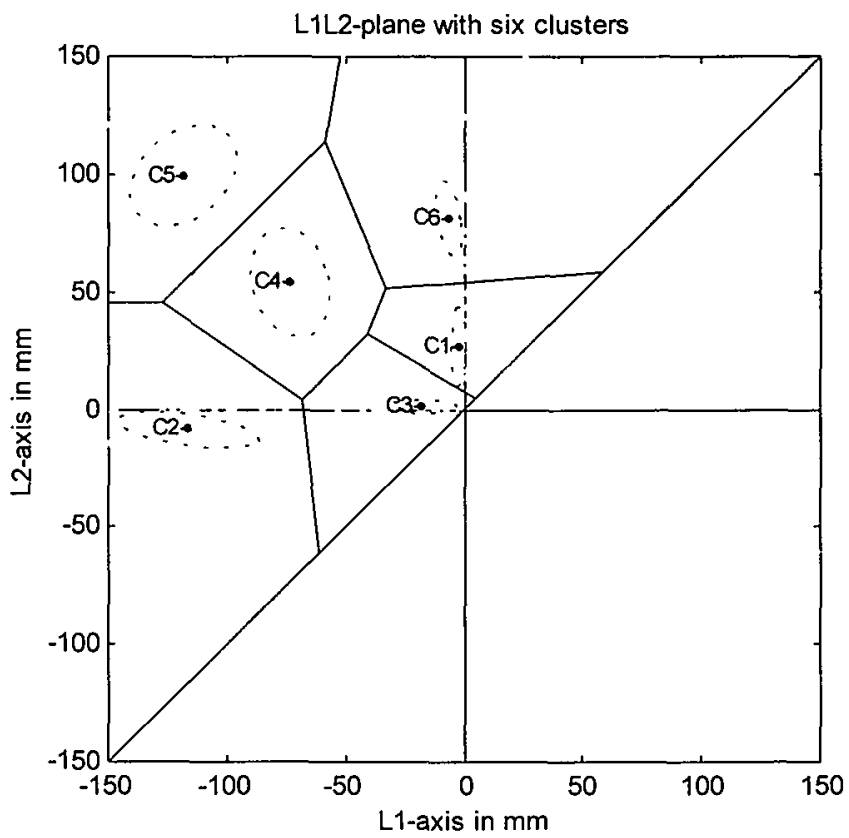

Fig. 4.22. Iterated $F M V$ clustering of empirical data in the $L_{1}, L_{2}-$ representation leads to six well-separated clusters, most consisting of multiple defect-classes.

\begin{tabular}{|c|c|l|}
\hline Cluster & Number of Classes & \multicolumn{1}{|l|}{ Defect Characterisation } \\
\hline$C 1$ & 4 & outside print \\
\hline$C 2$ & 7 & inside print \\
\hline$C 3$ & 2 & inside print near border \\
\hline$C 4$ & 1 & large defects all over print \\
\hline$C 5$ & 2 & small defects all over print \\
\hline$C 6$ & 2 & near borders \\
\hline
\end{tabular}

Table 4.7 The six visible clusters in the $L_{1}, L_{2}$-plane. 


\subsection{Main Conclusions}

Testing of the $F M V$ clustering approach on several sets of artificial and real data shows that FMV is faster than FCM in the sense of the number of iteration steps, and that the resulting energy of the clustering is somewhat lower. This indicates that FMV results in equivalent prototypes, but that it proceeds faster to its final result. On average, for higher $K$ this effect becomes stronger.

Iterated FMV is an automatic cluster analysis technique that can find the relevant number of clusters in a data set. The price for this efficiency is a high computational cost. The IFMV algorithm uses the assumption of the embedded clustering technique on the typical cluster shape. More complex models, based on $\mathrm{N}$-dimensional ellipsoidal distributions and non-linear criteria, rather than the simple linear model used here, may improve the performance regarding decomposition. Iterated FMV leads to the number of significant clusters present in an unlabeled data set. This stands in contrast to most clustering techniques like Fuzzy Cmeans, Kohonen's self-organising feature map, and subtractive clustering that demand some beforehand assumptions on the topology of the data space.

Neural networks have the benefit of fast design and quick implementation, at the price of long training times, and large training sets. The latter requirement may pose a problem regarding the knowledge acquisition. Neural networks have the ability to adapt to changing environments. An important problem associated to learning systems, however, is how to maintain consistency in the training set. They are not suited for interactive classification, because they require the entire input-vector.

The last two sections of this chapter showed that condensed representations based on the CIDtransform, combined with Kohonen clustering or fuzzy clustering, can be successfully used for simulation and pre-classification. 



\section{Chapter 5}

\section{Interactive Classification of Numerous Weakly Separated Many-Parameter Prototypes with Noise}

\subsection{Introduction}

Classification is the act of assigning an observed object to the most appropriate element of a finite set of classes, using the available information. Real-time (or online) classification means that the classification is performed during the time the data is collected. Interactive classification means that while the real-time data-collection unfolds, decisions can be made that influence the selection of future data. In order to perform interactive classification a set of specialised and analytical tasks is required. These can be divided in parameter selection tasks and object analysis tasks. In parameter selection, based on the available information and currently most likely hypothesis, the not-explored parameter is determined that will probably generate the most decisive information. In object analysis this selected parameter is measured on a semantical and statistical relevant way from the presently observed object. Because of its straight dependence on direct measurement, we henceforth refer to object analysis as interactive sensing.

As an example, consider the determination of a printing defect based on a suitable CCD-image. In that case, parameter selection refers to establishing the most promising feature-parameter, such as a scanning parameter, texture parameter, or a specialised feature parameter as defined in appendix A. In the same context, interactive sensing means: $i$. the identification of significant regions on the $Z$ representation of the image for the selected feature-parameter, ii. the subsequent extraction through scanning or texture analysis of these regions in a statistical significant way, and well-distributed over the relevant print-contexts, and finally: iii. 
the integration of all these measurements into the most likely value-domain for the requested feature parameter.

Together, the parameter selection and interactive sensing tasks constitute a diagnostic reasoning process in which information is actively acquired and processed, until a final conclusion is reached. Main objective of this reasoning process is to converge as quickly as possible to the best possible identification of the state of the object, i.e. its object class. It is the time constraint that justifies the interactive classification. If there was no time constraint, the object would be analysed in what ever time-consuming way, and all possible measurements supporting or rejecting the accumulated information would be performed, until finally all information was available for an integral assessment. The quality of the classification process can be expressed in terms of the quality of the resulting classification and its duration relative to the maximal possible duration.

The reasoning process described here is both 'dynamic' and 'interactive'. It is called 'dynamic' not so much because of the inherent sequential nature of the diverse reasoning steps, but because of the fact that in focussing on significant information, the optimal sensing action is selected. Therefore, there is no one and fixed order of analytical tests that controls the overall process. The reasoning process is called 'interactive', because the interaction between the reasoning process and the examination of the object. Firstly, it is able to select and 'zoom in' on the relevant parts of the object, and secondly, because of its control of the sensing process for the selected feature parameter in the selected parts of the object.

In the case of computer vision, this interactive sensing and reasoning creates a process of 'active vision', mimicking to some extent the 'focus of attention' in human vision.

The basic principle for classification is the statistical correlations between certain features of the object and the set of object classes. Therefore, a basic starting point of the interactive classification process is the clustering of the objects in parameter space as discussed in Chapter 4.

The problem with classifying many-parameter classes is the large amount of information that must be accumulated and processed before a full decision can be made. This can cause intolerable delays, and thus justify the application of interactive classification. Likewise, classification becomes more difficult when the object classes overlap. With increasing imprecision the object classes in parameter space gradually expand, and at a certain point they will significantly overlap. Therefore, it is important to express the amount of class-overlap in the currently explored region of parameterspace in a general, class-independent way. The same problem is encountered with noise in the information, for noise smears out the position of the current measurement in parameter space, potentially encompassing multiple classes within its realm.

This chapter's prime subject is interactive classification and sensing, given a highdimensional feature space with numerous weakly separated clusters. The central question in this chapter is whether a new feature needs to be extracted, and if so, which feature-parameter would be most proficient given the currently acquired information. To this end, in Section 5.2 a suitable symmetric normalised metric is proposed that complements the non-symmetric Mahalanobis-distance. Also in Section 5.2, utilising this metric, a dynamic algorithm is proposed for Functional Parameter Selection, called FPS, in which the topology of feature-space is combined with the current set of hypothesis to generate the best feature-parameter to be examined next. In Section 5.3, the fusion with other kinds of (sensor) information is considered. Essential element in the control of the reasoning process is a set of criteria that determine when the reasoning should terminate. These criteria are discussed in 
Section 5.4. In Section 5.5, the FPS-approach is integrated in a formalism for InterActive Sensing and Classification, called IASC. Finally, in Sections 5.6 and 5.7, much effort is devoted to the convergence, stability, and dynamic properties of the IASC-algorithm.

This chapter is based entirely on original work. It is also independent of the rest of the thesis, as it is likewise applicable for on-line diagnosis in chemical analysis or medical examination.

\subsubsection{General Reasoning Strategy}

The reasoning process described here is largely inspired by the interactive way human vision actively analyses a new image. While most computer vision systems at present are 'fixed input - fixed output', humans engage in a dynamical interaction between cognitive and perceptive tasks that in practice quickly converges to a correct identification of an image. A central place in human vision plays the 'focus of attention', mentioned in Chapter 1. Inspired by the great skill and ease with which human operators are able to swiftly identify defects, our approach was constructed as a process that reasons about possible interpretations of the image, and actively generate tests to confirm or reject these beliefs. The objective of the reasoning process was to facilitate the identification of the defect in comparison with random or exhaustive analysis of the image. The reasoning process was designed with an algorithm that - to a certain extent - emulates the 'focus of attention' in human vision.

The interactive classification process is based on a strategy consisting of the following steps.

- Pre-classification of the information using previously detected cluster hierarchies.

- Identification of feature-parameters that can optimally confirm or reject the present most likely hypothesis.

- Extraction of the selected parameter from significant parts of the object until its weighted result stabilises or fails.

- Integration of all information present to update the present set of hypotheses.

- Determination whether enough information is accumulated in order to come to a definite conclusion, or whether more measurements are necessary, or whether the process has failed.

The interaction of these steps are represented in Figure 5.1.

Our method differs from the approach most commonly encountered in literature. In literature, 'on-line control' or 'on-line classification' is usually based on the processing of the entire feature vector. Thereby, the classification or control action is generally obtained from a neural network operating on this vector. Typical examples of this approach are: [Stavrakakis et al.,1997] and [Vogel,Jäger, 1997]. 


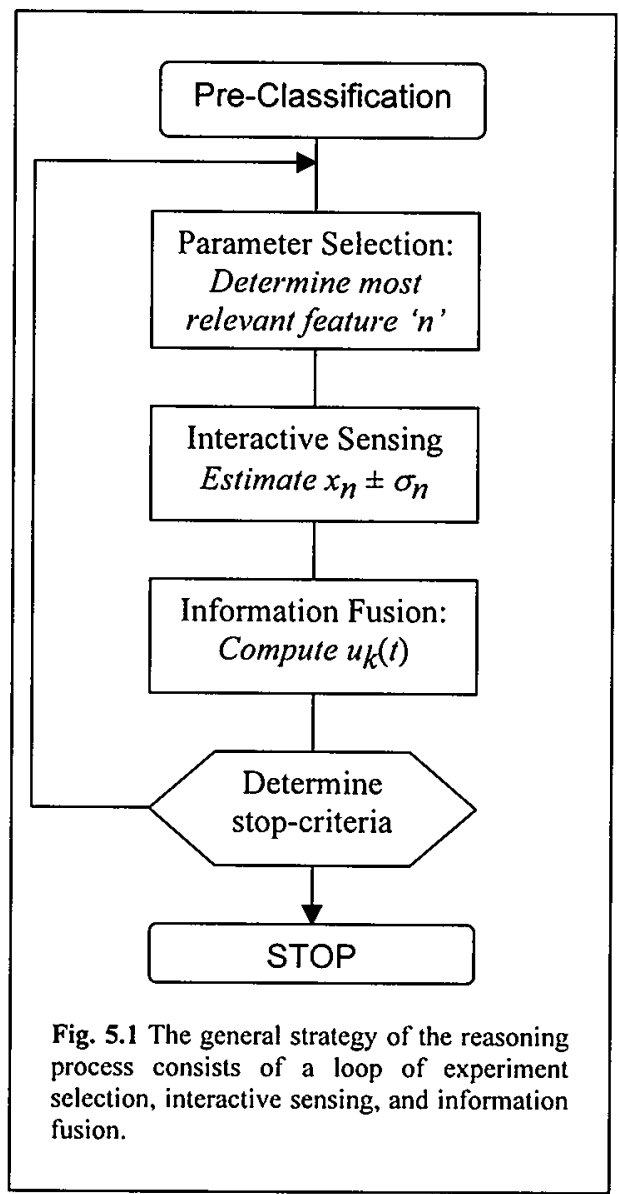

\subsubsection{Major Steps in the Interactive Classification Process}

Starting point for the interactive classification is the zero-hypothesis, in which no state is assumed for the object. A better starting point can be obtained in case there exists a hierarchical clustering in parameter space, as described in Chapter 4 . Such a clustering can be exploited to classify the current object to a super-cluster, using only a few parameters. This 'pre-classification' generates an initial hypothesis that can 'kick-start' the subsequent reasoning process in the right direction. This hypothesis is represented by a probability distribution over the known object classes. Equipped with this initial hypothesis, the system enters in the principle reasoning loop, indicated in Figure 5.1. This is similar to chemical analysis and medical examination.

First, the most informative feature parameter is determined. This is the parameter that contains the largest amount of information, based on the current available information. Next, the selected parameter is measured from the object. For this reason the system employs 'Interactive Sensing', which largely depends on the domain of application. In this analysis the major parts of the object are examined in order of 
decreasing relevance, while the selected parameter is measured. This is pursued until the parameter has been established with enough statistical precision, or the procedure fails.

During the reasoning process a large amount of information is collected. In case of printing defect classification with computer vision, the information concerns the distribution of defects over the image and over the different types of print-areas; similar for the distribution of the intensity of the defect over the image; information of textures and scanprofiles of the defect in hot spots; and shape parameters corresponding to matching with specific defect characteristics. In general, this poses the problem of the best-suited representation of knowledge. Next, all this information must be integrated to one new hypothesis. This complex problem, is the 'Information Fusion'.

The last step concerns the control of the reasoning process. The major problem in terminating the reasoning process is to prevent premature convergence on the one hand, and, on the other hand, to keep the number of reasoning steps as small as possible to optimise the computational performance of the system. Moreover, the system should be coerced to explore the relevant parts of the object as extensively as possible.

\subsubsection{Representation of a Hypothesis on the State of an Object}

The aim of an integrated approach for concurrent sensing and classification, depicted in Figure 5.1, is in elucidating the state of a physical object, provided that this object can exist in a discrete number of known states. In our principal context these states are the quality classes, or alternatively, the defect classes of the object. To deduce this state, one or more sensors are available to extract information from the object. The sensing operation itself may contain an active part, as in the chemical analysis of a fluid, or a passive part, as in imaging the object. The sensor outputs are represented as a state vector - denoted $\mathbf{x}$ - or an ellipsoid in the space of all possible measurements, called parameter space. Suppose that $K$ represents the a priori possible states of the object, and $N$ the maximum number of parameters that can be extracted based on sensor information. Consequently we have that parameter space is a sub-set of $\mathbb{R}^{N}$ : $\mathbf{x} \in \mathbb{R}^{N}$. The known states of the physical object are represented as collections in parameter space, denoted $\omega_{k} \subset \mathbb{R}^{N}$, and $\omega=\left\{\omega_{1}, \omega_{2}, \ldots, \omega_{K}\right\}$ is the set of all known states. If we allow the possibility of hierarchies in $\omega$, no further constraints are defined.

A hypothesis on the state of an object can now be represented as a distribution $\mathbf{u}=$ $\left\{u_{1}, u_{2}, \ldots, u_{K}\right\}$, with $0 \leq u_{k} \leq 1$, over the $K$ object states. Each value $u_{k}$ indicates the degree to which $\mathbf{x}$ can be considered as a member of $\omega_{k}$. For each specific application the best possible mathematical form - such as Gaussian or uniform, and most suitable semantic interpretation for $\mathbf{u}$ must be designed. For this moment we do not specify whether $\mathbf{u}$ must be interpreted as a probability for state $k$, or a fuzzy membership to state $k$, the perception of the object as belonging to state $k$, or the possibility that state $k$ may be applicable. The final result of the approach is a hypothesis on the most likely state of the object, given by:

$$
k=\arg \max _{k \in\{1, \ldots, K\}} u_{k}
$$




\subsubsection{The Quality of a Conclusion}

We will call the final distribution $\mathbf{u}$ of the reasoning process the conclusion. The associated defect class: $k^{*}=\arg \max _{k} u_{t}(k)$ is called the final hypothesis. A useful criterion to indicate whether the iteration has reached a hard conclusion is based on the composition of the final defect probability distribution $\mathbf{u}$. We call this the quality ' $q$ ' of the conclusion. First, the possibilities are renamed and sorted in decreasing order: $1 \geq u_{1} \geq u_{2} \geq u_{3} \geq \ldots \geq u_{K} \geq 0$. If the maximum $u_{1}$ is zero (as can be the case in the uncoupled model UM), then $q$ is also set to zero, otherwise $q$ is defined as the difference between the largest two, relative to the highest value: $q=\left(u_{1}-u_{2}\right) / u_{1}$. Summarising:

$$
\text { if } \mathbf{u}=\mathbf{0} \text { then } q=0 \text { else } q=\left(u_{1}-u_{2}\right) / u_{1}
$$

with $u_{1}=\max (\mathbf{u})$, and $u_{2}=\max \left(\mathbf{u} \backslash\left\{u_{1}\right\}\right)$. Since $0 \leq u_{2} \leq u_{1} \leq 1$, this definition ensures that $q \in[0,1]$. The quality of a conclusion increases monotonously from 'insufficient' for $q=0$ to 'excellent' for $q=1$. Quality can also be considered as a transformation $q:[0,1]^{K} \rightarrow[0,1]: \mathbf{u} \rightarrow q(\mathbf{u})$ that solely depends on the present hypothesis $\mathbf{u}$.

\subsubsection{Pre-Classification of the Measurement}

As depicted in Figure 5.1, the reasoning process can initiate with a pre-classification of the available information, when present. This pre-classification is based on detected properties in the clustering of empirical data, such as hierarchies. For this reason, this is domain dependent. This pre-classification system therefore has two modes; a training-mode, and a normal operation mode. In the training mode the system is offered a sufficiently large collection of empirical data representing the full spectrum of possibilities, with known object type. These examples were used to obtain a statistical distribution of each defect type ' $k$ ' over the selected representation. This distribution was subsequently used to calculate: (i) a probability distribution $u_{k}$ of class ' $k$ ' in the representation space, and (ii) a partition of the representation space in hyper-sectors, such that in each sector one defect type was predominant over all others. The latter could simply be obtained by a Voronoi-partitioning of the representation space with the given prototypes $\left\{\mathbf{m}_{\mathbf{k}}\right\}$, where $\mathbf{m}_{\mathbf{k}}$ is the average representation space-coordinate of the defects of type ' $k$ '. This was caused by the radial symmetric representation of $u_{k}$. These sectors follow from: $\omega_{k}=\left\{\mathbf{x} \mid u_{k}(\mathbf{x})>\right.$ $u l(\mathbf{x}), k \neq l\}$, where $\mathbf{x}$ represents a vector in the representation space. This mode of training is called 'supervised learning'. A regular element of the training phase is the validation phase, where a independent and typically smaller set of known object types is used to assess the performance of the classifier.

In the operation mode the system uses this acquired information to classify the object from information from a currently observed object. This information can be represented by a point $\mathbf{x}_{0}$, or an error-ellipse, in the representation space. Then the computed probability-distribution can be used to obtain the most likely defect class: $k_{0}=\arg \max _{k} u_{k}\left(\mathbf{x}_{0}\right)$. Similarly, the sector $\omega_{k 0}$, corresponding to the position $\mathbf{x}_{0}$, indicates the same object class $k_{0}$. 


\subsection{Parameter Selection by Functional Optimisation}

The main objective of parameter selection is choosing that experiment which will generate the most decisive information under the current circumstances and in this stage of the interactive classification process ${ }^{1}$. This is performed during the reasoning process as an increasing amount of information accumulates. For this reason the same criteria that determine Functional Parameter Selection can also be used to evaluate termination criteria for the reasoning process. A 'test' or 'experiment' in this context is defined as the statistical relevant empirical measurement of a single parameter. Parameter selection is instrumental in shortening and focusing the reasoning process. Effective parameter selection contributes to faster reasoning and a higher quality of its conclusions. In addition to these, certain conditions can demand for a crucial reduction of the number of tests. This is the case when:

- there is a large number of possible discriminatory tests,

- there is also a large number of object classes,

- object classes partially overlap,

- there are 'costs' involved in the evaluation of a test, such as delays between the start of the experiment and the availability of its results.

In all these cases an effective parameter selection is desired. The situation is applicable to chemical analysis and medical diagnosis, where these conditions are valid. In those cases there exist an ample choice of alternative tests, and the significance of a particular test depends on the entire information already present. In addition, tests will differ in costs and delay before a result is available. The formalism is also applicable on interactive classification of images of printing defects. In that light, the feature extraction algorithms, presented in appendix A, must be interpreted as tests that can be performed on an image of an unknown defect. Some of these tests require considerable computation times. In this context the significance of a test also depends on the information already available ${ }^{2}$.

Starting point of the parameter selection is the clustering of defects in the highdimensional parameter space. We assume that each class can be represented by a (set of) class-prototype(s), that collectively partition the parameter-space. In the remainder of this section we make an assumption that fits in well with our observations; each class can be reasonably well approximated with a Normal Distribution. Based on this assumption, we now can define normalised distance measures in the parameter space. In this section we first define distance measures in the metric parameter space. Next, we define some quantities dependent on such distance measures, such as the relevance of a parameter for a specific defect class. Then we treat parameter selection as the Optimisation of a set of functionals over the parameter space. We define a selection procedure and investigate under which circumstances this procedure converges to a single conclusion.

\footnotetext{
1 Part of this work has been published in [Westra,2000b].

2 If for instance the present hypothesis is that the defect is in the cluster 'defect totally inside the print', more specific that the choice is between defect 'air bubbles' and defect 'ink missing', then the most adequate parameter that can be selected is: 'elipsness', for the only difference between these two defects is the circular shape of the faulty patches in the former case.
} 


\subsubsection{Normalised Metric Distance Measures}

First, we define some normalised metric measures in the parameter space. 'Normalised' indicates the independence of the distance of some of the specific characteristics of the type of distribution that governs the defect-class. The distance $d$ of a point $\mathbf{x}$ to the centre $\mathbf{m}$ of a cluster of points has little relevance without our understanding of the shape and distribution of the individual points of the cluster. By 'comparing' the distance $d$ to a measure $\lambda$, typical for this type of distribution, we obtain a measure $d / \lambda$ that informs us about the relation of $\mathbf{x}$ to the cluster. In this way normalised distances offer a different approach to classification than one based on the membership to a Voronoi-partitioning of the parameter space, even if a class-specific metric is used as decision criterion. In Voronoi-partitioning membership to class $k$ is decided if: $\left\|\mathbf{x}-\mathbf{m}_{k}\right\|_{k}<\|\mathbf{x}-\mathbf{m} /\|$ for all other classes $l$. Here $\|.\|_{k}$ can be a classdependent metric; $\|\mathbf{x}\|_{k}^{2}=\mathbf{x}^{\mathrm{T}} A_{k} \mathbf{x}$. An approach based on normalised distances is also more appropriate for a probabilistic or fuzzy formalism, as $d / \lambda$ can be used as gaugefree argument of a probability measure. A condition for the applicability of normalised distances is the availability of formal descriptors of the type of distribution, such as mean and standard-deviations. For this reason we now focus on multivariate normal distributions. A multivariate normal distributed group of points $\left\{\mathbf{x}_{k}\right\}$ or distribution $\rho(\mathbf{x})$ can be adequately described by its mean $\mathbf{m}=\sum_{k} \mathbf{x}_{k} / n$, or in the continuous case: $\mathbf{m}=\int \mathbf{x} \rho(\mathbf{x}) d^{N} \mathbf{x}$, indicating the centre of mass of the distribution, and its covariance-matrix: $C=\sum_{k}\left(\mathbf{x}_{k}-\mathbf{m}\right)\left(\mathbf{x}_{k}-\mathbf{m}\right)^{\mathrm{T}} / n$, or: $C=\int \rho(\mathbf{x})(\mathbf{x}-\mathbf{m})(\mathbf{x}-\mathbf{m})^{\mathrm{T}} d^{N} \mathbf{x}$, indicating the ellipsoid shape and the principle orientation of the distribution. For multivariate normal distributions a normalised distance between points in space and class centres is given by the well-known Mahalanobis-distance or eccentricity S:

$$
\mathrm{S}_{k}(\mathbf{x})^{2}=\left(\mathbf{x}-\mathbf{m}_{k}\right)^{\top} C_{k}^{-1}\left(\mathbf{x}-\mathbf{m}_{k}\right)
$$

Here $\mathbf{x}$ represents the vector in parameter space corresponding to the actual measurement, and $C_{k}$ is the positive semidefinite covariance-matrix of class $k$. Both $\mathbf{m}_{k}$ and $C_{k}$ are computed over a large ensemble of empirical data for that class. An alternative definition of normalised distance is the geometric distance $D_{k}^{n}(\mathbf{x})$ from a particular point $\mathbf{x}$ to prototype $k$ measured with parameter $n$ :

$$
D_{k}^{n}(\mathbf{x})=\frac{\left|x_{n}-\mu_{k n}\right|}{\sqrt{\sigma_{n k}^{2}+\sigma_{n}^{2}}}
$$

Here $\mu_{k n}$ is the class-centre, and $\sigma_{k n}$ the standard deviation of class $k$ in dimension $n$. $x_{n}$ represents the average result of measurement of parameter $n$, and $\sigma_{n}$ is the standard deviation in that measurement. The denominator utilises that the distance between two normal distributions $N\left(x_{n}, \sigma_{n}\right)$ and $N\left(\mu_{k n}, \sigma_{k n}\right)$ is itself a normal distribution ${ }^{3}$ : $N\left(\left|x_{n}-\mu_{k n}\right|, \sqrt{ } \sigma_{k n}{ }^{2}+\sigma_{n}{ }^{2}\right)$.

${ }^{3}$ If the distribution is not Normal, then the distance is defined as: $D_{k}^{n}(\mathbf{x})=\frac{\left|x_{n}-\mu_{k n}\right|}{\sigma_{n k}+\sigma_{n}}$ 


\section{Separation of Class Prototypes}

These two definitions can be readily extrapolated to the next measure that we are interested in, a measure for the separation between two classes. In the case of the Mahalanobis distance, the separation of two classes $k$ and $l$ can be defined as:

$$
\mathrm{S}_{k l} \equiv \mathrm{S}_{k}\left(\mathbf{m}_{l}\right)
$$

This definition has as disadvantage that it is not-symmetric, as in general $S_{k l} \neq S_{l k}$. As we prefer a symmetric distance definition for the separation of classes, we look at the generalisation of definition [5.2], and thus define the geometric distance $d$ as:

$$
d_{k l}^{n} \equiv D_{k}^{n}\left(\mathbf{m}_{l}\right)
$$

The geometric distance $d_{k l}^{n}$ represents the distance in dimension (i.e. parameter) $n$ between the two classes $k$ and $l$. Following our assumption of normal distributions $N\left(\mu_{k n}, \sigma_{k n}\right)$, and $N\left(\mu_{l n}, \sigma_{l n}\right), d_{k l}^{n}$ can be expressed as the distance between the two class-prototypes normalised to the variances of their distribution.

$$
d_{k l}^{n}=\frac{\left|\mu_{k n}-\mu_{l n}\right|}{\sqrt{\sigma_{k n}^{2}+\sigma_{l n}^{2}}}
$$

This expression again exploits the difference between two normal distributions $N\left(\mu_{1}, \sigma_{1}\right)$ and $N\left(\mu_{2}, \sigma_{2}\right)$ into a new normal distribution $N^{4}\left(\left|\mu_{1}-\mu_{2}\right|, \sqrt{ } \sigma_{1}{ }^{2}+\sigma_{2}{ }^{2}\right)$. This measure indicates whether two distributions are well-separated in dimension $n$. In that case $d>1$, if $d \leq 1$ the classes overlap. The geometric distance between the two classes in the multi-dimensional parameter space cannot simply be combined as: $d_{k l}^{2}=\sum_{n \in E} d_{k l}^{n^{2}}$, because the dependence on the relative orientation of the ellipsoids defined by the covariance-matrices. Incorporation of such a consideration leads to ${ }^{5}$ :

$$
d_{k l}=\frac{\left\|\mathbf{m}_{k}-\mathbf{m}_{l}\right\|}{\sigma_{c f f}}
$$

The main difference between geometric and Mahalanobis distance is the normalisation factor in the denominator, as in [5.7] and [5.8]. This difference makes that the geometric distance is symmetric to classes and the Mahalanobis is not.

${ }^{4}$ If the distribution is not Normal, then the separation is defined as: $d_{k l}^{n}=\frac{\left|\mu_{k n}-\mu_{\ln }\right|}{\sigma_{k n}+\sigma_{\ln }}$

5 The multi-dimensional geometric distance follows from the following consideration. Let $M$ be the line-segment between prototype $\mathbf{m}_{1}$ of class $k$ and prototype $\mathbf{m}_{2}$ of class $l:\left\{\mathbf{x} \mid \mathbf{x}=\mathbf{m}_{1}+\alpha r \wedge \alpha \in[0,1]\right\}$, with: $r=\left(\mathbf{m}_{2}-\mathbf{m}_{1}\right)$. The ellipsoid $H_{1}:\left(\mathbf{x}-\mathbf{m}_{1}\right)^{\top} C_{1}^{-1}\left(\mathbf{x}-\mathbf{m}_{1}\right)=1$ indicates points with equal distance to the prototype of class $k$. Similarly for ellipsoid $H_{2}$ for class $l$. The effective standard deviation in direction $r$ follows from the intersection between $H_{1}$ or $H_{2}$ and $M$, yielding: $\sigma_{\mathrm{l}, e f f}=\left(\mathbf{r}^{\top} C_{k}^{-1} \mathbf{r}\right)^{-1}$, and: $\sigma_{2, e f f}=\left(\mathbf{r}^{\top} C_{l}^{-1} \mathbf{r}\right)^{-1}$. Combination of the normal distributions representing of the two classes gives the effective variance of the difference: $\sigma_{e f f}{ }^{2}=\sigma_{e f f, 1^{2}}+\sigma_{e f f, 2^{2}}$. Thus the separation of class $k$ and $l$ is: $d_{k l}=\|\mathbf{r}\| / \sigma_{e f f}$. 


Fig. 5.2. Representation of three
classes 1,2 , and 3 for two parameters.
The distances between the centers of
the classes ' $i$ and ' $\mathrm{j}$ ' for this
parameter is $d_{i j}$. The distance from
the actual measurement $\mathrm{x}$ to these
classes for parameter ' $\mathrm{n}$ ' is $D_{k}$.

The Relevance of a Parameter for Identifying a Defect Class

Not all parameters are equally relevant for all defect classes. Some parameters are

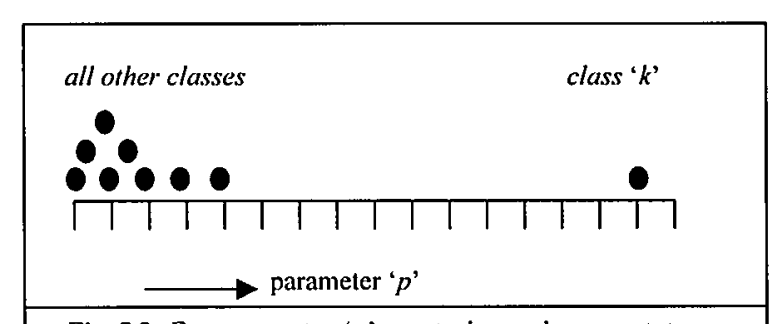

Fig 5.3. For parameter ' $p$ ' most classes have prototypes near $p=0$. The prototype for class ' $k$ ', however, has value near $p=1$. Therefore, $p$ is able to set class ' $k$ ' apart from the rest, and $p$ has high relevance for ' $k$ '. All other classes have low relevance for $D$. extremely important for identifying certain defect classes, while hardly conveying any information for all others ${ }^{6}$. Thus we are looking for a quantity that indicates the relevance from parameter $n$ for defect class $k$, denoted as $r_{k n}$. It is desired to define relevance $r_{k n}$ such that $0 \leqslant r_{k n} \leqslant 1$, increasing from ' $r_{k n}=0$ ',

indicating 'total irrelevance' of parameter $n$ for defect class $k$, to ' $r_{k n}=1$ ', indicating the highest degree of relevance. We define relevance as the ability of a parameter $n$ to set class $k$ apart from all other classes ${ }^{7}$. This is achieved by the following ratio:

$$
r_{k n}=\frac{L_{k n}}{\Delta_{n}}
$$

where:

$$
\Delta_{n}=\max _{k l} d_{k l}^{n}
$$

is the maximum observed distance between any two defect classes for parameter $n$. This value can always be considered to be non-zero, for $\Delta_{n}=0$ would not represent a meaningful clustering. The variable $L_{k n}$ represents a measure to set class $k$ apart. The most forthright proposal would be to let $L_{k n}$ be equal to the normalised distance to the closest class: $L_{k n}=\min _{j} d_{j k}^{n}$. This measure works well if class $k$ is the only class set apart from all other classes by parameter $n$. Especially when all other classes coincide we obtain the desired value ' $r_{k n}=1$ ' for all defect classes for this parameter $n$. But in the case where two classes $k$ and $l$ are set far apart from all others, while in addition these two do coincide, this definition of $L_{k n}$ would give a global relevance $r_{k n}=0$ for all classes for this parameter $n$. This contrasts with the perception that this parameter

\footnotetext{
${ }^{6}$ Most of the parameters constructed for image-analysis where constructed to have a value near 1 for one specific defect class, and practically 0 for almost all others.

${ }^{7}$ For an alternative definitions of relevance see: [Bezdek, 1992].
} 
is able to discern two classes - the ones that coincide - very well from all others. For this reason $L_{k n}$ is defined here as the average normalised distance of class $k$ to all other classes:

$$
L_{k n}=\frac{\sum_{j \neq k} d_{k j}^{n}}{K-1}
$$

where $K$ represents the total number of defect classes. If class $k$ is set apart from all other classes by a maximum distance that equals $L_{k n}$, as in the first example described above, then this definition too leads to a relevance of 1 . This definition however has the disadvantage that the lower value ' $r=0$ ' is never reached, apart from degenerate cases. Since for all meaningful classifications $d_{k l}^{n}>0$ for $k \neq l$, this means that $\left\langle d_{: k}^{n}\right\rangle>0$ for all $k$, therefore $r_{k n}>0$. Renormalisation of $r_{k n}$ with the minimum possible value is not particularly useful because it would make the definition of relevance problem dependent. It would also obstruct comparison between relevances for different parameters.

Notice that, because of the normalisation with $L$, now $r$ only represents the relative separation of the outlier-class. This is because we explicitly required $r$ to fall in the range $[0,1]$. Therefore, $r$ is now resistant to scaling. If we scale the situation depicted in Figure 5.3 with a scaling-factor 10, this does not affect the value for $r$.

\section{Distances in a Clustering of Classes}

A cluster is a group of classes that lie closer to each other than to all other classes. We define the geometric distance in between classes according to definitions [5.7] and [5.8]. The intra-cluster distance in one cluster $C$ is defined as:

$$
d_{C}=\left\langle d_{k l}\right\rangle_{k, l \in C}=\frac{\sum_{k<l \in C} d_{k l}}{c(c-1) / 2}
$$

where $c$ is the number of classes in cluster $C$. Accordingly, the inter-cluster distance for clusters $A$ and $B$ is defined as:

$$
d(A, B)=\left\langle d_{k l}\right\rangle_{k \in A, l \in B}=\frac{\sum_{\substack{k \in A, l \in B \\ k l}}}{a b}
$$

where $a$ and $b$ are the number of classes in clusters $A$ and $B$ respectively. The concepts 'well-separated', and 'overlapping' are defined analogous to class-separation. $d(A, B)$ $>1$ means that the clusters $A$ and $B$ are well-separated. Similarly, $d_{C}<1$ means that the cluster $C$ consists of partially overlapping classes.

\subsubsection{Functional Optimisation Approach to Parameter Selection}

Based on the criteria defined in the past sections we now define an approach for the selection of the optimal test. Optimality follows from the computation of a performance measure, denoted $J$, over all relevant parameters, and the assessment of the subset of parameters that maximise this measure. If the subset is empty the reasoning terminates. If the subset contains multiple elements, other considerations can be used to further restrict the set of suitable parameters. One of these considerations called 'Global Dynamic Relevance' is treated later in more detail. After all considerations have been used, a random element from the remaining set is 
selected. Parameters that have already been selected before are expelled for reexamination: each test is performed only once. This approach is referred to as the functional optimisation method. The symbol $J(n)$ denotes the value of the functional for the parameter with index $n$.

During the classification information is collected regarding the state of the system. Let $E$ be the set of explored parameters $\left\{n_{l}, n_{2}, n_{3}, \ldots\right\}$, and $\widetilde{\mathbf{x}}$ the partial state vector; $\widetilde{\mathbf{x}}=\left(x_{n_{1}}, x_{n_{2}}, x_{n_{3}}, \ldots\right)$. Let $n^{*}$ be the index of the parameter that maximises $J$, so: $J\left(n^{*}\right)=\max _{n \notin E} J(n)$, and let $U^{*}$ be the set of all possible values $n^{*}$. Define $n_{\text {opt }}$ as a random element of $U^{*}$, denoted by: $n_{\text {opt }}=\arg U^{*} 8$. Using this notation we can define optimality of a functional $J$ as the parameter with index $n_{\text {opt }}$ that satisfies:

$$
n_{\text {opt }}=\arg \max _{n \in E} J(n)
$$

In order for this approach to work, we must construct optimality criteria that actually influence the quality and duration of the classification process. Here we consider the following candidates, based on the definitions in the previous sections. The optimal parameters is in all cases given by $[5.14]^{9}$.

\section{Nearest Prototype Selection}

$$
J_{N P}(n \mid \widetilde{\mathbf{x}})=-K^{-1} \sum_{k=1}^{K} D_{k}^{n}(\widetilde{\mathbf{x}})
$$

Functional $J_{N P}(n)$ corresponds to the distance of the actual partial measurement vector $\tilde{\mathbf{x}}$ to the defect class $k$, using the definition of normalised distance from equation [5.2]. Here we have normalised the functional to the number of its summations $K$ to facilitate the optimisation of linear combination of functionals. The minus sign ensures that $J_{N P}$ can be treated as maximisation problem. This functional has as disadvantage that it has only significance for parameters that have already been computed. This is caused by the partial vector $\tilde{\mathbf{x}}$ has values only for tests already performed. If re-examination would be allowed, this functional would lead to the parameter $n$ representing the dimension where the shortest distance from the partial measurement to any of the class-prototypes is found. This represents the basic nearest prototype selection.

\section{Optimal Separation of Classes.}

$$
J^{s e p}(n)=\frac{2}{K(K-1)} \sum_{k_{1}<k_{2}} d_{k_{1} k_{2}}^{n}
$$

The functional $j$ sep is based on the definition of normalised class separation $d_{k_{1} k_{2}}^{n}$ from formula [5.7] above. Optimisation of this functional leads to the parameter $n$ that maximises the distance between the class prototypes. This represents the dimension corresponding to parameter $n$ that shows the largest normalised distance between any pair of defect classes. This functional is extremely practical to prune the search tree of possible experiments. It first focuses on the extreme possibilities, i.e. the parameter with index $n$ that exhibits the most extreme classes $k_{1}$ and $k_{2}$. After this parameter has

\footnotetext{
8 If $\mathrm{U}^{*}=\phi$ then $\mathrm{n}_{\mathrm{opt}}$ has a predefined fake value, for instance -1

${ }^{9}$ Notice that constants and positive factors may be omitted: $\arg \max J+C=\arg \max J$, and $\arg \max \lambda J=\arg \max J$ for $\lambda>0$.
} 
been used, the functional leads to the parameter that gives $J$ sep the second largest value - thus to an experiment that separates the next-extreme choice, and so on. This leads to a methodological approach to the reasoning that performs better and faster solutions than random selection or exhaustive exploitation of measurements.

\section{Most Relevant Parameter}

$$
J^{r e l}(n)=\frac{1}{K} \sum_{k=1}^{K} r_{k n}
$$

The relevance functional $\mathrm{frel}$ follows from the definition of relevance in equation [5.9]. Optimisation of this functional leads to the selection of the most relevant parameter. That is the parameter in which dimension one particular class is maximally set apart from all other classes. This is different from $J s e p$, that concentrates on separating two classes maximally. $J^{\cdot e l}$ favours outliers; classes that lie far away from the rest of the classes. Tests on membership to such peripheral classes provides useful information, making $\mathrm{Jrel}$ a useful functional. The disadvantage with this functional, however, is that it is sensitive to the imperfection contained in $r_{k n}$. The relevance matrix $r_{k n}$ is computed from a large number of empirical observations, and thus represents an average over all possible scenarios of measurements and analyses of all defect classes. In specific cases the factual relevance of a parameter may differ from $r_{k n}$.

\section{Computation Time}

Let $\tau_{k n}$ represent the computation time for measuring parameter with index $n$ for class $k$, averaged over a large set of empirical data. It is empirically observed that $\tau_{k n}$ hardly depends on class $k$, therefore a functional for generating fast computation times could be defined as:

$$
J^{\text {lemp }}(n)=-\tau_{n}
$$

Application of this functional leads to the selection of the fastest experiment for a specific defect class. However, the total computation time of the reasoning process is determined not only by optimising the speed of individual experiments, but also - and even more - by the effective accumulation of decisive information. The parameter choice generated by [5.18] will in general not contribute to such an information buildup. Therefore does the application of this functional not lead to globally shorter computation times or high-quality solutions. Nor does it conduct so in combination with other functionals.

\section{Random and Exhaustive Selection}

In order to evaluate the performance of the functionals we compare their results with random and exhaustive selection. In random selection a random element of the not yet explored parameters is selected: $n^{*}=\operatorname{rand}(\{1, . ., K\} \backslash E)$. In exhaustive selection all parameters are explored. 


\subsubsection{Dynamic versus Static Optimisation}

The definitions of parameter selection above hardly utilise any up-to-date information generated during the reasoning process. They are 'static' in the sense that they use fixed criteria for selection. Application of these functionals lead to a fixed sequence of

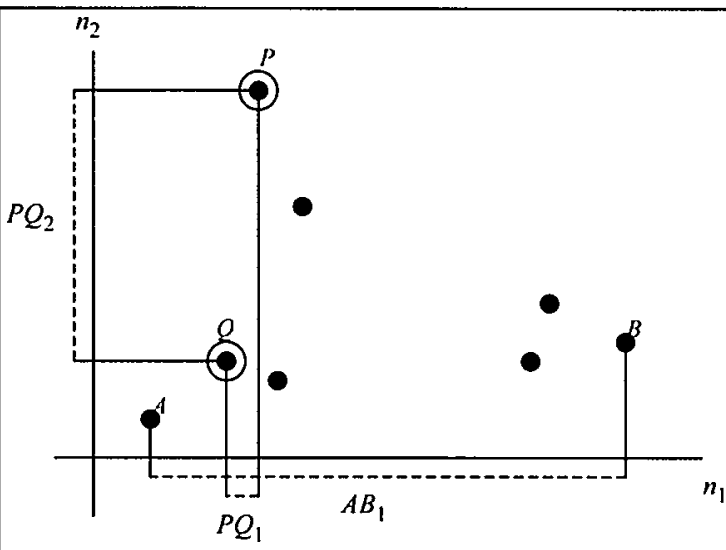

Fig. 5.4. Example of the influence of exponent $\alpha$. In the static scenario $(\alpha=0)$, the largest distance $A B_{1}$ would cause selection of parameter $n_{1}$. If the actual possibilities are included in the dynamic scenario $(\alpha>0)$, all classes have possibility zero, except $P$ and $Q$. This will cause the selection of $n_{2}$, for $P Q_{2}>P Q_{1}$. parameters every iteration. If the state of the classification process until now can be expressed as a hypothesis represented by a possibility distribution $P_{k}$, or equivalently, by the membership functions $u_{k}$, this information could be used to enhance and focus the selection. Therefore a logical approach is to multiply the components of the functional containing a summation over the defect classes with the value of the present possibility of that class: $u_{k}$. In contrast to the previous - static definitions, this creates a scenario where the selection of the optimal parameter is influenced by the possibility distribution $u_{k}$. This latter scenario is named dynamical. This however creates the danger of premature convergence. Especially in the initial stages of the reasoning process, when $u_{k}$ can be expected to contain a high degree of uncertainty, the present hypothesis should not be allowed to influence the parameter selection too much. Such influence could create circular reasoning; enforcing the present hypothesis by focussing on those experiments that would confirm it rather than the ones that could contradict it, or confirm a competing hypothesis. The intermediate between these two extremes can be expressed by attributing a 'dynamicallity exponent' ' $\alpha$ ' to the factor $u_{k}$ : can be expressed by attributing an exponent ' $\alpha$ ' to the membership function: $u_{k}^{\alpha}$, relating to the uncertainty in $u$. If $\alpha=0$, causing $u_{k}^{\alpha}$ to be 1 , we have a static scenario where no actual information is used. If $\alpha=1$, causing $u_{k}^{\alpha}$ to be equal to $u_{k}$, we obtain an scenario where all information is used - including the danger of premature convergence. In order to control the classification process to some extent and prevent premature convergence, this exponent should in fact start as 0 and gradually increase towards 1 as the classification process progresses. However, in practice classification processes are often too short to give general evolution schemes for $\alpha$. Alternatively, an optimal constant value for $\alpha$ in each stage of the classification can be calculated from considerations on the amount of uncertainty in $\alpha$. Figure 5.4 shows an example of the influence of dynamic selection on the selection process. 
Application of the term $u_{k}^{\alpha}$ leads to the following dynamic functionals:

The Dynamic Separation Functional

$$
J_{\alpha}^{s e p}(n)=\frac{2}{K(K-1)} \sum_{k_{1}<k_{2}} d_{k_{1} k_{2}}^{n} u_{k_{1}}^{\alpha} u_{k_{2}}^{\alpha}
$$

For $\alpha=0$ this expression degenerates to [5.16] and the reasoning is static. This leads to the selection of the parameter that generates the largest a priori separation between the classes. For $\alpha=1$ the reasoning is fully determined by actual probabilities. Distances between two classes are multiplied with the product of their probabilities. Classes with low probabilities therefore are virtually cancelled from the summation. This leads to the selection of the parameter that leads to the largest relevant separation of classes, so to the maximal pruning of allowable classes. Therefore, the reasoning process focuses on relevant test parameters that cut away the most viable competing classes given the present measurements.

\section{The Dynamic Relevance Functional}

$$
J_{\alpha}^{r e l}(n)=\frac{1}{K} \sum_{k=1}^{K} r_{k n} u_{k}^{\alpha}
$$

This functional promotes selection of the parameter which sets the current most likely class maximally apart from all other classes. A comparable consideration on the effect of incorporating actual probabilities can be given as above.

The nearest prototype functional can not be generalised with the actual possibility distribution in a similar way. The reason is that $u_{k}^{\alpha}$ increases while $D_{k}^{n}(\mathbf{x})$ decreases with increasing significance. Therefore the term $D_{k}^{n}(\mathbf{x}) u_{k}^{\alpha}$ is meaningless for optimisation.

\subsubsection{Weighted Multi-Criteria Optimisation}

Consider the problem of finding a parameter $n$ that both maximises relevance, and minimises computation time. Simply averaging the corresponding functionals as: $J m c=(J r e l+J l e m p) / 2$ appears like a suitable candidate. However, this will normally not lead to the desired solution. The optimum of a linear combination of functionals is in general not optimal for any of its constituents. So, the solution $n_{m i x}^{*}$ is generally non-optimal for both $\mathrm{j}$ rel and Jemp. Such combinations of functionals lead to complex multi-criteria problems, mostly leading to inferior parameter selection. Table 5.1 shows an example. More general, a mixed functional $f^{m i x}$ can be expressed as a weighted sum of

\begin{tabular}{|c|l|l|l|}
\hline param index & \multicolumn{1}{c|}{$\mathrm{J}_{1}$} & \multicolumn{1}{c|}{$\mathrm{J}_{2}$} & \multicolumn{1}{c|}{$\mathrm{J}_{\text {mc }}$} \\
\hline 1 & 0.46 & 0.4 & 0.43 \\
\hline 2 & 0.55 & 0.25 & 0.4 \\
\hline 3 & 0.21 & 0.55 & 0.38 \\
\hline 4 & 0.5 & 0.5 & 0.5 \\
\hline
\end{tabular}

Table 5.1 Concurrent optimisation of functionals. Optimal values are indicated by bold characters. The index that optimises the mixed functional is not optimal for either of the two functionals. individual functionals: $J^{m i x}=\sum_{i} \lambda_{j} J_{i}$. With an additional constraint on the weights: 
$\sum_{i} \lambda_{i}=1$. The weights $\left\{\lambda_{k}\right\}$ directly determine the parameter which generates the solution. The weight space $\left\{\lambda_{k}\right\}$ is partitioned in regions where different parameters generate the optimum of the mixed functional. This is true in each stage of the reasoning process, represented by the actual measurement vector $\tilde{\mathbf{x}}$. Though the optimisation of the mixed functional does not optimise any of the original criteria, in practice, this approach leads to useful solutions. It may be observed that some functionals 'cooperate' better then others. A specific case is if we let the weights depend on the stage in the reasoning process. Each of the functionals has it optimal phase in the reasoning process; in the beginning the separation functional may lead to good results while in later stages the relevance of the parameter becomes more important. The best values for the weights depend on the stage of the reasoning process. In this respect they are similar to the dynamicallity exponent $\alpha$ that depends on the uncertainty in the membership functions $u_{k}$. For this reason it is useful to base the values of the weights on $\alpha$ as: $\lambda_{k}(\alpha)$. A more profound approach is the fusion of functionals, as described in the next section.

\subsubsection{Fusion of Functionals}

The relevance functional $f r c l$ leads to a parameter index $n_{\text {opt }}$ with the most likely optimal relevance. The nearest-prototype functional $J N P$ leads to a parameter in which dimension the smallest distance of the measurement to any of the prototypes can be found. In the first functional the summant increases monotonously with $r_{k n}$ and $u_{k}^{\alpha}$. In the second functional, however, the summant decreases with increasing distance $D_{k}^{n}(\mathbf{x})$. The measurement $\boldsymbol{x}$ with parameter index $n$ is most significant for class $k$ when $D_{k}^{n}(\mathbf{x})=0$, so when it coincides with the class-prototype $\boldsymbol{m}_{k}$. If measurement $\boldsymbol{x}$ moves from prototype $m_{k}$, so $D_{k}^{n}(\mathbf{x}) \rightarrow \infty$, then parameter $n$ becomes totally irrelevant for class $k$. For that reason $J N P$ is equipped with a minus sign. This inconsistency is particularly awkward, as it is attractive to fuse these two functionals. The reason is that as $J^{N P}$ is restricted to parameters that have already been established, the relevance $r_{k n}$ is applicable to all classes. Thus by 'chaining' the normalised distance $D_{k}^{n}(\mathbf{x})$ with the relevance $r_{k n}$, a Nearest Prototype-like functional could be obtained that applies to all parameters. We therefore look for considerations that can harmonise $J N P$ with $\mathrm{Jrel}^{\mathrm{r}}$. Let us take as objective to find a modelling of $J^{N P}$ that resembles $J$ Nrel. The range of the summant of $J^{r e l}$, the expression $r_{k n} u_{k}^{\alpha}$, is the interval $[0,1]$. So we are looking for a function $\psi: \mathbb{R} \rightarrow[0,1]$ of $D=D_{k}^{n}(\mathbf{x})$, such that the significance of $\psi$ increases monotonously from $\psi=0$ for insignificant $D=\infty$, to $\psi=1$ for significant $D$ $=0$; the case where $x$ coincides with the class prototype. Of the infinite amount of possibilities we select $\psi(D)=\exp (-D)$. The combination of our choice for $\psi(D)$, relevance and possibility thus leads to a dynamical functional for Global applicable Nearest Prototype selection.

$$
J_{\alpha}^{G N P}(n \mid \widetilde{\mathbf{x}})=\frac{1}{K} \sum_{k=1 j \in E}^{K} \sum_{j \in E} r_{k n} \exp \left(-D_{k}^{j}(\widetilde{\mathbf{x}})\right) u_{k}^{\alpha}
$$


Where $E$ represent the set of indices of the examined parameters. This functional chains the limited range of $D_{k}^{n}(\mathbf{x})$ to the general applicability of $r_{k n}$. The chaining causes that for a specific examined parameter $j$, the factor $\exp \left(-D_{k}^{j}(\widetilde{\mathbf{x}})\right)$ quantifies its significance for any specific defect class $k$. Through the summation this is linked with the relevance matrix $r_{k n}$ that conveys the relevance of class $k$ for any parameter $n$. Thus the summation generates a Nearest Prototype-like functional that now applies to all parameters. However the same objection to the sensitive dependence on $r_{k n}$ can be made as above.

The result can be interpreted as follows. $\exp \left(-D_{k}^{j}(\widetilde{\mathbf{x}})\right)$ indicates the defect classes that are important for the parameters $j$ that have already been established - because of their distance to the respective defect centres. $r_{k n}$ relates to the parameters $n$ that convey relevant information for class $k$. Thus the chaining of both indicates those tests $n$ that convey information on the most likely hypotheses generated by the measurements $\{j\}$ up till now.

In a similar way the separation functional [5.19] can be combined with relevance functional [5.20]:

$$
J_{\alpha}^{\text {seprel }}(n)=\frac{4}{K^{2}(K-1)^{2}} \sum_{k=1 /=1}^{K} \sum_{k l}^{K} d_{k n}^{n} r_{k n} \eta_{n} u_{k}^{\alpha} u_{l}^{\alpha}
$$

In this separation/relevance functional the actualised term for the separation of two classes in dimension $n ; d_{k l}^{n} u_{k}^{\alpha} u_{l}^{\alpha}$, is multiplied by their relevances for parameter $n$ : $r_{k n} r_{l n}$. This definition will bias towards the selection of the most likely relevant parameters to separate classes. An argument against such an integration would be that both $d_{k l}^{n}$ and $r_{k n}$ are based on normalised metric distances in the parameter space. Mingling them would lead to a functional, with different expressions for comparable and partially redundant meaning. Moreover, this would multiply the inaccuracy contained in the relevance matrix, as described above.

\subsubsection{Global Constraints on Parameter Selection}

As the classification process progresses other constraints may be used to limit the domains of the remaining unexplored parameters. Ideally, we are looking for meaningful entities that alter monotonously as the process 'homes in' on the solution. An example of such a quantity is the information entropy $E_{k}$ [Shannon, 1948]:

$$
E(t)=\Sigma_{k} E_{k}(t)=-\Sigma_{k} u_{k}(t) \ln u_{k}(t)
$$

with $u_{k}(t)$ the current possibility distribution. This quantity could be used in limiting the choice of suitable parameter domains to a subset for which after establishment of the appropriate measurement and computation of $E(t+1)$ is found:

$$
\Delta E(t+l) \equiv E(t+1)-E(t)>0
$$

This could be performed as an expectation value, based on the known correlations between performed and open measurements. This would have as computational disadvantage that first the resulting distribution $u_{k}(t+l)$ for each possible parameter- 
selection and each possible outcome for this parameter should be calculated to see whether this constraint applies.

\section{Relation between Global Constraints and Functional Optimisation}

Suppose that $Q_{t}$ is a meaningful quantity that can be used to constraint the searchspace in iteration $t$. In analogy with entropy, the constraint is defined as the selection of that decision which maximises $\Delta Q_{t+1} \equiv Q_{t+1}-Q_{t}$. Suppose furthermore, that $Q$ can be written as a summation over all explored parameters: $Q_{t}=\sum_{n \in E_{t}} q_{n}$. Let $n_{t+1}$ be the parameter selected at step $t+1$. Then $Q_{t+1}=Q_{t}+q_{n_{t+1}}$, so optimisation of $\Delta Q$ implies optimisation of $q_{n}$. Therefore, these types of constraints can be understood as a functional optimisation of a functional $J(n)=q_{n}$.

Alternatively, useful global quantities can be constructed from the functionals defined in the previous sections. One such quantity is based on the relevance matrix, defined in equation [5.9]. Define the total actual relevance for parameter $n$ as:

$$
R_{n}(t)=\sum_{k=1}^{K} r_{n k} u_{k}(t)
$$

and define:

$$
R(t)=\sum_{n \in E_{f}} R_{n}(t)
$$

as the global actual relevance for the set $E_{t}$ of explored parameters. $R$ represents the total relevance of the present hypothesis, represented by $u_{k}$, for the set of explored parameters. Now, in line with such global constraints, allow only those selections that maximise: $\Delta R(t)$. This expresses the increase of the total relevance of the explored parameters given the present hypothesis $u_{k}(t)$. Clearly, the application of the constraint 'maximise $\Delta R$ ' is equivalent to the optimisation of the relevance functional for $\alpha=1$.

This entity can also be used to visualise the selection procedure. Let $\boldsymbol{u}$ be the vector containing the hypothesis at step $t+1 ; \mathbf{u}=\left(u_{k}(t+1)\right)_{k=1: K}$. Vector $\boldsymbol{u}$ is restricted to a sub-space $\Omega$; at least the hypercube $[0,1]^{K}$, or - if multiple defects are not allowed the hyperplane $\Sigma_{k} u_{k}=1$. The constraint [5.26] is a linear form in $u: \Delta R_{n}=r^{(n)} \cdot u-\mathrm{R}$, where $n$ is the candidate parameter for selection. Vector $r(n)$ is a vector with components: $r(n)_{k}=\Sigma_{m \in E} r_{m k}+r_{k n}$, and $R=R(t)$, which is a constant for step $t+1$. For each $n$ this is an optimisation problem that can be solved by polyhedral search or gradient descent in subspace $\Omega$. The solution to this optimisation problem is the parameter with index: $n_{\text {opt }}^{*}=\arg \max _{n \notin E} E_{t} \max _{\mathbf{u} \in \Omega} \Delta R_{n}$. This is the same parameter that maximises $J_{\alpha}^{r e l}(n)$ for $\alpha=1$. This optimisation is visualised in the figure below, where $\Omega$ is the subspace $\Sigma_{k} u_{k}=1$, and $H_{n}$ is the hyperplane $r(n) . u=$ constant. In the upper-half of $\Omega$ lines parallel to $H_{n}$ are indicated. Also indicated is the gradient of $H_{n}$, the vector $r(n)$. Optimisation of $\Delta R_{n}$ on $\Omega$ is determined by shifting $H_{n}$ in the direction of $r(n)$. This leads to the corner point with coordinates: $k=(0,0,1)-$ so a choice for class 3. Therefore, in this case the optimal value of $\Delta R_{n}$ for candidate $n$ is: $\Delta R_{n}=$ 
$\Sigma_{m \in E} r_{m k_{3}}+r_{k_{3} n}-R(t)$. For each candidate $n$ in a similar way the edge or corner that maximises $\Delta R_{n}$ can be calculated.

Yet another constraint on the reasoning process can be based on the global relevance $R(t)$. This constraint must prevent premature convergence by requiring that the process may not terminate as long as $R(t)$ is below a certain threshold $R_{\min }$. This constraint ensures that a significant fraction of the relevant parameters for the actual hypothesis $u$ have to be explored. This constraint can be used as one of the termination criteria as a negative criterion:

$$
\text { continuation constraint: while }\left(R(t)<R_{\min }\right) \text { "do not terminate" }
$$

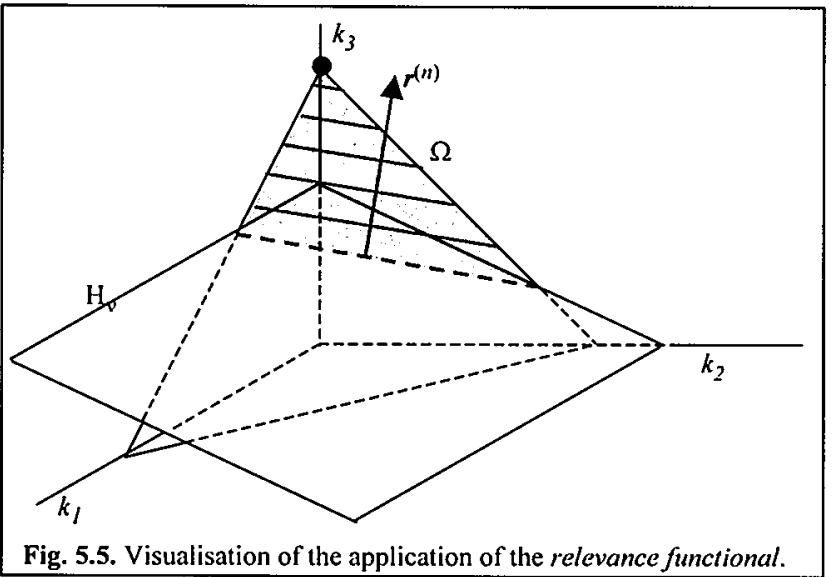

\section{Maximal Reduction of the Parameter Space}

For each parameter $x_{n}$ there is an associated domain $X_{n}$, containing its possible values. Let $X_{n}^{0}$ denote the $a$ priori domain of the parameter. As the reasoning process proceeds, correlations of parameter $n$ with the already measured parameters may cause the domain to shrink monotonously: $X_{n}^{t+1} \subseteq X_{n}^{t} \subseteq . . \subseteq X_{n}^{0}$. Let $V_{t}$ be the volume of the remaining parameter domains in step $t$. This quantity is a monotonously decreasing function of $t$. Now an extra constraint on the reasoning process would be to favour the selection of that parameter that maximises $\Delta V_{t+1} \equiv V_{t+1}-V_{t}$. Based on empirical data a probability distribution can be constructed on the product of the domains, given the currently performed measurements. Let $p_{n}\left(\xi \mid\left(x_{n_{1}}=a_{1}\right) \wedge\left(x_{n_{2}}=a_{2}\right) \wedge . .\left(x_{n_{t}}=a_{t}\right)\right)$ be the conditional probability that $x_{n}$ has a value between $\xi-\mathrm{d} \xi$ and $\xi+\mathrm{d} \xi$, given the values of the explored parameters with indices $E_{t}=\left\{n_{1}, n_{2}, . ., n_{t}\right\}$. This could be given in the form of Bayesian network. With these conditional probabilities it is possible to estimate the most probable value of $x_{n}$ and its associated standard deviation using: $\left\langle x_{n}\right\rangle=\int \xi p_{n}\left(\xi \mid A_{t}\right) d \xi \quad$ and $\left\langle x_{n}^{2}\right\rangle=\int \xi^{2} p_{n}\left(\xi \mid A_{t}\right) d \xi$. Here, $A_{t}$ denotes the set of measured parameter values: 
$A_{t}=\left\{\left(x_{n_{1}}=a_{1}\right) \wedge\left(x_{n_{2}}=a_{2}\right) \wedge . .\left(x_{n_{t}}=a_{t}\right)\right\}$. Moreover, this model can be used to calculate the new conditional probabilities for the remaining parameters, and thus the expectation value of the remaining volume $V_{1+1}$. The new probabilities are calculated as: $p_{j}\left(\xi \mid A_{t} \wedge\left(x_{n}=a\right)\right)=p_{j}\left(\xi \mid A_{t}\right) p_{n}\left(a \mid A_{l}\right)$, where $n$ is the selected parameter in step $t+1 ; n=n_{t+1}$, and $a$ is the expectation value of $x_{n} ; a=\left\langle x_{n}\right\rangle$. This equation can also be used to expand $p_{n}\left(a \mid A_{t}\right)$, and so iteratively expand $p_{j}\left(\xi \mid A_{t+1}\right)$ in a product of all selected parameter values. For each possible selection $n$, the volume is estimated by the product of the standard deviations:

$$
\left\langle V_{t+1}\right\rangle=\prod_{j} \sigma_{j}(t+1)
$$

with:

$$
\sigma_{j}(t+1)^{2}=\omega_{j 2}(t+1)-\omega_{j 1}^{2}(t+1)
$$

and:

$$
\omega_{j k}(t+1)=\int \xi_{j}^{k} p_{j}\left(\xi_{j} \mid A_{t} \wedge\left(x_{n}=a\right)\right) d \xi_{j}
$$

The integration is performed over the a priori domains. For measured parameters $\sigma_{j}$ is the obtained standard deviation. In this way we can find $\left\langle\Delta V_{t+1}\right\rangle_{n}$ for each notexplored parameter $n$, and thus treat this quantity as a normal functional optimisation as discussed before, with associated dynamic functional:

$$
J^{v o l}(n, t)=\left\langle\Delta V_{t}\right\rangle_{n}
$$

This leads to the selection of parameter with index:

$$
n_{\text {opt }}^{*}(t)=\arg \max _{n}\left\langle\Delta V_{t}\right\rangle_{n}
$$

Here we have assumed that the empirical data satisfies a normal distribution, as described in Chapter 4 . In this case the probability distribution for each class $k$ is given by: $p_{k}(\widetilde{\mathbf{x}})=\operatorname{det}\left(\widetilde{C}_{k}\right)^{-1} \exp \left(-1 / 2\left(\widetilde{\mathbf{x}}-\widetilde{\mathbf{m}}_{k}\right)^{\mathrm{T}} \widetilde{C}_{k}^{-1}\left(\widetilde{\mathbf{x}}-\widetilde{\mathbf{m}}_{k}\right)\right)$, where a constant factor is omitted that is equal for all classes. The tilde $(\sim)$ indicates that only a subset of the indices is used. For $\widetilde{\mathbf{m}}_{k}$ and $\widetilde{C}_{k}$ the indices are in subset: $E_{t} \cup\{n\}$, where $n$ is a candidate not-explored parameter. The partial vector $\tilde{\mathbf{x}}$ consists of the measured values in $A_{t}$ plus one variable $x_{n} ; \widetilde{\mathbf{x}}=\left(\widetilde{\mathbf{a}}, x_{n}\right)$. Combination of these equations leads to:

$$
p_{k}\left(x_{n}\right)=\operatorname{det}^{-1 / 2}\left(\widetilde{C}_{k}\right) \exp \left(-1 / 2\left(c_{0}+c_{1}\left(x_{n}-m_{k n}\right)+c_{2}\left(x_{n}-m_{k n}\right)^{2}\right)\right)
$$

with coefficients: $\left.c_{0}=\left(\tilde{\mathbf{a}}-\tilde{\mathbf{m}}_{k}\right)^{\mathrm{T}} \widetilde{C}_{k}^{-1}\left(\tilde{\mathbf{a}}-\tilde{\mathbf{m}}_{k}\right)\right), c_{1}=\sum_{i \in E_{l}}\left(C_{i n}^{-1}+C_{n i}^{-1}\right)\left(a_{i}-m_{k i}\right)$, and $c_{2}=C_{n n}$. In this way a probability distribution for variable $x_{n}$ can be constructed: $p_{n}\left(x_{n}\right)=\sum_{k} w_{k} p_{k}\left(x_{n}\right) / \sum_{k} w_{k}$, where $w_{k}$ is the a priori probability of defect class $k$. The volume $V_{t}$ thus calculated depends on the distance between the partial measurements and the class centres $\widetilde{\mathbf{m}}_{k}$, and the non-zero off-diagonal elements of $\widetilde{C}_{k}^{-1}$. Using equations [5.28] to [5.33], the best value for $n_{\text {opt }}^{*}(t)$ can be obtained. The functional $J \mathrm{vol}$ thus explicitly utilises the history of the reasoning process. It generates a solution that maximally reduces the remaining search-space. A disadvantage of this approach is its computational effort, as the solution to [5.30] must be numerically approximated. 


\subsection{The Fusion of Heterogeneous Information}

During the reasoning process an increasing amount of information is accumulated, representing a variety of probability measures with different kinds of degrees of belief and uncertainty, and originating from a number of unrelated information sources. Some of these sources, such as the feature parameters introduced in appendix A, can best be regarded as fuzzy measures. Others express statistical information, or stem from noise or randomness created by the physical characteristics generated in the sensors, and are therefore better modelled as probability measures. Therefore, one must combine quantitative measurements, probabilistic expressions, and qualitative criteria into one single hypothesis about the state of the system. First, we make some general remarks about information fusion. Next, some attention is devoted to Fuzzy Integration. Finally, Multivariate Covariant Analysis is described as basis for probability distribution and fuzzy memberships to the defect classes.

\subsubsection{Probability Fusion by a Weighted Average}

The most simple and straightforward approach to information fusion is in combining the probabilities of the different information sources. The integrated hypothesis is then expressed as a weighted average of the probabilities originating from the relevant sources. This approach thus relies on a probabilistic modelling of the underlying concepts. If the weights are equal this approach is referred to as a 'voting committee' [Grabisch, 1995]. For instance, when an image is segmented in a set of SearchBoxes $B_{j}$ each with an energy $w_{j}$, and the analysis of a specific set of parameters on each block results in a probability $\mathbf{p}_{j}$, then the cumulative information is a linear combination of these probabilities as:

$$
\mathbf{P}=\Sigma_{j} \mathbf{p}_{j} w_{j} / \Sigma_{j} w_{j}
$$

Where $\mathbf{P}$ is the vector $\left(P_{1}, P_{2}, \ldots, P_{K}\right)$ representing the integrated probability distribution over the $K$ defect classes. Another more specialised approach is not to look at the probability distributed over the collection of SearchBoxes for the ensemble of parameters, but to the probability distributed over the parameters for the ensemble of SearchBoxes. Let $\mathbf{p}_{n j}$ represent the probability resulting from a statistical correct measurement of parameter ' $\mathrm{n}$ ' for SearchBox $B_{j}$. The computation of the cumulative probability $\mathbf{P}$ as defined above, then leads to a second weighing of the probabilities with the relevance $r_{k n}$ of parameter ' $n$ ' for the specific defect class ' $k$ ':

$$
P_{k}=\Sigma_{n j} \mathbf{p}_{n j} w_{j} r_{k n} /\left(\Sigma_{j} w_{j} \Sigma_{n} r_{k n}\right)
$$

\subsubsection{Application of Fuzzy Integrals to Defect Classification.}

In this section we apply the theoretic framework developed by Sugeno [Sugeno,1977] and Grabisch et al. [Grabisch et al,1995] on fundamentals of uncertainty calculus, especially the parts devoted to its application to pattern recognition. The task to which we want to apply this framework is the classification of the state of a physical object as observed by a set of sensors to one of $K$ classes. The outputs of the sensors are considered as information 'sources' containing some contextual understanding about the object. Confronted with the unknown vector $\mathbf{x}$, each source $s_{n}$ provides a degree of confidence that $\mathrm{x}$ belongs to class $k$, denoted by $\phi_{\mathrm{n}}(k \mid \mathbf{x})$. Main objective of the 
framework is to integrate the beliefs of the sources into one coherent belief. For this reason it employs the method of 'fuzzy integration' [Dubois, Prade,1996; Grabisch et al., 1995]. Fuzzy integration is based on 'averaging' the 'opinions' of the sources in a consensus-like manner, weighted with the relative significance of the source. Let $\Phi(k \mid \mathbf{x})$ denote the integrated degree of confidence that $\mathbf{x}$ belongs to class $k$, then $\Phi$ can be computed from:

$$
\Phi(k \mid \mathbf{x})=F_{\mu}\left(\phi_{1}(k \mid \mathbf{x}), \ldots, \phi_{N}(k \mid \mathbf{x})\right)
$$

Where $F_{\mu}$ indicates a specific fuzzy integral type like Choquet or Sugeno ${ }^{10}$, and $\mu$ represents a fuzzy measure on the set of sources. The classification of $\mathbf{x}$ can now be assigned to the class $k^{*}$ that maximises [5.34], employing the Nearest Prototype Rule (NPR);

$$
k^{*}=\arg \max _{k} \Phi(k \mid \mathbf{x})
$$

In the discussions below, for simplicity we use this NPR rule, though in fact we employed a more sophisticated method that takes into account the relative and normalised distances between the degrees as described in Section 5.2. First we describe a general approach to multisource classification. Two significant cases can be distinguished:

1. Direct Information Integration. Here each source depends on one different trait of the image. If the number of sources equals the total number of distinguished traits this case is referred to as Multisensor Classification.

2. Indirect Information Integration. Here each source uses all available traits itself. This makes each source a classifier on its own. For that reason this case is called the Multiclassifier Integration.

An example of an indirect integration is a probability measure $p$ over the set of sources, where $p_{n}$ denotes the probability depending on feature ' $n$ '. Then the weighted sum $P_{k}=\Sigma_{n j} p_{n j} w_{j} r_{k n} /\left(\Sigma_{j} w_{j} \Sigma_{n} r_{k n}\right)$ (see equation [5.2]) is an indirect integration. An example of a direct integration is the Multivariate Covariant Probability $P$ over
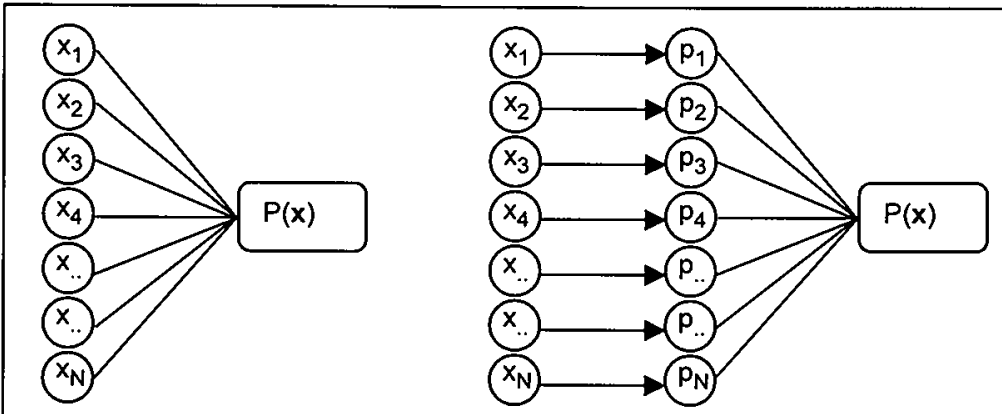

Fig. 5.6. Direct versus Indirect Probability Integration

Left: Direct Information Integration, where all values are directly translated to one belief function over the set of classes. Right: Indirect Information Integration, where all values are first translated in a specific belief function over the set of classes. Second these belief functions are integrated into one, global belief function.

${ }^{10}$ See: [Grabisch et al,1995] 
the set of values $\left\{\mathbf{x}=\left(x_{1}, \ldots, x_{N}\right)\right\}$ of the sources, that is discussed in the next section. Further examples are: Bayesian, classification, rule based systems, and ID3. The latter approach has as disadvantage that first the value $x_{n}$ of source $s_{n}$ is translated into a belief matrix $\phi_{n}(k \mid \mathbf{x})$. Second, the set of beliefs is transformed to one global belief function $\Phi$. In this two-step approach information can be easier lost than in the onestep approach of Direct Integration, where the global belief function directly depends on the set of values of the sources.

A disadvantage of Fuzzy Integration is that the election of only the maximal value under exclusion of all other information often leads to unwanted conclusions. In the application of this formulation to our problem the performance of this approach was inferior to the more classic Multivariate Covariant Analysis that is discussed next.

\subsubsection{Information Fusion for Multivariate Gaussian Mixtures}

Let us now study in detail how information is combined in the case that all individual class-conditional probability distributions are Gaussian. This means that the we interpret the sampling as a Gaussian mixture, i.e. a composite multivariate normal distribution, as defined in section 4.3.

Gaussian distributions are - literary - the normal distributions found in nature. Whenever variations in a measurement are independent, the Gaussian distribution is appropriate. For this reason, but also for its convenient mathematical shape, it is frequently explicitly assumed in parametric clustering and classification methods. Numerous computational vision models and applications based on multivariate Gaussian distribution are reported in literature. For instance, Moghaddam and Pentland [Moghaddam,Pentland,1997] present an unsupervised technique for visual learning and object recognition, based on unimodal and multimodal Gaussian models. W.J. Hwang et al. [Hwang et al.,2000] describe a competitive learning algorithm for parametric classification, explicitly based on a mixture of Gaussian distributions. J.L. Marroquin et al. [Marroquin et al.,2001] construct a class of Gaussian distributed Markov random field models for image reconstruction and classification. C.Biernacki et al. [Biernacki et al.,2000] estimate model parameters of mixed clusters, assuming Gaussian distributions. Moreover, multivariate Gaussian mixtures are a central topic in current research in pattern recognition. Numerous recent publications are available, see [Jain et al.,2000] for a thorough discussion.

Applied to our context, Gaussian mixtures provide an eloquent framework for deeper analysis. In the case that the classes can be adequately described by multivariate normal distributions in the space of the sensor parameters, the information fusion becomes relatively simple. However, here an additional problem is posed by the proper interpretation, and the normalisation of the distribution. We present two different interpretation models that have a distinct normalisation, and exhibit different performance characteristics for clustering and classification.

\section{i. Normalised Multivariate Gaussian Mixtures (NM)}

The first probability model that we present is based on a normalised multivariate normal probability of finding class $k$ given a measurement $\mathbf{x}$. Conform Section 4.2 , in this case the probability $u_{k}$ is given by:

$$
u_{k}=c_{k} \exp \left(-1 / 2\left(\mathbf{x}-\mathbf{m}_{k}\right)^{\mathrm{T}} C_{k}^{-1}\left(\mathbf{x}-\mathbf{m}_{k}\right)\right)
$$


where $m_{k}$ and $C_{k}$ are respectively the prototype, and the covariance-matrix of class $k$. $c_{k}$ is a normalisation constant $c_{k}=\mu(2 \pi)^{-N / 2} \operatorname{det}^{-1 / 2} C_{k}$, where $\mu$ is a normalisation constant such that for each measurement $\mathbf{x}: \sum_{k} u_{k}=1$. Due to this normalisation the distribution elements $u_{k}$ are directly linked, as a change in one element $u_{k}$ causes a chance in all others. For this reason we call this probability model the Normalised Model, short NM. It can be interpreted as the probability of finding the object in state $k$. It was successfully applied in the Fuzzy Multivariate Clustering algorithm FMV in Chapter 4, as an alternative to the Fuzzy C-means (FCM) membership function. Likewise, this function performs well when used for Functional Parameter Selection (FPS), defined in Section 5.2. The coupling through $\mu$ ensures that the for each measurement $\mathbf{x}$ the total probability is 1 , therefore also: $\operatorname{Prob}(k)+\operatorname{Prob}(\neg k)=1$, so $u$ is a valid probability measure.

\section{ii. Uncoupled Multivariate Gaussian Mixtures (UM)}

The second multivariate normal probability model is based on the fuzzy membership of the object to class $k$, given measurement x. Again, $u_{k}$ is given by equation [5.38], but now the coefficient $c_{k}$ assumes the value 1 . In contract to the previous model, this form satisfies all conditions of metric fuzzy membership functions by Diamond and Kloeden [Diamond,Kloeden, 1991]:

- $u_{k} \in[0,1]:$ true.

- $u_{k}$ is normal, i.e. there exist $\mathbf{x}^{*} \in \mathbb{R}$ such that $u_{k}\left(\mathbf{x}^{*}\right)=1$ : true for $\mathbf{x}^{*}=\mathbf{m}_{k}$.

- $u_{k}$ is fuzzy convex, i.e. $\forall{ }_{\mathbf{x}, \mathbf{y} \in \mathrm{IR}} N^{u_{k}}(\lambda \mathbf{x}+(1-\lambda) \mathbf{y}) \geq \min \left\{u_{k}(\mathbf{x}), u_{k}(\mathbf{y})\right\}$ for $\lambda \in[0,1]$ : true, for $f(\lambda)=u_{k}(\lambda \mathbf{x}+(1-\lambda) \mathbf{y})$ itself is a segment of a normal distribution, and therefore fuzzy convex.

- $u_{k}$ is upper semi-continuous: true for $u_{k}$ is continuous.

- the closure of: $\left\{\mathrm{x} \in \mathrm{IR}^{N} \mid u_{k}(\mathbf{x})>0\right\}$ is compact: true, if we assume that all domains of the components $x_{n}$ are compact.

For sets defined by such membership functions they proved that they yield complete, separable, and locally compact metric spaces, in which closed sets ${ }^{11}$ are compact. The functions u presented in expression [5.38] with $c_{k}=1$ are not directly coupled through a normalisation factor. Now there is no direct mathematical link between the classmemberships, as the value of one $u_{k}$ is not influenced by the other $u_{l}$ 's. However, they do interact indirectly through the measurement $\mathbf{x}$, for a small change in $\mathbf{x}$ will influence the values of all components of $\mathbf{u}$. For that reason the multivariate normal probability model defined by these functions is called the Uncoupled Model, short UM. This particular form can successfully be applied to Functional Parameter Selection (FPS). However, when used in the Fuzzy Multivariate Clustering algorithm (FMV) it utterly fails. This is caused by the fact that only in a relatively small region

\footnotetext{
11 provided they obey the so-called Blaschke property
} 
near $\mathbf{x}=\mathbf{m}_{k}$ the value of $u_{k}(\mathbf{x})$ differs significantly from zero ${ }^{12}$. For FPS, in most practical cases a measurement $\mathbf{x}$ will be near one of the prototypes $\mathbf{m}_{k}$, so for at least one class it will have a significant non-zero value. This will favour selection of this class, especially when the other classes generate a value that is almost zero. In the case of FMV, the random initialisation will most probably generate starting-points for the prototypes far outside the collection of data points. Therefore, the values of $\mathbf{u}$ caused by these prototypes at the data points will be extremely small. Consequently, the 'pulling' effect of the actual prototypes on the evolving centroids will be extremely small. As a result the algorithm is likely to terminate before any sensible clustering is achieved.

A more detailed description of information fusion with multivariate Gaussian mixtures is presented in a later section.

\footnotetext{
12 the region: $\left\{\mathbf{x} \mid u_{k}(\mathbf{x})>\varepsilon\right\}$ is the ellipsoid defined by: $\left\{\mathbf{x} \mid\left(\mathbf{x}-\mathbf{m}_{k}\right)^{\top} C_{k}^{-1}\left(\mathbf{x}-\mathbf{m}_{k}\right)>\ln \left(1 / \varepsilon^{2}\right)\right\}$
} 


\subsection{Control of the Reasoning Process}

\subsubsection{Termination Criteria}

The final step in the reasoning process is the decision whether the process could or should be terminated. Again this is a process that must balance carefully between convergence and exploration. Convergence should be promoted, but premature convergence should be avoided. Nor should convergence be gained at the expense of an insufficient exploration of the data, e.g. the image. For our system, the reasoning process is defined at two levels, represented by the Figure 5.1, the general reasoning level, and the interactive analysis level. Both levels are discussed below. First we define a useful quantity regarding the amount of certainty in the final conclusion.

\subsubsection{The General Reasoning Level}

The General Reasoning Level deals with the quality of the actual hypothesis and the exploration in parameter space. Its concerns are to find a balance between focus and exploration. Its objectives are promoting convergence to a high-quality conclusion, whilst avoiding premature convergence. Therefore, this level is domain-independent. Termination on this level can be formalised as a two-valued logical or Boolean function ' $T$ ', its both values being: $\{$ false, true $\}$. These values translate $1-1$ to the continuation of the reasoning process itself: $T(f a l s e)=$ continue, $T(t r u e)=$ stop. Termination of the process can be expressed as a function of the computation time ' $t$ ' spent so far, the search space explored, the quality of the result, and the convergence of the result. Let $X_{n}$ represent the domain of all values of the input parameter with index ' $n$ '. Let ' $t$ ' be represent the number of feature parameters examined so far, and let $E_{t}$ represent the set of indices of the explored parameters: $E_{t}=\left\{n_{1}, n_{2}, \ldots, n_{t}\right\}$; Let $S$ represent the product of the parameter domains: $S=\otimes_{\mathrm{j}} X_{\mathrm{j}}$. Let $S_{t}$ denote the subset of $S$ corresponding to the explored parameter spaces. Let $\mathbf{u}$ represent a possibility distribution over the $K$ defect classes. Then $\Omega=[0,1]^{K}$ is the space of possible values of $\mathbf{u}$. Let $P(\Omega)$ be the powerset of $\Omega$. Then $T$ is the following transformation:

$T: \mathbb{N x} S_{l} \times P(\Omega) \rightarrow\{0,1\}:\left(t, x_{n l}, \ldots, x_{n}, \mathbf{u}_{0}, . ., \mathbf{u}_{1}\right) \rightarrow T\left(t, x_{n}, \ldots, x_{n}, \mathbf{u}_{0}, . ., \mathbf{u}_{1}\right)[5.39]$

A number of efficient termination criteria for the general reasoning level are:

Terminate when the number of iterations ' $t$ ' becomes too large. This represents thresholding the computation time. Since the iteration stops when all possible featureparameters have been used for examination the upper limit to $t$ is $N$.

If the quality $q$ (defined in equation 5.2) of the conclusion exceeds a certain given threshold. In order to prevent premature convergence, this threshold is initially set to 1 and slowly decreases with time.

If the defect-distribution u converges within a given precision. In order to prevent premature convergence this threshold is initially set to 0 and slowly increases with time.

The total relevance, defined in equation [5.9], exceeds a given threshold, indicating sufficient feature-parameters have been explored as in [5.27].

The construction of, and interaction between these and other termination criteria are an important part of the design of the iteration algorithm, that have a large impact on the resulting conclusion. More specifically, rules must be defined which criterion can overrule another under certain conditions. 


\subsubsection{The Interactive Analysis Level}

The Interactive Analysis level is application dependent. Its main objective is to explore the significant parts of the data, and obtain a statistic relevant measurement. In the case of chemical or bio-medical applications this may involve some analyticalchemical experiments of a sample. In the case of computer vision this includes image analysis tests of an image. For the interactive analysis level two loops can be distinguished. This is high-lighted later, in discussing a real-world application in Section 7.4. The principle of the interactive analysis level is shown in Figure 7.12.

The inner-loop represents the detection of convergence. For the measurement of parameter $x$ this loop continues to generate a set of values $\left\{x_{1}, x_{2}, \ldots, x_{m}\right\}$ until the measurement stabilises, i.e. the value $<x>$ converges within the desired precision, or a statistically relevant number of measurements have been performed. Premature convergence is avoided by prescribing a minimal value $m_{\min }$ for $m$. If the convergence has not been attained after a certain while $m_{\max }$, the loop is also terminated. The termination criterion for the inner-loop can therefore be expressed as a function $T_{1}\left(x_{l}, \ldots, x_{m}\right)$.

The outer-loop controls the extent of exploration of the data. In the context of computer vision control this is the CCD-image. Here termination is only allowed if the sum of the energies of the examined SearchBoxes $\left\{B_{1}, B_{2}, \ldots, B_{m}\right\}$ is a minimal portion of the total energy $E$ of the defect. The total energy of the image is: $E_{f o t}=\int_{\text {image }} Z(\mathbf{x}) d_{2} \mathbf{x}$, with $Z$ according to [2.1]. The energy $E_{i}$ from SearchBox $B_{i}$ is:

$E_{i}=\int_{B_{i}} Z(\mathbf{x}) d_{2} \mathbf{x}$. Then a useful termination criterion is given by: $\sum_{i} E_{i} / E_{t o t}>\varepsilon$, where $\varepsilon$ denotes a threshold in $[0,1]$. The outer-loop termination criterion can thus be expressed as a function $T_{2}\left(E_{1}, \ldots, E_{m}, E\right)$. To prevent infinite looping a maximum number is set to the number of cycles.

\subsubsection{Static and Dynamic Termination-Criteria}

The objective of interactive classification is a high-quality conclusion. A direct application of this goal is termination criterion 2 from Section 5.4.2:

$$
\text { terminate if } q(t)>q_{\min } \text {. }
$$

This criterion does not involve any 'memory' of the earlier history of the iteration. For this reason it is a 'static' criterion. During an iteration it can be observed to swap discretely between high and low values, until it finally encounters a situation where it reaches its threshold value. This can be observed in Figure 5.7 below, that shows the evolution of $q$ during the iteration. This disadvantage can be resolved by including some sort of cumulative memory in the criterion. Thus we obtain a 'dynamic' criterion that is a weighted sum of previous terms. In fields where this kind of memory occur, two types of memories are used. One type of dynamic stop-criterion is based on exponentially decaying memory in time, as in Jordan-Elman recursive networks [Elman, 1988]:

$$
c_{\lambda}(t)=\sum_{s=0}^{n} q(t-s) e^{-\lambda s} / \sum_{s=0}^{n} e^{-\lambda s}
$$

A disadvantage is that parameter $\lambda$ must be tuned to the area of application. An other type is simply based on the average over the last $n$ values, as in life-time evaluation fitness functions in Genetic Algorithms [Hillis, 1992],[Whitley, 1989b] : 


$$
c(t)=\sum_{s=0}^{n} q(t-s) /(n+1)
$$

Figure 5.7 shows the evolution of a dynamic stop-criterion, based on the average value of the present plus the last two quality values $(n=2)$. Empirically, a significant difference between static and dynamic criteria is found, showing that a dynamic criterion gives better results. This is not surprising, for it guards the iteration for premature convergence. On the other hand, there was no significant difference between the two dynamic schemes. The average-scheme was slightly better, but within the accuracy. This can be understood from the fact that if the reasoning changes its hypothesis for the most-likely defect, that q restarts from a low value, but the two schemes inherit higher values, especially if the previous hypothesis was already converging to 1 . This is in certain sense a 'false' memory, for the criterion converged to another defect. This unwanted effect persists somewhat larger for exponentially decaying memory, which therefore is slightly inferior.

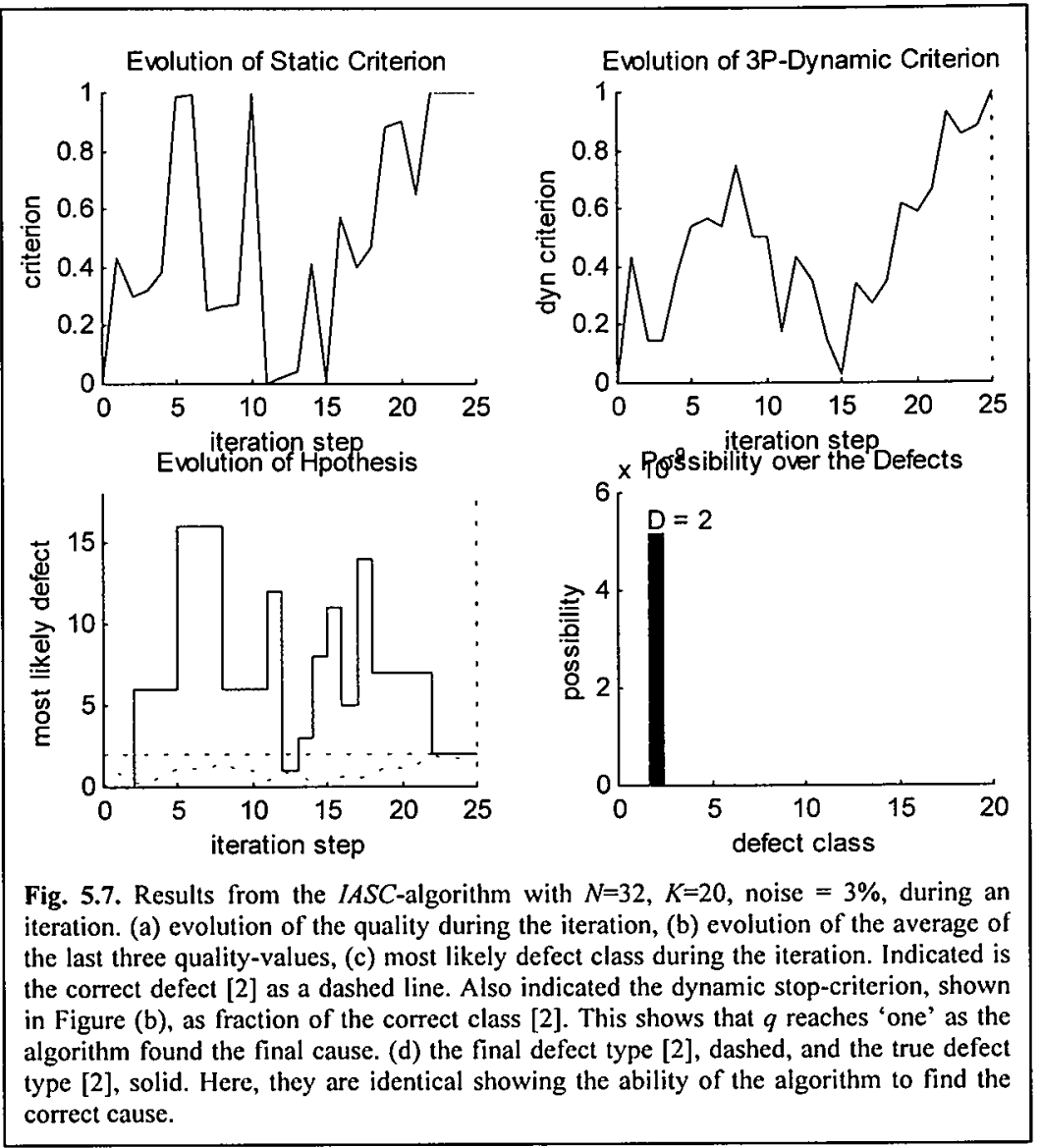




\subsection{IASC: InterActive Sensing and Classification Algorithm}

\subsubsection{Specification of IASC as an Iteration Algorithm}

The topics of the previous sections form the ingredients of an integrated approach for concurrent sensing and classification. This approach can be applied in all situations where one is interested in elucidating the state of a physical object, provided that the object can exist in a discrete number of known states. Moreover, one or more sensors must be available to extract information from the object. The sensing operation itself may contain an active part, as in chemical analysis of a fluid, or a passive part, as in imaging the object. The sensor outputs can be represented as a state vector - or ellipsoid - in the space of all possible measurements, called parameter space. The known states of the physical object are represented as collections in parameter space. Based on the position of the available part of the state-vector in parameter-space relative to the known states, the best subsequent sensing operation can be planned. In this section we describe an application-independent algorithm for InterActive Sensing and Classification, short: IASC. The scope of the IASC-algorithm is the immediate classification of an object based on selective sensor measurements. The objective is to generate the best possible conclusion in the shortest possible time. Examples are: the deduction of the chemical composition of a solid using a collection of physical and chemical tests; remote sensing using a collection of sensors; physical measurements using multi-sensors; and quality monitoring using a CCD-camera. The latter case is discussed in more detail in a later section on the application of $I A S C$.

The general outline of this algorithm was sketched in the introduction 5.1 , and is represented in Figure 5.1. The algorithm consists of three main components. In the Initialisation all constants and parameters receive their initial values. Next, an Iteration Cycle starts in which, firstly, the optimal sensing operation is established given the present set of hypotheses. This is one of the key-steps in IASC, and uses Functional Parameter Selection (FPS) $J:[0,1]^{K} \rightarrow \mathbb{N}: \mathbf{u} \rightarrow n_{o p t}$, as defined in Section 5.2. Deliberately we do not specify what specific combination of functionals is used, for the efficiency of the entire algorithm depends on the relative positions and distributions of the object-states in parameter-space, and may vary for each field of application. Secondly, after the optimal sensing-test has been established, this action is performed on the object using one of the sensors. This may also include a specific algorithmic action on data already available from the object, such as a spectral analysis on a CCD-image. Thirdly, this new measurement is joined with all available information, resulting in a new hypothesis on the state of the object. Like in the case of Functional Parameter Selection this is not further specified, as it depends on the selected representation of uncertainty. Finally, Termination of the iteration cycle is based on the new hypothesis and the extended set of measurements. The final result of the algorithm is a hypothesis on the most likely state of the object.

We can now describe the essential steps in the IASC-algorithm. 


\section{IASC-Algorithm:}

\section{Initialisation}

i. $\quad t=0 ; \mathbf{u}=\mathbf{u}_{0}: E_{0}=\{\} \quad \%$ iteration step $=0$, initial hypothesis, no measurements

\section{Update u with Optimal Test}

$$
\begin{array}{lll}
\text { ii. } & t \leftarrow t+1 & \text { \% increase iteration step with l } \\
\text { iii. } & n_{1+1}=\arg \max J\left(u_{1}, E\right) & \% \text { new test follows from Functional Parameter Selection } \\
\text { iv. } & E_{t+1}=\left\{E_{1}, n_{t+1}\right\} & \% \text { update list of performed measurements } \\
\text { v. } & \left(x_{1+1}, \sigma_{t+1}\right)=\operatorname{Sensors}\left(n_{1+1}, \text { Object) \% measure sensor-output } n_{1+1}\right. \text { from the object } \\
\text { vi. } & X_{t+1}=\left\{X_{1},\left(x_{t+1}, \sigma_{t+1}\right)\right\} & \% \text { update the set of measurements } \\
\text { vii. } & u_{1+1}=p\left(X_{t+1}\right) & \% \text { new hypothesis based on information fusion }
\end{array}
$$

\section{Termination}

viii. $\quad T\left(u_{t+1}, E_{t+1}\right) \quad \%$ calculate the termination criterion

Here, we used the following notations. $K$ represents the a priori possible states of the object, and $N$ the maximum number of parameters that can be extracted based on sensor information. Internally, the algorithm represents a hypothesis by a distribution $\mathbf{u}=\left(u_{1}, u_{2}, \ldots, u_{K}\right), 0 \leq u_{k} \leq 1$, over the $K$ object states. For each specific application the best possible mathematical form - such as Gaussian or uniform, and most suitable semantic interpretation for $\mathbf{u}$ must be designed. For this moment we will not specify whether $\mathbf{u}$ must be interpreted as a probability for state $k$, or a fuzzy membership to state $k$, the perception of the object as belonging to state $k$, or the possibility that state $k$ may be applicable. In addition, a list $E$ is kept of (indices of) parameters that have been sufficiently explored. The measurements ' $x$ ' and the associated standard deviations ' $\sigma$ ' are represented by a set called $X$, that has values only for the components of $E$. The step in the iteration, identical to the number of extracted parameters, is represented by ' $t$ '.

A flow diagram of the IASC-algorithm is shown in Figure 5.8. 


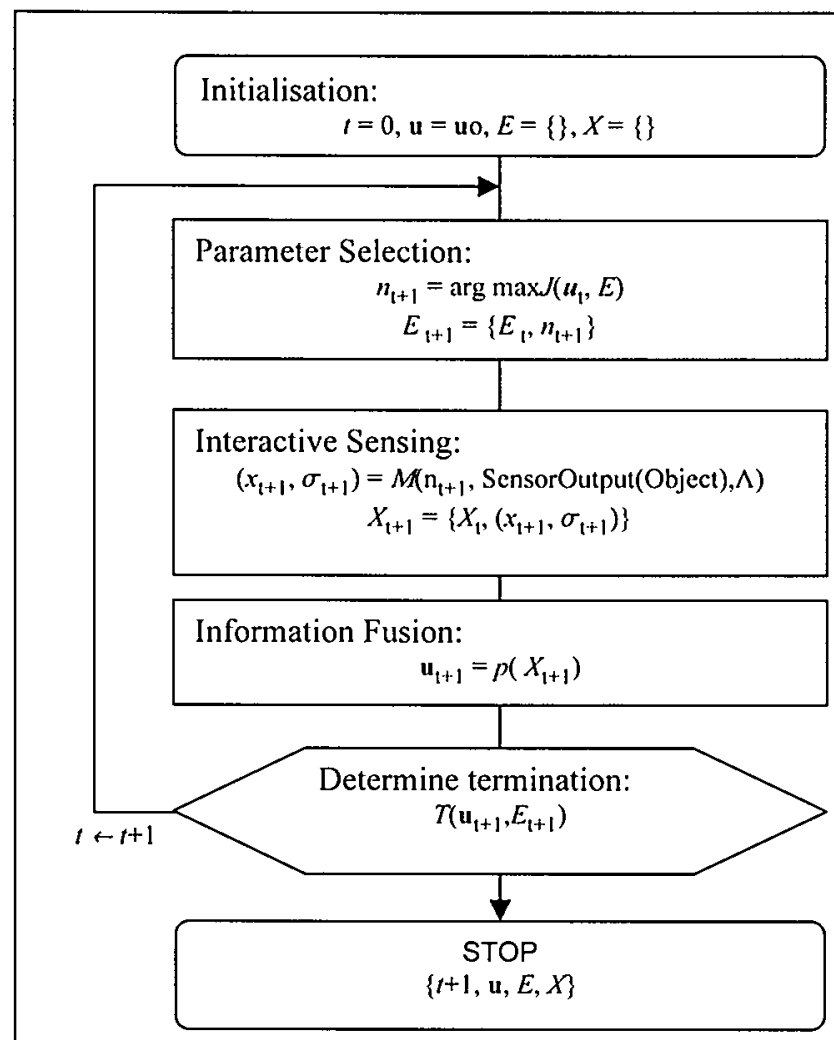

Fig. 5.8. The general $I A S C$ algorithm. 


\subsubsection{IASC as a Transformation on the Space of Hypotheses}

Any outcome of the IASC-algorithm can be represented as an element in the space of all possible hypotheses $\left\{\left(u_{1}, . ., u_{K}\right)\right\}=[0,1]^{K}$. Therefore, the IASC-algorithm induces a mapping ' $\Phi$ ' from $[0,1]^{K}$ on itself. This mapping consists of three components:

(1) The Functional Parameter Selection $n_{\alpha}^{*}(\mathbf{u})=\arg \max _{n \notin E} J_{\alpha}(n, \mathbf{u})$ is a partitioning on the hyper-cube $[0,1]^{K} ; n_{\alpha}^{*}:[0,1]^{K} \rightarrow\{1,2, . ., N\}$. This partitioning is used to define the parameter for measurement.

(2) Let $n \equiv n_{t+1}=n_{\alpha}^{*}(\mathbf{u})$, so the list $E$ of completed measurements is updated as: $E_{t+1} \leftarrow E_{t} \cup\{n\}$. Let us assume that available measurements corresponding to $E_{t}$ do not influence the outcome of the measurement of $x_{n}$. Then the object can be represented by a set of measurements with mean $x_{m}$ and a standard deviation $\sigma_{m}$ : $\left\{\left(x_{m}, \sigma_{m}\right) \mid m=1, . ., N\right\}$. Thus, the object corresponds to a state-vector $\mathbf{x}$ in parameter-space, with: $\mathbf{x}=\left(x_{1}, x_{2}, \ldots, x_{N}\right)^{\top}$. In iteration step $t$ only the $t$ elements in $E_{t}$ are known, so the measurements correspond to a partial state-vector, denoted $\widetilde{\mathbf{x}}$, with: $\widetilde{\mathbf{x}}[i]=\mathbf{x}\left[E_{l}(i)\right]$, and $i=1, \ldots, t$. In iteration step $t+1$ this partial statevector is updated as: $\quad \widetilde{\mathbf{x}}_{t+1}=\left(\begin{array}{l}\widetilde{\mathbf{x}}_{t} \\ x_{n}\end{array}\right)$

(3) The object-state distribution $\mathbf{u}$ is updated as: $\mathbf{u}_{t+1}=f\left(\widetilde{\mathbf{x}}_{t+1}, \tilde{\sigma}_{t+1}\right)$, here $\mathrm{X}$ and $\mathrm{S}$ are the partial state- and standard deviation-vectors. Function $f$ is the appropriate information-fusion of the partial measurements $\left\{\widetilde{\mathbf{x}}_{t+1}, \widetilde{\boldsymbol{\sigma}}_{t+1}\right\}$ into the object-state distribution $\mathbf{u}$.

The entire mapping can thus be represented as a transformation:

$$
\left\{\begin{array}{l}
\mathbf{u} \leftarrow \Phi(\mathbf{u}, \mathbf{x}, E) \\
E \leftarrow \Psi(\mathbf{u}, \mathbf{x}, E)
\end{array}\right.
$$

\subsubsection{IASC Information Fusion for a Multivariate Gaussian Mixture}

If the distribution for sampling a measurement $x \in \mathbb{R}^{N}$ is described by a multivariate Gaussian mixture (see section 4.3.e), then each individual single-state distribution $u_{k} \in[0,1], k=1 . . K$, follows a multivariate normal distribution, represented by classconditional partial centroid $\widetilde{\mathbf{m}}_{k}(t)$ and partial covariance-matrix $\widetilde{C}_{k}$. The 'tilda' $(\sim)$ indicates a partial vector that is composed of measured - hence known elements. These are the elements of set $E_{t}$, so: $\widetilde{\mathbf{m}}[i]=\mathbf{m}\left[E_{t}(i)\right]$, where $E_{t}(i)=n_{t_{i}}$, i.e. the $t_{i}$-th parameter-index. $\widetilde{C}_{k}$ is calculated likewise. Conform Section 5.3.3, the elements of $\mathbf{u}$ satisfy:

$$
u_{k}(t)=c_{k} \exp \left(-1 / 2\left(\widetilde{\mathbf{x}}_{t}-\widetilde{\mathbf{m}}_{k}(t)\right)^{\mathrm{T}} \widetilde{G}_{k}(t)\left(\widetilde{\mathbf{x}}_{t}-\widetilde{\mathbf{m}}_{k}(t)\right)\right)
$$

For $c_{k}=1$ this represents the uncoupled model (UM), and for $c_{k}=\mu_{t}(2 \pi)^{-t / 2} \operatorname{det}^{1 / 2} \widetilde{G}_{k}(t)$ we obtain the normalised model (NM), where $\mu_{t}$ is a 
normalisation constant such that: $\forall_{t} \sum_{k} u_{k}(t)=1$. Matrix $\tilde{G}_{k}$ is the metric tensor for class $k$, and the inverse of the covariance matrix; $\widetilde{G}_{k}=\widetilde{C}_{k}^{-1}$. This entity $G_{k}$ is known from a large set of empirical data. The $[i, j]$-th component of $\widetilde{G}_{k}(t)$ can be computed as: $\widetilde{G}_{k}(t)[i, j]=G_{k}\left[E_{t}(i), E_{t}(j)\right]$. For this particular case, information fusion is simply based on the computation of $\mathbf{u}$ with the updated partial state-vector $\widetilde{\mathbf{x}}_{t+1}$, using equation [5.44]. In iteration step $t+1, \widetilde{G}_{k}$ can be written as:

$$
\widetilde{G}_{k}(t+1)=\left(\begin{array}{cc}
\widetilde{G}_{k}(t) & \mathbf{g}_{k} \\
\mathbf{g}_{k} \mathrm{~T} & \gamma_{k}
\end{array}\right)
$$

with vector $g_{k}: g_{k}[i]=G_{k}\left[E_{t}(i), n\right]$, and scalar: $\gamma_{k}=G_{k}[n, n]$. Similar to [5.42] we define:

$$
\tilde{\mathbf{m}}_{k}(t+1)=\left(\begin{array}{c}
\tilde{\mathbf{m}}_{k}(t) \\
m_{k n}
\end{array}\right)
$$

Using expressions [5.42], [5.45], and [5.46], we can rewrite [5.44] and obtain:

$$
u_{k}(t+1)=A_{k} u_{k}(t) \exp \left(-\beta_{k} \xi_{k n}-1 / 2 \gamma_{k} \xi_{k n}^{2}\right)
$$

with parameter: $\xi_{k n}=\left(x_{n}-m_{k n}\right)$, and constant:

$$
\beta_{k}=\mathbf{g}_{k}^{\mathrm{T}}\left(\widetilde{\mathbf{x}}_{t}-\widetilde{\mathbf{m}}_{k}(t)\right)=\sum_{i=1}^{t} G_{k}\left[E_{t}(i), n\right] \xi\left[k, E_{t}(i)\right] .
$$

In the UM we find $A_{k}=1$, while in the NM we obtain:

$$
A_{k}=\frac{1}{\sqrt{2 \pi}} \frac{\mu_{t+1}}{\mu_{t}}\left(\frac{\operatorname{det} \widetilde{G}_{k}(t+1)}{\operatorname{det} \widetilde{G}_{k}(t)}\right)^{1 / 2} .
$$

In the dynamic scenario $(\alpha>0)$ selection is driven by the classes with the highest probabilities. However, the measurement resulting from this selection need not necessarily favour an increase of the possibilities of these classes. The true direction of change depends on the resulting probability distribution of $u_{k}(1+1)$ as function of the actual measurement $\xi \equiv \xi_{k n}$ :

$$
P\left(u_{k}(t+1)\right)=p(\xi) A_{k} u_{k}(t) \exp \left(-\beta_{k} \xi-1 / 2 \gamma_{k} \xi^{2}\right)
$$

Here $p(\xi)$ is the probability to measure $\xi$ in the interval $\langle\xi-\mathrm{d} \xi, \xi+\mathrm{d} \xi\rangle$, and equation [5.47] is used to denote the conditional probability to measure $\xi$ given the partial measurement $\tilde{\mathbf{x}}$. The expectation value of class $k$ in the next iteration, $\left\langle u_{k}(t+1)>\right.$, follows from integration over $\xi$ :

$$
\left\langle u_{k}(t+1)\right\rangle=A_{k} u_{k}(t) \int p(\xi) \exp \left(-\beta_{k} \xi-1 / 2 \gamma_{k} \xi^{2}\right) d \xi
$$

In case $\xi$ is subject to a normal distribution $N\left(\xi_{0}, \sigma\right)$, then [5.49] can be computed as:

$$
\left\langle u_{k}(t+1)\right\rangle=A_{k} u_{k}(t) \sqrt{\frac{2 \pi}{\gamma_{k}+\sigma^{-2}}} \exp \left(\frac{-2 \beta_{k} \xi_{0}-\gamma_{k} \xi_{0}^{2}+\beta_{k}^{2} \sigma^{2}}{2+2 \gamma_{k} \sigma^{2}}\right)
$$

As apparent from expressions [5.49] and [5.50], it is not possible to predict without additional knowledge whether the probability of an individual class will increase or decrease. This, however, is intuitively clear, for each new measurement can 
potentially alter the emerged picture, as it indicates that some of the hitherto probable classes do not apply, and other previously improbable ones, suddenly become the most likely. For the same reason it is not possible to predict the quality $q$ of the next hypothesis $\mathbf{u}(t+1)$. Therefore, these results can not directly be used to deduct convergence and stability properties of the iteration. 


\subsection{Convergence and Stability of the IASC Algorithm}

In this section we examine the convergence and stability properties of the interactive classification-algorithm IASC. Numerical simulations, that are presented in Chapter 6 , empirically suggest that the approach in most cases finds the correct solution within a fraction of the maximal computation time. Here, we explore the convergence and stability properties in a more formal way. In our context 'convergence' indicates that the algorithm reaches a hard final conclusion on the physical state of the object, e.g. its defect class. 'Stability' is understood as the resistance of the algorithm to noise and small perturbations in the input. In contrast to normal dynamical systems, here it is not necessary to study the behaviour as time increases to infinity, for the final result will always emerge in finite time: the algorithm simply terminates if all $N$ parameters are explored. The first step in our exploration concerns the definition of appropriate criteria to quantify the performance of on-line classification algorithms. Next, we formulate necessary and sufficient conditions for reaching a high-quality, stable conclusions of the algorithm. This also includes considerations on the stability of the iteration, and the optimality of the generated solution. Considerable attention is devoted to studying the volume-decrease in probability-space, represented by the Jacobian, but a conventional proof of convergence based on such an approach fails by its inappropriateness. For that reason we analyse the convergence of the algorithm as a function of its distance to the nearest prototype. The section closes with the description of three models for the evolution of the dynamicallity exponent $\alpha$.

\subsubsection{Criteria for the Performance of On-Line Classification Algorithms}

The quality $q$, defined in Section 5.1 , reflects the uncertainty in the final conclusion. If this uncertainty is low, then the generated conclusion is of high quality, and vice versa. However, the final conclusion of the algorithm must not necessarily coincide with the true state of the object. The potential of the algorithm to find the real state is another important performance criterion. Moreover, we wish to understand how the result of the algorithm depends on small variations in the input. In order to describe the performance of the algorithm, we define the following performance criteria:

- quality ' $q$ ': this parameter is defined in Section 5.1. It indicates whether the algorithm has come to one clear conclusion in its final hypothesis $\mathbf{u} . q$ is defined as the difference between the largest and the second-largest possibilities $u_{1}$ and $u_{2}$, relative to $u_{1}: q(u)=\left(u_{1}-u_{2}\right) / u_{1}$, except when the maximum $u_{1}$ is zero, in which case $q$ is defined as zero.

- success rate ' $S$ ': the probability, conditional for the set of input parameters, that the inferred conclusion is identical to the $t r u e$ state $k^{*}$. So, if a trial with otherwise fixed input parameters, like $K$ and $N$, results in fraction $f$ of correct conclusions, then the success rate, denoted $S$, is equal to $f$.

- relative number of iterations ' $n_{\mathrm{rel}}$ ': this is denoted by the parameter $n_{\mathrm{rel}}$, and indicates the quotient of the realised number of iterations $t$ and the maximal number of iterations $N$; so: $n_{\text {rel }}=t / N$.

- relative computation time ' $\tau_{\text {rel' }}$ ' this equals the quotient of the realised computation time $\tau$ and the maximal computation time $\tau_{\max }: \tau_{\text {rel }}=\tau / \tau_{\max }$. The 
maximal computation time $\tau_{\max }$ follows from: $\tau_{\max }=\sum_{i=1}^{N} \tau_{i}$, where $\tau_{n}$ represents the average computation time of measuring the parameter with index $n$. The realised computation time $\tau$ after $t$ iterations with explored parameter-list $E$, is given by: $\tau=\sum_{i \in E} \tau_{i}$. If all $\tau_{n}$ are equal, then $n_{\text {rel }}=\tau_{\text {rel }}$.

- stability: the tolerance of the algorithm to small perturbations in the input. More specific: if $I A S C$ for a certain observation $\boldsymbol{a}$ in parameter-space converges to a class $k^{*}$, then we call $I A S C$ ' $\delta$-stable' in $\boldsymbol{a}$ if for all $\boldsymbol{x}$ in parameter-space with: $\|x-a\|<\delta, I A S C$ also converges to the same class $k^{*}$.

Most of these performance criteria are contained in $[0,1]$. 


\subsubsection{Visualisation of Reasoning as a Trajectory in Probability Space}

It is illustrative to visualise the IASC-iteration as a trajectory in the space of all possible hypotheses. The properties of this path are a function of the position of the actual measurement $\mathbf{x}$ in parameter space. Let $U$, called the representation space, represent the set of all possible hypotheses $\mathbf{u}=\left(u_{1}, u_{2}, \ldots, u_{K}\right) . U \subset \mathbb{R}^{K}$ is equal to the Cartesian product of the ranges of all object-states, so: $U=[0,1]^{K}$, where $K$ is the number of classes. If we furthermore apply the constraint $\Sigma_{k} u_{k}=1$, we can reduce the number of dimensions by 1 . In this way each possible hypothesis $\mathbf{u}$ can be uniquely represented as a single point in a hyper-isoeder (HIE) contained in $U$. The corners of the HIE represent 'pure' hypotheses; e.g. $[1] \sim(1,0,0, \ldots, 0), \ldots,[K] \sim$ $(0,0, \ldots, 1)$. 'No Defect', represented by: $[0] \sim(0,0,0, \ldots, 0)$, can not be represented in this way. The intermediate steps in the reasoning process can be visualised by the positions of their hypotheses in the HIE. In this way the whole reasoning process is represented by a set of points $\mathbf{u}(t)$ on a trajectory $P$, depicted in Figure 5.9. In case of hard conclusion this trajectory terminates in a corner.

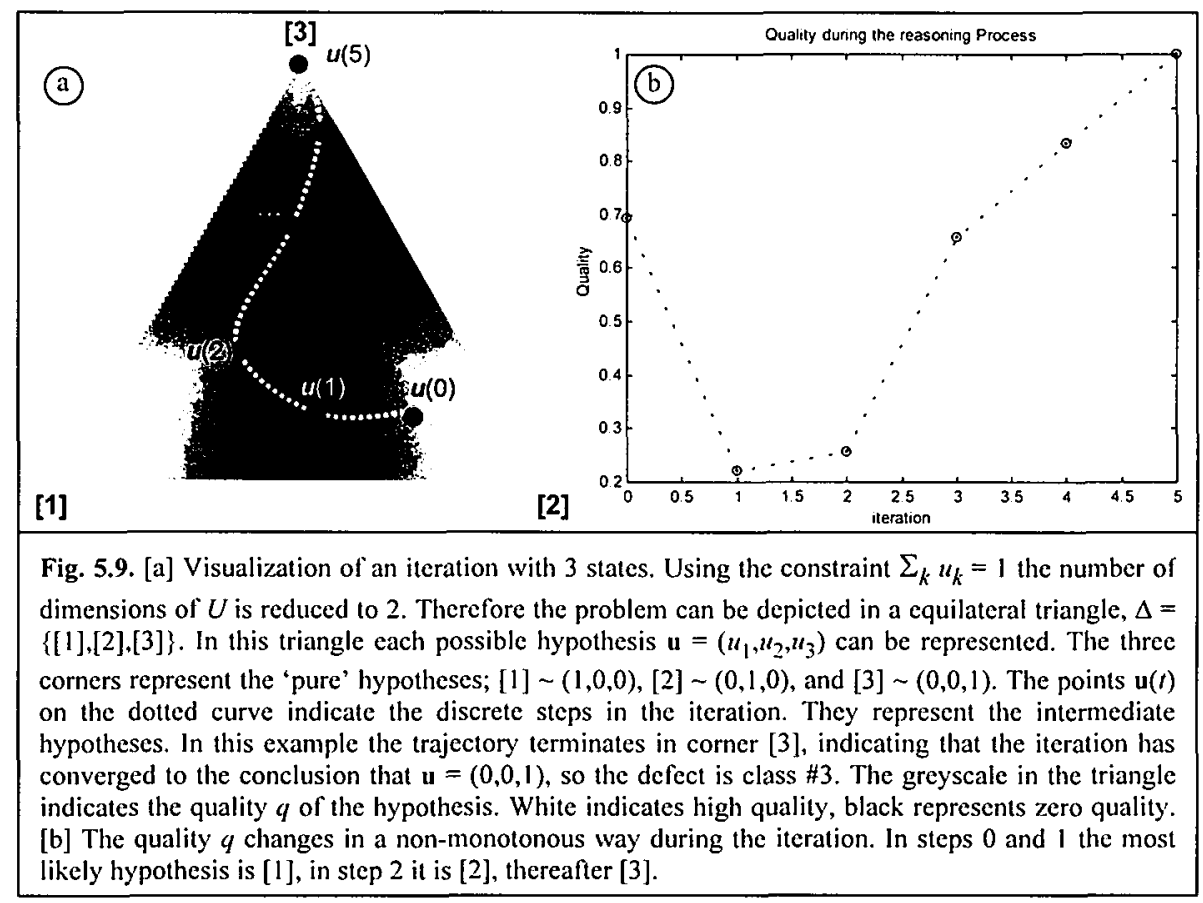

We define parameter space $\Xi \subset \mathrm{IR}^{N}$ as the space of all parameter-values $\mathbf{x}=$ $\left(x_{1}, x_{2}, \ldots, x_{N}\right)$, representing the $N$ possible measurements on the object. This representation differs qualitatively from the $U$ representation. In $U$ each hypothesis can be uniquely represented by one point, and vice versa each point in $U$ uniquely indicates one single hypothesis. In $\Xi$, however, during the iteration only partial vectors $\widetilde{\mathbf{x}}$ exist, as many of the parameters have not yet been measured. However, a state where only $x_{1}$ has been evaluated to be $v_{1}$, cannot be represented in $\Xi$ in a 
forthright way. This measurement can not be represented by a single point $\left(v_{1}, 0,0, \ldots, 0\right)$, for this point would rather represent a measurement where all other parameters would have exactly the value ' 0 ', which is not the case. Alternatively, a hyperplane defined by ' $x_{1}=v_{1}$ ' can not be simply transformed to one single vector in $U^{13}$. Consequently, it is not practical to consider the iteration as a trajectory in an $\Xi^{14}$. However, there exists a deterministic transformation ' $F$ ' from $\Xi$ to $U$, such that for each point $\mathbf{x}^{*}$ in $\Xi$ there is exactly one hypothesis $\mathbf{u}^{*}$ in $U$. Using expression [5.43] from Section 5.5.2, we can express $F$ in terms of the IASC-transformation $\Phi$ at the moment when all parameters have been measured:

$$
F\left(\mathbf{x}^{*}\right)=\Phi\left(\mathbf{u}_{0}, \mathbf{x}^{*},\{1,2, \ldots, N\}\right)
$$

with: $\mathbf{u}_{0}$ the initial hypothesis, $\mathbf{x}^{*}$ the full set of measurements, and $\{1, \ldots, N\}$ the set of all parameter indices.

It is illustrative to depict the quality $q$ as a scalar field in $U$, following the definition in Section 5.1. In the corners of the HIE the quality $q=1$. In Figure 5.9 the quality $q$ is depicted as greyscales in a triangular representation. Observe the progress of $q$ during the trajectory in Figure 5.9. Desired is a trajectory that terminates with $q=1$; i.e. in one of the corners of the HIE. This objective, however, meets two complications.

Primo, in many representations, the state functions $u$ are globally defined as non-zero, e.g. the Gaussian distribution. Therefore, in regions where the most-likely state function 15 assumes a local maximum, the quality $q$ also attains a local maximum that is somewhat smaller than 1.

Secundo, $q$ will in general not increase monotonously during the iteration. Depending on the sequence of measurements, during the iteration different hypotheses may act as 'most likely', i.e. $\arg \max _{k} u_{k}$. Consequently, $q$ may first decrease and only later progress to a value near 1 . This means that in the sense of $q$ the iteration is non-monotonous.

For the latter reason, an extra constraint on the parameter selection as discussed in Section 5.2 based on $\Delta q>0$ would in general lead to inferior conclusions, and premature convergence. It would restrict the evolution in $U$ to the initial basin of attraction, caused by the starting hypothesis $\mathbf{u}_{0}$, so always lead to the conclusion $k^{*}=$ $\arg \max _{k} u_{0 k}$. On the other hand, the resulting $u^{*}$ would be invariant for all permutations of the explored parameter list $E$.

The situation in $\Xi$ is comparable to a dimension-reduction process. Before the iteration, all points in $\Xi$ are equally probable as potential measurements. So the subspace $A$ of admissible points in $\Xi$ before the iteration is: $A_{0}=\Xi$, with dimension: $\mu_{0}=$ $\operatorname{dim}\left(A_{0}\right)=N$. After the first iteration of the IASC-algorithm one parameter with index $n^{*}$ has been assessed to a specific value $v$. Therefore, the space of admissible values in $\Xi$ has been reduced to: $A_{1}=A_{0} \cap\left\{x_{n^{*}}=v\right\}$, therefore: $\mu_{1}=\operatorname{dim}\left(A_{1}\right)=\mu_{0}-1=N-1$. The IASC-algorithm therefore induces a dimension-reduction process in $\Xi$ defined by:

\footnotetext{
13 This hyper-plane corresponds to a subspace in $U$ that contains the final solution $\mathbf{u}^{*}$.

$14 \Xi$ would be a useful representation in the case of Concurrent Interactive Analysis, discussed in Section 4.3.1

15 i.e. the state function corresponding to the most likely class.
} 


$$
\mu_{\mathrm{t}}=\operatorname{dim}\left(A_{\mathrm{t}}\right)=\mu_{\mathrm{t}-1}-1=\mu_{0}-\mathrm{t}=N-\mathrm{t}
$$

If the iteration is not terminated prematurely, this dimension reduction continues until $\mu_{\mathrm{t}}=0$ at $t=N$. Then the iteration is represented by one single point $\mathrm{x}^{*}$ in $\Xi$. This point represents the result of the measurement of all parameters.

\subsubsection{Definitions of Convergence of IASC to a High Quality Solution}

When the termination criteria (defined in Section 5.4) apply, the IASC-algorithm will terminate, and the elements of $\tilde{\mathbf{x}}$ will determine - through the information fusion (step vii in the definition of $I A S C$ ) - the final hypothesis $\mathbf{u}^{*}$. In that sense there is always a final result to IASC. Our main interest is whether there exist situations where the iteration terminates without one single conclusion. The 'conclusion' is defined above as the most likely state of the final hypothesis $\mathbf{u}^{*}: k^{*}=\arg \max _{k} \mathrm{u}^{*} k$. One single conclusion, expressed in terms of the quality $q$, means that $q=1$. When this is not feasible for the reasons mentioned in 5.6.2, we demand that $\mathbf{u}$ is above a certain lower limit $\mathrm{Q}_{\min }<1: q\left(\mathbf{u}^{*}\right)>\mathrm{Q}_{\min }$. Therefore, in our context we are not only interested in convergence to a solution $\mathbf{u}^{*}$, but also in convergence of the quality $q$ to a high value, or at least that $q$ reaches a minimal quality $Q_{\min }$. For these reasons we define both convergence of $\mathbf{u}$ and $q$.

Definition 5.6.1: Let $U$ be the state space $[0,1]^{K}$ and $\Phi$ the $I A S C$ transformation $\Phi$ : $U \rightarrow U$. Then $\Phi$ induces the $I A S C$-iteration in $U: \mathbf{u}_{n+1}=\Phi\left(\mathbf{u}_{n}\right)$, with $\mathbf{u}_{i} \in U$ and step $0 \leq i \leq N \in \mathbb{N}$. Let $\|\cdot\|$ be the Euclidean metric in $U$. Then $\Phi$ converges to $\mathbf{u}^{*}$ in $U$ if:

$$
\begin{aligned}
& \text { either: } \exists T<<\forall_{t>T}\left\|\mathbf{u}_{t+1}-\mathbf{u}^{*}\right\|<\left\|\mathbf{u}_{t}-\mathbf{u}^{*}\right\| \\
& \text { or: } \exists T<N \forall_{t>T} \mathbf{u}_{t}=\mathbf{u}^{*}
\end{aligned}
$$

Definition 5.6.2: (Same conditions as above). Let $Q_{\min }$ be a suitable constant that satisfies: $0 \ll Q_{\min }<1$. Now $\Phi$ is said to converge to a high-quality solution $\mathbf{u}^{*}$ if:

1. $\Phi$ converges to $\mathbf{u}^{*}$ in $U$, and

2. $\exists_{(T<N)} \forall(t>T) \quad q_{t+1}>q_{t}$,

3. With the same $T$ as in $2 .: \forall{ }_{(t>T)} q_{t}>Q_{\min }$.

So, essentially we are looking for the stationary points of mapping $\Phi$ that are attractors. We will later see that a $Q_{\min }$ satisfying: $Q_{\min }<\min k q \mathrm{o} F\left(\mathbf{m}_{k}\right)$ is such a suitable constant. So, from a certain moment $T$ onwards the quality $q$ increases monotonously, and at termination of the iteration $q$ satisfies: $q>Q_{\text {min }}$. Condition 1 in the latter definition is necessary, because otherwise it is possible that $q$ would converge to a high value, but the hypothesis $\mathbf{u}$ itself intermittently switches from prototype to prototype. On the other hand, convergence to $\mathbf{u}^{*}$ is only relevant if it is associated with a definite conclusion, i.e. that $q$ lies above the specified value $Q_{\min }$.

These definitions do not imply that the conclusion has necessarily reached the correct solution, for this will be unknown in practical situations. The definitions merely imply that the algorithm has arrived at a high-quality solution, and is likely to remain there. The success of the algorithm in finding the correct defect cause is described by the success rate, defined above. 
bound $Q_{\min }$. This in its turn means that the distance between $\mathbf{x}^{*}$ and the stateprototype $\mathrm{m}_{k^{*}}$ in the metric of class $k^{*}\|\cdot\|$ is below another small threshold $\delta . \delta$ and $Q_{\min }$ are related by the specific properties of $F$ and the class-metric.

More difficult is the case when not all parameters $x_{k}$ have been assessed. Then, the

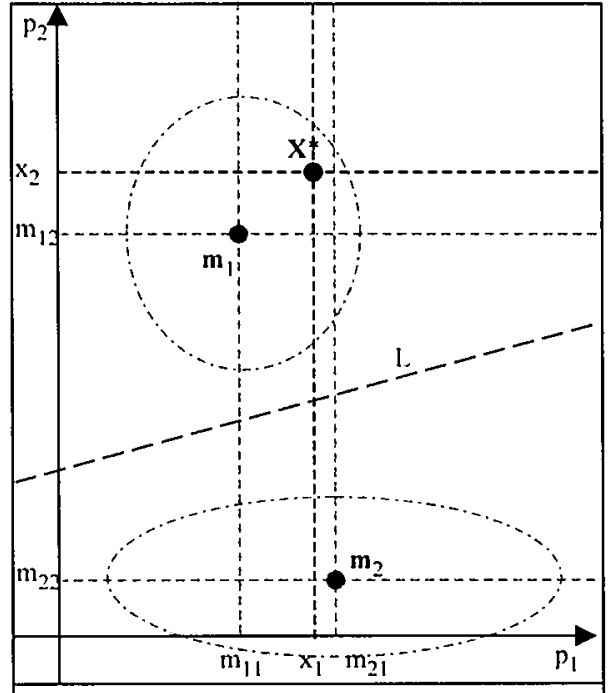

Fig. 5.11. Example where partial measurement will result in a wrong conclusion. convergence depends on the accidental sequence of measurements generated by the $I A S C$-algorithm. An example may illustrate this. Figure 5.11 shows an example where a partial measurement will result in a wrong conclusion. In this diagram two parameters $\left\{p_{1}, p_{2}\right\}$ are represented. Two classes are shown with prototypes $\left\{\mathbf{m}_{1}, \mathrm{~m}_{2}\right\}$ and ellipses defined by their covariance-matrices. Let $\mathbf{x}^{*}$ represent the actual state of the object. From the figure it is clear that the position of $\mathbf{x}^{*}$ lies well within the scope of class ' 1 '. However, the situation is such, that if only parameter $p_{1}$ would be measured, it would be classified as belonging to class ' 2 ' for:

$$
\left|x_{1}^{*}-m_{21}\right|<\left|x_{1}-m_{11}\right| \text {. }
$$

The border for classification between class 1 and 2 is represented in Figure 5.11 by a separatrix ' $L$ '. This curve indicates the decision boundary. Observe that states ' 1 ' and ' 2 ' are badly separated for $p_{1}$, but well-separated for $p_{2}$, as: $d_{12}^{1}<d_{12}^{2}$, using the class-separation defined in Section 5.2. Therefore, a 'good' parameter selection algorithm would suggest parameter $p_{2}$ for testing, rather than parameter $p_{1}$. This example shows the entanglement of the steps of $I A S C$; the quality of the present hypothesis depends both on the information fusion and the merits of the parameter selection. As the example demonstrates, an inferior result for parameter selection can propose a parameter that will falsely activate the termination criteria. This will occur as the partial measurement vector $\widetilde{\mathbf{x}}$ is found to fall within a critical distance $\delta$ of a particular class-prototype.

In practice, the convergence of $I A S C$ near the correct prototype is always observed. Thus we come to the following conjecture:

\section{Conjecture 5.1:}

For a given constant $Q$ with: $0 \ll Q<Q_{\max } \equiv q_{0} F\left(\mathbf{m}_{k^{*}}\right)$, the IASC-algorithm converges to a state $k^{*}$ with a high-quality solution for those measurements $\mathbf{x}^{*}$, that satisfy:

$$
\forall_{\left(0<Q<Q_{\max }\right)} \exists_{(\delta>0)} \forall_{\left(\left\|\mathbf{x}^{*}-\mathbf{m}_{k} *\right\|<\delta\right)} \quad q \circ F\left(\mathbf{x}^{*}\right)>Q .
$$

In Figure 5.12 this Conjecture is illustrated with a simplified example. 
Once a suitable distance $\delta$ has been found for a given $Q$, this is a necessary and sufficient condition for high-quality convergence. The problem in proving this conjecture lies in the unspecified and composite character of the transformation $F$. Without additional knowledge, it is difficult to make any definite statement about the convergence properties. Notice again that convergence does not necessarily mean that the correct solution has been found.

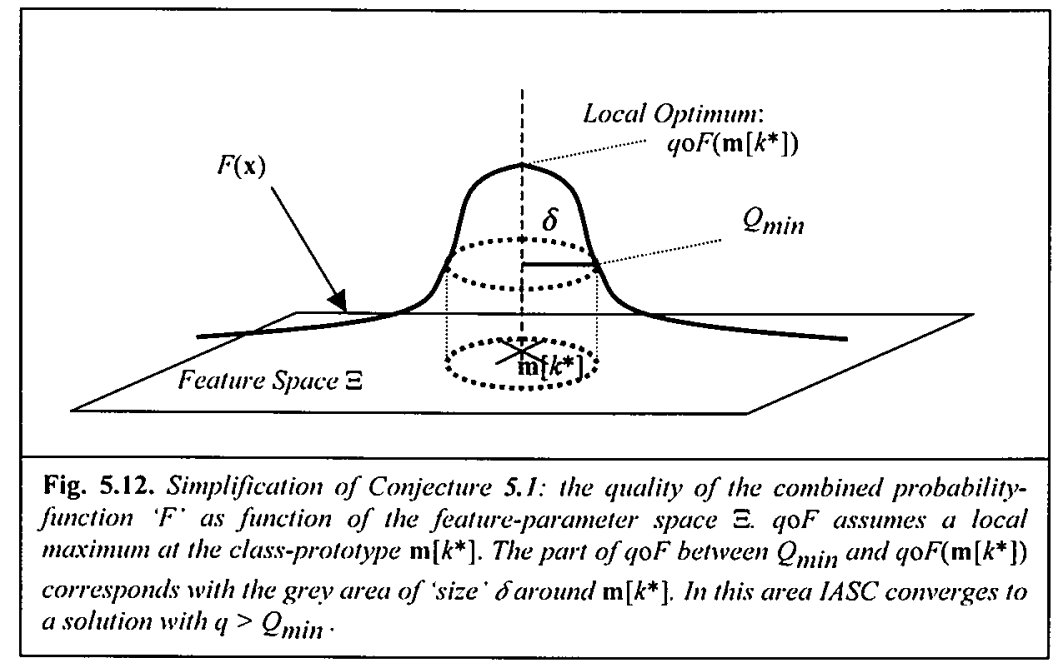

We can state a similar conjecture on the stability of the $I A S C$-algorithm.

\section{Conjecture 5.2:}

The following statements are equivalent:

1. For: $\left\{\mathbf{x} \mid\left\|\mathbf{x}-\mathbf{m}_{k^{*}}\right\|<\delta\right\} \quad$ IASC converges with a high-quality solution to $k^{*}$.

2. The IASC-algorithm is $\delta$-stable in $\mathbf{m}_{k^{*}}$.

Here, we used the definition of $\delta$-stability as defined in Section 5.6 .1 on the performance criteria. If Conjecture 5.1 is true, the proof of Conjecture 5.2 is trivial.

So, in the general case, where ' $F$ ' is not specified, we can only conjecture on the convergence and stability of $I A S C$. In order to make more definite statements about the convergence and stability of the $I A S C$-algorithm, we introduce the following restriction. In the remainder, we assume that the states in parameter-space can be reasonably well approximated by a multivariate Gaussian mixture. Based on this assumption, we analyse both multivariate Gaussian mixture models: UM and NM.

We first examine the Jacobian of the transformation $\Phi$ that induces the IASCalgorithm, and proof that under our new assumption it is a contraction. Second, we study the convergence for three scenarios of the distance of the observation $\mathbf{x}$ to the nearest prototype. In those cases, we are able to say more about convergenceproperties. 


\subsubsection{Convergence of the IASC Algorithm Based on the Jacobian of $\Phi$}

In this section we interpret the IASC algorithm as an iteration $u_{t}$ in IR ${ }^{K}$, induced by equation [5.43]: $\mathbf{u}_{t+1}=\Phi\left(\mathbf{u}_{t} \mid \mathbf{x}, E\right)$, where $\mathbf{x} \in \mathbb{R}^{N}$ acts as a parameter. For such dynamical systems the convergence properties relate to the Jacobian of the transformation $\Phi$, defined as:

$$
j_{k l}(\mathbf{u})=\frac{d \Phi_{k}\left(\mathbf{u}_{t}, \mathbf{x}, E_{t}\right)}{d u_{l}}=\frac{d u_{k}(t+1)}{d u_{l}(t)}
$$

The first theorem concerning the convergence of an iteration induced by a transformation relates to the set of eigen-values $\left\{\lambda_{1}, . ., \lambda_{K}\right\}$ of its Jacobian:

$$
\text { the iteration defined by: } \mathbf{x}_{t+1}=\Phi\left(\mathbf{x}_{t}\right) \text { is convergent if: } \forall_{k=1 . . K}\left|\lambda_{k}\right|<1
$$

Less stringent for convergence is the rate of change of an infinitesimal volume $d V \equiv d_{K} \mathbf{u}$ in IR $\mathbb{R}^{K}$. If a transformation $\Phi$ locally is a contraction at $\mathbf{u}=\mathbf{a}$, then the iteration $\mathbf{u}_{t}$ induced by $\Phi$ is convergent also at $\mathbf{u}=\mathbf{a}$. This rate of change depends on the absolute value of the determinant of the Jacobian ' $j$ ' associated with transformation.

$$
d V_{t+1}=|\operatorname{det} j(\mathbf{u})| d V_{t}
$$

Therefore:

$$
\text { () is a contraction if: }|\operatorname{det} j(\mathbf{u})|<1
$$

This is, however, not sufficient for the convergence of the iteration, as the decrease in some components of $\mathbf{u}$ can overcome the increase of others. However, contraction is an important indicator for convergence. If the iteration does not a contract at $\mathbf{u}=\mathbf{a}$, then the iteration does also not converge at $\mathbf{u}=\mathbf{a}$.

Applied to our context, this means that the IASC algorithm is convergent in representation space $U$, if the iteration generated by equation [5.43] obeys condition [5.52]. However, an attempt to prove convergence of IASC based on this condition suffers from the same problems as encountered in the previous section. This is specially caused by the fact that $\Phi$ is completely unspecified. Therefore, in the remainder of this section we restrict IASC to the case where $\mathbf{u}$ is represented by a multivariate normal distribution. This concretises $\Phi$ to the set of equations presented in Section 5.5.3. Below, we investigate both the multivariate normal models UM and NM.

\section{i. Uncoupled model (UM)}

In the UM the states $k$ and $l$ do not directly interact via their representations $u_{k}$ and $u_{l}$. Starting point for finding an expression for the Jacobian are the equations [5.44] and [5.47] for $u_{k}(t)$ and $u_{k}(t+1)$ in Section 5.5.3. We can rewrite these expressions as:

$$
\begin{aligned}
& u_{k}(t)=\exp \left(-1 / 2 \widetilde{\xi}_{k}(t)^{\mathrm{T}} \widetilde{G}_{k}(t) \widetilde{\xi}_{k}(t)\right) \\
& u_{k}(t+1)=\Phi_{k}\left(u_{k}(t)\right)=B_{k} u_{k}(t)
\end{aligned}
$$

Using the following definitions:

$$
\tilde{\xi}_{k}(t)=\widetilde{\mathbf{x}}_{t}-\tilde{\mathbf{m}}_{k}(t)
$$




$$
B_{k}=\exp \left(-\beta_{k} \xi_{k n}-1 / 2 \gamma_{k} \xi_{k n}^{2}\right)
$$

Depending on the interpretation of measurement $\mathbf{x}$ we can give two different expressions for the Jacobian.

\section{i.i. Measurement is an Initially Unknown Constant}

In this interpretation the measurement vector $\mathbf{x}$ is understood as a constant. The values of $\mathbf{x}$ are initially unknown, but during the iteration constantly more components of $\mathbf{x}$ become involved in the iteration through the partial measurement vector $\tilde{x}_{t}[i]=x\left[E_{l}(i)\right]$. As stated before, the functions $u_{k}(t)$ are not directly coupled, for there is no expression for $u(t)$ when $l \neq k$ in the right-hand sides of equations [5.55] and [5.56]. Therefore, the Jacobian can be calculated by direct differentiation of expression [5.56] for $u_{k}(t+1)$ to $u_{k}(t)$ :

$$
\frac{d \Phi_{k}}{d u_{l}}=\frac{\partial \Phi_{k}}{\partial u_{l}}=B_{k} \delta_{k l}
$$

where $\delta_{k l}$ is the Kronecker-delta. So, $j_{k l}(\mathbf{u})=B_{k} \delta_{k l}$ is a diagonal matrix, therefore, the $K$ eigen-values of $j$ are:

$$
\lambda_{k}=B_{k}=\exp \left(-\beta_{k} \xi_{k n}-1 / 2 \gamma_{k} \xi_{k n}^{2}\right)
$$

Condition [5.2]: $\quad \forall_{k}\left(\left|\lambda_{k}\right|<1\right)$ for convergence, therefore becomes: $\beta_{k} \xi_{k n}+1 / 2 \gamma_{k} \xi_{k n}^{2}>0$, which implies:

$$
\left(\xi_{k n}<a_{1}\right) \text { or }\left(\xi_{k n}>a_{2}\right)
$$

with: $a_{1}=\min \left(0,-\frac{2 \beta_{k}}{\gamma_{k}}\right), a_{2}=\max \left(0,-\frac{2 \beta_{k}}{\gamma_{k}}\right)$. Here, $\gamma_{k}$ is always a positive quantity, but $\beta_{k}$ can be both positive and negative. So, convergence is attained in: $\xi_{k n} \in<\leftarrow, a_{1}>\cup<a_{2}, \rightarrow>$. The direction of the convergence is also clear; since in this domain $\left|\lambda_{k}\right|<1$, we have that $u_{k} \downarrow$. This result corresponds with the notion that $\xi_{k n}$ denotes the distance of the measurement of parameter with index $n$ for class $k$. If the measurement $x_{n}$ coincides with the prototype $m_{k n}$, then the associated representation $u_{k}(t)$ will not decrease. If, on the other hand, the measurement lies relatively far from the class-prototype, resulting in a relatively high value of $\xi_{k n}$, then $u_{k}(t)$ indeed will decrease. Therefore, the states $u_{k}(t)$ will evolve according to the distance between the measurements and the corresponding coordinates of the prototypes. So, following this interpretation, conditions for convergence of IASC can be easily and plausibly formulated.

\section{i.ii. Measurement as a Dynamic Quantity}

Can the partial measurement $\widetilde{\mathbf{x}}$ be regarded as a dynamic quantity, that should be incorporated in the calculation of the Jacobian? In such an interpretation, the measurement $\mathbf{x}$ is seen as a variable that has a binding effect on the representation $\mathbf{u}$. This is because indirectly the functions $u_{k}(t)$ interact through $\mathbf{x}$, as a small change $\Delta \mathbf{x}$ in $\mathbf{x}$ will influence the values of all $u_{k}(t)$. The effect of an infinitesimal change $\mathrm{d} \mathbf{x}$ in $\mathbf{x}$ on the resulting Jacobian $j$ follows from the partial derivatives of $\Phi$ to $\mathbf{u}$ as well as the 
partial derivatives of $\Phi$ to $\mathbf{x}$. Using this consideration the Jacobian should be expanded as:

$$
j_{k l}(\mathbf{u})=\frac{d \Phi_{k}}{d u_{l}}=\sum_{j \in E} \frac{\partial \Phi_{k}}{\partial x_{j}} \frac{\partial x_{j}}{\partial u_{l}}
$$

Using expressions [5.56] - [5.58] this can be written as (see appendix C.1):

$$
j_{k l}(\mathbf{u})=B_{k} \frac{u_{k}}{u_{l}}\left(\sum_{j \in E} \frac{\widetilde{\mathbf{g}}_{k j}^{\mathrm{T}}}{\left(\widetilde{\mathbf{g}}_{l j}^{\mathrm{T}} \widetilde{\xi}_{l}\right)}\right) \widetilde{\xi}_{k}
$$

Notice that this expression is singular for $u_{l}=0$. Moreover, this result leads to conflicting situations, as in some cases the determinant $\operatorname{det}(j)$ grows linearly in time, though the system converges to a solution, see appendix C.2. Finally, it is important to notice that, though $\mathbf{u}$ depends on $\mathbf{x}$ as in equation [5.55], the outcome of measurement of $x_{n}$ is a random process that does not depend on the present hypothesis $\mathbf{u}$. Clearly, this interpretation can not satisfactory be applied to our context, and we can conclude that $\mathrm{x}$ may be regarded as a constant for the IASC-algorithm.

\section{ii. Normalised model (NM)}

In the normalised model NM, the normalisation of the $u_{k}$ 's causes a stronger coupling than in the UM. First, we rewrite the expressions for the NM from Section 5.5.2 as:

$$
\begin{aligned}
& z_{k}(t)=c_{k}(t) \exp \left(-1 / 2 \widetilde{\xi}_{k}(t)^{\top} \widetilde{G}_{k}(t) \widetilde{\xi}_{k}(t)\right) \\
& z_{k}(t+1)=B_{k} u_{k}(t) \\
& u_{k}(t)=z_{k}(t) / \sum_{l} z_{l}(t)
\end{aligned}
$$

Using the following definitions:

$$
\begin{aligned}
& c_{k}(t)=(2 \pi)^{-t / 2} \operatorname{det}^{1 / 2} \widetilde{G}_{k}(t) \\
& \widetilde{\xi}_{k}(t)=\widetilde{\mathbf{x}}_{t}-\widetilde{\mathbf{m}}_{k}(t) \\
& B_{k}=A_{k} \exp \left(-\beta_{k} \xi_{k n}-1 / 2 \gamma_{k} \xi_{k n}^{2}\right) \\
& A_{k}=\sqrt{2 \pi}\left(\frac{\operatorname{det} \widetilde{G}_{k}(t+1)}{\operatorname{det} \widetilde{G}_{k}(t)}\right)^{1 / 2}
\end{aligned}
$$

The $u_{k}$ 's are now coupled via their normalisation, therefore, we can write:

$$
\left(\frac{d \Phi_{k}}{d u_{l}}\right)_{N M}=\sum_{i=1}^{K} \frac{\partial u_{k}(t+1)}{\partial z_{i}(t+1)} \frac{\partial z_{i}(t+1)}{\partial u_{l}(t)}
$$

Where we follow the interpretation of $\mathbf{x}$ as a constant. The right-hand term describes the coupling between possibilities $u_{k}(t+1)=\Phi_{k}(\mathbf{u}(t))$ and $u_{l}(t)$ via their normalisations: $u_{k}=z_{k} / \sum_{l} z_{l}$. Utilising expressions [5.65] and [5.67] in the right-hand side of [5.71] we obtain:

$$
j_{k l}(\mathbf{u})=\hat{B}_{l}\left(\delta_{k l}-u_{k}\right)
$$

where: $\hat{B}_{l} \equiv B_{l} / \sum_{i} z_{i}$. The eigen-values of this equation are difficult to obtain analytically. However, the determinant of the Jacobian can be readily computed as: 


$$
\operatorname{det} j(\mathbf{u})=\left(1-\sum_{k=1}^{K} u_{k}\right) \prod_{l=1}^{K} \hat{B}_{l}=0
$$

Therefore, condition [5.54] can be applied, and we can conclude that $\Phi$ is a contraction. This contraction is a direct result from the normalisation, as clear from equation [5.73]; if the sum of $\mathbf{u}$ was not equal to 1 , then the determinant of the Jacobian would not be zero. It can be explained from the reduction of the $K$ dimensional hypercube to the $(K-1)$-dimensional hyper-isoeder HIE, described in Section 5.6.2. The convergence-properties of the iteration in this HIE, however, is not clear from this conclusion.

Summarising, an approach to prove the convergence of the iteration induced by the IASC algorithm suffers from mathematical complexities associated in calculating the eigen-values of the associated Jacobian. Intuitively, it is also clear that during an iteration the state-vector $\mathbf{u}$ can switch position until the final moments, though on average a volume in representation space $U$ may decrease. Therefore, in the next section we analyse three different scenarios for the convergence of the iteration, based on the distance of measurement $\mathbf{x}$ to the prototype $\mathbf{m}_{k}$ of the 'true' state ' $k$ '. 


\subsubsection{Three Scenarios for the Convergence and Stability of IASC}

We now examine three scenarios based on the position of the measurement-vector relative to the state-prototypes. In order to obtain some quantitative understanding of the convergence and stability properties of the IASC algorithm during an iteration, we make a number of assumptions. Suppose that sensor data of an object is available, such that (1) the data represents a known state, (2) the characteristic features of the state are clearly present in the sensor data, (3) the sensor data is of sufficient quality, (4) all states can be sufficiently approximated by a multivariate normal distribution, and (5) the states are independent, meaning that the metric tensors, the inverse of the covariance matrices: $G_{k}=C_{k}^{-1}$, are diagonal: $G_{k}[i, j]=\sigma_{k i}^{-2} \delta_{i j}$. Condition (5) is a reasonable assumption, for if two sensors were correlated one could be discarded. Under these five assumptions the hypothesis regarding the state of the object can be represented by a vector $\mathbf{u}$ located near a corner-point of the hyper-cube $[0,1]^{\mathrm{K}}$ in $U$. Now, suppose exactly one single defect, say ' $l$ ', is present in the data, so the object is represented by its prototype $\mathbf{m}_{l}$ in parameter-space, and the actual state is a vector $\mathbf{u}^{*}$ in representation-space, with: $u^{*}{ }_{k}=\delta_{k l}$. Let the entire sequence of measurements reflecting the state of the system be represented by the vector ' $x$ '. Here we assume that the order of the measurements does not influence the value of $x$. Due to imperfections in the sensors and noise in the sensing process, however, the measurement $\mathbf{x}$ will not directly coincide with the prototype $\mathbf{m}_{/}$. We now distinguish three cases for the convergence of IASC, based on the location of $\mathbf{x}$ relative to the actual defect-prototype $\mathbf{m}_{\mathrm{l}}$. These cases can be interpreted as an increasing amount of noise or imperfection in the sensing process.

(i) $\mathbf{x}$ coincides with a prototype $\mathbf{m}_{l}$,

(ii) $\mathbf{x}$ is close - but not identical - to a prototype $\mathbf{m}_{l}$,

(iii) $\mathbf{x}$ is not close to a prototype $\mathbf{m}_{l}$.

Here, we interpret 'close' in terms of the normalised distance measures, defined in Section 5.2.

\subsubsection{Scenario i. Convergence on a Prototype}

First, we consider the convergence of the IASC-algorithm, when the input exactly equals a prototype, free of noise and imperfections in the sensing process, and study how the imprecision in $\mathbf{u}$ develops during the iteration. Starting point of our analysis is the set of clustered states: $\left\{\omega_{k} \mid k=1 . . K\right\}$. The actual state $\mathbf{u}^{*}$ in the set of all hypotheses $U$ is represented by a corner-point $\mathbf{e}_{/}$. in the hypercube $[0,1]^{K}$. In this ideal situation, the measurement - represented by vector $\mathbf{x}$ - is exactly identical to a prototype ' $l$ ', in other words: $\mathbf{x}=\mathbf{m}_{l}$. As state functions $\mathbf{u}$ we follow our assumption of a multivariate normal distribution, both the UM and NM. Thus, after $t$ measurements, $\mathbf{u}$ is given as:

$$
u_{k}(t)=c_{k} \exp \left(-1 / 2\left(\widetilde{\mathbf{x}}-\widetilde{\mathbf{m}}_{k}\right)^{\mathrm{T}} \widetilde{G}_{k}\left(\widetilde{\mathbf{x}}-\widetilde{\mathbf{m}}_{k}\right)\right)
$$

with $c_{k}=1$ for the uncoupled model (UM), and $c_{k}=\mu_{t}(2 \pi)^{-t / 2} \mathrm{det}^{1 / 2} \widetilde{G}_{k}(t)$ for the normalised model (NM). $\mu_{t}$ is a normalisation constant such that: $\forall, \sum_{k} u_{k}(t)=1$. The tilda-symbol ' $\sim$ above a vector $a$ indicates that the $[i]$-th component follows: 
$\tilde{a}[i]=a\left[E_{t}(i)\right]$, where $E_{t}$ is the list of explored parameters in iteration step $t$. In the case that $\mathbf{x}$ coincides with a prototype $\mathbf{m}_{l}$ expression [5.74] becomes:

$$
u_{k}(l)=c_{k} \exp \left(-1 / 2\left(\tilde{\mathbf{m}}_{l}-\widetilde{\mathbf{m}}_{k}\right)^{\top} \widetilde{G}_{k}\left(\widetilde{\mathbf{m}}_{l}-\widetilde{\mathbf{m}}_{k}\right)\right)=c_{k} \exp \left(-1 / 2 \tilde{S}_{k l}^{2}\right)
$$

where $S_{k l}$ is the Mahalanobis class-separation, defined in equation [5.5] in Section 5.2.1. In order to compare successive steps in the iteration, we expand the Mahalanobis class-separation from step $t$ to step $t+1$ as:

$$
\widetilde{S}_{k l}^{2}(t+1)=\widetilde{S}_{k l}^{2}(t)+\sum_{i=1}^{1} G_{k}\left[E_{t}(i), n\right] \mu\left[k, E_{t}(i)\right] \mu[k, n]+G_{k}[n, n] \mu_{k n}^{2}
$$

with: $\mu_{k i}=\left(m_{l i}-m_{k i}\right)$, and $n$ the selected parameter index at step $t+1$. The second term in this expression can be both positive or non-positive, the rest only semipositive. We can now apply assumption (5) above, stating that $G_{k}[i, j]=\sigma_{k i}^{-2} \delta_{i j}$. Since $n \notin E$, we obtain:

$$
\widetilde{S}_{k l}^{2}(t+1)=\widetilde{S}_{k l}^{2}(t)+s_{k l}^{n}
$$

Here we have introduced the I-dimensional Mahalanobis separation for parameter index $n$ :

$$
s_{k l}^{\prime \prime}=\frac{\left|m_{k n}-m_{l n}\right|}{\sigma_{k n}}
$$

Notice that in general: $s_{k j}^{n} \neq s_{j k}^{n}$, and that: $s_{k j}^{n}=d_{j k}^{n}\left(1+\frac{\sigma_{k j}}{\sigma_{k n}}\right)$, with $d$ the geometric distance defined in Section 5.2.1. Thus, the sequence $\widetilde{S}_{k l}(t)$ is monotonous nondecreasing, since: $\widetilde{S}_{k l}^{2}(t+1) \geq \widetilde{S}_{k l}^{2}(t)$. This result, however, can not directly be translated to a conclusion for the sequence $u_{k}(t)$, because from Section 5.6.5, the iteration of $u_{k}(t)$ follows:

$$
u_{k}(t+1)=A_{k} u_{k}(t) \exp \left(-1 / 2 s_{k l}^{n^{2}}\right) \equiv \lambda_{k} u_{k}(t)
$$

with $A_{k}=1$ in the UM, and: $A_{k}=\frac{1}{\sigma_{k n} \sqrt{2 \pi}} \frac{\mu_{t+1}}{\mu_{t}}$ in the NM. So the factor: $\lambda_{k}=A_{k} \exp \left(-1 / 2 s_{k l}^{n}\right)$ determines decrease or increase of state $u_{k}(t)$. To examine the evolution of $u_{k}(t)$ we now study the two multivariate normal models separately.

\section{i. Uncoupled Model}

In the UM model the factor $\lambda_{k}$ becomes: $\exp \left(-1 / 2 s_{k l}^{\prime \prime}{ }^{2}\right) \leq 1$, so, according to [5.79]: $u_{k}(t+1) \leq u_{k}(t)$. The identity in this equation is reached only for $s_{k l}^{n}=0$. For each state $k \neq l$, by definition, there exists a component $n$ such that: $s_{k l}^{n}>0$, otherwise states $k$ and $l$ would be identical. Therefore, a $T \in \mathbb{N}$ exists, such that: $u_{k}(t+1)<u_{k}(t)$ for all $t \geq T$. For the actual object state ' $l$ ' we have: $\widetilde{S}_{/ l}(t)=0$, so expression [5.74] becomes: $u_{l}(t)=1$, since $c_{k}=1$ in the UM. For the UM therefore, the actual state $l$ 
maintains a value 1 , while on average the other states decrease monotonously to zero $^{16}$. Therefore, ultimately $\mathbf{u}$ reaches the corner of the hyper-cube that corresponds to the actual state of the object $\mathbf{u}^{*}$, so the IASC algorithm converges to the correct solution. To quantify the convergence, we rewrite equation [5.74] in iteration-step $t$ as:

$$
\begin{aligned}
& u_{j}(t)=1 \\
& u_{k \neq l}(t)=\exp \left(-\sum_{n \in E} s_{k l}^{n^{2}} / 2\right)
\end{aligned}
$$

Let $<s^{2}>$ denote the average squared Mahalanobis separation:

$$
<s^{2}>=\frac{2}{N K(K-1)} \sum_{n} \sum_{k \neq l} s_{k l}^{n^{2}}
$$

Furthermore, let us assume that the standard deviations $\left\{\sigma_{k n}\right\}$ are independent and uniformly distributed. Then, according to: $s_{k j}^{n}=d_{j k}^{n}\left(1+\frac{\sigma_{k j}}{\sigma_{k n}}\right)$, we obtain: $\left.\left.<s_{k j}^{n}\right\rangle \approx 2<d_{j k}^{n}\right\rangle$, and $\left\langle s^{2}>\approx 4<d^{2}>\right.$. Thus, we replaced the non-symmetric $s_{k l}^{n}$ by the symmetric geometric distance $d_{k l}^{n}$. Using this result, equation [5.81] can now be approximated by:

$$
<u_{k \neq l}(t)>\approx \exp \left(-2<d^{2}>t\right)
$$

Next, let us use this result to estimate the length of the iteration. The quality $q(t)$, defined in equation [5.2] in Section 5.1.4, can in this case be approximated with:

$$
q(t) \approx \frac{u_{l}(t)-<u_{k \neq l}(t)>}{u_{l}(t)}=1-\exp \left(-2<d^{2}>t\right)
$$

The static termination criterion, defined in Section 5.5.3, becomes true if $q$ exceeds a certain threshold $\theta: q>\theta$. In our example, this means that the threshold is reached for $t$ $>T_{\max }$. With equation [5.83] and the definition of $q$ we obtain:

$$
T_{\max }=\frac{|\ln (1-\theta)|}{2\left\langle d^{2}\right\rangle}
$$

As can be expected, the convergence time $T_{\max }$ decreases with the average distance, and increases as the selected threshold approaches 1 .

\section{ii. Normalised Model}

In the NM, the normalisation constant $\mu_{f}$ slightly complicates the matter. In iterationstep $t$ equation [5.79] can be written as:

$$
\begin{aligned}
& u_{l}(t)=\mu_{t} \prod_{i \in E} \sigma_{k i}^{-1} \\
& <u_{k \neq l}(t)>=\frac{\mu_{t}}{K-1} \sum_{k \neq l} \exp \left(-\sum_{n \in E} s_{k l}^{n^{2}} / 2\right) \prod_{i \in E} \sigma_{k i}^{-1}
\end{aligned}
$$

\footnotetext{
${ }^{16}$ Since $u_{k}(t) \in[0,1]$ we have: $0 \leq u_{k}(t+1)<u_{k}(t) \leq 1$ for $t>T$, so: $u_{k}(t) \downarrow 0$ as $t$ increases.
} 
where $\langle\bullet\rangle$ denotes the average performed over all states $k \neq l$. In order to make some qualitative and quantitative statements, we make two approximations. First, let the distances $s_{i j}^{n}$ follow a probability distribution $\rho$. Then using [5.86],[5.87], we obtain:

$$
<u_{k \neq l}(t)>=\frac{\mu_{1}}{K-1} \int \exp \left(-t s^{2} / 2\right) \rho(s) d s \sum_{k \neq l} \prod_{i=1}^{l} \sigma_{k n(i)}^{-1}
$$

Second, let the standard deviations $\sigma$ be governed by a distribution $\varphi$. Furthermore, we assume the standard deviations $\left\{\sigma_{k n}\right\}$ to be independent. Then, averaged over the entire ensemble of combinations $\left\{\sigma_{k n}\right\}$ we can approximate:

$$
\left\langle\prod_{i=1}^{t} \sigma_{\ln (i)}^{-1}\right\rangle=\prod_{i=1}^{t}\left\langle\sigma_{\ln (i)}^{-1}\right\rangle \approx\left\langle\sigma^{-1}\right\rangle^{\prime}=\int \varphi(\sigma) \sigma^{-1} d \sigma
$$

Combining [5.86] to [5.89], we find:

$$
\begin{aligned}
& u_{l}(t)>=\mu_{t} a^{t} \\
& <u_{k \neq l}(t)>=u_{l}(t) \mathrm{z}(t)
\end{aligned}
$$

with: $a=\left\langle\sigma^{-1}\right\rangle$, and:

$$
z(t)=\int_{0}^{\infty} \exp \left(-t s^{2} / 2\right) \rho(s) d s
$$

This $z(t)$ is a monotonous decreasing ${ }^{17}$ function from $z(0)=1$ to $z \downarrow 0$ for $t \rightarrow \infty$. This means that the other possibilities $u_{k \neq j}(t)$ decay relative to $u_{j}(t)$. To make an estimation of the length of the iteration let us again apply the static termination-criterion from Section 5.4. In our example we can approximate the termination-criterion as:

$$
q(t)=\frac{u_{l}(t)-<u_{k \neq l}(t)>}{u_{l}(t)}=1-\mathrm{z}(t)
$$

The termination-criterion is true if $q$ exceeds a certain threshold $\theta: q>\theta$. With this expression we see that the termination-criterion is reached for $t>T_{\max }$ with $z\left(T_{\max }\right)=$ $1-\theta$. Again we observe that the convergence time $T_{\max }$ decreases with the average distance, and increases as the selected threshold approaches 1 from below.

\section{iii. The Effect of Pre-Classification}

For simplicity we have neglected the effect of pre-classification, and set: $\mathbf{u}(0)=$ $(0, \ldots, 0)^{\top}$. Since $0^{0} \equiv 1$, this will not cause any problem for the optimisation functional $J$ for $\alpha=0$. From the definition of a multivariate normal distribution one can easily prove that whatever first parameter $n_{1}$ is selected, the next value $u_{1}$ will always be unequal to $\mathbf{0}$ - provided the domains of all observables are finite. So $\mathbf{0}$ acts as a global repellor, and will never reoccur again during an iteration. In the case that the states represent defects of the object, above consideration is valid under the condition that no special defect-class 'No Defect' is defined, indicated by $\mathbf{u}=\mathbf{0}$. In our context we shall adopt this condition.

${ }^{17} z(t)$ is monotonous decreasing because: $z^{\prime}(t)=-1 / 2 \int s^{2} \exp \left(-t s^{2} / 2\right) \rho(s) d s<0$ 


\section{iv. Upper and Lower Values of State Functions}

So, the distribution $\mathbf{u}$ can never reach $\mathbf{0}$, because of its definition in equation [5.74], and the fact that all observables $x_{n}$ will have a finite value. We can make this statement more precise if we take into consideration the range of the distribution over its domain. If for a certain parameter $n$ the observed or defined 18 values $x_{n}$ are within its associated domain $X_{n}$, we can define the upper and lower values, $U$ and $L$, of the representation $u_{k}(t)$ of state $k$ as $19: U_{k}=\max \left\{u_{k} \mid x_{n} \in X_{n}\right\}=1$, and $L_{k}=\min \left\{u_{k} \mid x_{n}\right.$ $\left.\in X_{n}\right\}$. Values above $U_{k}$ or below $L_{k}$ will never occur during the iteration, apart from initialisation ${ }^{20}$. Since logically $\mathbf{m}_{l}$ belongs to $X_{l}$, the constraints on representation functions demand that $U_{l}=1$. Therefore, though the 'real' state of an object may be represented by $\mathbf{m}_{l}$ and $\mathbf{u}^{*}$, the best what this formalism can do is a representation $u_{k}$ with: $L_{k} \leq u_{k} \leq 1$. Since the functions $u_{k}$ are independent, the lower limit of $L_{k}$ is given by the product of all minima over their specific domain, so $L_{k}$ will indeed be a small number:

$$
L_{k}=\min \left\{c_{k} \exp \left(-1 / 2\left(\mathbf{x}-\mathbf{m}_{k}\right)^{\mathrm{T}} G_{k}\left(\mathbf{x}-\mathbf{m}_{k}\right)\right) \mid x_{j} \in X_{j}\right\}
$$

If al domains $X_{n}$ are equal to $[0,1]$ so: $\left|x_{j}-m_{j k}\right| \leq 1$, and if all metrical matrices $G_{k}$ are diagonal, and the 2nd moment of the variances is equal to $\left\langle\sigma^{2}\right\rangle$, then in the UM the lower value of $L_{k}$ can be approximated by:

$$
L_{k} \geq L_{\min }=\exp \left(-\frac{N}{2<\sigma^{2}>}\right)
$$

So $L_{k}$ decreases exponentially with the number of test-parameters $N$, and will in realistic cases be close to zero ${ }^{21}$. Note that there is no influence from the 'dynamicallity'-exponent $\alpha$, as each measurement $n$ will confirm the hypothesis 'the state is l', parameter-selection is not so important. However, parameter-selection can favour well separated classes, and thus accelerate the increase of the terminationcriterion $q(t)$ relative to random selection.

Summarising, in the case that a measurement exactly coincides with a prototype, then the IASC-algorithm will quasi-exponentially converge to the correct conclusion $\mathbf{u}^{*}$, i.e. the hypothesis close to the appropriate corner $\mathbf{e}_{k}=\left(\delta_{k l}\right)$ of the hyper-cube $[0,1]^{K}$.

\footnotetext{
18 Most of the parameters, defined in appendix A, can be understood as fuzzy memberships to a specific characteristic, and thus have a value between 0 and 1 .

19 A representation with domain $[0,1]$ can be obtained by a renormalisation: $u_{k}^{\prime}=\left(u_{k}-L_{k}\right) /\left(1-L_{k}\right)$.

20 Even from equation [5.81] it is clear that the lowest value of $\langle u\rangle$ is above zero.

21 This means that the convergence time must be adapted accordingly, for instance the termination condition in the SCM becomes: $z\left(T_{\max }\right)=1-L_{\min }-\theta$, so the threshold must obey: $\theta<1-L_{m i n}$.
} 


\subsubsection{General Expressions for State Functions}

Suppose that a sequence of measurements with parameter-indices $E=\left\{n_{1}, n_{2}, . ., n_{t}\right\}$ has been performed. This sequence is represented by a vector $\widetilde{\mathbf{x}}$ consisting of $t$ components, representing the values of the collected parameters. Likewise, we define partial prototypes $\widetilde{\mathbf{m}}_{k} \in \mathrm{IR}^{t}$, and partial metric $t \times t$ matrices $\widetilde{G}_{k}$, all composed from parameter-indices in set $E$. In order to study the general behaviour of the IASCalgorithm, let us again follow the simplification that $\widetilde{G}_{k}$ is diagonal: $\widetilde{G}_{k}=\operatorname{diag}\left(\sigma_{i k}^{-2}\right)$, with indices $i \in E$, and states $k=1 . . K$. Then, at iteration $t$, the state function for state $k$ is given by:

$$
u_{k}(t)=c_{k} \exp \left(-\sum_{n \in E} \frac{\left(x_{n}-m_{k n}\right)^{2}}{2 \sigma_{k n}^{2}}\right)
$$

with $c_{k}=1$ for the UM, and: $c_{k}=\mu_{t} \prod_{n \in E}^{l} \sigma_{k n}^{-1}$ for the NM, and normalisation constant $\mu_{t}$. Next we define the position of $\mathbf{x}$ relative to prototype $l$ by the vector ' $\mathbf{r}_{l}$ ' as: $\mathbf{x}=\mathbf{m}_{l}$ $+\mathbf{r}_{l}$. Furthermore, let ' $\mathrm{y}_{k l}$ ' represent the vector from $\mathbf{m}_{l}$ to another prototype ' $k$ '; $\mathbf{y}_{k l} \equiv$ $\mathbf{m}_{l}-\mathbf{m}_{k}$. Using this notation, we can now write the vector from state ' $k$ ' to the measurement $\mathbf{x}$ as: $\mathbf{x}-\mathbf{m}_{k}=\mathbf{r}_{l}+\mathbf{y}_{k l}$. Therefore, [5.96] becomes:

$$
u_{k}(t)=c_{k} \exp \left(-\sum_{n \in E} \frac{\left(\eta[n]+y_{k l}[n]\right)^{2}}{2 \sigma_{k n}^{2}}\right)=c_{k} \prod_{n \in E} \exp \left(-1 / 2\left(s_{l k}^{n}+v_{l k}^{n}\right)^{2}\right)
$$

Here we used the 1-dimensional Mahalanobis distance $s_{/ k}^{n}$ from equation [5.78], and $v_{l k}^{\prime \prime}$, defined as the normalised $n$-th component of vector $\mathbf{r}_{l}$ from state ' $l$ ' to the measurement:

$$
v_{l k}^{\prime \prime} \equiv \frac{x_{n}-m_{l n}}{\sigma_{k n}}
$$

Notice, however, that this vector is normalised to the metric of state ' $k$ ', as we are now interpreting the measurement as an occurrence of state ' $k$ ' rather than the actual state ' $l$ '. Vector $\mathbf{v}_{/ k}$ provides a gauge-free representation of the position of the measurement $\mathbf{x}$ relative to the state-prototype $\mathbf{m}_{l}$. Under the basic assumption of multivariate normal distributed states, this aberration is caused by either noise or imperfections of the sensors. Therefore, we can interpret vector $\mathbf{v}_{l k}$ as a normalised, or 'relative' noise for state ' $k$ '. We can now study the state functions as a function of increasing 'noise', i.e. as function of $\left\|\mathbf{v}_{l k}\right\|$. For $\left\|\mathbf{v}_{l k}\right\|=0$ we again obtain scenario i, as to be expected.

Actually, we are only interested in the value of the 'true' state ' $l$ ', and an expectation value of all other 'incorrect' states. Therefore, we distinguish between state ' $l$ ', and an average value for the $(K-1)$ other states. For the 'true' state ' $l$ ', $s_{l k}^{\prime \prime}=0$, so expression [5.97] becomes: 


$$
u_{l}(t)=c_{l} \prod_{n \in E} \exp \left(-1 / 2 v_{l}^{n^{2}}\right)
$$

This immediately shows that the correct state rapidly decays with increasing relative noise, indicating the sensibility to defects in the sensing process. Notice that $v_{\| \prime \prime}^{\prime \prime}$ is the normalised distance defined in equation [5.4] in Section 5.2.1, of $\mathrm{x}$ to prototype ' $l$ ': $v_{l l}^{n} \equiv D_{l}^{\prime \prime}(\mathbf{x})$. The expectation values of the other states are defined similarly to equation [5.88] above as:

$$
<u_{k \neq l}(t)>=\frac{1}{K-1} \sum_{k \neq l} c_{k} \prod_{n \in E} \exp \left(-1 / 2\left(s_{l k}^{n}+v_{l k}^{n}\right)^{2}\right)
$$

Similarly, this exhibits a fast decay with increasing relative noise, but here the function also decreases exponentially with the Mahalanobis distance between the state prototypes. Therefore, a good clustering with high $s_{l k}^{n}$ leads to fast iterations of the IASC algorithm.

Now, assume a distribution ' $\eta$ ' for the relative noise level $\mathbf{v}_{/ k}$, and similarly to scenario $i$, we assume a probability distribution ' $\rho$ ' for the distances $s_{i j}^{n}$, and independent standard deviations with: $\left\langle\prod_{i=1}^{t} \sigma_{\ln (i)}^{-1}\right\rangle \approx\left\langle\sigma^{-1}\right\rangle^{\prime}$. Next, we study the NM and UM separately.

\section{i. Uncoupled Model}

In the $\mathrm{UM}$, the factor $c_{k}=1$, so, after averaging over the relative noise $v$, the expression for state ' $l$ ' becomes:

$$
u_{/}(t)=z(t)
$$

with ' $z$ ' a monotonous decreasing function similar to [5.92]:

$$
z(t)=\int_{-\infty}^{\infty} \exp \left(-1 / 2 \mathrm{t} v^{2}\right) \eta(v) d v
$$

For the other states ' $k$ ' we obtain:

$$
<u_{k \neq l}(t)>=Z(t)
$$

with: $\quad Z(t)=\int_{0-\infty}^{\infty} \int \rho(s) \eta(v) \exp \left(-1 / 2 t(s+v)^{2}\right) d v d s$

Like in scenario $\mathrm{i}$, the 'incorrect' states $u_{k}$, with $k \neq l$, monotonously approaches zero, because: $Z^{\prime}(t)=-1 / 2 t \int(s+v)^{2} \rho(s) \eta(v) \int \exp \left(-1 / 2 t(s+v)^{2}\right) d s d v<0$. However, here the 'true' state $u_{l}$ itself also monotonously decreases during the iteration, because $z^{\prime}(t)<0$, as shown above.

To estimate the length ' $T_{\max }$ ' of the iteration, we once again apply the static termination-criterion based on the quality $q$. Using [5.100]-[5.103], we can approximate $q$ by: 22 :

\footnotetext{
22 Here for simplicity the small quantity $L_{m i n}$, defined in the previous section, is set to zero.
} 


$$
q(t)=1-Z(t) / z(t)
$$

Termination is achieved if: $q(t)>\theta$, for given $\theta$ just below 1 . This means that: $Z\left(T_{\max }\right) / z\left(T_{\max }\right)=1-\theta$. Since $Z(0)=1$ and $z(0)=1$, it follows that $q(0)=0$. The direction of change of $q$ relates to: $q^{\prime}(t)=\left(z(t) Z^{\prime}(t)-z^{\prime}(t) Z(t)\right) / z(t)^{2}$. Therefore, $q(t)$ increases in $t$ if: $\frac{Z^{\prime}(t)}{Z(t)}>\frac{z^{\prime}(t)}{z(t)}$.

It is not possible to further elaborate equation [5.104], for in this general scenario no convergence of $\mathbf{u}$ or $q$ is guaranteed.

\section{ii. Normalised Model}

In the NM the development of the state representation $\mathbf{u}$ becomes:

$$
\begin{aligned}
& u_{l}(t)=\mu_{t} z(t) a^{t} \\
& <u_{k \neq l}(t)>=\mu_{t} Z(t) a^{t}
\end{aligned}
$$

with: $a=\left\langle\sigma^{-1}\right\rangle$, normalisation constant $\mu_{t}$, and functions $\mathrm{Z}(t)$ and $Z(t)$ defined above. Since the factor: $\mu_{t} a^{t}$ drops out in the quotient $u_{k} / u_{l}$, the results of the UM can be directly applied. Here, the normalisation coefficient $\mu_{t}$ determines the relative strength of state ' $l$ ' compared with the others. therefore, in the general case no prediction is possible. An estimate of the length of the iteration, again follows from the static termination-criterion: $q$. Using [5.105] and [5.106], $q$ is again approximated by the UM-result 23 :

$$
q(t)=1-Z(t) / z(t)
$$

${ }^{23}$ Again, $L_{\text {mirm }}$ is set to zero. 


\subsubsection{Scenario ii. Convergence near a Prototype}

Now suppose that the actual measurement $\mathrm{x}$ represents a point close to - but not identical to - a certain prototype ' $l$ '. This can be caused by noise or imperfections in the sensing process. Now, suppose that $\mathrm{x}$ is much closer to ' $l$ ' than to any other prototype ' $j$ ': $\left\|\mathbf{x}-\mathbf{m}_{/}\right\| \ll\left\|\mathbf{x}-\mathbf{m}_{j \neq \|}\right\|$. We extend this condition to all $N$ individual components, so: $\underset{k \neq 1}{\forall}\left|x_{n}-m_{l n}\right| \ll\left|x_{n}-m_{k n}\right|$. This condition implies that: $\left|x_{n}-m_{k n}\right| \approx\left|m_{l n}-m_{k n}\right|$, so: $\underset{k \neq l}{\forall}\left|x_{n}-m_{l n}\right| \ll\left|m_{l n}-m_{k n}\right|$. Using the 1-dimensional Mahalanobis distance $s_{l k}^{n}$ and relative noise $\mathbf{v}_{l k}$ defined above, this condition can be written as:

$$
\underset{n \in E}{\forall} \underset{k \neq l}{\forall} v_{l k}^{n} \ll s_{n} \equiv \min \left\{s_{l k}^{n}>0 \mid k \neq l\right\}
$$

Starting point for this scenario are the equations [5.100],[5.102] and [5.105],[5.106]. We analyse the behaviour of $z(t)$ and $Z(t)$ under approximation [5.100], for both models UM and NM relate to these two entities. The fact that $v_{l k}^{n} \ll s_{n}$ means that all deviations $\mathbf{r}$ from prototype ' $l$ ' are contained within a small sphere around $\mathbf{m}_{/}$with $\operatorname{radius}^{24}$ :

$$
R_{l} \equiv \min \left\{\left|m_{l n}-m_{k n}\right| / 2 \mid s_{k l}^{n}>0\right\}
$$

This means that the distribution ' $\eta$ ' of $v_{l k}^{n}$ has a compact support; there exists an $\mathrm{A}$ with: $0<A \ll s_{n}$, such that $\eta(v)=0$ if $v \notin[-A, A]$. Now we assume that $\eta(v)$ is a $\mathrm{C}^{\infty}$ diffeomorphism, and has a Taylor expansion at $v=0$. In the limit $A / s_{n} \downarrow 0$, we approximate $Z(t)$ by the first term of its Taylor expansion:

$$
Z(t)=\int_{0}^{\infty} d s \rho(s) \int_{-A}^{A} d v \eta(v) e^{-t(s+v)^{2} / 2} \approx \eta(0) \int_{0}^{\infty} d s \rho(s) e^{-t s^{2} / 2}
$$

This function has the characteristics: $Z(0)=\eta(0) \geq 0, \lim _{t \rightarrow \infty} Z(t)=0$, and $Z^{\prime}(t)<0$ if: $\eta(0)>0$. Therefore, the states $k \neq l$ monotonously decease to zero. Similarly, for large $t$ or small $A$ the function: $v \rightarrow \exp \left(-1 / 2 t v^{2}\right)$ becomes a spline at $v=0$, so expression [5.101] for $z(t)$ can be approximated as:

$$
z(t)=\int_{-A}^{A} \exp \left(-1 / 2 t v^{2}\right) \eta(v) d v \approx \eta(0)
$$

So, for small relative noise $v$, state ' $l$ ' remains almost unchanged, while the other states rapidly decrease to zero. An estimate for the length of the iteration ' $T_{\text {max }}$ ', once again stems from the quality $q$ :

$$
q(t)=1-Z(t) / z(t) \approx 1-\int_{0}^{\infty} d s \rho(s) \exp \left(-1 / 2 t s^{2}\right)
$$

\footnotetext{
24 In the case that some $s_{l k}^{n}=0$, this means that $m_{l n}=m_{k n}$, so there is no discrepancy if $x_{n}$ converges to either of the two prototypes. Therefore, this case does not influence our argument.
} 
Notice that: $q(0)=0, \lim _{t \rightarrow \infty} q(t)=1$, and $q^{\prime}(t)>0$. Therefore, the quality $q$ monotonously increases from zero to one, which - due to the finite length of the iteration - is never reached. The static termination criterion: $q(t)>\theta$, for a given $\theta$ just below 1 , determines the iteration length from the numerical solution of:

$$
\int_{0}^{\infty} d s \rho(s) \exp \left(-1 / 2 T_{\max } s^{2}\right)=1-\theta
$$

Summarising, we see that if a measurement is very close to a prototype, the situation is identical to scenario i. For both multivariate normal models UM and NM, the IASC algorithm quasi-exponentially converges to the correct conclusion $\mathbf{u}^{*}$, i.e. the appropriate corner $\mathbf{e}_{k}=\left(\delta_{k l}\right)$ of the hyper-cube $[0,1]^{K}$.

\section{iii. Expression in Geometric Distance}

We can use the assumption of independent distributed standard deviations $\left\{\sigma_{k n}\right\}$, to replace the ungainly non-symmetric 1 -dim Mahalanobis distance $s_{k l}^{n}$ by the symmetric geometric distance $d_{k l}^{n}$. Using: $s_{k j}^{n}=d_{j k}^{n}\left(1+\frac{\sigma_{k j}}{\sigma_{k n}}\right)$ and averaged over the entire ensemble of distributions $\varphi(\sigma)$, we obtain: $\left\langle s_{k j}^{n}\right\rangle=2\left\langle d_{j k}^{n}\right\rangle$. We can use this result to make some crude estimations. First, $z(t)$ from equation [5.92] can be approximated by: $\mathrm{z}(t) \approx \int \exp \left(-2 t d^{2}\right) \rho(d) \mathrm{d} d \approx \exp \left(-2 t<d^{2}>\right)$. Similarly, $Z(t)$ and $z(t)$ from equations [5.101] and [5.103] become: $\mathrm{Z}(t) \approx \exp \left(-2 t<d^{2}>\right) \exp \left(-1 / 2 t<v^{2}>\right)$, and $z(t) \approx \exp \left(-1 / 2 t<v^{2}>\right)$. Thus, in this crude approximation the static termination-criterion evolves like: $q(t)=1-\mathrm{Z}(t) / z(t) \approx 1-\exp \left(-2 t<d^{2}>\right)$.

Therefore, under condition: $\underset{n \in E}{\forall} v_{n} \ll \min _{k} d_{k l}^{n}$ convergence to the correct state $l$ proceeds approximately in exponential time, and: $T_{\max }=\frac{|\ln (1-\theta)|}{2\left\langle d^{2}\right\rangle}$

\section{iv. Role of alpha in selection}

Similarly to the noise-free scenario i, the influence of the 'dynamicallity'-exponent $\alpha$ is limited. The condition: $\underset{n \in E}{\forall} v_{n} \ll \min _{k} d_{k l}^{n}$ defines a base of attraction state ' $l$ ', and all parameters will indicate this true origin of the defect. Again, parameter-selection will slightly accelerate the iteration by favouring well separated states. 


\subsubsection{Scenario iii. Behaviour far from a Prototype}

Now suppose that condition [5.107] is not satisfied. This means that there exist parameter-indices $n$ for which the normalised noise $v_{n}$ is of the same order, or even exceeds, $R_{l}$ from equation [5.108]. In that case the measurement $x_{n}$ is closer to some incorrect prototype-value $m_{k n}$ than it is to the actual defect class value $m_{l n}$. For such parameters the outcome of that measurement will contribute to $k$ as the most likely defect class, and diminish the value $u_{\text {l }}$ of state $l$. The fraction of such errors increases with the 'noise' $|\mathbf{v}|$, as more and more clusters fall within the sphere $\left(\mathbf{m}_{l},|\mathbf{v}|\right)$. Therefore, the fraction of clusters as function of $|\epsilon|$ gives some indication of the amount of such errors as 'noise' increases. This fraction is a monotonous notdecreasing function, that increases from 0 for $\min _{n k l} d_{k l}^{n}$, to 1 for $\max _{n k l} d_{k l}^{n}$. Figure 5.13 shows the fraction of clusters within a normalised distance $d$ of a prototype for real data. The figure shows the relation for all defect classes, which form a relative narrow band on the figure, indicating the similarity of the clusters in this respect. This figure shows the large extent of cluster-overlap, as for $d \approx 1$ the fraction is almost 1 .
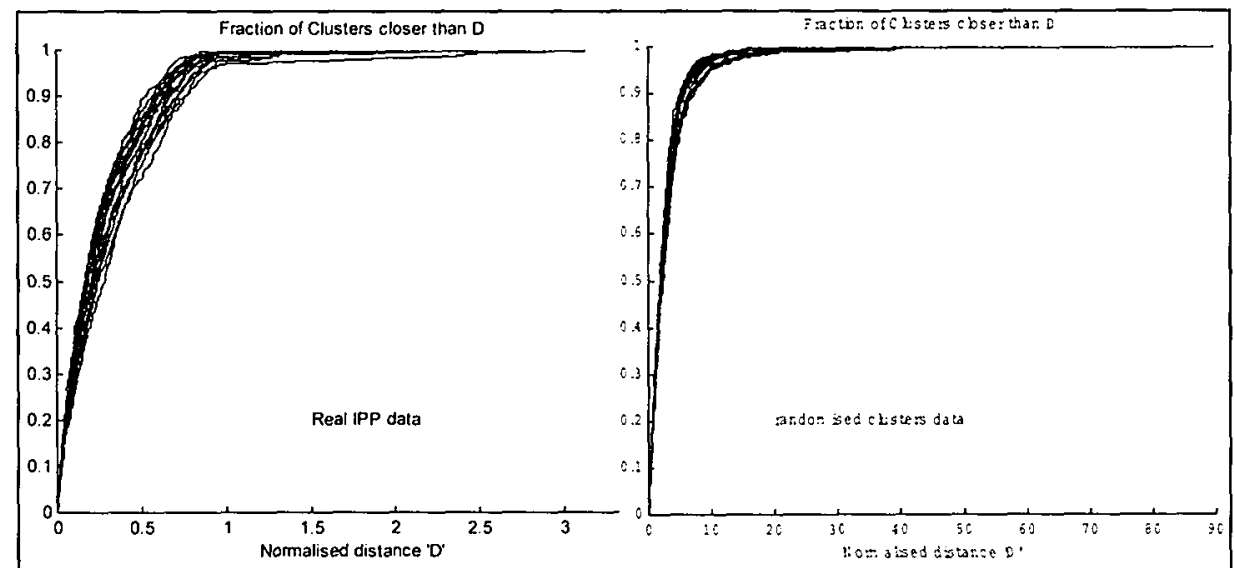

Fig. 5.13. Figure, showing the fraction of clusters within geometric distance $d$, for: (left) real experimental data, and: (right) a randomised cluster. This shows that the distances between clusters in the real data is small. caused by a high degree of cluster-overlap. In the randomised cluster the distances are much larger.

Above about $d=3.1$ all fractions are 1 , indicating that for such normalised distances no correct classification is possible. More problematic is that close to $d=0$ already the nearest prototypes can be found, as evident from Figures 5.13 and 5.14. So even for small noise some parameters will favour other prototypes. Therefore, even for small noise state-function $\mathrm{u}_{\mathrm{l}}$ will not reach 1 . However, as it remains higher than the other states, ' $l$ ' continues to be the most likely defect class for IASC. This is a direct consequence from the construction of many of the parameters described in appendix A. They were designed as fuzzy membership-functions in a way that they had a low value for most classes, and only for one or a small number of classes reached a high value. Therefore, many defects will share the value $x=0$. For those classes a measurement $x=0$ has no diagnostic value. 
We conclude with some remarks on the behaviour far from the actual prototype. This is the case when for many $n: v_{n} \gg \max _{k} d_{k l}^{n}$. In that case the corresponding parameters will all favour their nearest prototype. In general, those prototypes will in general differ, and there will be no consensus between these measurements, so overall the states will decrease. This situation can be caused by a low-quality image, or by an inferior parameter-selection. For a low-quality image no definite conclusion is possible. Relatively good parameter-selection is the strong point of the combination of the IASC-algorithm and the fuzzy-based definition of many class parameters. If some classes are highly likely, their contribution to the dynamic parameter selection, especially with the separation- or relevance-functional, will indicate new parameters that will have the greatest potential for testing whether the hypothesis about these parameters is correct, and also to distinguish between the competing classes. Therefore, there is a high probability that such relevant parameters are selected first ${ }^{25}$.

\begin{tabular}{|c|c|c|c|}
\hline Cluster & Dmin & $D \max$ & Dmean \\
\hline 1 & 0.001 & 2.434 & 0.315 \\
\hline 2 & 0.000 & 2.889 & 0.315 \\
\hline 3 & 0.000 & 2.114 & 0.269 \\
\hline 4 & 0.000 & 3.128 & 0.267 \\
\hline 5 & 0.000 & 2.113 & 0.250 \\
\hline 6 & 0.000 & 2.412 & 0.264 \\
\hline 7 & 0.000 & 1.452 & 0.241 \\
\hline 8 & 0.000 & 3.128 & 0.289 \\
\hline 9 & 0.000 & 2.889 & 0.262 \\
\hline 10 & 0.000 & 1.869 & 0.234 \\
\hline 11 & 0.000 & 2.460 & 0.245 \\
\hline 12 & 0.001 & 2.367 & 0.246 \\
\hline 13 & 0.000 & 3.128 & 0.346 \\
\hline 14 & 0.000 & 1.903 & 0.308 \\
\hline 15 & 0.000 & 2.144 & 0.260 \\
\hline 16 & 0.000 & 1.879 & 0.310 \\
\hline 17 & 0.000 & 1.269 & 0.245 \\
\hline 18 & 0.000 & 2.490 & 0.338 \\
\hline
\end{tabular}

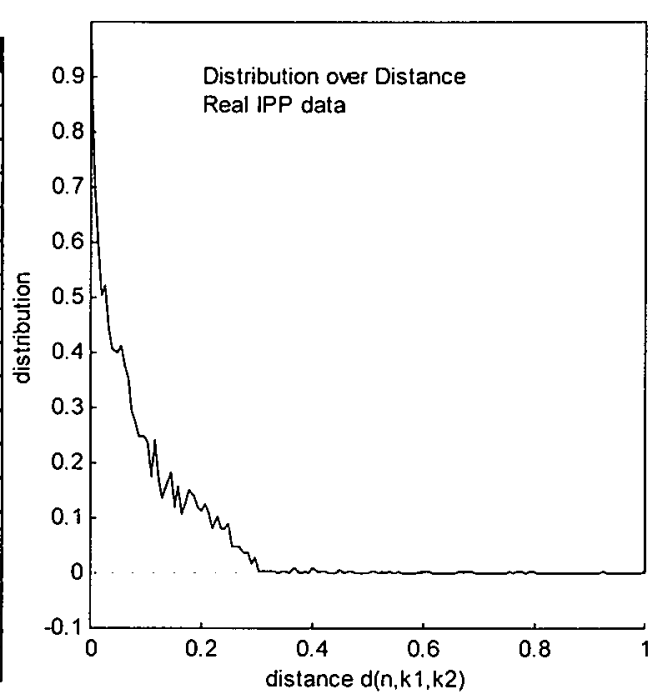

Fig. 5.14. Geometric distance distribution for real sensor data. Left: Table of normalised distances for 18 main classes, and right: the frequency distribution of the geometric distances.

\footnotetext{
2s To emphasise this point a special test-distribution was created, such that such the parameter relevance was high for only a few classes, and zero for all others. Logically, this test-set gave very good results, as it was fine-tuned for the IASC algorithm.
} 


\subsection{Dynamic Properties of the IASC Algorithm}

In this section we examine other aspects relevant to the dynamic properties of the IASC algorithm. The main emphasis is on the modes of evolution of the state vector $u$, especially the state function of the true class ' $l$ '. We investigate under which conditions u will evolve to a correct or an incorrect value - i.e. near zero or one - or into a 'mixed' state. We investigate the role of broad distributions with regard to the efficiency of FPS, and evaluate the probability that the main hypothesis changes during the iteration. Likewise, the influence of the termination criteria on the performance parameters is studied. Finally we propose models for the evolution of the exponent.

\subsubsection{Typology of the Evolution of the True State Function}

The average evolution of the function of the correct object state $u_{f}(t)$ is given by equations [5.98],[5.99]. This represents the main type of evolution if the noise level is below the average cluster distance. Empirically, however, more types of development of the true state are encountered. In order to construct a typology of evolution types, an experiment was performed with 10,000 different runs on a clustering based on scaled real data with: $\langle a\rangle=0.278$, absolute noise: $v=0.300$, using the separation functional with $\alpha=1$. The results of these experiments were quantified relative to two criteria:

1. $x$ : the average gradient of $u_{l}(t)$ over the time interval ${ }^{26}: x=\left\langle\dot{u}_{l}\right\rangle$.

2. $y$ : the average value of $u_{l}(t)$ over the time interval $27: y=\left\langle u_{l}\right\rangle$.

In Figure $5.14 a$ below all functions are depicted as individual points in the $(x, y)$-plane. This collection was clustered using Fuzzy Multivariate Clustering. Good clustering results were obtained with 4 prototypes. These main types are called I, II, III, and IV, depicted as Voronoi partition in Figure 5.15a. The composition among the types is: type I: $19.9 \%$, type II: $25.9 \%$, type III: $5.7 \%$, type IV: $48.6 \%$.

Figure $5.15 b$ shows the typical development of these types, averaged over all elements of the class: the class prototypes. These types can be arranged in two major groups. Types I and II together comprise almost half of the set. Functions in this group typically start as a quasi-exponential approach towards the value 1, but eventually decrease towards (almost) zero. In type I this decrease is gradually after about $20 \%$ of the iteration length $N$, in group II the decrease is late and sudden. The second major group consists of types III and IV, and constitutes more than half of the set. The typical evolution, visualised in Figure $5.15 b$, is characterised by an initially quasi-exponential growth, to about $20 \%$ of the iteration length $N$, after which the function quasi-exponentially converges towards a constant value. For type III this value varies between zero and one. This type constitutes only $6 \%$ of the entire set. For type IV - with $49 \%$ the largest of all - the terminal value is practically one. Group IV is explained by the theory for the average evolution of the true-state function in Section 5.7, the other types result from 'mixed' states that are explained below.

${ }^{26}$ Since $u(0)=0$ and $t$ is in $[0,1], x$ is equal to the terminal value of the true state; $x=u(N)$.

${ }^{27}$ Since $u_{l}$ and $t$ are in $[0,1], y$ is equal to the area under the true state; $y=\int^{1} u_{l}(t N) d t$. 

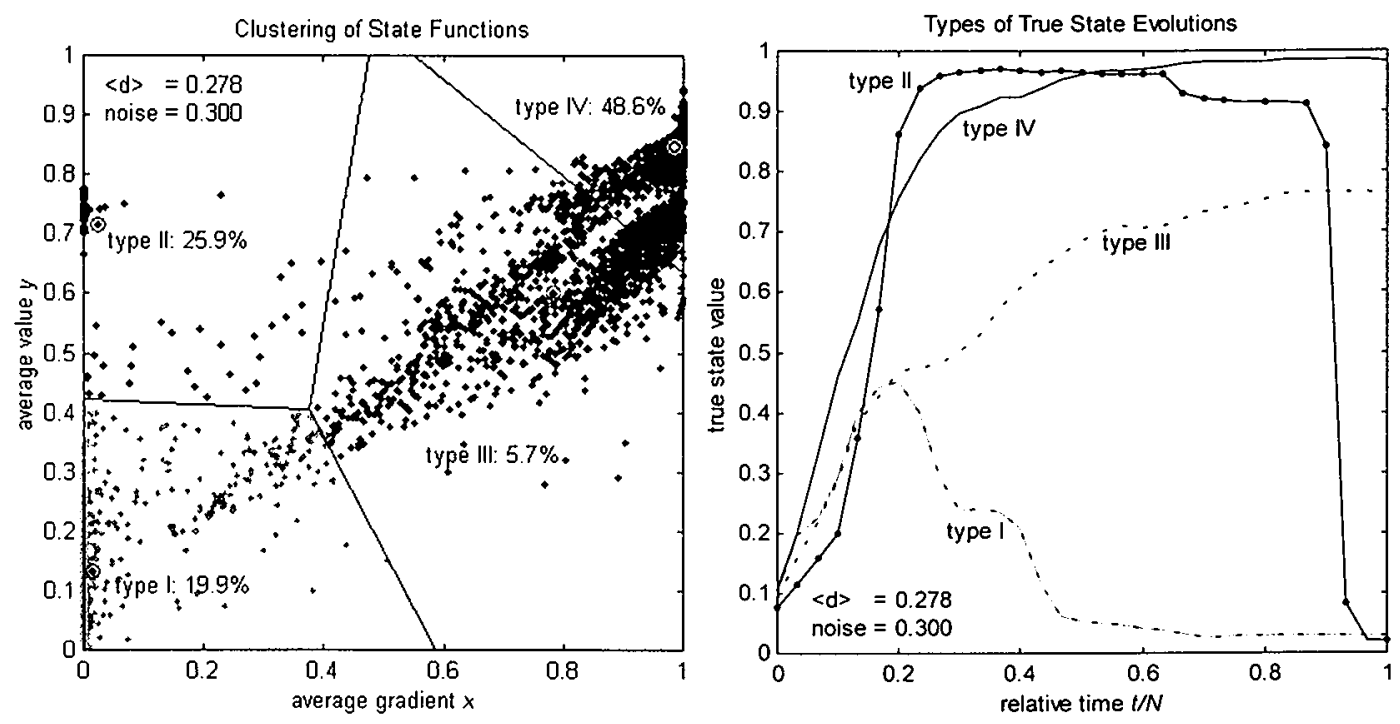

Fig. 5.15. $a$ (left): clustering of 10,000 points in $(x, y)$-plane, disks indicate prototypes. $b$ (right): four prototype evolution types for true state function $u(t)$.

From Figure $5.15 a$ it is also clear that for types III and IV, and part of type I, there is an almost linear relation between $x$ and $y$, so: $\left\langle\dot{u}_{l}\right\rangle=\lambda\left\langle u_{p}\right\rangle$ with $\lambda=0.84$ and a correlation coefficient of 0.77 . This relation supports the observed exponential character of the convergence.

An explanation for the anomalous types I and II lie in the character of the absolute noise $v=\left\|\mathbf{x}-\mathbf{m}_{\|}\right\|$that was used to generate these measurements. The fact that the absolute noise is 0.3 does not imply that the measurement $\mathbf{x}$ is actually closer to the true state ' $l$ ' than to some other defect class ' $k$ '. The relative sensor noise $f$, defined in equation [5.98], gives a better indication of the position of $\mathbf{x}$ relative to the true cluster and its competitors. Figure 5.16 shows the distribution of the $f$ over the entire set. In this distribution three near-Gaussian groups can be observed. The multi-member group $A$ has a very low relative noise, meaning that the nearest prototype to ' $l$ ' is relatively far away. Consequently, it should exhibit good convergence properties to the true state ' $l$ '. Table 5.2 lists the conditional probabilities for finding a specific function type I, II, III, or IV for the three groups $A, B$, and $C$. From this table it is clear that

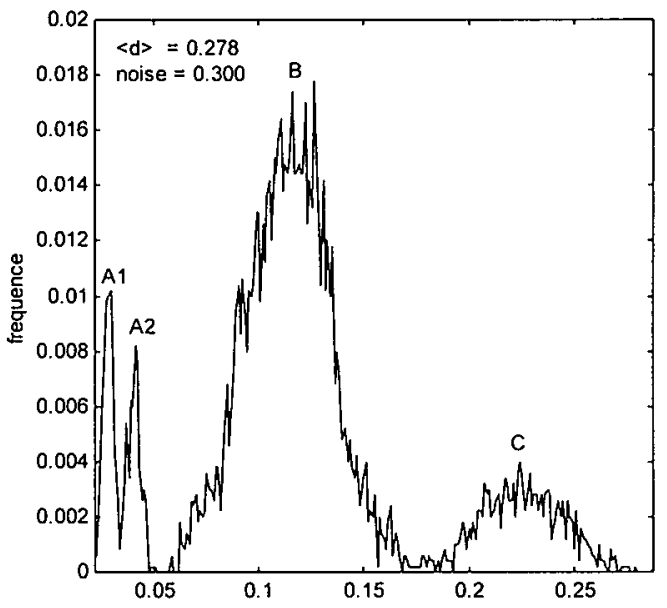

Fig. 5.16. Distribution of the relative sensor noise in the 10,000 measurements. 
indeed $100 \%$ of the functions found in group $A$ belong to the strongly convergent type IV. The large group $B$ represent measurements which lie somewhat closer to a competing prototype in parameter space. Still, the largest single group is type IV. But now the influence of the competing prototype confuses the algorithm. This results in either a mixed equilibrium between the correct and competing prototype, like type III, or in an eventual failure of the algorithm as the conclusion $\mathbf{u}$ becomes fully absorbed by the competing prototype, like the types I and II. In group C, representing an even more adjacent - though incorrect - cluster in parameter space, these effects are still stronger. Here, only $18 \%$ of the functions evolve towards the correct state ' $l$ '. The rest is eventually attracted towards the competing prototype, or gets stuck in an equilibrium point somewhere in between. In the next section we analyse the evolution to such equilibrium points in more detail.

\begin{tabular}{|c|c|c|c|c|}
\hline group & \multicolumn{1}{c}{ type I } & \multicolumn{1}{c}{ type II } & \multicolumn{1}{c|}{ type III } & \multicolumn{1}{c|}{ type IV } \\
\hline A & 0 & 0 & 0 & 1 \\
\hline B & 0.174 & 0.290 & 0.087 & 0.449 \\
\hline C & 0.435 & 0.386 & 0 & 0.179 \\
\hline
\end{tabular}

Table 5.2. Distribution of function types over the three main image quality groups

\subsubsection{The Role of Diffuse Classes and Mixed Equilibrium Points}

In the previous section we encountered a type of evolution of the state function towards a mix of several prototypes. A consideration regarding these mixed equilibria is the following. Suppose that we have a great number of classes $K$ and parameters $N$. Furthermore, suppose that we have a severe class overlap, such that $\langle d\rangle \ll 1$. Under these circumstances, the current measurement $\mathbf{x}$ may fall within the realm of various prototypes. This becomes even worse in the case of noise, as $\mathbf{x}$ will lie some distance away from its true class prototype. Such a situation is depicted in Figure 5.17, where the current measurement $\mathbf{x}$ belongs to class $\mathrm{I}$, but also falls within the diffuse classes II and III. Such diffuse clusters represent broad distributions of the class memberships function for a measurement of the current parameter $n$. Membership to a broad class $k$ is hardly indicative for the presence of the characteristic property associated with $n$, since the characteristic area of $k$ may well cover a substantial

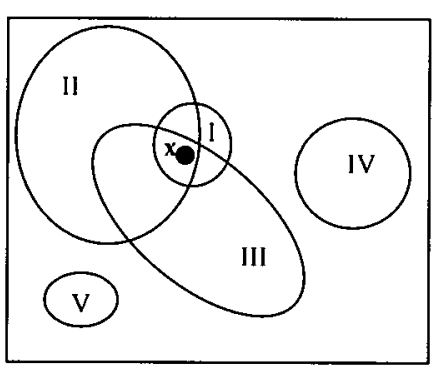

Fig. 5.17. Partially overlapping cluster distribution with focussed (I, V) and diffuse classes (II, III). part of the domain of $n$. Efficient parameter selection will concentrate first on the focussed classes, like class $I$ in Figure 5.17. The separation functional, for instance, will start with the sharpest class, and afterwards will increasingly involve broader classes, with small geometric distance $d$. However, above a certain value $d$-typically $d=1-$ the mentioned diffuse classes will become involved in the evaluation. This will dissolve the emerging hypothesis that is based on the sharper classes. As the measurement $\mathbf{x}$ for this parameter $n$ lies within the realm of a broad class $k$, a non-zero value is assigned to the memberships-function of $\mathbf{x}$ to $k$. It is a small value, for the class is broad and the distance to this incorrect class is non-zero. However, worse than assigning a small value to an incorrect class is the fact that - in case of the normalised 
model NM - it will also diminish the value of the correct class. This depends on whether in this particular dimension $n$, the correct class is diffuse or sharp. In the first case, the probability or class-membership of the correct class was already small altogether. It may be expected, however, that a efficient parameter selection procedure like FPS, will initially propose parameters wherein the most likely class is sufficiently narrow. In both cases, however, the cumulative effort of the broad and incorrect classes will quickly annihilate whatever sensible hypothesis was build. Therefore it is paramount to terminate the process at the right moment. If the process is terminated too early, it is sensitive to the non-informed state, hence the random initial choices of the algorithm. If the termination is too late, then broad, incorrect classes will quickly evaporate the, potentially correct, established hypothesis.

\section{Mixed Equilibria}

As argued in Section 5.6.2, we can understand the IASC algorithm as a dynamical system in the state space $u$. The pure states: $u_{l}=1$, and $u_{k}=0$ for $k \neq l$, are attractors when the position of the measurement $x$ coincides with the one of the prototypes $\mathbf{m}_{l}$. The separatrices between these prototypes, forming a Voronoi partitioning of parameter space, constitute the basins of attraction. Within these partitionings the main conclusion of the IASC algorithm:

$$
l^{*}=\arg \max _{k} u_{k}
$$

will always evolve to the most adjacent prototype $k^{*}$ to $\mathbf{x}$ in parameter space:

$$
k^{*}=\arg \min _{i}\left\|\mathbf{x}-\mathbf{m}_{i}\right\|
$$

In the case that point $x$ lies exactly on the separatrix of classes in some set $V$, the final values $u_{k}(N)$ for states $k \in V$ are all equal and

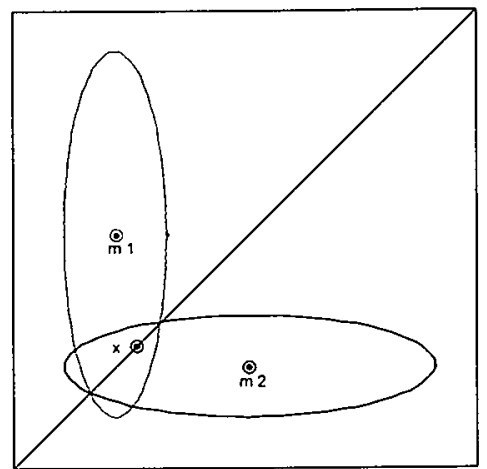

Fig. 5.18. $x$ is an equilibrium point for the classes with prototypes $\boldsymbol{m}_{1}$ and $\boldsymbol{m}_{2}$. there exists no unique solution to [5.113]. In this case $\mathbf{x}$ is called a 'mixed equilibrium point' of these classes. An example is shown in Figure 5.18. In general, for each point $\mathbf{x}$ in parameter space we can define $T(\mathbf{x})$, the iteration time for IASC necessary to reach the conclusion state $l^{*}=k^{*}$. In the case that $\mathbf{x}$ is an equilibrium point, then $T(\mathbf{x})$ $=\infty$. However, due to the finite length $N$ of the iteration, all points $\mathbf{x}$ in parameter space with: $T(\mathbf{x})>N$ can in effect be considered equilibria. Such factual equilibria cause mixed states, where the final state vector $\mathbf{u}(N)$ shows a non-trivial distribution over the clusters $k$.

\section{Role of Mixed States on the Convergence of the State-vector}

Let us consider a simple model to obtain some qualitative understanding of mixed equilibria. Assume a Normalised Model, where the state-vector $\mathbf{u}$ has initial values $\left(u_{l}, u_{2}, . ., u_{K}\right)$. Now, for simplicity suppose that each iteration step every state ' $k$ ' is multiplied by a constant $\lambda_{k}$ and subsequently normalised. In this case the evolution of the state-vector is given by:

$$
u_{k} \leftarrow \frac{\lambda_{k} u_{k}}{\sum_{i} \lambda_{i} u_{i}}
$$


This iteration leads to the following dynamical behaviour:

$$
u_{k}(n)=\frac{\lambda_{k}^{n} u_{k}}{\sum_{i} \lambda_{i}^{n} u_{i}}
$$

Consider the supremum $\lambda^{*}=\max \left\{\left|\lambda_{k}\right|\right\}$, and the set $L^{*}=\arg \max \left\{\left|\lambda_{k}\right|\right\}$. Then we can rewrite $[5.114 b]$ as:

$$
u_{k}(n)=\frac{\left(\lambda_{k} / \lambda^{*}\right)^{n} u_{k}}{\sum_{i}\left(\lambda_{i} / \lambda^{*}\right)^{n} u_{i}}
$$

Therefore, there are only two modes for the evolution of a state-function ${ }^{28}$. All states $k \in L^{*}$ satisfy: $\left|\lambda_{k} / \lambda^{*}\right|=1$, so: $\left|u_{k}\right| \rightarrow \mathrm{I} / n_{K}$, with: $n_{K}=\# L^{*}$, the number of states with the maximal lambda. All other states obey: $\left|\lambda_{k} / \lambda^{*}\right|<1$, and consequently converge to zero: $\left|u_{k}\right| \downarrow 0$ if $k \notin L^{*}$. In this model the state-vector evolves to the exact average location of the pure states in $L^{*}: \mathbf{u} \rightarrow\left\langle\mathbf{e}_{k}\right\rangle_{k \in L^{*}}$, with unit-vectors: $e_{k}[i]=\delta_{i k}$. Therefore, a equilibrium point is the centre of mass of the states that exert influence on the state vector by a multiplication factor $\left|\lambda_{k} / \lambda^{*}\right|=1$. Hence, these points are called mixed states.

We can use this simple model to obtain some understanding of the real iteration, defined by equation [5.47] from Section 5.5.3:

$$
u_{k}(t+1)=A_{k} u_{k}(t) \exp \left(-\beta_{k} \xi_{k n}-1 / 2 \gamma_{k} \xi_{k n}^{2}\right)
$$

where $A_{k}$ follows from the normalisation. Here, we can interpret this equation in the sense of our simple model if we equate:

$$
\lambda_{k}=\exp \left(-\beta_{k} \xi_{k n}-1 / 2 \gamma_{k} \xi_{k n}^{2}\right)
$$

So, all lambda's are positive. The maximal possible value of $\lambda_{k}$ follows from differentiation and is:

$$
\lambda_{\max }(k)=\exp \left(\frac{\beta_{k}(\widetilde{\mathbf{x}})^{2}}{2 \gamma_{k n}}\right)
$$

Consider the highest value $\lambda^{*} \leq \lambda_{\max }$ defined by [5.116], and the associate set $L^{*}$. Let $l \in L^{*}$. Now, let us compare a state $k$ with this optimal state. Following our simple model discussed above, we find convergence of $u_{k}$ to a mixed state if:

$$
\lambda_{k}=\lambda_{l} \Rightarrow \beta_{k} \xi_{k n}+1 / 2 \gamma_{k} \xi_{k n}^{2}=\beta_{l} \xi_{l n}+1 / 2 \gamma_{l} \xi_{i n}^{2}
$$

This equation defines a hyperbola in the $\left(\xi_{k n}, \xi_{m}\right)$-plane. If all measurements obey [5.118], then $k$ is also an optimal state, resulting in a mixed state generated by $k$ and $l$ and all others that obey equation [5.118]. Convergence to zero follows from: $\lambda_{k}<\lambda_{l}$. This defines an area in the $\left(\xi_{k n}, \xi_{l n}\right)$-plane, bounded by the hyperbola defined by equation [5.118]. Depending on a quantity $r$, defined as:

$$
r=\frac{\beta_{l}^{2}}{\gamma_{l}}-\frac{\beta_{k}^{2}}{\gamma_{k}}
$$

\footnotetext{
28 Because in our case all lambda's are positive there are no alternating states with cycle 2 solutions.
} 
this area consists of one connected area near the $\xi_{l}$ axis if $r \geq 0$, or two disconnected convex areas near the $\xi_{l}$ axis if $r<0$. Using expression [5.116] and the definition for $\xi$, we can write this case as:

$$
\lambda_{k}<\lambda_{l} \Rightarrow \gamma_{l}\left(x_{n}-m_{l n}^{*}\right)^{2}<\gamma_{k}\left(x_{n}-m_{k n}^{*}\right)^{2}+r
$$

with: $m_{k n}^{*} \equiv m_{k n}-\frac{\beta_{k n}}{\gamma_{k}}$. This means that the prototypes have been shifted by an amount $-\beta_{k n} / \gamma_{k}$. For the design of an efficient analysis process, the parameters averaged over all classes - should be constructed near independent. This implies that the off-diagonal components of the metric tensor of class $k, G_{k}$, should be near zero, so, because of the definition of $\beta$, it also implies $\beta \approx 0$. In that case $r$ can also be expected to be relatively small, and we can approximate [5.120] as:

$$
\frac{\left|x_{n}-m_{l n}\right|}{\sigma_{l n}}<\frac{\left|x_{n}-m_{k n}\right|}{\sigma_{k n}} \Rightarrow D_{l}^{n}(\mathbf{x})<D_{k}^{n}(\mathbf{x})
$$

Thus, the geometric distance from the measurement for parameter index $n$ should be closer to state $l$ than to state $k$. So, state $k$ converges to zero if equation [5.121] holds for all measurements, so: $\forall D_{l}^{n}(\mathbf{x})<D_{k}^{n}(\mathbf{x})$, indicating that measurement $\mathbf{x}$ is closer to state $/$ than to state $k$.

\subsubsection{Estimates of the Entire Measurement Based on Partial Information}

If we possessed total information about the sensed object, we would know all components of measurement $\mathbf{x}$, and hence the final distribution $\mathbf{u}^{*}=N(\mathbf{x},\{\mathbf{m}\},\{C\})$. During the iteration, however, only sequential information is available that gradually builds up to $\mathbf{u}^{*}$. Let us analyse the probability that a new measurement will generate totally unexpected information. From the typology of state functions in Section 5.7.1 it is clear that this probability mostly decreases gradually in time. The main exception is type II, that after an apparent convergence of the criterion $q$ to near 1 , abruptly collapses to 0 . The other types hardly change after their initial evolution, because in most FPS-functionals start with utilising the focussed classes that assure the highest class separation. The exceptions are situations where newly selected parameters exhibit broad distributions over the set of competing classes. As shown in the models above, the actual class ' $l$ ' is expected to win if the measurements lie closer to its prototype for all parameters.

The question whether it is possible to alter the current hypothesis during the remainder of the iteration depends on the degree of freedom present in the notperformed measurements. In other words; what is the volume of the remaining search space, and how many prototypes - besides the true class ' $\%$ ' - are contained therein? The partial state vector $\tilde{\mathbf{x}}$ represents the set of measured parameter values. Let $\mathbf{y}$ represent the set of not measured parameters, so $\mathbf{y}$ is the complement of $\widetilde{\mathbf{x}}$ relative to the entire set of measurements $x$. Then we can write the expectation value of the final value of state $k$ as:

$$
\left\langle u_{k}(N)\right\rangle=u_{k}(t) \operatorname{det}^{1 / 2}\left(G_{k}(\mathbf{y})\right) \int_{R} \exp \left(-1 / 2\left(\mathbf{y}-\mathbf{m}_{k}\right)^{\mathrm{T}} G_{k}(\mathbf{y})\left(\mathbf{y}-\mathbf{m}_{k}\right)\right) d_{N-t} \mathbf{y} / C
$$

where $C$ is a normalisation coefficient as we assume normalised state functions $u$ : 


$$
C=\sum_{k=1}^{K} \operatorname{det}^{1 / 2}\left(G_{k}(\mathbf{y})\right) \int_{R} \exp \left(-1 / 2\left(\mathbf{y}-\mathbf{m}_{k}\right)^{\mathrm{T}} G(\mathbf{y})\left(\mathbf{y}-\mathbf{m}_{k}\right)\right) d_{N-t} \mathbf{y}
$$

This is a very broad argument; in principle it is always possible that a new measurement overturns the present hypothesis. In practice, however, we may assume that the object belongs to one of the $K$ known states, and that the confusion is caused by either unfortunate parameter selection due to class overlap, or to the degree of noise in the sensor output. We therefore assume that the measurement reflects a distribution over the classes caused by noise as: $P_{\lambda}(y)$. Then the expectation value for class $k$ becomes:

$\left\langle u_{k}(N)\right\rangle=u_{k}(t) \operatorname{det}^{1 / 2}\left(G_{k}(\mathbf{y})\right) \int_{R} P_{k}(\mathbf{y}) \exp \left(-1 / 2\left(\mathbf{y}-\mathbf{m}_{k}\right)^{\mathrm{T}} G_{k}(\mathbf{y})\left(\mathbf{y}-\mathbf{m}_{k}\right)\right) d_{N-1} \mathbf{y} / C$

with:

$$
C=\sum_{k=1}^{K} \operatorname{det}^{1 / 2}\left(G_{k}(\mathbf{y})\right) \int_{R} P_{k}(\mathbf{y}) \exp \left(-1 / 2\left(\mathbf{y}-\mathbf{m}_{k}\right)^{\mathrm{T}} G_{k}(\mathbf{y})\left(\mathbf{y}-\mathbf{m}_{k}\right)\right) d_{N-1} \mathbf{y}
$$

As an example consider $\left\langle u_{k}(N)>\right.$ in case of Gaussian noise: $P_{l}(\mathbf{y})=N\left(\mathbf{m}_{l},\left\{\sigma_{l}, . ., \sigma_{N}\right\}\right)$. This is analogous to equation [5.50] of Section 5.5.3, where we calculated the expectation of the next iteration step as function of the next measurement under a Normal distribution. Therefore, the solution is a product of all intermediary steps:

$$
\left\langle u_{k}(N)\right\rangle=u_{k}(t) \prod_{j=t+1}^{N} A_{k} \sqrt{\frac{2 \pi}{\gamma_{k}+\sigma^{-2}}} \exp \left(\frac{-2 \beta_{k} \xi_{0}-\gamma_{k} \xi_{0}^{2}+\beta_{k}^{2} \sigma^{2}}{2+2 \gamma_{k} \sigma^{2}}\right)
$$

where all factors after the product sign are time dependent.

A more efficient approach is based on the expectation value of the entire measurement in step $t$, based on the partial information available. Assume that at iteration step $t$ we have a partial measurement vector $\widetilde{\mathbf{x}}$. Then the best estimate $\mathbf{x}^{*}$ for the entire sensor representation of the object is:

$$
\mathbf{x}^{*}=\sum_{k=1}^{K} u_{k} \mathbf{m}_{k} / \sum_{k=1}^{K} u_{k}
$$

Similarly, the probability distribution $P$ for the entire vector $\mathbf{x}$, conditional to the partial measurement $\widetilde{\mathbf{x}}$, is given by:

$$
P(\mathbf{x} \mid \widetilde{\mathbf{x}})=\sqrt{2 \pi} K \operatorname{det}^{-1 / 2}(C) \exp \left(-1 / 2\left(\mathbf{x}-\mathbf{x}^{*}\right)^{\mathrm{T}} C^{-1}\left(\mathbf{x}-\mathbf{x}^{*}\right)\right)
$$

with covariance matrix:

$$
C_{k}=\sum_{k=1}^{K} u_{k}\left(\mathbf{m}_{k}-\mathbf{x}^{*}\right)\left(\mathbf{m}_{k}-\mathbf{x}^{*}\right)^{\mathrm{T}} / \sum_{k=1}^{K} u_{k}
$$

In this formalism the expectation for the final state distribution is simply given by:

$$
\left\langle u_{k}(N)\right\rangle=P\left(\mathbf{m}_{k} \mid \tilde{\mathbf{x}}\right)
$$

In the case of a multivariate normal distributed clusters we can use expression [5.44] for $\mathbf{u}$ from Section 5.5.3 to calculate [5.127] to [5.130]. This provides a one-step approach for estimating $\mathbf{x}$. Successive repetition of these steps until $\mathrm{x}^{*}$ converges, defines an iteration algorithm for estimating $\mathbf{x}$ based on partial information $\tilde{\mathbf{x}}$. This algorithm is presented in Figure 5.19. 


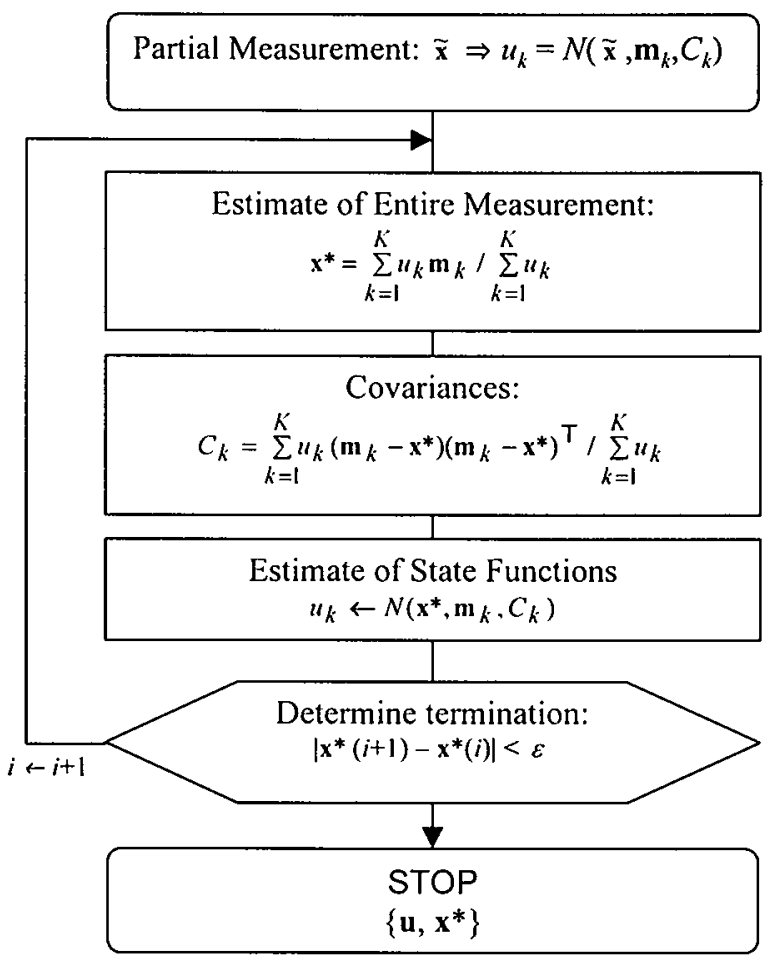

Fig. 5.19. Algorithm for estimation of $x$.

The one-step approach as function of the iteration step $t$ in IASC can also be used to interpret the IASC iteration as a trajectory of $\mathrm{x}^{*}$ in parameter space, or more appropriately a set of ultimately shrinking ellipsoids in parameter space, defined by the covariance matrix. Here, we are only interested in quantifying the possibility that a measurement $x_{n}$ is performed in iteration step $t+l$ of IASC that an entirely differs from the emerged picture. In the next section we shall use the estimator for $x^{*}$ for this purpose. 


\subsubsection{Probability to Overturn the Presently Established Hypothesis}

It is relevant to notice that the estimate $x^{*}$ at step $t$ should equal the partial measurement vector $\widetilde{\mathbf{x}}$ for the set of explored parameter indices $E(t) ; \mathbf{x}^{*}[E(i)]=\widetilde{\mathbf{x}}[i]$ for $i=1$..t. More specific, this should be the case as the entire measurement $\mathrm{x}$ is available at $t=N ; \mathbf{x}^{*}(N)$. This would imply:

$$
\mathbf{x} \stackrel{?}{=} \sum_{k=1}^{K} u_{k}(\mathbf{x}) \mathbf{m}_{k} / \sum_{k=1}^{K} u_{k}(\mathbf{x})
$$

where $u_{k}(\mathbf{x})$ follows from the multivariate normal distribution for class $k: N\left(\mathbf{m}_{k}, C_{k}\right)$. Differentiation to $\mathbf{x}$ left and right shows that this is not the case. On the other hand, significantly high correlations, ranging from 0.9 to 1.0 , were empirically found between $\mathbf{x}^{*}(N)$ and $\mathbf{x}$, especially in the case of low to medium noise, non-severe cluster overlap, and $N$ and $K$ not too small. Over the entire length of the iteration the correlation between the estimate $\mathbf{x}^{*}$ and the actual measurement $\mathbf{x}$ builds up to 1 . Therefore, this approach does indeed provide a high quality estimator.

More important than the actual correlation coefficient between the estimate and the actual measurement is the fact that they should be identical. This is expressed by the match between the unexplored coordinates of $\mathbf{x}$, and the equivalent coordinates of $\mathbf{x}^{*}$. This is the match between: $\{x[i] \mid i \notin E(t)\}$, and: $\left\{x^{*}[i] \mid i \notin E(t)\right\}$. This match is quantified by the following quadratic criterion:

$$
Q^{2}=\sum_{i \notin E(t)}\left(x(i)-x^{*}(i)\right)^{2} /(N-t)
$$

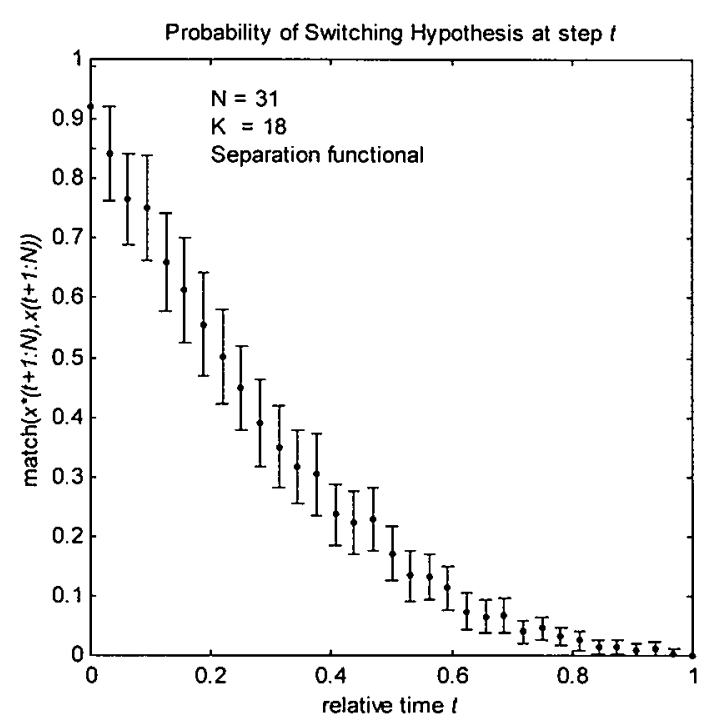

Fig. 5.20. Probability of finding a measurement at step ' $t$ that changes the current hypothesis, based on the match between the not explored parameter indices of the actual measurement $x$ and the estimate $x^{*}$. Based on 2500 simulations/measurement. where $Q=0$ indicates equivalence between $\mathbf{x}$ and $x^{*}$. This match is empirically observed to evolve monotonously from initially high values to zero. This match provides a measure for the probability that an entirely new measurement will overturn the presently established hypothesis. Therefore, we can interpret the plot as the probability that the present hypothesis will change as function of the iteration step. An example is shown in Figure 5.20. From this figure we can conclude that the probability that an entirely unexpected measurement is made, decreases monotonously in

time. Because of the correspondence between the estimate $x^{*}$ and the state functions $\mathbf{u}$, we can conclude that similarly the probability that the state functions - and hence 
the main conclusion of IASC - will change, decreases quasi-exponentially during the iteration.

\subsubsection{Impact of the Termination Condition on the Performance of IASC}

As mentioned in the introduction of Section 5.7 , it is paramount to terminate the iteration process at the right moment. If the process is terminated too early, it is sensitive to the insufficient amount of aggregated information, hence the random initial choices of the algorithm. If the termination is too late, then broad, incorrect classes can possibly deteriorate the, potentially correct, established hypothesis. We now try a qualitative modelling the optimal termination of the iteration in the case of closely packed clusters, so $\langle d\rangle \ll 1$. We discuss two simple models of the influence of the termination criterion on the success rate $S$ and iteration length $T$.

\subsubsection{Model I: state ' $l$ ' differs from average state value}

Let us assume the following simplifications:

1. suppose, we have a $(N, K)$-model, and measurement $\mathbf{x}$ close to the correct class ' $l$ '.

2. suppose, the state functions of all states are equal to a constant ' $u$ ', only state ' $l$ ' has a value ' $u+\varepsilon$ '

3. for simplicity, we use the weakly bound, not-normalised state functions ${ }^{29}$;

4. we use the separation functional with exponent $\alpha=1$.

Under these simplifications, we can write the separation functional $J$ from equation [5.19] in Section 5.2.3 as:

$$
J(n)=1 / 2<d^{n}>u^{2}+\frac{<d_{l}^{n}>u \varepsilon}{K}
$$

with averages: $\left\langle d^{n}\right\rangle \equiv \sum_{i, j} d_{i j}^{n} / K(K-1)$, and: $\left\langle d_{l}^{n}\right\rangle \equiv \sum_{i} d_{i l}^{n} / K$, using: $d_{k k}^{n}=0$.

Let us suppose that this represents an initial situation, and let us study the development of $\varepsilon$ during the iteration. Suppose that the clustering of the states is such, that the distances $d$ range from values below to values above one. Under these conditions we can distinguish the following possibilities.

\section{i. $\varepsilon$ is very small or even negative}

Let: $\varepsilon \ll 1^{30}$. In that case, the selection is fully determined by the first term in [5.131]: the average cluster distances for parameter $n:\left\langle d^{n}\right\rangle$. This results in a sequence $\left\{n_{1}, n_{2}, n_{3}, \ldots, n_{1}\right\}$ with monotonous non-increasing cluster separations: $\left\langle d^{n(1)}\right\rangle \geq\left\langle d^{n(2)}\right\rangle \geq\left\langle d^{n(3)}\right\rangle \geq \ldots \geq\left\langle d^{n(t)}\right\rangle$. In case of negligible noise, and nearly

\footnotetext{
29 Otherwise: $u=(1-\varepsilon) / K$.

${ }^{30}$ More correct: $\varepsilon<K u\left(d^{n}\right\rangle /\left(2\left\langle d_{l}^{n}\right\rangle\right)$
} 
unrelated parameters, using equation [5.47] of Section 5.5.3, we can approximate the development relative to the other states as:

$$
\left\langle\frac{u_{l}(t+1)}{u_{k}(t+1)}\right\rangle \approx \exp \left(-1 / 2\left(D_{l}^{n}(\mathbf{x})^{2}-<d^{n}>^{2}\right)\right)\left\langle\frac{u_{l}(t)}{u_{k}(t)}\right\rangle
$$

with $n=n_{t+1}$. Because of the low noise we made the following approximations: $D_{k}^{n}(\mathbf{x}) \approx d_{k l}^{\prime \prime} \approx\left\langle d^{n}\right\rangle$. Here $\left\langle d^{n}\right\rangle$ follows the non-decreasing sequence described above, so initially has a large value, while $D_{l}^{n}(\mathbf{x})$ is small as the measurement is close to prototype ' $l$. So: $\exp \left(-1 / 2\left(D_{l}^{n}(\mathbf{x})^{2}-<d^{n}>^{2}\right)\right)$ is initially relatively large, and the expectation of state ' $l$ ' consequently increases. So even in this case, when the statevalue of the correct class ' $l$ ' is not much larger than the mean state value $u$, the probability of the ensemble average of the correct class ' $l$ ' increases because the measurement for each parameter $n$ is close to the correct prototype ' $l$, and the class separation $\left\langle d^{n}\right\rangle$ is large due to the result of the separation functional.

\section{ii. $\varepsilon$ is positive and not too small}

Now suppose that $\varepsilon$ becomes sufficient large to make the second term of equation [5.131] leading. In that case the selection is fully determined by the average cluster distance to class ' $l$ ' for parameter $n:\left\langle d_{l}^{n}\right\rangle$. This results in a sequence $\left\{n_{1}, n_{2}, n_{3}, \ldots\right.$ ,$\left.n_{\mathrm{t}}\right\}$ with monotonous non-increasing distances:

$\left\langle d_{l}^{n(1)}\right\rangle \geq\left\langle d_{l}^{n(2)}\right\rangle \geq\left\langle d_{l}^{n(3)}\right\rangle \geq \ldots \geq\left\langle d_{l}^{n(t)}\right\rangle$. So now the functional $J$ favours selection of parameters that maximally separate class ' $l$ ' from all others. This leads to:

$$
\left\langle\frac{u_{l}(t+1)}{u_{k}(t+1)}\right\rangle \approx \exp \left(-1 / 2\left(D_{l}^{\prime \prime}(\mathbf{x})^{2}-<d_{l}^{n}>^{2}\right)\right)\left\langle\frac{u_{l}(t)}{u_{k}(t)}\right\rangle
$$

with $n=n_{t+1}$. Now, the distance $<d_{l}^{n}>$ to one specific cluster ' $l$ ' is less than or equal to the general average cluster distance $\left\langle d^{n}\right\rangle ;\left\langle d_{l}^{n}\right\rangle \leq\left\langle d^{n}\right\rangle$, because the former lacks all the small inter-class distances of the latter. Therefore: $\exp \left(-1 / 2\left(D_{l}^{n}(\mathbf{x})^{2}-<d_{l}^{n}>^{2}\right)\right) \geq \exp \left(-1 / 2\left(D_{l}^{n}(\mathbf{x})^{2}-<d^{n}>^{2}\right)\right)$, and the increase of the expectation of state ' $l$ ' consequently increases faster than in the case of small $\varepsilon$.

\section{iii. Transient effects under high relative noise}

Initially the expectation value of the correct state, averaged over all possible clusterings and initial values, quickly increases. Initially in specific cases a parameter may be selected, such that another incorrect class is closer to the measurement than the correct one, see also Figure 5.11 from Section 5.6.4, but as information accumulates during the iteration, on average the correct class increases. The average increase becomes especially true as its value $u+\varepsilon$ exceeds the average state value $u$, such that the second term in [5.131] becomes leading, and the increase becomes exponential. This depends on the assumption that initially there are many parameters $n$ with $\left\langle d_{l}^{n}\right\rangle \gg 1$ and $\left\langle d^{n}\right\rangle \gg 1$. As these parameters have been used for sensing, they are excluded from further selection. As the list of explored parameters $E(t)$ 
grows, the associated orderings $\left\langle d_{l}^{n}\right\rangle$ and $\left\langle d^{n}\right\rangle$ decrease in value, and at a certain moment all averages $\left\langle d_{l}^{n}\right\rangle$ and $\left\langle d^{n}\right\rangle$ are well below 1 . Consequently, in the set of unexplored parameters the class-membership functions are represented by broad distributions. Actually, they are so broad that now large state values are instigated for the membership-functions $u_{k}$ of incorrect classes. As the sequences $\left.<d\right\rangle$ further decrease, the growth factors $\left.\exp \left(-1 / 2\left(D_{l}^{n}(\mathbf{x})^{2}-<d_{l}^{n}\right\rangle^{2}\right)\right)$ and $\exp \left(-1 / 2\left(D_{l}^{n}(\mathbf{x})^{2}-<d^{n}>^{2}\right)\right)$ will even become smaller then 1 . This again will cause the value of the correct class $u$, to decrease, and of some incorrect classes $u_{k}$ to increase. The cumulative effect of this hastens the relative decrease of $\langle u\rangle$. Between the initial phase and the latter phase there is a transition period, where the two effects overlap, giving on average a stasis in the evolution of all state-functions $\left\langle u_{k}\right\rangle$. This stasis determines the optimal moment for termination. To estimate that moment, we discuss a more general model based on the average values of the state functions.

\subsubsection{Model 2: General Average Case}

In the general case, we have expressions for the average development of $u_{k}$ and $u_{l}$ given in equation [5.100] and [5.101] from Section 5.6.6: $u_{t}(t)=z(t)$, and: $\left\langle u_{k \neq l}(t)\right\rangle=\mathrm{Z}(t)$. This allows us to estimate the average value of the functional $J$ as:

$$
\left\langle J_{n}(t)\right\rangle=\sum_{i, j \neq l}\left\langle d^{n}\right\rangle_{t} Z(t)^{2}+\sum_{i \neq l}\left\langle d_{l}^{n}\right\rangle_{t} Z(t) z(t)
$$

There are $(K-1)(K-2)$ off-diagonal components not on the $l$-th row or column. Due to the symmetry of $d_{i j}^{n}=d_{j i}^{n}$ this is halved. Likewise, there are $(K-1)$ independent offdiagonal components on the $l$-th row or column. Thus, the expectation value of the functional becomes after omitting a factor $(K-1)$ :

$$
\left\langle J_{n}(t)\right\rangle=1 / 2(K-2)\left\langle d^{n}\right\rangle_{t} Z(t)^{2}+\left\langle d_{l}^{n}\right\rangle_{t} Z(t) z(t)
$$

Analogous to the first model, this gives rise to non-increasing sequences in the cluster distance. Similarly, if state ' $l$ ' is above average the second term becomes leading. In that case the selection is driven by the non-increasing sequence $\left\langle d_{l}^{n}\right\rangle$, giving rise to even faster increase of state ' $l$ '. In order to calculate the effect of the termination criterion, we approximate the state-functions as:

$$
\left\langle u_{k \neq l}(t)\right\rangle=\exp \left(-1 / 2 \sum_{n \in E(t)}\left\langle d_{l}^{\prime \prime}\right\rangle^{2}\right) \text {, and } u_{l}(t)=\exp \left(-1 / 2 \sum_{n \in E(t)} D_{l}^{n}(\mathbf{x})^{2}\right) \text { [5.136] }
$$

Next, we make a crude estimate for the static termination criterion $q(t)$. Since it is difficult to estimate an individual state like the second largest state $u 2$, we observe as criterion the difference with the average state $\langle u\rangle$. Thus, the static termination criterion $q$ from Section 5.6.1 can be approximated as:

$$
\langle q(t)\rangle=1-\left\langle u_{k}(t)\right\rangle / u_{l}(t)=1-\exp \left(-1 / 2 \sum_{n \in E(t)}\left(\left\langle d_{l}^{n}\right\rangle^{2}-D_{l}^{n}(\mathbf{x})^{2}\right)\right)
$$

Termination is achieved if $\langle q\rangle$ exceeds a given threshold $\vartheta$, so: 


$$
\sum_{n \in E(t)}\left(\left\langle d_{l}^{n}\right\rangle^{2}-D_{l}^{n}(\mathbf{x})^{2}\right)>-\ln (1-\vartheta)^{2}
$$

\subsubsection{Dependence of Performance on the Termination Threshold}

Now, define the sequence $a_{i} \equiv\left\langle d_{l}^{n(i)}\right\rangle$. Because of the FPS-parameter selection $a_{i}$ is an ordered sequence: $a_{1} \geq a_{2} \geq \ldots \geq a_{N}$. Suppose, $a_{1}>1$, and $a_{N}<1$, a reasonable assumption for clusterings with severe cluster overlap. Furthermore, let: $\delta_{n}=D_{l}^{n}(\mathbf{x})<1$ represent a small relative noise level. Then the termination condition [5.138] becomes:

$$
\sum_{n \in E(t)}\left(a_{n}{ }^{2}-\delta_{n}{ }^{2}\right)>C(\vartheta) \equiv-\ln (1-\vartheta)^{2}
$$

Where $C$, the generalised threshold, can be any non-negative number. Let us now study the sequence:

$$
b_{n} \equiv \sum_{i=1}^{n}\left(a_{i}^{2}-\delta_{i}^{2}\right)
$$

Define: $\delta=\max \delta_{n}$, so: $0 \leq \delta_{n} \leq \delta \ll 1$, with small $\delta$ because we assumed a low noise-level. Therefore, sequence $b$ is bounded by: $b_{1 n} \leq b_{n} \leq b_{2 n}$, with: $b_{1 n} \equiv \sum_{i=1}^{n}\left(a_{i}{ }^{2}-\delta^{2}\right)$, and: $b_{2 n} \equiv \sum_{i=1}^{n} a_{i}{ }^{2}$. Sequence $b_{2 n}$ is monotonously increasing, since $a_{n}$ is a non-increasing sequence, and all non-degenerate clusterings obey $a_{n}>0$. Sequence $b_{l n}$, exhibits a different behaviour. Suppose that: $\min _{n} a_{n}<\delta$. This is again a reasonable assumption since we are dealing with closely packed clusters and the average geometric distance is much smaller than one. Then, there exists an $n_{o} \in$ $[0, N]$ such that: $a_{n}{ }^{2}-\delta^{2}<0$ for all $n>n_{o}$. Consequently, the sequence $b_{l n}$ first increases, and later decreases with $n$. The general shape of the cumulative sums $b_{l n}$ and $b_{2 n}$ is shown in Figure 5.21 below. So, the development of $b_{n}$ is bound between $b_{l n}$ and $b_{2 n}$, while all individual IASC-sessions will differ as they depend on the accidental sequences $a_{n}$ and $\delta_{n}$ generated during the session.

As the termination is determined by the $n$ that satisfy: $b_{n} \geq C(\vartheta)$, the termination time $T$ follows from: $T=\min \left\{n \in[1 . . N] \mid b_{n} \geq C(\vartheta)\right\}$. This relation between the threshold and the iteration time is shown in Figure 5.22 below. This is the inverse relation as depicted in Figure 5.21. The value of $T(C)$ is bound between the inverses of the bounds $b_{1 n}$ and $b_{2 n}$. These bounds exhibit jumps at the thresholds $C_{1}$ and $C_{2}$, that were defined in Figure 5.22. 


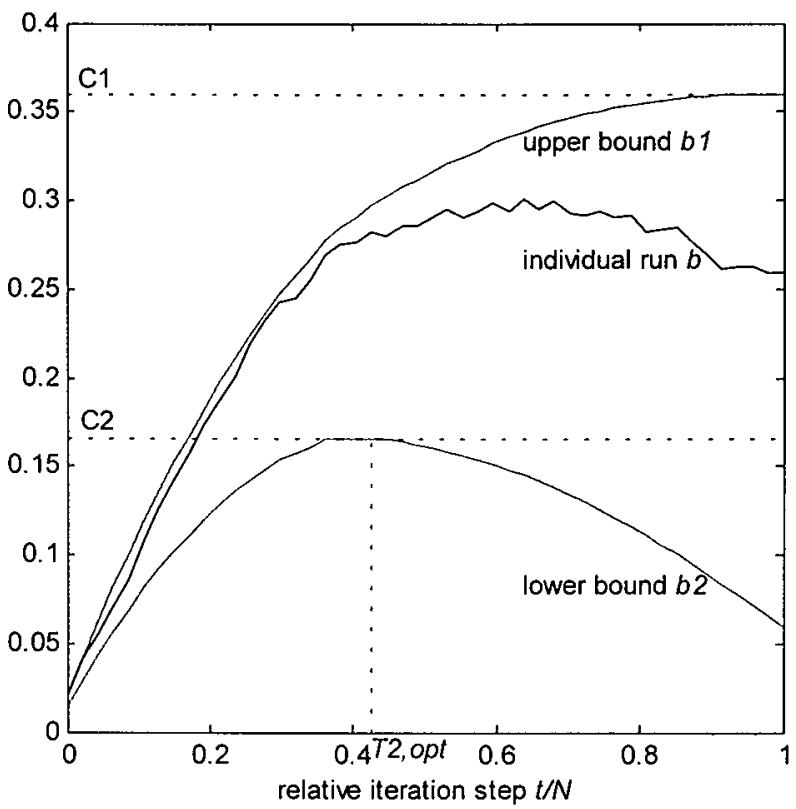

Fig. 5.21. Evolution of sequence $b$ bounded between $b_{1}$ and $b_{2}$. The bounds themselves are also bounded by respectively $C_{1}$ and $C_{2}$.

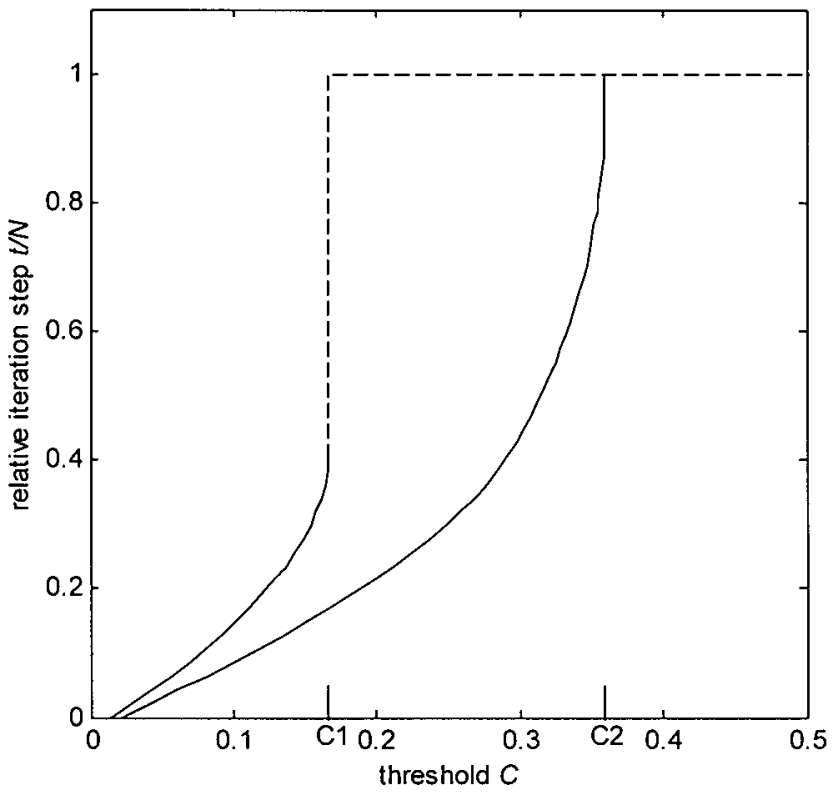

Fig. 5.22. Termination time as function of the generalised threshold $C$. Shown are the two bounds, indicating the lowest and highest possible termination time for a given noise level. Beyond the value $C_{l}$ the iteration will not be interrupted before the maximum time $N$. 
To obtain more insight in the average behaviour of $b_{n}$, we average expression [5.140] over all clusters:

$$
\left\langle b_{n}\right\rangle=\sum_{i=1}^{n}\left(h_{i}^{2}-\eta^{2}\right)
$$

with: $h_{i}^{2}=K^{-1} \sum_{l=1}^{K}\left\langle d_{l}^{n(i)}\right\rangle^{2}$, and: $\eta^{2}=(N K)^{-1} \sum_{n=1}^{N} \sum_{l=1}^{K} D_{l}^{n}(\mathbf{x})^{2}$, the squared effective relative noise ${ }^{3 I}$. Notice that $h_{n}$ is an ordered non-increasing sequence: $h_{1} \geq h_{2} \geq \ldots \geq$ $h_{N}$. The expression for $\left\langle b_{n}\right\rangle$ is morphologically identical to the expression for the lower-bound sequence $b_{2 n}$. Therefore, the same conclusions hold, and consequently sequence $\left\langle b_{n}\right\rangle$ initially increases, and later decreases with $n$. Likewise, the termination time follows from the 'inverse' from sequence $\left\langle b_{n}\right\rangle$. If the generalised threshold $C$ exceeds the maximum value of $\left\langle b_{n}\right\rangle$, the termination condition is never reached, and the iteration continues uninterrupted to its maximum value $N$. In case that: $C<\max _{n}<b_{n}>$ the iteration stops ahead of time. Hence, there exists an optimal time for terminating the iteration, given by:

$$
T_{o p t}=\arg \max \left\langle b_{n}\right\rangle
$$

The success rate of the algorithm for a specific clustering and noise-level follows from the probability that the true class $l$ is elected as most likely cause. Therefore, the expectation value of the quotient of the state functions: $\left\langle u_{j} / u_{k}\right\rangle$ is a good measure for the most probable success rate. This quotient is approximated in equation [5.133] in Section 5.7.5 as:

$$
\left\langle\frac{u_{l}(t)}{u_{k}(t)}\right\rangle \approx \prod_{n \in E(t)} \exp \left(-1 / 2\left(D_{l}^{n}(\mathbf{x})^{2}-<d_{l}^{n}>^{2}\right)\right)
$$

Averaged over all possible clusters, we notice that in the exponent we have obtained the expression for sequence $\left\langle b_{p}\right\rangle$. Therefore, we can write:

$$
<S(t)>=\left\langle\frac{u_{l}(t)}{u_{k}(t)}\right\rangle \approx \exp \left(1 / 2<b_{l}>\right)
$$

So $\langle S(t)\rangle$ follows the same qualitative behaviour as for sequence $\langle b\rangle$ discussed above. The dependence of $\langle S(t)>$ on $t$ is depicted in Figure 5.23.

31 The relation between the absolute noise $v_{l}$ and the effective relative noise $\eta$ follows from the following consideration. Let $\Omega$ be the generalised set of angles, and $P_{\Lambda}(\Omega)$ the generalised polar coordinates in $N$ dimensions. Then we can use the definition of absolute noise to write: $x_{n}=m_{l n}+v_{l} P_{N}(\Omega)$. Therefore: $D_{l}^{n}(\mathbf{x})^{2}=\left(\frac{x_{n}-m_{l n}}{\sigma_{l n}}\right)^{2}=v_{l}^{2}\left(\frac{P_{N}(\Omega)}{\sigma_{l n}}\right)^{2}$. So: $\left\langle\eta^{2}\right\rangle=\omega\left\langle v^{2}\right\rangle$, with: $\omega=\frac{1}{N K} \sum_{l} \sum_{n} \int\left(\frac{P_{N}(\Omega)}{\sigma_{l n}}\right)^{2} \frac{d \Omega}{\Omega_{t o t}}$, and: $\Omega_{t o t}=\int d \Omega$. Thus, for a given clustering, the expectation values $\left\langle\eta^{2}\right\rangle$ of the relative noise, and $\left\langle v^{2}\right\rangle$ of the absolute noise are linearly dependent. 


\subsubsection{Sequences of the Type: $b_{n}=\Sigma_{n}\left(a_{n}{ }^{2}-\delta_{n}{ }^{2}\right)$}

We conclude this section on the influence of the termination criterion on the performance, with a closer look on the type of sequences defined in equation [5.141], that govern the development of the quality $q(t)$ and the success rate $S(t)$. We impose the same basic restrictions as in the previous section;

1. $a_{n}$ is an ordered non-increasing sequence: $a_{1} \geq a_{2} \geq \ldots \geq a_{N}$,

2. all elements are positive: $a_{n}>0$, for all $n$,

3. the first element of $a_{n}$ is larger than 1: $a_{1}>1$,

4. the average is smaller than one: $\left\langle a_{n}\right\rangle<1$,

5. there exists a small $\delta$, such that: $0 \leq \delta_{n} \leq \delta \ll 1$,

6. the minimum element of $a_{n}$ is smaller than $\delta: \min _{n} a_{n}<\delta$.

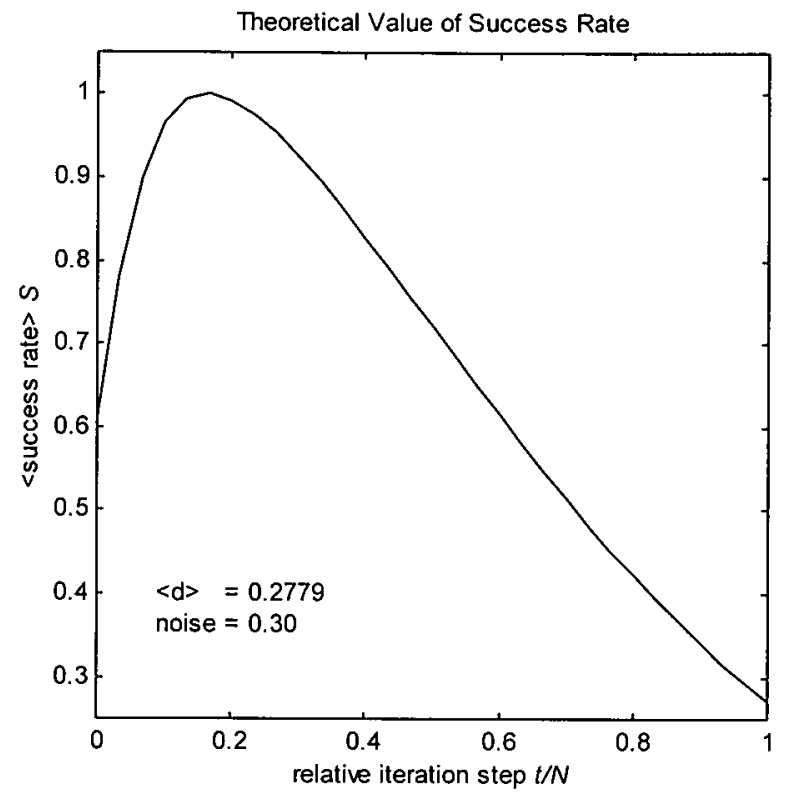

Fig. 5.23. Expectation value of the success rate $S$, as the quotient of the statc-functions: $\ln \langle S(I)\rangle^{2} \approx\langle b\rangle$.

Under these restrictions we have shown that each possible sequence $b_{n}$ is limited between a lower bound $b_{I n}$, and an upper bound $b_{2 n} ; b_{I n} \leq b_{n} \leq b_{2 n}$, with: $b_{1 n} \equiv \sum_{i=1}^{n}\left(a_{i}^{2}-\delta^{2}\right)$ and: $b_{2 n} \equiv \sum_{i=1}^{n} a_{i}^{2}$. These definitions do not impose any restriction on the length of the sequence. However, we now confine the length of the sequence to a - possibly large - number $N$, as we examine the special case where the elements in $a_{n}$ are uniformly distributed. In that case, and following the constrictions (1) and (2), we can write: $a_{i}=p-q i$, where $p$ and $q$ are constants. Let the sequence 
decrease with a certain amount $\Delta$, and let it end with a small but positive number $\varepsilon$. Therefore, $p$ and $q$ are defined as: $p=N \Delta+\varepsilon$, and: $q=\Delta$. Following restriction (5), we require that: $\varepsilon<\delta$. Restriction (3) implies that: $\Delta<2(1-\varepsilon) /(N-1)$. For this particular sequence $a_{n}$ the upper bound $b_{2 n}$ becomes:

$$
b_{2 n}=\sum_{i=1}^{n}(p-q i)^{2}=u_{3} n^{3}+u_{2} n^{2}+u_{1} n
$$

with: $u_{3}=\Delta^{2} / 6, u_{2}=-\Delta(\varepsilon+(N-1) \Delta)$, and:

$$
u_{1}=N(N-1) \Delta^{2}+\Delta \varepsilon(2 N-1)+\varepsilon^{2}+\Delta^{2} / 6 \text {. }
$$

Because of the values of its coefficients $u$, sequence $b_{2 n}$ is a third order polynomial that monotonously increases from zero to $b_{2 \max }$, while the derivative $b_{2}^{\prime}(n)$ is always positive but monotonously decreasing. It reaches its maximum value before $b_{2}{ }^{\prime}=0$, as its increase $a_{n}$ has become very small, but still positive. The point of inflexion follows from: $b_{2} "=0 \Rightarrow n=-u_{2} /\left(3 u_{3}\right)=2 N+2(\varepsilon / \Delta-1)>2 N-2$. This value is not reached during the iteration if $N>2$, which may be assumed safely. The general form of $b_{2}$ is depicted in Figure 5.24.

The lower bound sequence $b_{1 n}$ can be written as:

$$
b_{1 n}=b_{2 n}-\delta^{2} n=u_{3} n^{3}+u_{2} n^{2}+\left(u_{1}-\delta^{2}\right) n
$$

The most important difference between the sequences $b_{l n}$, and $b_{2 n}$ is when $n \uparrow N$. Since in that case $b_{2}(n)$ is slowly approaching its maximum value $b_{2 \max }$ from below, the sequence $b_{l}(n)$ is determined by the term $-\delta^{2} n$, and so almost linearly decreasing. The slope of its descent near $n=N$ is fully determined by the squared effective relative noise $\delta$. For each different effective noise $\delta$ a different negative slope and a different resulting final value $b_{2}(N)$ will arise. If the noise exceeds a lower threshold value, $\delta>\delta^{*}$, this will even cause the resulting lower bound $b_{2}(n)$ to intersect the horizontal axis during the iteration. 


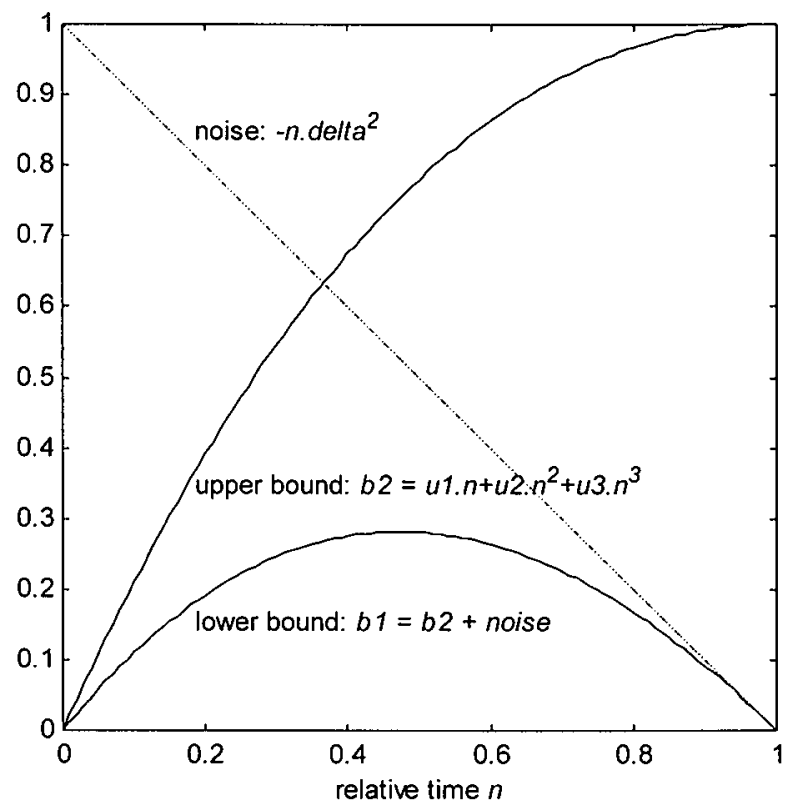

Fig. 5.24. The lower- and upper bound of a uniformly distributed sequence an are third-order polynomials. The upper bound has a local optimum just bcyond the domain of the sequence. The dashed line indicates the influence of the noise. Near the end of the iteration the lower bound is fully determined by this influence. 


\subsubsection{Models for the Evolution of the $\alpha$-Exponent}

The dynamicallity exponent $\alpha$ was introduced in order to find a trade-off between exploration and convergence in the parameter space. It relates to the imprecision contained in the present hypothesis represented by $u_{k}$. The relation is inverse; initially, as there is a high degree of uncertainty in the hypothesis, $\alpha$ should be zero in order to use a priori information like the separation of classes. Later, as the hypothesis converges to the solution, it may be utilised to steer the selection process and $\alpha$ assumes the value 1 . The most straightforward approach is to use the fraction of the explored parameters $t$ to the entire number of parameters $N$ :

$$
\alpha \text {-model } 1: \alpha(t)=\frac{t-1}{N-1}
$$

This is a monotonous increasing function in $t$, such that $\alpha(1)=0$ and $\alpha(N)=1$. However, in practice not all $N$ parameters are used, and the actual number of iterations can be much smaller than $N$. Therefore, in this simple approach $\alpha$ will have intermediate values, and would never utilise the potential to explore the parameter space based on current information.

In this section we present two additional models for the evolution of $\alpha$, based directly on the uncertainty in the actual hypothesis $\boldsymbol{u}=\left(u_{l}, . ., u_{K}\right)$. Let us assume a situation with $K$ defect classes and $N$ parameters. The best estimate for the exponent $\alpha$ depends on the imprecision in the actual hypothesis, represented by $u_{k}$. This imprecision depends on the distance of the actual hypothesis $u$ to the 'real' defect situation of the imaged object, represented by $u^{*}$. In the case that all $K$ defect classes are known and represented, the image is of sufficient quality, and the features are clearly present, this real situation is always represented by a corner-point of the hyper-cube $[0,1]^{K}$. The number of concurrent defects in the actual image therefore is: $\Sigma_{k} u_{k}{ }^{*}$. Now, suppose exactly one defect, $l$, is present, so the image is represented by: $\mathbf{x}=\mathbf{m}_{l}$ in parameterspace, and: $u_{k}{ }^{*}=\delta_{k l}$ in representation space. We now propose two methods for the evolution of $\alpha$, based on an estimation of the imprecision in $u_{k}$.

\subsubsection{A Model Based on Constant Speed in Parameter Space}

The first method is based on the assumption that the progress in parameter space is constant during the iteration. Therefore we make an estimate for the average velocity $V$ in $U$ during the iteration. We compare this with the actual speed of the iteration, in order to estimate the total duration $\mathrm{T}_{t o t}$. First, we calculate the average distance $S$ of a random point $u_{\text {rand }}$ to the 'target' $u_{k}{ }^{*}$. This is the average distance of a random hypothesis $u_{\text {rand }}$ to the real situation $u_{k}{ }^{*} . S$ follows from the average distance of a vector in the hyper-cube $[0,1]^{\mathrm{K}}$ to one of the edges $\delta_{k l}$, i.e. $(0,0, \ldots, 0,1,0, \ldots, 0,0)$ :

$$
S_{K}=\left\langle\left\|\mathbf{u}_{\text {rand }}-\mathbf{u}^{*}\right\|\right\rangle=\int_{0}^{1} d p \int_{0}^{1} d q_{1} \ldots \int_{0}^{1} d q_{K-1} \sqrt{(1-p)^{2}+\sum_{k=1}^{K-1} q_{k}^{2}}=\int_{0}^{1} d q_{1} \ldots \int_{0}^{1} d q_{K} \sqrt{\sum_{k=1}^{K} q_{k}^{2}}
$$

Expression [5.148] can not be solved analytically ${ }^{32}$. Since $0 \leq q_{k}^{2} \leq 1$ we have that: $0 \leq S_{K} \leq \sqrt{ } K$. Let furthermore $T(N)$ be the number of steps, averaged over the entire

\footnotetext{
32 A useful approximation is to calculate the integral over the squares, and then apply the square-root. This leads to an approximation: $S_{K} \approx \sqrt{ } K / 3$.
} 
ensemble of iterations ${ }^{33}$. This provides a rough estimate for the average velocity $V$ from a random point in the hyper-cube to the target:

$$
V=\frac{S_{K}}{T(N)}
$$

The actual speed $v$ in $U$ at step $t$ follows from the distance between the actual hypothesis $u_{t}$ and the initial hypothesis $u_{0}: v=\left\|u_{t}-u_{0}\right\| / t$. In our context $u_{0}$ is either determined by pre-classification, or set to some initial value like 0 or $(1 / 2,1 / 2, . ., 1 / 2)$. Now we can compare the actual speed $v$ with the average speed $V$. Let us assume that the motion in $U$ is approximately linear. Then we can estimate the total duration of the present iteration as: $\mathrm{T}_{t o t}=T(N) V / v$. Using the definitions of $V$ and $v$ we find:

$$
T_{t o t}(t)=\frac{S_{K}}{\left\|\mathbf{u}_{t}-\mathbf{u}_{0}\right\|} t
$$

The expected remaining time for the iteration is: $\theta\left(\mathrm{T}_{t o t}(t)-t\right)$. Let us require that during the iteration $\alpha$ increases from $\alpha=0$ at $\ell=0$ to $\alpha=1$ at the end of the iteration. Therefore, we set $\alpha$ to:

$$
\alpha-\text { model } 2: \alpha(t)=\min \left(1, \frac{t}{T_{t o t}(t)}\right)
$$

Notice that - using equation [5.150] - this definition allows a time-independent formulation of $\alpha$ :

$$
\alpha_{K}\left(\mathbf{u} \mid \mathbf{u}_{0}\right)=\min \left(1, \frac{\left\|\mathbf{u}-\mathbf{u}_{0}\right\|}{S_{K}}\right)
$$

This method, however, will in many situations under-estimate $\alpha$. For example, when the initial hypothesis - due to good pre-classification - is closer to the real situation that an average random hypothesis, or when - due to good parameter-selection - the duration of the actual iteration is shorter than the average iteration length $T(N)$. The basic assumption of constant speed, however, is a gross simplification of the reality, where the position of $\boldsymbol{u}$ is observed to first move quasi chaotically before converging to its final value.

\subsubsection{A Model Based on the Behaviour near the Solution}

The starting point of the second method is the average behaviour of $\boldsymbol{u}$ near a prototype, as discussed in the method 1. For this method we also require that $\alpha$ increases from 0 to 1 during the iteration. In this case, we base the increment scheme on the observation leading to equation [5.83]. In the simplification applied there, the quotient of the largest function $u_{1}$ with second-largest function $u_{2}$ increases as $\exp \left(2 d^{2} t\right)$. Therefore, we propose the following scheme:

$$
\alpha-\text { model } 3: \alpha(t)=\min \left(1, \exp \left(G<d^{2}>(t-1)\right)-1\right)
$$

$G$ is a general constant, introduced in order that $\alpha$ on the one hand does not flip directly from 0 to 1 , and on the other hand reaches 1 before the end of the iteration. The value of $G$ was based on a calculation over a large number of real clusters. A good value was found to be $G=0.75 .\left\langle d^{2}\right\rangle$ is the average normalised distance between the clusters:

${ }^{33}$ Since $0<T(N) \leq N$, a reasonable choice is: $T(N)=1 / 2 N$. 


$$
<d^{2}>=\frac{2}{N K(K-1)} \sum_{n=1}^{N} \sum_{k<l=1}^{K} d_{k l}^{n^{2}}
$$

For a clustering with well-separated classes, the average distance is $\langle d\rangle\rangle\rangle$, and $\alpha$ increases rapidly. For partially overlapping clusters the increase is much slower, $\left\langle d^{2}\right\rangle$ $<1$. Applied to our context, the average distance was found to be: $\left\langle d^{2}\right\rangle=0.181 \pm$ 0.020 . This means that after approximately 5 iterations $\alpha$ reached 1 .

\subsubsection{A Model Based on a Constant Value for $\alpha$}

We compare the three $\alpha$-scenarios with a scheme where $\alpha$ assumes a constant value during the iteration. In Section5.2.4 we mentioned some disadvantages to both $\alpha=0$, and $\alpha=1$. Therefore we look at the performance of the algorithm as function of the constant $\alpha$. To see the effect of $\alpha$ on the performance, let us first consider two examples

\section{Example 1}

Suppose that $h$ hypotheses remain, all with equal probability: $u_{k}=1 / h$. For the separation functional this means that there are two distinct possibilities.

Case 1: $a=0$

$$
\begin{aligned}
& n_{o p t}^{*}(0)=\arg \max _{n} \sum_{k_{1}<k_{2}} d_{k_{1} k_{2}}^{n} \text {. Sequence }\left\{n^{*}\right\} \text { is determined by the order of } \\
& \text { decreasing }\left\{d_{k_{1} k_{2}}^{n}\right\} .
\end{aligned}
$$

Case 2: $\alpha>0$

$$
n_{\text {opt }}^{*}(\alpha)=\arg \max _{n} h^{-2 \alpha} \sum_{k_{1}<k_{2} \in H} d_{k_{1} k_{2}}^{n}=\arg \max _{n} \sum_{k_{1}<k_{2} \in H} d_{k_{1} k_{2}}^{n} \text {, where } H \text { is the }
$$

set of hypotheses. In this case the sequence $\left\{n^{*}\right\}$ is independent of $\alpha$ and depends solely by the order of decreasing $\left\{d_{k_{1} k_{2}}^{n}\right\}_{k_{1} k_{2} \in H}$ within the set of hypotheses $H$.

The same reasoning can be applied to the relevance functional, because $\arg \max _{n} \sum_{k \in H} r_{n k} h^{-\alpha}=\arg \max _{n} \sum_{k \in H} r_{n k}$. In both cases the performance is constant for all $\alpha>0$, only for $\alpha=0$, the performance will show a discrete jump.

\section{Example 2}

In reality the relevant hypotheses - defined by $u_{k}>0$ - will have dissimilar values. This causes a partitioning of the alpha domain in intervals with different optimal parameters. This partitioning will result in different parameters sequences per alpha domain interval, as explored parameters are excluded, and the probabilities are constantly changing during the iteration. Consequently, the performance of the algorithm depends on alpha. In order to find the performance of the IASC-algorithm for a constant alpha, a number of simulations was performed, that will be described in detail in Section 5.9. These simulations conclusively showed that $i$. for alpha $=0$ the 
algorithm has a significant longer iteration length and lower success rate than for alpha $>0$, ii. for $\alpha>0$ the performance remains constant within the experimental accuracy. Therefore, all $0<\alpha \leq 1$ can be used as constant, and within this range there is no factual optimal value for alpha.

\subsection{Main Conclusions regarding Interactive Classification}

In this chapter we provided a theoretical foundation for interactive sensing and classification. The approach was eventually integrated as the InterActive Sensing and Classification (IASC) algorithm. The basis of the IASC algorithm is a symmetric distance measure in the feature space, as defined in equations [5.4] and [5.7]. Similarly, we defined the relevance of a parameter for a specific class or state in equation [5.9]. Using these indicators for distance and relevance, we defined a procedure for selecting the most significant feature parameter for a given state (class). This procedure is called Functional Parameter Selection (FPS). By including the present hypothesis, this approach could be made dynamic. The degree of dynamic reasoning can be modified by the exponent $\alpha$. Sensing is domain dependent. Interactive sensing must be able to explore the object, yet converge to a relevant value at the same time. Integration of FPS with automatic interactive sensing leads to the IASC algorithm.

By defining IASC as a transformation from feature space to the continuous space of possible hypothesis, we were able to study its convergence and stability. If the measurement is within the associated error-ellipse of a class (state) prototype, the IASC algorithm will converge to the correct conclusion. Noise has a detrimental effect, but can be translated to a smearing out of the measurement in feature space.

The dynamic properties of IASC can be classified into a few types of behaviour of the time evolution of the state (class) functions ('probabilities'). Diffuse classes and mixed equilibrium points can lead to incorrect conclusions of IASC.

The termination criteria of the IASC algorithm have a decisive role on its performance. Given the noise and average cluster overlap, an optimal threshold for the quality based termination criterion can be defined.

Based on different assumptions several evolution schemes for the dynamicallity exponent $\alpha$ could be defined.

In the next two chapters we will test the IASC approach. First, in Chapter 6, we test IASC on a wide range of theoretical problems. Finally, in Chapter 7, we look at a complex real-world application of IASC in the context of the automatic quality control of an industrial printing robot, based on computational vision. 


\section{Chapter 6}

\section{Numerical Simulations with the IASC Algorithm}

\subsection{Introduction}

In this chapter we present a number of experiments based on the approach, sketched in the previous chapter. Main objective of this chapter is to gain insight in the performance of $I A S C$, and to compare it with other methods such as exhaustive and random parameter-selection. Moreover, we can test theoretical predictions of chapter 5. First, we provide some useful definitions. For a thorough description of the performance, it is useful to regard the IASC algorithm as a black box control system. In this interpretation, three main information steams can be identified.

1. the input-information, consisting of the off-line clustering results $\left\{\mathbf{m}_{k}, C_{k}, k=1 . . K\right\}$, and the real-time sensoring-output vector $\mathbf{x} \in \mathbb{R}^{N}$.

2. the output-information, consisting of the vector $u \in \mathbb{R}^{K}$, i.e. the probability distribution over the $K$ object states.

3. the control-data $p$, a feed-back based on the performance and some characteristics of the input data such as the noise.

In this representation, we can represent the algorithm as a system: $\mathbf{u}=\Phi(\mathbf{x}, \mathbf{p})$. Moreover, we define descriptors for the data and the efficiency and performance of the algorithm. These descriptors convey information about the performance and accuracy of the classification process, and provide a structuring and characterisation of the data. They provide a framework for the evaluation of the algorithm, and the interpretation of the results. In Figure 6.1 a schematic representation of the various descriptors are depicted in their role in the IASC algorithm. In the next section, we present a number of suitable descriptors for the data and the performance of the algorithm. In the subsequent sections, we employ these descriptors as a guideline in the discussion of the evaluation of the algorithm. 


\subsection{Descriptors for the IASC Algorithm}

To quantify the performance of IASC, we first define descriptors for the input, output, and control parameters of the algorithm.

\subsubsection{Descriptors for the Input Parameters}

The input of the system can be characterised by:

1. the set $\Omega$ of the $K$ clustered states in the space of the $N$ assessable parameters,

2. the representation of the actual sensor information relative to $\Omega$.

The first category consists of the elements of cluster $\Omega$; the object states or the defect classes. It can be characterised by the clustering descriptors $\{K, N,\langle d\rangle\}$, where $\langle d\rangle$ denotes the average geometric cluster-distances between the elements of $\Omega, d_{k l}^{\prime \prime}$, defined in Section 5.2.1.

Based on the average geometric cluster-distance $\langle d\rangle$, three situations can occur for the relative position of the clusters:

situation 1: clusters are well separated; the average geometric distance is larger than 1 ,

situation 2: clusters are partially overlapping, the average geometric distance is about equal to 1 ,

situation 3: clusters are strong overlapping, the average geometric distance is smaller than 1 .

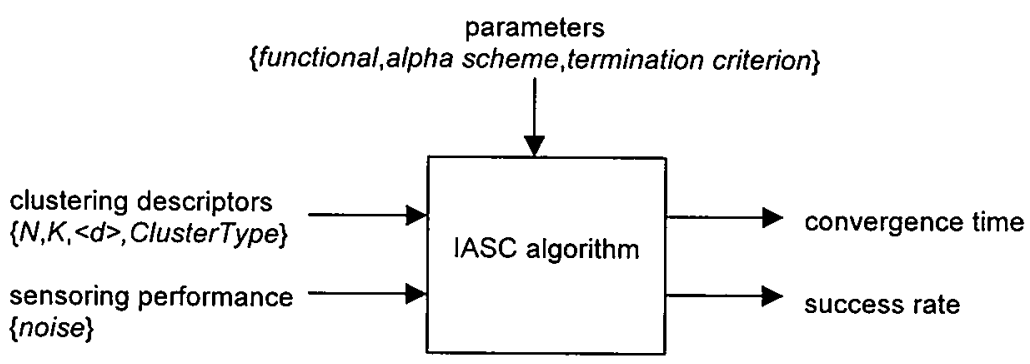

Fig. 6.1. Main descriptors of the IASC Algorithm.

Another descriptor is the typology of the distribution of the classes in parameter space. We define three cluster types;

1. Random, the distribution of the classes follows some spatial probability distribution,

2. Special, for each parameter there are a few classes - possibly one - that have a value near 1, all other classes have a near-zero value for this parameter. Simultaneously, for each class there is one, or a few, parameters that have near-one values for this class, while all other parameters have value near zero. This type is based on the design of the image analysis parameters in Chapter 1, where most defect classes had specific fuzzy descriptors. 
3. Real. In principle, all clusterings can be classified by the extent to which they are random or special. This type is introduced to distinguish the performance of the algorithm on real data from the two former, constructed sets.

Figure $6.2 a$ shows the distribution of a well separated random clustering, generated with a uniform spatial distribution. Figure $6.2 b$ shows the distribution of the geometric distance for a real clustering, exhibiting strong overlap. This distribution represents a case where $N=31$, and $K=18$. About $45 \%$ of the parameters and $39 \%$ of the classes fit with the type 'special cluster'.
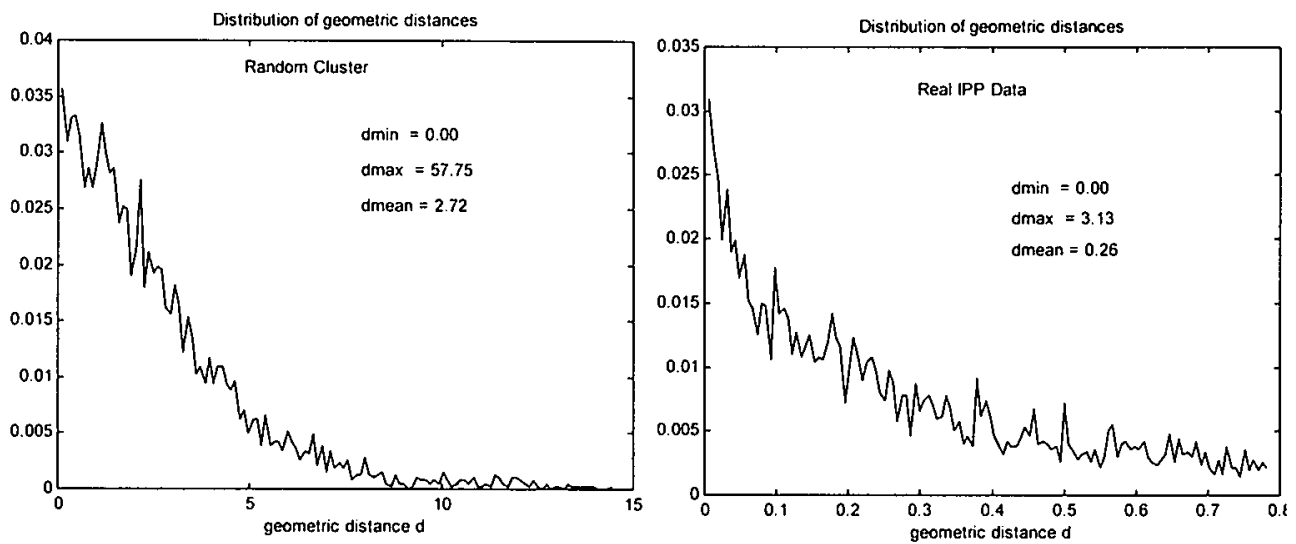

Fig. 6.2. Distribution of geometric disfances a (left): random-generated clusters. b (right): clusters from a from a real data-set, stemming from image analysis of printing defects.

The second category of input parameters concerns the information produced by the sensors with respect to the actual object. This data can be characterised by the position of the actual measurement in parameter space, relative to the clustered states $\Omega$. This can be quantified by the absolute noise $v=\left\|\mathbf{x}-\mathbf{m}_{k}\right\|$, or the relative noise, defined in equation [5.98] in Section 5.6.6. An alternative definition, that well quantifies the relative position of the measurement with respect to the actual object state is the normalised quality of the sensor output, that we define next. Consider an actual state ' $l$ ', and a measurement $\mathbf{x}$ in parameter space. Now we compare the distance of $\mathbf{x}$ the actual state ' $l$ ' with the distance of the closest state to ' $l$ '. The distance of the closest state to ' $l$ ' is: $\Delta_{l}=\min _{n} \min _{k \neq l} s_{k l}^{n}$, so we calculate:

$$
f=\frac{2 S_{l}(\mathbf{x})}{\Delta_{l}}
$$

If $f<1$, the actual measurement is closer to the real state ' $l$ ' than to any other state, and the quality of the sensor data is good. Quantity $f$ is called the relative sensor noise. The sensor data quality can be defined as some inverse function of the sensor noise, like:

$$
Q_{\text {sens }}=\frac{1}{1+f^{2}}
$$




\subsubsection{Main Input Descriptors: Average Cluster Separation and Absolute Noise}

The main descriptor of the clustering is the average geometric distance between the clusters: $<d\rangle$. The noise and the sensor data quality provide a suitable descriptor for the sensor output. Together, they influences the main IASC performance. Therefore, the performance is measured as a mapping of these two entities.

\subsubsection{Descriptors for the Output Parameters}

In Section 5.6.1 a number of criteria for the performance of the IASC algorithm were defined.

1. the quality of the final conclusion.

2. the success rate ' $S$ ', i.e. the probability to find the true state represented by the input data.

3. the relative number of iterations or relative convergence time of the iteration: $T_{\text {con }} / N$.

4. the relative computation time of the iteration.

5. the stability, i.e. the tolerance of the output to small perturbations in the input.

In the evaluation of the algorithm we employ especially the second and third criterion, the success rate and the relative convergence time. The quality of the conclusion is studied separately in the analysis of the termination criteria. The relative computation time has a clear relation to the relative convergence time, though in practice it may be more interesting. Moreover, it depends on the used platform and implementation.

A consideration with respect to the relative convergence time would be to involve the result of the algorithm. Let $T_{1}$ represent the relative convergence time in case the algorithm succeeds in finding the correct solution, and $T_{0}$ when it does not. Within the experimental accuracy no difference was found between $T_{0}$ and $T_{1}$, therefore we only analyse the general quantity $T_{\text {con }} / N$.

In our evaluation, we measure the performance of the various types of IASC-algorithms according to the two parameters $S$ and $T_{c o n} J N$. In certain situations, there is an intuitive appeal to represent the performance of the algorithm with one single identifier. The difficulty in such situations is that the cost of one unit 'relative convergence time' has to be expressed in one unit 'success rate'. This can be done by adding the success rate with the 'speed' of the classification as: $Q=\lambda_{1} S+\lambda_{2}(1-T \operatorname{conv} / N)$, with: $\lambda_{1}+\lambda_{2}=1$. Here we chose: $\lambda_{1}=\lambda_{2}=1 / 2$. Though this represents just one arbitrary choice, it gives an integrated measure for the result of the IASC algorithm. We call this quantity ' $Q$ ' the quality of the classification process. 


\subsubsection{Control Parameters of the Algorithm}

Three entities that control the output of the algorithm can be freely selected. These entities are:

1. The functional type for the Functional Parameter Selection (FPS). These were defined in Section 4.2. Examples are: $\{$ nearest prototype, separation, relevance, ... .

2. The alpha-scheme, i.e. the dependence of the 'dynamicallity exponent' $\alpha$ as function of the state of the algorithm. These were defined in Sections 5.2.3 and 5.7.6. Examples are: \{constant, linear, exponential, ...\}.

3. The termination criterion as function of the state of the algorithm. These were defined in Sections 5.4.4, 5.7.5. Examples are: \{convergence rate, output based with(out) memory\}.

These entities were analysed in great detail, and the results are presented in the subsequent sections.

\subsubsection{Relation with number of clusters $\mathrm{K}$ and number of parameters $\mathrm{N}$}

The basic descriptors of the clustering are the number of clustered states $K$ and the number of assessable parameters $N$. It is found empirically that, for a given range of the main input descriptors $\langle d\rangle$ and noise, the output-parameter Success Rate, does not significantly depend on $N$ or $K$, as clearly visible in Figure $6.3 a$. This figure shows the empirical values for a large set of experiments, based on rescaled real data. The surface shows the average value per segment of the $\mathrm{N} \times \mathrm{K}$-plane. The success rate can have a wide range of values in $[0,1]$, depending on the other input and control variables. The output-parameter Tconvergence/ $N$, on the other hand, shows a clear relation with $N$ and $K$, as depicted in Figure $6.3 b$. This shows that the relative convergence time Tconvergence/ $N$ increases monotonically with $K$ and $N$ from $K=1, N=1$, until the maximum value: Tconvergence/ $N=1$ is reached.
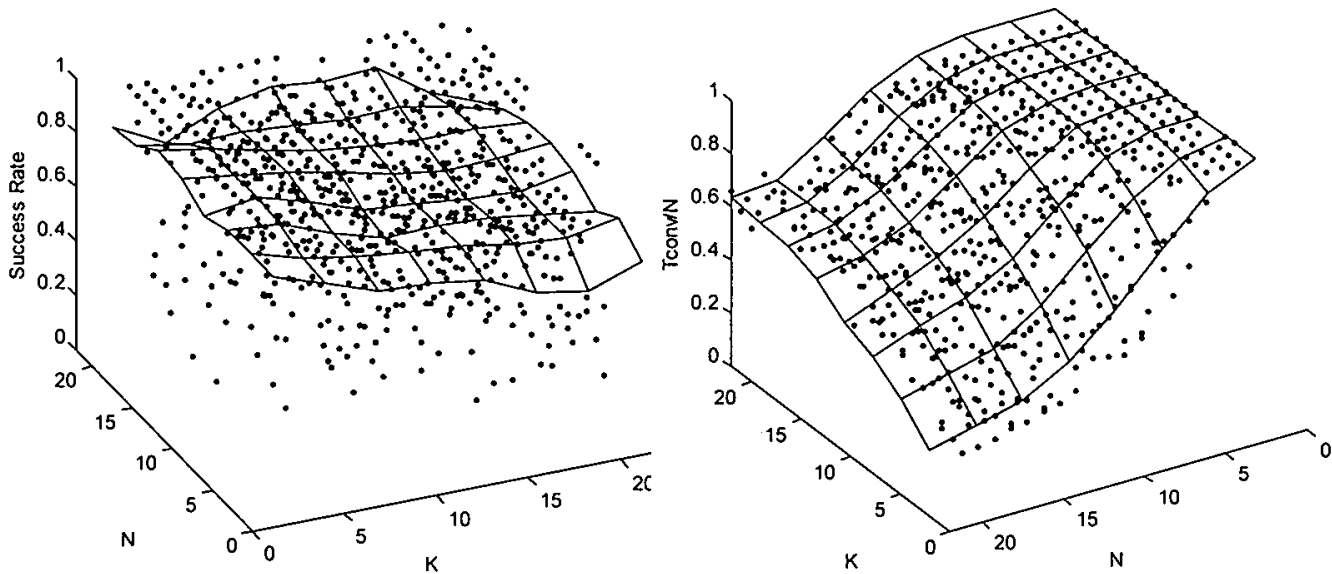

Fig 6.3. Relation of the output parameters $S$ (Success Rate) (left) and $T / N$ (Relarive Convergence Time) (right) with the clustering descriptors $N$ and $K$. The surfaces show the average value per segment of the $N \times K$-plane. Simulations based on separation functional, with noise 0.15 , scaled artificial data with $\langle d\rangle=1$, and alpha-model 4 . 


\subsubsection{Relation with the Cluster Type}

Below the diagram of success rate versus relative convergence time shows the relation for three different cluster types. All calculations have been made with: the separation-functional, $\alpha$ jumping from 0 at $t=1$ to $\alpha=1$ for $t>1$, and noise in the interval $[0.15,0.2]$. The real data has: $N=31$, and $K=18$. The covariance matrix of the real data was artificially rescaled, such that the average geometric distance was 1 . The other clusters have: $\langle d\rangle=1, N=8$, and $K=$ 8. The diagram shows that there is indeed some dependence on the cluster type. Random clusters have a higher success rate and lower relative convergence time than special clusters. This is caused by the fact that more parameters correlate with the object types than in the case of special clusters, resulting in somewhat longer computation time but higher success rates.

\begin{tabular}{|c|c|c|}
\hline Cluster Type & Relative Convergence Time & Success Rate \\
\hline Random & $0.97 \pm 0.03$ & $0.64 \pm 0.18$ \\
\hline Special & $0.91 \pm 0.05$ & $0.44 \pm 0.14$ \\
\hline Scaled Real & $0.65 \pm 0.09$ & $0.81 \pm 0.13$ \\
\hline
\end{tabular}

Table 6.1

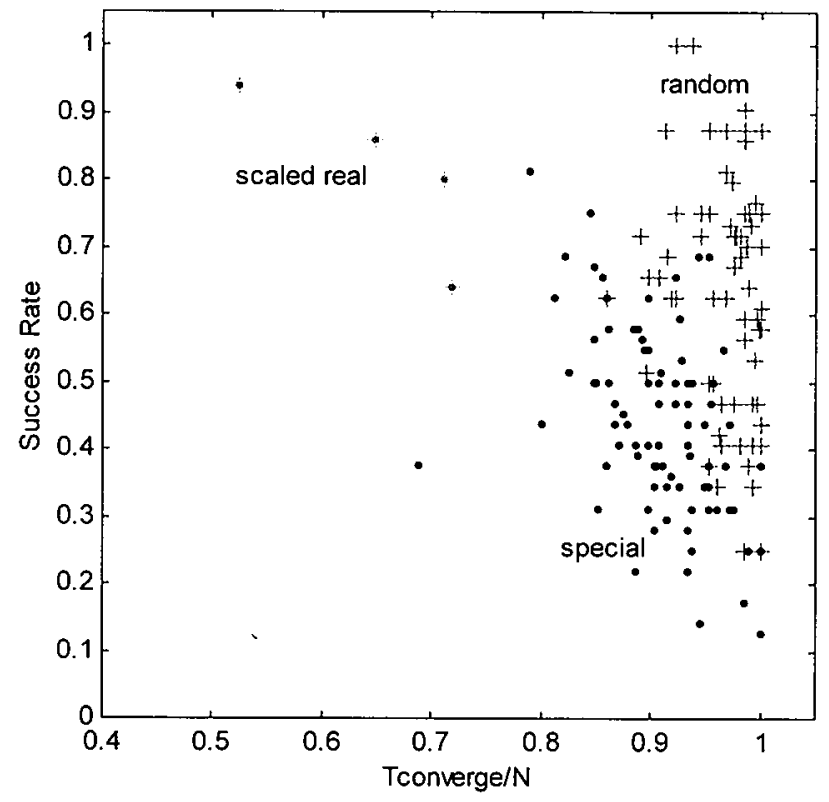

Fig. 6.4. Position of cluster-types in $(T c o n / N, S)$-plane based on simulations with separation functional on scaled data with $\langle A\rangle=1$, noise in $[0.15,0.25]$, and alpha-model 4 .

\subsubsection{Mapping the performance of the Algorithm.}

The average geometric cluster distance $\langle d\rangle$ together with the absolute sensor output noise $v$ constitute a basic but adequate pair of input descriptors for the algorithm. They exert the principal influence on the IASC performance; the relative convergence time $T_{\text {rel }}=T_{\text {conv }} / N$, and the success rate ' $S$ '. In the next sections, we analyse the relation between the input $\{<d$, noise $\}$ and the output $\left\{T_{r e l}, S\right\}$, for various control descriptors, like the FPS functionals. 


\subsection{Comparison of FPS Functionals}

In this section we compare the performance of four functionals for Functional Parameter Selection (FPS), described in Section 5.2. As performance criteria we use the success rate, and the relative number of iterations or convergence time, defined in Section 5.6.1. As gauge we use the corresponding results of random and exhaustive selection. As a first result, the performance of random and exhaustive selection was found to be equivalent. Exhaustive selection can be regarded as one specific instance of random selection, i.e. one with a predefined, fixed order. The following FPS functionals were involved in the comparison:

1. random/exhaustive selection, defined in Section 5.2.2, as yardstick for the comparison;

2. the 'dynamic separation' functional, defined in Section 5.2.3;

3. the 'dynamic relevance' functional, also defined in Section 5.2.3;

4. the 'global nearest prototype' functional defined in Section 5.2.5, a mix of the functionals for 'nearest prototype' $(N P)$ and 'relevance', and

5. a mix of the dynamic functionals for 'separation' and 'relevance', defined in Section 5.2.5.

The comparison focussed on the two output-parameters success rate ' $S$ ' and the relative convergence time ' $T$ conv $/ N$ ', as function of the two input-parameters average geometric cluster distance ' $\angle d>$ ' of the clustering, and noise ' $v$ ' in the sensor output. In order to quantify the performance, a large number of numerical simulations was performed on real data with $N$ $=31$ and $K=18$. The data consisted of the prototypes and covariance matrices of a clustering, stemming from CCD-image analysis of defects in industrial pad-printing. Noise was artificially added by generating a measurement vector $\mathbf{x}$ that had a distance ' $v$ ' to the - known - prototype ' $l$ ', so: $v=\left\|\mathbf{x}-\mathbf{m}_{\|}\right\|$. In order to obtain all possible cluster distances, the covariance matrices of the prototypes were rescaled to fit the desired cluster separation $\langle\alpha\rangle$, using equation [5.7] from Section 5.2.1. In total a number of 2287 combinations of noise $v$ and cluster separation $\langle d\rangle$ were generated, using a uniform random distribution on: $(v,\langle d\rangle) \in$ $[0,1] \times\left[2^{-7}, 2^{5}\right]$. For each combination $(v,<\Delta>)$, the IASC algorithm was applied 100 times for each of the five functionals, in order to obtain significant mean values and standard deviations. The IASC used a model for alpha, called model 4, where $\alpha$ jumped from $\alpha=0$ in the first step, to $\alpha=1$ during the rest of the iteration. Furthermore, the dynamic termination criterion of Section 5.4.4 was used in the simulations.

The quality $q$ is not studied separately, because it is expected to exhibit a strong correlation with the success rate $s$. 


\subsubsection{General Input-Output Relations for FPS Functionals}

Figure 6.5 shows the relation of, respectively, the success rate $S$, and the relative convergence time $T$ conv $/ N$, as function of $\langle d\rangle$ and $v$, for random/exhaustive selection (grey surface), and the dynamic separation functional (black surface). Such plots are the basis of the evaluation. As the figures show, the spread in the data is relatively low. Therefore, the indicated surfaces represent a fair approximation of the relation between the performance parameter and the input-parameters $v$ and $\langle d\rangle$. Similar surfaces are available for all four FPS functionals. In all cases, the $S$-surface lies above, and the $T$ con $/ N$-surface lies below the equivalent surface of random/exhaustive selection. This immediately demonstrates that the FPS functionals outperform random selection, both in success rate and computation steps. Though the figures for the individual FPS functional differ, they share the following properties of the separation functional as compared with random selection, visible in the Figure 6.5;

- for significant cluster overlap, i.e. for low values of $\langle d\rangle$, the surfaces of the FPS functional and random selection flow together;

- in the same way, for very low noise values the two surfaces coincide;

- for higher values of $\langle d\rangle$ the two surfaces deviate monotonously;

- similarly for increasing noise values, the two surfaces monotonously separate.

It is therefore useful to divide the $(v,<d>)$-plane into a small number of segments, where the behaviour of the functional is relatively constant. We will later analyse the performance of FPS relative to random selection in such a partitioning of the $(v,<d>)$-plane in more detail. From a practical perspective this partitioning is useful, because in concrete applications the clustering is available, so $\langle d\rangle$ is known, and $v$ can normally be estimated from experience with the sensors.

Depending on the specific segment of the $(v,<d>)$-plane, the selected optimisation criterion determines the most appropriate FPS functional or random selection for the IASC algorithm. This optimisation criterion can be the highest possible success rate $S$, or the largest possible speed N/Tconv. An intermediate solution is a mixed performance measure, like the combination of $S$ and $T c o n v / N$ in the quantity $Q$, denoting the quality of the classification process, defined earlier in this section.

In order to avoid confusion, there are no error-bars depicted in the two Figure 6.5. The errors were found to be practically zero in the regions where the noise was near zero, or for where $<d>$ was small. The size of the error increased with increasing noise and $\langle d\rangle$, to a maximum of about 0.1 in the extreme regions, both for $S$ and $T c o n v / N$. 

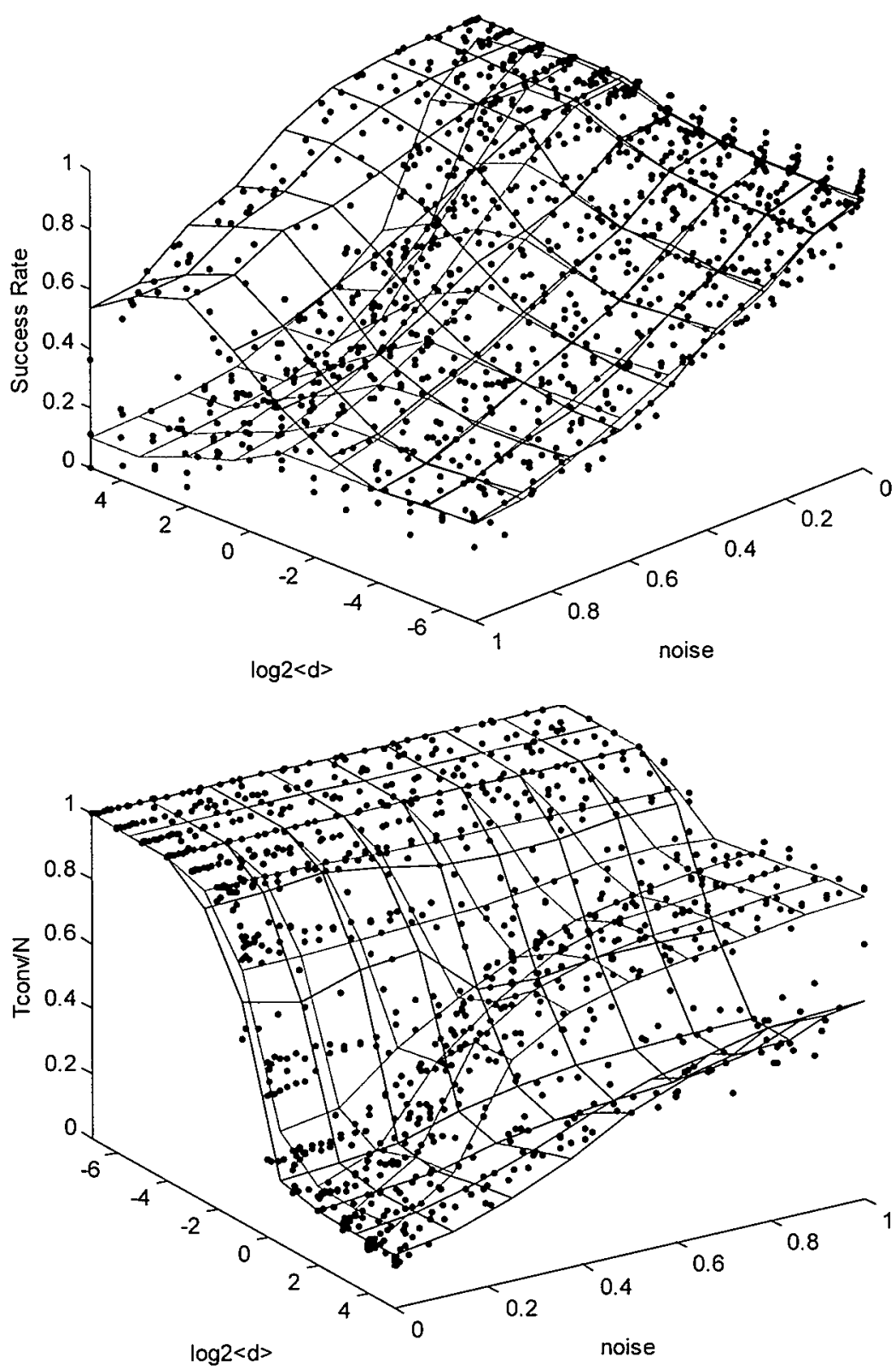

Fig. 6.5. Relation between performance parameters of IASC and problem descriptors $<d>$ and noise for scaled real data, with $N=31$ and $K=18$, alpha-model[4], both for FPS with 'separation' functional (dark surface), and 'random/cxhaustive' selection (light surface). a (above), the relation with the average success rate for 2287 uniformly random generated points. with 100 trials per point. The upper plane represents FPS. b (below), ditto for the relative convergence time. Here, the upper plane represents random/exhaustive selection. 


\subsubsection{Averaged 1-Dimensional FPS Input-Output Relations}

The surfaces in Figure 6.5 can be projected over one dimension on a side-plane, and subsequently averaged. This gives the average relation between an input and an output parameter. Two examples are shown in Figure 6.6, where the data for random/exhaustive selection from Figure $6.5 b$ is averaged respectively over the $\log _{2}(<d>)$ and noise-parameters.
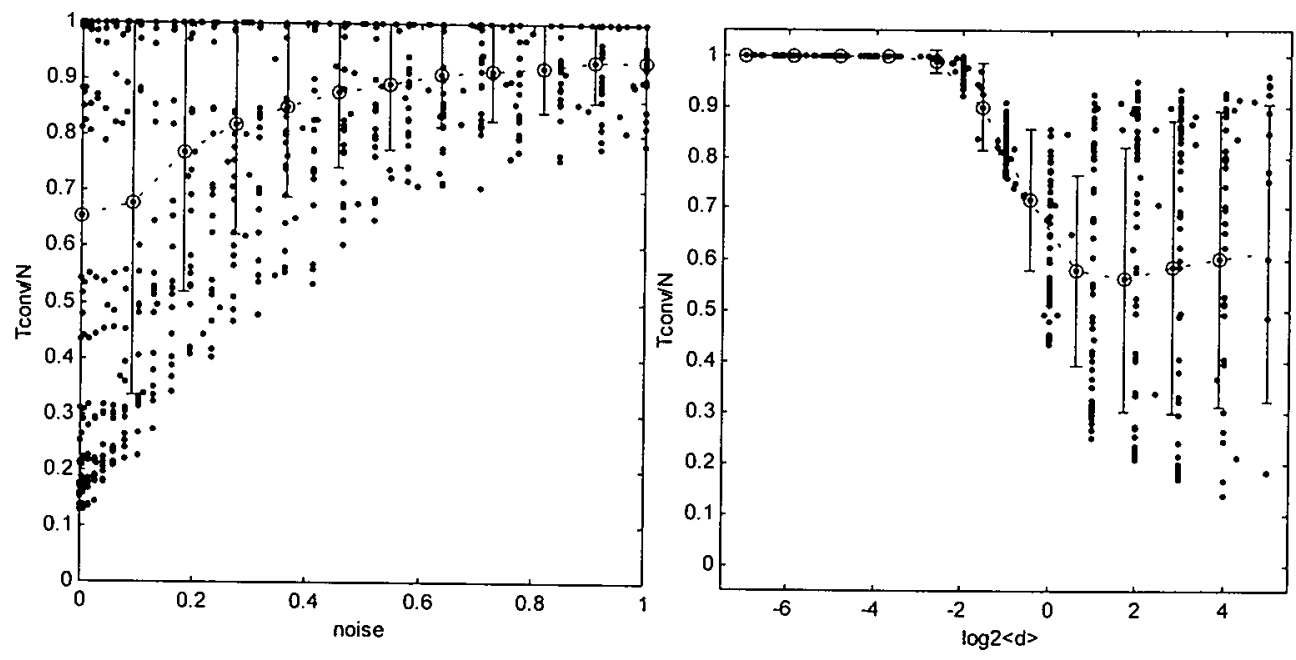

Fig. 6.6. Performance of random/exhaustive selection. The left-hand figure shows the relation of the relative convergence time versus noise. This is derived from a projection of Figure 6.5 . The right-hand figure shows the relation of the relative convergence time versus the cluster spacing $\langle d\rangle$, also derived from a projection of Figure 6.5. Based on scaled real data with alpha-model 4.

Such averaged data provides a qualitative picture on the relation between the two input and the two output parameters. In Figure 6.7 on the next page all four of these average relations for all five functionals are shown. These figures give the following information:

- for all functionals the success rate decreases with increasing noise,

- for all functionals the convergence time increases with increasing noise,

- for all functionals the convergence time decreases with increasing cluster separation $\langle d\rangle$,

- for all FPS functionals the success rate slightly increases with increasing cluster separation $\langle d\rangle$.

Like in the original data like Figure 6.5, these figures show that all FPS functionals outperform random/exhaustive selection. 

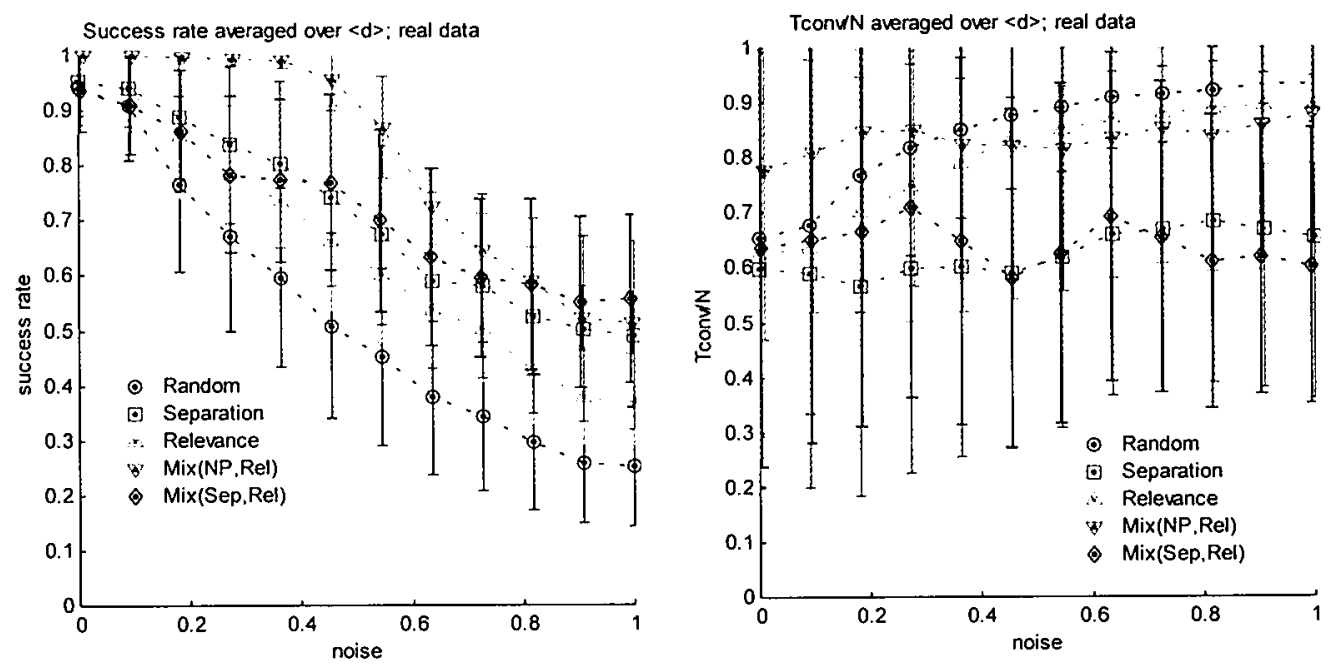

Success Rate averaged over noise; scaled real data
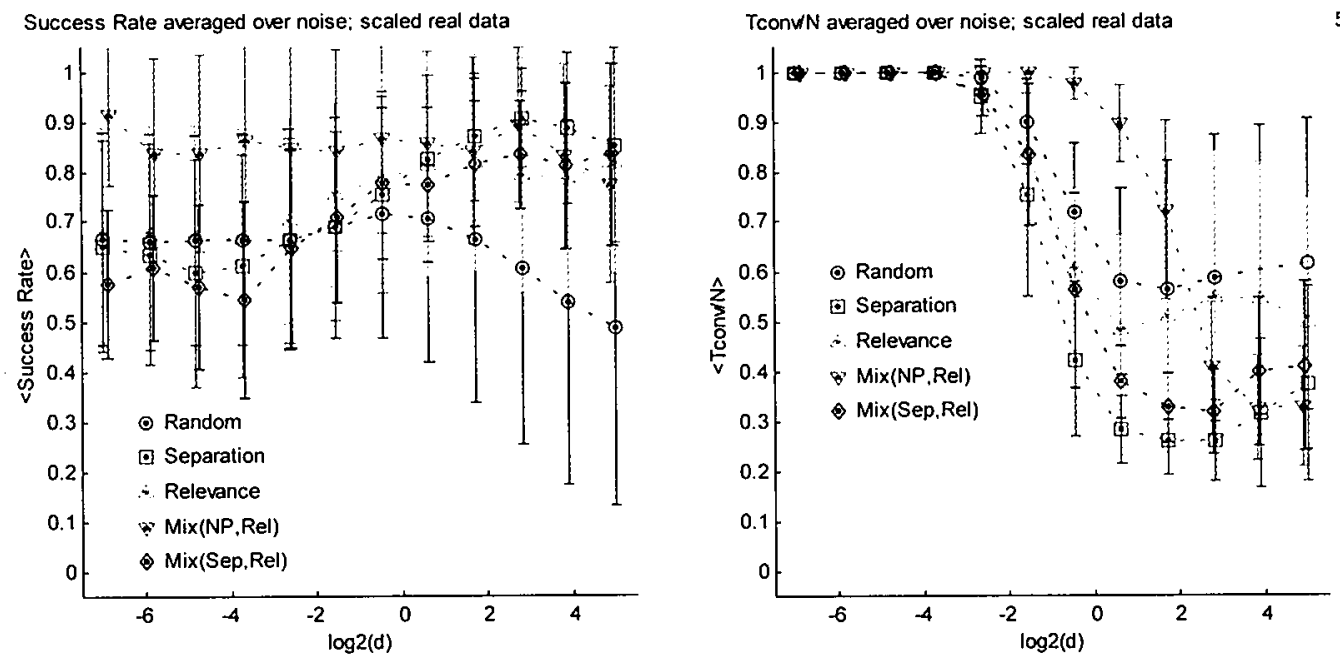

Fig. 6.7. Performance of four functionals for Functional Parameter Selection, compared with random/exhaustive selection. The results are based on of 2287 uniformly random generated points, with 100 trials per point, using scaled real data, with $N=31$, and $K=18$. The left-hand figures show the relation with the average success rate, the right-hand figures the relation with the relative convergence time. Above, the relation versus noise, below, the relation versus the cluster spacing $\langle d\rangle$. These figures are derived from projections of Figure 6.5 . Based on scaled real data and alpha-model 4. 


\subsubsection{FPS Performance Averaged Over All Input Parameters}

Similarly, the data for each output parameter can be averaged over both input parameters. This averaging must be corrected for inhomogenities of the distribution of generated experiments in the $(v,<\infty)$-plane. This results in the average performance for each functional on the $(v,<d>)$-plane. The table below gives these average values for the performance each of the five functionals. Included in the table is the 'classification quality', defined above, and the effective computation time for the numerical simulation.

Results averaged over noise and $\langle a b$

\begin{tabular}{|c|c|c|c|c|}
\hline Functional & $<$ success rate $>$ & $<$ Tconv/N $>$ & $<$ Quality $>$ & $\begin{array}{c}\text { Computation } \\
\text { Time }^{\prime}\end{array}$ \\
\hline Random/Exhaustive & $0.53 \pm 0.27$ & $0.84 \pm 0.20$ & $0.35 \pm 0.20$ & $0.09 \pm 0.02 \mathrm{sec}$ \\
\hline Separation & $0.70 \pm 0.21$ & $0.65 \pm 0.32$ & $0.53 \pm 0.24$ & $0.02 \pm 0.02 \mathrm{sec}$ \\
\hline Relevance & $0.64 \pm 0.22$ & $0.80 \pm 0.23$ & $0.42 \pm 0.20$ & $0.04 \pm 0.02 \mathrm{sec}$ \\
\hline Mix(NP,Rel) & $0.82 \pm 0.19$ & $0.80 \pm 0.28$ & $0.51 \pm 0.17$ & $0.04 \pm 0.02 \mathrm{sec}$ \\
\hline Mix(Sep,Rel) & $0.70 \pm 0.20$ & $0.67 \pm 0.30$ & $0.52 \pm 0.23$ & $0.03 \pm 0.02 \mathrm{sec}$ \\
\hline
\end{tabular}

Table 6.2

From this table several conclusions can be drawn:

- for all performance criteria all FPS functionals outperform random/exhaustive selection,

- the iteration length and the actual computation times are correlated,

- in an equal appraisal of success rate and convergence time, in form of the 'quality', the best FPS functional is 'separation', mainly because it is the fastest with a reasonable success rate.

Histograms, showing this information, are presented in Figure 6.8. In the same figure, the location is shown of each functional in the plane of the output parameters $S$ and $T \operatorname{con} v / N$.

${ }^{1}$ Calculations performed on a $600 \mathrm{MHz}$ Athlon Processor using Matlab-interpreter under WindowsNT 

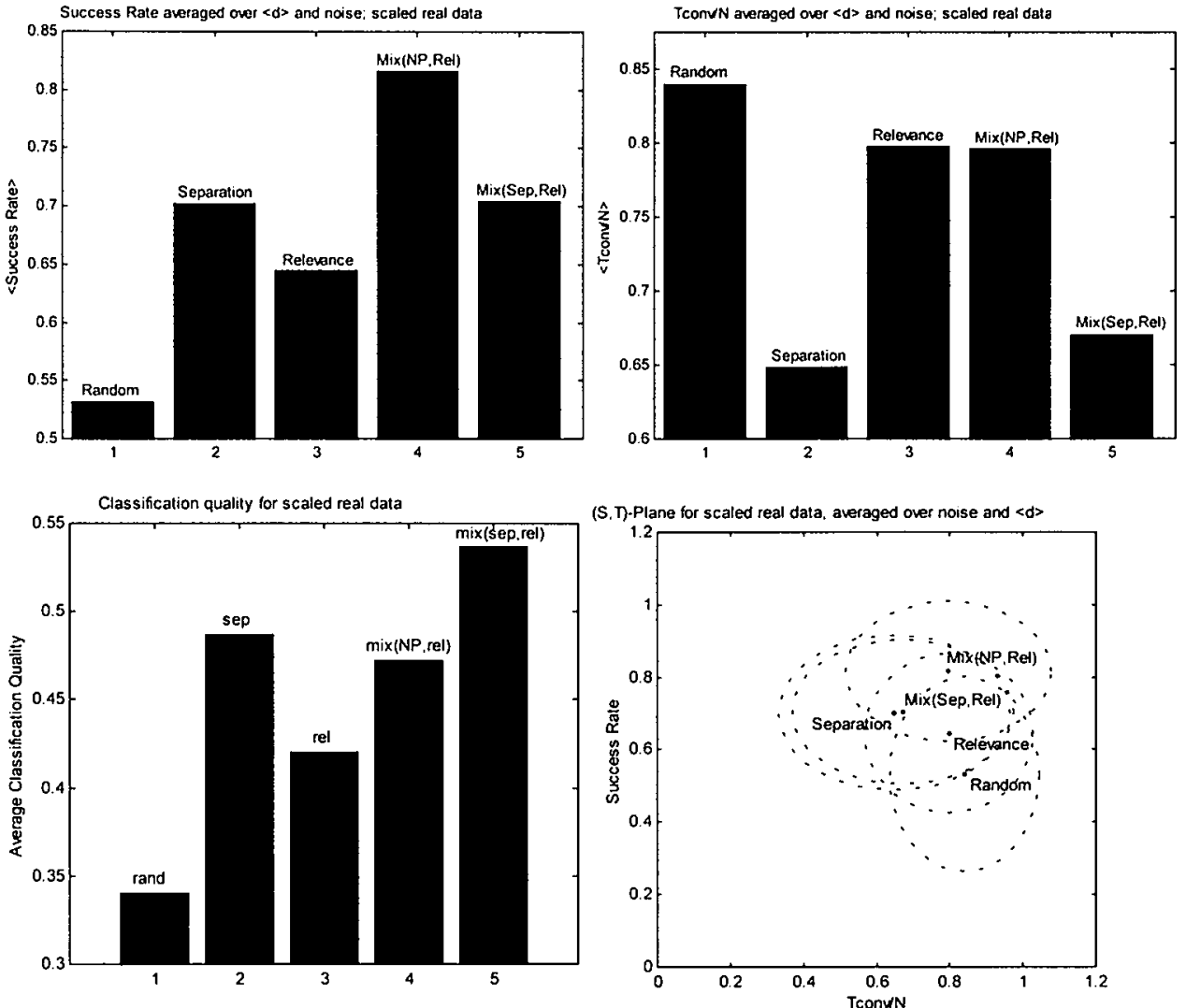

Fig. 6.8. Performance measures lor the IASC algorithm, using of four types of functionals for Functional Parameter Selection, and compared with random/exhaustive selection. The results are based on of 2287 uniformly random generated points, with 100 trials per point, using scaled real data, with $N=31$, and $K=18$. The performance is averaged over all noise and $\langle d\rangle$. Above left the average success rate $S$, showing that all four FPS functionals outperform random/exhaustive selection by some $25 \%$. Above right the average relative convergence time $T$. From this figure it is clear that all FPS functionals are at least $15 \%$ faster compared to random/exhaustive selection. Below left, the parameter 'classilication quality' is defined as: $Q=(S+(1-T)) / 2$. This gives a 1-dimensional measure for the performance. The figure shows that the FPS functionals have on average a $33 \%$ higher quality than random/exhaustive selection. Based on alpha-model 4. 


\subsubsection{FPS Performance In 9 Segments of the Input Space}

Averaging over all input-parameters gives a convenient estimate for the performance of the functional, but does not involve detailed behaviour in parts of the input space. As noted before, the input-plane $(v,<d>)$ can be divided in non-overlapping segments, where the behaviour of the functionals can be characteristically described.

Based on the results from our analysis, we propose the following typology for noise and cluster overlap.

- The noise $v$ can be divided in \{LOW NOISE, MEDIUM NOISE, HIGH NOISE\}.

- The cluster separation $\langle d\rangle$ can be divided in: \{SEVIERE OVERLAP, OVERLAP, NO OVERLAP\}.

This gives a partitioning of the input plane in 9 parts.

The boundary between these intervals are based on Figures 6.5 and 6.6. Thus, the following values were assumed for the domains of noise and cluster overlap:

- LOW NOISE: : [0,0.2>, MEDIUM NOISE : [0.2, 0.4], HIGH NOISE: $<0.4,1]$, and:

- SEVERE OVERLAP : $<-\infty, 0.2>$, OVERLAP : $[0.2,1.2]$, NO OVERLAP $:<1.2, \infty>$.

Based on this partitioning, and the results of the previous sections, we can give the following characterisation of the output parameters.

\begin{tabular}{|c|c|}
\hline Input Space Segment & FPS Performance \\
\hline$\{*$, SIEVIERE OVIERLAP $\}$ & $\begin{array}{l}\text { 'nothing works', as all FPS functionals give the same high } \\
\text { convergence times and low success rates as } \\
\text { random/exhaustive selection. }\end{array}$ \\
\hline \{LOW NOISE, OVERLAP\} & FPS somewhat outperforms random/exhaustive selection. \\
\hline $\begin{array}{l}\text { \{MIEDIUM-HIGH NOISE, } \\
\text { OVERLAP\} }\end{array}$ & 'nothing works'. \\
\hline \{LOW NOISE, NO OVERL $A P\}$ & $\begin{array}{l}\text { 'everything works', as all FPS functionals give the same low } \\
\text { convergence times and high success rates as } \\
\text { random/exhaustive selection. }\end{array}$ \\
\hline $\begin{array}{l}\text { \{MEDIUM-HIGII NOISE, NO } \\
\text { OVIERLAP\} }\end{array}$ & FPS clearly outperforms random/exhaustive selection. \\
\hline
\end{tabular}

Table 6.3

To demonstrate this partitioning, the three tables below give the quality of the classification for each of these nine segments. In Figure 6.9 histograms of the quality are arranged in a $3 \times 3$ matrix, corresponding to the nine segments. 
$<$ Quality $>$ with $<$ d $>$ in: $<-\infty, 0.2>$

\begin{tabular}{|l|l|l|l|} 
Functional & noise $=[\mathbf{0 , 0 . 2}>$ & noise $=[\mathbf{0 . 2}, \mathbf{0 . 4}]$ & noise $=<\mathbf{0 . 4 , 1}]$ \\
\hline Random & $0.42 \pm 0.03$ & $0.35 \pm 0.02$ & $0.23 \pm 0.05$ \\
\hline Separation & $0.44 \pm 0.02$ & $0.35 \pm 0.02$ & $0.22 \pm 0.03$ \\
\hline Relevance & $0.43 \pm 0.02$ & $0.32 \pm 0.02$ & $0.22 \pm 0.04$ \\
\hline Mix(NP,Rel) & $0.50 \pm 0.00$ & $0.50 \pm 0.00$ & $0.36 \pm 0.08$ \\
\hline Mix(Sep,Rel) & $0.42 \pm 0.03$ & $0,33 \pm 0.03$ & $0.22 \pm 0.02$ \\
\hline
\end{tabular}

\section{Table 6.4}

$<$ Quality $>$ with $<\mathrm{d}>$ in: $[0.2,1.2]$

\begin{tabular}{|l|c|c|c|} 
Functional & noise $=[\mathbf{0 , 0 . 2}>$ & noise $=[\mathbf{0 . 2 , 0 . 4}]$ & noise $=<\mathbf{0 . 4 , 1 ]}$ \\
\hline Random & $0.54 \pm 0.09$ & $0.50 \pm 0.10$ & $0.30 \pm 0.08$ \\
\hline Separation & $0.70 \pm 0.13$ & $0.54 \pm 0.15$ & $0.48 \pm 0.13$ \\
\hline Relevance & $0.65 \pm 0.13$ & $0.58 \pm 0.13$ & $0.39 \pm 0.08$ \\
\hline Mix(NP,Rel) & $0.51 \pm 0.02$ & $0.50 \pm 0.00$ & $0.37 \pm 0.08$ \\
\hline Mix(Sep,Rel) & $0.57 \pm 0.31$ & $0.62 \pm 0.10$ & $0.45 \pm 0.11$ \\
\hline
\end{tabular}

\section{Table 6.5}

$<$ Quality $\rangle$ with $\langle$ d $>$ in: $\langle 1.2, \infty\rangle$

\begin{tabular}{|l|c|c|c|}
\hline Functional & noise $=[\mathbf{0 , 0 . 2}>$ & noise $=[\mathbf{0 . 2 , 0 . 4}]$ & noise $=<\mathbf{0 . 4 , 1}]$ \\
\hline Random & $0.78 \pm 0.11$ & $0.45 \pm 0.13$ & $0.20 \pm 0.06$ \\
\hline Separation & $0.91 \pm 0.01$ & $0.86 \pm 0.03$ & $0.69 \pm 0.07$ \\
\hline Relevance & $0.86 \pm 0.03$ & $0.67 \pm 0.04$ & $0.38 \pm 0.08$ \\
\hline Mix(NP,Rel) & $0.78 \pm 0.10$ & $0.72 \pm 0.15$ & $0.57 \pm 0.15$ \\
\hline Mix(Sep,Rel) & $0.89 \pm 0.01$ & $0.81 \pm 0.21$ & $0.67 \pm 0.06$ \\
\hline
\end{tabular}

\section{Table 6.6}



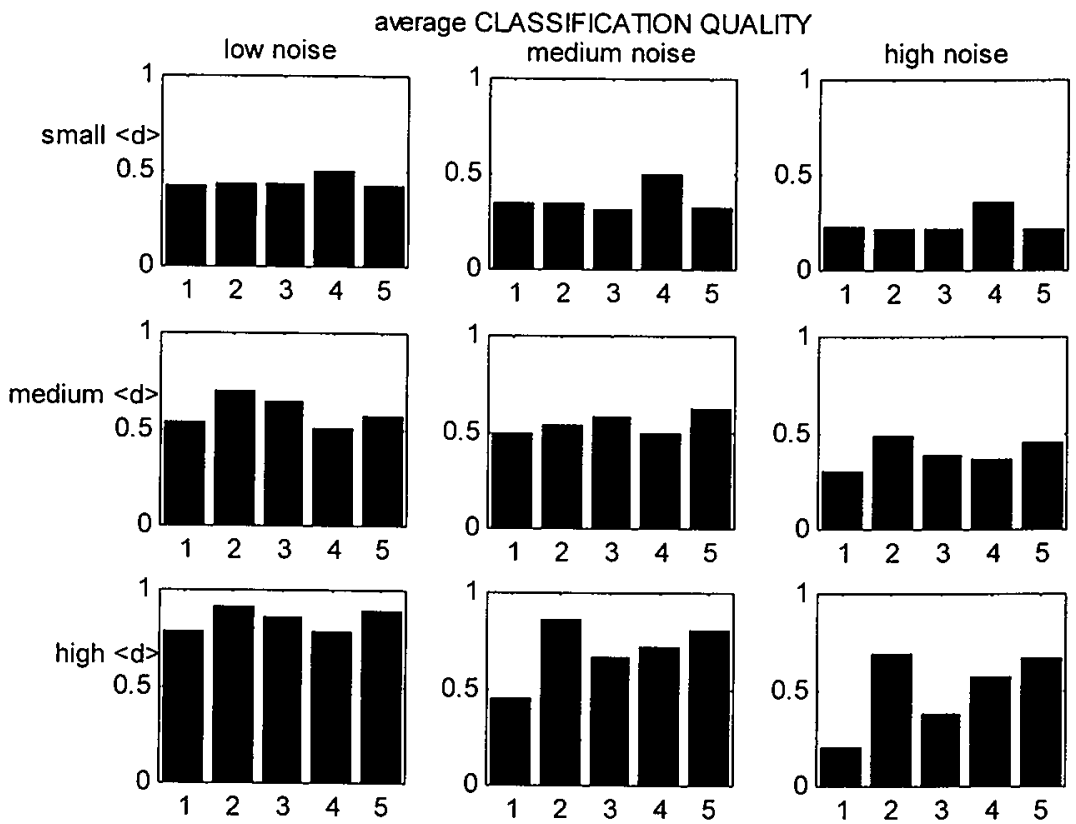

Fig. 6.9. Performance of the IASC algorithm in nine sections of the input space. Three types of noise $v$ are defined: LOW: $0 \leq v<0.2$, MEDIUM: $0.2 \leq v<0.4$, and HIGH: $v \geq$ 0.2 . Likewise, the clustering was divided as: SEVERE OVERLAP: $\langle d\rangle \leq 0.2$, OVERLAP: $0.2 \leq\langle d\rangle \leq 1.2$, and NO OVERLAP: $\langle d\rangle>1.2$. The histograms show the 'Classification Quality', defined as: $Q=(S+(1-T)) / 2$, in each combination of these divisions. The functionals are: 1: random/exhaustive, 2: separation, 3: relevance, 4: $\operatorname{mix}(\mathrm{NP}, \mathrm{rel}), 5$ : mix(rel,sep). This survey shows that: $i$. in the case of SEVERE OVERLAP or LOW NOISE, FPS does not outperform random/exhaustive, ii. in the case MEDIUM-NO OVERLAP and MEDIUM-HIGH NOISE, FPS is superior to random/exhaustive selection. 


\subsection{Comparison of Alpha-Increment Schemes}

\subsubsection{General Influence of the Exponent on the Selection Process}

Next, we investigate the effect of the dynamicallity exponent ' $\alpha$ ', defined in Sections 5.2.3 and 5.7.6, that expresses the influence of the current hypothesis on the parameter selection. In Figure 6.10, an example is shown with $K=3$ and $N=3$, for a separation functional. The left figure shows the value of the three parameter-functionals $J(n)$ as function of $\alpha$, for a given probability distribution $u(k)$ over the object states $k$. Indicated in this figure are three areas corresponding to the resulting optimal parameter $n \in\{1,2,3\}$ as function of $\alpha$. This figure shows the principle effect of the exponent. For each value of $\alpha$ one specific $J(n)$ will be the maximum of the set of functionals. The corresponding $n$ is the selected parameter for this value of $\alpha$. This parameter $n$ may optimise the success rate or convergence time, depending on the given clustering and the ensemble of input vectors $\mathbf{x}$. So for each $\alpha$, there is a specific sequence of parameters $\left\{n_{1}, n_{2}, \ldots\right\}$, for the current probability distribution $u$. One iteration step later this probability distribution will be changed, and so does the parameters sequence. Moreover, each iteration step the selected parameter is omitted from the evaluation, causing a steady decrease of the number of functionals involved in the optimisation.

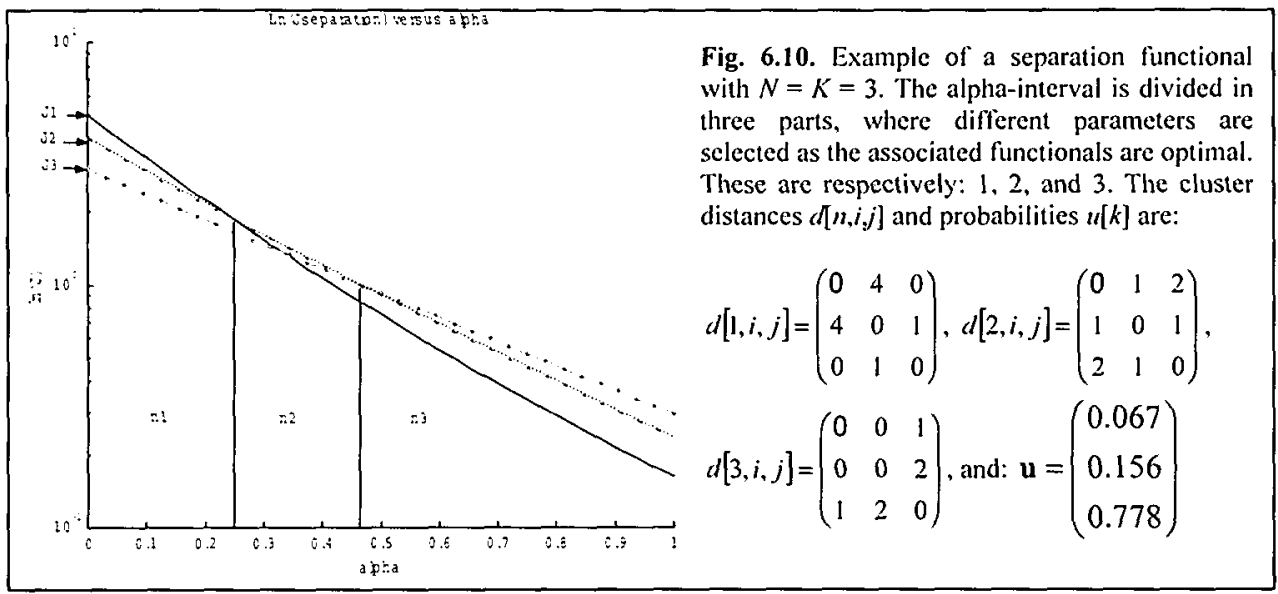




\subsubsection{Optimal Value of a Constant Exponent}

In the first scheme that we investigate, the dynamicallity exponent $\alpha$ is a constant during the iteration. Figure 6.11 shows the relation between the principle performance descriptors, success rate and convergence time, as function of a constant $\alpha$. From these figures it is clear that the two relations are comparable. From a certain inferior value for $\alpha=0$, the performance measure jumps - within the experimental precision - to a superior value, and maintains that value all the way to $\alpha=1$, again within the observed accuracy. In the case of the success rate, the jump is from: $S=0.55 \pm 0.12$ for $\alpha=0$, to $\langle S\rangle=0.69 \pm 0.04$ for $\alpha$ in the interval [0.01, 1]. In the case of the relative iteration length, the jump is from: Tconv $/ N=0.75 \pm 0.13$ for $\alpha$ $=0$, to $\langle T$ conv $/ N>=0.67 \pm 0.06$ for $\alpha$ in the interval $[0.01,1]$. This measurements were performed on the artificially scaled real data, described in the previous section. In total a number of 10 values for $\alpha$ were investigated, visible in the figure. Similarly to the previous section, a total number of 5120 combinations of noise $v$ and cluster separation $\langle d\rangle$, were generated with a uniform random distribution on: $(v,<\Delta) \in[0,1] \times\left[2^{-7}, 2^{5}\right]$, and for each combination $(v,<d>)$ the IASC algorithm was applied 100 times.

These figures clearly demonstrate the effect of dynamic reasoning. For all $\alpha>0$ the current hypothesis is, and for $\alpha=0$ is not involved in the iteration. For $\alpha>0$, the actual value of $\alpha$ has, as it seems, no discernible influence on the performance.
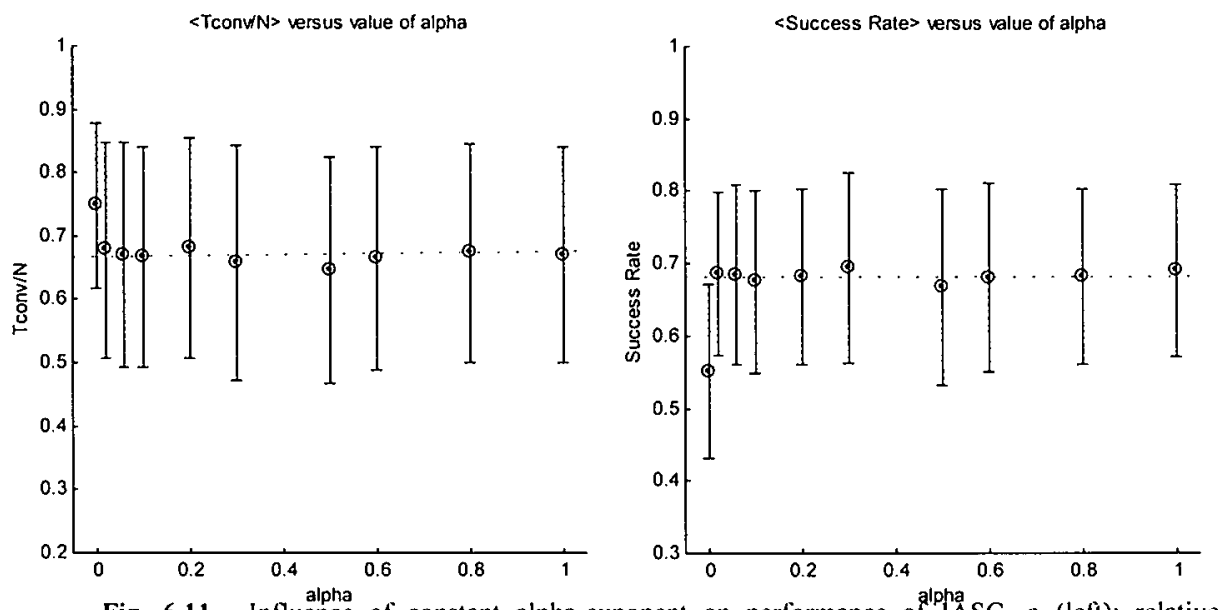

Fig. 6.11. Influence of constant alpha-exponent on performance of lAs convergence time. b (right): success rate. 


\subsubsection{Comparison of Several Models for the Evolution of the Alpha- Exponent}

In Section 5.7.6 three models for the evolution of the alpha-exponent were introduced. Based on the results of the experiments with constant alpha, one more alternative is interesting for further evaluation. This fourth model is based on the observation that there are only two types of alpha: 'zero' and 'non-zero'. Therefore, instead of an intricate evolution from zero to one, this model jumps directly from $\alpha$-type 'zero' in the first step, to $\alpha$-type 'one' ever-after. Together, these four models can be summarised as.

model 1: $\quad \alpha$ increases linearly from 0 to 1 in $N$ steps

model 2: $\quad \alpha$ increases linearly from 0 to 1 in $T(N)$ steps (see equation [5.150])

model 3: $\quad \alpha$ increases exponentially from 0 to 1 in $1+\ln 2 / G<d^{2}>$ steps

model 4: $\quad \alpha=0$ at $\mathrm{t}=1$, and $\alpha=1$, otherwise.

In the table and diagrams below the results of the comparison are presented. The linear models 1 and 2 were observed to give identical empirical results within the experimental accuracy.

\begin{tabular}{|l|l|l|l|}
\hline \multicolumn{1}{|c|}{ alpha scheme } & success rate & Tconv/N & Qual \\
\hline constant 0 & $0.55 \pm 0.12$ & $0.75 \pm 0.13$ & $0.40 \pm 0.13$ \\
\hline constant 1 & $0.69 \pm 0.12$ & $0.67 \pm 0.17$ & $0.51 \pm 0.14$ \\
\hline model 1, 2 & $0.71 \pm 0.10$ & $0.63 \pm 0.17$ & $0.54 \pm 0.13$ \\
\hline model 3 & $0.70 \pm 0.11$ & $0.64 \pm 0.16$ & $0.53 \pm 0.13$ \\
\hline model 4 & $0.69 \pm 0.10$ & $0.65 \pm 0.16$ & $0.52 \pm 0.13$ \\
\hline
\end{tabular}

Table 6.7

From these results the following conclusions can be drawn:

i. a constant alpha $=0$ gives the worst results,

ii. the evolution models give somewhat better results as the constant alpha $=1$,

iii. the linear models ( 1 and 2) are slightly better than the exponential model (3),

iv. the jump-model (4) is only slightly better than a constant 1-model, but slightly inferior to the continuous evolution models.

The difference between the evolution models is small compared with the difference between constant 0 and constant 1 model, as visible in Figure 6.12. That there is a difference at all between the continuous evolution models 1,2,3 and the discrete jump-model 4 is remarkable, because, based on the observation from the constant alpha's, there is only a difference between $\alpha=0$ and $\alpha>0$. 


\subsubsection{Comparison of Several Models for the Evolution of the Alpha- Exponent}

In Section 5.7.6 three models for the evolution of the alpha-exponent were introduced. Based on the results of the experiments with constant alpha, one more alternative is interesting for further evaluation. This fourth model is based on the observation that there are only two types of alpha: 'zero' and 'non-zero'. Therefore, instead of an intricate evolution from zero to one, this model jumps directly from $\alpha$-type 'zero' in the first step, to $\alpha$-type 'one' ever-after. Together, these four models can be summarised as.

model 1: $\quad \alpha$ increases linearly from 0 to 1 in $N$ steps

model 2: $\quad \alpha$ increases linearly from 0 to 1 in $T(N)$ steps (see equation [5.150])

model 3: $\quad \alpha$ increases exponentially from 0 to 1 in $1+\ln 2 / G<d^{2}>$ steps

model 4: $\quad \alpha=0$ at $\mathrm{t}=1$, and $\alpha=1$, otherwise.

In the table and diagrams below the results of the comparison are presented. The linear models 1 and 2 were observed to give identical empirical results within the experimental accuracy.

\begin{tabular}{|l|l|l|l|}
\hline alpha scheme & success rate & Tconv/N & Qual \\
\hline constant 0 & $0.55 \pm 0.12$ & $0.75 \pm 0.13$ & $0.40 \pm 0.13$ \\
\hline constant 1 & $0.69 \pm 0.12$ & $0.67 \pm 0.17$ & $0.51 \pm 0.14$ \\
\hline model 1, 2 & $0.71 \pm 0.10$ & $0.63 \pm 0.17$ & $0.54 \pm 0.13$ \\
\hline model 3 & $0.70 \pm 0.11$ & $0.64 \pm 0.16$ & $0.53 \pm 0.13$ \\
\hline model 4 & $0.69 \pm 0.10$ & $0.65 \pm 0.16$ & $0.52 \pm 0.13$ \\
\hline
\end{tabular}

Table 6.7

From these results the following conclusions can be drawn:

i. a constant alpha $=0$ gives the worst results,

ii. the evolution models give somewhat better results as the constant alpha $=1$,

iii. the linear models ( 1 and 2 ) are slightly better than the exponential model (3),

iv. the jump-model (4) is only slightly better than a constant 1-model, but slightly inferior to the continuous evolution models.

The difference between the evolution models is small compared with the difference between constant 0 and constant 1 model, as visible in Figure 6.12. That there is a difference at all between the continuous evolution models $1,2,3$ and the discrete jump-model 4 is remarkable, because, based on the observation from the constant alpha's, there is only a difference between $\alpha=0$ and $\alpha>0$. 

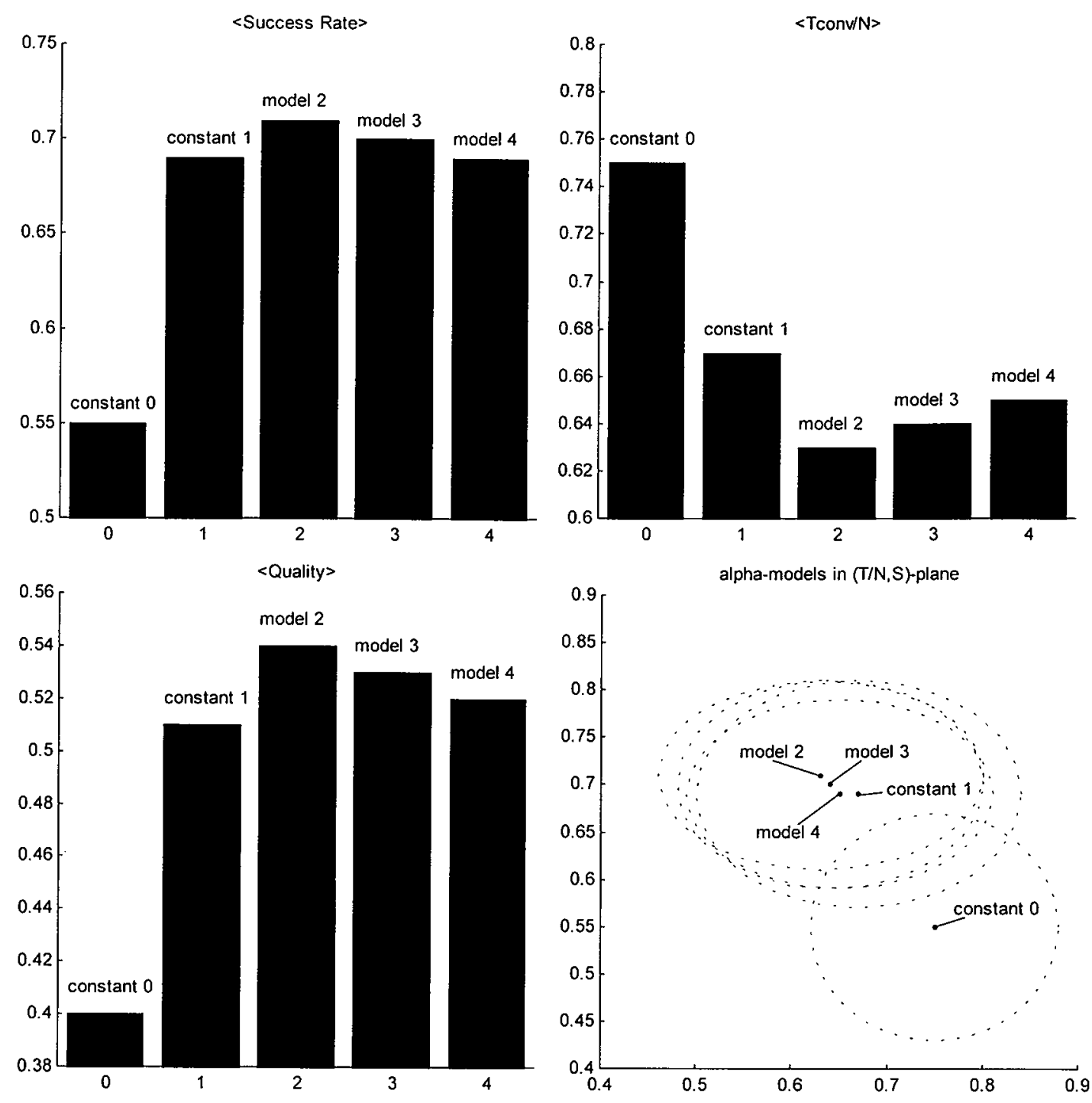

Fig. 6.12. Comparison of three models for alpha-evolution, and two models based on constant alpha's. Above left: the average 'success rate', above right: the average 'relative interval Iength' $T c o n v / N$, below left: the 'classification quality'. The results are based on of 4688 uniformly random generated points, with 100 trials per point, using scaled real data, with $N=31, K=18$, and the Separation functional. The perlormance is averaged over all noise and $<C<$. Below right, the positions and error-ellipses of the models in the ( $T_{c o n v} / N$, successrate)-plane. 


\subsection{Performance of Multi Criteria Optimisation Functionals}

In Section 6.3 we studied the performance of a number of parameter functionals, in order to find the sequence that gave the best results for a given clustering and sensoring process. There we found that there is no overall optimal functional, but only local ones, depending on the descriptors of the data and the desired optimisation criteria. A simple extension to this set of functionals lies in the linear combination of some of the more suitable functionals. Here, we present a simple example of mixed optimisation. Let the functional $j(\lambda)$ be given as a linear combination of:

- the separation functional $J_{\alpha}^{s c p}(n)$, with $\alpha=1$, and

- the functional equal to the computation time required for measuring parameter with index $n: J^{\text {cenp }}(n)=-\tau_{n}$, defined in Section 5.2.2.

More specific:

$$
j(\lambda)=\lambda J_{\alpha}^{s e p}(n)+(\lambda-1) \tau_{n}
$$

So, for $\lambda=0$ we obtain the separation functional, and for $\lambda=1$ we obtain the pure computation time functional. The latter is a static functional, that will always generate the identical sequence of parameter indices, regardless of the state and history of the algorithm. In order to have a 1-dimensional optimisation criterion, we use a variant on the classification quality, based on the computation time rather than the number of iterations:

$$
Q=\left(S+\left(\tau_{\max }-\sum_{k \in E} \tau_{k}\right) / \tau_{\max }\right) / 2
$$

with success rate $S$, set of explored parameter indices $E$, and maximum computation time: $\tau_{\max }=\sum_{n} \tau_{n}$.

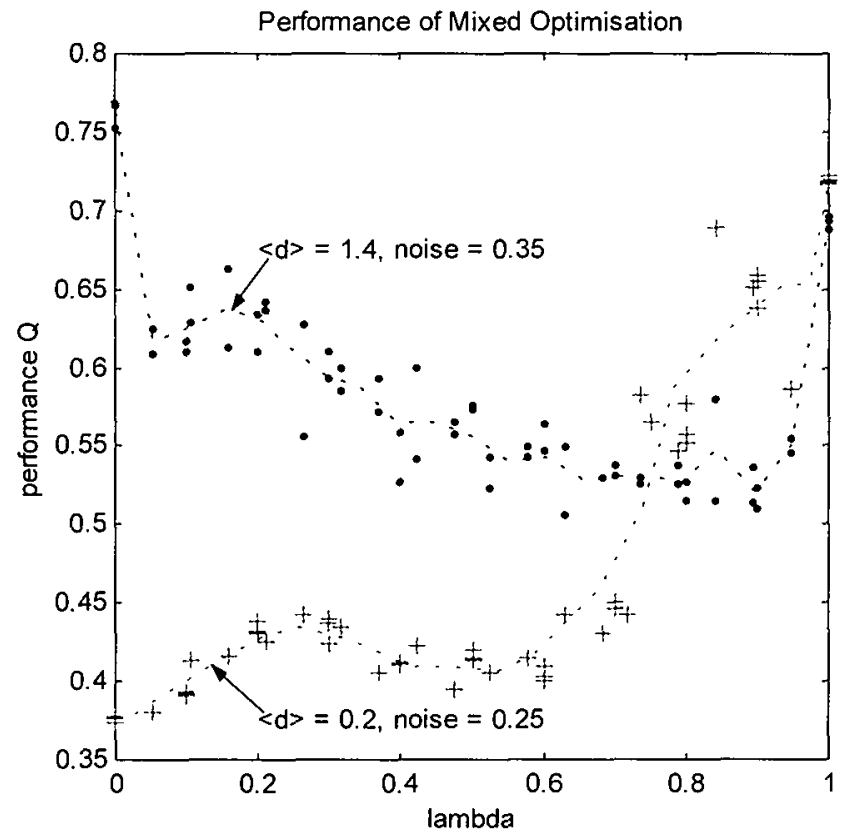

Fig. 6.13. Performance of IASC for mixed optimisation. 
The performance of the algorithm as function of the mixing parameter $\lambda$ was studied for two instances of tuples $(\langle d\rangle, v)$, with $\langle c|>$ the average class separation, and $v$ the noise. The results are depicted in the figure below. For clarity the error-bars (approximately constant 0.04 ) are not indicated.. The first instance was in the partition 'severe cluster-overlap and low noise', indicated with ' + '. The optimum performance is achieved for $\lambda=1$, so for optimisation purely directed towards computation time. The second instance was in the partition 'partial cluster-overlap and medium noise', indicated with '.'. Here, the best performance is achieved for $\lambda=0$, so for a pure separation optimisation.

In both cases solely the extremes $\lambda=0,1$ are global optima. So, no actual gain was achieved from the mix, as in each case one specific functional would have sufficed. In general, there may indeed exist specific linear combinations of functionals that generate optimal solutions, compared with single functionals, but there exists no clear-cut strategy fir obtaining them. This can only be achieved by in advance mapping all possible combinations and identifying the coefficients of the optimal combinations. Another disadvantage of mixed optimisation is that it profoundly depends on $\langle d\rangle$ and noise. Therefore, for all pairs $(<d\rangle, v)$ there are different coefficients for the optimal mix of functionals. A practical disadvantage is that the noise can often only be roughly estimated. Summarising, mixed optimisation poses more problems than it actually solves. 


\subsection{Evolution of Static and Dynamic Termination Criteria}

A termination condition in our context is a dynamic entity $a$ that passes through a pre-set threshold $\vartheta$. We study termination criteria of the type: $(a(t)<\vartheta)$ and analyse their influence on the performance parameters of the algorithm. An example of such a termination condition is the convergence of the state vector $\mathbf{u}$ :

$$
\|\mathbf{u}(t+1)-\mathbf{u}(t)\|<\varepsilon
$$

or the approach of the maximum state to 1 :

$$
\max u_{k}(t)>1-\varepsilon
$$

where in both cases $\varepsilon$ is a small number. Other examples are the static and dynamic termination criteria defined in Section 5.4.4. We discuss three aspects of the termination condition;

1. termination based on convergence type criteria, like condition (i) above, versus quality type criteria, like condition (ii);

2. static versus dynamic termination criteria based on the quality $q$ of the conclusion; and

3. the influence of the value of the pre-set threshold $\vartheta$.

In situations where optimal termination conditions can be specified we compare the performance of the algorithm relative to non-optimal termination.

\subsubsection{Comparison of Convergence-based versus Quality-based Termination}

Figures 6.14 and 6.15 below show two different runs of the IASC algorithm. Both figures show as function of the time - i.e. the step ' $t$ ' in the iteration: (1) the evolution of the static and dynamic criteria (static is shown solid, exponential memory dashed, and 3-steps memory dotted), (2) the convergence of the state vector as: $\log _{2}\|\mathbf{u}(t)-\mathbf{u}(t-1)\|$, (3) the evolution of the most likely defect: $\arg \max _{k} u_{k}(l)$, where a dashed horizontal line indicates the true class ' $l$ '. Moreover, the figure shows: (4) the final conclusion of the algorithm, where the true class ' $l$ ' is indicated by a dashed bar. In both cases the entire state vector $\mathbf{u}(t)$ converges more or less exponentially to its final value. However, in the case of Figure 6.14 the algorithm eventually fails in identifying the correct object class as state 9, while in Figure 6.15 the system drifts towards a mixed state between states 16 and 17 . This fact is documented in the evolution of the termination criteria. In the first case the quality ultimately converges to a high value, while in the second case the criterion, after an initial increase, remains decreasing. In all empirical situations it is found that the passage to a crisp conclusion is reflected much better by the evolution of a quality-based criterion than by its (logarithmic) rate of convergence; $\log _{2}\|\mathbf{u}(t)-\mathbf{u}(t-1)\|$. This is hardly surprising, as the state vector $\mathbf{u}$, in both correct and incorrect identification of the IASC algorithm, converges quasi-exponentially to its ultimate value $\mathbf{u}(\mathbf{x})$, whereas quality based termination criteria depend on the relative difference of the two most competing hypotheses. Quality based termination criteria convey more information on the aptitude of the current hypothesis, whereas convergence based types merely conclude that a solution is reached. Therefore, quality based criteria are more suitable as termination condition than convergence based ones. 

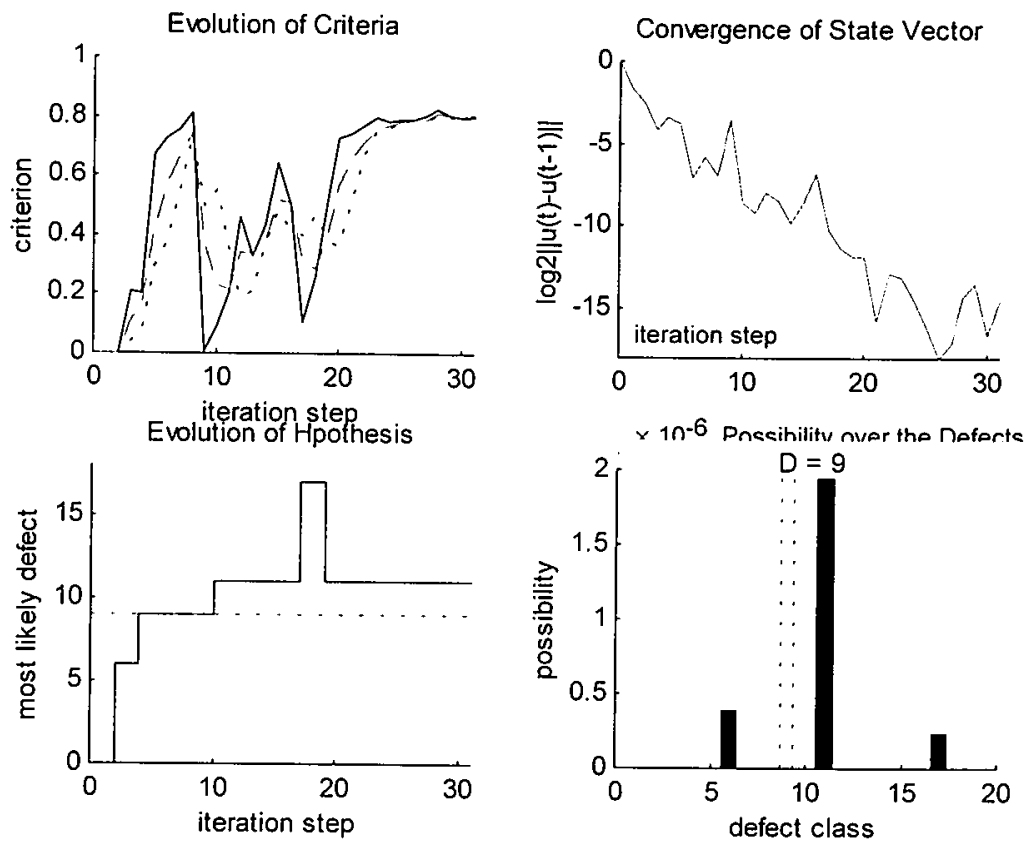

Fig. 6.14
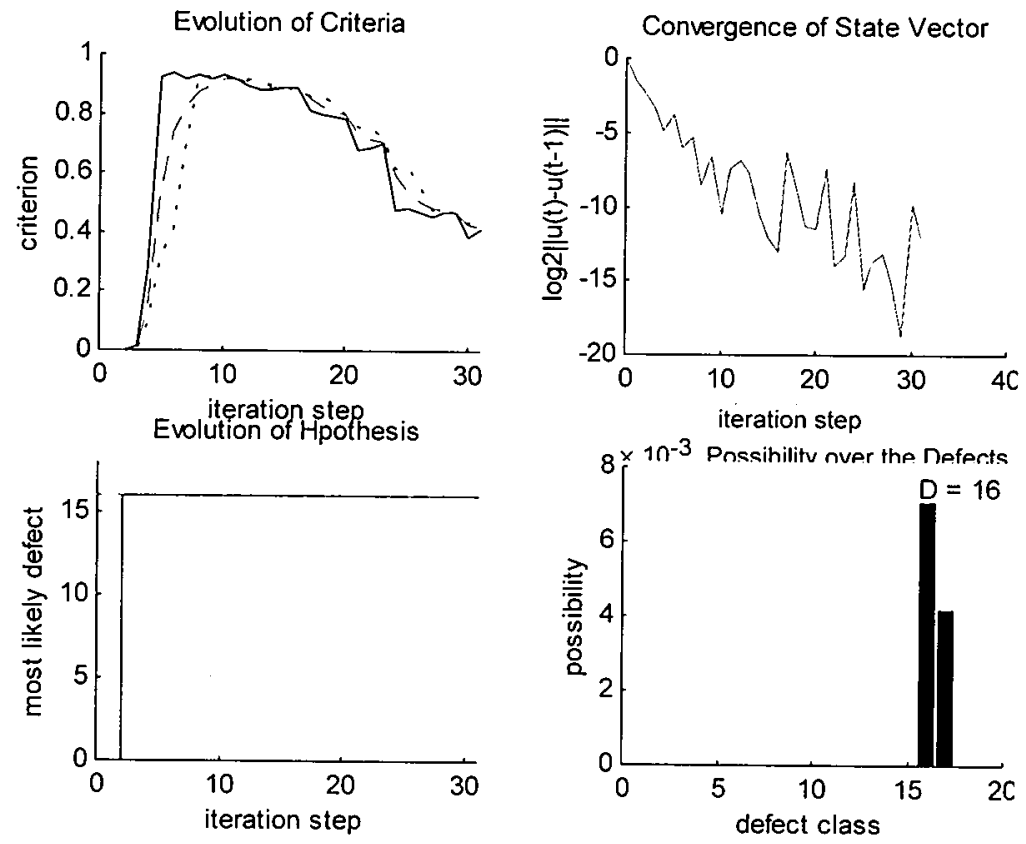

Fig. 6.15 


\subsubsection{Comparison of Static and Dynamic Termination Criteria}

The difference between the static termination criterion from Section 5.4.4, and a dynamic termination criteria concerns the history of the iteration process. The static criterion is based solely on the actual quality of the conclusion $q$ from equation [5.2] from Section 5.1.4:

$$
\text { if } \mathbf{u}=\mathbf{0} \text { then } q=0 \text { else } q(t)=\left(u_{1}(t)-u_{2}(t)\right) / u_{1}(t)
$$

where the states are sorted in decreasing order. A dynamic criterion, however, explicitly retains some form of memory of the history in order to prevent premature convergence. The objectives of termination criteria are threefold; primo, to shorten the computation time of the iteration, secundo, to prevent diffuse classes from contaminating the final hypothesis as FPS yields less significant parameter-information, and finally, to prevent premature convergence. We discuss two types of memory. First, a dynamic criterion based on an exponentially decaying memory:

$$
c_{\lambda}(t)=\sum_{s=0}^{n} q(t-s) e^{-\lambda s} / \sum_{s=0}^{n} e^{-\lambda s}
$$

Second, a dynamic criterion based on the average value of the quality during the last $n$ steps $^{2}$ :

$$
c_{n}(t)=\sum_{s=0}^{n} q(t-s) /(n+1)
$$

In the experiments the parameters were chosen as: $\lambda=\ln 2$ and $n=3$. Thus we compare the three following termination conditions:

$$
\begin{array}{ll}
\text { condition 1: } & \text { stop if } q(t)>\vartheta \\
\text { condition 2: } & \text { stop if } c_{\lambda}(t)>\vartheta \\
\text { condition 3: } & \text { stop if } c_{n}(t)>\vartheta
\end{array}
$$

The nature of the criteria are visible in Figures 6.14 and 6.15. Whereas the static criterion jumps instantaneous between high and low values, the dynamic criteria are more robust and exhibit a more graduate evolution due to their mnemonic capacities. The average performance of the three termination models is shown in the table below.

\begin{tabular}{|c|c|c|}
\hline criterion & success rate & relative iteration length \\
\hline static quality based & $0.74 \pm 0.04$ & $0.21 \pm 0.04$ \\
\hline 3 last steps memory & $0.76 \pm 0.04$ & $0.26 \pm 0.04$ \\
\hline exponential memory & $0.76 \pm 0.04$ & $0.27 \pm 0.04$ \\
\hline
\end{tabular}

Table 6.8. Performance of dynamic and static termination

In comparing the static and dynamic criteria a small gain in success rate can be observed at the expense of a somewhat bigger loss in iteration length. Moreover, the two dynamic models hardly differ within the experimental accuracy. More interesting than the average performance over the entire input space is to observe the performance in selective parts of the (noise, $<$ ( $>$ )-plane. As noted in the discussion on the performance of the FPS functionals, it is useful to divide the noise $v$ as: \{low noise, medium noise, high noise\}, and the cluster separation $\langle d\rangle$ as: \{severe overlap, overlap, no overlap $\}$. Here, we use the same boundaries for $v:\{0.2,0.4\}$, and $\langle d\rangle:\{0.2,1.2\}$. For that partitioning of the input plane in 9 parts we find the following comparison of the termination criteria.

\footnotetext{
' if $t<n$ then the average is taken over the last $t$ steps.
} 
Table 6.9. Table indicating the relative difference ' $r$ ' between the termination length of the 3-step memory ' $T_{3 m e m}$ ' and the static criterion ' $T_{0}$ ', detined as: $r=\left(T_{3 m e m}-T_{0}\right) / T_{0}$

\begin{tabular}{|c|c|c|c|}
\hline$\times 10^{-2}$ & \multicolumn{1}{c}{ low noise } & \multicolumn{1}{c|}{ medium noise } & \multicolumn{1}{c|}{ high noise } \\
\hline no overlap & 28 & 46 & 67 \\
\hline medium overlap & 9 & 29 & 24 \\
\hline severe overlap & 13 & 10 & 14 \\
\hline
\end{tabular}

Table 6.10. Table indicating the relative dilference ' $r$ ' between the success rate of the dynamic 3-step memory ' $S_{3 m e m}$ ' and the static criterion ' $S_{0}$ ', delined as: $r=\left(S_{3 m e m}-S_{0}\right) / S_{0}$

\begin{tabular}{|c|c|c|c|}
\hline$\times 10^{-2}$ & low noise & medium noise & high noise \\
\hline no overlap & 2 & 10 & 15 \\
\hline medium overlap & 0 & 0 & 0 \\
\hline scvere overlap & -1 & -3 & -5 \\
\hline
\end{tabular}

Table 6.11. Table with the relative difference ' $\mu$ ' between success rate of the 3-step memory ' $S_{3 m \cdot m}$ ' and the exponential decaying memory ' $S_{\text {exxy }}$ ', delined as: $r=\left(S_{3 m e m}-S_{\text {exp }}\right) / S_{\text {exp }}$

\begin{tabular}{|c|c|c|c|}
\hline$\times 10^{-2}$ & low noise & medium noise & high noise \\
\hline no overlap & 0 & 3 & 1 \\
\hline medium overlap & 0 & 0 & -1 \\
\hline severe overlap & 0 & -1 & -2 \\
\hline
\end{tabular}

These tables show that in most parts there is no significant difference between static and dynamic termination models. Only in case of medium to high noise and non-severe cluster overlap the dynamic criteria clearly outperform the static criterion. Moreover, there is no important difference between the two dynamic termination models, be it that the 3 last step average memory model performs somewhat better than the exponential decaying memory. This is because the latter model caries the entire history of the iteration process, including all distracting information, while the former merely focuses on the last few iteration steps. This explanation is strengthened by analysing the performance of $n$-step memory as function of $n$. For $n=0$ this model is identical to the static criterion and subject to severe jumps in its value. For large $n$ the dynamic criterion becomes the average of all values $q$ generated during the iteration, and will hardly evolve. Optimal values for a clustering with $N=31$ and $K=18$ are obtained in the range $n=3$ to 5 .

\subsubsection{Influence of the Value of the Pre-Set Threshold}

The value of the threshold $\theta$ has an vital impact on the performance of the IASC algorithm. If the algorithm is executed without whichever termination criterion, or better, if the value of the threshold $\vartheta$ was set to its maximal value 1, all FPS-functionals generated the same result. This is because in that case the entire amount of sensor information in the form of vector $\mathbf{x}$ is used, leading to a conclusion $\mathbf{u}=N(\mathbf{x}, \mathbf{m}, C)$. This is equal to exhaustive selection, resulting in the maximal number $N$ of iteration steps, and the same success rate for all FPS functionals. The importance of the threshold can further be illustrated by two examples shown in Figures 6.14 and 6.15 , with $N=31, K=18, v=0.3$, and $\langle d\rangle=0.2779$.

1. The process depicted in Figure 6.14 shows a case where in an early part of the iteration, from step 4 to 10 , the algorithm was targeted towards the correct solution, class nr. 9. If the criterion would have been somewhat smaller, the algorithm would have been terminated and this correct solution would have been found. Later in the iteration, however, diffuse clusters were selected by the FPS that slowly engulfed the correct conclusion as they drew the state vector into a mixed state of the incorrect and broad classes 6,11 , and 17 . 
2. In the iteration shown in Figure 6.15 the decision for the correct class 16 was made relatively early in the process, at step nr. 2 . A lower threshold in this case would have stopped the iteration at an early moment, and thus prevented a whole range of superfluous computations without impairing the value of the conclusion. It would also have prevented the broad class 17 from entering the classification process, which more and more dragged the state vector into a mixed equilibrium in-between class 16 and 17 . This caused a gradual decline of the criteria that prevented any termination criterion to stop the process from that moment on. Moreover, it led to a continuous growth of the probability of class 17 , as less and less significant information was generated by FPS. This process was only stopped when all 31 parameters were used.

Therefore we analyse the performance of the algorithm as function of the value of the threshold $\vartheta$ in the termination condition $(a(t)<\vartheta)$. Figure 6.16 shows the influence of the threshold on the performance parameters $S$ (success rate) and $T / N$ (relative iteration time). In graph $a$ and $b$ these parameters are plotted against the value of threshold $\vartheta$. Using these two plots, in Figure $c$ a trajectory is drawn in the $(S, T / N)$-plane as a parameterisation of $\vartheta$. This shows that there exists a clear optimal value $\vartheta^{*}$ that yields the highest success rate $S^{*}$ and associated iteration length $T^{*}$. In a similar manner the optimal values $\left(\vartheta^{*}, S^{*}, T^{*}\right)$ can be obtained for all points in the input-plane $(v,<\phi>)$ and all possible combinations of control parameters. Since in all practical applications the clustering is known and the noise can be estimated, certainly within the three noise-levels $\{l o w$, medium, high $\}$, it will be possible to use this information to select a near-optimal value for the termination threshold $\vartheta$.

All the tests presented earlier in this chapter were performed with a high value of the threshold, $\vartheta=0.9999$, in order to highlight the effect of the control parameters, like the $J$ type (type of FPS functional), $\alpha$-type (type of alpha scheme). For the entire average performance we find:

$$
\begin{array}{ll}
\text { for } \vartheta=0.9999: & <S>=0.68 \pm 0.21, \quad<T / N>=0.63 \pm 0.33, \\
\text { for } \vartheta^{*}: & <S^{*}>=0.78 \pm 0.21, \quad<T^{*} / N>=0.25 \pm 0.21 .
\end{array}
$$

In tables below the performance parameters of $\vartheta=0.9999$ are compared with the corresponding values of the optimal value $\vartheta^{*}(v,\langle d\rangle$, Jtype, $\alpha t y p e)$. Again, we used the classification of noise $v$ in three levels: \{low noise, medium noise, high noise , and cluster separation $\langle\phi\rangle$ in three degrees of overlap: \{severe overlap, overlap, no overlap\}, with boundaries for $v:\{0.2,0.4\}$, and $\langle d\rangle:\{0.2,1.2\}$. These tables show the significant gain in success rate and loss in iteration length when using the optimal values. The benefit was maximal in case of medium to high noise and non-severe overlap. Figure 6.17 shows the relative iteration time as function of the input-space $(\nu,\langle d\rangle)$ for both $\vartheta=0.9999$ and $\vartheta^{*}$, showing the much performance at the optimal value. 


\begin{tabular}{|c|c|c|c|c|c|c|}
\hline \multicolumn{4}{|c|}{ Success Rate for threshold $=0.9999$} & \multicolumn{3}{|c|}{ Optimal Success Rate } \\
\hline & low noise & medium noise & high noise & low noise & medium noise & high noise \\
\hline severe overlap & 0.90 & 0.73 & 0.45 & 0.90 & 0.73 & 0.54 \\
\hline medium overlap & 0.93 & 0.83 & 0.58 & 1.00 & 0.96 & 0.75 \\
\hline no overlap & 1.00 & 0.98 & 0.77 & 1.00 & 0.99 & 0.80 \\
\hline
\end{tabular}

\section{Table 6.12}

\begin{tabular}{|c|c|c|c|c|c|c|}
\hline \multicolumn{4}{|c|}{ Relative Iteration Length for threshold $=0.9999$} & \multicolumn{3}{|c|}{ Optimal Relative Iteration Length } \\
\hline & low noise & medium noise & high noise & low noise & medium noise & high noise \\
\hline severe overlap & 1.00 & 1.00 & 1.00 & 0.45 & 0.46 & 0.43 \\
\hline medium overlap & 0.62 & 0.58 & 0.59 & 0.09 & 0.16 & 0.15 \\
\hline no overlap & 0.17 & 0.23 & 0.39 & 0.07 & 0.12 & 0.18 \\
\hline
\end{tabular}

\section{Table 6.13}
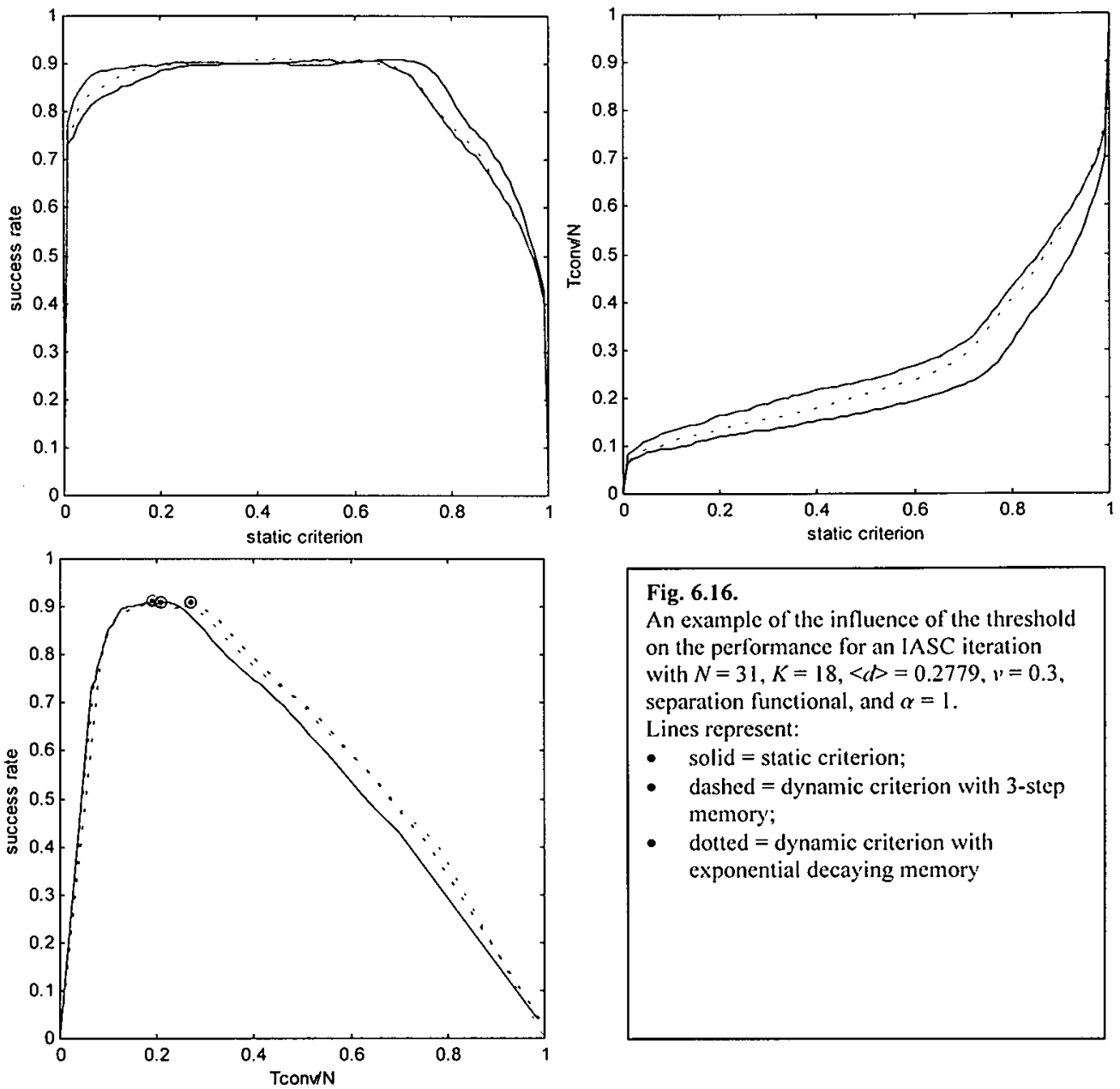

Fig. 6.16.

An example of the influence of the threshold on the performance for an IASC iteration with $N=31, K=18,\langle d\rangle=0.2779, v=0.3$, separation functional, and $\alpha=1$. Lines represent:

- $\quad$ solid = static criterion;

- $\quad$ dashed = dynamic criterion with 3-step memory;

- $\quad$ dotted = dynamic criterion with exponential decaying memory 


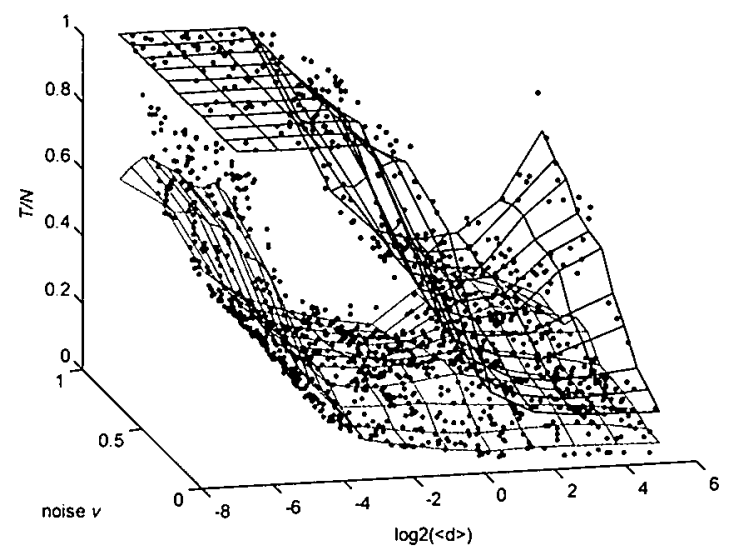

Fig. 6.17. The relative iteration time as function of the input-space $(v,<d>)$ for an IASC iteration with $N=31, K=18,\langle d\rangle=0.2779$, separation functional, and $\alpha=1$. The higher plane represents a threshold with $\vartheta=0.9999$, the lower plane the optimal threshold $\vartheta^{*}$.

\subsubsection{Comparison of Observed and Theoretical values for the Performance as Function of the Threshold}

In the last part of Section 5.7.5 a theoretical model was constructed for the relation between the actual value of the threshold $\vartheta$, and the observables 'success rate' $S$ and 'relative iteration length' $T / N$. We shall now evaluate this model with some numerical experiments. The first observation that we can make is that there exists a reasonable superficial agreement between the Figures 5.20 to 5.23 of Section 5.7.5, and the corresponding plots in the Figures 6.16 above, giving the relations between threshold, success rate, and relative iteration length.

In order to obtain a more profound understanding of the relation, a large number of experiments with fixed IASC-problem descriptors was carried out. The experiments were performed with a fixed noise-level 0.3 , using a real clustering stemming from CCD-quality control, with $N=31, K=18$, and $\langle\not b=0.2779$. Selected were 101 different values for the threshold $\vartheta$, and 100 different runs per threshold. For each threshold the success rate was calculated as the fraction of correct solutions, and the iteration length as the mean iteration length over the 100 runs. The empirically observed relation between the success rate $S$ and relative iteration length $T / N$ was compared with the predictions of the theoretical model. In Figure 6.18a the theoretical model and the observed values are depicted. Based on the known prototypes and covariances in this data, and using equation [5.141] from Section 5.7.5, the sequences $h_{i}^{2}$ and $\left\langle b_{n}\right\rangle$ were calculated. In this formula an effective relative noise (defined in equation [5.98] in Section 5.6.6.2) of 0.395 is used, rather than the absolute noise-level of 0.30 . The obtained expectation value for the success rate $\langle S\rangle$ is given by equation [5.144] as:

$$
<S(t)>\approx\left\langle\frac{u_{l}(t)}{u_{k}(t)}\right\rangle \approx \exp \left(1 / 2<b_{t}>\right)
$$

This theoretical sequence is plotted as a solid line in Figure 6.18a. The observed data are indicated by individual points, connected by a dashed line in the same figure. Figure $6.18 a$ shows that the theory provides a good approximation for the relation between $S$ and $T / N$, especially during the later parts of the iteration. As described in the last part of Section 
5.7.5.1, this behaviour originates primarily from the influence of the effective relative noise. For lower iteration lengths, representing about the first quarter of the iteration, the qualitative match is fair, but the curve-fit is poor.

Relation [6.3] between the expected success rate $\langle S\rangle$ and sequence $b_{n}$ can also be used to reconstruct the generated sequence of selected cluster-distances $h_{n}=\langle d(n)\rangle$. From equation [6.3] we can write:

$$
\ln \left\langle S_{n}\right\rangle^{2} \approx \sum_{i=1}^{n}\left(\left\langle d^{n}\right\rangle^{2}-\delta^{2}\right)
$$

Therefore, the generated sequence of cluster-distances follows from the increase of the success rate $\langle S\rangle, \Delta \ln \left\langle S_{n}\right\rangle^{2}=\ln \left\langle S_{n+1}\right\rangle^{2}-\ln \left\langle S_{n}\right\rangle^{2}$, as:

$$
\left\langle d^{n}\right\rangle^{2} \approx \Delta \ln \left\langle S_{n}\right\rangle^{2}+\delta^{2}
$$

In this expression $n$ represents the (absolute) iteration step. However, our experimental data was in the form of a set of triplets: $\left\{\left(T_{i}, S_{i}, \vartheta_{i}\right) \mid i=1\right.$..\# experiments $\}$, ordered in ascending termination time $T_{i}$. In this case the accurate expression becomes:

$$
\langle d\rangle^{2} \approx \frac{\Delta \ln \left\langle S_{i}\right\rangle^{2}}{\Delta T_{i}}+\delta^{2}
$$

This expression must be interpreted as the estimate of $\langle d\rangle$ as function of the iteration length $T$. In Figure $6.18 b$ the experimentally reconstructed cluster-distances $\langle d(n)\rangle$ using this expression, are plotted versus the theoretically calculated values $h_{n}$, based on the prototypes and covariances of the clustering. Since the experimentally reconstructed values are based on averages over large runs of IASC with the same settings, the values of $T_{i}$ are generally noninteger. Therefore, the associated theoretic value of $h_{i}$ is based on a linear interpolation. This plot in Figure $6.18 b$ exhibits a markedly different behaviour left and right of $\langle d\rangle$ theor $\approx 0.40$. This value is remarkably close to the effective relative noise $\eta=0.395$. Since this model was constructed under the approximation of low noise, as described in Section 5.7.5, it is tempting to interpret this fact as a consequence of this assumption.

Below the value of $\langle d\rangle_{\text {theor }} \approx \eta$, there exists an almost perfect identity between the two values, exhibiting a linear correlation of 0.71 . In addition, the plot falls almost together with the diagonal of the plot - indicated as a dashed line. Both arguments imply that indeed: $\langle d\rangle_{\text {observed }}=\langle d\rangle_{\text {theor. }}$ Above this value, however, the observed value increases stronger than the theoretically predicted value, and they quickly separate. This separation is almost linear with a coefficient of 2.49 and a linear correlation of 0.81 . As the largest $\langle d\rangle$ values are selected first, due to FPS, the difference is most noticeable in the initial behaviour of the iteration. From this fact one can conclude that the theory fails to adequately predict the initial part of the generated sequence $h_{n}$ of average cluster-distances. This can be caused by the way the averages $\langle d\rangle$ are calculated, because they are systematically underestimated.

We can also use the thus obtained experimental relation between $\langle d\rangle_{\text {observed }}$ and $\langle d\rangle_{\text {theor }}$ to slightly adjust the theoretical model for $\langle S\rangle$ as function of $\langle T\rangle$. To do so, we alter the first three elements of sequence $h_{n}$ according to the relation in Figure $6.18 b$. The other 28 elements of $h_{n}$ remain unaltered. The resulting changes are given in table 6.14 below. 


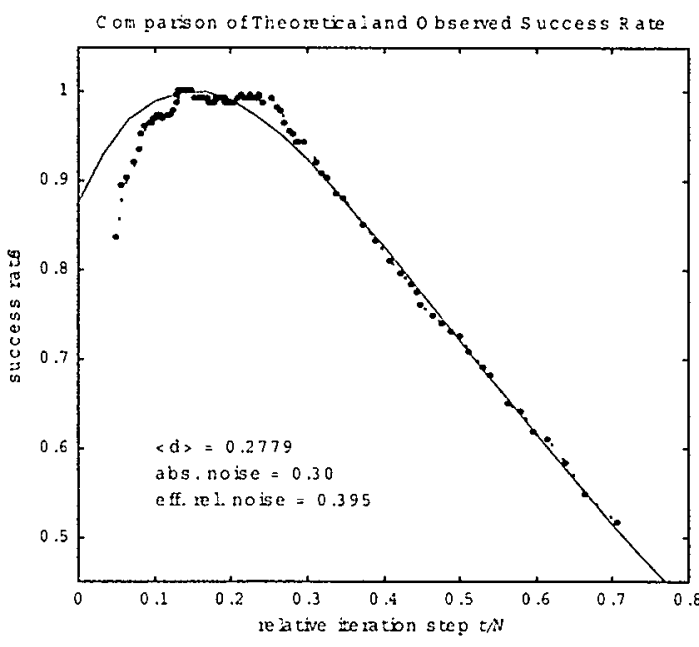

Fig. 6.18a. Comparison of theoretical and observed values of the relation between success rate and iteration length. Data rescaled to maximum valuc.

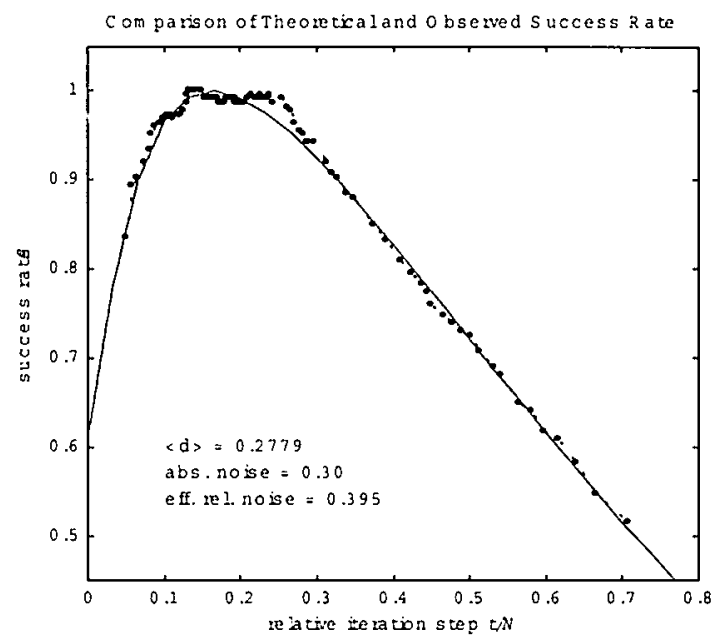

Fig. 6.18c. Adjusted theoretical model (solid linc), based on the first three selected cluster-distances as reconstructed from the empirical performance data. The relative slight change in the sequence results in a much better fit with the observed $(S, T)$-values, indicated with points, as compared with sub-figure $a$.

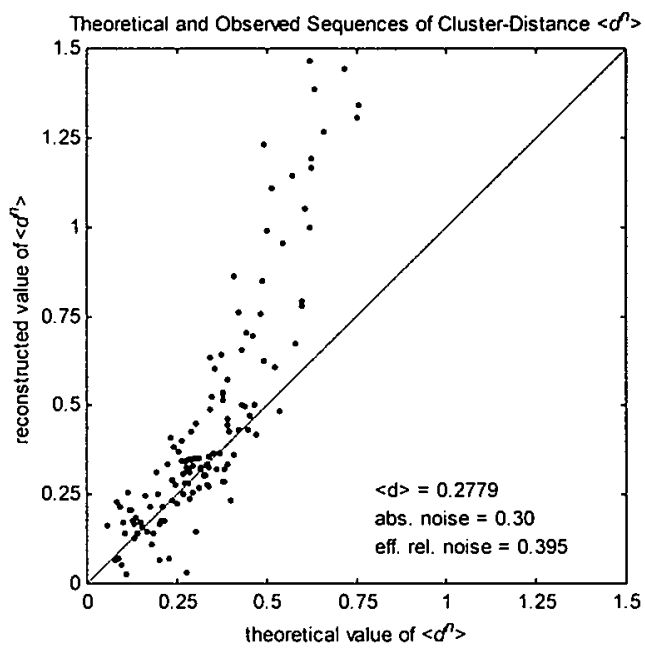

Fig. 6.18b. Comparison of theoretically predicted and empirically reconstructed sequences. Theoretical $\langle A$-value interpolated for non-integer empirical $\langle T$.

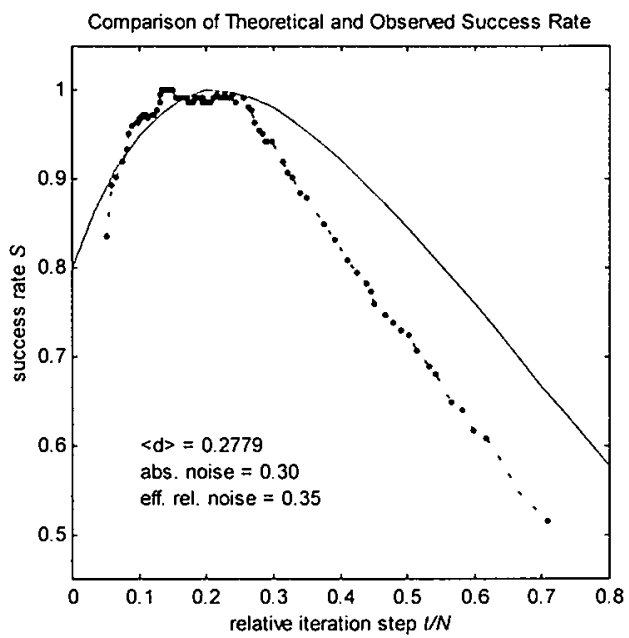

Fig. 6.18d. Similar plot, now the theoretical curve is made with the originally predicted sequence $h$, but with a slightly lower effective relative noise level of 0.35 in stead of 0.395 . This causes a better map for the initial part. but the fit with the later part - caused by the influence of noise - has deteriorated. 


\begin{tabular}{|c|c|c|}
\hline Averaged Cluster Distances & predicted by theory & reconstructed from data \\
\hline$<\mathrm{d}(1)>$ & 0.664 & 1.30 \\
\hline$<\mathrm{d}(2)>$ & 0.442 & 0.53 \\
\hline$<\mathrm{d}(3)>$ & 0.275 & 0.30 \\
\hline
\end{tabular}

Table 6.14

This slight adjustment caused a significant improvement of the theoretical predictions. The new relation is depicted in Figure $6.18 \mathrm{c}$. Now the predicted curve almost fully coincides with the observed values. One can, however, rightfully remark that now the theoretic model is adjusted with the experimental data itself. At least this near-perfect match shows that this approach can be used to satisfactory fit the empirical data with a suitable sequence $h_{n}$. Therefore, it is accurate to conclude that the formalism as expressed in equation [6.4] can predict the correct behaviour, but that the expression for the coefficients $h_{i}$ as average cluster distances is initially incorrect.

Another way was found to improve the match with the initial phase of the iteration, while maintaining the original calculated sequence $h_{n}$. A slightly lower effective relative noise of 0.350 instead of the calculated 0.395 gave a better fit with the initial part of the curve, but at the expense of a deteriorated fit with the later part. This is more remarkably because the later part is primarily caused by the influence of noise, as demonstrated in Section 5.7.5. In all, this modification only worsens the fit. Moreover, there is no theoretical justification for adjusting the noise. Without changing sequence $h_{n}$, it is not possible to find a noise-value that fits with the entire range of the empirical data.

\subsubsection{Conclusions Regarding Termination Conditions}

We close this section with the following conclusions:

1. quality-based termination conditions are more useful than convergence-based ones,

2. dynamic termination conditions give a marginal better success rate at the expense of a higher iteration length,

3. for each combination of clustering, noise-level, FPS-functional type 'J-type', and alphascheme ' $\alpha$-type' it is possible to off-line obtain an optimal value for the threshold: $\vartheta^{*}(v,<d>$, Jtype, $\alpha t y p e)$, resulting in a significant better performance than any other value of the threshold, and

4. The model, proposed in Section 5.7.5, provides a good qualitative and fair quantitative understanding for the relation between the threshold value and the output parameters 'success rate' $S$ and 'relative iteration length' $T / N$.

In a later chapter we will describe the application of Functional Parameter Selection to the direct control of a printing robot, based on the analysis of a CCD-image of a printed product. 


\subsection{General Conclusions}

We have presented an on-line classification approach, called IASC, for interpreting noisy sensor data of weakly separated clusters. Multivariate normal functions based on the prototypes and covariances of the clusters were used to limit and focus this process. An approach was proposed based on the maximisation of functionals expressed in measures that include normalised distances and class memberships. The approach was applied on several test-sets of artificially generated data with all degrees of class-separation, and real data stemming from image analysis of print quality with considerable class overlap. This showed that Functional Parameter Selection (FPS) outperforms Random and Exhaustive Selection when the clusters overlap considerable and/or medium to high noise-levels, and - our basic assumption - they can be reasonably well approximated by multivariate normal distributions.

It was also shown that better results can be obtained by including actual hypotheses at the current stage of the classification process, expressed as the state membership functions. The results show that the exponent $\alpha$ of the state membership function has a small but significant influence, be it that the value zero gives definitively inferior results.

There is significant variation between the set of FPS-functionals that were studied, and the choice for a specific FPS-functional is one effective control mechanism of the algorithm. As the data consisted of various degrees of noise and class-separation, not all classifications led to the correct result. In the case of significant class-overlap, as present in the real data, Functional Parameter Selection clearly leads to better and faster results.

A classification into three noise-levels and three degrees of cluster-overlap leads to the following characterisation:

- none to medium overlap - low noise:

everything works

- none to medium overlap - medium to high noise:

parameter selection is better than random and exhaustive selection

- severe overlap - low to medium noise:

parameter selection better than random and exhaustive selection

- severe overlap - high noise:

nothing works.

Termination conditions are effective tools to control the performance of the algorithm. For each known clustering of states and estimate of the noise, it is possible to obtain an optimal value for the value of the termination threshold, that will prevent both premature convergence and contamination by not relevant sensor information. 



\section{Chapter 7}

\section{On-line Control of Printing Quality Using Computational Vision}

\subsection{Introduction}

In this chapter we discuss the application of the ideas presented in the previous chapters on a complex and widespread industrial problem. The area of this application is on-line visual quality control of padprinting processes. The application serves as a testbed for the consistency of our approach, and for their evaluation in an intricate and demanding environment. This work was performed in the framework of an international joint research project, aimed at the development of a new generation of 'intelligent', autonomous and flexible padprinting machines, hence its name 'IntelPadPrint'. The research was an intense cooperation between six industrial and scientific partners from four European nations, performed in the course from late 1996 to early 2000. During this project two successive integrated prototype printers were developed and tested in an industrial setting.

Padprinting is currently the most efficient and reliable technique for high-yield/low cost printing on practically all types of substrates and shapes. Therefore, it is the most extensively used technique for industrial decoration. However, due to partially understood physicalchemical reasons, a variety of printing defects may occur while the process was assumed to be stable. Numerous printing defects are now known and catalogued. These defects are all characterised by their specific visual effect on the print. For this reason the application of computational vision, image analysis and pattern recognition appeared sensible techniques to contribute to a better understanding and hence an improvement of the padprinting process. However, computational vision has a number of recognised deficiencies, that were also valid in this context. Computer vision involves the processing of lots of sizeable images, causing long computation times, while the intended development had to meet rigorous speed constraints. Additionally, image analysis tests were found to have, in general, weak correlations with most defect classes. Therefore, specific image analysis tests had to be designed, that had significant correlations with all but a few particular printing defects. This approach led to an immense inflation of the number of tests, especially in a research project that was liable to significantly increase the number of known printing defects and their underlying mechanisms. For this reason, two other scientific fields were involved in the 
research. First, efficient and speedy clustering techniques were required to detect relevant structures in the output of the numerous image analysis tests. In this way hierarchies of printing defects could be constructed in terms of these parameters, which could be used for pre-classification. This meant the clustering of extensive multi-parameter image analysis data, notorious for its high degree of cluster-overlap. Second, on-line classification techniques could combine the information available for an early identification of the defect. In this way the number of tests performed - and thus the identification time - could be minimised drastically.

Several attempts were made to develop suitable clustering and on-line classification systems. These included elements from computational neural networks, self organising maps, fuzzy clustering, and rule based reasoning systems. Ultimately, targeted clustering techniques and a devoted on-line classification system were developed specifically for this application. These methods were described earlier in this thesis.

Before and during the project various domain experts acquired an extensive amount of knowledge of the physical, chemical and mechanical processes involved in the padprinting process. This included a complete catalogue of padprinting defects, called the process encyclopaedia, containing all known printing defects, their physical or chemical causes, and suitable measures to resolve them. Based on this process encyclopaedia a simple idea emerged for the control of padprinting processes. The main principle for the control of a padprinting process was the assumption that the correct identification and classification of the printing defect would directly define the most suited control action. The direct control would thus be based on the sequence of four central activities: 1. identification that a defect had occurred, 2. correct automatic classification, 3. diagnosis of the underlying chemical, physical, or mechanical causes, 4 . definition of suitable process control actions. The last two steps of this sequence were directly defined by the process encyclopaedia. The first two steps would have to be performed by the sensors and software of the system. Most notably, the early identification of a printing defect should be performed by the computer vision subsystem. Hence the great importance of the correct visual identification of a printing defect, and the significance of computational vision.

The main objection against this line of thought was that it perhaps could assuage the symptoms, but did not cure the underlying disease. In addition to the computational vision, many more sensors were available on the prototype printers. Preferably, these sensors - like for instance the ink viscosity sensor - should transmit early warnings signals that the process was heading towards an unstable state.

There were two reactions to this objection. First, the fundamental physical, mechanical, and chemical mechanisms for defects were - if at all - only poorly and qualitatively understood, while the process encyclopaedia was considered to be quite complete. Second, the prospect of obtaining a prototype padprinter system, equipped with a wealth of accurate and sensitive sensors, was considered as an opportunity to acquire a great wealth of new process knowledge. This knowledge could be extracted from information stored during the process. This information consisted of the input and process parameters, defined by the sensor readings, machine settings, and material properties of substrate, cliché, and pad, and the resulting output parameters in the form of the quality descriptors of the printing process. This information would be stored on-line in a database system. For this reason a suitable database structure was designed that could combine the outcome of the computational vision system on the diagnosis of the quality of the print with the observed setting of the machine, and sensed values of the process parameters. This database was called the Principal Process Database System. During the testing of the machines, voluminous amounts of information could thus be accumulated in this archiving system. Later, this immense database could be used off-line for 
data-mining. In this way, relevant structures and patterns in the space of process and input parameters could be identified. These structures could on the one hand be interpreted by the domain experts. On the other hand, this information could be used as a black box for direct process control, for instance using the same on-line sensing and classification system, IASC, as used for the computer vision. All these components were developed for, and integrated in the second and final and major prototype padprinter.

In the section 7.2 we present a brief overview of the padprinting process, as far as necessary for understanding the setting of our application. We also shortly present the outlines, objectives, and results of the IntelPadPrint project, in which most research activities took place. Then, we briefly discuss the defects that can occur during a padprinting process. In Section 7.3 we present a survey of image analysis techniques for recognising the visual characteristics of these defects. In Sections 7.4 and 7.5 the two prototypes stemming from the research are discussed in detail. We close this chapter with the main conclusions regarding the practical lessons learned from the application of IASC and computational vision to this domain.

\subsection{Intelligent Control of Padprinting Processes}

\subsubsection{A Brief Description of a Padprinting Process}

Padprinting is an industrial process for printing a decoration on an object. It consists of a electro-mechanical machine for printing, the 'printer', and a conveyer belt for transporting the object to and from the printer. Padprinting is a simple and straightforward process suitable for a wide variety of industrial products. This process has a number of outstanding and attractive characteristics for industrial decoration.

- it is an extremely high-yield/low cost process,

- it is the only decoration technique that allows printing on curved or irregular substrates,

- it is able to print on a large variety of substrates including most synthetic materials, metals, glass, and even food,

- it can print almost every colour or pattern on a coloured substrate,

- it is relatively fast as one typical printing cycle takes only a few seconds,

- because of its straightforwardness it does not cause frequent or complex machine problems,

- once the process is stable it is highly efficient and reliable.

This simple and cheap process allows more flexibility than any other printing process. Not surprisingly, padprinting is one of the most widely used decoration techniques throughout the world. The process is mechanised and automatically controlled to a high perfection. The principles of electro-mechanic padprinting were invented in 1965 by Wilfried Philipp in Stuttgart (Germany), originally for the decoration of the faces of clocks. The three principal components in the padprinting process are the pad, the cliché, and the ink. Below we briefly consider the major steps and components. 


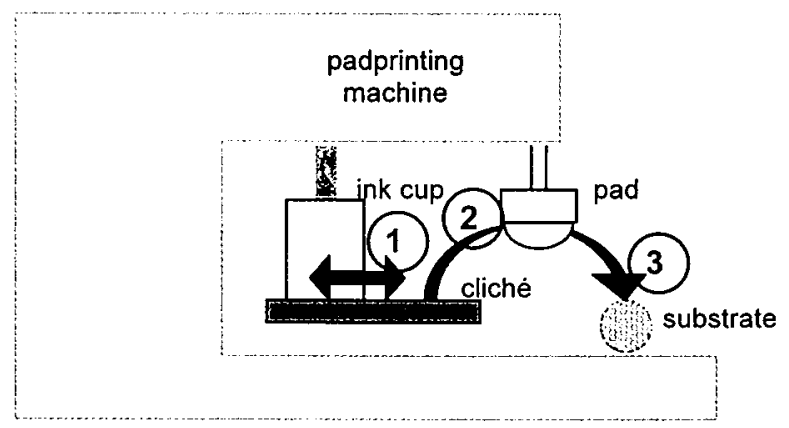

Fig. 7.1. Schematic drawing of a padprinting machine, indicated are the three major process steps.

The three principal steps in the padprinting process are illustrated in the figure above. In chronological order these steps are:

1. A hermetically sealed inkcup moves over the template of the cliche, which thereby is filled with ink. At the same time the silicon rubber pad moves in the direction of the cliché.

2. The pad is pressed with adequate force on the inked negative image from the cliché, and thereby picks up the ink. Subsequently, it moves in the direction of the substrate.

3. The pad is pressed down with great accuracy and adequate force on the substrate, thereby transferring the inked image on the intended position of the object.

After these three steps the substrate is replaced by an undecorated one, and the process resumes from start. For each colour one full padprinting cycle is required. Sometimes it is necessary to print several times, e.g. on dark substrates.

The two most defining components in the printing process are the pad and the inks.

\section{Pad Properties}

The material properties of the pad define the quality and constrain the efficiency of the printing process. The pad must obey a number of contradicting properties: 1 . The pad must quickly and fully absorb the ink from the cliché, 2 . During the transfer the absorbed inked image must stick firm to the pad surface, while at the same time it must not flow on, or diffundate in its surface, 3 . When the pad is pressed on the substrate the inked image must be fully released, and: 4 . The pad must be pressed forcefully on the substrate, but the inked image must not deform. Most pads are made of a silicon rubber and have varying degrees of stiffness, depending on the characteristics of the substrate and the decoration. During the process the pad may become fairly hot, and attract a substantial amount of static electricity. Again, this must not affect the quality of the printing process.

\section{Padprinting Inks}

There are no universal padprinting inks that will attach to every kind of material. Therefore, a wide variety of suitable inks are available for different materials, colours and textures. Constraints for the ink are dictated by the material, texture and colour of the substrate, as well as by the colour and graphics of the intended decoration. Close attention must be paid to the composition of the ink as substrates may interact physically or chemically with specific 
constituents of the inks. In addition to the normal one-component inks, there exist twocomponent inks that will react only on the substrate, and ultra-violet inks, that need a certain dose of UV-light to attain its ultimate colour, gloss, and adhesion with the surface of the substrate. The final quality of the decoration depends on physical-chemical processes during the absorption of the ink. In these processes the viscosity and evaporation characteristics of the ink play a decisive role. Besides the usual decoration of consumer products, there exist special inks for printing in medical industries, as well as edible inks for printing on food products, such as chocolate confection.

\section{Clichè's}

The cliche forms an important obstacle for reducing the lead time between design of the decoration, and the beginning of the actual padprinting process. This is related to the time required to produce a high quality cliché. Traditionally, the design is etched into a cliché consisting of a polymer coating on a metal backing, or of hardened steel. Within the IntelPadPrint-project described below, laser engraving was applied to successfully decrease the lead time. The cliche is inked by either an open inkwell 'doctoring system', or, with more advanced printers, by a closed inkcup sliding across the cliché.

\subsubsection{Important Impediments of Padprinting Processes}

Once a padprinting process is stable it can remain so for long undisturbed periods. In such periods padprinting is highly efficient and reliable. A characteristic trait of padprinting processes is the rather sudden emergence of misprints after long periods of process stability. This can be caused by a wide range of physical, chemical, or human reasons. However, the most important source of rejected prints is the set-up of the machine for a new process. A new set-up is required when a new product, a new decoration, or a different colour must be printed. In this phase up to $66 \%$ of the total reject-rate during the entire production run is generated [Legierse,2000]. These initial rejects are mainly caused by the trial-and-error type of regulation of the process parameters. This conduct is caused by a lack of process expertise, mainly due to insufficiently experienced personnel. Consequently, the initial reject rate for entirely new processes is found to be higher than for processes which are more familiar.

Another source for product rejects is visual fatigue of the human controller inspecting the print quality of the process output. Visual inspection is subject to large variations in skill from person to person, between age and gender groups, or for one person throughout a working day, and throughout the seasons of a year. After some time human visual scrutiny will inevitably decline and subtle printing defects will be able to pass unnoticed. Printing defects are superpositioned upon the design of the print. Therefore, defect characteristics are expressed as general features and not in terms of well-defined shapes. This is an additional complication for the detection of printing defects, for it requires a mental subtraction of the observed pattern with the design of the decoration. Since print quality is a visual and cognitive criterion, misprints are defined by a notable difference between the realised decoration and the design as defined by the graphic engineer. The difference is marked by a deviation from the intended colour palette, gloss, texture, position, or orientation of the printed image on the product. Depending on the residing quality standards, these deviations can be nearly imperceptible for the untrained eye. Experienced padprinting experts regularly evaluate product decorations with respect to their quality standards and so detect even minute defects. The central observation in our work is the ability of such experts to interpret subtle properties of a misprint, and from these to deduce the original cause of the defect. From this observation it became important to meticulously catalogue the visual features of all known misprints, and to record their specific causes. 


\subsubsection{A Visual Taxonomy of Padprinting Defects}

Padprinting defects can have various physical, chemical, or mechanical origins, they can be caused by operator faults, or instead have an external cause. A large number of padprinting defects is presently known and catalogued. In Table 7.1 below some examples are listed for several types of causes.

\begin{tabular}{|c|c|}
\hline Physical & $\begin{array}{l}\text { Static electricity; incorrect ink viscosity; evaporation of solvent; } \\
\text { incomplete ink transfer; irregular deformation of pad. }\end{array}$ \\
\hline Mechanical & $\begin{array}{l}\text { Incorrect pad pressures; incorrect positioning of pad; incorrect inking of } \\
\text { cliché; surface roughness too high; }\end{array}$ \\
\hline Chemical & $\begin{array}{l}\text { Chemical properties of ink, thinner, substrate or pad; interaction } \\
\text { between ink and substrate, pad, or cliché; incorrect UV-reaction of ink. }\end{array}$ \\
\hline Human operator & $\begin{array}{l}\text { ink pot empty; wrong cliché depth; wrong pad shape or hardness; } \\
\text { product or jig not well fixed. }\end{array}$ \\
\hline Graphical design & $\begin{array}{l}\text { Bad film outline; wrong raster size; wrong etch roughness; wrong ink } \\
\text { specification; }\end{array}$ \\
\hline Other & $\begin{array}{l}\text { Dirt on pad, cliché, or substrate; curved substrate too smooth; print } \\
\text { speed too high; }\end{array}$ \\
\hline
\end{tabular}

Table 7.1

Printing defects are characterised by their visual effects on the print. Therefore, it is possible to make a classification merely based on the visual characteristics of the defect. In Figure 7.2 below an example of a typical padprinting defect is shown.

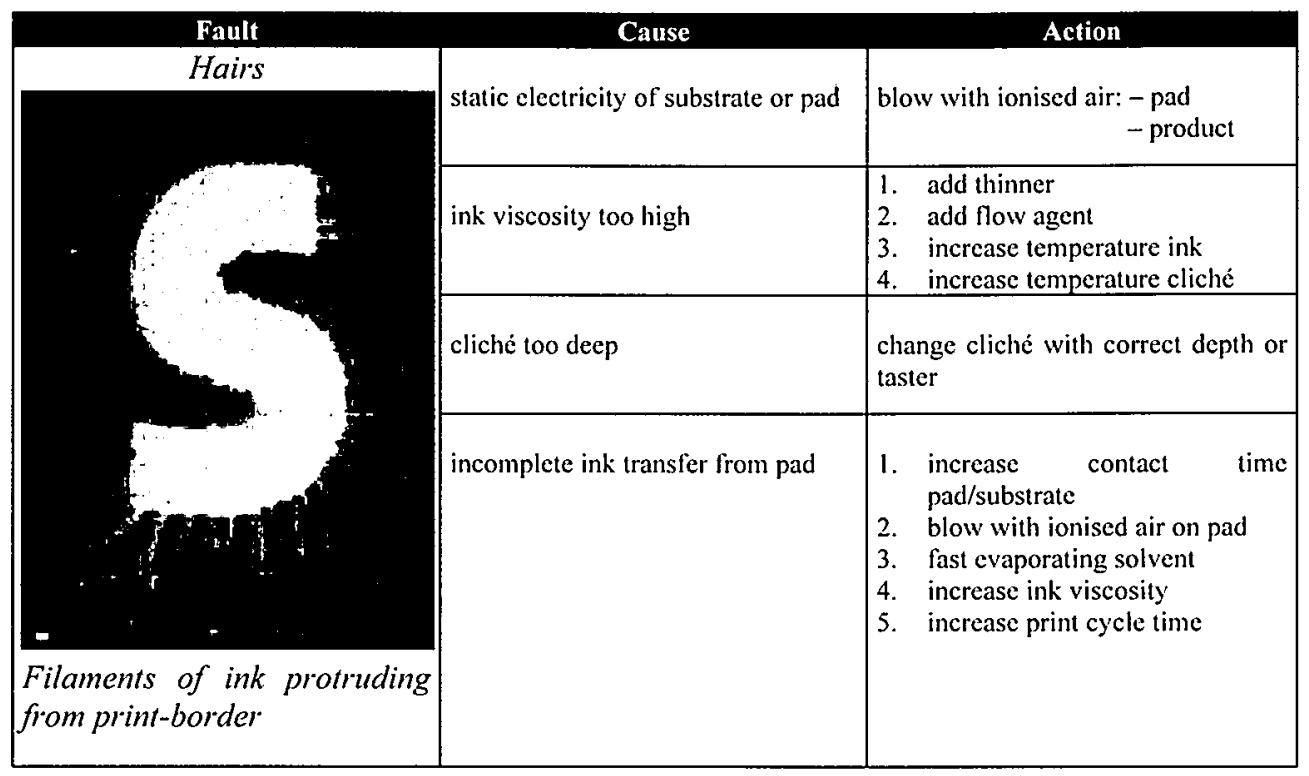

Fig. 7.2. Example of padprinting defect 'hairs'. its possible causes, and suitable control actions.

This defect is called 'hairs' for obvious reasons, and is characterised by protruding filaments of ink almost perpendicular to the print-border. 'Hairs' can have various causes; static electricity can cause ink to form filaments directed according to the electrostatic field-lines. 
When the charge has leaked away the filaments fall down and form the defect. Otherwise, the ink viscosity can be too high, causing the ink to spray away from the printed area under the high pressures realised during the printing of the pad. This out-going ink flows in discrete filaments perpendicular to the print-border. For each of these causes a suitable reaction can be defined; blowing ionised air in case of static electricity, or adding thinner in case of too high ink viscosity. For all known padprinting defects such visual characterisations are available, together with a verbal definition, their known causes, and for each cause a suitable control action. This is the so-called padprinting process encyclopaedia. Figure 7.3 shows one complete record of this encyclopaedia.

At this point it must be said that the participating physicists and chemists in many instances disagreed with padprinting experts over a more detailed visual classification. The objective was to have only one cause per visual defect category. For instance, some insisted that static electricity typically gave other shapes of 'hairs' than high viscosity. In our context, this is an area where the texture-analysis discussed in Chapter 3 could shred some new light.

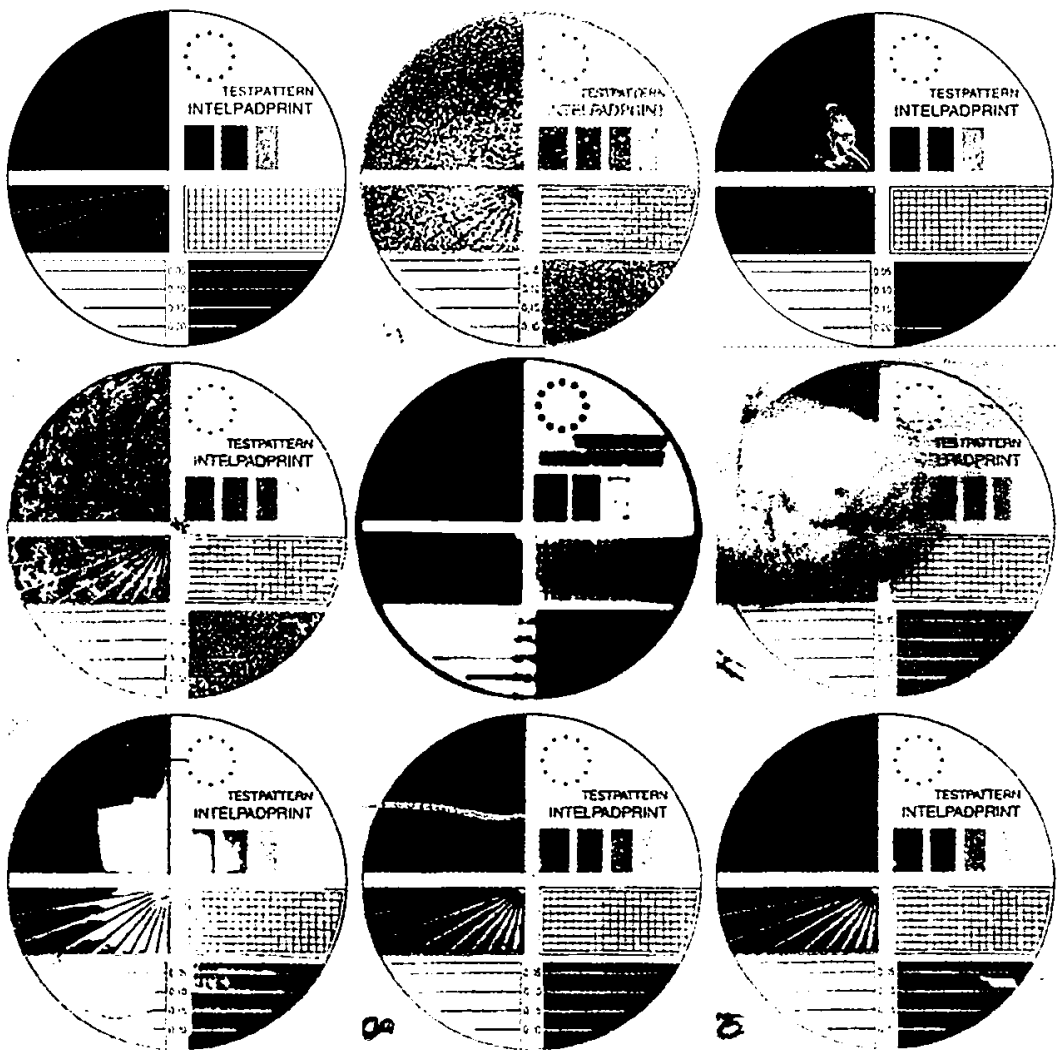

Fig. 7.3 CCD-images of real samples of padprinting defects. Left to right from above: 1. Perfect print (template), 2. air foam, 3. ink missing, 4. orange peel, 5 . ink flow, 6. smearing, 7. constriction, 8 . scratch or lines, 9. edge roughness.

The visual characteristics of padprinting defects as provided by the experts are essentially based on subjective criteria. Many authentic specimen of misprints, nevertheless, are of an ambiguous nature and can not be simply labelled as one or another defect class. In-between 
the defect classes intermediates of all degrees of similarity exist. In that respect our printing defect classes are of an inherently fuzzy nature. For that reason a fuzzy modelling proved especially promising. In the figure below some typical padprinting defects are shown, together with the template - i.e. the graphical design of the print.

The highly efficient, yet subjective human vision is a solid criterion for print quality, for ultimately it is the human consumer that founds his purchase on his appraisal of the visual appearance of the product. The visual characteristics of padprinting defects correlate so well with the more fundamental origins that the defect can be exclusively deduced from visual analysis. Ultimately, however, all padprinting defects are of course defined by their underlying physical-chemical processes, especially by the rheological and hydrodynamical properties of the ink, and the material properties of the pad, substrate and the cliché. Naturally therefore, the clustering of padprinting defects based on visual criteria as in Section 4.7.2, is an entirely different one as a clustering based on the ultimate causes of the defect.

\subsubsection{Original Objectives of the 'IntelPadPrint' Project}

For many years, padprinting proved to be the most effective, in fact, the only, way to print and decorate the curved surfaces of a wide range of products, and also the surfaces of threedimensional objects. Near the end of the previous century, production line requirements had increased in such a way that they resulted in unacceptably high rejection rates of about $5 \%$. The estimated cost of the loss and regranulation of that quantity of basic material amounted to some $20 \mathrm{MECU} / \mathrm{yr}$ in the European Union alone [Legierse,Witgrefe, 1995]. Moreover, the labour-intensive nature of padprinting accounted for a further $50 \mathrm{MECU} / \mathrm{yr}$ in the EU. Therefore, there was a high risk that this type of industry would be moved to low-wage areas like south-eastern Asia. Also technical requirements and consumer demands on print quality intensified, such as the need to print very fine lines and textures on surfaces with smaller radii. Finally, other printing techniques emerged, such as ink jet and laser printing, causing potential competition for padprinting. Since its invention, the padprinting-process had proved to be so successful that little or no research had been undertaken to improve it. The main improvement had been the introduction of 'closed' as opposed to 'open' printing, but the process remained heavily labour-intensive. These and other realisations led a number of producers to form a consortium aimed at the development of a new type of high-yield padprinting process that could be installed at the end of production lines. This would be essential for allowing flexible decorative printing that gives acceptable rejection rates of less than $0.1 \%$. To realise this objective essentially required intense research into all aspects of the padprinting process, such as inks, substrates, cliché, and pad design and materials. This should be accompanied by a close examination of the print process parameters, including visual control of prints, with ultimate feedback to the printer. Adaptive and intelligent control of the parameters seemed to be the required direction to follow, as it allows off-line selection of inks, clichés, and pads, and on-line monitoring of the process, with real-time adjustment of control variables. The original industrial objectives of the research project were the following:

1. to develop a new, high-yield, self-learning production process for padprinting, comprising computer-controlled movement of the printing pad, and single-print technique of new inks. The self-learning process is multi-sensor assisted with sensors for optical inspection, pressure, humidity, temperature measurement, and viscosity. Sensor information is fed back to the process by adaptive and flexible algorithms.

2. to develop materials and inks that will give high contrast after a single printing cycle, and have good resistance to chemicals, abrasion, and light of various colours and luminosities. 
Table 7.2 compares the practice at start of the project in 1996 with the original project targets.

\begin{tabular}{|c|c|c|}
\hline Feature & Current Practice & Project Target \\
\hline minimum line width & $100 \mu \mathrm{m}$ & $30 \mu \mathrm{m}$ \\
\hline dry layer thickness & $5 \mu \mathrm{m}$ & $10 \mu \mathrm{m}$ \\
\hline print cycle & $1 \mathrm{sec}$ & $0.5 \mathrm{sec}$ \\
\hline change-over times & 10 minules & 1 minute \\
\hline reject rate & $5 \%$ & $0.1 \%$ \\
\hline adhesion & $1-3 \mathrm{ISO}$ & 0 ISO \\
\hline wear resistance & limited. 1-3 Rda & very good, 0 Rda \\
\hline chemical resistance & limited, 1-2 Rhe & very good, 0 Rhc \\
\hline inks & solvent based & little or no solvent \\
\hline other leatures: & $\begin{array}{l}\text { - operator-assisted with } \\
\text { empirical knowledge } \\
\text { - high ink usage } \\
\text { - labour-intensive hence } \\
\text { expensive }\end{array}$ & $\begin{array}{l}\text { - computer-controlled, operator- } \\
\text { independent process } \\
\text { - low ink use, robust process, } \\
\text { - computer-controlled, } \\
\text { inexpensive process. }\end{array}$ \\
\hline
\end{tabular}

Table 7.2

The research was submitted as part of the European research programme 'BriteEuram' as an industrial applied research project, titled: 'Development of an intelligent padprinting system', acronym 'IntelPadPrint', short 'IPP', funded under grant BE3180 [Legierse, Witgrefe, 1995]. Partners in the project were: Philips CFT (project coordinator) (physical/chemical research), and Philips DAP-consumer products (end-user) in the Netherlands; LEGO engineering (testing, end-user) in Denmark; TampoPrint $\mathrm{GmbH}$ - the original inventor of automatic padprinting and so the oldest manufacturer - (development of two prototypes, electromechanical research) in Germany; Marabu Inks (producer of high-quality and specialised inks, chemical research and development of special inks), also in Germany; and the University of Birmingham, United Kingdom, for Dynamic Finite Elements analysis of the padprinting-process, and the development of the camera system, and sensor-actuator interfaces. The Universiteit Maastricht was a partner with 3 man-years ${ }^{l}$ involvement aimed at the development of an on-line control system based on multi-sensor integration - including computer vision. TampoPrint sub-contracted ESD GmbH (Germany) for the development of a computer controlled electro-mechanic control system. In the following sections we restrict the description of the development of the IntelPadPrint-prototypes to our own contribution, unless stated explicitly. The IntelPadPrint-project started late 1996, and was successfully completed after a brief extension early 2000 [Legierse,2000].

\subsubsection{Realised Course and Results of the 'IntelPadPrint' Project}

Within the scope of the IPP-project, and based on the original problem definition, the partners early-on agreed on the basic configuration of the machine. In the course of the project two fully integrated prototype machines based on this configuration were constructed, called PT1 and PT2. PT1 was realised after 18 months in the project. The design of PT2 was based on the lessons learned during the construction and evaluation of PT1. PT2 was near completion at the end of the project. Both PTs were constructed and built by TampoPrint. Marabu developed special inks to meet the constraints of the target printing process. The testing of the prototypes were performed both at Philips DAP, and LEGO Engineering. Both universities contributed to the construction of the multi-sensor integration and actuator control, and to the realisation of a suitable CCD-camera with built-in image processing hardware. Specific tasks of Universiteit Maastricht were: 1. the development of algorithms for deducing the

\footnotetext{
'Later extended with two man-months.
} 
padprinting defect class from a calibrated $C C D$-image of an recognised misprint; 2 . the development of algorithms for the integration of multi-sensor information - including computer vision - for efficient on-line classification of the printing defect; 3 . development of a database structure for on-line storage of process information and suitable tools for off-line data-mining of these data-bases.

The two prototypes PT1 and PT2 are discussed in detail in the later sections. First, however we have to discuss in detail the feature-parameters that were designed to identify the padprinting defect classes.

\subsection{Defining Features of Padprinting Defects}

In previous sections we have remarked that padprinting defects are essentially defined by a set of characteristic features. The basic strategy in our approach is to translate a specific digital image of a padprinting defect into a set of these features. Therefore, it is of outstanding importance to define a set of adequate and characteristic features of padprinting defects. In this section we briefly discuss a number of algorithms that are specially designed for the detection of a specific feature of a padprinting defect. In appendix $A$ these algorithms are presented in more detail. First, we characterise the defining features of a defect, and then construct a test suited specifically for the detection of these features. Therefore, the outcome of such tests conveys information on the likelihood that a certain defect is present. This information is represented in the form of feature parameters. We make a distinction between scanprofile features that are valid for $1 \mathrm{D}$-intensity profiles over curves on an image, and texture parameters defined on 2D-patches from an image. Another useful distinction that we can make is between the continuous quantitative feature parameters, and the discrete qualitative feature parameters. Qualitative feature parameters are necessary if the values obtained by calculation can only be assigned meaningfully to a limited number of cases. An intermediate solution is to define adequate fuzzy membership functions in terms of the parameters. A fuzzy representation is in its own also a parameter in the interval $[0,1]$ that expresses the degree to which a specific feature is present in the image. The basic philosophy behind the design of such tests is to derive a large number of meaningful feature parameters from the image that correlate well with specific characteristic features of a defect. These parameters can then be used to calculate the correlation with specific defect types - or alternatively to discriminate between two otherwise similar defects. Moreover, the representation in feature parameters has the benefit of producing a symbolic representation of an image.

\section{Features Defined by Basic Image Characteristics}

Standard Image Analysis provides a number of basic feature parameters intended to characterise images. Examples of such parameter are the centre-of-mass of the image, the average size of the image - defined as the average distance to the centre-of-mass, and the average orientation of the image. Examples of such tests are provided in appendix A.1.

\section{Mean Density of the Fault}

A basic feature parameter that is especially simple to compute is the mean density of the $Z$ representation of the image. This is presented in appendix A.2. 


\section{Feature parameters for Scan Profiles}

For certain padprinting defects, relevant features can be identified in scanprofiles resulting from known scancurve-types and from known print-contexts. In appendix A.3 a number of specially designed tests are presented for the extraction of such information. The following approaches are discussed.

\section{i. $\quad$ Optimal Match with a Characteristic Profile \\ ii. Peak Partitioning and Decomposition Statistics}

\section{Detecting Oscillatory Signals with 'hairity'}

The defect 'hairs' is characterised by the typical 'hair-like structures, emanating almost perpendicularly from the print border. This defect can easily be identified by a scanprofile taken from a scancurve parallel to the printborder, just outside the print - that is Curve Type $\Gamma 2$ according to table 2.2 in Section 2.3.2. An example of such a scan and the resulting scanprofile is presented in Figure 7.4. Therefore, the detection of 'hairs' focuses on the analysis

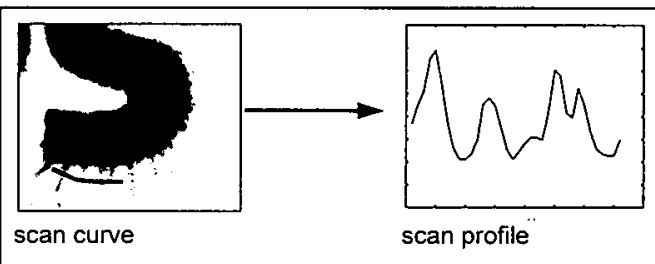

Fig. 7.4. Typical scan profile of defect 'hairs', taken over a scancurve of type $\Gamma 2$ : 'just outside print and parallel to printborder'.

of such specific scanprofiles. A number of such approaches are presented in appendix A.4.

\section{Statistical Distribution over a Partition of Image Cells.}

Most often the defect is not concentrated in one localised area, but distributed over the entire image as a collection of disconnected fragments. The defect can thus be envisaged as a partitioning of cells covering these fragments. In appendix A.5 two aspects of partitionings are presented. First, the average shape properties of the individual fragments. Second, the form of the statistical distribution of these properties over the ensemble of fragments. Two methods are employed.

\section{i. $\quad$ Statistical Cell Distribution Parameters \\ ii. Fractal Scaling Laws in the Cell Size Spectrum}

\section{Identification of Spherical versus Irregular Shapes.}

With the application of a focused scanning method to an image it is in general not possible to distinguish between a number of more-or-less similar defects. One example is the set of defects defined by unwanted intensity variations occurring at specific locations within the printed area. This may be caused by 'air bubbles', 'absence of ink', 'inhomogenities in the ink', or 'dirt particles' enclosed in the ink. In order to discriminate between these possibilities, specific tests are designed that describe the general shape of the inhomogenities. Particular relevant criteria in this context are tests that convey whether the inhomogenity is of more-orless spherical shape. Such tests can discriminate 'air bubbles' from other defects. In appendix A.6 a number of such 'shape-parameters' are defined. The following shape-parameters are presented.

i. Convexity: detect whether a shape is circular is to determine 'how' convex it is. It is defined as a parameter in $[0,1]$. 
ii. Eccentricity: the degree of 'circularity' of an object. For an elliptical orbit with radii $r_{\min }$ and $r_{\max }$ - representing the small and large radius - the eccentricity is defined as: $r_{\min } / r_{\max }$. Notice that eccentricity lies in $[0,1]$.

iii. Perimeterity: for a cell with area $\mathrm{A}$ and perimeter $\mathrm{E}: 4 \pi \mathrm{A} / \mathrm{E}^{2}$, the 'perimeterity' is defined as: $p=4 \pi A / E 2$. For all other shapes is $0 \leq p<1$.

Finally, we can utilise the correlation between these three parameters. They have in common that they attain high values for the defect 'air bubbles'. On the other hand, high values need not necessarily imply that the defect is 'air bubbles'. Combined these parameters reveal more information than on their own.

\section{Identification of Rasters versus Irregular Patterns}

Under certain conditions the raster of the cliché may be visible. It is useful to have an indicator for the detection of this unwanted phenomenon. For this reason the specific test 'rasterity' is designed. This parameter indicates the degree in which a structure exhibits a raster-like appearance. This quantity is defined in appendix A.7. In order to distinguish rasters from random aggregations of dots, as may occur for instance in the defect 'dirt particles' and in noise, the sensitivity of the feature parameter to disorder in the raster is studied. Another practical matter is that the raster on the CCD-image is in general somewhat rotated. Therefore, the behaviour of the rasterity with respect to rotations with an angle $\phi$ is investigated. An alternative approach is based on the Peak Partitioning and Decomposition approach, described in appendix A.3.

\section{Identification of Streaks, Lines and Scratches}

Lines, streaks, and scratches on an image can be identified with an approach similar to the identification of spherical objects using eccentricity. Like some of the other methods this approach depends on an interactive focussing on the individual defect cells. This approach is presented in appendix A.8.

\section{Identification of Dirt Particles.}

Images showing the defect 'dirt particles' are characterised by small isolated and irregular spots of intermediate greylevel, that are randomly distributed over the image. In appendix A.9 three methods are presented for the identification this defect. These methods are relevant both for identifying 'dirt particles', and distinguishing them from 'air bubbles' foam, 'rasters' and random spikes generated by noise. All three methods utilise the characteristics of dirtparticles such as their small size, their random distribution in the plane, and their sharply peaked distribution with narrow flanks. These three methods are:

\footnotetext{
i. Statistical Cell Size Distribution

ii. Medial Fourier Filter

iii. Peak Decomposition and Partitioning
} 


\section{Conclusions}

In this section a number of specific tests for the quantification of specific features of padprinting defects were briefly discussed. In appendix A they are presented more thoroughly. These algorithms result in quantities called feature parameters that indicate the degree to which the image exhibits certain important features. These parameters can be grouped as scanprofile parameters that act on $1 \mathrm{D}$-scanprofiles, and texture parameters that represent features of (parts of) 2D-images. General image analysis techniques and statistical analysis can be seen as basic texture parameters. Some of the methods discussed depend on an interactive focussing on individual defect cells, others act on the image as a whole.

The presented parameters give reasonable to good results on the available set of test images. Important defects like 'hairs' and 'air bubbles' can be identified efficiently. During the evaluation of the two prototypes in an industrial environment, discussed below, however, there were major problems caused by lighting conditions, the CCD-arrays, and the image-preprocessing. These problems significantly reduced the performance of these feature parameters. An important problem in that respect is the role of false negatives and false positives. As images became unfocused, the feature parameters deviate from their 'true' value. Some parameters drift up rather than down. The result is that during the reasoning process false evidence accumulates and the wrong tests are proposed. This can result in longer evaluation times per image, and worse, the wrong conclusions. These effects were discussed in Chapter 5 with regard to the reasoning process. In all, there is a strong demand on a close connection between the image analysis, defect diagnosis, and required CCD-camera actions like area selection on the printed element, active focussing, active positioning to minimise ghost-images, and zooming-in.

Another aspect of these parameters is the potential wealth of data that would result if we would perform all tests described in this section. This would generate two problems, first an unmanageable cumulative computation time, second, the open question how to find structure in the data, and use it to select the optimal next test. In several examples we saw that a combination of tests was necessary to discriminate competing cases. Which specific combination of tests is best depends on the present balance of evidence and the actual most likely hypothesis. For that reason Functional Parameter Selection (FPO) was developed, as discussed in Section 5.2.

An entire different set of features is based on fractal properties of the defects. These features, alongside the importance of wavelets for the detection and extraction of texture properties, were discussed in Chapter 3.

The relation of the many extractable features discussed here, with the set of known padprinting defect classes is the basis of the two prototypes constructed during the IntelPadPrint-project, and they are the subject of the next two sections. 


\subsection{The IPP-PT1 Approach: Fuzzy Correlations between Scan-features and Defect Classes}

The principle for identifying and classifying padprinting defects in the first prototype PT1 is the efficient generation and analysis of scan-profiles. In the previous sections it was shown how scan-profiles relate to the padprinting defect classes, and earlier in this thesis, especially in Section 2.2, the basic structure of printed images was described. Based on relative locations in the print, called 'print-contexts', five suitable types of scan-profiles were identified, called 'curve-topologies'. The mechanism for PT1 essentially consists of three components. [1] An algorithm for generating relevant scan-curves on the print, and from these curves extract the intensity profiles called 'scan-profiles', [2] an algorithm for extracting significant features from the scan-profile, given the print-context and curve-topology of the scan-curve, [3] an algorithm for computing the most likely padprinting defect class from these features. This three-stage algorithm is called the 'Fuzzy Correlation Approach', or FCA, for step three employs the fuzzy correlation between scan-profiles and defect classes. Furthermore, a reasoning mechanism is required to steer the scanning of the image and to decide when to derive a final conclusion.

Below, we describe these stages in more detail. First, we describe the computation of the defect class-membership function from the feature-vector using the fuzzy correlation matrix. Next, we describe the image analysis and feature extraction based on scanning. Third, we describe the integration of multiple FCA-experiments into one final conclusion. We close this section with an evaluation of this approach.

\subsubsection{Linear Fuzzy Correlations between Scan-features and Padprinting Defect Classes}

Suppose that we want to establish the padprinting defect class from an CCD-image of a padprinted object. With that purpose we obtain a scanprofile from a curve of certain curve-topology $i(i=1 \ldots 5)$. We can match this scanprofile with the possible featuretype profiles for curve-type ' $i$ ' and print-context ' $l$ ', as described in Section 7.3.3. We can represent this match by a number between 0 and 1 , that can be considered as the fuzzy membership function of the scan to that feature-type. Since the result depends on the topology-type $i$ and print-context l, we can represent the match of our scanprofile with feature-type $j$ as: $X_{i j l} \in[0,1]$. Now, independent of our actual image - and even independent of the template - a certain result of a scan on a curve of specific type in a certain print-context, contributes an a priori amount of support to the hypothesis that failure class $k$ is occurring. This conjecture is the starting point of our further analysis. We shall call the likelihood of the hypothesis that failure class $k$ is occurring $p_{k}$. Since the observed result of the scan is represented by $X_{i j l}$, we can consequently write the likelihood $p_{k}$ for one scan-line as some function:

$$
p_{k}=\Phi_{k}(X)
$$

We can approximate $p_{k}$ by expanding $\Phi_{k}$ near $X_{i j l}=0$, observing that $\Phi_{k}(0)=0$. As a first approximation we only consider the first term, and write $p_{k}$ as a linear equation in $X$ :

$$
p_{k}=\Sigma_{i j l} S_{i j / k} \cdot X_{i j l}
$$


The matrix $S_{i j l k}$ represents the amount of support (hence ' $S$ ') that a scan on a curve with topology type ' $i$ ' in print-context ' $l$ ', having possible feature ' $j$ ' as its result, contributes to the hypothesis that this image belongs to failure class $k$. Though this support can be either positive or negative $\left(S_{i j l k}=-1\right.$ could indicate that combination $(i, j, l)$ definitely does not support inclusion in failure class $k$ ), we here demand that the matrix satisfies: $S_{i j l k} \in[0,+1]$ - because otherwise $p_{k}$ could become negative. The vector $p_{k}$ and the feature matrix $X_{i j l}$ can be understood in terms of membershipfunctions: $p_{k}$ as the membership of the defect in failure class $k, X_{i j l}$ as the membership of the scan in the class of possible feature ' $j$ '. In this fashion $S_{i j l k}$ can be regarded as the (first order approximation of) the correlation between these two membershipfunctions. For this reason, and because the starting point are the $1 \mathrm{D}$-scans, we call $S$ the ID Fuzzy Correlation Matrix. The matrix $S$ is independent of the template and observed image and therefore needs to be calculated only once for all combinations of the five curve topologies, four print-contexts, $f$ possible features, and $K$ padprinting defect classes. Therefore, $S$, has $20 f K$ elements. An example of the calculation of the probability vector ' $p$ ' is presented below. The calculation of the support-matrix $S$ is performed by linear least-squares from a large set of empirical data, containing scans of known curve-topology, known print-context, and known padprinting-defect class.

\subsubsection{Calculation of the fuzzy membership-function for multiple scans}

For one specific curve of known type ' $i$ ' in print-context ' $j$ ', we can calculate the match with feature ' $j$ ' and find $X_{i j /}$. If we calculate $\Sigma_{j} S_{i j / k} X_{i j l}$ - where the sum extends over all possible features ' $j$ ', while ' $i$ ' and ' $l$ ' are fixed, for the topology of the scan-line and its print-context are known - we find the probability, based on this one measurement, that defect class ' $k$ ' has occurred. If we have performed several scans on curves $\gamma_{1}, \gamma_{2}, \ldots, \gamma_{N}$, with topology types $i(1), i(2), \ldots, i(N)$, in print-contexts $l(1)$, $l(2), \ldots, l(N)$, we have to add all of the probabilities, and thus find $p_{k}$, representing the probability that defect class $k$ has occurred as:

$$
p_{k}=\Sigma_{n j} S[i(n), j, l(n), k] X[i(n), j, l(n)]
$$

Next we determine the index number of the maximum value of $p_{k}$ and thus we find the index of the most likely padprinting defect class $k^{*}$.

$$
k^{*}=\arg \max _{n} p_{n}
$$

If more than one element of $p$ is found, we could conclude that multiple defects have occurred simultaneously. Now the basic theory for our approach is introduced, we apply and test it on a realistic example. 


\subsubsection{Example of a calculation using the 1D-fuzzy-correlation approach}

In the figure below an example of a measured scan of defect type 'hairs', somewhere outside the printed area, just crossing the 'hairs', as indicated in the left image below.

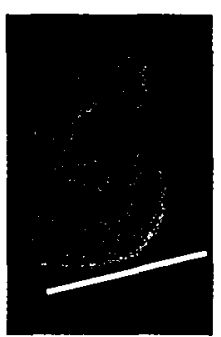

Figure 7.5.

Above: scan-curve of type $\Gamma$, right: resulting scamprofile (solid) and expected result (dotted)

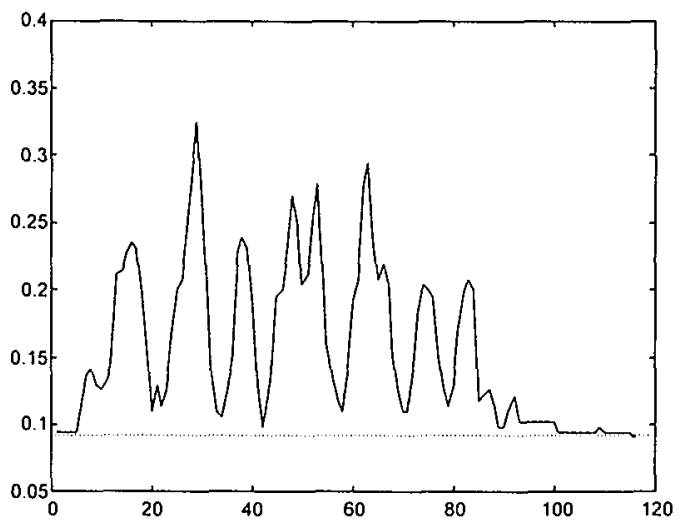

The dashed line in the left figure is the expected result if the print was perfect: a straight line with the greyscale of the substrate. The scan line is of type $\Gamma_{2}$, the printcontext is 2 ('just outside the print') and so we can calculate the match with the following 6 feature-types:

\begin{tabular}{|c|l|c|}
\hline \multicolumn{1}{c|}{$\boldsymbol{j}$} & Feature-typeljl & Match $X_{2 j 2}$ \\
\hline 1 & straight line & 0.02 \\
\hline 2 & step function from high to low & 0.01 \\
\hline 3 & step function from low to high & 0.35 \\
\hline 4 & block-function & 0.36 \\
\hline 5 & oscillating pattern & 0.91 \\
\hline 6 & bell-shaped curve & 0.04 \\
\hline
\end{tabular}

\section{Table 7.3}

At once it is visible that the largest match is found with an oscillating pattern. Next, we calculate the probability vector - or membership function - that the failure belongs to class $k$ as: $p_{k}=\Sigma_{j} S_{2 j 2 k} X_{2 j 2}$. Given the values of correlation matrix $S$, we find the values as shown in the table below:

\begin{tabular}{|l|l|}
\hline Padprinting Defect & \multicolumn{1}{|l|}{ Likelihood } \\
\hline hairs & 0.38 \\
\hline recess & 0.07 \\
\hline double print & 0.04 \\
\hline edge roughness & 0.08 \\
\hline smearing & 0.09 \\
\hline slreaks of lines & 0.20 \\
\hline ink droplets & 0.10 \\
\hline haze & 0.02 \\
\hline no defect & 0.02 \\
\hline
\end{tabular}

Table 7.4

Thus, the most probable conclusion is the padprinting defect class 'hairs' with a probability of 0.38 . 


\subsubsection{Integration of Image Analysis and the FCA Approach}

In this section we describe the major stages of image analysis and FCA in the PT1 in more detail.

\section{Phase i. Pre-Matching and Subtraction}

The first step in the image analysis, the subtraction of the template from the CCDimage, has a considerable impact on the subsequent classification process. The quality of the subtraction is defined by a chain of complicated hardware and software constraints. First, the lighting is devoid of ambient light or reflections, and the light level matches the template-luminosity stored in the computer. Second, the optical and electronic processing in the CCD-system is free of flaws. Third, pre-matching software is able to translate, rotate, and scale the resulting CCD-image to the template co-ordinate-system. Even in the optimal case, there are always a residual image after subtraction, called the ghost-image. This ghost-image generally outlines the borders of the template, for there the effects of pre-processing will be the most felt. The dependency of the subtracted CCD-image on the template implies that our approach not totally template-independent. Finally, the pre-processed image $F$ is compared with the template $T$. If the integrated absolute difference between them is below a certain threshold, the image is accepted as defect-free, or formally:

$$
\int_{\Omega}|F(\mathbf{x})-T(\mathbf{x})| \mathrm{d}_{2} \mathbf{x} / \int_{\Omega} \mathrm{d}_{2} \mathbf{x}<\theta \Rightarrow \text { conclude: "no padprinting-defect" }
$$

where ' $\Omega$ ' represents the entire area of the image, and $\theta$ is a pre-defined noisedependent threshold. This is Equation [2.3] from Section 2.2.4.

\section{Phase ii. searchboxes: Intelligent Binary Search}

The next step is a focussed decomposition of the image. First, isolated areas of the image are selected where the difference $Z(\mathbf{x})=|F(\mathbf{x})-T(\mathbf{x})|$ is above a predefined value. The collection of these areas is called a SearchMap. The image then is decomposed into a union of disjunct compact areas called searchboxes, where the 'energy' of the image is sufficiently high. The energy of a searchbox $B$ is defined as: $\int_{B}|F(\mathbf{x})-T(\mathbf{x})| \mathbf{d}_{2} \mathbf{x}$. For this decomposition an algorithm has been developed that iteratively covers the SearchMap with a set of searchboxes. This algorithm involves binary search on the absolute difference $Z$. Figure $9 a$ shows the iterative search of the algorithm to an interesting area on the image. In Figure $9 b, 75$ of these searches are performed, resulting in a covering of the 'interesting' areas of the image. The algorithm must balance between finding the smallest significant areas, and cutting relevant areas in multiple fragments. Attempts to add some intelligence to the algorithm improved its performance, but also significantly decreased its speed. Therefore, empirically a balance was sought between 'SearchBox-quality' and 'speed'. This resulted in an 'Intelligent Binary Search' algorithm called FindAreas. The basic trick of FindAreas is to iteratively dispose the $k$-th found SearchBox $S_{k}$ from the actual SearchMap $Z_{k}: Z_{k+1} \leftarrow Z_{k} S_{k}$. Therefore, the algorithm exhaustively covers the SearchMap $Z$ with a set of searchboxes $\left\{S_{k}\right\}$, until the remaining cumulative energy of $Z_{k}$ falls below a certain pre-set threshold:

$$
\int_{Z[k]}|F(\mathbf{x})-T(\mathbf{x})| \mathrm{d}_{2} \mathbf{x}<\epsilon \cdot \int_{Z[k]} \mathrm{d}_{2} \mathbf{x}
$$




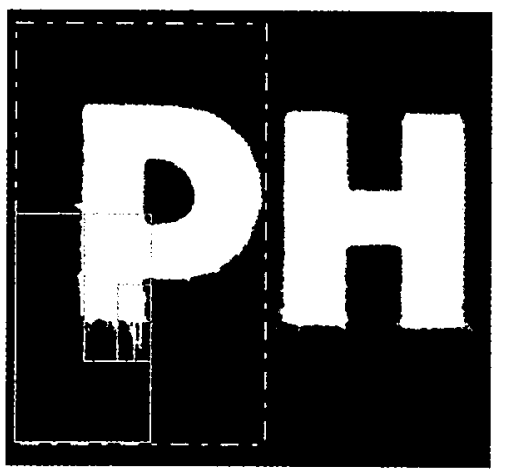

Fig 7.6a Iterative computation of one SearchBox

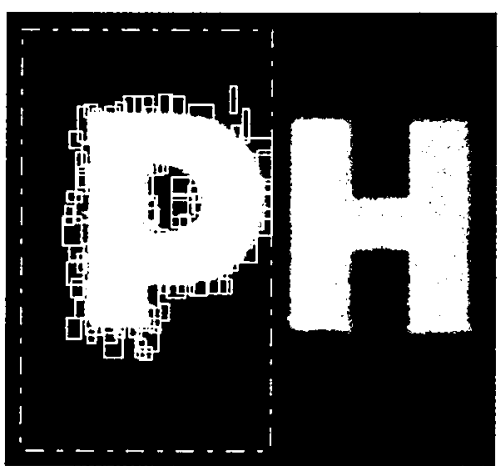

Fig $7.6 b$ Covering with 75 SearchBoxes

A partitioning algorithm based on finding the location of the highest discrepancy on the SearchMap, rather than binary search did not yield better results. Another interesting alternative is by employing Multi Resolution Analysis (MRA). MRA generates wavelets with translations $\left(k_{1}, k_{2}\right)$ and dilatations $\left(\lambda_{1}, \lambda_{2}\right)$ from a mother wavelet. So box ' $i$ ' is defined by corners: $\left(k_{1} / \lambda_{1}, k_{2} / \lambda_{2}\right)$ and sides: $\left(\lambda_{1}, \lambda_{2}\right)$. In this way a covering of boxes of SearchMap $Z$ is generated. This, however, did not necessarily improve the covering of the FindAreas algorithm.

\section{Phase iii. Automatic Generation of ScanCurves}

After an adequate SearchBox has been generated by FindAreas, an algorithm called GenerateScanCurve generates a scan-curve $\gamma$ of pre-defined topology CurveType (defined in table 2 in Section 2.1) within the SearchBox.

\section{Phase iv. scanprofile over the ScanCurve}

A scan-profile is defined as the intensity of the image $F$, measured along the scancurve $\gamma$. If the scan-curve $\gamma$ is defined by its parameterisation $\mathbf{x}=\left(x_{1}(\lambda), x_{2}(\lambda)\right)$ with $\lambda \in[0, \Lambda]$, then the scan-profile is defined by: $I(\lambda)=F(\mathbf{x}(\lambda))$.

\section{Phase v. Match scanprofiles and calculate the Correlations with DefectClasses}

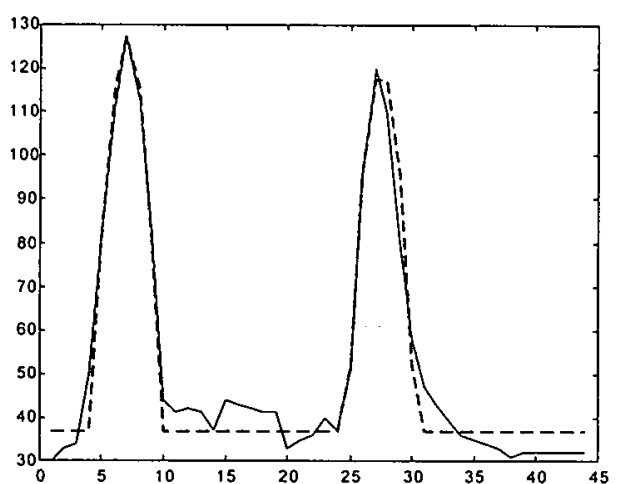

Fig 7.7 Optimal fit of a scan-profile of defectclass 'hairs' with $2^{\text {nd }}$ order B-spline wavelets.
Now relevant padprinting features are extracted from the scanprofile. The feature is for instance calculated from an optimal fit between a parameterised feature-type profile and the scanprofile, using least squares optimisation. This is described in appendix A.4. Figure 7.7 shows a scanprofile of a defect 'hair' (solid) and a matching 'hair' approximation (dashed). Their optimal fit defines a feature called 'multiple oscillations'. This feature is defined such that it falls in $[0,1]$, and thus can serve as a fuzzy membership function. 


\subsubsection{Combining Information of Multiple FCA Experiments}

Performing one single FCA experiment will in general not be sufficient to establish the exact nature of the printing defect, as the imperfections in a single scan-profile will still allow a number of possibilities. Therefore, a number of different FCA'experiments' will be necessary. Moreover, it is necessary to determine exactly what kind of FCA-experiment should be performed next. The control parameters are the location on the image and the curve-topology of the scan-curve that will be generated next.

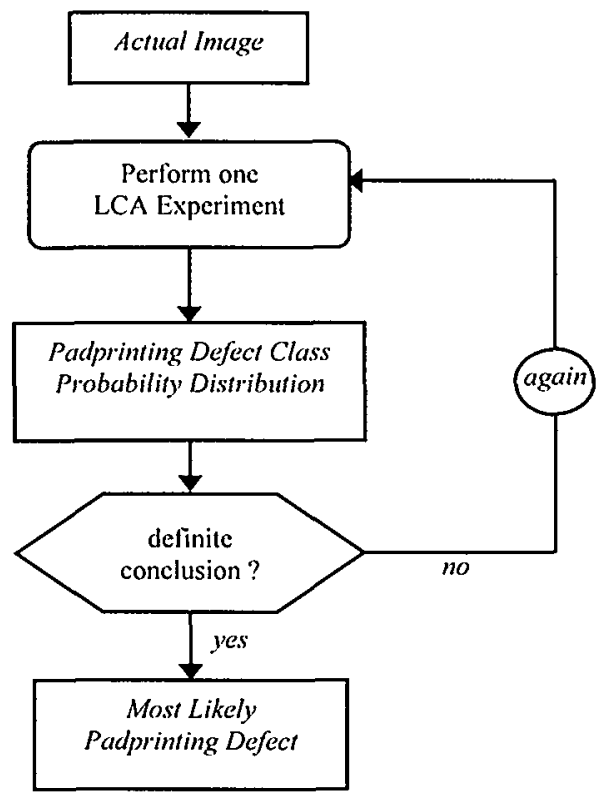

Fig. 7.8. Multi-FCA-diagnosis.

This choice is determined by the total set of experiments performed until now, and the resulting combined probability-distribution over the padprinting-defects. For this reason, a special diagnostic hypothesis evaluation module was designed and built. The results of all FCA-experiments must be combined in a suitable manner, and gradually a conclusion is 'built up'. Alternatively, the series may never converge towards a definite conclusion, and the cycle of FCA-experiments is terminated. The result of all the FCA-experiments is a combined probability-distribution over the padprintingdefects, that still has to be transformed (de-fuzified) to one single conclusion. This may include that two or more defects have occurred simultaneously. This algorithm is a primitive forerunner of the IASC-algorithm.

Figure 7.9 below shows a typical example of a multi-FCA-diagnosis. For sake of simplicity, we only distinguish three printing defects, called: 'hairs', 'double print', 'restriction', besides 'normal' (no defect). The upper part of the figure shows the absolute difference of a defect 'hairs'. This is covered with (in this case) 342 rectangles (the SearchBoxes automatically generated using FindAreas), with in each rectangle a number (varying from $1-5$ ) scan-curves (automatically generated with 
GenerateCurve). The lower part of the figure shows the evolution of the defect probability distribution as function of the number of performed FCA-experiments. The likelihood of the correct defect class 'hairs' slowly increases in 'time', and only after about 130 experiments 'wins' from double print. After about 220 steps the algorithm has - more or less - converged.
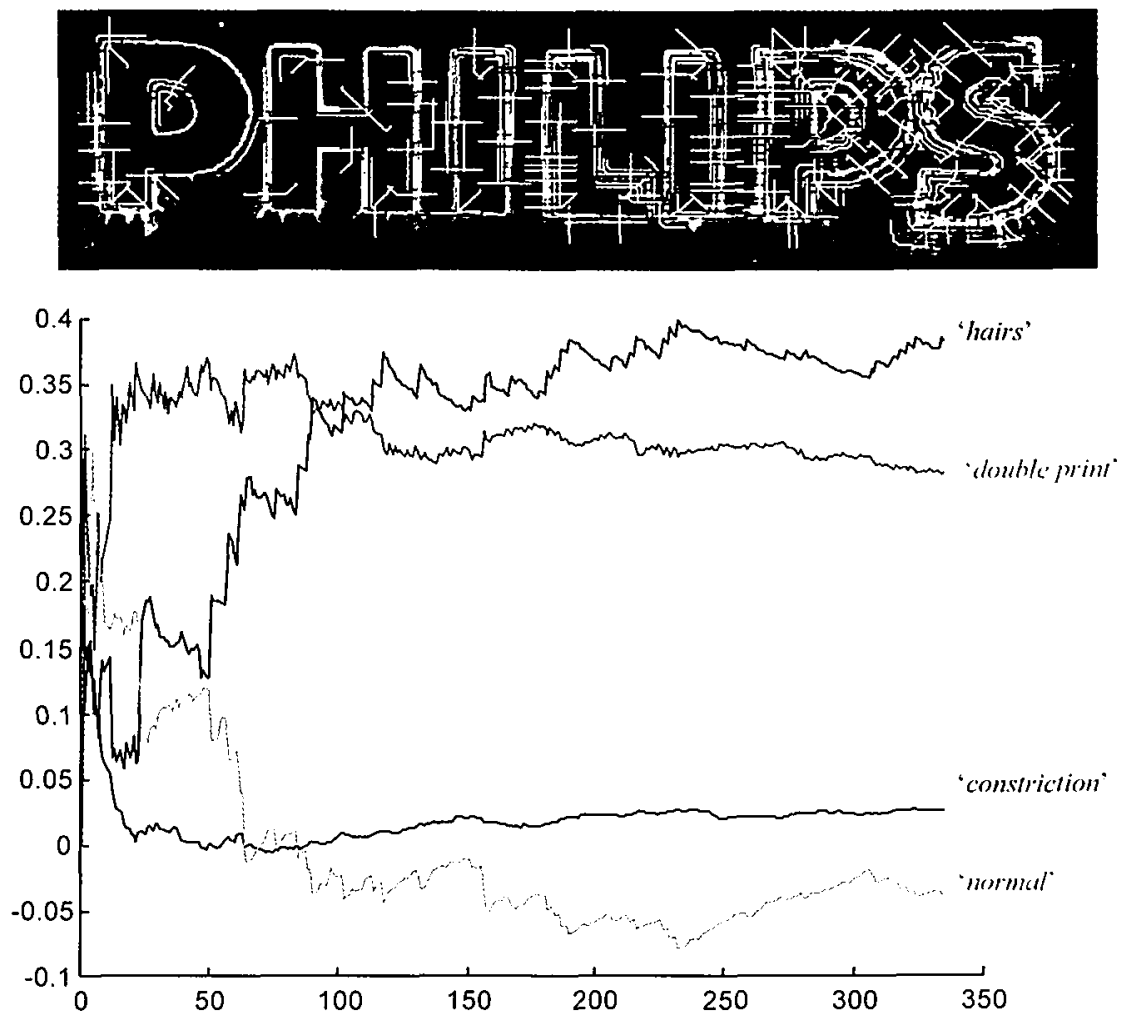

Fig. 7.9. Example of the multi-FCA evaluation of a defect 'hairs'. (above) CCD-images with generated scan-lines, (below) evolution of the probabilities for four defect-types during 350 iterations of FCA.

\subsubsection{Evaluation of the PT1-Prototype Computer Vision System}

The first prototype system was realised after 18 months in the project. Besides the newly designed electro-mechanical configuration and inks, it contained a large number of sensors, such as several mechanical pressures and temperatures, ink viscosity and temperature, and a built-in CCD-camera system. The multi-sensor integration and actuator control were performed using PC-control via a CAN-bus system. This allowed the efficient translation of high-level algorithmic output with basic electro-mechanical control signals to the prototype-system.

Initially, there was a shortage of suitable CCD-images of real padprinting defects. During the testing of PT1-system gradually real CCD-images became available. 
However, the quality of these images was insufficient. Therefore, it was not practically possible to test the camera system on-line. The evaluation of the prototype vision system was therefore restricted to a laboratory environment. In the laboratory evaluation upgraded real images of the CCD-camera system were used for classification. Ultimately, a collection of 556 laboratory examples was available. Of this set, 324 specimen were used for training the system (= computing the $S$-matrix), and the rest $(232$ specimen) for testing. For a representative evaluation of the approach, the number of defect classes was restricted to 7 , and the number of features to 6 . Therefore, $S$ contained $5 \times 4 \times 6 \times 7=840$ elements. From each CCD-image the FCA-approach generated a large number of scanprofiles. Thus, from the 324 specimen, the FindAreas and GenerateCurve algorithms automatically generated 11021 scan-profiles. In this way, a ratio of $11021 / 840 \approx 13$ scans/matrix-element was obtained, sufficient for a reliable computation of matrix $S$.

Under these assumptions the following results were obtained:

2 defect classes were identified with a success rate of: $>95 \%$

11 defect classes were identified with a success rate of: $75-95 \%$

2 defect classes were identified with a success rate of: $50-75 \%$

2 defect classes were identified with a success rate of: $40-50 \%$

0 defect classes were identified with a success rate of: $20-40 \%$

1 defect class was identified with a success rate of: $<20 \%$

Averaged over all experiments, the success rate of FCA was therefore: $79 \pm 22 \%$.

It is clear that in a laboratory setting the integrated FCA-Computer Vision System was able to correctly identify most padprinting defects. First of all, the huge sensitivity of the approach on the quality of the CCD-system output became clear. Since many padprinting-defects are very subtle, small effects on the CCD-image could potentially lead to completely different conclusions of the system. The evaluation showed that within the small margin of acceptable CCD-image quality - the performance of the system was reasonably good. At the same time, a number of major limitations of this FCA approach became visible.

1. The system is extremely sensitive to the image quality and vulnerable to noise.

2. The linear computation of the most likely defect class from the feature-vector with the fuzzy correlation matrix $S$ is a plain oversimplification. In later versions a computational neural network was trained to map the feature-vector into the defect probability-distribution. This approach is described in Section 4.4.3. Not surprisingly, this network outperformed the linear FCA computation. However, the disadvantage of the neural network was its parallel nature - it required all information to generate its output. Therefore, it was not suitable for on-line control, as all features must first be extracted from the image, some of which were very time-consuming, before it could identify the most-likely defect. The linear FCA technique was more practical in this respect, as it could use any number of features, using the associated part of the $S$-matrix to generate a full probability distribution over the defects. 
3. The computation time of the approach is long compared to the padprinting cycle. Generating the scan itself is relatively fast, but the subsequent processing and computation is slow.

4. A deficit is that in restricting the analysis to one-dimensional scans, unwanted ambiguity is introduced, as much of the original two-dimensional information is lost. Therefore, in practical cases it is impossible to distinguish certain defect classes. Such cases can only be resolved by using 2D-measurements. This induced the development of special tools for texture-analysis.

5. During the scanning analysis a number of errors accumulate, significantly decreasing the quality of the resulting probability distribution. The uncertainty originates from:

(i) errors in generating the relevant parts of the image, called the SearchBoxes;

(ii) errors in generating appropriate scan-curves in the SearchBox. In some cases it was not possible to generate suitable scan-curves, especially for complex printpatterns.

(iii) errors in comparing scanprofiles using a reasoning algorithm that takes into account geometrical and chemical-physical padprinting expertise.

(iv) FCA Diagnosis is rigid, it cannot adapt to newly obtained insights.

6. There is a necessity for a comprehensive set of adequate feature-types. The mapping of characteristic scanprofile-features to adequate padprinting-defect features can be considerably improved by using automatic clustering techniques.

7. An important omission of this approach concerns a reasoning system that is able to combine all knowledge available; i.e. information that can be extracted from the actual image, together with the observed values of the sensors, e.g. viscosity, load on the central axis, and so on.

These realisations eventually stimulated the development of the IASC-algorithm described in Chapter 5, and fractal and wavelet based tools for texture-analysis described in Chapter 3. 


\subsection{The IPP-PT2 Approach: Interactive Sensing and Classification}

\subsubsection{Final Prototype as Synthesis of Different Lines of Research}

The second prototype PT2 was based on the insights gained during the implementation and evaluation of PT1. The design of PT2 was largely built on the same concepts of PT1 regarding image processing, focussed image analysis, and feature extraction. The reasoning mechanism of PT2, however, was based on a entirely different philosophy. The model for PT2 was the interactive vision that humans employ in actively analysing a new image. While most computer vision systems at present are 'fixed input - fixed output', humans engage in a dynamical interaction between cognitive and perceptive tasks that in practice quickly converges to a correct identification of an image. A central place in human vision plays the 'focus of attention'. Therefore, the PT2 computer vision system was equipped with a process that could 'reason' about possible interpretations of the image, and actively generate tests to confirm or reject these beliefs. The objective of the reasoning process was to facilitate the identification of the defect in comparison with random or exhaustive analysis of the image. The reasoning process was designed with an algorithm that - to a certain extent - emulates the 'focus of attention' in human vision. This idea inspired the development of an interactive sensing and reasoning system that could identify the mostlikely printing defect, or alternatively determine the most informative featureextraction to be performed next. This interactive analysis and sensing method for classification is the IASC-algorithm, extensively described in Chapter 5 . The basis for IASC was the representation of padprinting defects as sets in feature space and a current measurement as one single point in feature-space. Hierarchical clustering of these defect-collections using multivariate normal distributions led to a useful partitioning of feature-space for pre-classification, but also to a suitable representation of defects in terms of

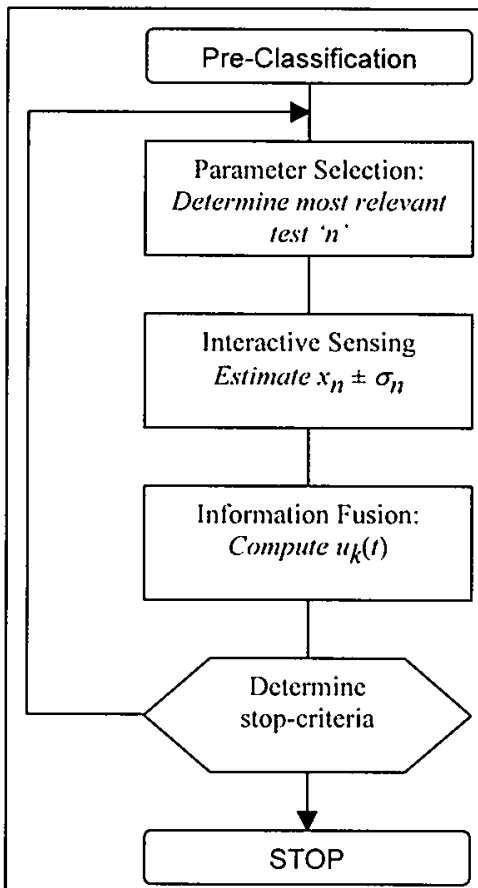

Fig. 7.10. The general strategy of the interactive reasoning and sensing process consists of a loop of experiment selection, interactive sensing, and information fusion.

its features. In the resulting defect distribution in feature-space, some defects were not linearly separable and most defect-classes exhibited a large degree of overlap.

Moreover, the components of PT2 were greatly extended and enhanced compared to PT1. In addition to the scan-analysis now powerful techniques for texture analysis were developed. These were based mainly on fractal wavelet-analysis, as described in Chapter 3. Also, the full set of 1D- and 2D-feature-extractions described in Section 7.3 were implemented. The implementation of new image features, especially those characteristic for certain defects, led to a large number of feature-parameters. Furthermore, much work had been performed on clustering the defects in feature space. This laid the foundation for the IASC approach, but 
also led to efficient pre-classification techniques. Moreover, the entire system was made more robust towards noise and mis-positioning of the printed element.

The IASC algorithm implemented in the PT2-vision system consisted of the following steps.

1. Pre-classification of the image as belonging to a super-cluster of defect-classes.

2. Generation of a set of hypotheses regarding the possible defect class as defectmembership functions.

3. Identification of significant areas in the image based upon the present hypotheses, and arranging weights to these areas.

4. Identification of feature-measurements that can optimally confirm or reject the present hypothesis.

5. Measurement of the selected feature on the significant areas until its weighted result stabilises or fails.

6. Integration of all information present to update the set of hypotheses.

7. Determination whether a final conclusion can be derived, whether more measurements are necessary, or whether the reasoning process has failed.

In the following sections we describe the main innovative features of the PT2.

\subsubsection{Pre-Classification Techniques as Kick-Start for the IASC-Algorithm}

The IASC-algorithm is an efficient method for interactively constructing a sound hypothesis on the defect of a print. This process can start from scratch with the 'null-hypothesis' - all defects are equally likely, or employ some a priori default values. During the clustering of defects in feature-space, however, it was observed that certain features grouped the defects in small aggregations. Some of these features were relatively easy to obtain. Application of this observation leads to a pre-classification of the image, and assigns an initial value to the defect-memberships function: $\mathbf{u}=\mathbf{u}_{0}$. This effectively shortens the subsequent reasoning process considerably. For that reason, the reasoning process starts with a pre-classification of the actual image, as depicted in Figure 7.10. For this purpose we can choose between two classification techniques that were developed in the research, and were described earlier in this thesis. A global flow diagram depicting the mutual relations between the entities is shown in Figure 7.11.

The first pre-classification technique relies on the clustering of the block-match of the IDCID-transform. This is called: ' $\mathrm{L}_{1} \mathrm{~L}_{2}$-clustering', and is extensively described in Sections 2.6.2.2, and 4.7.2. In this approach a CCD-image is translated, after pre-processing, into one point ' $L$ ' in the $L_{1} L_{2}$-plane. This point is used to compute the most likely defect class. The clustering is based on a large set of empirical observations. Thus, a partitioning of the $\mathrm{L}_{1} \mathrm{~L}_{2}-$ plane in Voronoi-cells was obtained. The obtained clustering can be used for either a direct assignment of the defect by its resident Voronoi- cell. Alternatively, an initial probability distribution can be computed from the closest prototype and its covariance-matrix.

The second pre-classification technique relies on the distribution of the fault on the image over the four 'print-contexts'. This is called the $\xi^{4}$-clustering, and is described in Section 2.6.2.1, and 4.7.1. Similar to the $L_{1} L_{2}$-pre-classification, the distribution of the defect over the PrintContexts was used to obtain a position in $\mathrm{IR}^{4}$-space, and consequently to compute the most likely defect class. 
In the PT2 we ultimately chose for the $L_{1} L_{2}$-method, because of its higher efficiency and ease of computation. An example of a pre-classification of a defect in class 1 'hairs' using the $\mathrm{L}_{1} \mathrm{~L}_{2}$-method is depicted in Figure 7.12.

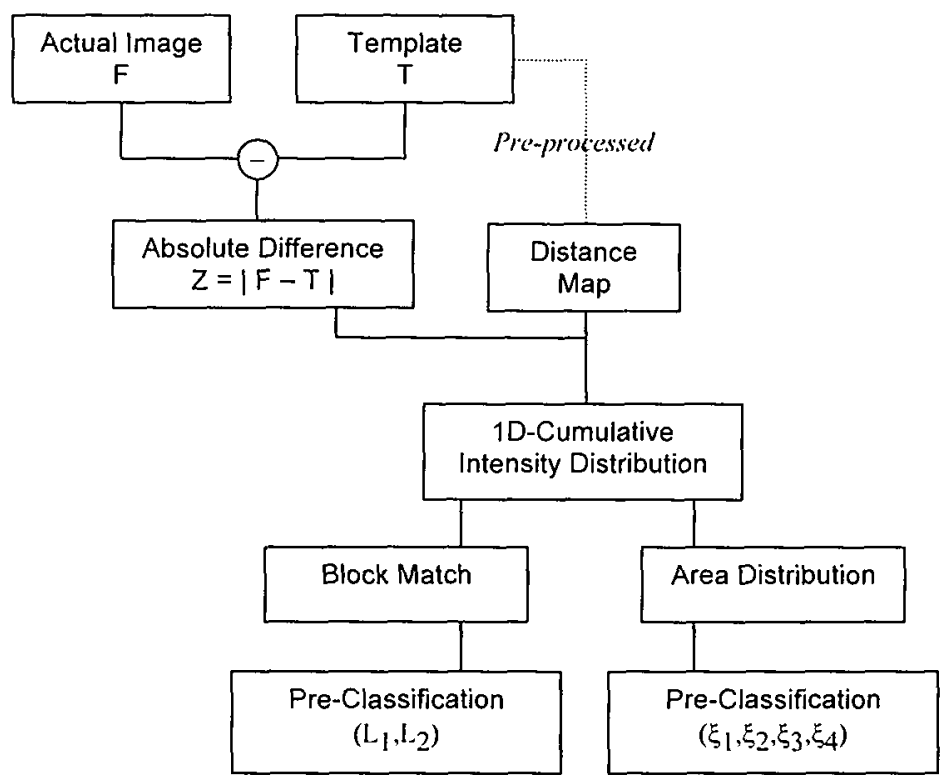

Fig. 7.11. Pre-classification of the defect is based on comparing the defect with the template, and using the distance-map to transform it to a ID-cumulative intensity distribution. Two techniques for pre-classification are described in the text. 

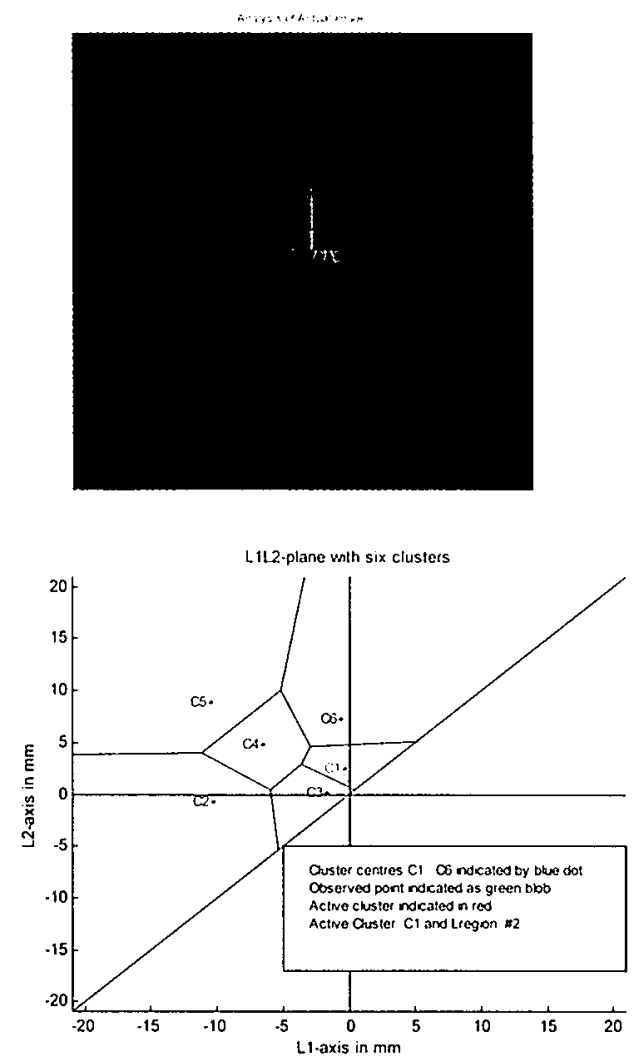
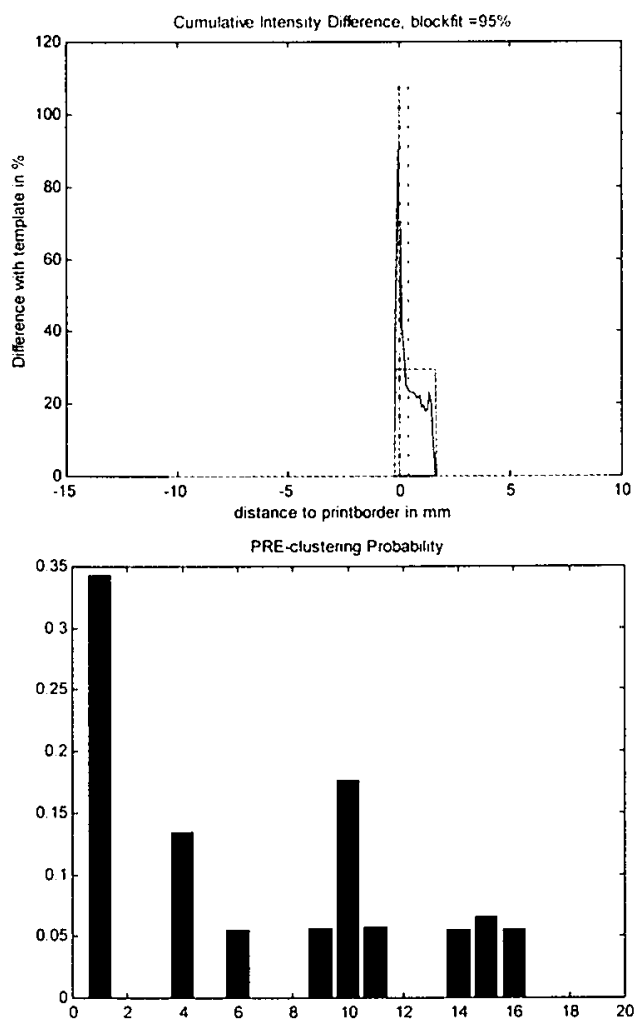

Fig 7.12. Pre-Classification of a defect in the class 'hairs'.

Clockwise: $1 . Z$, the absolute difference between an actual image and the template. 2. The cumulative ID-intensity distributions of $Z$, with the optimal blockmatch $\left(L_{1}, L_{2}\right)$. 3. The position of the $L_{1} L_{2}$ in the $L_{1} L_{2}$-plane in relation to the position of the main $L$-clusters $C 1$..C6. This provides the initial classification of the defect. 4. Based on the position in the L-plane using multivariate covariant approach to $\left(\mathrm{E}, \mathrm{L}_{1}, \mathrm{~L}_{2}\right)$ the initial value of the probability distribution is computed. This gives as starting hypothesis the most likely hypothesis [1] 'hairs' with $p=0.34$, and second best hypothesis [10] 'smearing' with $p=0.18$. The quality of this hypothesis is therefore $Q=47 \%$. 


\subsubsection{Interactive Analysis of CCD-Images Using the IASC-Algorithm}

Once a specific feature-parameter is selected as supposedly providing the most conclusive evidence, the necessary information must be extracted from the image. This is performed in an interactive analysis. Here, 'interactive' refers to the interaction between the reasoning process and the examination of the image. Firstly, the system is able to select and 'zoom in' on the relevant parts of the image, and secondly, it controls both the convergence and exploration of the sensing process. The resulting measurement of the feature-parameter is a trade-off between meaningful exploration of the image versus preventing premature convergence of the reasoning process. The control of the analysis balances between these two extremes in order to come to the best possible solution. In order to measure the selected feature-parameter the system determines the most significant areas of the actual image. This is obtained by investigating $\mathrm{Z}$, the absolute value of the difference between the actual image and the template. Depending on the present hypothesis, the Intelligent Binary Search-algorithm (IBS), described in Section 7.4.2, is employed to find the most relevant areas of $Z$. The IBS results in a number of rectangular areas on the image called 'SearchBoxes'. The IBSalgorithm determines the parts of the image for the feature-extraction. After this evaluation, the SearchBox is deleted from the SearchMap. The efficiency of the IBS algorithm constraints the efficiency of the subsequent analysis.

\subsubsection{Sequential versus Concurrent Analysis}

There are two modes for the interactive analysis. The difference concerns the way in which the feature-parameters are established over the ensemble of potential SearchBoxes $\left\{B_{i}\right\}$.

In the first method, termed 'concurrent', the values of all feature-parameters are measured simulianeously per SearchBox $B_{i}$. Then the next SearchBox $B_{i+1}$ is analysed in this way. The result is a set of complete feature-vectors $\left\{\mathbf{x}_{i}\right\}$, where $x_{i n}$ represents the value of featureparameter ' $n$ ' for SearchBox ' $i$ '. The resulting measurement of the entire image can then be represented as the weighted average over the ensemble search-boxes: $\langle\mathbf{x}\rangle=\Sigma_{j} w_{j} \mathbf{x}_{j}$. Here $w_{j}$ denotes the relative weight of SearchBox $B_{j}$, based on its fraction of the entire 'energy' $E$ of the image: $E=\int Z(\mathbf{x}) \cdot \mathrm{d}_{2} \mathbf{x}$. So the weight can be expressed as: $w_{j}=\iint_{B[j]} Z(\mathbf{x}) \cdot \mathrm{d}_{2} \mathbf{x} / E$.

In the second method, termed 'sequential', the values of the feature-parameters are evaluated sequentially for each parameter. Once a feature-parameter has been selected for evaluation, it is evaluated over the ensemble of SearchBoxes, without any consideration to the other parameters. The process of evaluation feature-parameter ' $n$ ' is driven by convergence of the weighted average: $\left\langle x_{n}\right\rangle=\Sigma_{j} w_{j} x_{n j}$, and sufficient exploration of the image: $\left.\Sigma_{j} w_{j}\right\rangle \theta$, where the summation is over the set of explored SearchBoxes. ' $\theta$ ' represents the minimal portion of the total energy that must have been explored. For the next feature-parameter this whole procedure is repeated over the same set of SearchBoxes.

It is clear that the first method is more time-consuming than the second. In the first method, convergence is determined by the slowest converging measurement. Moreover, in the first method all feature-parameters are measured. Not all parameters, however, are equally interesting, and certainly not all parameters are necessary to come to a reliable conclusion of the reasoning process. Furthermore, measurement of all feature-parameters causes tremendous computational effort. In the second method the measurement concentrates on one, but relevant parameter. This consequently generates a disproportionate amount of evidence in this stage of the reasoning process - compared with a random selected parameter. A disadvantage, 
however, is that now for each feature-parameter the collection of SearchBoxes must be visited anew.

For all these reasons the most efficient and only interactive method is the sequential analysis. Therefore, sequential analysis was chosen as the mechanism for the interactive analysis.

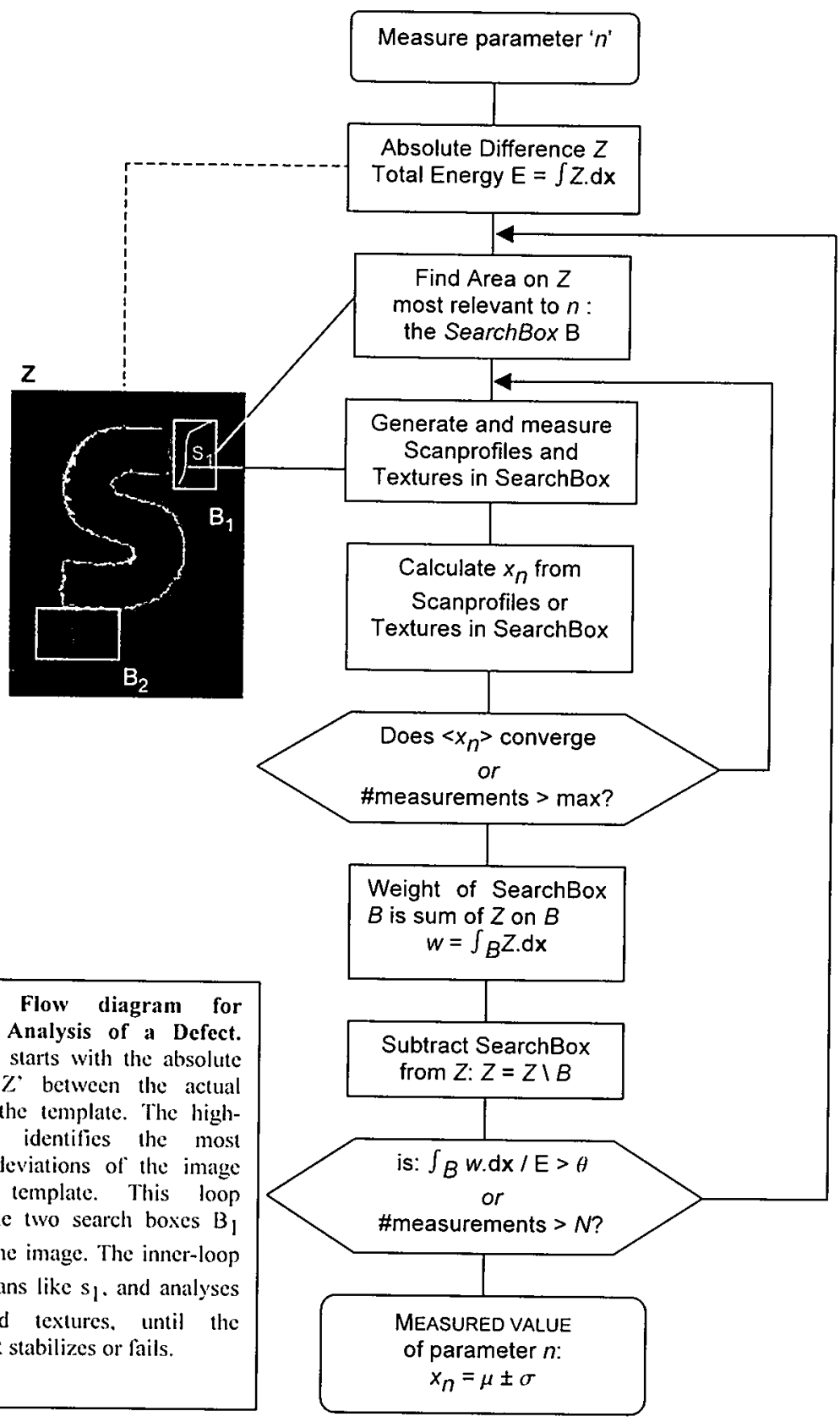

Fig. 7.13 Flow diagram for Interactive Analysis of a Defect. The process starts with the absolute difference ' $Z$ ' between the actual image and the template. The highlevel loop identifies the most significant deviations of the image from the template. This loop generated the two search boxes $B$ and $\mathrm{B}_{2}$ on the image. The inner-loop generates scans like $s_{1}$. and analyses profiles and textures, until the measurement stabilizes or fails. 


\subsubsection{Management of Feature Extraction}

A number of control variables can be employed to manage the feature-extraction in a methodological and statistical manner.

- Comergence of the feature-parameter: within the set of SearchBoxes the featureparameter is measured. Feature-parameters can be classified as 1D-profiles, or 2Dtextures, or statistical correlations, as described in Section 7.3. Generation of measurements is performed both within SearchBoxes (intra SearchBox) and among SearchBoxes (inter SearchBox). The $k^{\text {th }}$ measurement $x_{n k}$ is weighted with the energy $w_{k}$ of the SearchBox $B_{k}$, and so the measurement can be represented as a set $\left\{\left(x_{n k}, w_{k}\right) \mid k=\right.$ $1, \ldots, N\}$, with $N$ the number of measurements. The number of measurements $N$ increases until the weighted average value of the measurement $\mu$ converges or the measurement fails because a maximal number of measurements is exceeded. The result of the measurement is given by its average $\mu=\langle x\rangle$ and its variance $\sigma^{2}=\left\langle x^{2}\right\rangle-\langle x\rangle 2$, both weighted with the SearchBox-energies $w_{k} ;<Y>=\Sigma_{k} Y_{k} w_{k} / \Sigma_{k} w_{k}$.

- Adequate exploration: in order to promote significant exploration of the image another criterion is introduced; the cumulative energy of the explored SearchBoxes must be at least a given proportion $\vartheta$ of the entire energy $E$ of the image, so: $\Sigma_{k} w_{k} / E>\vartheta$, with: $0<\vartheta<1$, and $E=\int Z(\mathbf{x}) \mathrm{d}_{2} \mathbf{x}$. This guideline acts as a termination-criterion and must both prevent premature convergence and stimulate extensive exploration of the image.

- Relative relevance: the sites on the image relevant for the selected feature-parameter must be identified. From feature clustering it is known that certain defects can only occur in specific parts on the map, the 'PrintContexts' defined in Chapter 2. Likewise, for featureparameters some PrintContexts may be more appropriate than others. This relation is defined by ' $" n k$ ', called the relevance of feature-parameter ' $n$ ' for defect class ' $k$ '. This entity is discussed in Section 5.2.1. From the clustering of the defects over the four specific PrintContexts ' $i$ ' as defined in Chapter 4 , we can compute $C_{i k}$ denoting the correlation between PrintContext ' $i$ ' and defect class ' $k$ '. The relevance $G_{i n}$ of a specific PrintContext ' $i$ ' for a given feature-parameter ' $n$ ' then follows from: $\Sigma_{k} C_{i k} r{ }_{n k}$.

The general flow of the process is depicted in Figure 7.13. Summarising, the interactive analysis can be represented as an algorithm with input: the selected feature-parameter with index $n$, the absolute difference $Z$, the maximal allowed number of measurements $N_{\max }$, and the minimal portion $\theta$ of the total energy that must be explored. The output then is the weighted average and standard deviation of the selected parameter:

$\{\mu, \sigma\}=$ InteractiveAnalysis $\left(n, Z, N_{m a x}, \theta\right)$.

\subsubsection{Data Mining and Statistical Process Control}

A second field of activities was aimed at the realisation of an adaptive system for estimating the optimal values for the process parameters from the process description and material constants. Results from the Knowledge Acquisition of the expertise of padprinting specialists turned out to be unsatisfactory. For this reason, a new approach was proposed. A design was made for a database management system - called IPP-DBMS - for storing all relevant printing process and associated quality parameters. Initially, this database system would be empty, but during the testing it would quickly grow with all encountered testing conditions and results. Additionally, a set of data mining tools were developed for - off-line - analysing 
this database. The results could be used for Statistical Process Control (SPC). In this way an adaptive control system would be realised. The system was developed in association with ESD, sub-contracted by TampoPrint. ESD provided the coupling with the sensors and the implementation of the database structure. UM developed an Adaptive Statistical Process Control System, equipped with a reasoning system that would indicate the most probable reason for the printing defect. The UM developed a tool that applied Multivariate Covariant Analyses to the actual sensor data, and used the deviation of the defect class prototypes relative to the variances as an indication for sufficient quality. In the case of large deviations the system could reason: [1] whether to collect more information i.e. to inspect for some more prints, or [2] take some action, and [3] indicate the most likely physical/chemical/mechanical cause of the defect based on the actual sensor information, and [4] use the IPP-DBMS to estimate the strength and direction of the selected control action. As interactive reasoning tool an adaptation of the IASC-algorithm was chosen.

\subsubsection{Evaluation of the PT2-Prototype Vision Control System}

The second prototype was near completion at the end of the project [Westra,2000c]. The huge technological problems involved in connecting the sensors and actuators caused for an insurmountable obstacle. Consequently, the sensors could transmit their signals to the appropriate software-modules, but the generated output could not be sent back to the associated actuators. For that reason the evaluation of the prototype computer vision and SPC system was once again performed in a laboratory environment. As the IPP-databases remained empty, the adaptive SPC could only be tested and evaluated on artificial example. There it performed satisfactory. The off-line testing of the IASC algorithm is described comprehensively in Chapter 6.

In the mean time, the CCD-camera system had been considerably improved, and now large quantities of excellent CCD-images of most defect classes were available. This greatly favoured the efficient development of the approach, and provided a solid basis for the realistic evaluation of our approach. In order to use those CCD-images directly for the evaluation, we first define the concept of image quality. Image quality is merely a special case of sensor data quality, as in expression [6.2] in Section 6.2, defined as the inverse of sensor noise - here image noise ' $f$, so:

$$
Q_{\text {image }}=\frac{1}{1+f^{2}}
$$

with image noise:

$$
f=\frac{2 S_{l}(\mathbf{x})}{\Delta_{l}}
$$

and Mahalanobis separation:

$$
\Delta_{l}=\min _{n} \min _{k \neq l} s_{k l}^{n}
$$

Here, $\mathbf{x}$ is the feature vector of the actual image, ' $l$ ' is the correct padprinting defect class, and $s$ and $S$ are Mahalanobis-distances. Figure 7.14 shows the observed distribution of the image quality over a large sample of real CCD-images. This distribution shows that more than $75 \%$ of the images have a quality 0.8 or higher. 


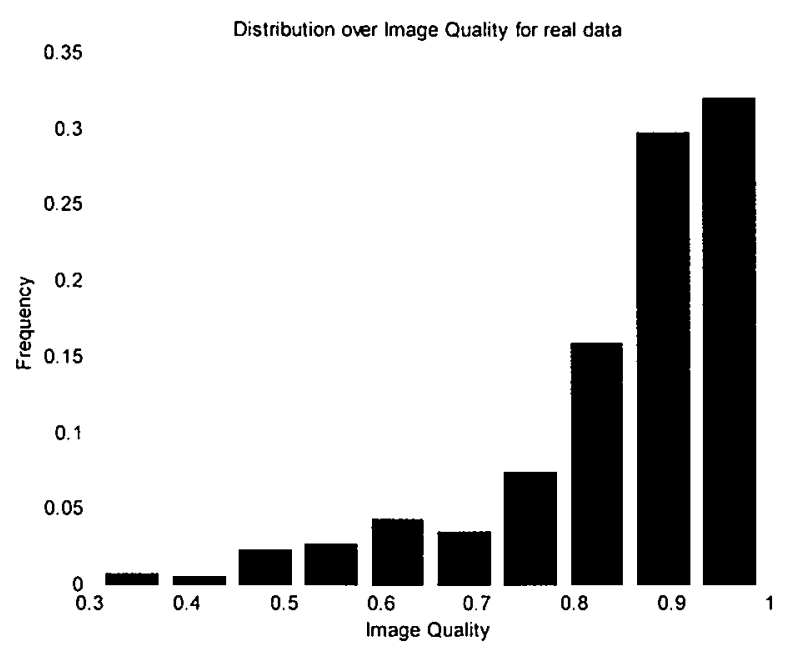

Fig. 7.14 Observed distribution of Image Quality over a set of 1066 CCD-images, with quality range $[0.3,1]$ divided in ten equal parts.

The table below shows the success rate of the integrated approach in IntelPadPrint for different types of image quality. Here, we distinguished three gradations of defect recognition.

1. At the basic level, a defect has to be detected at all. This introduces the notion of true identification, false negatives, and false positives. False negatives occur especially in case of minute instances of subtle defect types, such as 'hairs', provided the image quality is high enough, $Q \geq 0.8$. False positives occur in case of low image quality, especially when $Q<0.6$. The high amounts of noise are then simply interpreted as faults, and trigger the identification. Within these margins; i.e. high image quality and substantial faults, the defect is noticed correctly with a high success rate of $99 \%$.

2. When a defect is detected, first the integrated approach attempts to pre-classify the CCDimage with the pre-classification method described in Section 7.5.2. This means that the image is assigned to one of six clusters that contain visually similar defects. In case of sufficient image quality, this pre-classification has a high success rate of about $97 \%$. For lower image qualities, it is observed that the uniform distributed noise favours an interpretation for one specific cluster type with faults uniformly distributed over the image. Hence, this specific defect cluster - incorrectly - increases in probability, while the others quickly decrease.

3. At the highest level, the IASC-algorithm performs an interactive scanning and classification of the CCD-image. As described in Chapter 6, the success rate depends on the noise - so here the image quality, and the average class-separation. For our featurespace the average cluster-separation was found to be: $\langle d\rangle \approx 0.2779$. This means that for high image quality, $Q>0.8$, the average success rate is $95 \%$.

These results show that for high quality CCD images the final PT2-computer vision system achieved a good performance of identifying and subsequently correctly classifying a padprinting defect. With decreasing image quality these results quickly deteriorate. In the idealised case of perfect image quality, $Q=1$, and strict prototype printing defects, the first 
two modes of identification even performed with an accuracy of almost $100 \%$. The performance of the third mode, the IASC-classification, was only restricted by the power of the feature-extraction techniques. This is because for $Q=1$ the feature-vector $\mathbf{x}$ coincides with the class-prototype $\mathbf{m}_{k}$. As described in Section 5.6.6.1, the IASC-algorithm will always transform a measurement $\mathbf{x}=\mathbf{m}_{k}$ to the class with index ' $k$ '. It is, however, the quality of the feature-extraction techniques that determine whether this observation $\mathbf{x}=\mathbf{m}_{k}$ corresponds with the physical reality.

\begin{tabular}{|c|c|c|c|c|}
\hline Image Quality & Fraction of images & Defect Identification & Pre-Classification & IASC-Classification \\
\hline 1.0 & 0 & 1 & I & -1 \\
\hline$\langle 0.8,1.0\rangle$ & 0.77 & 0.99 & 0.97 & 0.95 \\
\hline$<0.6,0.8]$ & 0.16 & 0.87 & 0.82 & 0.65 \\
\hline$<0.4,0.6]$ & 0.06 & 0.12 & 0.35 & 0.23 \\
\hline$[0.0,0.4]$ & 0.01 & 0 & 0.16 & 0.06 \\
\hline weighted average & - & 0.91 & 0.90 & 0.85 \\
\hline
\end{tabular}

Table 7.5. Success rates of integrated PT2 approach for different image qualities

The table shows that for high image quality, about $77 \%$ of the samples, the IASC-success rate is $95 \%$. Averaged, with the distribution of image qualities as weights, we find that the IASCclassification classifies $85 \%$ of the samples correctly. This evaluation once again underlines the necessity to increase the image quality even further.

An example of a IntelPadPrint-IASC session is presented in appendix D. 


\subsection{General Conclusions regarding the Integrated Approach for Interactive Vision and Control in the 'IntelPadPrint' Project}

In this chapter we discussed the application of the formalisms presented earlier in this thesis for image analysis, feature extraction, clustering and interactive classification, to the area of automatic visual quality control of padprinting processes. This application was performed in the framework of an extensive and collaborated applied research project, called 'IntelPadPrint'. During this project, two prototype padprinting robots, equipped with a large number of sensors, including an interactive CCD-camera system, were realised. The testing and evaluation of these prototypes resulted in a large quantity of data, including suitable CCD-images, that could be used as test-data for our means. From the evaluation of our integrated vision control approach applied on these test-data, the following conclusions can be drawn.

1. There is a high degree of sensitivity of the success rate of our approach on the quality of the input data, especially the image quality.

2. The observed image qualities varied between 0.3 and 1.0 , and was as such hardly sufficient for our means.

Therefore the emphasis for further implementation of the approach should focus on further improvement and stabilisation of the image quality, and simultaneously improving the robustness of the approach towards noise and insufficient image qualities. Given a suitably high data and image quality, additionally the following concluding remarks can be made.

3. Processing with the CID-transform is a strong, though time-intensive, transformation of the (template subtracted) image, that provides strong clues to the defect super-cluster. This method - in combination with the iterated FMV-clustering technique - is very suitable for a reliable pre-classification of the defect.

4. The focussed image analysis with the FindAreas-algorithm provides a collection of SearchBoxes of the most relevant faulty areas of a (template-subtracted) image.

5. Feature extraction with the various image analysis tests described in Section 7.3, focussing on peculiar traits of defect-groups, are efficient and fast. The resulting features display reasonable correlations with the defect classes. These correlations can be strengthened by increasing the variety in PrintContext and position of the samples, and the sample-size.

6. In the space constructed from this set of feature-parameters, the padprinting defect classes are but weakly separated. Though the dimension of the feature-space is high, the average normalised cluster separation is only 0.2779 .

7. Efficient clustering provides a necessary starting point of the classification. Iterated FMVclustering provides good clustering results of weakly separated many-parameter feature spaces, as observed for the padprinting-defect classes in our feature-parameter space.

8. The focussed 1D-scanning and Fuzzy Correlation Approach, as implemented in the first prototype, provides a consistent and efficient framework for defect classification. The approach is, however, unable to distinguish defects that are primarily defined by shape or texture. The average success rate on a set of high-quality CCD-images was $79 \%$. 
9. The Fractal Wavelet Analysis of selected SearchBoxes exhibits a strong correlation with the padprinting defect class.

10. Computational Neural Networks outperform the linear Fuzzy Correlation Approach in mapping feature vectors into most likely defect. However, since they require the complete set of input-parameters, they are not so useful for on-line classification. In this sense a linear approach is more useful, for it can provide an estimate based on partial inputvectors.

11. Because of the curse-of-dimensionality (Section 2.4.2) Interactive Classification in highdimensional feature-spaces is only possible if large numbers of empirical observations are available. These observations can be labelled by human experts, or independently by automatic clustering-techniques like Iterated FMV (Section 4.6.2).

12. The interactive sensing and analysis using the IASC-algorithm provides an efficient approach for on-line classification of padprinting defects captured in CCD-images. It outperforms the Fuzzy Correlation Approach in success rate, and is more reliable in its conclusion. For the set of available CCD-images of all image qualities, the average success rate was about $85 \%$. For a set of high-quality images $(Q>0.8)$, the average success rate was $95 \%$.

The IntelPadPrint project was performed with great determination and devotion by all participants. Notwithstanding the problems experienced in the integration of the second prototype, the project was considered successful by both the partners and the Commission of the European Community [Legierse,2000]. The most essential aims of the project were achieved, namely, to demonstrate the basis of the technology for completely new padprinting processes. The main achievements and results of the entire project, as officially listed, included:

- Vision equipment for in-process analysis of the print quality by improving hardware, software, and illumination.

- Algorithms for analysing the print quality and analysing the print failures.

- Algorithms for feedback of the print failures for process optimisation.

- High-speed padprinting equipment with sensors, actuators, and vision analysis and control was demonstrated. 
9. The Fractal Wavelet Analysis of selected SearchBoxes exhibits a strong correlation with the padprinting defect class.

10. Computational Neural Networks outperform the linear Fuzzy Correlation Approach in mapping feature vectors into most likely defect. However, since they require the complete set of input-parameters, they are not so useful for on-line classification. In this sense a linear approach is more useful, for it can provide an estimate based on partial inputvectors.

11. Because of the curse-of-dimensionality (Section 2.4.2) Interactive Classification in highdimensional feature-spaces is only possible if large numbers of empirical observations are available. These observations can be labelled by human experts, or independently by automatic clustering-techniques like Iterated FMV (Section 4.6.2).

12. The interactive sensing and analysis using the IASC-algorithm provides an efficient approach for on-line classification of padprinting defects captured in CCD-images. It outperforms the Fuzzy Correlation Approach in success rate, and is more reliable in its conclusion. For the set of available CCD-images of all image qualities, the average success rate was about $85 \%$. For a set of high-quality images $(Q>0.8)$, the average success rate was $95 \%$.

The IntelPadPrint project was performed with great determination and devotion by all participants. Notwithstanding the problems experienced in the integration of the second prototype, the project was considered successful by both the partners and the Commission of the European Community [Legierse,2000]. The most essential aims of the project were achieved, namely, to demonstrate the basis of the technology for completely new padprinting processes. The main achievements and results of the entire project, as officially listed, included:

- Vision equipment for in-process analysis of the print quality by improving hardware, software, and illumination.

- Algorithms for analysing the print quality and analysing the print failures.

- Algorithms for feedback of the print failures for process optimisation.

- High-speed padprinting equipment with sensors, actuators, and vision analysis and control was demonstrated. 


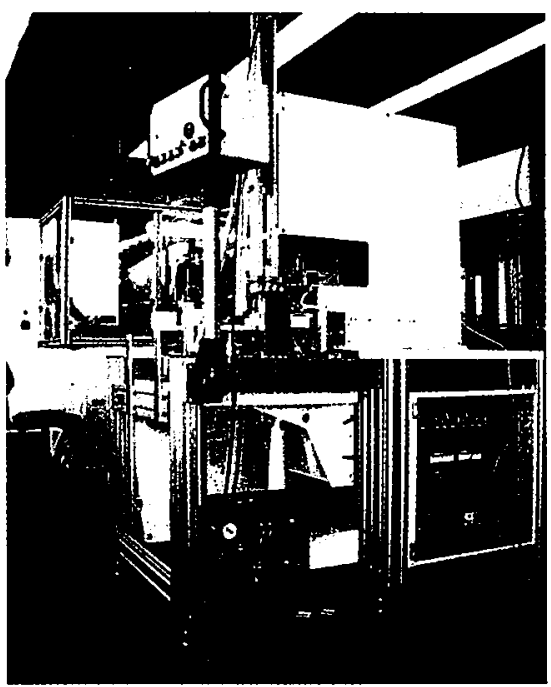

Fig. 7.15 Prototype PT1, global view ${ }^{2}$

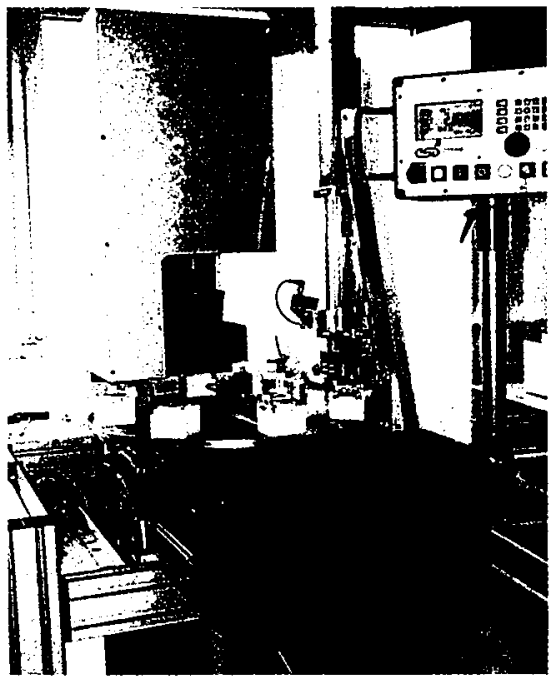

Fig. 7.17 Prototype PT1, printing pad and SBS-monitor

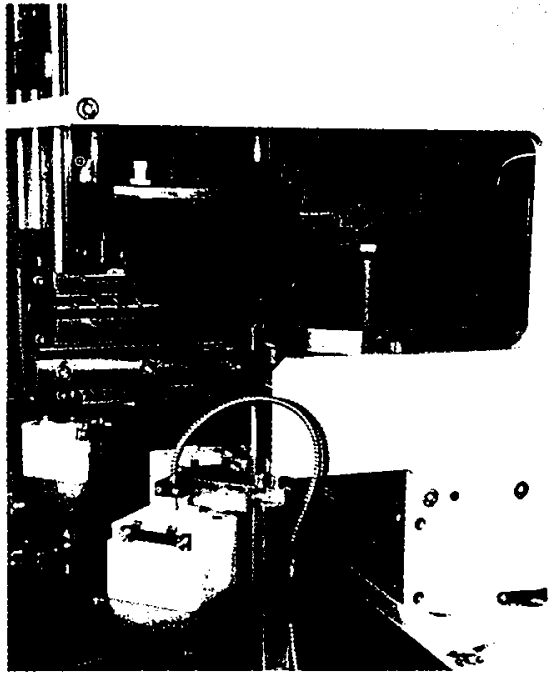

Fig. 7.16 Prototype PT1, the printing pad

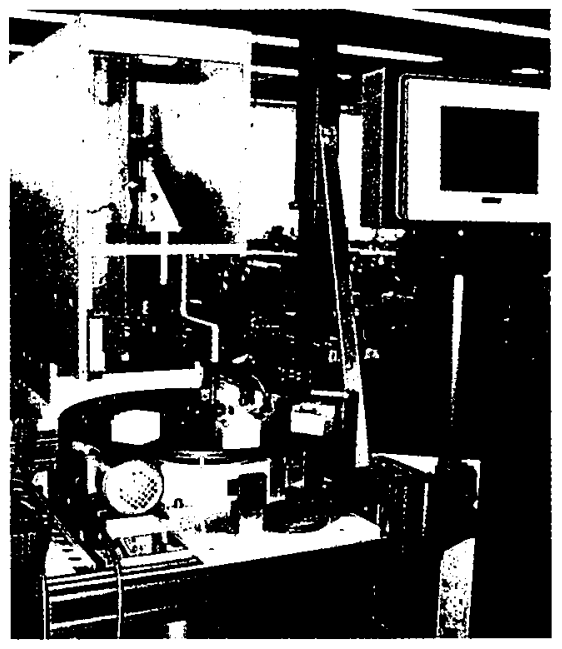

Fig. 7.18 Prototype PT2, during construction

\footnotetext{
? All pictures courtesy Andreas Reiss, TampoPrint GmbH.
} 


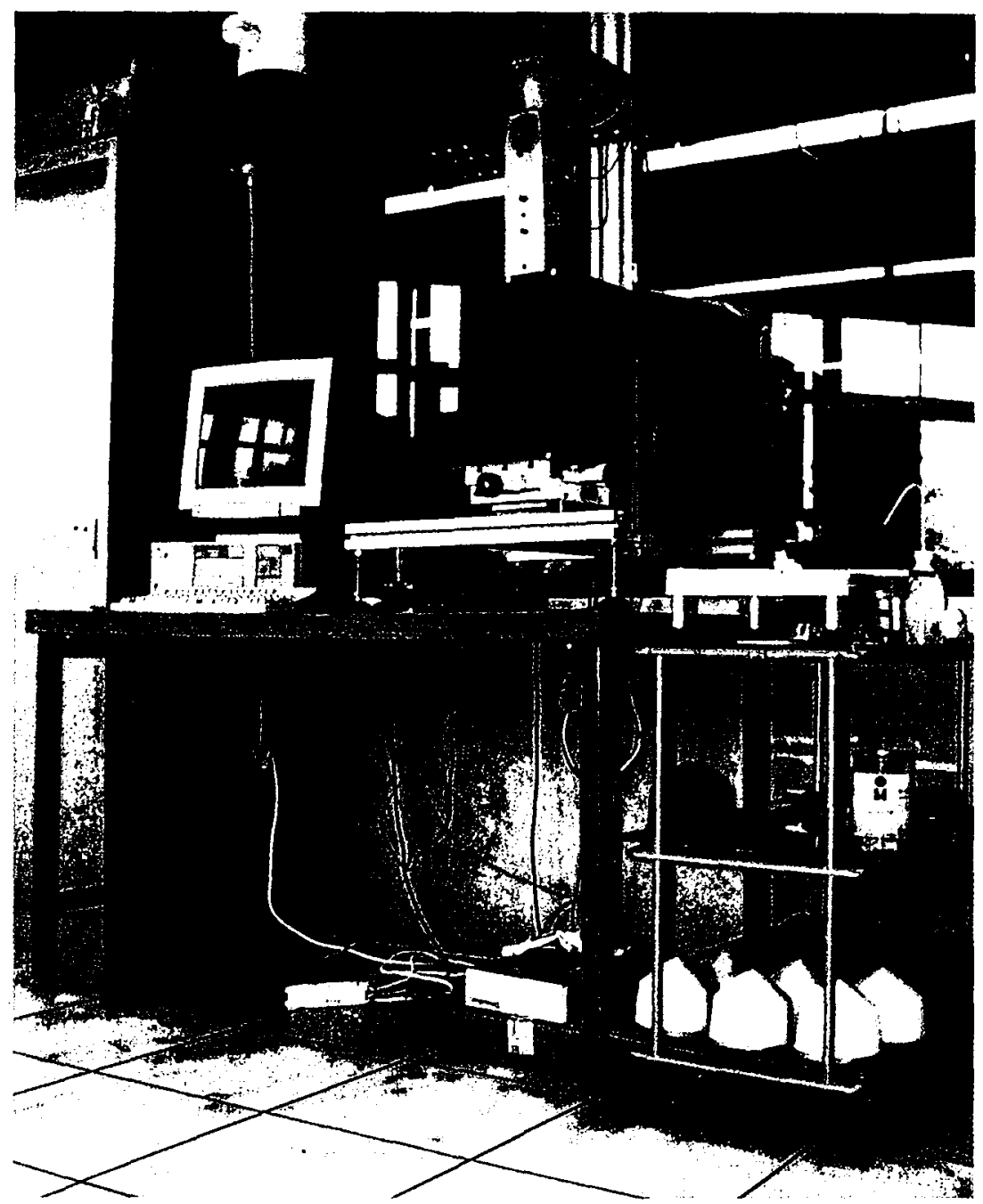

Fig. 7.19 Off-line testing of CCD-camera system, built by N. Freear and J.M. Shippen from Birmingham University. Camera not visible due to black box to shield false lighting. 


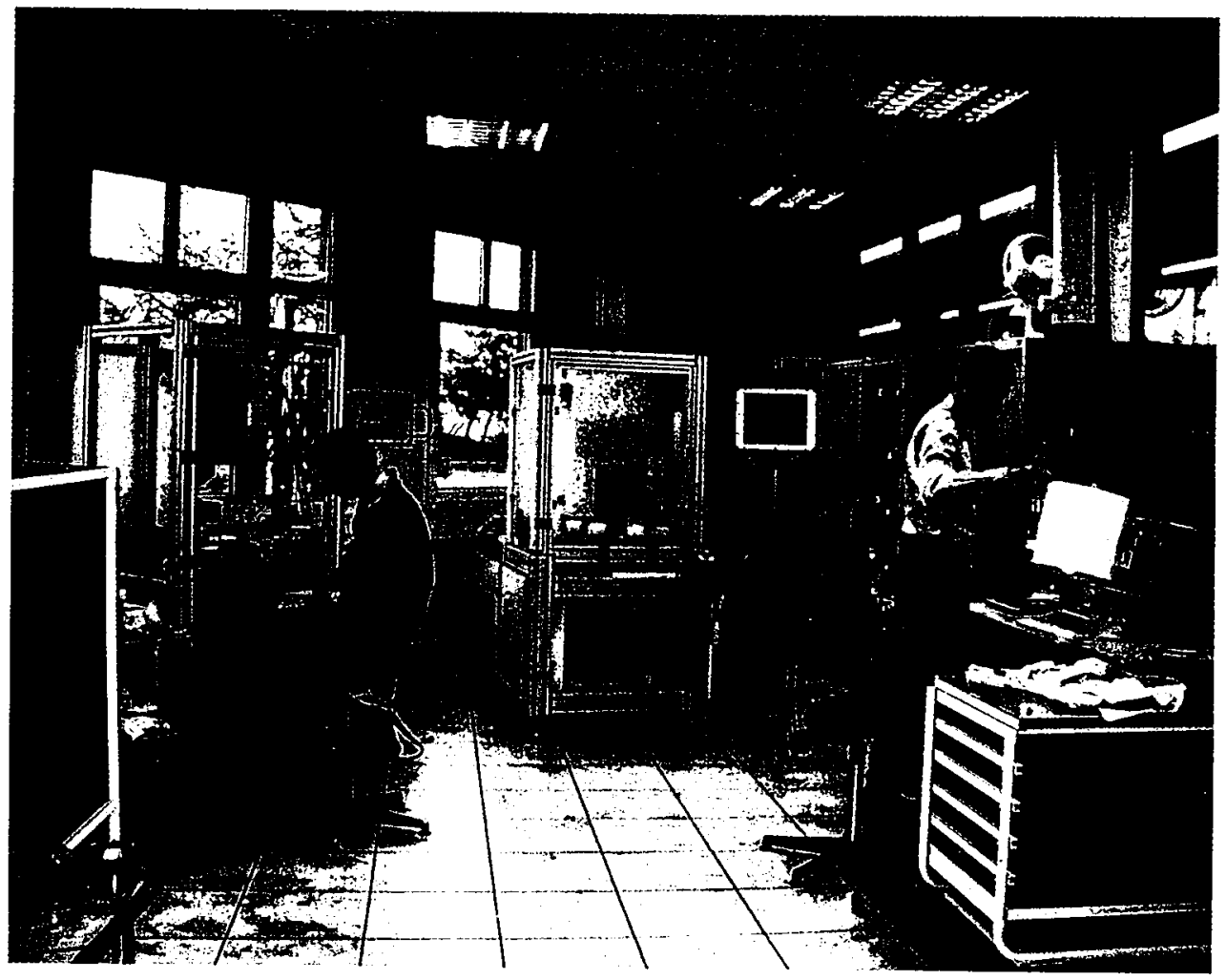

Fig. 7.20 Preparation of final system tests at TampoPrint GmbH, Stuttgart-Münchingen (Germany). Right: Nick Freear of Birmingham University off-line optimising a prototype interactive CCD-camera system, middle: prototype PT2. Left: prototype PT1, with author, making last minute revisions to vision control system software. 



\section{Evaluation and Conclusions}

The subject discussed in this thesis is the automatic and real-time interpretation of printed images based on visual information. This topic was studied in the context of quality control of printing processes using digital images. In this study we constructed an approach for this objective based on the following premises and assumptions:

i. Domain experts (are able to) provide sufficient background-knowledge of the physical, chemical, and mechanical principles involved in the printing process. More concretely: printing defects can be related to known (i.e. empirically observed, but not necessarily understood) inconsistencies in the physical/chemical/mechanical process.

ii. Digital images consist of a RGB-composition of three separate greyscale-matrices. The structure of printed images can be described geometrically in terms of PrintContexts, DistanceMaps, and ScanCurveTypes.

iii. Printing defect classes are fully characterised by their visually observable features. Printing defects can be adequately described as multi-fractal textures.

The objective of fast and effective on-line classification of printing defects was achieved by the application of the following scientific and technological principles.

i. Focused image analysis using PrintContexts, ScanCurveType-profiles, and DistanceMaps (see Sections $2.2-2.5$ ), applied on the template-subtracted image using Intelligent Binary Search (see Section 7.4).

ii. Geometric grey-scale transformations of an image to a unique 1D- or 2Drepresentation using CID (see Sections $2.6-2.7$ ).

iii. Interpretation of wavelet spectra of image faults as fractals, and using its multi-fractal dimensions for identification (Chapter 3).

iv. Feature-extraction - within the context of focused image analysis - of selected scans or patches of digital images with a large set of specialised domain-specific algorithms (Section 7.3, appendix A).

v. Structuring feature-space representations of defect classes using automatic fuzzy clustering techniques (Chapter 4).

vi. Considering printing defect classes as fuzzy sets (Section 4.2).

vii. Approximating printing defect class-membership by a multivariate normal distribution (Section 5.5.3).

viii. Introduction of symmetric metric in feature-space (Section 5.2.1). 
4. With the Iterative Local Least Squares Approximation (ILLSQ) it is possible to represent a 1D-signal in the Global Wavelet Decomposition Approach (GWDA) (both in Section 3.3.7). The latter is a generalisation of MRA (Multi Resolution Analysis).

5. Fractal wavelet analysis can be used for template-independent defect classification (see Chapter 3).

6. FMV-clustering is able to cope with not-linear cluster separability by treating it as cluster overlap (see Chapter 4).

7. The iterated FMV automatic cluster analysis technique can find the relevant number of isolated clusters in a data set. (see Section 4.6)

8. Neural networks for computing defect class membership outperform linear approximations, provided sufficient training data is available. A good guideline is: \#testdata/\#connections $>\sim 10$ (see Section 4.4.4).

9. Exact Parameterisations of printing defects can be used for direct process control (see Section 4.7.1).

10. Hierarchical clustering of printing defect can be used for efficient pre-classification (see Section 4.7.2).

11. Dynamic FPS combines feature-space topology with the current most likely defect class (see Section 5.2.3).

12. 'Dynamic' FPS outperforms 'static' FPS, but there is no significant influence by the way the alpha-parameter converges to one (see Section 6.3).

13. 'Separation' is the best dynamic FPS-functional in sense of computation-time and success-rate (see Section 6.2).

14. Mixed FPS should be avoided (see Section 6.5).

15. Because of the curse-of-dimensionality (Section 2.4.2) Interactive Classification in high-dimensional feature-spaces is only possible if large numbers of empirical observations are available. These observations can be labelled by human experts, or independently by automatic clustering-techniques like Iterated FMV (Section 4.6.2).

16. For a given minimally required quality $Q_{\min }$ of the conclusion (Section 5.1), there exists a maximal absolute noise-level $v$ for which the sequence of measurements converge to the proper defect class (Conjecture 5.1 in Section 5.6).

17. A distinction must be made between the convergence of IASC to the correct solution and the correct classification of the actual printing defect, because the image analysis and feature extraction can misrepresent the physical-chemical reality.

18. IASC outperforms random selection under low to medium noise and/or none to medium cluster-overlap (see Section 6.7). This is true both for theoretical problems (see Chapter 6) and real world data (see Chapter 7). 
19. Quality-based termination conditions of the IASC algorithm are more useful than convergence-based ones, but there is only a marginal difference between static and dynamic termination-conditions (see Section 6.6).

20. The threshold of the termination-condition of IASC defines the efficiency of the algorithm. For each pair of noise-level and average cluster-overlap there exists a unique value for the threshold that minimises IASC-computation time and maximises IASC-success rate (see Section 6.6).

We conclude this thesis with some recommendations.

- Before designing a computational vision system, first the feasibility of the required image quality should be deduced.

- Human vision provides a good model for image analysis, such as in the case of printing defects. Therefore, in designing a computational vision system, an interaction should be constructed between image analysis and context interpretation. In this way 'seeing' becomes 'understanding'.

- More research should be devoted to the interpretation of defect classes as textures, and their resulting multifractal properties. These properties could be used as principal features of the defects. Combined with an adequate process data archiving system, a suitable clustering technique (like FMV) could 'learn' the mapping between these fractal properties and the physical and chemical state of the process. Together with suitable knowledge of the process states of high-quality processes, this would enable both a self-learning classification system, and the outset for process control based on computational vision. 




\section{APPENDix A. EXTRACTING FEATURES FROM Printing DefeCtS}

In this appendix special-purpose algorithms are described for the extraction of printing defect features.

\section{A.1 Features Defined by Basic Image Characteristics}

Standard Image Amalysis provides a number of basic feature parameters intended to characterise images. After a number of such parameters have been obtained it is perhaps possible to use clustering techniques to segregate the relevant padprinting defect groups. Examples of such parameter are the centre-of-mass of the image. the average size of the image - defined as the average distance to the centre-of-mass, and the average orientation of the image. Rather than the entire image, we will here employ the absolute difference $Z$ between the image $F$ and its associated template $T: Z=|F-T|$. For sake of a simple notation we will regard the image with continuous coordinates $x$ rather than the discrete coordinates $\left(i_{i}\right)$ provided by the CCD-image. As a first step these basic image analysis parameters were applied to a set of absolute differences $Z$ to evaluate their usefulness in our context. Below a number of standard image analysis feature parameters are defined and their usefulness for our context is discussed.

1. The parameter $z$ denotes the 'Centre of Mass' of the image. and represents the average position of the image. $z$ is calculated according to:

$$
z=\left\langle x>=\int_{\Omega} x Z(x) d_{2} x / \int_{\Omega} Z(x) d_{2} x\right.
$$

Where ' $\Omega$ ' represents the entire area of the image.

2. The parameter $d$ denotes the 'Average Size' of the image, and represents the average distance from parts of the image to the centre-of-mass of the image. $d$ is calculated according to:

$$
\mathrm{d}=\left\langle|\mathbf{x}-\mathbf{z}|>=\int_{\Omega}|\mathbf{x}-\mathbf{z}| Z(\mathbf{x}) \mathrm{d}_{2} \mathrm{x} / \int_{\Omega} Z(\mathbf{x}) \mathrm{d}_{2} \mathrm{x}\right.
$$

3. The parameter $A v$ Dist denotes the Average Distance and represents the average distance between the specific parts of the image. AvDist is calculated according to:

$$
A v \text { Dist }=\left\langle|x-y|>=\int_{\Omega}|x-y| Z(x) \cdot Z(y) d_{2} x d_{2} y / \int_{\Omega} Z(x) \cdot Z(y) d_{2} x d_{2} y\right.
$$

A general disadvantage of all these parameters is that they are extremely computation-time intensive. Moreover, it is observed that the separation between the defeet classes, relative to these paraneters, is relatively small. Similarly, the overlap of the defects is considerable. Therefore these parameters are not particularly useful for identifying the defect class. However, if not sufficient evidence is available from other tests, to be presented later, they can be applied as a last resort. The decision to select and employ suitable tests is controlled by the main reasoning system to be discussed in part IV. based on the most likely hypothesis concerning the nature of the defect, the main reasoning system can execute specific tests on demand.

\section{A.2 Mean Density of the Fault}

A basic feature parameter that is especially simple to compute is the mean density of $Z$ of the image, denoted $\mathrm{D}_{\text {dens. }}$. It represents the integral size of the defect divided by the size of the image:

$$
D_{\text {dens }}=\int_{\Omega} Z(x) d_{2} x / \int_{\Omega} d_{2} x
$$

where ' $\Omega$ ' represents the entire area of the image.

$A$ feature based on the mean density of $Z$ can be represented directly by the 'feature parameter' $D_{\text {dens, }}$ or, alternatively, some fuzzy membership function can be constructed using $D_{\text {dear. }}$ For example, we could define a fuzzy membership function as some bell-shaped function on the interval $\left\langle a_{k}-\sigma_{k}, a_{k}+\sigma_{k}\right\rangle$. where $a_{k}$ is the average observed mean density of defect class $k$, and $\sigma_{k}$ its associated standard deviation.

From an analysis of $495 \mathrm{CCD}$-images it became clear that only in a few cases $\mathrm{D}_{\text {dens }}$ correlates significantly with the padprinting defect. Here, we encounter a typical characteristic of feature 
parameters in the context of computer vision; i.c. the fact that features typically correlate with but a few defect classes while all other defect classes have about the same value.

Though the mean density correlates but weakly with the defects class, it can be computed fast and efficiently. Therefore, this method can be used as a 'trigger' to employ more powerful - but timeconsuming - image analysis techniques. For that reason. this method is implemented in both prototypes to identify a misprint when $D_{\text {dens }}$ is observed to exceed a certain empirically determined threshold.

\section{A.3 Feature parameters for Scan Profiles}

In section 2.3 we discussed the scanning of an image and - depending on its location on the print as expressed by its PrintContext - the resulting expected and possible scanprofile. It was found within our definition of printed elements that the expected scanprofile can be easily classified as merely two types: $\left\{\mathrm{E}_{1}, \mathrm{E}_{2}\right\}$, a constant and a step-function. The possible scanprofile was more difficult to classify, but depending on the actual printing defect a number of likely outcomes were defined as the "featuretypes'. In order to find the best fitting of these feature-types with a given scanprofile, and thus extract information about the possible printing defect, we present in this appendix $\Lambda$ number of specially designed tests.

\section{i. Optimal Match with a Characteristic Profile}

Some of these tests give the optimal fit of the scanprofile with one of the feature-types, parameterised by a set $\left\{a_{1}, a_{2}, \ldots, a_{n}\right\}$. The fit - called feature-fit - conveys the degree that the scanprofile can be considered as belonging to this feature-type. The general approach for computing the 'feature-fit' of the actual scanprofile $\mathrm{s}(\mathrm{x})$ with one of the feature-types can be summarised by the following steps:

1. Approximate scan $\mathrm{s}(x)$ with a parameterised feature-function $H$ with parameters $a=\left(a_{1}, a_{2}, \ldots, a_{n}\right)$.

Apply Lecost Squares with quadratic criterion $V\left(a_{1}, a_{2}, \ldots, a_{n}\right)=\int\left(s(x)-H\left(x, a_{1}, a_{2}, \ldots, a_{n}\right)\right)^{2} \mathrm{~d}$, and use the resulting constraints to find a solution $a^{*}$.

2. Establish the minimal value of the criterion $\nu^{*}=V\left(a^{*}\right)$. Note that if $s$ fully complies with the feature-type $H$ that $r^{*}=0$.

3. Construct a continuous monotonous decreasing function ' $f$ ' such, that $f$ obeys: [i] $f(0)=1$, and [ii] $f(x) \downarrow 0$ for $x \rightarrow \infty$. Now define the feature-fit as: $H f i t=f\left(\mathrm{~V}^{*}\right)$. Base the choice of $f$ on empirical data such that the feature-fit is on average high for evident feature-type-scanprofiles. This matching to the test-set can be expressed by a tuneable parameter $\lambda$ and likewise $f_{\lambda}$.

'Feature-fit' is a number between zero and one, and thus can be seen as a fuzzy membership function of the scanprofile to the feature-type. Besides the feature-fit, also the resulting parameters $a^{*}$ can be used for reasoning about the defect. If the expected profile is a step-function, while the observed profite is also a step-function, but shifted lightly to the left, this may indicate the defect 'ink flow' at the edge of the print.

\section{ii. Peak Partitioning and Decomposition Statistics}

Finally we present a simple but effective algorithm specially designed to identify 'spikes' or 'bumps' above a given threshold in a given scanprofile. This method operates on 'peaked' iD-functions $s(x)$ with domain $S$, and generates the collection of intervals for which the function is equal to or above a given criterion $C$. Here we assume that $S$ is given as a closed interval [a,b]. First, the function $s$ is thresholded with a threshold defined by $y^{\prime}: C_{\epsilon}=m+\epsilon(M-m)$, with $m=m_{x} s(x)$, and $M=\max _{x} s(x)$, so $0 \leq \epsilon \leq 1$. The advantage of this representation is that while the value of $C$ depends on the context, the value of $\epsilon$ is restricted to $[0,1]$. Therefore general applicable values of $\epsilon$ can be specified that provide good results. The thresholded function $u_{\epsilon}$ is given by:

$$
u_{\epsilon}(\mathrm{x})=\theta\left(\mathrm{s}(\mathrm{x})-C_{\epsilon}\right)
$$

with threshold function $\theta(x)=x$ if $x>0$, and zcro otherwise. The best results were obtained for values $0.25 \leq \epsilon \leq 0.4$. In our context $\epsilon$ was set to $1 / 3$. The thresholding can also be regarded as a constraint that reduces the domain $S$ of $s$ to a subset $R$ :

$$
R=\{\mathrm{x} \in \mathrm{S} \mid \mathrm{s}(\mathrm{x}) \geq \epsilon\}
$$

\footnotetext{
'In certain cases it is more useful to express $C$ as: $C_{E}=\mu+\epsilon(\mathrm{M}-\mu)$, where $\mu=\langle\mathrm{s}\rangle$, , especially in the case of sharp and high peaks, because such representation is more resistant to high amounts of noise.
} 
We assume that subset $R$ can be expressed as a union of $N_{\epsilon}$ closed intervals $\left[a_{k} \cdot b_{k}\right]$. with $I \leq k \leq N_{\epsilon}$.

$$
R=\mathrm{U}_{\mathrm{k}=1: \mathrm{N \epsilon}}\left[\mathrm{a}_{\mathrm{k}}, \mathrm{b}_{\mathrm{k}}\right]
$$

Let function $p$ be a partitioning algorithm that applied on $R$ generates this collection of closed intervals $\left[\mathrm{a}_{\mathrm{k}}, \mathrm{b}_{\mathrm{k}}\right]: p: R \rightarrow\left\{\left(\mathrm{a}_{\mathrm{k}}, \mathrm{b}_{\mathrm{k}}\right) \mid 1 \leq \mathrm{k} \leq N_{\epsilon}\right\}$. This approach is the ID-analogon of the $2 \mathrm{D}$-methods as the Cell Parritioning approach or the FindAreas algorithm to be discussed later. This representation allows the computation of some useful statistical quantities to characterise the defect.

In the context of the identification of specific defects characterised by peaks and bumps, we are interested in statistical parameters describing the partition of intervals. Important quantities in that respect are the length of the intervals: $D_{k}=\operatorname{size}\left(\left[a_{k}, b_{k}\right]\right)=b_{k}-a_{k}$. The average interval-Iength is expressed by $\langle\mathrm{D}\rangle$ and its corresponding standard deviation $\delta \mathrm{D}$. Another important quantity is the peakcentre $\mathrm{c}_{\mathrm{k}}$ delined as the centre-of-mass in interval $k$ :

$$
\mathrm{c}_{\mathrm{k}}=\int_{[\mathrm{ak}, \mathrm{lk} \mid \mathrm{k}]} \xi \mathrm{s}(\xi) \mathrm{d} \xi
$$

In a similar way, using the second moment, the standard deviation $\delta \mathrm{c}_{\mathrm{k}}$ can be defined. that provides another measure for the width of the interval. Particularly relevant is the spacing $\lambda_{k}$ between the peakcentres:, $\lambda_{k}=c_{k+1}-c_{k}$. In a similar way the average spacing can be expressed as: $\langle\lambda\rangle \pm \delta \lambda$.

Finally, in certain cases it can be relevant to express the match of $s(x)$ in each interval $k$ with a characteristic peak or bump shape for a specific defect. Let $/ \mathrm{k}$ represent the lit of $s(x)$ in interval $k$ with a characteristic peak-shape $\phi$, such that $f_{\mathrm{k}} \in[0,1]$, and $f_{\mathrm{k}}=1$ indicates a perfect match. This leads to an average match $\langle f\rangle_{ \pm} \delta f$. This approach can be casily extended to $2 \mathrm{D}$-images $Z\left(\mathrm{x}_{1}, \mathrm{x}_{2}\right)$ by decomposition in its two constituent dimensions. Let us define: $z_{1}(\xi)=\int_{\Omega} Z\left(\xi . x_{2}\right) d x_{2}$, and $z_{2}(\xi)=\int_{\Omega}$ $\mathrm{Z}\left(\mathrm{x}_{1}, \xi\right) \mathrm{dx_{1 }}$, and apply the 'peak partitioning' approach independently to $z_{1}$ and $z_{2}$. For each of the parameters ' $\alpha$ ' described above this results in a pair of quantities $\alpha_{x}$ and $\alpha_{y}$. Dependent on the characteristics of the defect a combined parameter ' $\alpha$ ' for the entire image can be expressed as a function of $\alpha_{\mathrm{x}}$ and $\alpha_{\mathrm{y}}$, for instance: $\alpha=\alpha_{\mathrm{x}} \alpha_{\mathrm{y}}$.

The peak-partitioning approach proofed to be an effective method for the identification of 'spiky' defects like 'hairs', 'dirt particles' and rasters. Compared with the other approaches it has the highest computational efficiency and speed, and in average a strong correlation with the defect. An example of PeakPartitioning applied on the defect 'hairs' is shown in Figure A.8. In the table below $s$ is the observed scanprofile, $s^{*}$ is the expected scanprofile according to the feature-type, and $\theta$ is a threshold function: $\theta(x)=x$ if $x>0$. and zero otherwise. In the next section we will discuss in detail the fit of the

\begin{tabular}{|c|c|}
\hline Parameter & Description \\
\hline Equal & $\begin{array}{l}\text { The degree in which the observed ScanProfile } s(x) \text { is equal to the expected } \\
\text { ScanProfile } \mathrm{s}^{*}(\mathrm{x})\end{array}$ \\
\hline TooHigh & $\begin{array}{l}\text { The degree in which the observed ScanProfile is consequently higher than } \\
\text { the expected ScanProfile }\end{array}$ \\
\hline TooLow & $\begin{array}{l}\text { The degree in which the observed ScanProfile is consequently lower than the } \\
\text { expected ScanProfile }\end{array}$ \\
\hline Meamlintensity & Mean intensity of the scanprofile \\
\hline Stdlntensity & Standard deviation in Mecumlmensity \\
\hline LineFit & Fit of the observed ScanProfile with a horizontal line \\
\hline StepFit & Fit of the observed ScanProfile with a StepFunction \\
\hline BlockFit & Fit of the observed ScanProfile with a BlockFunction \\
\hline$D . c . \lambda . f$ & PeakPartioning of ScanProfile identifies 'peaks' and 'bumps'. \\
\hline Hairity & $\begin{array}{l}\text { The degree in which the observed ScanProfile exhibits a quasi- } \\
\text { pattern }\end{array}$ \\
\hline
\end{tabular}
scanprofile with the feature-type of quasi-periodic peaks.

Table A1 Some useful scanprofile parameters 

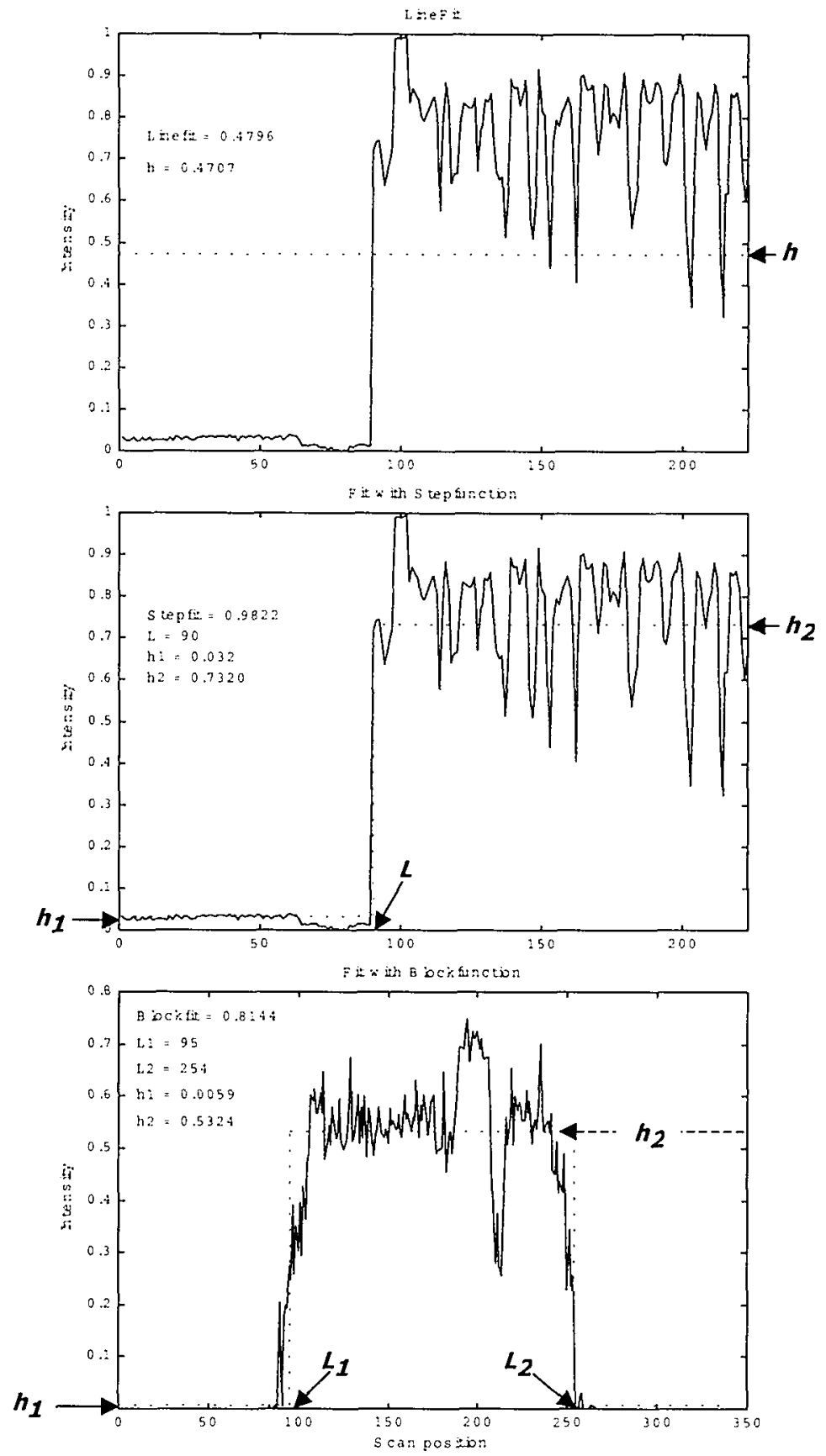

Fig. A I. Examples of 'feature-type' functions with delinitions of its parameters. Depicted is an observed scanprofile (solid) and the optimal fitting feature-type-function. respectively [1] a horizontal line, [2] a stepfunction, and [c] a blockfunction. The values of the parameters and the fit is shown in the ligure. 


\section{A.4 Detecting Oscillatory Signals with 'hairity'}

The defect 'hairs' is characterised by the typical hair-like structures, emanating almost perpendicularly from the border of the print. This defect can easily be identilied by a scanprofile taken from a

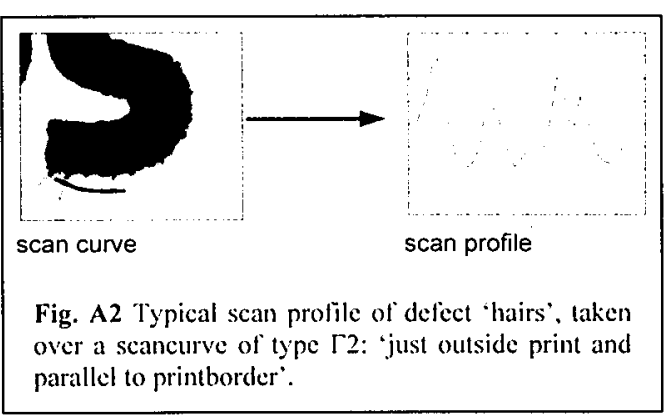

scancurve parallel to the printborder, just outside the print - that is Curverype $\Gamma 2$ according to table 2 in section 2.1.7. An example of such a scan and the resulting scamprofile is presented in Figure $\mathbf{A . 5}$. Therefore the detection of 'hairs' focuses on the analysis of such specific scamprofiles $\sigma(\lambda)$.

In order to quantify the concept of hairity we are looking for a suitable definition of a quantily $h$. Ideally, $h$ should take the value $\%$ for evident cases of scans over hairs, and ${ }^{\circ}$ ' for scans that are evidently not hairs. Thus, $h$ can be considered as a

fizzy measue for the degree we consider the scan as a member of the class of 'hairy profiles'.

As a first approach Fourier analysis was employed in order to distinguish the quasi-periodic signals occurring in "hairs" from non-periodic signals.

For this reason a uniform filter was applied on the Fourier transform $\mathrm{F}[\sigma]$ from $\sigma$ :

$\psi(\omega)=\mathrm{F}[\sigma](\omega)=(2 \pi)^{-1 / 2} \int \sigma(\lambda) \mathrm{e}^{\mathrm{i} \omega \lambda} \mathrm{d} \lambda$

that operated on trequencies between $\omega_{1}=3.3$ $\mathrm{mm}^{-1}$ and $\omega_{2}=0.75 \mathrm{~mm}^{-1}$ - corresponding 10 wavelengths from $0.3 \mathrm{~mm}$ to $1.3 \mathrm{~mm}$. These figures are based on empirical evidence. The energy within this frequency band $E_{1}$ :

$$
E_{l}=\int_{\omega \mid}^{\int \omega^{2}}|\psi(\omega)|^{2} \mathrm{~d} \omega
$$

was compared with the entire energy $E$ :

$$
E=\int|\psi(\omega)|^{2} \mathrm{~d} \omega
$$

and a quantitative feature parameter $h_{\mathrm{f}}$ called hairity was defined as $\mathrm{h}_{\mathrm{F}}=\mathrm{C}$. $\mathrm{E}_{\mathrm{l}} / \mathrm{E}$, where $C$ is a scaling constant. Figure $\wedge .6$ shows an example where $\mathrm{E}_{1} / \mathrm{E}=0.357$. This definition of hairity had a number of serious disadvantages. First, the value of the scaling constant was difficult to calibrate. In above example for instance, where we have a clear example of defect 'hairs'. we could chose $C$ such that $h_{1}=1$, so $C=1 / 0.357 \approx 2.8$. In other comparable cases, however, we might find higher $E_{l} / E$ values, causing $h_{f}$ to execed 1 , which is against its definition. Second, based on empirical evidence, the value of the filterband were often found not to coincide with the actual relevant area of the frequency spectrum.

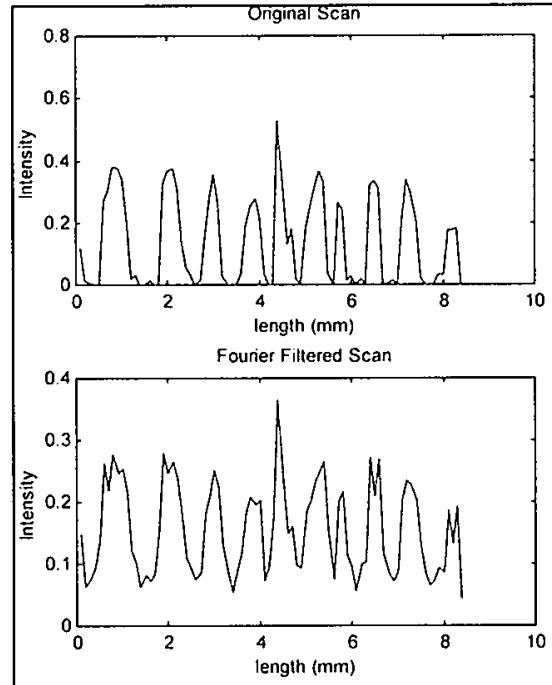

Fig A3. Example of lourier reconstruction. Above scan is taken from a sample exhibiting defect 'hairs'. The lower figure is reconstructed from the Fouric transform of the original scan restricted to a frequency band from 3.3 to $0.75 \mathrm{~mm}^{-1}$. The quotient of the spectral energy of the constructed and original scan is 0.357 .

Therefore the filter could miss the relevant part of the spectrum causing $E_{[} / E$ to be relatively low whilst the energy was actually quite localised. Thirdly, even with an optimal choice for $C, \omega_{1}$ and $\omega_{2}$, the measure $h_{F}$ was not quite able to distinguish noisy signals fiom real hairy profiles. Therefore, other alternatives for $h_{F}$ were considered. 
As mentioned in appendix A.3 during the discussion of matching scanprofiles with standard featuretypes. the oscillatory behaviour of a "hairy' signal was detected with an adaptation of the 'Multi Resolution Approach' (MRA), that will be discussed in section 2.7. Two methods were considered. Both approaches used a quadratic scaling function: $\phi(x)=\gamma x(1-x)$ if $x \in[0,1]$, and $\phi(x)=0$ elsewhere. Here $\gamma=\sqrt{ } 30$ is a suitable scaling constant such that $\|\phi\|=1$ in the norm of $L^{2}$-integrable functions. In fact $\phi$ is related to the Quadratic Cardinal B-Spline Wavelet function ${ }^{2}$ [Ch.Chui.1996].

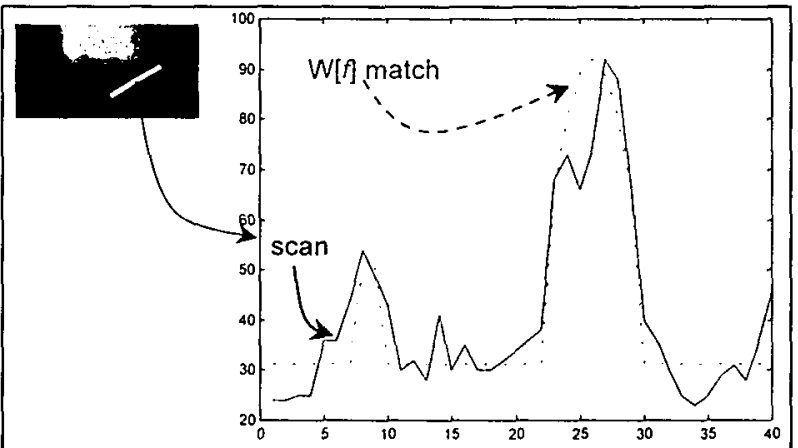

Fig. A4. Example of a $\mathrm{H}_{\mathrm{s}} \mathrm{l} / \mathrm{l}$ approximation of a scanprofile. Left: the image and the scancurve. Right: the resulting scanprofile (solid) and the approximation $\mathrm{H}_{d}[$ (dotted). The threshold $x=0.3$.

In order to reduce the influence of noise a threshold $\alpha$ was introduced. Only those values of the scanprolite above threshold $\kappa$ were considered. This delines a thresholded profile: $f(x)=\theta(\sigma(x)-\kappa)$, where $\theta(x)=x$ if $x>0$, and zero othervise. Next. the thresholded scanprofile $f$ was approximated with the following series in the scaling function $\phi$ :

$$
H_{\alpha}[](x)=\Sigma_{k=1: N} \alpha_{k} \phi\left(\lambda_{k} x-\beta_{k}\right)
$$

with $\lambda$ restricted to $\left[3.3 \mathrm{~mm}^{-1}, 0.75 \mathrm{~mm}^{-1}\right]$, and $\mathrm{N} \leq \mathrm{N}_{\max } . \mathrm{H}_{6}[\cap$ is a wavelet transform called ODA, and will be discussed in more detail in section 2.7.2.5. In our experiments we restricted $\mathrm{N}_{\max }$ to 16.
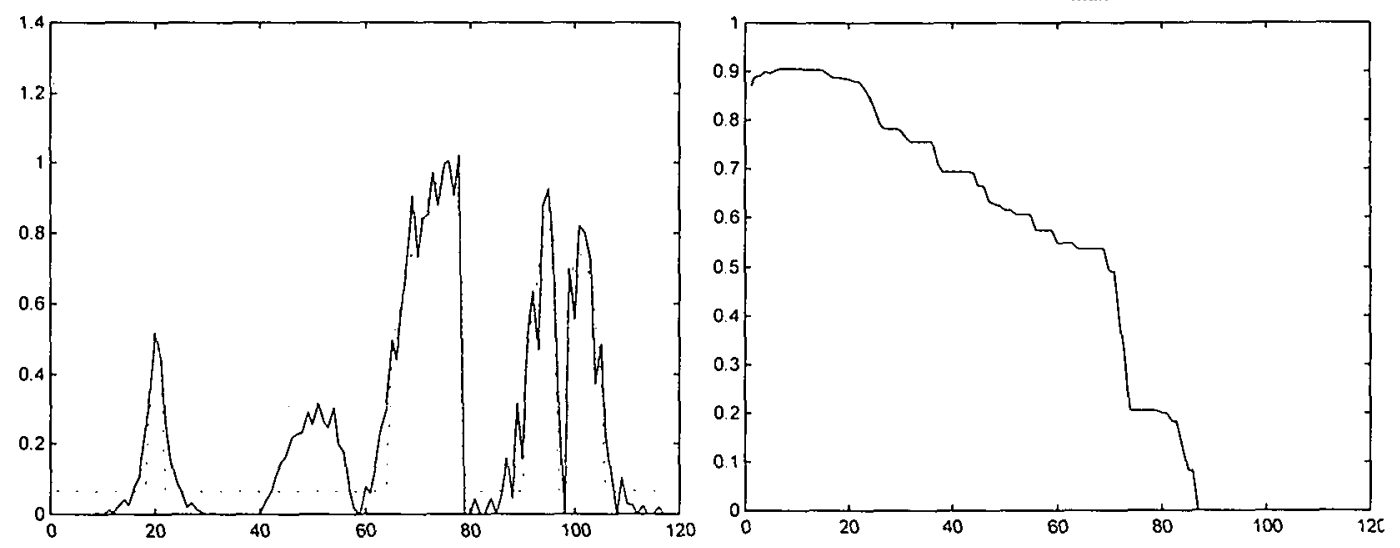

Fig. A5. Effect of the threshold $\kappa$. Left: a 'hairy' scanprolile (solid). The threshold $\kappa$ was set to 0.3 (dotted horizontal line). The part of the profile above the threshold is approximated by the best fitting parabolas $\mathrm{H}_{\phi}[f]$ (dotted). From this figure it is clear that bumps in the scanprofile below the threshold are not considered in $\mathrm{H}_{6}[\mathrm{f}$. Right: The effect of the threshold $\alpha$ on the value of hairity $h_{W}$ for this scanprofile. The horizontal axis denotes $100^{*} k$, the vertical axis denotes the value of $h_{W}$. From this figure it is clear that at about $k=0.7$ the value of $h_{W}$ suddenly drops to low values. Best values for $h_{W}$ were obtained with $k$ between 0.2 and 0.4 .

\footnotetext{
${ }^{2}$ Cardinal $B$-splines are a family of functions $B_{k}(x)$ with $B_{0}(x)$ the characteristic function on $[0,1]$ and
} 
The actual approximation was performed with Least Squares (LSQ) with a quadratic criterion $v$ :

$$
\mathrm{V}=\left\|f-\mathrm{H}_{\mathrm{o}}[\mathrm{b}]\right\|^{2}
$$

Application of $L S Q$ leads to a series of complexly entangled cquations.

$$
\begin{aligned}
& <f \mid \phi\left(\lambda_{k} x-\beta_{k}\right)>=\sum_{n=1: N} \alpha_{n} P\left(\lambda_{k}, \beta_{k}, \lambda_{n}, \beta_{n}\right) \\
& <f \mid \phi^{\prime}\left(\lambda_{k} x-\beta_{k}\right)>=\sum_{n=1: N} \alpha_{n} Q\left(\lambda_{k}, \beta_{k}, \lambda_{n}, \beta_{n}\right) \\
& <f \mid x \phi^{\prime}\left(\lambda_{k} x-\beta_{k}\right)>=\sum_{n=1: N} \alpha_{n} \mathrm{R}\left(\lambda_{k}, \beta_{k}, \lambda_{n}, \beta_{n}\right)
\end{aligned}
$$

Where $<\cdot 0>$ represents the inner product in $L^{2}$, and the constants $P . Q$. and $R$ depend on $\left(\lambda_{k}, \beta_{k}, \lambda_{n}, \beta_{n}\right)$ and are defined by:

$$
\begin{aligned}
& \mathrm{P}\left(\lambda_{\mathrm{k}}, \beta_{\mathrm{k}}, \lambda_{\mathrm{n}}, \beta_{\mathrm{n}}\right)=\left\langle\phi\left(\lambda_{\mathrm{k}} \mathrm{x}-\beta_{\mathrm{k}}\right) \mid \phi\left(\lambda_{\mathrm{n}} \mathrm{x}-\beta_{\mathrm{n}}\right)\right\rangle \\
& Q\left(\lambda_{k}, \beta_{k}, \lambda_{11} \beta_{n}\right)=\left\langle\phi^{\prime}\left(\lambda_{k} x-\beta_{k}\right) \mid \phi\left(\lambda_{n} x-\beta_{n}\right)\right\rangle \\
& \mathrm{R}\left(\lambda_{\mathrm{k}}, \beta_{\mathrm{k}}, \lambda_{11}, \beta_{\mathrm{n}}\right)=\left\langle\mathrm{x} \phi^{\prime}\left(\lambda_{\mathrm{k}} \mathrm{x}-\beta_{\mathrm{k}}\right) \mid \phi\left(\lambda_{\mathrm{n}} \mathrm{x}-\beta_{\mathrm{n}}\right)\right\rangle
\end{aligned}
$$

Since $\phi\left(\lambda_{k} x-\beta_{k}\right)$ is delined on $x \in\left[\beta_{k} / \lambda_{k},\left(1+\beta_{k}\right) / \lambda_{k}\right]$ the left part $\langle f| \cdot>$ of above equations also contain the parameters $\left(\lambda_{\mathrm{k}}, \beta_{\mathrm{k}}\right)$. Moreover, $N$ can be considered as an extra optimisation parameter. resulting in even more complex equations. Therefore, these equations cannot be solved in general. However, instead of performing $L S Q$ on the entire range of $f$, equivalent results can be obtained by local $L S Q s$ restricted to intervals were $f$ is above the threshold: $f>\theta$. In this way the best litting sealingfunction $\phi$ on each sub-interval can be simply determined using standard textbook procedures [Tuma90]. For instance, in Figure A.8 the 4 peaks above the dotted horizontal line, indicating the criterion, are individually matched with the best-litting parabola $\phi\left(\lambda_{\mathrm{k}} \mathrm{x}-\beta_{\mathrm{k}}\right)$. This calculation leads to a set of coefficients $c_{w}$ :

$$
\mathrm{c}_{\mathrm{W}}=\left\{\left(\alpha_{1}, \beta_{1}, \lambda_{1}\right),\left(\alpha_{2}, \beta_{2}, \lambda_{2}\right), \ldots,\left(\alpha_{N}, \beta_{\mathrm{N}}, \lambda_{N}\right)\right\}
$$

In the first approach a measure for hairity was based on the rescaling of criterion $\nu$ to $\|f\|^{2}$ :

$$
\mathrm{h}_{W}=1-\left\|f-\mathrm{H}_{\phi}[f]\right\|^{2} /\|/\|^{2}
$$

This gave satisfactory results, provided that the threshold $\kappa$ was chosen sufficiently high. This, however, introduced a problem concerning the subjective choice of $k$ similar to the choice of $C$ for $h_{F}$. The method for $h_{W}$ gave satisfactory results. For scans of hairy profiles the value of $h_{W}$ ranged normally between 0.7 and 1 , giving a clear indication of the condition 'hairity'. Best values were obtained when $\kappa$ was chosen between 0.2 and 0.4 . Lower values made $h_{W}$ too sensitive to noise, higher values of $k$ deleted too much information from the profile and so decreased the value of $h_{W}$. In that sense this method is much more independent of $\alpha$ than $h_{F}$ depends on $C$. Figure $A .7$ shows an example of a calculation of $h_{W^{\prime}}$ In Figure A.8 the effect of the threshold $k$ on $h_{W}$ is demonstrated, clearly showing that (1) for $0.2<k<0.4 h_{W}$ is nearly independent of $k$, (2) for such $k$ values $h_{W}$ is not too far from 1 .

Correction factors $h_{l}$ and $h_{2}$, based receptively on: [1] $n=$ the number of peaks $/ \mathrm{mm}$, and [2] $l_{a}=$ the average greylevel of the peaks, further increased the performance of $h_{W}: h_{W}{ }^{*}=h_{l} h_{2} h_{W}$.

Starting from the coefficient set $c_{W}$ a second method can be defined. Rather than on the parameter $\beta$ this approach would consider the distribution on the spacing between the spikes: $\delta_{\mathrm{ij}}=\beta_{\mathrm{i}}-\beta_{\mathrm{j}}$. Based on the $c_{W}$ distributions $\rho_{\alpha}(\alpha), \rho_{\delta}(\delta)$ and $\rho_{\lambda}(\lambda)$ can be calculated. Next, this method depends on the availability of a large empirical set $D$ of such distributions for given defect types. Based on this set $D$ a clustering algorithm can be cmployed to make a mapping $\mu$ from, for instance $\langle\alpha\rangle,\langle\delta\rangle$ and $\langle\lambda\rangle$, to the defect-type $K$. In general: $\mathrm{K}=\mu\left(\mathrm{c}_{W}\right)$. The concept of hairity in this context can be defined as the 'confidence' or 'probability' we have that the mapping $\mu$ assigns the set $c_{w}$ correctly to defect class 'hairs'; hairity $=\mathrm{P}\left[\mu\left(\mathrm{c}_{\mathrm{W}}\right)=\right.$ 'hairs' $]$.

A similar approach using neural networks for mapping is introduced in section 2.7.

$B_{k+1}(x)=\int_{\{0.1\}} B_{k}(x) d x$ 
There are a number of general problems involved in determining hairity based on the scanning approach. First, the scans must be of sufficient length, otherwise there will not be sufficient 'bumps' on the profile in order to be recognised as 'hairy'. This is a problem that must be mastered by the algorithm that generates scancurves. Too long scancurves, however, may include extensive parts where no heirss occur, and thus likewise affect the evaluation of the profile.

\begin{tabular}{|l|l|l|}
\hline Parameter & False Negatives & False Positives \\
\hline$h_{\boldsymbol{F}}$ & $32 \pm 5$ & $43 \pm 5$ \\
\hline$h_{\boldsymbol{W}}$ & $4 \pm 5$ & $39 \pm 5$ \\
\hline
\end{tabular}

Table A2 Success-rate as percentage of profiles identified as such.
A second problem concerns the fact that definitive non-hairy profiles are incidentally identilied as being 'hairs'. This is the case for both $h_{w}$ and $h_{k}$. These false positives thus pose a larger threat than the false negatives, that in the case of $h_{\mathbb{I}}$. hardly occur. This effect is shown in table 2 below.

A problem more specilic to $h_{W}$ is the fact that $N_{\text {max }}$ must be restricted to relatively small sizes. Otherwise the quality of the approximation $\mathrm{H}_{\mathrm{l}} \mathrm{l} / \mathrm{f}$ will approach $100 \%$ as wavelets are very elficient in approximating arbitrary functions $f$ to what ever desired precision - given sufficient terms.

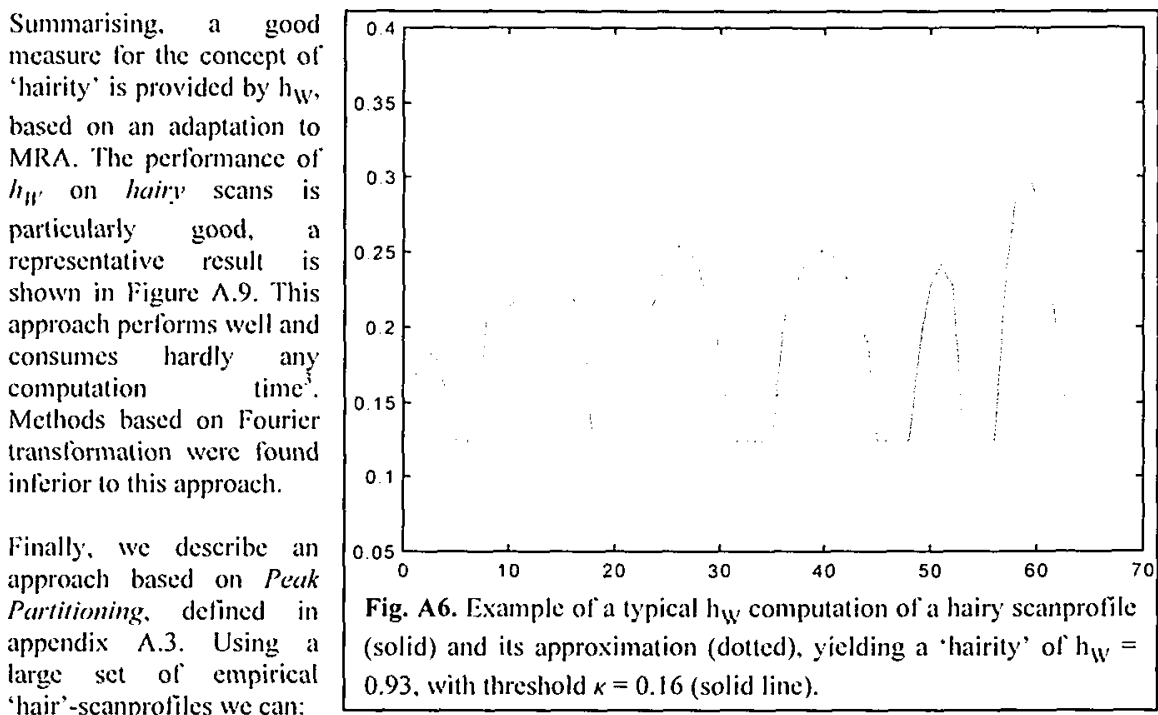

i. Calculate the distributions for peak width $\mathrm{D}$ and peak spacing $\lambda$ :

ii. Establish the average shape of one 'hair'-peak.

Figure $A .10$ depiets an averaged 'hair'-peak and tables the average width and spacing of hairs. For an observed scanprofile its measure for 'hairity' can then be based on:

i. Its particular distributions for $D$ and $\lambda$;

ii. The average fit with the archetypal 'hair'-peak.

Based on these values, and the average hair-properties depicted in above figure, the probability that the present scanprofile represents "hairs' can be calculated. This involves the fundamental problem of fusing two different probabilities; $p /$ stemming from the statistical distributions, and $p 2$ from the average peak-match. $A$ combination like max $(p l, p 2)$ ignores small values of the other probability, while a combination like mean(pl.p2) or pl*p2 can decrease a high value of one probability.

An entirely different approach to identification of hairs is offered by [Cucchiana.Filicori. 1998]. who construct a 2D-l lough transform for detecting clongated shapes in greyscale images.

"On average $0.1 \mathrm{sec}$ with MatLab on a $400 \mathrm{MHz}$ PentiumPro PC under Windows NT. 


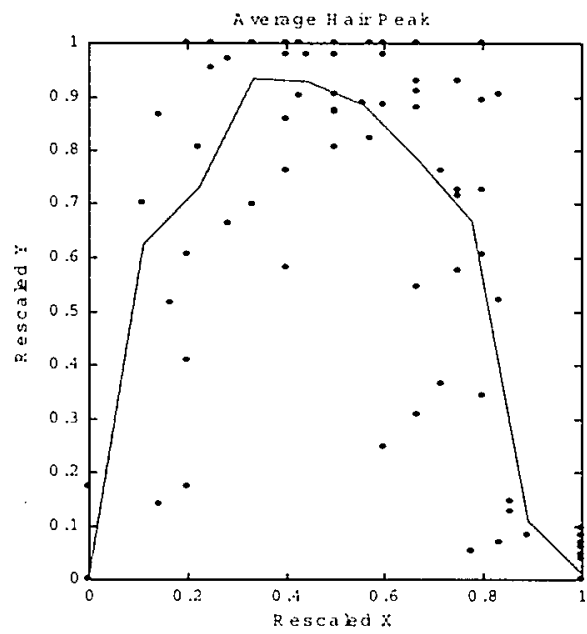

Average values for Hair-peaks:

\begin{tabular}{|l|l|}
\hline Hair-width & $\mathrm{D}=0.069 \pm 0.024 \mathrm{~mm}$ \\
\hline Hair-spacing & $\lambda=0.12 \pm 0.04 \mathrm{~mm}$ \\
\hline $\begin{array}{l}\text { Center-of-mass } \\
\text { (relative to width) }\end{array}$ & $\mathrm{c}=0.50 \pm 0.18$ \\
\hline
\end{tabular}

Fig. A7 Average 'Hair' peak. The points show rescaled values averaged over a large set of hairprofiles, thresholded with $\epsilon=0.05$. The solid line is the optimal 9 point piece-wise lincar fit.

\section{Scanprofile from Hairs}

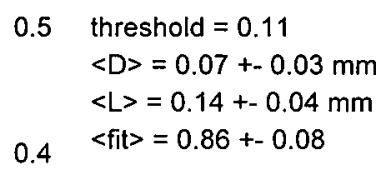

0.3

0.2

0.1

0

$\begin{array}{llccc}0.2 & 0.4 & 0.6 & 0.8 & 1 \\ & & & \text { Scanposition }[\mathrm{mm}] & \end{array}$

$1.2-1.4$

Fig. A8. Example of the matching of an actual scanprofile with average values. Based on a Normal Distribution with the values in figure 10 we find probabilities based on $D$ and $\lambda: P[D]=0.97, P[\lambda]$ $=0.94$. This must be combined with the feature-fit of $0.86 \pm 0.08$. Three models for integration of the probabilities differ largely: [i] max: 0.97 , [ii] product: 0.78 , [iii] average: 0.93 . 


\section{A.5 Statistical Distribution over a Partition of Image Cells.}

In many cases the defect is not situated in one localised area within the absolute difference $Z$, but rather distributed over the image as a collection of disconnected fragments. We will call such an individual fiagment a cell. The defect represented by $Z$ can thus be envisaged as a partitioning in cells. This can be obtained by thresholding $Z$ with threshold $\theta$ generating $\{x \mid Z(x)>\theta\}$ and labelling the resulting cells with standard image analysis techniques, or with the algorithm FindArets - described before - that produces a collection of SearchBoxes. In many cases we are interested in the shape properties of individual cells. If $s_{k}$ represents a certain shape parameter of cell $k$ we can calculate the average and standard deviation of $s$ over the collection of cells, the average $<s>$ and its standard deviation $\delta$ s. In other cases we are more interested in the properties of the statistical distribution of $s$ over the cells itself. In the sections over circular and linear shape of the defect we will concentrate on averages of the shape parameters, here we will present two approaches that utilise the specific characteristics of the distribution over a parameter $s$.

\section{i. Statistical Cell Distribution Parameters}

Once we have obtained a collection of cells that practically cover $Z$, we can extract a number of useful statistical properties. Especially useful are the average size, intensity, shape, and orientation of the cells, and their standard deviations. More useful parameters that are relevant for specilic defects are discussed in the following sections. Table 3 presents a summary of parameters that can be computed for one cell, and able 4 presents a overview of statistical paramelers defined for an ensemble of cells.

\begin{tabular}{|c|c|c|c|}
\hline Cell Parameter & Symbol & Description & Definition \\
\hline Cellsize & $A_{k}$ & Size of cell $\omega_{k}$ & $\Lambda_{k}=\int_{t w k} d_{2} x$ \\
\hline Cellintensity & $\mathrm{I}_{\mathrm{k}}$ & Intensity of cell $\omega_{k}$ & $I_{k}=\int_{c u k} Z(x) d_{2} x$ \\
\hline Cellshape & - & Shape parameters of cell $\omega_{k}$ & see later sections \\
\hline Col/COM & $z_{k}$ & Centre-of-mass of cell $\omega_{k}$ & $z_{k}=\int_{(2) k} z(x) d_{2} x / I_{k}$ \\
\hline Cellorientation & $\phi_{k}$ & Mean orientation of cell $\omega_{k}$ & $\cos \phi_{\mathrm{k}}=\int_{\mathrm{u}, \mathrm{k}}<\mathrm{x}-\mathrm{z} \mid \mathrm{u}_{1}>\mathrm{Z}(\mathrm{x}) \mathrm{d}_{2} \mathrm{x} / \mathrm{I}_{\mathrm{k}}$ \\
\hline
\end{tabular}

Table A3 Some uscful cell parameters

\begin{tabular}{|c|c|c|}
\hline Ensemble Parameter & Description & Definition \\
\hline$N_{u} m C_{c} / / s$ & The total number of cells & - \\
\hline AvCellsize & Average size of the cells & $\left\langle A_{k}\right\rangle$ \\
\hline StdCellSize & Standard deviation in size of the cells & $\delta \mathrm{A}^{2}=\left\langle\mathrm{A}_{\mathrm{k}}\right\rangle^{2}-\left\langle\Lambda_{k}^{2}\right\rangle$ \\
\hline AvCellhnensify & Average intensity of the cells & $\left\langle\mathrm{I}_{\mathrm{k}}\right\rangle$ \\
\hline StdCellmensity & Standard deviation in intensity of the cells & $\delta \mathrm{I}^{2}=\left\langle\mathrm{I}_{k}\right\rangle^{2}-\left\langle\mathrm{I}_{k}^{2}>\right.$ \\
\hline CellDensity & The average density of cells over the image & NumCells/lmageSize \\
\hline AvOrientation & Average cell orientation & $\phi=\left\langle\phi_{k}\right\rangle$ \\
\hline StdOrientation & Standard deviation in cell orientation & $\delta \phi^{2}=\left\langle\phi_{k}\right\rangle^{2}-\left\langle\phi_{k}^{2}\right\rangle$ \\
\hline
\end{tabular}

Table A4 Some statistical cell parameters

Another useful criterion is the type of the statistical distribution $P$ that describes the frequency of observed values of a parameter $\xi$ over the collection of cells. In this respect we are especially interested in the relation of $P$ to the Normal Distribution $N(\mu, \sigma)$. Therefore we detine the distance to the Nomal Distribution as:

$$
\mathrm{E}_{\mathrm{N}}=\int_{\Xi}(P(\xi)-N(\mu, \sigma, \xi))^{2} \mathrm{~d} \xi / \oint_{\Xi} \mathrm{d} \xi
$$

With $\mu=\langle\xi\rangle$, the average over the data set, and $\sigma$ the standard deviation in the data set. The fit of the distribution with the Nomal Distribution can then be defined as:

$$
f_{\mathrm{N}}=f\left(\mathrm{E}_{\mathrm{N}}\right)
$$


with any continuous monotonous decreasing function ' $f$ ' that obeys $f(0)=1$ and $f(x) \downarrow 0$ for $x \rightarrow \infty$. Similarly, the standard deviation in $f_{N}$ can be calculated. This fit $f_{N}$ conveys the degree to which the observed frequency distribution obeys a Normal Distribution. This is particularly relevant for the distinction between small-scale defects like for instance 'air bubbles', 'foam' and 'dirt particles'. In experimental studies it was found that 'air bubbles', 'foam' and 'dirt particles' fit very well with Normal Distributions, while for instance 'ink missing' dies not. Moreover, 'dirt particles are characterised' by sharp peaks located at the average particle-size, whilst 'air bubbles' and 'foam' have broad flanks. another observation is that the sharply peaked average particle size is highly variable. whilst the broadness of the peak always is very low. This spread in observed average size holds in a Iesser degree for 'foam' and 'air bubbles'. Some examples of observed distributions and their fit with Normal Distributions are represented in Figure A.10 and table 5. Here, however, the standard deviation is much larger. The fit with a Normal Distribution can, as explained in appendix A.3, calculated with any continuous monotonous decreasing function $f$ that complies with the empirical data. In this case the fit-lunction is expressed as:

$$
f_{\mathrm{N}}=f\left(\mathrm{E}_{\mathrm{N}}\right)=1 /\left(1+\left(\mathrm{E}_{\mathrm{N}} / \mathrm{K}\right)\right)
$$

with $\mathrm{K}=0.1225$ to obtain the best fit to empirical data.

In Figure $A .10$ three examples of small-scale defects are shown.

\begin{tabular}{|l|l|l|l|}
\hline Defect & $\boldsymbol{\mu}(\boldsymbol{\mu m})$ & \multicolumn{1}{c|}{$\boldsymbol{\sigma}(\boldsymbol{\mu m})$} & \multicolumn{1}{c|}{$\boldsymbol{f}_{N}$} \\
\hline 'dirt particles' DP1 & 70 & 4 & 0.76 \\
\hline 'dirt particles' DP2 & 28 & 3 & 0.81 \\
\hline 'dirt particles' DP3 & 120 & 7 & 0.78 \\
\hline 'foam' F1 & 71 & 12 & 0.95 \\
\hline 'foam' F2 & 170 & 21 & 0.91 \\
\hline 'foam' F3 & 102 & 16 & 0.92 \\
\hline 'air bubbles' AB1 & 535 & 62 & 0.70 \\
\hline 'air bubbles' AB2 & 430 & 56 & 0.75 \\
\hline
\end{tabular}

Table A5 Average sizes and standard deviation of some defects and fit with Normal Distribution 

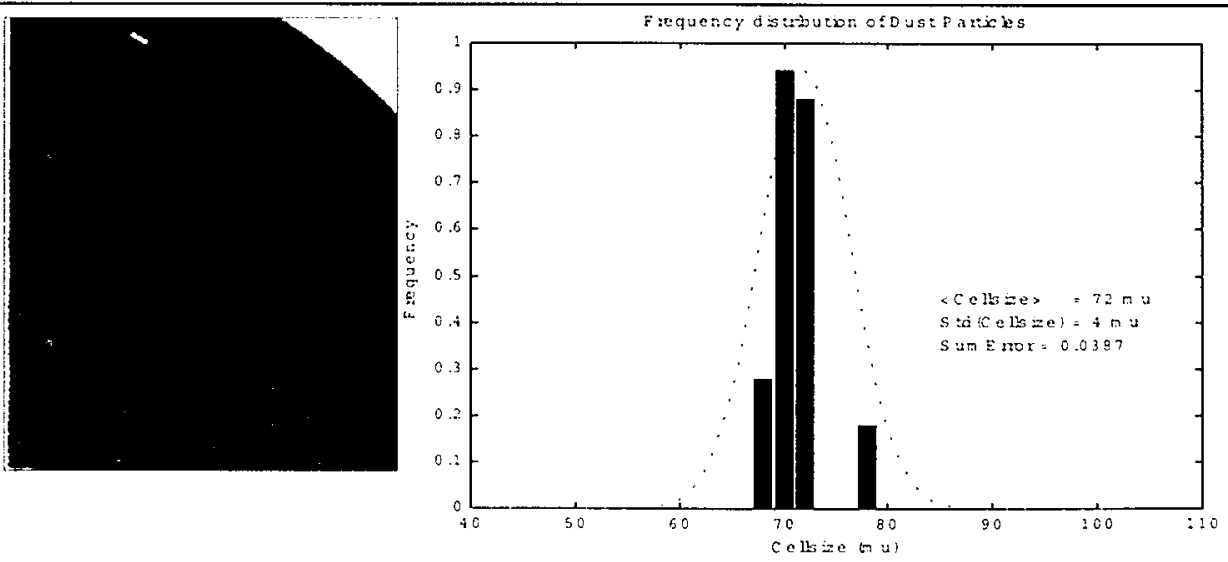

Fig. A9 |a|. Typical image of 'dirt particles' and relative frequency distribution with best fitting Normal Distribution (rescaled to same size). Fit $=0.760$.
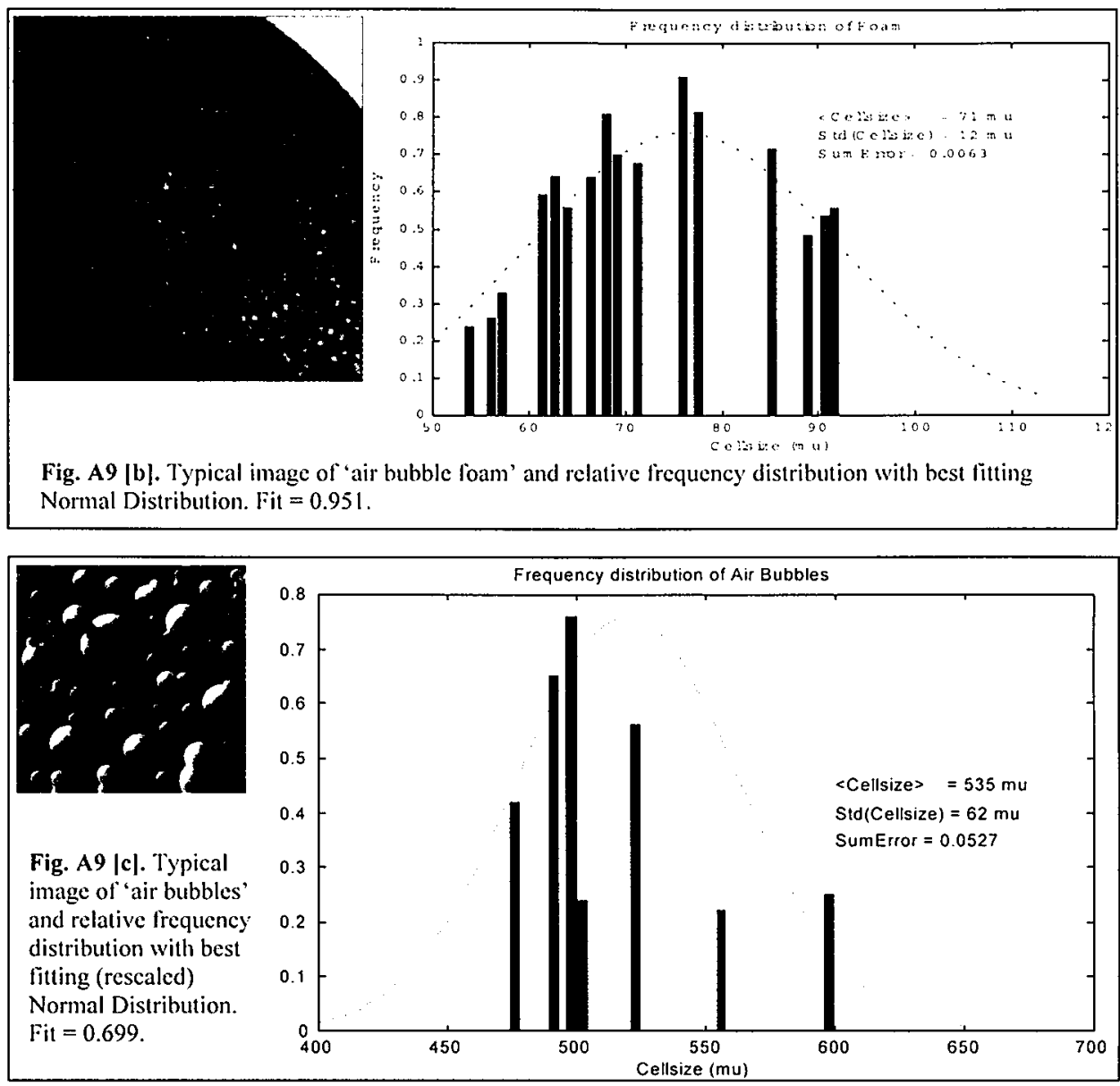

Fig. A9 |c|. Typical image of 'air bubbles' and relative frequency distribution with best fitting (rescaled) Normal Distribution. $\mathrm{Fit}=0.699$. 


\section{ii. Fractal Sealing Laws in the Cell Size Spectrum}

In this section we will present a special kind of statistical relation in an image; the relation between the defect class and the frequency distribution over the cell sizes. Like the average Z-density presented in the previous section, this method operates on the global $Z$. This quantity is determined from the statistical properties of the difference $Z$. The method stems from the experimental observation that $Z$ often consists of a number of smaller and larger fragments with varying intensity and size. as shown in Figure A.11. This fact is reminiscent of many fractal images. These kinds of relations are governed by scaling laws, like the famous Mandelbrot-Prick law. Here we investigate whether this relation correlates with the delect class. A typical example (from a real object) is given in Figure A.11. From this higure it becomes clear that the method can distinguish 'hairs', but has difficulty in distinguishing 'misregistration' from 'double print'.
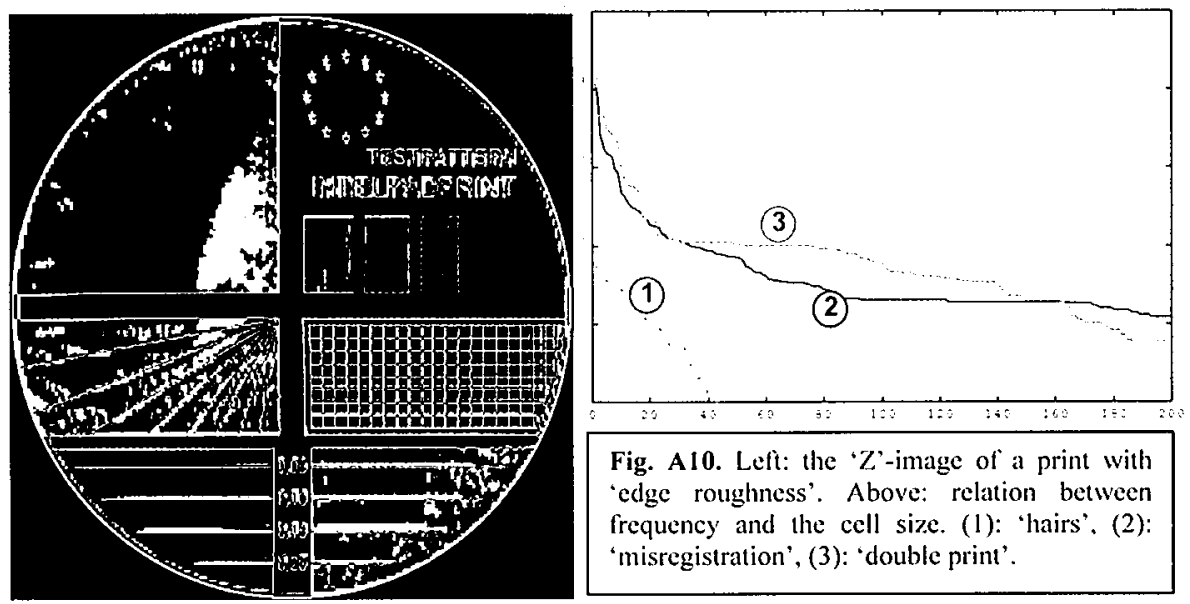

Fig. A 10. Left: the ' $Z$ '-image of' a print with 'edge roughness'. Above: relation between frequency and the cell size. (1): 'hairs', (2): 'misregistration', (3): 'double print'.

An actual inage may differ on several not-connected areas from the template. Therefore the absolute difference $Z$ will reveal a large collection of cells of varying size. This is represented in the left part of Figure A.11. Next, the cells - i.e. the isolated areas visible in this image - are labelled, and then sorted in decreasing cell size. These cell-sizes are represented in a logarithmic plot. This plot is called the cellsize fiequency-spectrum of the defect. The frequency-spectra lor three different defect classes are depicted in the right part of Figure $A .11$. Here, the $X$-axis indicates the cell size, and the $Y$-axis indicates the number of area's with this specific cell size. The frequency-spectrum was calculated for a

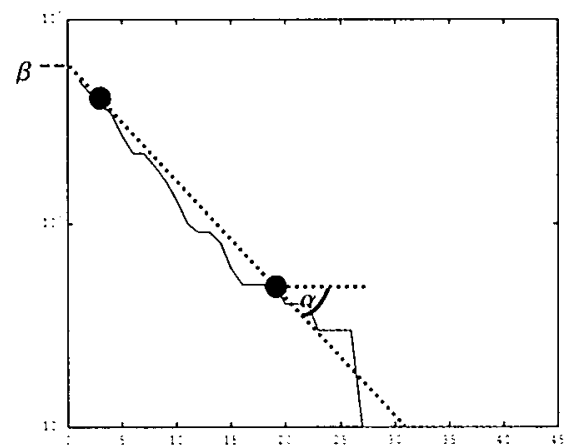

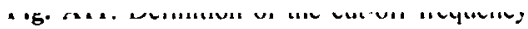
' $\beta$ ' and the gradient ' $\alpha$ ' in frequency-size set of 85 examples. For this set indeed a correlation was found between the spectrum and the defect class. However, the correlation was at best weak. In order to measure the strength of the relation, two parameters were delined to describe the spectrum. The spectrum was fit using LSQ with a line with gradient $-\alpha$ (in order that $\alpha$ was positive), and $\beta$ the cut-off' frequency for cell-size ' 0 '. So the equation of the line was $\mathrm{Y}=\alpha \mathrm{X}+\beta$, with $X$ the cell-size and $Y^{\prime}$ the cell-size frequency. and $\alpha=<d \ln (Y) /$ $\mathrm{dX}>$. Figure $A .12$ illustrates the definitions of $\alpha$ and $\beta$. Figure $A .13$ shows the values of $\alpha$ and $\beta$ with their standard deviation for the defect classes in the test-set. From Figure $\Lambda .13$ it is clear that most values lie within their deviation in a narrow band, therefore the diagnostic value is low. Similarly, it appears that parameter $\beta$ has somewhat more discriminative value than $\alpha$. Another disadvantage of this approach is that it consumes considerable computation time. In practice. however, it is found that this method ean 
contribute significant information that may just help in deciding between certain alternatives. Fractal scaling laws in both images and scanprofiles are discussed in detail in section 2.7.
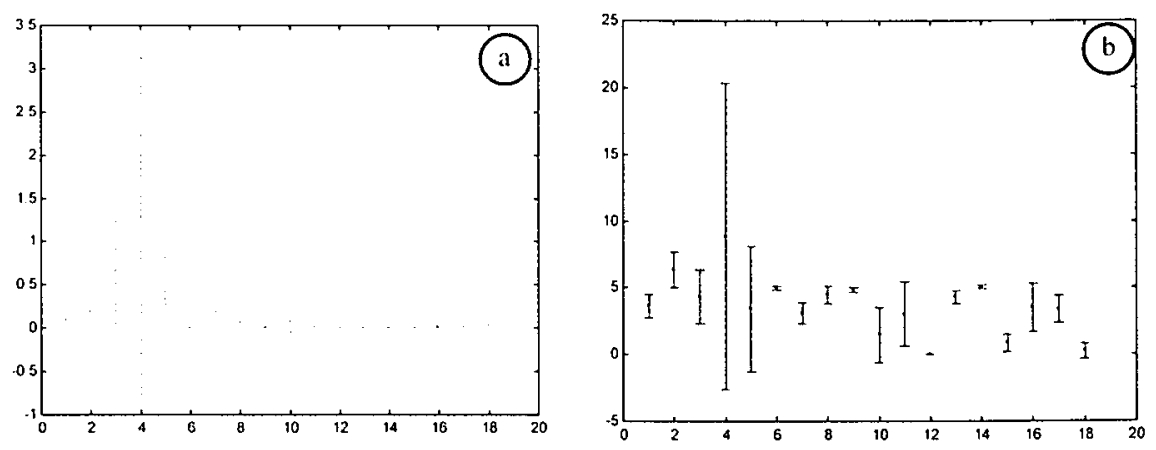

Fig. A12 (a): The value of the gradient ' $a$ ' as function of the -defect class for 85 examples. (b): 'The Value of cut-off frequency ' $b$ ' as function of the defect class for 85 cxamples 


\section{A.6 Identification of Spherical versus Irregular Shapes.}

With the application of a focused seanning method to an image it will in general not be possible to distinguish between a number of more-or-less similar defects. One example is the set of defects defined by unwanted intensity variations occurring at specific locations within the printed area. This may be caused by 'air bubbles', 'absence of ink', 'inhomogenities in the ink', or 'dirt particles' enclosed in the ink. In order to discriminate between these possibilities specific tests are designed that describe the general shape of the inhomogenities. Particular relevant criteria in this context are tests that convey whether the inhomogenity is of more-or-less spherical shape. Such tests could discriminate air bubbles' from other defects. In literature, some parameters and algorithms are presented for the detection and characterisation of ellipses through Projective Geometry and the Hough-Transform, see: [Bennet et al., 1999]. Below, we present a number of suitable 'shape-parameters' for spheral detection.
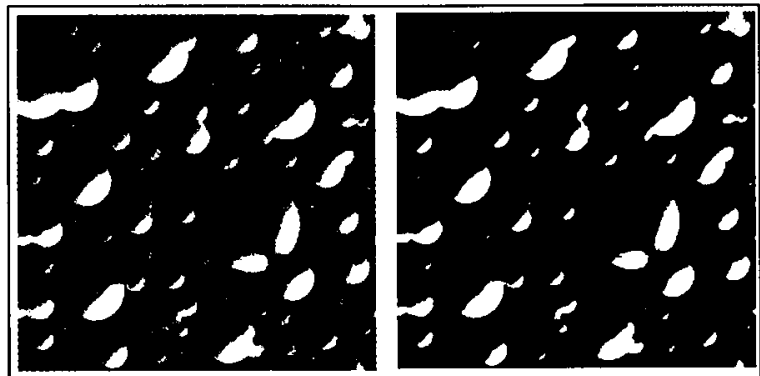

Fig A13. Effect of thresholding. Left: image with 'air bubbles'. right: image after thresholding with threshold $=0.7$ The typical circular shape of the bubbles is lost.

Figure A13 shows the dilemma typical for thresholding. On the one hand thresholding is necessary in order to limit the amount of information to manageable sets and obtain a meaningful partition of $Z$, on the other hand thresholding distorts images to an extent where the typical features are destroyed. This is the case in the image above where the circular features are almost annihilated after thresholding.

\section{i. Convexity}

A first useful test in order to detect whether a shape is circular is to determine 'how' convex it is. In order to do so, we first review the classical definition of the concept convex. A planar object $S$ is called convex if for each pair of points in $S$ the line fragment that connects them falls entirely inside $S$.

$$
\forall X, Y \in S \Rightarrow X Y \subset S
$$

Based on this definition we define the feature parameter convexity, denoted $C . C$ is a measure that expresses the degree in which $S$ can be considered as convex. $C$ must be defined such that $C \in[0,1]$, and $C=1$ implies that $S$ is convex in the 'traditional' sense. We review two possible definitions.

[a] First, we define convexity as the fraction of lines connecting border points of $S$ that fall entirely within $S$. Let $V_{1}=\{X, Y \in \partial S \mid X Y \subset S\}$ and $V=\{X, Y \in \partial S\}$. We restrict the calculation to $\partial S$ because the probability that $X Y$ falls (partly) outside $S$ is larger if $X, Y$ lie on the border of $S$. Now define $c_{1}$ as: $c_{1}=\# V_{1} / \# V$. Since $V_{1} \subseteq V$ we know that $c_{1} \in[0,1]$. This definition is a good extension of the classical concept convex. An important disadvantage of this approach. however, is that if a line fragment $X Y$ falls just a fraction outside $S$ the line is no member of $V_{1}$. For this reason a large number of points must be investigated before a reasonable value of $c_{l}$ is available. To ease this effect we consider the next definition of convexity.

[b] For a line fragment $X Y$, with $X, Y \in \partial S$, we define the fraction of the line that falls inside $S$ as: $\sigma(\mathrm{XY})$. No we consider the ensemble of these fractions of all line fragments:

$$
L=\{\sigma(\mathrm{XY}) \mid \mathrm{X}, \mathrm{Y} \in \partial S\}
$$

Here we define convexity $c_{2}$ as the average over the ensemble $L$ : 


$$
c_{2}=\langle L>
$$

For a true convex set $S$ we know that $L=\{1\}$, so $c_{2}=1$. The lower the average fraction of line fragments inside the object, the lower its convexity. Therefore this is also an adequate extension of the concept convex. This definition of $c_{2}$ will sooner reach a representative value, for it is continuous rather than the discrete definition of $c_{1}$.

Consequently we base our definition of convexity on the second definition: $C=c_{2}$. The quantity $C$ can be estimated with Monte Carlo integration. Figure A.15 shows two examples. Such examples illustrate

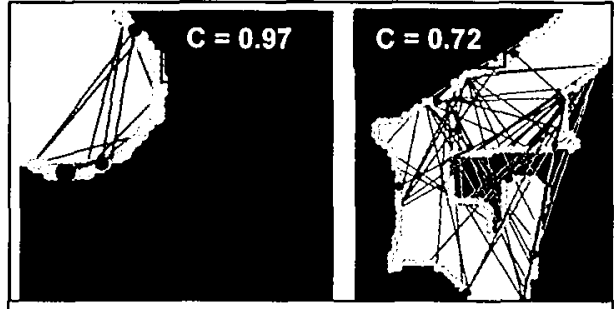

Fig. A14 Examples of a computation of Convexity. Right a spherical shape that yields a convexity of 0.97 , left an irregular shape, yielding a of 0.72 . that $C$ gives a value that corresponds well with our interition of the concept convexity.

For the morc realistic case that an image consists of a number of disconnected components the statistical approach as defined in appendix $A .3$ is applied. This means that we must estimate the statistical distribution $P_{C}$ of $C$ over the cells; where $P_{C}(C) d C$ represents the fraction of cells with convexity $C$ in the interval $\angle C, C+\mathrm{d} C\rangle$. In this way $C$ can be calculated as an average over the collection of cells: $\angle C>=\int C P_{C}(C) \mathrm{d} C$. Similarly, the standard deviation in $C$ can be calculated. Convexity is hardly sensitive to the effects of thresholding as defined in appendix $A .3$. 'This is caused by the fact that after thresholding images of 'air bubbles' may loose there typical circular shape, but retain a high degree of convexity. Therefore 'air bubbles' can be characterised by their high degrec of convexity. Certain problems are caused by the fact that the operation 'edge extraction' yields some noise, i.e. disconnected points around the object. This decreases incorrectly the fraction $\sigma(X Y)$ for certain points $X, Y$. For example for the true circle left in Figure A.15, this causes that $C$ does not exhibit its actual value ' 1 ' but attains the somewhat lower value ' 0.97 '.

Figure A. 14 shows an averaged frequency distribution for convexity based on a large number of images of 'air bubbles' and 'irregular shapes'. These frequency distributions are used to represent a fuzzy membership function $\mu_{C}(C)$ that denotes the degree to which an image $Z$ exhibiting an eccentricity $C$ can be considered as belonging to defect class 'air bubbles'. The expected degree that the collection of cells represent a defect with 'air bubbles' is then expressed by:

$$
\left\langle\mu_{C}\right\rangle=\int \mu_{C}(C) \mathrm{P}_{C}(C) \mathrm{d} C
$$

Similarly, the standard deviation $\mathrm{d} \mu_{C}$. can be calculated. The pair $\left\{<\mu_{C}>\mathrm{d} \mu_{C}\right\}$ can be used to convey a qualitative confidence in our belief that the defect belongs to the type 'air bubbles'.
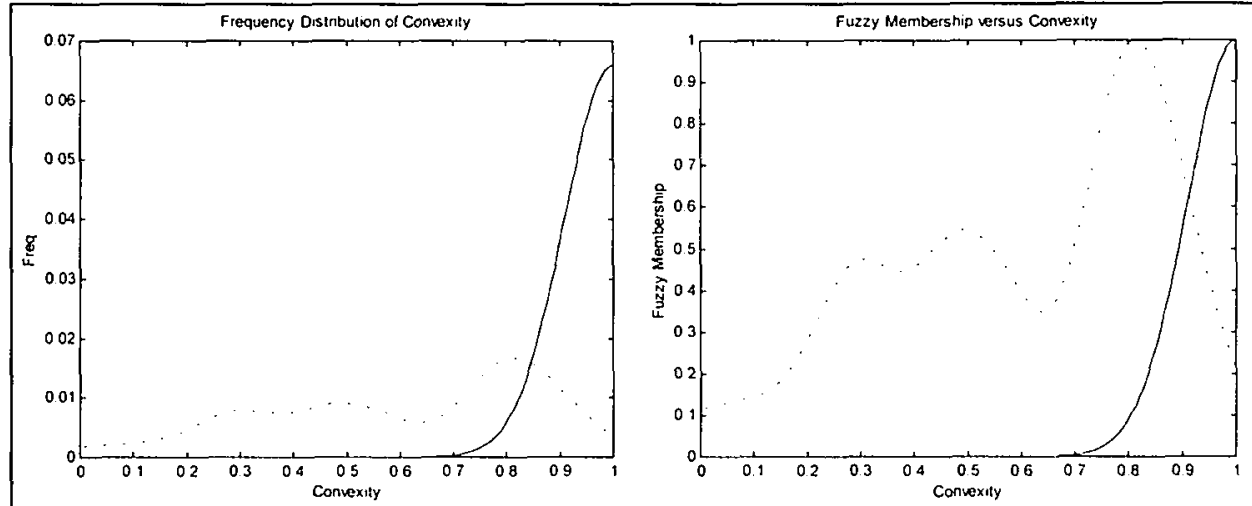

Fig. A15. Left: Frequency distribution versus convexity, solid: 'air bubbles', dotted: irregular shapes. The distribution is the weighted sum of the frequency distributions of individual images. Right: Fuzzy Membership-functions obtained from the frequency distributions by division by the maximum value attained. 
Summarising, the concept of convexity gives a good indication for 'air bubbles'. At least in the forward sense: the presence of "air bubbles" implies a high convexity. The other way is more troublesome as certain irregular shapes can have high convexity, even up to 1 . Therefore this method is not fully capable of discriminating 'air bubbles' from other defects. This can only be done by involving other sources of information. like for instance the distribution of cell-size. An advantage of this approach is that it can deal reasonably well with crescent-shaped bubbles like in Figure A.14b. Moreover, this method is reasonably fast and efficient. An other advantage is that the approach does not depend on the scale of the image used.

\section{ii. Eccontricity}

Another shape parameter that defines the degree of 'circularity' of an object is its eccentricity, as used normally in the description of elliptical orbits in planetary systems. For an elliptical orbit with radii $r_{\mathrm{min}}$ and $r_{\max }$ - representing the small and rage radius - the eccentricity ' $\xi$ ' is defined as [Unsöld1976]:

$$
\xi=r_{\min } / r_{\max }
$$

So $\xi \in[0,1]$, with $\xi=1$ defining a true circle, and $\xi=0$ defining a line segment. The essential steps in determining the eceentricity of a shape are shown in diagram 17.The starting point for the calculation of eccentricity is the absolute difference $Z$, of one more-or-less circular shape(s) $S$. The shape is defined by: $Z(x)>\theta$, for some given threshold $\theta \in[0,1]$, so $Z(x) \leq \theta$ implies that $x$ is no member of $S$. Next the edge $\partial S$ of $S$ is determined, resulting in a set of points $E=\left\{x_{n} \mid x_{n} \in \partial S\right\}$. In order to establish the degree to which $E$ fits with a circle. we first match these points on the edge with a quadric $Q$ :

$$
Q\left(q_{1}, q_{2}, q_{3}, q_{4}, q_{5}, q 6, x\right)=q_{1} x_{1}^{2}+q_{2} x_{2}^{2}+q_{3} x_{1} x_{2}+q_{4} x_{1}+q_{5} x_{2}+q_{6}=0
$$

using an additional constraint $C$ :

$$
C\left(q_{1}, q_{2}, q_{3}, q_{4}, q_{5}, q_{6}\right)=q_{1}+q_{2}+q_{3}+q_{4}+q_{5}+q_{6}-1=0
$$

In order to compute the best fitting values for $\mathbf{q}=\left(\mathrm{q}_{1}, \mathrm{q}_{2}, \mathrm{q}_{3}, \mathrm{q}_{4}, \mathrm{q}_{5}, \mathrm{q}_{6}\right)$ for litting $E$ with $Q$ we apply Least Stuctres (LSQ) with a quadratic critcrion function in $Q$ and Laplace multiplier in $C$. In this way we obtain a set of equations in $q$ that result in the best litting values for $\mathbf{q}^{*}$ with errors $\mathrm{dq}^{*}$. From the general expression for a quadric [2] we can subdivide the situations that represent an ellipse. They are defined by those $q$ that obey:

$$
q_{1} q_{2}>0
$$

If this condition is not fulfilled then the ecentricity is set to zero: $\xi=0$, otherwise $\xi$ is defined by:

$$
\xi=\left(q_{1}+q_{2}-\sqrt{ } D\right) /\left(q_{1}+q_{2}+\sqrt{ } D\right), \text { where } D=\left(q_{1}-q_{2}\right)^{2}+q_{3}^{2}
$$

Using the standard deviation dq we can likewise determine an expression for the standard deviation $\mathrm{d} \xi$.

In order to define the quality of the match, we lirst deline the distance $D$ from a point $x$ from $E$ to a true circle with centre $m$ and radius $R$ :

$$
D(\mathrm{x})=|\|\mathbf{x}-\mathbf{m}\|-R|
$$

So $D \geq 0$, with $D(\mathbf{x})=0$ implying that $\mathbf{x}$ is on the circle. Now we can define the $f i t f_{\mathrm{E}}$ of $E$ with circle (II.R) as:

$$
f_{\mathrm{l}}=\sum_{\mathbf{x} \in \mathrm{E}} \theta(1-D(\mathrm{x}) / \lambda R) / \mathrm{n}_{\mathrm{l}}
$$

where $\theta(x)$ is defined as $x$ if $x \geq 0$, and 0 elsewhere, and $n_{E}$ is the number of elements of $E$. This means that only points of $E$ closer than $\lambda R$ to the circle are involved, cach of those attributing a fraction $1-$ $D(x) / \lambda R$. In our calculation we chose $\lambda=0.25$. This means that $f_{1:} \in[0,1]$, with $f_{\mathrm{E}}=1$ defining a perfect hit, and $f_{1:}=0$ implying that all points of $E$ are more that $\lambda R$ away from the circle. $\Lambda$ similar expression can be derived for an ellipse.

Using the lit of $E$ with the quadric $Q$, we can now redefine the measure for eccentricity as $\xi_{\mathrm{E}}$, defined by:

$$
\xi_{1:}=\xi f_{1:}
$$

Again $\xi_{1:} \in[0.1]$, but now with $\xi_{1:}=1$ delining both a perfect lit with a circle, as also $r_{\min }=r_{\max }$. Parameter $\xi_{\mathrm{E}}$ is better able in defining a circular shape than $\xi$. An example is the case where $E$

\footnotetext{
${ }^{4}$ On average $\approx 0.1 \mathrm{sec}$ with Matlab on a $400 \mathrm{MHz}$ PentiumPro PC under Windows NT.
} 
represents a square. In that case the best fit with a quadric results in a true circle, so $\xi$ from equation [5] gives ' 1 '. However the fit $f_{\mathrm{E}}$ : will be less than 1 , resulting in an eccentricity $\xi_{\mathrm{I}}=f_{\mathrm{I}}<1$.

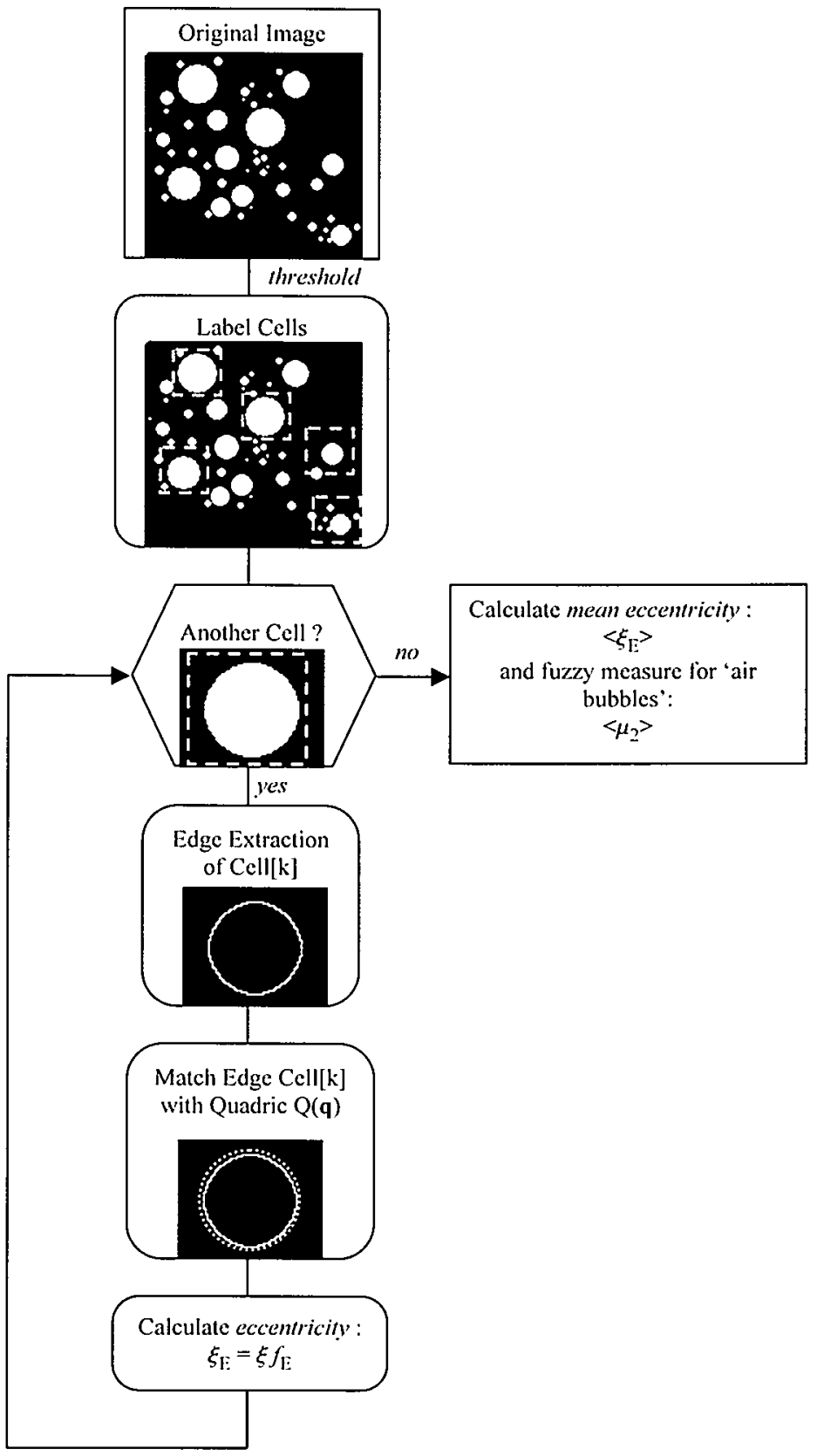

Fig. A.16. Schematic representation of the steps in the calculation of the eccentricity of an image. 
For the more realistic case where a number of fragments belonging to the absolute difference $Z$ need to be investigated - 'air bubbles' always occur in aggregations - we need a statistical approach as defined in appendix A.3. This means that we must explore the statistical distribution $P$ of $\xi_{\mathrm{E}}$ over the cells; where $P\left(\xi_{\mathrm{E}}\right) \mathrm{d} \xi_{\mathrm{E}}$ represents the fraction of cells with eccentricity $\xi_{\mathrm{E}}$ in the interval $\left\langle\xi_{\mathrm{E}}, \xi_{\mathrm{E}}+\mathrm{d} \xi_{\mathrm{F}}\right\rangle$. In this way $\xi_{\mathrm{E}}$ can be calculated as an average over the collection of cells: $\left\langle\xi_{\mathrm{E}}\right\rangle=\int \xi_{\mathrm{E}} \mathrm{P}\left(\xi_{\mathrm{E}}\right) \mathrm{d} \xi_{\mathrm{E}}$. Similarly, the standard deviation in $\xi_{\mathrm{F}}$ can be calculated.

In Figure $A .18$ a number of examples are shown that illustrate that this method is capable of identifying 'air bubbles' versus irregular shapes.

$\xi_{\mathrm{E}}=0.81 \pm 0.24 \quad \xi_{\mathrm{E}}=0.85 \pm 0.15$
$\mu_{2}=0.76 \pm 0.04 \quad \xi_{\mathrm{E}}=0.72 \pm 0.3$

For a large number of shapes the eccentricity was computed using equation [32]. In Figure A.18 the resulting frequencies of these experiments for respectively 'air bubbles' and 'irregular shapes' are shown. These frequency distributions are used to represent a fuzzy membership function $\mu_{2}\left(\xi_{\mathrm{V}}\right)$ that denotes the degree to which an image $Z$ with eccentricity $\xi_{1}$ can be considered as belonging to defect class 'air bubbles' ('2' because the defect number of 'air bubbles' is ' 2 '). This empirical measure is used to compute the expected degree that the image represents 'air bubbles'. If the statistical cell approach described above results in a distribution $\mathrm{P}\left(\xi_{\mathrm{E}}\right):$ the fraction of cells with eccentricity $\xi_{1}$, then the expected degree that the collection of cells represent 'air bubbles' is:

$$
\left\langle\mu_{2}>=\int \mu_{2}\left(\xi_{\mathrm{F}}\right) \mathrm{P}\left(\xi_{\mathrm{E}}\right) \mathrm{d} \xi_{!}:\right.
$$

Similarly, the standard deviation $\mathrm{d} \mu_{2}$ can be calculated. The pair $\left.\left\{<\mu_{2}\right\rangle, \mathrm{d} \mu_{2}\right\}$ can be used to establish a qualitative confidence we can have that the defect belongs to the type 'air bubbles'.
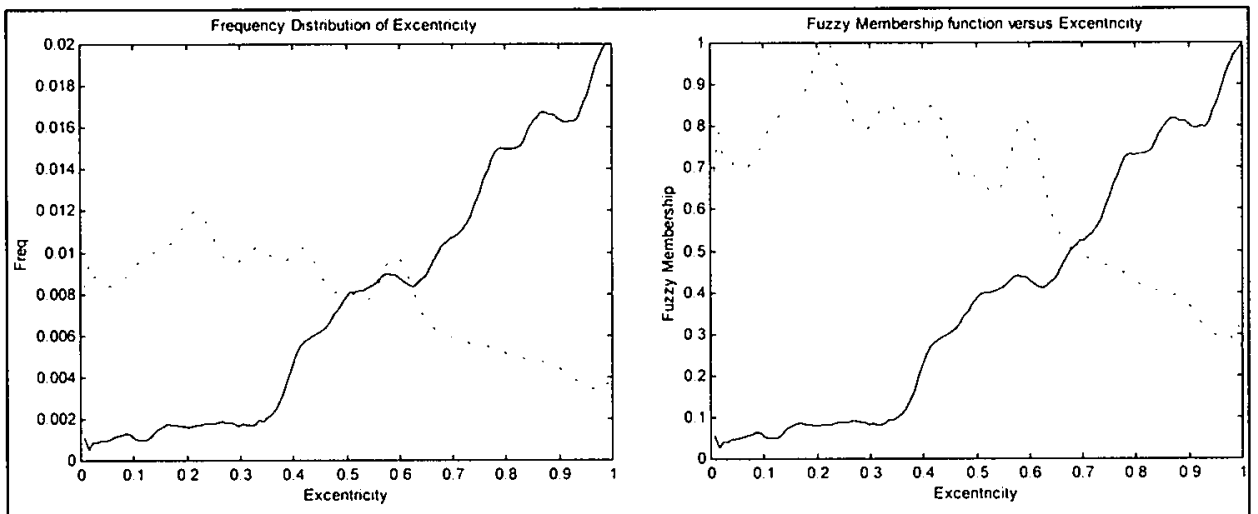

Fig. A.18.. Left: Frequency distribution versus eccentricity. solid: 'air bubbles', dotted: irregular shapes. The distribution is the sum of the frequency distributions of individual images. Right: Fuzzy Membershipfunctions obtained from the frequency distributions by division by the maximum value attained.

Summarising it was found that eccentricity defined by [32] is a good indication of 'air bubbles'. $A t$ least in the forward sense; it is that the presence of 'air bubbles' implies that $\left\langle\mu_{2}\right\rangle$ is high. The other way is more cumbersome. Certain irregular shapes can have $r_{\min } \approx r_{\text {max }}$, with low but still reasonable fit $f_{\mathrm{E}}$, for instance 0.7 . In that case the eccentricity $\xi_{\mathrm{E}}=1 \times 0.7$ is also reasonable high. and well within 
the range of 'air bubbles'. 'Therefore this method is not fully capable of discriminating 'air bubbles' from other defects. This can only be done by considering other sources of information, like for instance the distribution of cell-size. Moreover, this method is reasonably fast ${ }^{5}$ and efficient. $A$ disadvantage is that the approach depends on the scale of the image used. Since 'air bubbles' have characteristic cell size distributions, too large views show not enough detail. Fig 20 shows a real image of "air bubbles", where the individual bubbles are to small for identification and computation of $\xi_{1}$ :

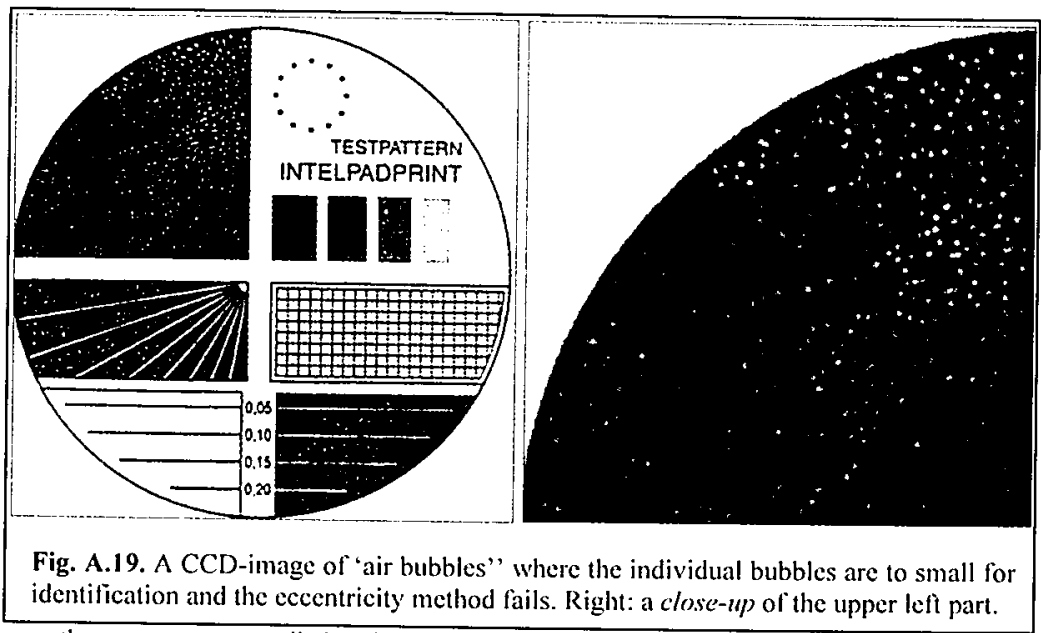

In this case the system cannot distinguish between similarly small defects like 'dirt particles' and 'orange peel'. Here the method clearly fails. What should happen in such a case is that the CCD-camera should focus in on these defects, such that the individual cells can be identified and the analysis can be applied successful.

\section{iii. Perimeterity}

The method for eccentricity described above is efficient but complex. Instead of the whole matehing process using $L S Q$ and computation of eccentricity, a more simple approach is possible. After thresholding and partitioning of the cells. at the stage where the individual cell is derived and its edge is inferred, a simple measure for the circular shape of the cell is: $4 \pi \mathrm{A} / \mathrm{E}^{2}$, a quantity that we will detine as perimeterity with symbol $p$.

$$
p=4 \pi \mathrm{A} / \mathrm{E}^{2}
$$

Here $A$ is the area of the cell, and $E$ is the perimeter of the cell. This value gives the quotient between the observed area $A$ of the cell, with the area $A_{\text {circle }}=\mathrm{E}^{2} / 4 \pi$ if the cell was a circle with the observed perimeter $E$. Since with a given perimeter $E$ the area $A$ is always smaller than or equal to $A_{\text {circle. }}$ the value $p=A / A_{\text {circle }}$ is ' 1 ' only for true circles. For all other shapes is $0 \leq p<1$.

The advantage of this approach is that it is much faster than the eccentricity method". The disadvantage of this approach is that it is quite sensitive for the inevitable thresholding. As discussed before. this may distort the circular shapes of air bubbles towards crescents or even amorphous blobs, with negative effect on perimeterity $p$. An observation is that the values of $p$ are often not far from 1 , but this does not have important effects on classification techniques using $p$. The result is that perimeterity has somewhat less discriminating power as eccentricity. In Figure A.18 a number of examples are shown that illustrate the capability of this method to identify 'air bubbles' versus irregular shapes.

\footnotetext{
5n average $\approx 1$ sec with Matlab on a $400 \mathrm{MHz}$ PentiumPro PC under Windows NT.

"On average $<0.2 \mathrm{sec}$ with Matlab on a $400 \mathrm{MHz}$ PentiumPro PC under Windows NT.
} 


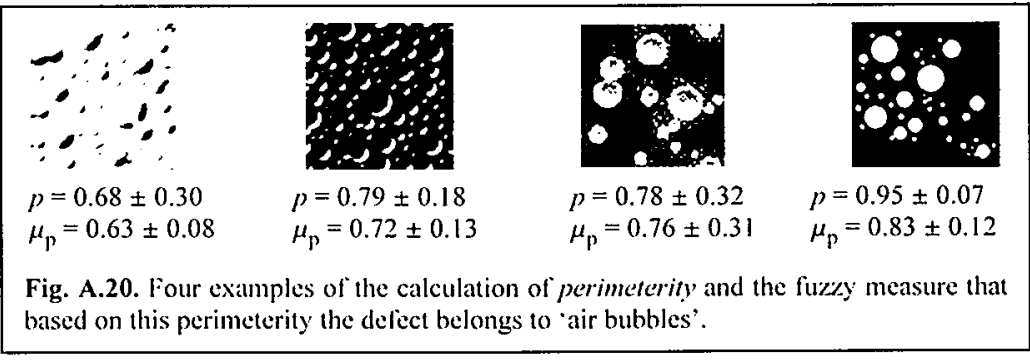

The quantity perimeterity was computed for a large number of shapes using equation [1]. In Figure A.22 the resulting frequencies of these calculations are shown for respectively "air bubbles" and 'irregular shapes', similar to Figure A.16. Similarly as above, these frequency distributions are used to compute the fuzzy membership function $v_{2}(p)$ that denotes the degree to which an image with perimeterity $p$ can be considered as belonging to defect class 'air bubbles'. This empirical frequency diagram is used to compute the expected degree that an image represents defect "air bubbles". If the statistical cell approach described above results in a distribution $F(p)$ : the fraction of cells with perimeterity $p$, then the expected degree that the collection of cells represent 'air bubbles' is:

$$
\begin{aligned}
& <p>=\int p F(p) d p \\
& <v_{2}>=\int v_{2}(p) F(p) d p
\end{aligned}
$$

Similarly, the standard deviations $\mathrm{d} p$ and $\mathrm{d} v_{2}$ can be calculated. The pair $\left\{\left\langle v_{2}\right\rangle, \mathrm{d} v_{2}\right\}$ can be used to establish a qualitative confidence we can have that the defect belongs to the type 'air bubbles' - give the average perimeterity $p$.

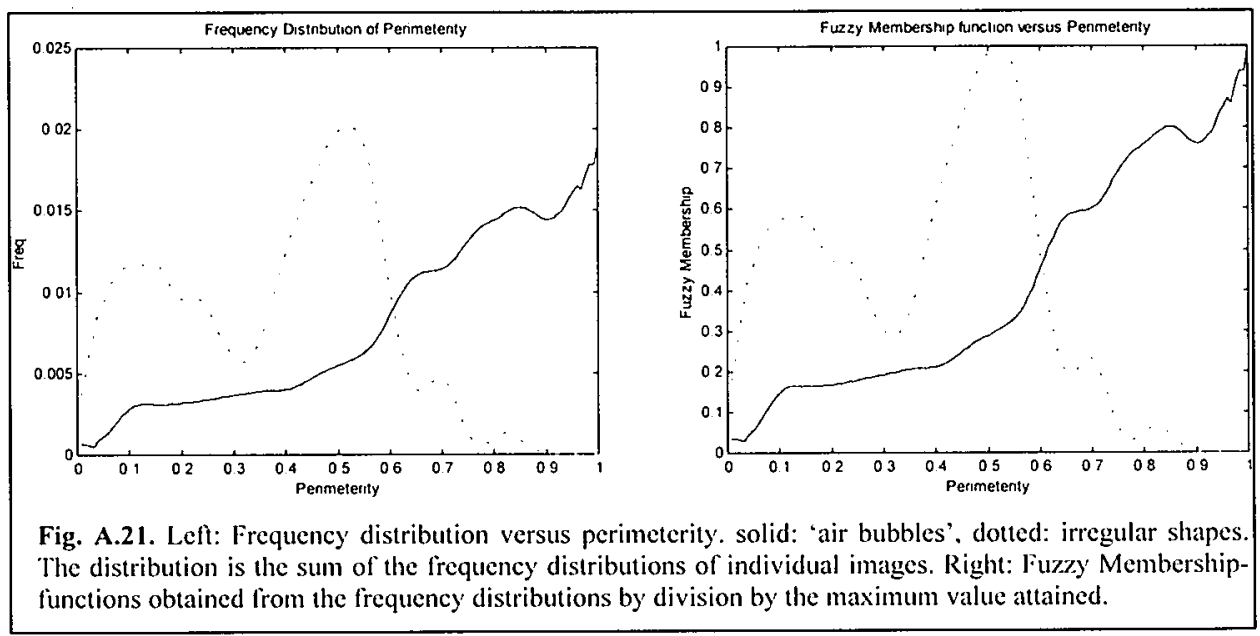

iv. Comelation, Integration and Synergy between Comexity, Eccentricity Perimeterily.

In this section three leature parameters were introduced that indicate the degree in which a defect looks like the defect 'air bubbles'. These parameters have in common that for the defect 'air bubbles' they attain high values. However, on the other hand high values of these parameters need not necessarily imply that the defect is unambiguously 'air bubbles'. Combined these parameters reveal more information than on their own. Still, the discriminatory power of these test are limited. This synergy is illustrated in Figure A.22. In that figure are a number of the defect 'air bubbles' versus 'irregular' shapes' are plotted in the $\left(\xi_{E}, p\right)$-plane. This representation clearly shows the relation in location between the two sets. An other useful representation is to plot the probability $P_{A B}$ as third axis over the 
$\left(\xi_{\mathrm{E}}, p\right)$-plane, indicating for each point on the plane the probability that it represents a defect 'air bubbles'. The plot in Figure $A .22$ is also helpful in visualising the correlation between the two parameters $\xi_{1}$ and $p$. From this figure. and similar plots involving convexity it becomes clear that these three parameters are uncorrelated.

Though the bulk of images with defect 'air bubbles' are located near the upper-right corner of the plane - i.e. near $\left(\xi_{1:}, p\right)=(1,1)$ - 'irregular shapes' is distributed diffusely over the plane, be it preferably at the left side. The areas covered by the defects overlap considerably. especially the central part of the plane. As an example of the synergy consider point $\mathrm{X}=\left(\xi_{\mathrm{E}}, p\right)=(0.6,0.8)$, represented in Figure A.23. Based solely on the ecentricity $p$ the probability that the defect is 'air bubbles' is just 0.4 - based on the fuzzy membership represented in Figure $\wedge .21$. Combined with the value of $\xi_{1:}$ the location of the point $\mathrm{X}$ is clearly within the area of 'air bubbles'.

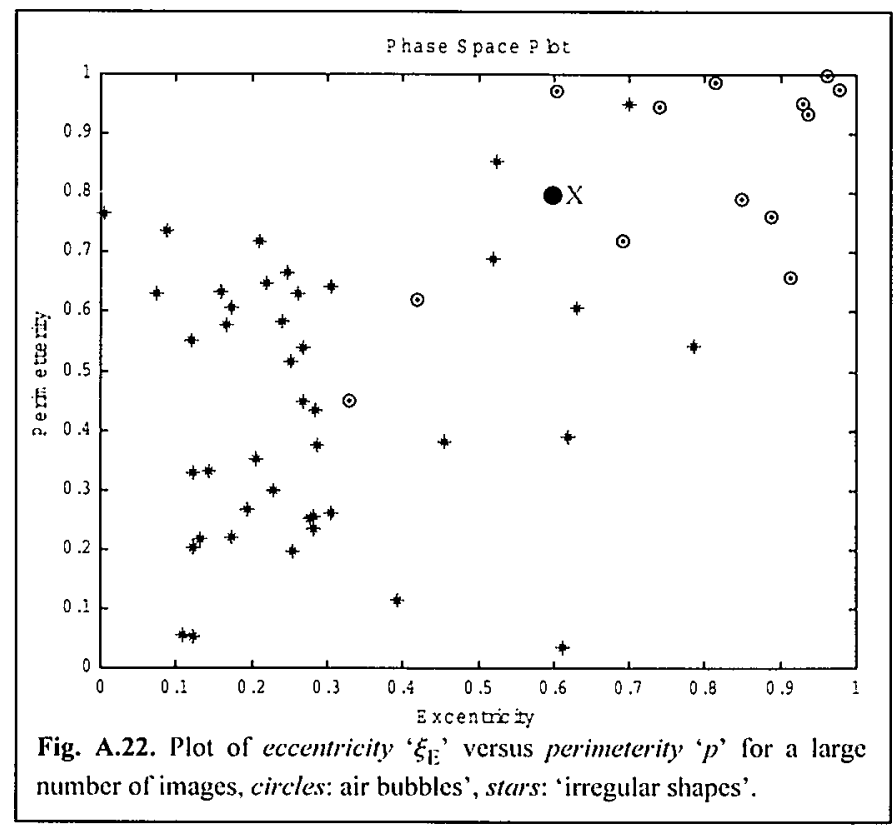

This matter of integrating sensor information represents a central problem in decision making in defect identification, where all information stems from tests performed on the image. The problem is that the defect classes represented in terms of the sensor parameters are not - or at best - weakly separated. For this reason it is important to involve as much information as possible. But this on the other hand will lengthen the decision making process, for some of these experiments are considerably time-intensive. Morcover, some experiments may not contribute essential new information. For this purpose in part IV a system will be introduced that is intended to minimise the number of tests and simultaneously optimise the quality of the decision. 


\section{A.7 Identification of Rasters versus Irregular Patterns}

Under certain conditions the raster of the cliche may be visible. It is useful to have an indicator for the detection of this unwanted phenomenon. For this reason the specific test 'rasterity' was designed. This parameter indicates the degree in which a structure exhibits a raster-like appearance. $A$ good criterion for 'rasterity' is the standard deviation in the mean distance between neighbours, the 'grid-size' of the lattice. In dealing with a lattice-like structure, however, it is more advantageous to inspect the Fourier transform of the grid - here called the K-grid - than the original grid itself (the X-grid). This is because the Fourier transform smears out all local effects and so represents a mean prototype of the grid - be it that the grid-size $b$ in K-space relates to the grid-size $a$ in X-space as $a b=2 \pi$. Therefore the Fourier transform gives a good indication of any periodicity in a structure. The emphasis on the Fourier transform rather than on the original image is also found in crystallography, when characterising lattices [Neder\&Proffen 1996]. Hence we base our definition of 'rasterity' on the Fourier transform of the image. Here we introduce a new method for calculating 'order' in a lattice. First we introduce a quantity $\phi$ that is computed as:

$$
\phi(\kappa)=\int_{\Omega} Z(\mathbf{x}) \delta(|k|-\kappa) \mathrm{e}^{\mathrm{ikx}} \mathrm{d}_{2} \mathbf{x}
$$

With $\delta$ is the Dirac-distribution, and ' $\Omega$ ' represents the entire area of the image. $|\phi|$ denotes the fraction of the Fourier transform in the $\mathrm{K}$-grid with a $\mathrm{K}$-grid-size of $\|\mathrm{k}\|=\kappa$. For lattice-like configurations $|\phi|$ will exhibit a high degrec periodicity. To avoid noise, we only observe peaks above a certain threshold c. We define $c$ as: $c=\mu+\epsilon(M-\mu)$, where $\mu=\min _{K} \phi(\kappa)$, and $M=\max _{K} \phi(\kappa)$, so $0 \leq \epsilon \leq 1$. With these definitions we can define the thresholded function $h_{\epsilon}$ as:

$$
h_{\epsilon}(\xi)=\theta(|\phi(\xi)|-\epsilon)
$$

with threshold function $\theta(x)=x$ if $x>0$, and zero otherwise. Next, the thresholded function of $|\phi|$ is used to calculate the average spacing of the spikes as $\lambda \pm \delta \lambda$.

$$
\lambda=g_{N} \int h_{\epsilon}\left(\xi_{1}\right) h_{\epsilon}\left(\xi_{2}\right)\left|\xi_{1}-\xi_{2}\right| \mathrm{d} \xi_{1} \mathrm{~d} \xi_{2} /\left(\int h_{\epsilon}(\xi) \mathrm{d} \xi\right)^{2}
$$

In this approach $h_{\epsilon}$ is considered as a distribution function, and ' $\lambda$ ' is calculated as the average of the distance between the centres of the spikes. This approach works best for spiked functions like Diracdistributions. In this approach distances between all centres are compared, so a correction factor ' $\mathrm{g}_{\mathrm{N}}$ ' is necessary to obtain the mean distance between neighbouring peaks. Here, ' $N$ ' is the number of peaks above the threshold $c$, and ' $\mathrm{g}_{\mathrm{N}}$ ' is equal to:

$$
g_{N}=N^{2} / \Sigma n_{n 1, n 2=1: N}|n 1-n 2|
$$

The standard deviation in the grid-size can now be defined in a similar manner:

$$
\delta \lambda^{2}=\mathrm{g}_{\mathrm{N}} \int h_{\mathrm{\epsilon}}\left(\xi_{1}\right) h_{\mathrm{\epsilon}}\left(\xi_{2}\right)\left(\left|\xi_{1}-\xi_{2}\right|-\lambda\right)^{2} \mathrm{~d} \xi_{1} \mathrm{~d} \xi_{2} /\left(\int h_{\epsilon}(\xi) \mathrm{d} \xi\right)^{2}
$$

Finally, the quantity $r$, the measure for the rasterity, is expressed in terms of $\delta \lambda \lambda$ (provided $\lambda>0$ ), with any continuous monotonous decreasing function ' $f$ ' that obeys $f(0)=1$ and $f(x) \downarrow 0$ for $x \rightarrow \infty$.

$$
r=f(\delta \lambda / \lambda)
$$

In practice the choice of $f$ is based on empirical data such that $r$ is on average high for obvious cases of visible rasters, and sufficiently low for competing defects as 'dirt particles'.

\section{i. Rasters versus Random Associations of Dots}

In order to distinguish rasters from random aggregations of dots, as may occur for instance in the defect 'dirt particles' and in noise, we investigate the sensitivity of this parameter to disorder in the raster. For this reason we consider a raster as an ordered lattice with basis $\left\{\mathbf{e}_{1}, \mathbf{e}_{2}\right\}$ and corresponding grid-sizes $\left\{a_{1}, a_{2}\right\}$. For rastered clichés oriented to the CCD-camera we assume $\mathrm{e}_{1}=(1,0)^{\mathrm{T}}, \mathbf{e}_{2}=(0,1)^{\mathrm{T}}$, and $a_{1}=$ $a_{2}=a$. Order in a raster is expressed by an order parameter $\eta \in[0,1]$, such that $\eta=0$ indicates total disorder and $\eta=1$ a perfect lattice. We model disorder in a lattice by assuming that the lattice points are shifted from their grid-positions by a randomly oriented vector $\mathbf{p}$, such that its size $p=\|\mathbf{p}\|$ obeys a uniform distribution in $[0,(1-\eta) a]$. Therefore the lattice points are represented by vectors: $x_{\mathrm{k} 1 \mathrm{k} 2}=$ $k_{1} a_{1} \mathbf{e}_{1}+k_{2} a_{2} \mathbf{e}_{2}+p u(\varphi)$, where $\mathbf{u}(\varphi)=\cos (\varphi) \mathfrak{e}_{1}+\sin (\varphi) \mathbf{e}_{2}$ with uniform distribution in $\varphi \in[0,2 \pi]$. In Figure $A .25$ a number of examples are presented of values of $r$ for increasing amounts of disorder in an otherwise perfect lattice. In the same figure is depicted the relation between rasterity $r$ and the lattice 
order parameter $\eta$, exhibiting a discrete order transition at $\eta \approx 0.7$. This jump in rasterity is a consequence of its definition in equation [5]. It is an artefact that depends on the peculiarities of $f$, because for this uniform probability distribution it can be simply calculated that $\lambda=a$ and $\delta \lambda \propto a(1-\eta)$. So according to the principal definition of rasterity $r=f(\delta \lambda / \lambda)=f(1-\eta)$, which is monotonous increasing in $\eta$. As a meaningful criterion for the visibility of a raster on an image we can therefore take a qualitative criterion like: $(r>0.5)$.

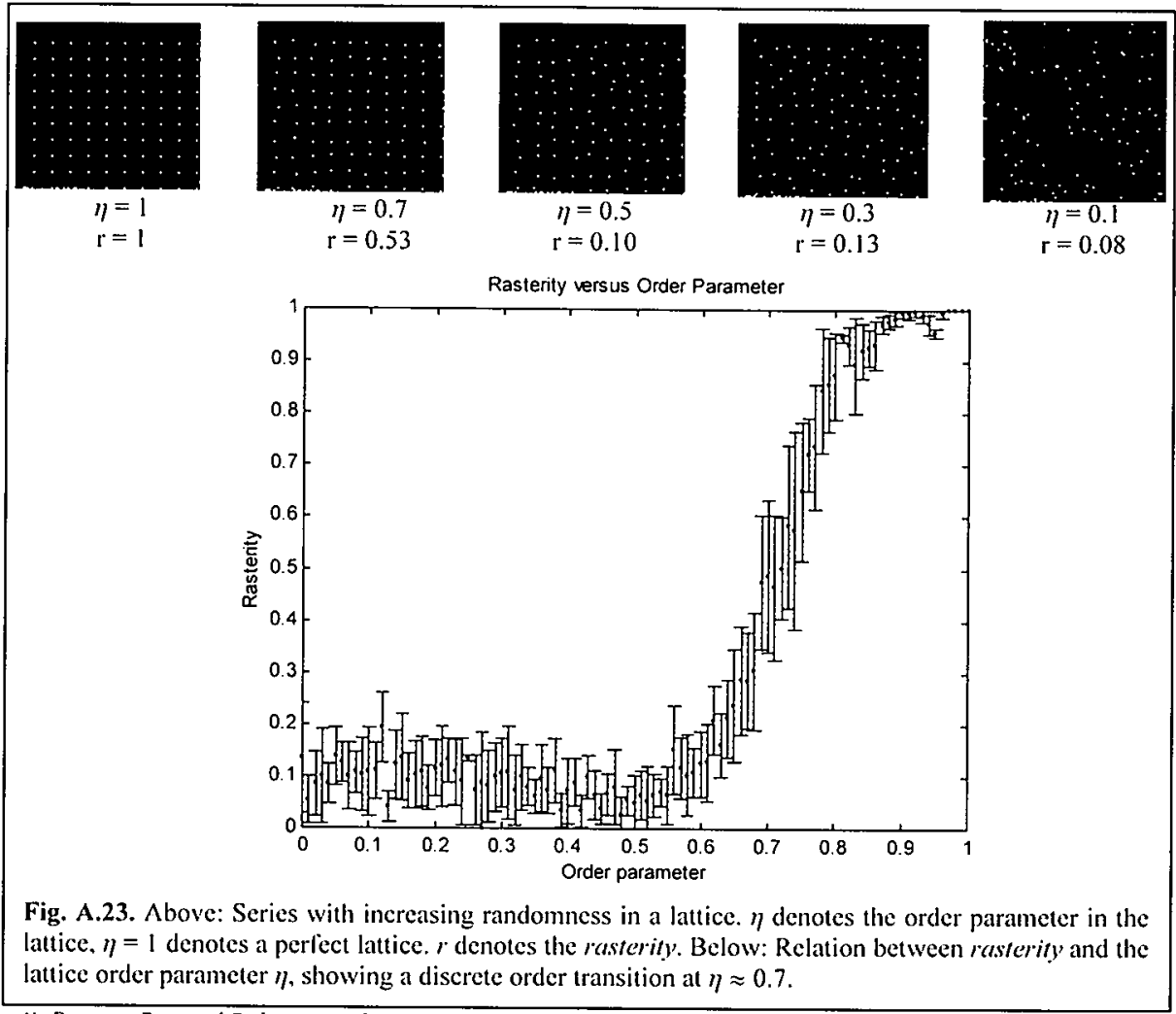

ii. Resters Rotated Relative to the CCD-Camera

Another practical matter is that the raster on the CCD-image will in general be somewhat rotated. Therefore we investigate the behaviour of the rasterity with respect to rotations with an angle $\phi$. Figure A.23 shows the effect of rotation on the rasterity. For rotations of a perfect lattice it was found that averaged over the rotation angles $r=0.82 \pm 0.11$. Though the quantity $r$ obviously depends on the angle $\phi$, the qualitative criterion $Q=(r>0.5)$ is rotation-invariant. The relation between rotation angle and rasterity is shown in Figure A.26. Similar to the dependence on the order parameter discussed above, a criterion $(r>0.5)$ would definitively indicate a lattice-like structure.

\section{iii. Other Methods}

A second approach was based on the Peak Partitioning and Decomposition approach, described in appendix A.3. First, $Z\left(x_{1}, x_{2}\right)$ is decomposed as: $z_{1}(\xi)=\int_{\Omega} Z\left(\xi . x_{2}\right) d x_{2}$, and $z_{2}(\xi)=\int_{\Omega 2} Z\left(x_{1}, \xi\right) d x_{1}$. Then the 'peak partitioning' approach was applied to $z_{1}$ and $z_{2}$ separately. On cach of these IDcomponents the method produced the average spacing $\langle\lambda\rangle \pm \delta \lambda$ between the peak-centres. In this way it could be used to estimate rasterity in the constituent dimension on a similar manner as described above based on $\delta \lambda\left\langle\langle\lambda\rangle\right.$. As described in appendix A.3. rasterity $\mathrm{r}_{\mathrm{k}}$ in component $k$ can now be expressed with any continuous monotonous decreasing function ' $f$ ' complying the empirical data as: $r_{k}=f(\delta \lambda /<\lambda>$ ). This results in two quantitics $r_{x}$ and $r_{y}$. Now we can present the degree that the image contains a raster as: 


$$
r=r_{x} r_{y}
$$

In the case of a rotated raster each of the decompositions $z_{1}$ and $z_{2}$ contains an interference of periodic peaks. The centres of the peaks occur at: $x_{k_{1} k_{2}}=\lambda k_{1} \cos \varphi+\lambda k_{2} \sin \varphi$, for $k_{1}, k_{2} \in Z, \lambda$ the rasterdistance, and $\varphi$ the angle of rotation. Using Fourier transform this periodicity can be easily identified as: $\lambda_{1}=\lambda \cos \varphi$ and $\lambda_{2}=\lambda \sin \varphi$. The standard deviations in these periods provided an even better basis for the definition of ressterity, for instance:

$$
\mathrm{r}=f\left(\delta \lambda_{1} /<\lambda_{1}>\right), f\left(\delta \lambda_{2} /<\lambda_{2}>\right)
$$

This expression gave excellent results. ' $f$ is a proper continuous monotonous decreasing function ' $f$ conform the empirical data

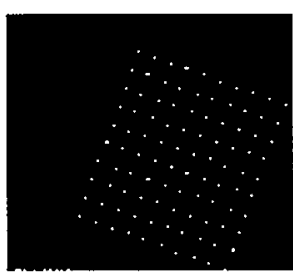

$\phi=20$

$r=0.82$

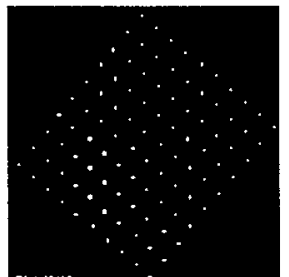

$\phi=40$

$r=0.68$

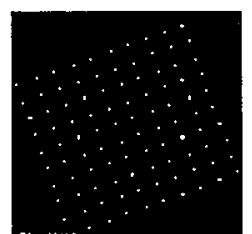

$\phi=70$

$\mathrm{r}=0.95$

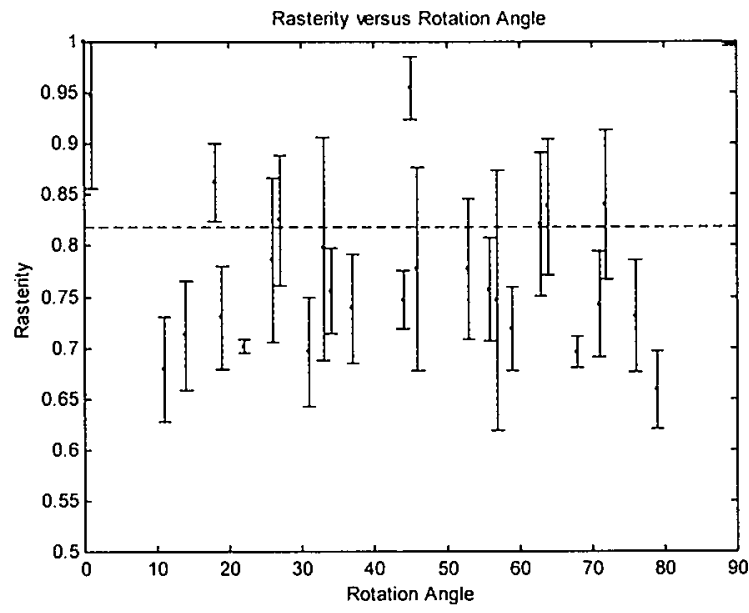

Fig A.24. Above: Rotations of perfect lattices with angle $\phi$ relative to the negative horizontal axis. (respectively 20, 40 and 70 degrees). $r$ denotes the rasterity value. Below: Relation between rotation angle and rasterity. The dotted line gives the average rasterity of 0.82 .

Two other possibilities that do not involve Fourier transform but rely on the spatial distribution in the supposed lattice were also considered for applicability as rasterity-parameter. These were $\rho_{1}(\mathbf{s})$, the 'spacing distribution', and the auto-correlation function $\rho_{2}(\mathbf{s})$.

$$
\begin{aligned}
& \rho_{1}(\mathrm{~s})=\int_{\Omega} Z(\mathbf{x}) \delta(|x-\mathrm{z}|-\mathrm{s}) \mathrm{d}_{2} \mathrm{x} / \int_{\Omega} Z(\mathrm{x}) \mathrm{d}_{2} \mathrm{x} \\
& \rho_{2}(\mathrm{~s})=\int_{\Omega} Z(\mathrm{x}) Z(\mathbf{x}-\mathrm{s}) \mathrm{d}_{2} \mathrm{x} / \int_{\Omega} Z^{2}(\mathrm{x}) \mathrm{d}_{2} \mathrm{x}
\end{aligned}
$$

Here $z$ denotes the centre-of-mass. Using these expressions, the spatial distance distribution function $\rho_{1}$ could be computed and, similarly to $\phi$, tested for equidistant spikes. This gave inferior results to the rasterity calculated above. Moreover, it consumed more computation time. The auto-correlation function $\rho_{2}$ is a 2D-distribution and as such poses the same problem for the calculation of $r$ as the original function $Z$. Summarising it can be said that $r$ is a good representation for rasterity. It is able to distinguish orderly structures from random configurations, and if we take as criterion $(r>0.5)$ it is also 
rotation invariant. This is an example where an originally quamitative feature parameter $r$ can be replaced by a more robust qualitaive criterion 'rasterity': $(r>0.5) \Rightarrow$ (rasterity $=\|$ uce).

\section{A.8 Identification of Streaks, Lines and Scratches}

Lines, streaks, and scratches on an image can be identified with an approach similar to the identification of spherical objects using eccentricity. Like some of the methods discussed in previous sections this method depends on an interactive focussing on the individual defect cells. First the individual defect on $Z$ must be identified and singled out - for instance using the statistical cell partitioning approach as defined in appendix $A .3$. Using Least Squares (LSQ) the best fitting line is calculated, given the collection $P$ of points in the identified cell. For this reason we fit the points $x \in P$ with a line $L$ :

$$
L\left(q_{1}, q_{2}, q_{3}, x\right)=q_{1} x_{1}+q_{2} x_{2}+q_{3}=0
$$

with an additional - non-linear - constraint $Q$ :

$$
Q\left(q_{1}, q_{2}, q_{3}\right)=q_{1}^{2}+q_{2}^{2}-1=0
$$

using a quadratic criterion $V$ :

$$
\mathrm{V}\left(\mathrm{q}_{1}, \mathrm{q}_{2}, \mathrm{q}_{3}\right)=\Sigma_{\mathrm{x} \in P} \mathrm{~d}_{\mathrm{L}}\left(\mathrm{x}, \mathrm{q}_{1}, \mathrm{q}_{2}, \mathrm{q}_{3}\right)^{2} / \mathrm{n}_{\mathrm{p}}
$$

Where $n_{p}$ is the number of elements of $P$, and $d_{L}$ is equal to the distance from an arbitrary point $\mathrm{p}$ to line $L, d_{L}\left(p, q_{1}, q_{2}, q_{3}\right)=\left|q_{1} p_{1}+q_{2} p_{2}+q_{3}\right|$. After applying LSQ we obtain a set of equations in the coefficients $q=\left(q_{1}, q_{2}, q_{3}\right)$ which generates the best fitting values $q^{*}=\left(q^{*}, q^{*}{ }_{2}, q^{*}{ }_{3}\right)$, with corresponding errors $\delta q_{k}, k=1 . .3$. Now we can relate the linefit $f_{\mathrm{p}}$ - contorm the remarks of appendix A.3 - of $P$ with line $L$ to the value of the criterion at the solution $\mathbf{q}^{*}$ :

$$
f_{\mathrm{P}}=1 /\left(1+\left(V^{*} / \mathrm{K}\right)\right)
$$

with: $v^{*}=\mathrm{V}\left(\mathrm{q}^{*}{ }_{1}, \mathrm{q}_{2}, \mathrm{q}_{3}, \mathrm{x}\right)$. Like so many fuzzy functions, this definition uses a free parameter $K$ that is determined by the subjective feeling of a 'good' fit'. This definition means that $f_{p} \in[0,1]$, with $f_{p}=1$ defining a perlect fit, and $f_{p}$ reaching 0 when the points of $P$ move to infinity. Using the fit of $P$ with the line $L$, we can now define the measure linity, denoted $\lambda$, simply by equating:

$$
\lambda=f_{\mathrm{p}}
$$

So $\lambda \in[0,1]$, with $\lambda=1$ defining a perfect fit with a line. In the case of multiple scratches or lines the statistical approach used. This results in the statistical distribution $P$ of $\lambda$ over the cells; $P(\lambda) \mathrm{d} \lambda$

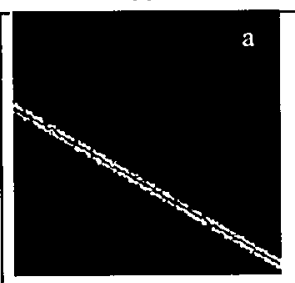

$\lambda=0.977 \pm 0.007$

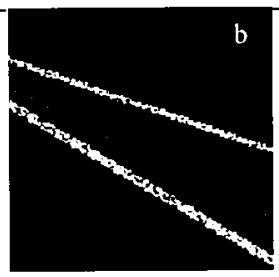

$\lambda=0.195 \pm 0.080$

Fig. A.25. Images with 'streaks', with best-fitting line, a: wide, straight stripe yields a good fit. b: effect of multiple lines yields the weighted average of the lines. representing the fraction of cells with linity $\lambda$ in $<\lambda, \lambda+d \lambda>$. In this way $\lambda$ can be calculated as an average over the collection of cells:

$$
<\lambda>=\int \lambda \mathrm{P}(\lambda) \mathrm{d} \lambda
$$

Similarly, the standard deviation in $\lambda$ can be calculated. In Figure $A .27$ two examples are shown that illustrate that this method is capable of identilying obvious scratches. streaks and lines. Similarly to the method for computing the eccentricity the frequency distributions for linity $\lambda$ can be calculated from a large number of adequate images. These frequency distributions can be used to represent a tuzzy membership function $\mu_{\text {linity }}(\lambda)$ that denotes the degree to which an image $Z$ with linity $\lambda$ can be considered as belonging to defect class 'streaks of lines or scratches'. This empirical measure can on its turn be used to compute the expected degree that an image represents defect 'streaks'. The expected degree that the collection of cells represent 'streaks' is then given by:

$$
<\mu_{\text {linity }}>=\int \mu_{\text {linity }}(\lambda) \mathrm{P}(\lambda) \mathrm{d} \lambda
$$

\footnotetext{
${ }^{7}$ In our context we used $K=33$.
} 
Similarly, the standard deviation $\mathrm{d} \mu_{\text {limity }}$ can be calculated. The pair $\left\{\left\langle\mu_{\text {linity }}\right\rangle, \mathrm{d} \mu_{\text {linity }}\right\}$ can be used to establish a qualitative confidence we can have that the defect belongs to the defect type 'streaks'.

In Figure $A .26$ a number of examples are given. From these examples it is clear that there can be some complications in the computation of the parameter linity. For clear, thin, linear scratches the method gives good results. i.e. $\lambda \approx 1$. For fat scratches like picture a in Figure $A .28 \mathrm{a}$, the average axis can be found reasonably well. but the spread around the line decreases the fit and increases the error. Unrelated 'blobs' like in Figure A.28b near a scratch will somewhat deteriorate the effect. In the case that more lines occur simultaneously in the cell, the method will produce a line something like the weighted average. This method operates on the absolute difference $Z=|F-1|$. If the actual image $F$ docs not align with the template $T$ then a residual image - the 'ghost-image' - appears. The ghost-

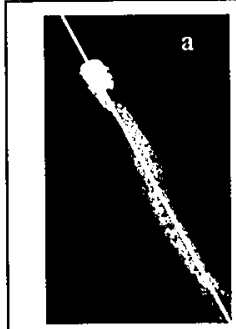

$\lambda=0.809$

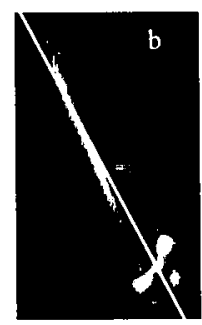

$\lambda=0.613$

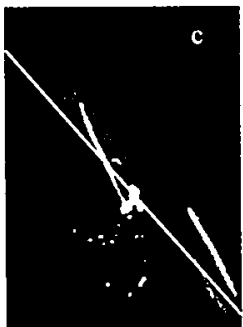

$\lambda=0.247$

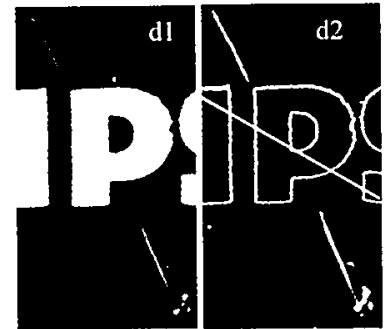

$\lambda=0.035$

Fig. A.26 Images with 'streaks', with best-litting line. a: wide, straight scratch yields a good fit. b: effect of 'blobs' reduces the fit. $\mathrm{c}$ : effect of multiple lines deteriorates the result. d: effect of a 'ghost-image' on line-lit elfectively annihilates the result, $\mathrm{d} 1$ : CCD-image, $\mathrm{d} 2: Z$ with strong residual 'ghost-image' lrom bad match between image and template.

image itself outlines the edge of the template. The method cannot distinguish the ghost-image from any occurring scratch, so the resulting line and line-fit will yield a meaningless result.

Summarising it was found that linity $\lambda$ gives an indication of the defect 'streaks. lines and scratches'. There are, however, a number of harmful factors that can significantly decrease this parameter. The most serious threat is the residual ghost-image, an effect that will always occur on the absolute difference $Z$. Therefore this method is not fully capable of discriminating the defect 'streaks, lines and scratches' from the other defects. This can only be done by involving other sources of information. specifically statistical and texture propertics of the image. A clear advantage of this approach is that it can deal reasonably well with most of the common scratches, streaks and lines like in fig 28 a and b. and $\mathrm{d}$. Moreover, this method is also reasonably fast ${ }^{8}$ and efficient. Another advantage is that the approach does not depend on the scale of the image used.

An entirely different approach to identilication of lines and streaks is offered by employing the Hough transform, see: [Bow, 1992]. [Cucchiana,Filicori, 1998] construct a 2D-Hough transform for detecting clongated shapes in greyscale images.

\footnotetext{
${ }^{8}$ On average $<0.003 \mathrm{sec}$ for a $256 \mathrm{pixcl} 256$ colour image pixel [MatLab/400 $\mathrm{MH} z \mathrm{PC} / \mathrm{Windows} \mathrm{NT}$ ].
} 


\section{A.9 Identification of Dirt Particles.}

Images showing the defect 'dirt particles' are characterised by small isolated and irregular spots of intermediate greylevel. that are randomly distributed over the image. In this section we will present three methods for the identification this defect. These methods are relevant both for identifying 'dirt particles', and distinguishing them from 'air bubbles' foam. 'rasters' and random spikes generated by noise. All three methods give rise to a quantity $s \in[0,1]$ called spikity that indicates the degree that the image contains 'dirt particles'. All three methods utilise the characteristics of dirt-particles such as their small size, their random distribution in the plane, and their sharply peaked distribution with narrow flanks.

\section{i. Stutistical Cell Size Distribution}

The first method is discussed in the section on statistical analysis of cell sizes. The defect dirt particles' is characterised by typical small sizes and narrow flanks of the cell size distribution. This can distinguish this defect from noise, but the overlap with small air bubbles (foam) is considerable.

\section{ii. Medial Fowrier Filter}

$\Lambda$ second method that can be constructed is based on the Fourier transform $\psi$ of the image $Z$ :

$$
\psi(\mathbf{k})=(2 \pi)^{-1} \int_{\Omega} Z(\mathbf{x}) e^{i \mathbf{k} x} \mathbf{d}_{2} \mathbf{x}
$$

Like the first method, this method uses the empirical information on average particle size between 10 $\mu \mathrm{m}$ to $100 \mu \mathrm{m}$ and the small variation in size. Therefore it filters out the higher and lower sizes. For this reason a uniform filter $f$ was defined, that - based on empirical evidence - operated on $\mathbf{k}$ with size $|\mathbf{k}|$ between $\kappa_{1}=0.01 \mu \mathrm{m}^{-1}$ and $\kappa_{2}=0.1 \mu \mathrm{m}^{-1}$, corresponding to sizes from $10 \mu \mathrm{m}$ to $100 \mu \mathrm{m}$.

$$
f(\mathbf{k})=\theta\left(|\mathbf{k}|-\kappa_{1}\right) \theta\left(\kappa_{2}-|\mathbf{k}|\right)
$$

with $\theta(x)=x$ if $x>0$, and zero otherwise. Application of this filter, combined with the inverse Fouricr transform yiclds:

$$
Z_{2}(x)=\int_{\Omega} \psi(\mathbf{k}) f(\mathbf{k}) \mathrm{e}^{-i k x} d_{2} k
$$

Typical examples of the application of this medial filter to 'dirt particles', 'foam', and large 'air bubbles' are shown in Figure A.29. These pictures confirm the hypothesis that the medial filter can distinguish between 'dirt particles'/ foam' on the one hand, and 'large air bubbles' on the other. Note from figure $A .27$ that the standard deviation in the large-size defect large 'air bubbles' in $Z_{2}$ is much smaller than in the small-size defects 'dirt particles' and 'foam'. From this point two approaches are possible:

[i] Founded on before mentioned observation we construct a measure called $s$, based on the variation in $\mathrm{Z}_{2}$. This defines spikity in terms of the standard deviation $\delta Z_{2}$ in $Z_{2}$, such that $\delta Z_{2}=0$ implies $\mathrm{s}_{1}=0$, and $s_{1} \uparrow l$ if $\delta Z_{2} \rightarrow \infty$. So we define: $s_{1}=f\left(\delta Z_{2} /\left\langle Z_{2}>\right)\right.$, where $' f$ ' is a continuous monotonous increasing function, that obeys $f(0)=0$ and $f(x) \uparrow 1$ for $x \rightarrow \infty$, based on empirical data. Figure A.27 shows some examples of $\delta Z_{2} /<Z_{2}>$ for three similar defects.

[ii] Similar to the definition of 'hairity' a method based on the fraction of the energy within the frequency band $\left[\kappa_{1}, \kappa_{2}\right]$. The energy within this band is $E_{1}$ :

$$
E_{l}=\int_{k}|\psi(\mathbf{k})|^{2} f(\mathbf{k}) \mathrm{d}_{2} \mathbf{k}
$$

that is compared with the entire energy $E$ :

$$
E=\int_{k}|\psi(\mathbf{k})|^{2} \mathrm{~d}_{2} \mathbf{k}
$$

This yields a definition for spikity - denoted $s_{2}-$ as $s_{2}=C . E_{1} / E_{\text {, where }} C$ is a suitable scaling constant based on empirical data. Figure A.27 shows some examples of $\mathrm{E}_{1} / \mathrm{E}$. 


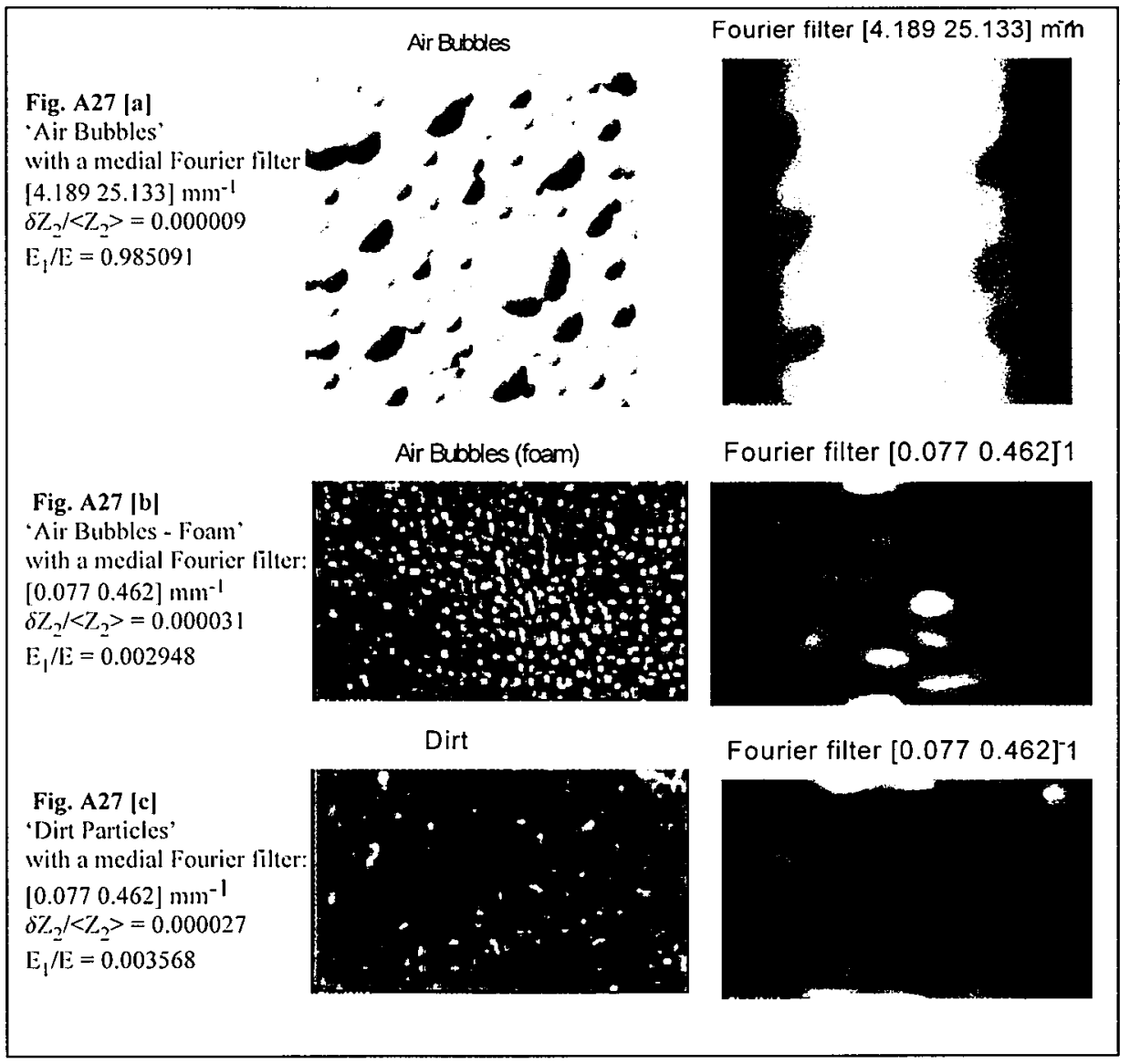

\section{iii. Peak Decomposition and Partitioning}

The third method proposed uses the Peak Decompesition and Partitioning method, described in appendix A.3. In the context of the identification of isolated spikes in the decompositions $z_{1.2}$ we are interested in the statistics of the size of the intervals: $D_{k}$. The average interval-length is expressed by $\langle D\rangle$ and its corresponding standard deviation $\delta \mathrm{D}$. Based on the observation mentioned before that 'dirt particles' typically exhibit narrow and sharp peaks, we base our delinition of 'spikity' on $\delta \mathrm{D} /\langle\mathrm{D}\rangle$. Therefore, we express $s_{11}$ as:

$$
\mathrm{s}_{1 \mathrm{D}}=f(\delta \mathrm{D} /<\mathrm{D}>)
$$

with ' $y$ - conform the remarks in appendix A.3. - a proper function based on empirical data that gives 'sensible' values for 'dirt particle' peaks. Using the decomposition of $Z\left(x_{1}, x_{2}\right)$ in $z_{1}\left(x_{1}\right)$ and $z_{2}\left(x_{1}\right)$, we obtain two quantities, the $x$ - and $y$-'spikity' $s_{x}$ and $s_{y}$, each based on equation [6]. Now we can present the degree that the image contains isolated 'dirt particles' by $s_{3}$. expressed as:

$$
s_{3}=s_{x} s_{y}
$$

This approach provides the best method available to identify 'dirt particles'. Compared with the other approaches it has the highest computational efficiency and speed, and has the strongest correlation with the defect. As min definition of spikity therefore was selected definition [60]:

$$
s=\mathrm{s}_{3}
$$


Summarising it was found that spikity $s$ delined by [11] provides a method for the identification of the defect 'dirt particles'. A general disadvantage of especially the last two methods, is that they have difficulty in distinguishing the defects 'dirt particles' and 'rasters'. For this reason it is necessary to involve the quantity rasterity for this takes low values for 'dirt particles' and high values for 'rasters', and so can distinguish the two defects. This effect is shown in table 6.

\begin{tabular}{|c|c|c|}
\hline spikity & rasterity & most likely defect \\
\hline$h i$ & $h i$ & 'rasters' \\
\hline$h i$ & lo & 'dirt particles' \\
\hline
\end{tabular}

Table A6; Discrimination between two competing defects 'dirt particles' and 'rasters'. 


\section{APPENDIX B.1 CID Transforms of 18 printing defects}
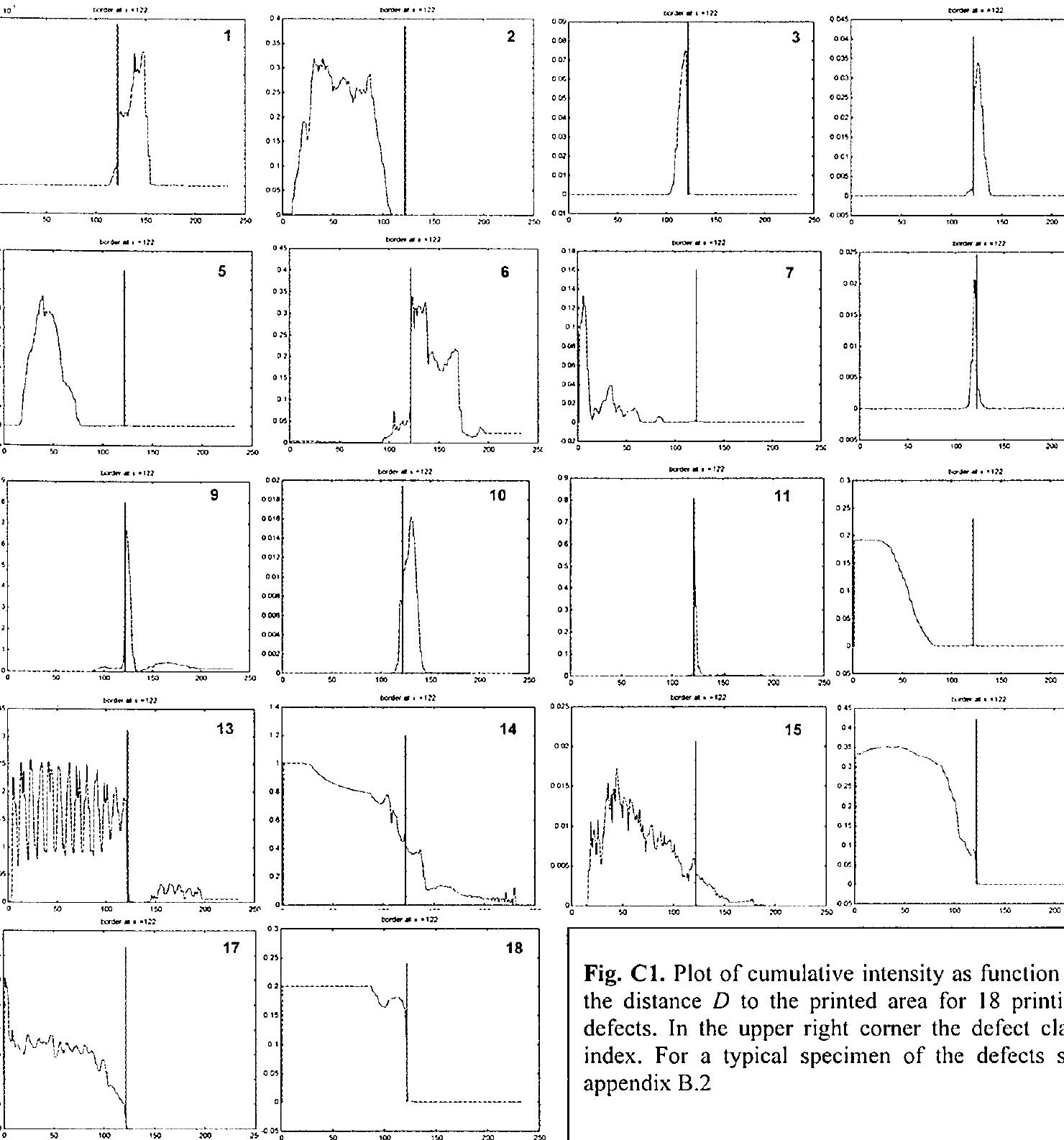

18

Fig. C1. Plot of cumulative intensity as function the distance $D$ to the printed area for 18 print defects. In the upper right corner the defect cl index. For a typical specimen of the defects $s$ appendix B.2 


\section{APPENDIX B.2 2DI Transforms of 18 padprinting defects}

defect \#1, hair:

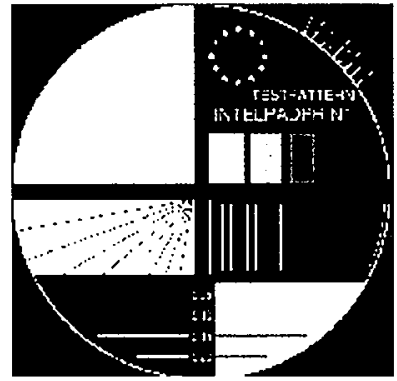

defect \#2: bubbles

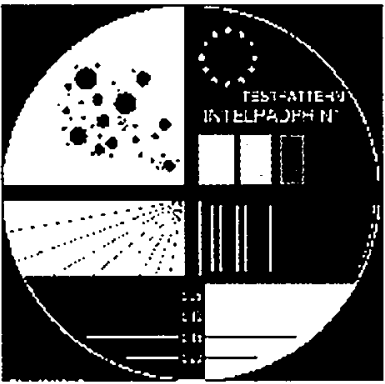

delect $\#$ 3: constriction

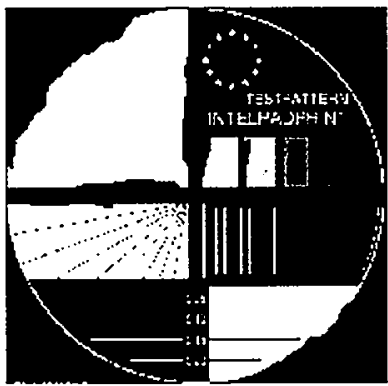

defect \#4: recess

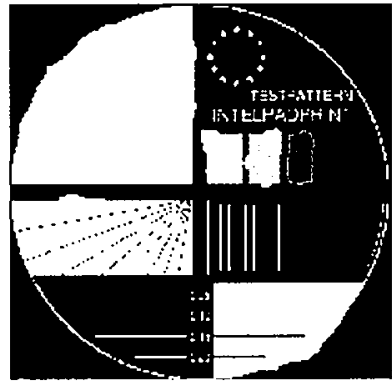

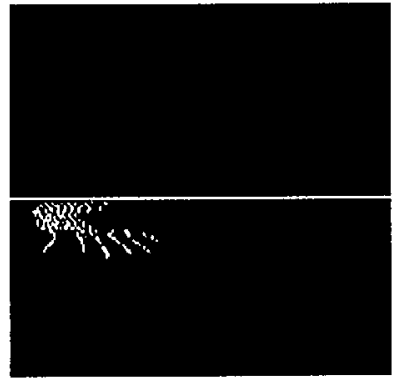
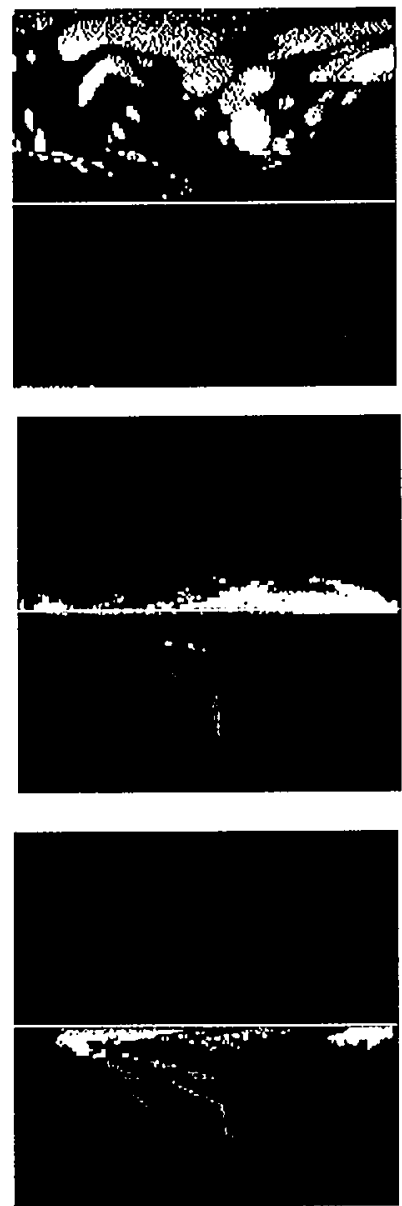


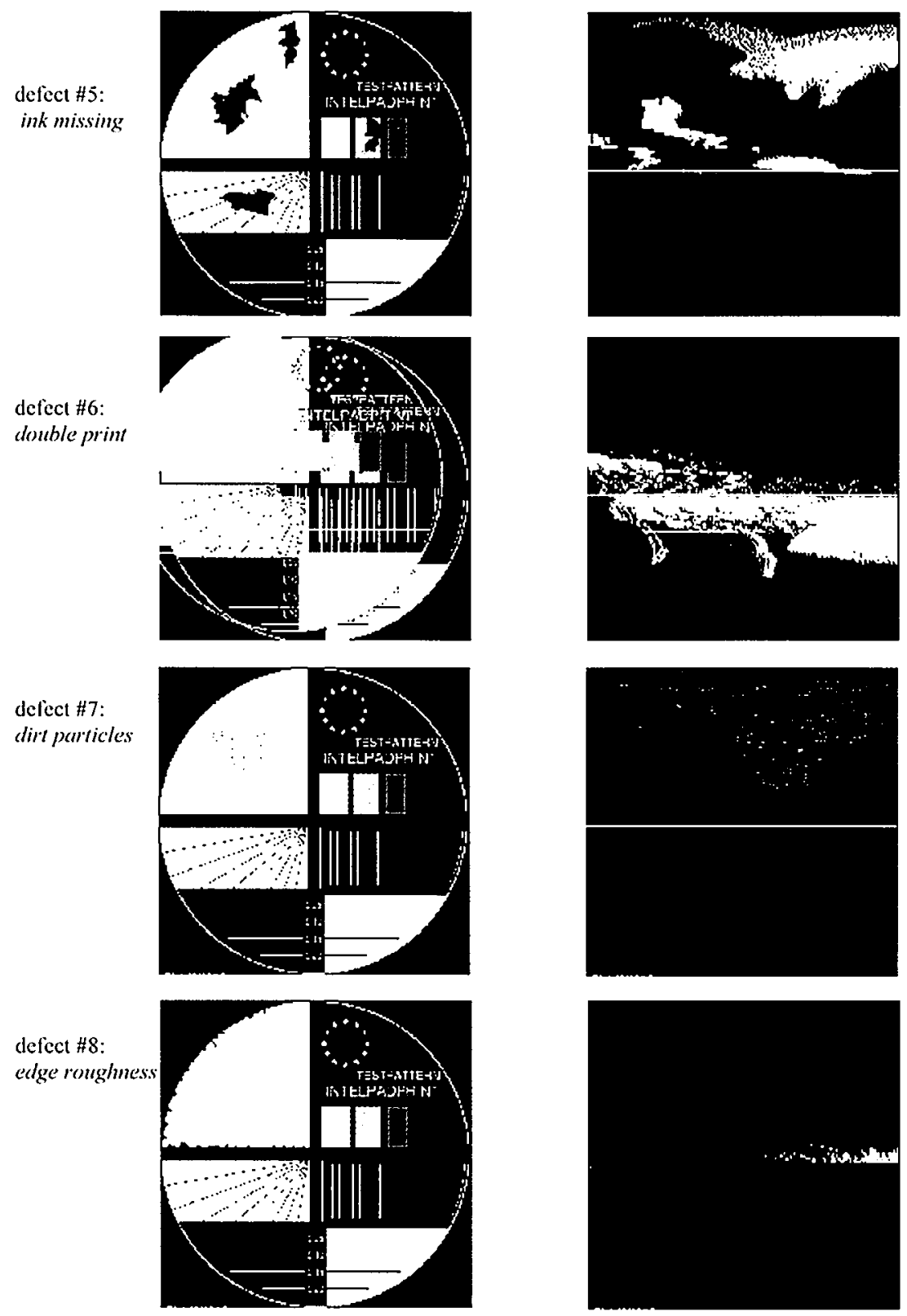



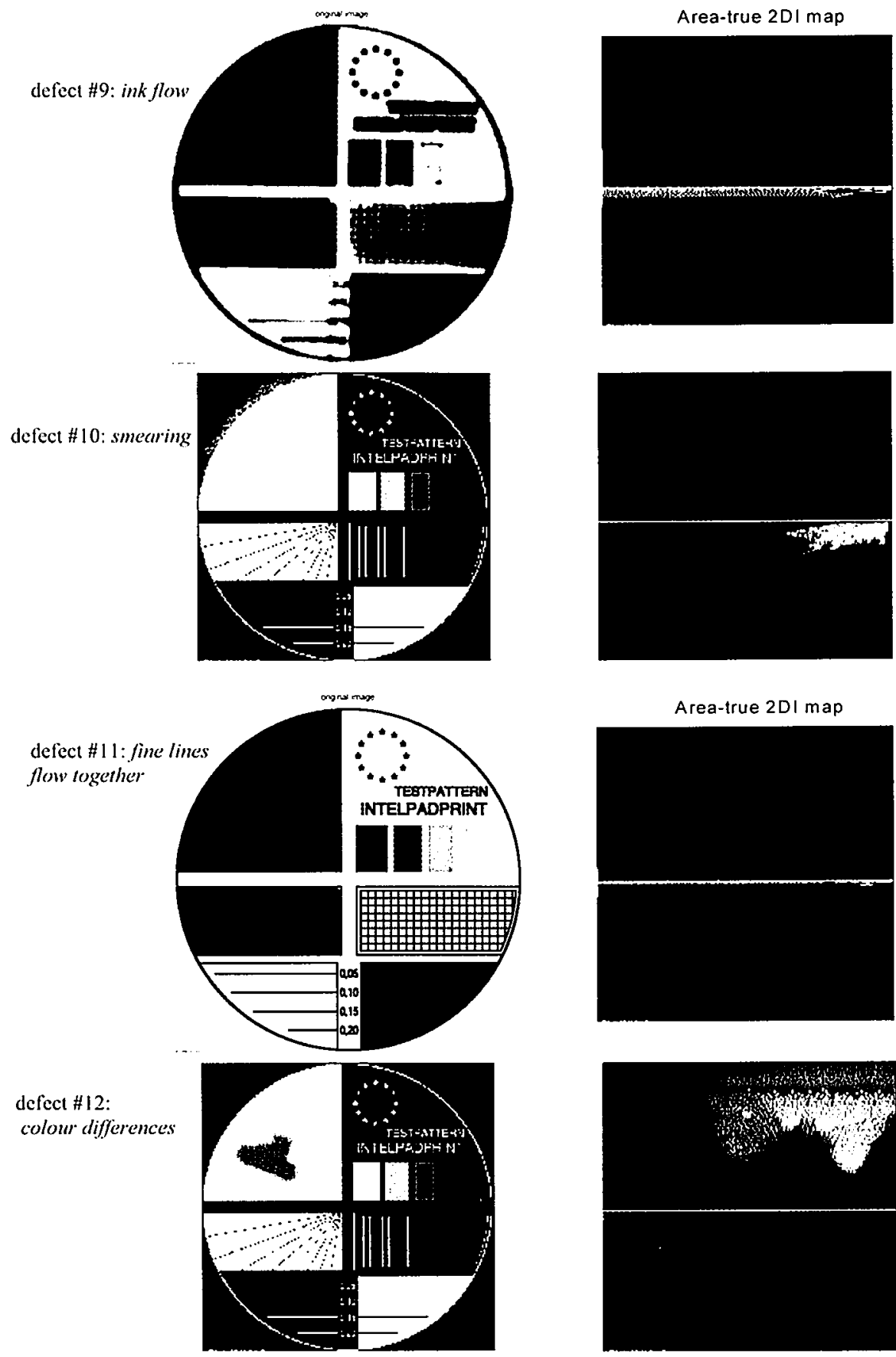

Area-true 2DI map
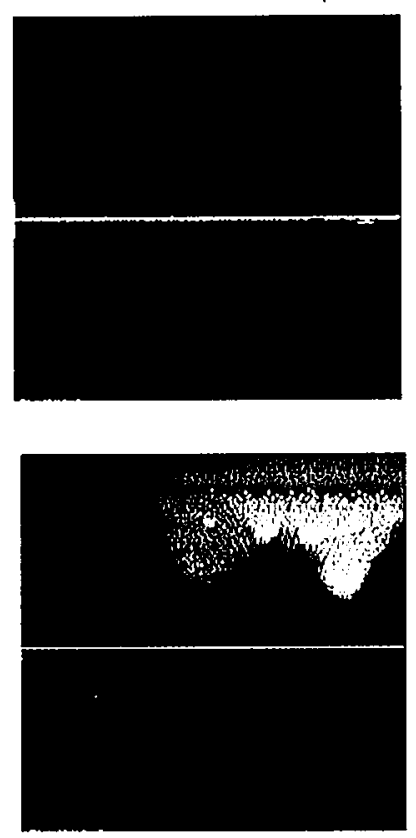

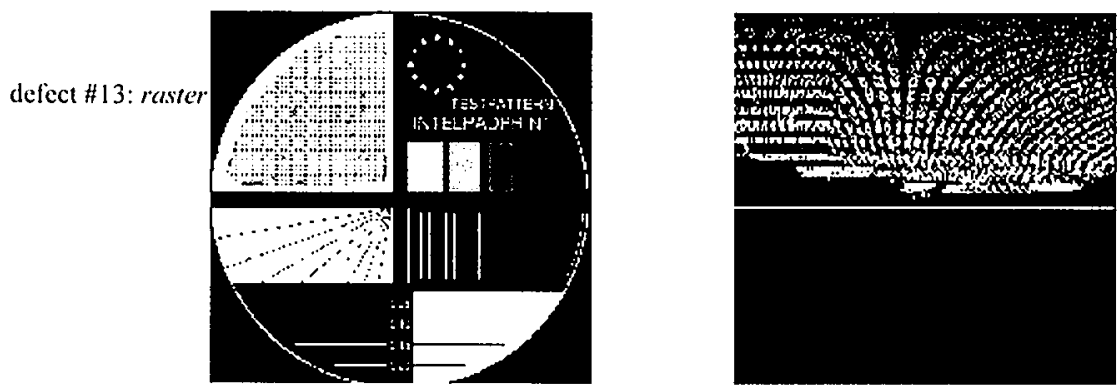

defect \#14: misregistration
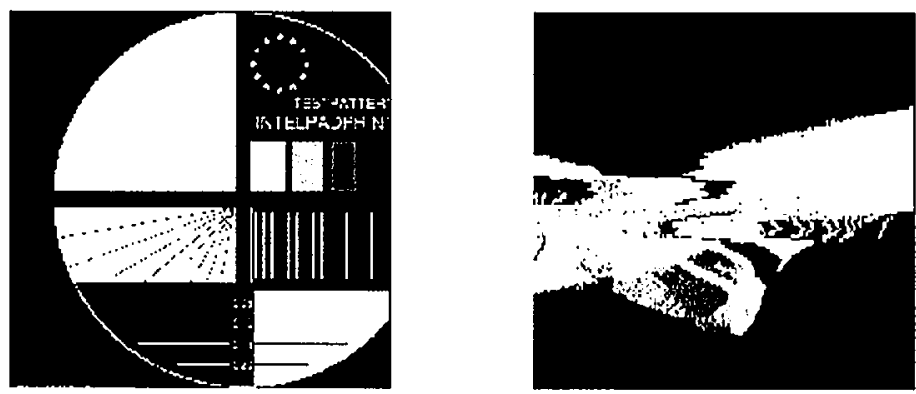

defect \#15: stripes
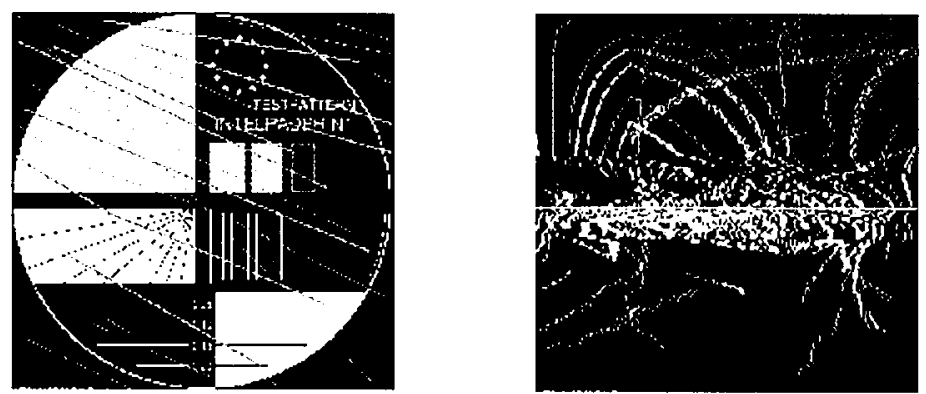

defect \#16: haze
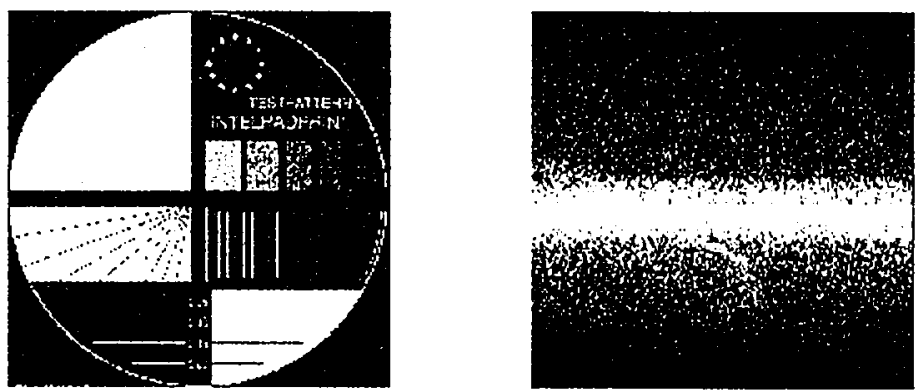

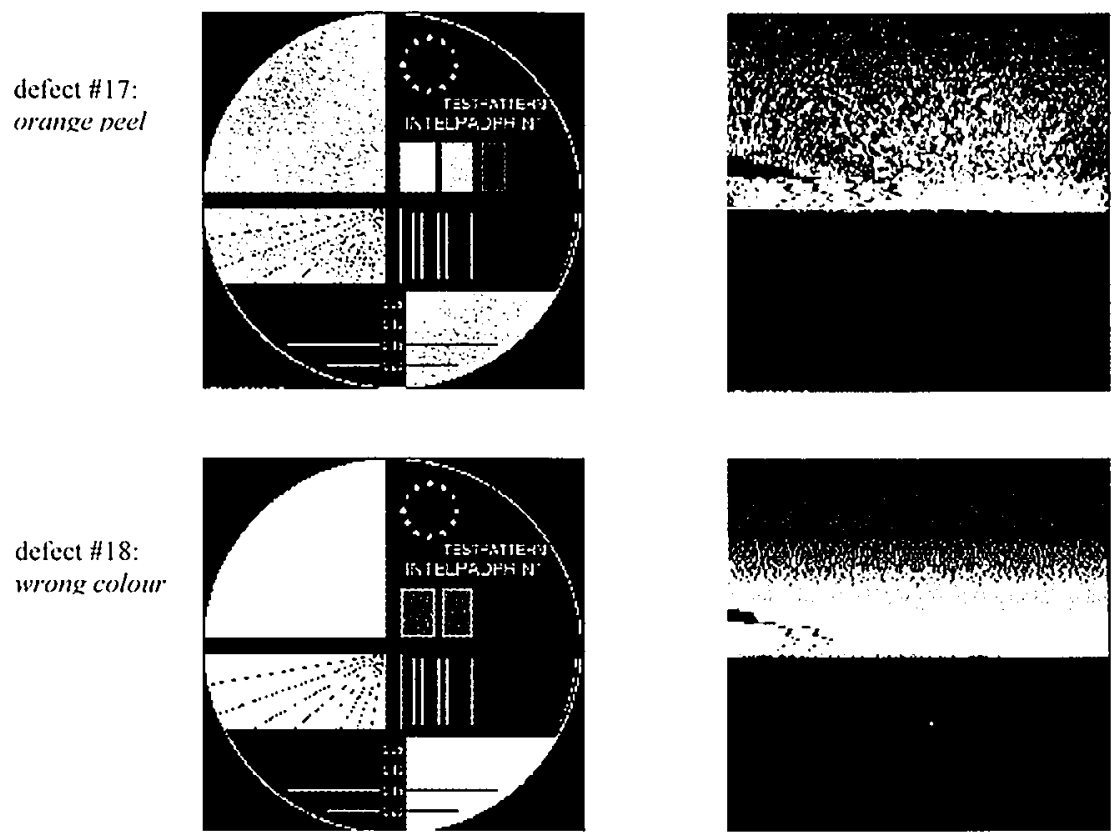

defect $\# 19$ normal
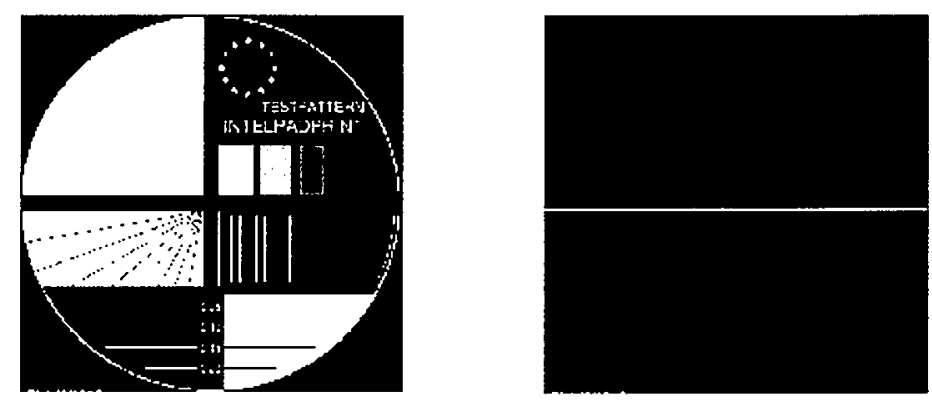


\section{APPENDIX C Dynamic Properties of IASC}

\section{C.1 Calculation of Jacobian for $\boldsymbol{x}$ as dynamic quantity.}

Using the consideration of $\mathbf{x}$ as parameter, and using expressions [5.51]-[5.55] we expand the Jacobean $j$ as:

$$
j_{k l}(\mathbf{u})=\frac{d \Phi_{k}}{d u_{l}}=\sum_{j \in E} \frac{\partial \Phi_{k}}{\partial x_{j}} \frac{\partial x_{j}}{\partial u_{l}}
$$

Applying expression [5.56]: $\Phi_{k}=B_{k} u_{k}$, and using [5.57]: $\xi_{k n}=\left(x_{n}-m_{k n}\right)$, we can rewrite the second term in [C1] as:

$$
\sum_{j \in E} \frac{\partial \Phi_{k}}{\partial x_{j}} \frac{\partial x_{j}}{\partial u_{l}}=\sum_{j \in E} \sum_{i \in E}\left(B_{k} \frac{\partial u_{k}}{\partial \xi_{k i}}+u_{k} \frac{\partial B_{k}}{\partial \xi_{k i}}\right) \frac{\partial \xi_{k i}}{\partial x_{j}} \frac{\partial x_{j}}{\partial u_{l}}
$$

The right-hand expression can be further simplified using the following considerations:

- from [5.55] follows: if $(i \in E)$ then $\frac{\partial u_{k}}{\partial \xi_{k i}}=-u_{k} \widetilde{\mathbf{g}}_{k i}^{\mathrm{T}} \cdot \widetilde{\xi}_{k}$ else $\frac{\partial u_{k}}{\partial \xi_{k i}}=0$

- here we used: $\widetilde{\mathbf{g}}_{l j}^{\mathrm{T}}[i]=\left(\widetilde{G}_{l}(E)[j, i]+\widetilde{G}_{l}(E)[i, j]\right) / 2$

- similarly: if $(j \in E)$ then $\frac{\partial u_{l}}{\partial x_{j}}=-u_{/} \widetilde{\mathbf{g}}_{l j}^{\mathrm{T}} \cdot\left(\widetilde{\mathbf{x}}-\widetilde{\mathbf{m}}_{l}\right)$ else $\frac{\partial u_{l}}{\partial x_{j}}=0$

- moreover: $\frac{\partial x_{j}}{\partial u_{l}}=\left(\frac{\partial u_{l}}{\partial x_{j}}\right)^{-1}$

- likewise, from [5.58] we obtain:

$$
\text { if }(n \in E) \text { then } \frac{\partial B_{k}}{\partial \xi_{k n}}=-B_{k}\left(\beta_{k}+\gamma_{k} \xi_{k}\right) \text { else } \frac{\partial B_{k}}{\partial \xi_{k n}}=0
$$

- from [5.57] follows: $\frac{\partial \xi_{k i}}{\partial x_{j}}=\delta_{i j}$

Using these simplifications in expression [C2], expression [C1] can now be written as:

$$
\left.j_{k l}(\mathbf{u})=B_{k} \delta_{k l}+B_{k} \frac{u_{k}}{u_{l}}\left(\sum_{j \in E} \frac{\tilde{\mathbf{g}}_{k j}^{\mathrm{T}}}{\left(\widetilde{\mathbf{g}}_{l j}^{\mathrm{T}} \widetilde{\xi}_{l}\right.}\right)\right) \widetilde{\xi}_{k}
$$

Equation [C3] is expression [5.63] in the main text, QED. Notice that this expression is singular for $u=0$. 


\section{C.2 Simplifications of Jacobian lead to conflicting results for convergence.}

In order to make some qualitative statements about the Jacobian, we make the simplification that $G$ is a diagonal matrix: $\tilde{G}_{k}[i, j]=\sigma_{k i}^{-2} \delta_{i j}$. In that special case $B_{k}=\exp \left(-1 / 2 \gamma_{k} \xi_{k n}^{2}\right)$ and [C2] becomes:

$$
j_{k l}(\mathbf{u})=B_{k} \delta_{k l}+B_{k} \frac{u_{k}}{u_{l}} \sum_{j \in E} \frac{\sigma_{l j}^{2} \xi_{k j}}{\sigma_{k j}^{2} \xi_{l j}}
$$

The diagonal elements of [C4] are: $j_{k k}(\mathbf{u})=(1+t) B_{k}$, where $t$ is the present step in the iteration. As a further simplification, let us assume that the last measured value $\xi$ follows a probability distribution $p$. Then the average elements of [C4] become:

$$
\left\langle j_{k l}\right\rangle=h\left(\gamma_{k}\right) \delta_{k l}+a h\left(\gamma_{k}\right) \frac{u_{k}}{u_{l}} \sum_{j \in E} \frac{\sigma_{l j}^{2}}{\sigma_{k j}^{2}}
$$

with: $h(\gamma)=\int p(\xi) \exp \left(-1 / 2 \gamma \xi^{2}\right) d \xi$, and: $a=\int p\left(\xi_{1}\right) p\left(\xi_{2}\right) \frac{\xi_{1}}{\xi_{2}} d \xi_{1} d \xi_{2}$. As an extreme assumption, consider a situation where all sigma's are equal. Then [C5] becomes:

$$
\left\langle j_{k l}\right\rangle=H \delta_{k l}+A \frac{u_{k}}{u_{l}} l
$$

with $H=h\left(\sigma^{-2}\right)$ and $A=a H$. Now consider the following two scenarios.

1. Initially, all $u_{k}$ 's are equal. Now, suppose they remain equal, so all object-states are equally probable. Then [C6] becomes: $\left\langle j_{k l}\right\rangle=H \delta_{k l}+A t$, with determinant: $\operatorname{det}\left\langle j_{k l}\right\rangle=H^{K}+K H^{K-1} A t$.

2. All $u_{k}$ 's are small and equal, except $u_{n}$ which is large. Again: $\operatorname{det}\left\langle j_{k l}\right\rangle=H^{K}+K H^{K-1} A t$ So, in both cases the determinant grows linearly in time, though the system converges to a solution, respectively: $u_{k}=1 / K$, and: $u_{k}=\delta_{k l}$. Clearly, this approach can not satisfactory be applied to our context. 


\section{Appendix D1. Example of Session with IPP-prototype 1}

\section{IPPdiagnose version 3.0}

SEIECT OPTION :

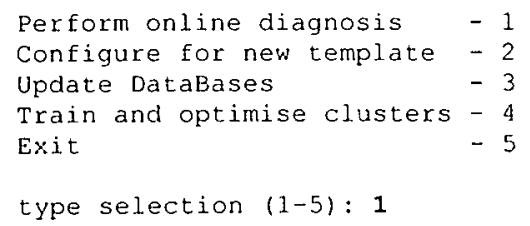

IntelPadPrint PadPrint Defect Identification and Classification Tool

type 1 to change the default configuration parameters

1: WHITE is ETCHED area

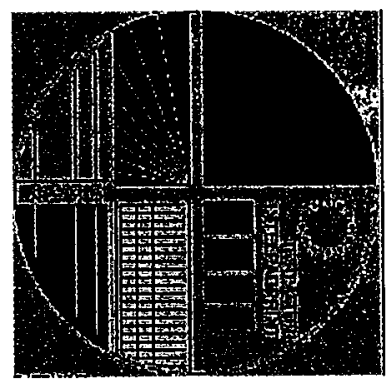

2. WHITE is ETCHED area

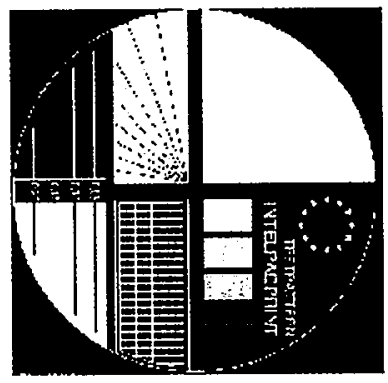

IPP - CLICHEE TYPE

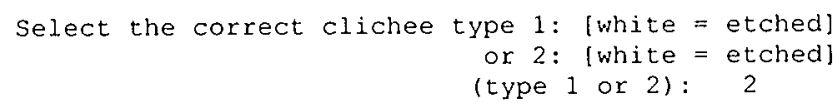

(type 1 or 2 ): 2 


\section{COLOR SCALING}

make sure a CCD-camera image is available !!!

select a specimen of a PERFECTLY printed specimen for optimal colorscaling

Press Enter when CCD-image is available

Loading: [C:\Ipp\Buffer\alignedBadGraphic.jpg] . .

Preprocessing actual image...

\section{COLOR SCALING}

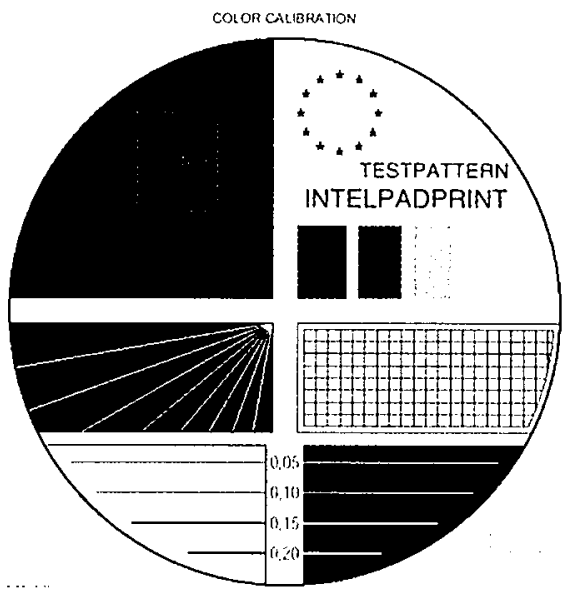

SELECT WITH MOUSE: * a patch that falls ENTIRELY inside an printed area (no raster) to establish the INK COLOR

ATTENTION: SELECT is done by double clicking the left mouse button

[1] select UPPER LEFT corner of square: (IN PRINTED AREA)

(2) select LOWER RIGHT corner of square: (IN PRINTED AREA)

NOW SELECT WITH MOUSE: * a patch that falls ENTIRELY outside the print (to establish BACKGROUND COLOR)

[1] select UPPER LEFT corner of square: (OUTSIDE PRINTED AREA)

(2) select LOWER RIGHT corner of square: (OUTSIDE PRINTED AREA) 
total defect is 18 ?

rescaling of template failed...

SELECT :

0 : Try again to rescale colors

1: Accept higher Noiselevel

[select 0 or 1]: 1

pLANAR scaling

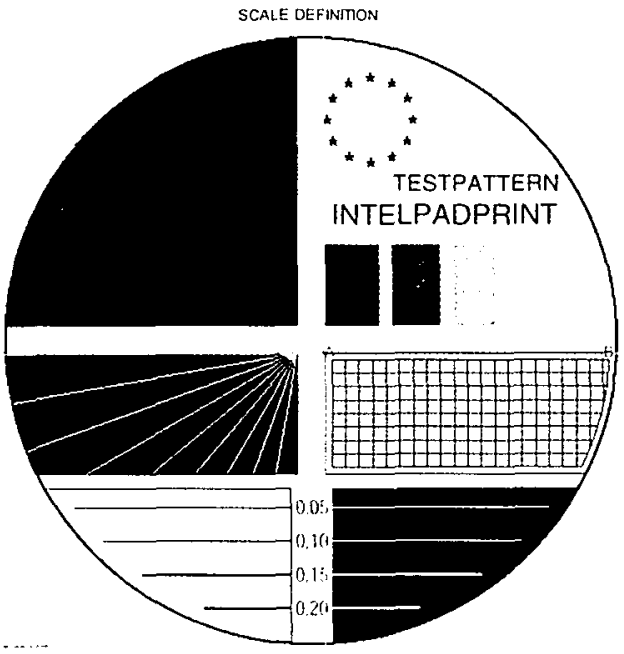

PLANAR SCALING

SELECT WITH MOUSE: * a line on the template with known length

ATTENTION: SELECT is done by double clicking the left mouse button

[1] select STARTPOINT of line:

[2] select ENDPOINT of line:

Give the EXACT length of line $A B$ in $\mathrm{mm}$

Enter length (in $\mathrm{mm}$ ): $\mathbf{2 5}$

Scale of image is $0.064408 \mathrm{~mm} / \mathrm{pixel}$

READY

... system installed 


\section{IMAGE ANALYSIS:}

significant difference between image and template detected.

total deficiency is 5673, def.density $=2.756754 \mathrm{e}+000$, def.quotient $=9$

analysing image ...

Most probable cluster \#2 = $\quad$ [2] "air bubbles",

[5] "ink missing",

[7] "dirt particles",

[12] "colour differences",

[13] "raster point visible",

[17] "orange peel",

[18] "colour match wrong" \}

Iregion $=4:$ "totally inside the print"

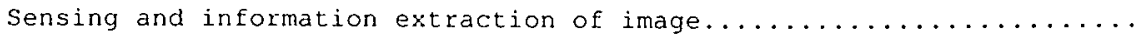

Measurement vector:

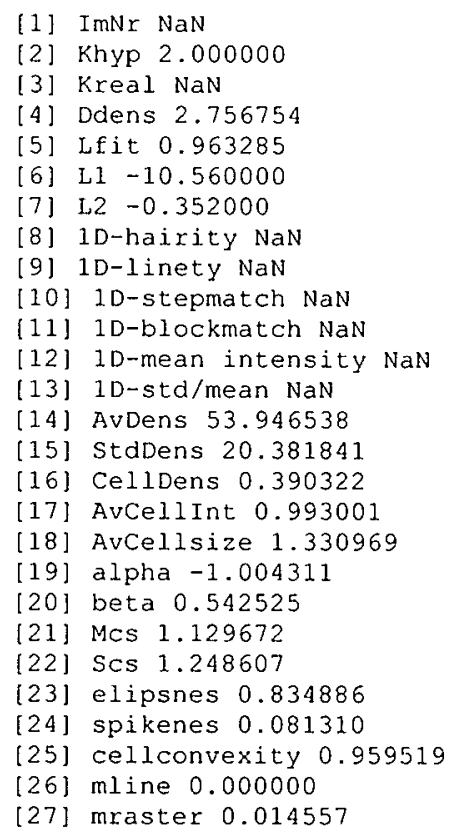

Dynamic reasoning on measurements, step: $1 \ldots 2 \ldots .3 \ldots 4 \ldots 5 \ldots 6 \ldots$ 
Results of the analysis, clockwise: 1. CIDZ-transformation of image. 2. Position in LiL2-plane of image, and allocation to cluster 2

3. searchbores on the image, 4. resulting probabilities: \#2 is most probable
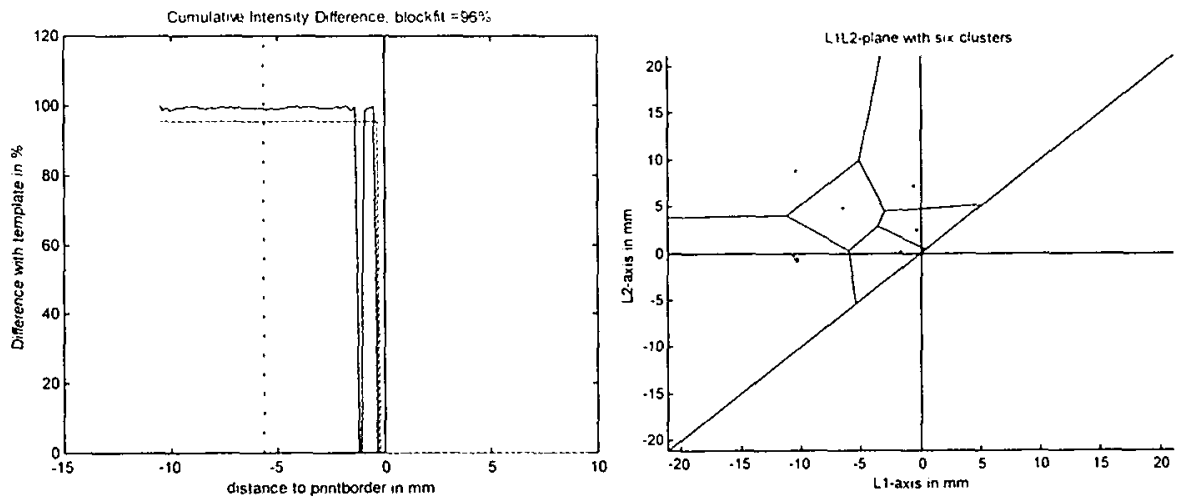

Ans'ysis of Aclual image
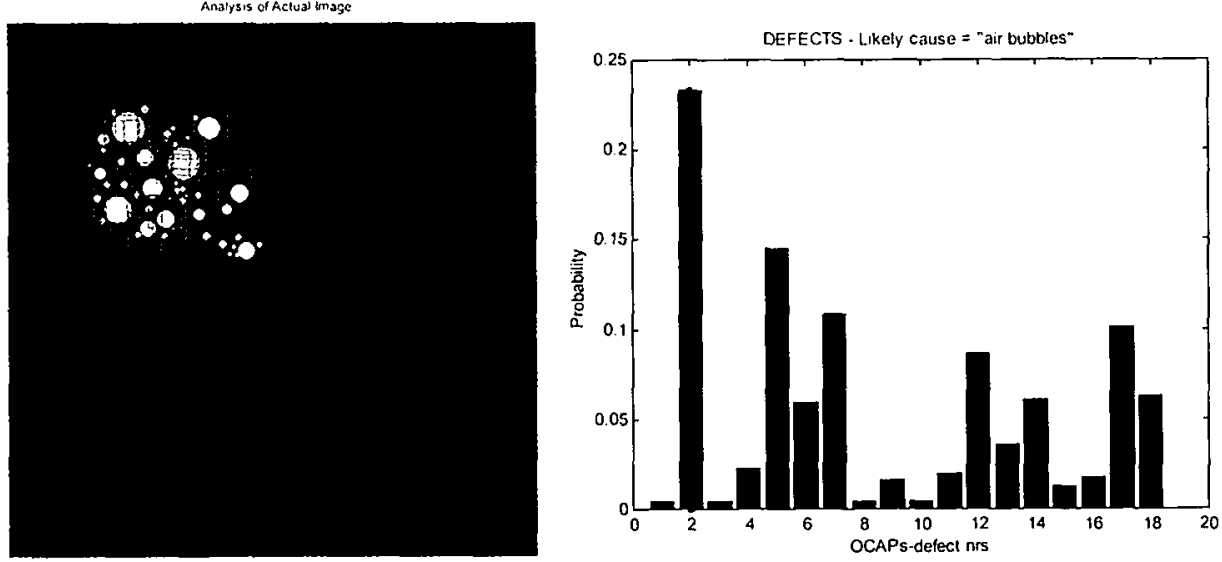
DIAGNOSIS and CONTROL ACTIONS -.---

\section{DIAGNOSIS :}

most likely cause: air bubbles

CLASSIFICATION as a probability distribution :

OCAPS

probability

$P[$ air bubbles $]=$

$P$ [ink missing $]=$

Pldirt particles]

P[orange peel]

$\mathrm{P}$ [colour differences] =

P [colour match wrong $]=$

P[colour or mis registration] =

P[misregistration or double print] =

P[raster point visible] $=$

$P[$ recess $]=$

$P[$ fine lines flow together $]=$

$P[$ haze $]=$

$P[$ ink flow $]=$

P[streaks of lines and droplets of ink] =

Possible CAUSES for defect: air bubbles

wrong pad shape,

pad hardness too weak,

ink viscosity too high,

doctor-blade speed too high

Necessary CONTROL ACTIONS for defect: air bubbles

use steeper pad,

increase pad hardness,

decrease ink viscosity by adding thinner,

add flow agent,

increase temperature of ink and/or clichee,

decrease doctor-blade speed

\section{ARCHIVING AND TRAINING}

Should this experiment be archived? $(0=$ no $/ 1=$ yes $): \ldots$ 


\section{Appendix D2. Example Sessions with IPP-prototype 2}

\section{IPPdiagnose version 4.0}

\section{SELECT ORTION:}

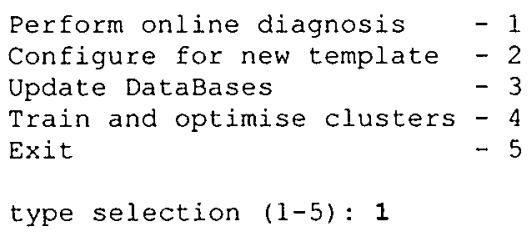

IntelPadPrint PadPrint Defect Identification and Classification Tool

type 1 to change the default configuration parameters

1: WHITE is ETCHED area

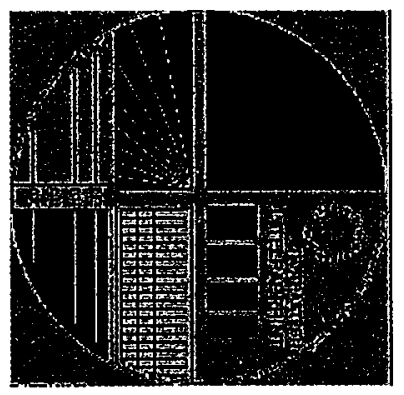

2: WHITE is ETCHED area

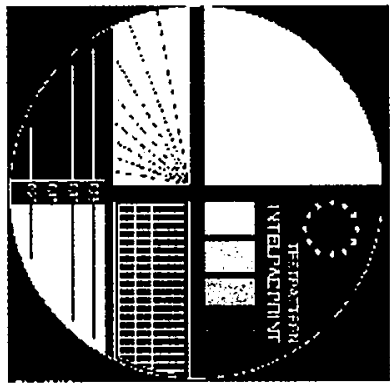

IPP - CLICHEE TYPE

Select the correct cliché type 1: [white = etched]

or 2: [white = etched]

(type 1 or 2): 2 


\section{COLOR SCALING}

make sure a CCD-camera image is available !!!

select a specimen of a PEREECTLY printed specimen for optimal color-scaling

Loading: [C:\Ipp\Buffer\alignedBadGraphic.jpg] ...

Preprocessing actual image...

total defect is 68

rescaling of template failed...

SELECT :

$0:$ Try again to rescale colors

1: ACCEPT higher NOISELEVEL

[select 0 or 1]: 1

MISMATCH estimation

Calculation of mismatch and noise [1] ...

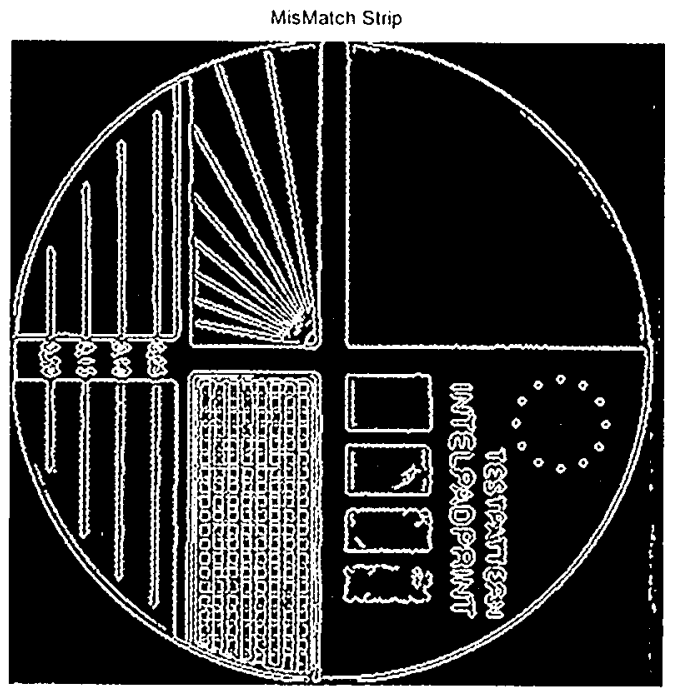

!!! MISMATCH DETECTED !!!

Mismatch between template and actual image is detected (see figure)

Range: from -2 pixels inside print to 1 pixels outside print

SELECT SOLUTION:

[0] ignore mismatch

[1] ignore red strip indicated in figure around printborder enter choice (0 or 1): 1

(2) .... ready 
PLANAR SCAIING

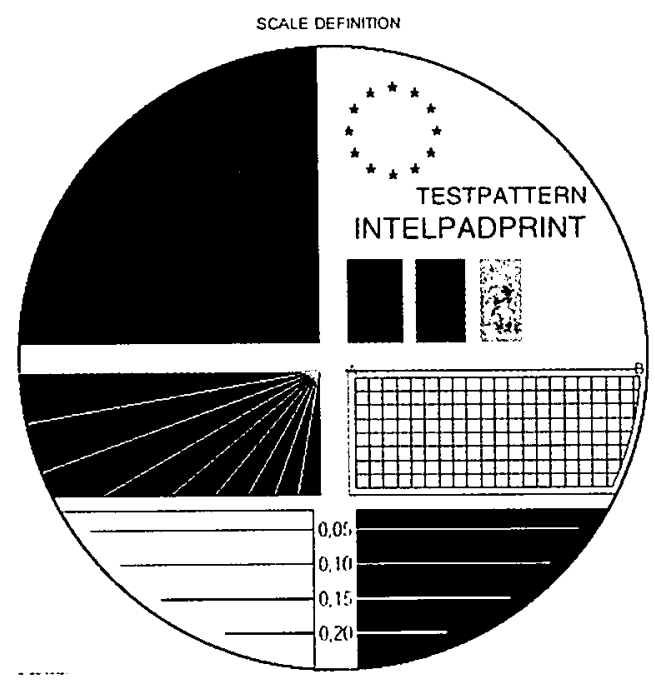

PIIANAR SCAIING

SELECT WITH MOUSE: * a line on the template with known length

ATTENTION: SELECT is done by double clicking the left mouse button

[1] select STARTPOINT of line:

[2] select ENDPOINT of line:

Give the EXACT length of line $A B$ in $\mathrm{mm}$

Enter length (in $\mathrm{mm}$ ): 25

Scale of image is $0.064408 \mathrm{~mm} / \mathrm{pixel}$.

READY

... system installed 


\section{EXAMPLE 1}

PHASE I: IS DEFECT ABOVE THRESHOLD?

TOTAL DEFICIENCY IS 8567, DEF. DENSITY $=4.1$, DEF. OUOTIENT $=13$

preclustering image ...

PHASE II: PRE-CLUSTERING

Lregion $=7$ : "distributed inside the print"

Most probable cluster \#7 = \{ [2] "air bubbles",

[3] "constriction",

[5] "ink missing",

[7] "dirt particles",

[8] "edge roughness",

[12] "colour differences",

[13] "raster point visible",

[14] "colour or misregistration",

[17] "orange peel",

[18] "colour match wrong" \}

RHASE III: DETAILED ANALYSIS

Sensing and information extraction of image...

Dynamic interactive reasoning on measurements:

\begin{tabular}{|c|c|c|c|c|c|c|c|c|}
\hline STEP: & SELEC? & T PARAMETER: & Num: & & ALUE: & DECISIC & -CRITERIUH : & MOST PROBABLE CAUSE: \\
\hline 1 & [31] & "rasterity & $"$ & 2 & 0.00 & +-0.00 & 0.2 & "colour match wrong" \\
\hline 2 & [19] & "StdDens & $"$ & 8 & 0.03 & +-0.03 & 0.4 & "colour differences" \\
\hline 3 & {$[18]$} & "AvDens & $"$ & 2 & 1.00 & +-0.00 & 0.2 & "ink missing" \\
\hline 4 & {$[27]$} & "elipsnes & $"$ & 2 & 0.00 & +0.00 & 0.9 & "ink missing" \\
\hline 5 & (29) & "cellconvexity" & & $\ddot{8}$ & 0.35 & +-0.37 & 0.5 & "ink missing" \\
\hline 6 & {$[28\}$} & "spikenes & $"$ & 8 & 0.01 & +-0.01 & 0.7 & "ink missing" \\
\hline 7 & {$[24]$} & "beta & $"$ & 3 & -3.66 & +-0.00 & 0.6 & "ink missing" \\
\hline 8 & {$[23]$} & "alpha & $"$ & 3 & -0.12 & +-0.00 & 0.8 & "ink missing" \\
\hline 9 & {$[25]$. } & MCS & $"$ & 2 & 1.05 & +-0.00 & 0.8 & "ink missing" \\
\hline 10 & {$[30]$. } & "linity & $"$ & 2 & 0.00 & +-0.00 & 1.0 & "ink missing" \\
\hline
\end{tabular}

IPPreason ends: decision criterion has been met: 1.00 , most probable cause:

"ink missing"

DIAGNOSIS and CONTROL ACTIONS

CLASSIFICATION as a probability distribution :

OCAPS

PROBABILITY

P[ink missing] $=$

\section{DIAGNOSIS:}

there is one most likely cause: [5] ink missing

Possible causes for defect: ink missing

ink viscosity too high,

dirt on pad,

too fast evaporating solvent,

too slow process,

small sized graphics,

pad surface too rough,

too much/irregular air blowing,

insufficient ink on cliché 


\section{Necessary CONTROL ACTIONS for defect: ink missing}

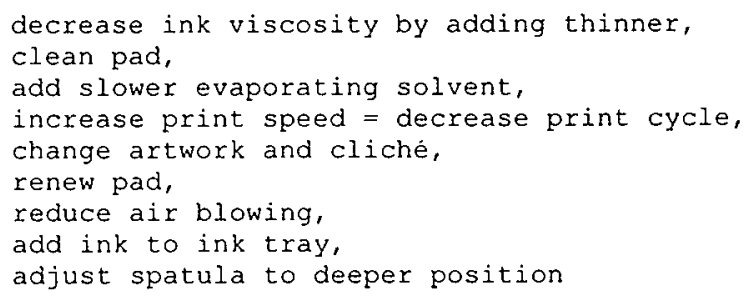

Results of the analysis, clockwise: 1. CIDZ-transformation of image.

2. Position in L1L2-plane of image, and allocation to cluster 2

3. searchboxes on the image, 4. resulting probabilities: \#2 is most
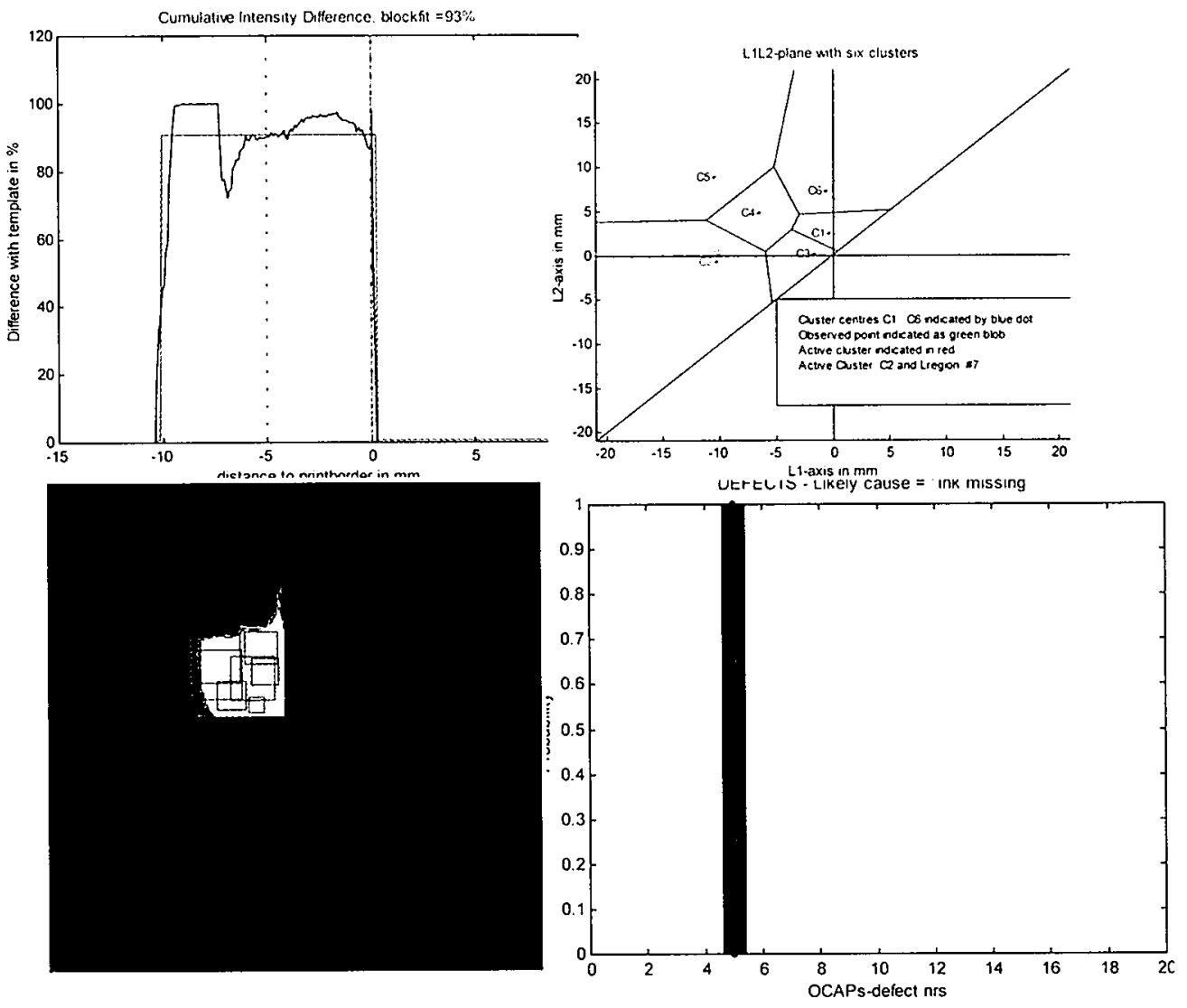

probable
DISCRIMATIVI
Noiselevel
MisMatch detected
$3 \%$
: NO

\section{ARCHIVING AND TRAINING}

Should this experiment be archived? $(0=$ no $/ 1=$ yes $): \ldots$ 


\section{EXAMPLE 2: real image * extract *}

\section{PHASE I: IS DEFECT ABOVE THRESHOLD?}

total deficiency is 1182 , def.density $=5.7$, def.quotient $=2$

\section{PHASE II: PRE-CLUSTERING}

Lregion = 1: "far outside the print"

Most probable Cluster \#1=\{[6] "misregistration or double print".

[15] "streaks of lines and droplets of ink", [16] "haze"]

\section{PHASE III: DETAILED ANALYSIS}

Sensing and information extraction of image...

Dynamic interactive reasoning on measurements:

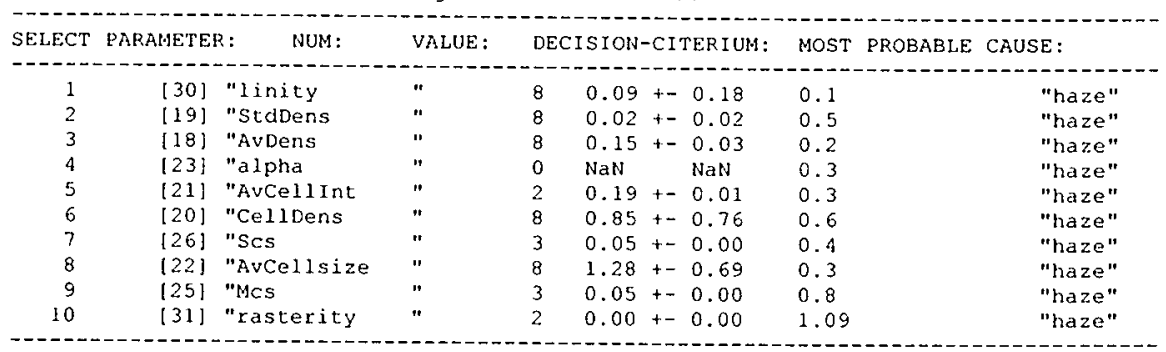

IPPreason ends: decision criterion met: 1.00, most probable cause: "haze"

STEP:

\section{DIAGNOSIS and CONTROI ACTIONS}

\section{DIAGNOSIS :}

there is one most likely cause: [16] haze

DISCRIMATIVE QUALITY of diagnosis: "very good" (4.327447e+035\%)

Noiselevel : $6:$

MisMatch detected from $-0.209790 \mathrm{~mm}$ (inside print) to $0.104895 \mathrm{~mm}$ (outside print) 
Results of the analysis, clockwise: 1. CIDZ-transformation of image. 2. Position in L1L2-plane of image, and allocation to cluster 2 3. searchboxes on the image, 4. resulting probabilities: \#2 is most probable
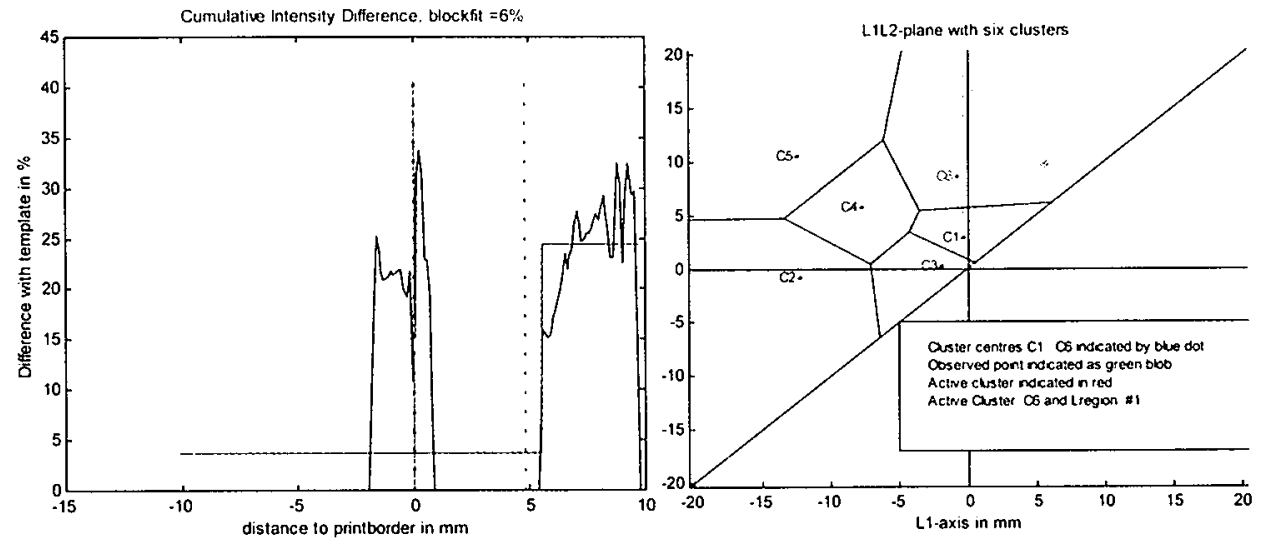

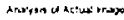
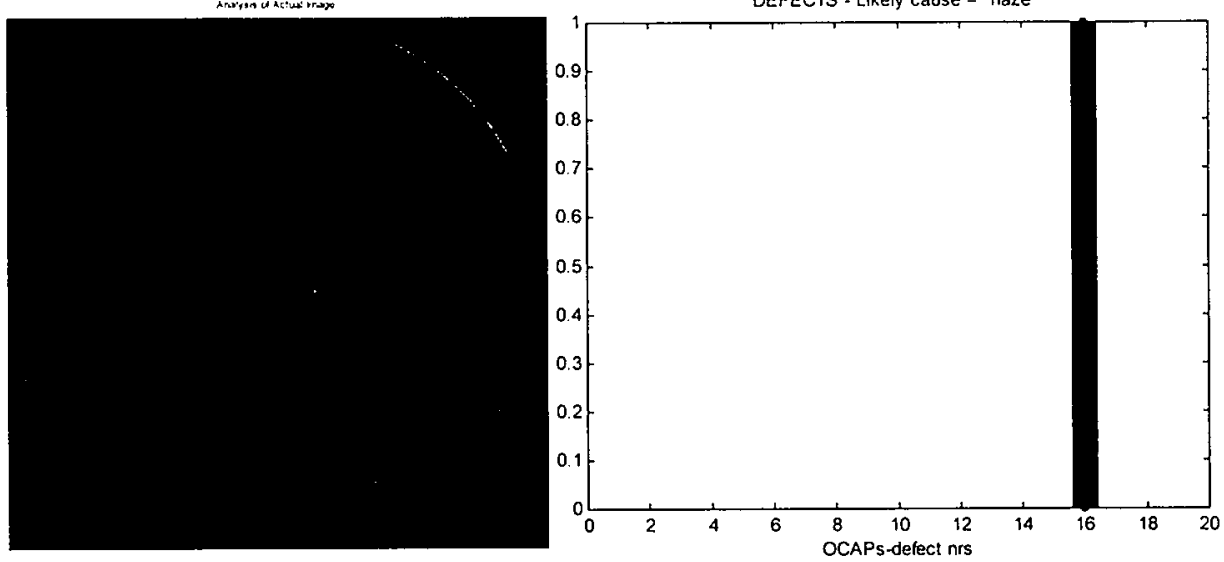



\section{GENERAL REFERENCES}

Altmann, M. Feilitzsch, F.v. Schanda U. (1996), A parameterized pulse shape analysis method for the GALLEX solar neutrino experiment, Nucl. Inst. Meth. A 381 (1996) 398

Amitrano, C. Coniglio, A. di Liberto F., Phys. Rev. Lett. 571016 (1986).

Argoul, F., Arneodo, A., Elezgaray, J., Grasseau, G., Murenzi, R. (1989) Wavelet transform of fractal aggregates. Phys. Lett. A. 135: 327-336.

Arneodo, A., Bacry, E., Muzy, J.F. (1995) The thermodynamics of fractals revisited with wavelets. Physica A. Vol.213, pp. 232-275

Arneodo (1996), Wavelet Analysis of Fractals in: 'Wavelets, Theory and Applications', Erlebacher et al. (Eds), Oxford University Press, Oxford (UK).

Arneodo, A., Bacry, E, Manneville, S., Muzy, J., (1998), Analysis of Random Cascades Using Space-Scale Correlation Functions, Physical Review Letters, Vol. 80. p. 4

Asvestas G., Matsopoulos G.K., Nikita K.S. (1999), Estimation of fractal dimension of images using a fixed mass approach, Pattern Recognition Letters, Vol 20, pp. , 347-354

Azencott R., Wang J. P., Younes L. (1997), Texture classification using Windowed Fourier filters, Vol 19, No 2, page 148, IEEE Transactions on Pattern Analysis and Machine Intelligence

Barron, A.R. (1984), Predicted squared error: a criterion of automatic model selection, in Self-Organising Methods in Modelling, S.J. Farlow (ed), Marcel Dekker NY, 1984.

Bellman, R.E. (1961), Adaptive Control Processes, Princeton (USA), Princeton University Press.

Bennett N., Burridge R., Saito N. (1999), A method to detect and characterize Ellipses using the Hough transform, Vol 21, No 7, page 652, IEEE Transactions on Pattern Analysis and Machine Intelligence

Beylkin, G. (1996), Wavelets, Multiresolution Analysis and Fast Numerical Algorithms. in 'Wavelets, Theory and Applications'. Ed. C. Erlebacher, M. Y. Hussani, L.M. Jameson. Oxford University Press, 1996.

Bezdek, J.C., Castelaz, P.F. (1971), Prototype classification and feature selection with fuzzy sets, IEEE Trans. Syst. man. Cyb., Vol. 7, pp. 87-92.

Bezdek, J.C. (1973), Fuzzy mathematics in pattern classification. Ph.D.Thesis, Applied Mathematics, Cornell University, Ithaca, New York (USA).

Bezdek, J. C. (1980), A Convergence Theorem for the Fuzzy ISODATA Clustering Algorithms. In: IEEE Trans. Pattern Anal. Machine Intell., Vol. PAMI-2, No. 1, pp. 1-8. 
Bezdek, J. C. (1981), Pattern Recognition with Fuzzy Objective Function Algorithms, Plenum Press, New York.

Bezdek, J. C., (Ed.) (1987): The analysis of fuzzy information, 3 Volumes, CRC Press, Boston (USA).

Bezdek, J.C., Pal, S.K. (1992), Fuzzy models for Pattern Recognition, IEEE-Press, New York (USA).

Bezdek, J.C. , Pal, N.R. (1998), Some new indexes for cluster validity, IEEE Transactions on Systems, Man, and Cybernetics, part B, Vol. 28, pp. $301-315$.

Biernacki C., Celeux G., Govaert G. (2000), Assessing a mixture model for clustering with the integrated completed likelihood, Vol 22, No 7, page 719, IEEE Transactions on Pattern Analysis and Machine Intelligence

Bijaoui, A. (1996), Vision Modeling and Information Coding, Vistas in Astronomy, Special issue, Vol. 40, part 4.

Bishop, C.M. (1995), Neural Networks for Pattern Recognition, Clarendon Press, Oxford (UK).

Bisoi, A.K., Mishra, J., (2001), On calculation of fractal dimension of images, Pattern Recognition Letters Vol. 22, pp. 631-637

Bock, H.H., (1979), Clusteranalyse mit unscharfen Partitionen in Klassification und Erkenntnis III: Numerische Klassification, H.H. Bock, Ed., Frankfurt a.M., pp 137-163.

Bow, Sing-Tze (1992), Pattern Recognition and Image Processing, ISBN 0-8247-8583-5, Dekker inc., New York (USA).

Bowyer, Kevin W., Jonathon Phillips, P. (1998), Empirical Evaluation Techniques in Computer Vision, June 1998, ISBN 0-8186-8401-1, IEEE Press.

Breu H., Gil J., Kirkpatrick D., Werman M. (1995), Linear Time Euclidean Distance Transform Algorithms, Vol 17, No 5, page 529, IEEE Transactions on Pattern Analysis and Machine Intelligence

Buchanan, B., Shortliffe, E.H., Rule-Based Expert Systems, $2^{\text {nd }}$ ed., ISBN 0-201-10172-6, Addison Wesley, Reading (Ma), 1985

Buckley, J.J., Hayashi, Y., Czogala, E., (1993), On the equivalance of neural nets and fuzzy expert systems, Fuzzy Sets and Systems 53, (1993), pp 129-134.

Calway, A. (1993) Image analysis using a generalised wavelet transform, Colloque institution of electrical engineers, vol. DIG009, 1993

Carlin M. (2000), Measuring the complexity of non-fiactal shapes by a fractal method, Pattern Recognition Letters, Vol 21, pp., 1013-1017

Chan, J. (1998), Print quality assessment by human perception, Proc. of Europto98, Zürich, 1998.

Chaudhuri B.B., Sarkar N. (1995), Texture Segmentation using Fractal Dimension, Vol 17, No 1, page 72, IEEE Transactions on Pattern Analysis and Machine Intelligence

Chiu, S. (1994), Fuzzy Model Identification Based on Cluster Estimation, Journal of Intelligent and Fuzzy Systems, Vol. 2, No. 3, pp. 267-278. 
Christensen, H., Foerstner, W., Special Issue on Performance Evaluation, Machine Vision and Applications, 9(5), 1997.

Chui, Ch. K., (1996) 'An Introduction to Wavelets', Academic Press, Boston.

Courtney, P., Thacker, N., Clark, A.F. (1997), Algorithmic Modelling for Performance Evaluation. Machine Vision and Applications, 9(5):219-228,.

Cucchiana R., Filicori F. (1998), The vector-gradient Hough transform, IEEE Transactions on Pattern Analysis and Machine Intelligence, Vol 20, No 7, page 746

Darwin, Ch., (1859), On the origin of species by means of natural selection, London, John Murray Publ.

Daubechies I. (1990), The Wavelet Transform, Time-Frequency Localisation and Signal Analysis, IEEE Trans. Information Theory., Sept. 1990

Dave, R. N. (1992): Generalized fuzzy c-shells chistering and detection of circular and elliptical boundaries. Pattern Recognition, Vol. 25, No. 7.

Diamond, P., Kloeden, P., (1991), Metric Spaces of Fuzzy Sets, Fuzzy Sets and Systems Vol.35, pp. 241-249.

Dijkstra, E.W. (1974), The Problem of the Shortest Subspanning Tree, Numerische Mathemathik.

Drolon H., Druaux F., Faure A. (2000), Particles shape analysis and classification using the wavelet transform, Pattern Recognition Letters, Vol 21, pp. , 473-482

Dubois, D., Prade, H. (1987), Fuzzy Sets in Approximate Reasoning, Fuzzy Sets and Systems 40, pp. 143-202.

Dubois, D., Prade, H. (1996), Fuzzy Sets Methods in Information Engineering, A Guided Tour of Applications, J. Wiley \& Sons, New York, USA.

Duda, R.O., Hart,P.E. (1973), Pattern Classification and Scene Analysis, Wiley Interscience, John Wiley \& Sons New York (USA), ISBN 0-471-22361-1.

Dunn, J.C. (1973), A Fuzzy Relative of the ISODATA Process and its Use in Detecting Compact Well-Separated Clusters, Journal of Cybernetics, Vol. 3, No. 3, pp. 32-57, 1973

Elman, $\mathbf{J},(1988)$, Finding structure in time, CRL Technical Report 8801, Center for Research in Language, University of California, San Diego, (1988).

Erlebacher G., M. Yousuff Hussaini, L.M. Jameson, (Eds) (1996), Wavelets, Theory and Applications, Oxford University Press, Oxford (UK), ISBN 0-19-509423-9.

Erwin, E., Obermayer K., Schulten K. (1992). Self-organising maps: Ordering, convergence properties and energy functions. Biological Cybernetics, 67(1):47-55, 1992.

Falconer, K. J. The Geometry of Fractal Sets. Cambridg University Press. Cambridge 1985.

Farmer J. D., Z. Naturforsch. Vol. 37 a, page 1304 (1982).

Farmer, J.D., Ott, E, Yorke.J.A. (1983), Physica D 7, page 153

Figueredo, M., Leitao, J., Jain, A.K. (1999), On Fitting Mixture Models, in: 'Energy Minimisation Methods in Computer Vision and Pattern Recognition', Hancock, E., Pellillo, M., Eds., Springer-Verlag. 
Firschein, O., Fischler, M., Kanade, T. Creating Benchmarking Problems in Machine Vision: Scientific Challenge Problems. DARPA Image Understanding Workshop, pages 177-182, 1993.

Fisher,R.A. (1936), The use of multiple measurements in taxonomic problems, Annual Eugenics, 7, Part II, 179-188 (1936); also in "Contributions to Mathematical Statistics" (John Wiley, NY, 1950).

Freear, N.D., Shippen, J.M., Westra, R.L., (1999), Applying Neural Networks to Fault Classification in Image Data, Proc. of the European Conference on Intelligent Techniques and Soft Computing, Aachen (Germany) September 1999.

Fukuyama, Y., Sugeno, M. (1989), A new method of choosing the number of clusters for the fuzzy C-means method, Proc. fifth Fuzzy Syst. Symp., pp. 247-250.

Gath, I., Geva,, A.B. (1989), Fuzzy chustering for the estimation of the parameters of the components of mixtures of normal distributions, Pattern Recognition Letters, Vol. 9, Nr. 3, pp. 77-86.

Geva A.B., Steinberg Y., Bruckmair Sh., Nahum G. (2000), A comparison of cluster validity criteria for a mixture of normal distributed data, Pattern Recognition Letters , Vol 21, pp. , $511-529$

Gimelfarb, G., (1999), Modeling image textures by Gibbs random fields. Pattern Recognition Letters 20 (1999), pp. 1123-1132.

Gleick, J. (1987), Chaos: Making a New Science (1987), Viking Science Publ. New York (USA), ISBN 90-254-6628-1.

Goldberg, D.E. (1989), Genetic Algorithms in Search, Optimisation, and Machine Learning, Addison-Wesley, Reading/Massachusetts (USA), 1989.

Gonzalez, R.C., Woods, R. E., (1992) Digital Image Processing, Addison-Wesley Publishing, Reading (USA), 1992.

Gould, S.J., (1991), Bully for Brontosaurus, Penguin books, ISBN 0140159851, London, pp 340-353.

Grabisch, M, (1996), Applications of Fuzzy Integral to Decision Analysis and Pattern Classification, EUFIT Conference, pp 1289-1294, Aachen 1996

Grabisch, M, Nguyen H.T, Walker E.A, (1995), Fundamentals of Uncertainty Calculi, with Applications to Fuzzy Inference, Kluwer Academic, 1995

Guan W., Ma S. (1998), A list-processing Approach to Compute Voronoi Diagrams and the Euclidean Distance Transform, Vol 20, No 7, page 757, IEEE Transactions on Pattern Analysis and Machine Intelligence

Gustafson, D.E., Kessel, W. (1979), Fuzzy Clustering with a Fuzzy Covariance Matrix, in: K.S. Fu, Ed., Proc. IEEE-CDC2, San Diego, CA, pp. 761-766, Jan. 10-12, 1979.

Guzman, A. (1968), Decomposition of a visual scene into three-dimensional bodies, in: AFIPS Conference Proceedings, Thompson, Washington (USA), Vol. 33, pp. 291-304.

Halsey, T. C. Jensen, M. H. Kadanoff, L. P. Procaccia I., Shraiman B. I., Phys. Rev. A 33, 1141 (1986). 
Hancock, E., Pellillo, M. (1999), Eds., Energy Minimisation Methods in Computer Vision and Pattern Recognition, Springer-Verlag.

Haralick, R.M. Statistical and structural approaches to texture, Proceedings of the IEEE, may 1979 , number 5, vol.67.

Haralick, R.M., Computer Vision Theory: The Lack Thereof. Computer Vision, Graphics, and Image Processing, 36:372-386, 1986.

Hati, S., Sengupta, S., (2001), Robust camera parameter estimation using genetic algorithm, Vol. , 22, pp. 289-298

Hausdorff, F. Dimension und äıßeres Maß, Mathematische Annalen 79,157 (1919).

Hecht, E., Geometrical and Physical Optics, Addison-Wesley Publ., New York, 1987.

Hentschel H. G. E., I. Procaccia, (1983), Physica D, Vol. 8, page 435.

Hildreth, E.C. (1998), Models of Recognition, Vision Research, Volume 38, Number 15/16, 1998

Ho, T. K., Baird, H. S. (1997). Large-scale simulation studies in image pattern recognition. IEEE Transactions on Pattern Analysis and Machine Intelligence, Vol 19, No 10, pp. 1067-1079.

Hoffbeck J.P., Landgrebe D.A. (1996), Covariance Matrix Estimation and Classification with Limited Training Data, IEEE Transactions on Pattern Analysis and Machine Intelligence, $\mathrm{Vol} 18$, No 7, page 763.

Holland, J.H. (1975), Adaptation in Natural and Artificial Systems, University of Michigan Press, Michigan (USA).

Holschneider, M., 1988, Journal Statistical Physics, Nr. 50, pp. 963-970.

Holschneider, M., 1990, Inverse Radon Transform through Inverse Wavelet Transform, J. Inverse Problems, 1990, pp. 134-143.

Hsu, T. Calway A., Wilson R. (1993), Texture analysis using the multiresolution Fourier transform, Proceedings at the 8th scandinavian conference on image analysis, Tromso, may 1993.

Huygens, Ch., Traité de la lumiere, Leiden, 1678.

Hwang W.J., Ye B.Y., Lin Ch.T. (2000), A novel competitive learning algorithm for the parametric classification with Gaussian distributions, Pattern Recognition Letters , Vol 21, pp. , 375-380

IEEE Standard 610.4-1990, (1990) IEEE Standard Glossary of Image Processing and Pattern Recognition Terminology, IEEE Press, New York, 1990

Jaffard, S. (1989), Conditions de Lipschitz Contimuité pour le Transformation Ondulettes, C.R. de Acad. des Sciences de Paris, Vol. 308, Series I, page. 97.

Jahne, B., 1997, Digital Image Processing: Concepts, Algorithms, and Scientific Applications, Springer Verlag, 1997.

Jain A.K., Chandrasekaran, B. (1982), Dimensionality and Sample Size Considerations in Pattern Recognition Practice, in: The Handbook of Statistics, by Krishnaya, P.R., and Kanal, L.N., (eds.), Vol. 2, pp. 835 - 855, North-Holland Pub., Amsterdam, 1982. 
Jain, A.K., Moreau, J.V. (1987), Bootstrap technique in cluster analysis, Pattern Recognition, Vol. 20, pp. 547-568.

Jain A.K., Tuceryan, M. (1992), Texture analysis, chapter 11 in: Handbook of Pattern Recognition and Computer Vision by C.H.Chen (Ed.) 1992.

Jain A.K., Duin R.P.W., Mao J. (2000), Statistical Pattern Recognition: A Review, Vol 22, No 1, page 4, IEEE Transactions on Pattern Analysis and Machine Intelligence

Jones, C.L., Lonergan, G.T., Mainwaring, D.E. (1996) Wavelet packet computation of the Hurst exponent. Journal of. Physics A, Vol. 29: pp. 2509-2527.

Jordan, D.W., Smith, P. (1987), Nonlinear Ordinary Differential Equations, Clarendon Press, Oxford, $2^{\text {nd }} \mathrm{ed} ., 1987$.

Kaplan L.M., Jay Kuo C.C. (1995), Texture Roughness Analysis and Synthesis via Extended Self-Similar Model, IEEE Transactions on Pattern Analysis and Machine Intelligence, Vol 17, No 11, page 1043.

Kaplanova, M., M. Fribert (1998), Image Analysis in Multicolour Printing, Proc. Europto98, Zürich, 1998.

Keller, J. M., Gray, M.R., Givens, J.A. (1985), A fuzzy K-Nearest Neighbour algorithm. IEEE Trans. Syst. Man., Cybern. Vol. 15, pp. 580-585.

Keller, J. M., Gader, P., Tahani, H., Chiang, J. H., Mohamed, M. (1994): Advances in fuzzy integration for pattern recognition. In: Fuzzy Sets and Systems, Vol. 65, pp. 273-283.

Khalil M.I., Bayoumi M.M. (2000), Invariant 2D object recognition using the wavelet modulus maxima, Pattern Recognition Letters, Vol 21, pp. , 863-872

Khotanzad, A., Lu, J.H. (1990). Classification of Invariant Image Representations Using a Neural Network, IEEE Transactions on Acoustic Speech and Signal Process., Vol. 38, No. 6, pp. 1028-1038.

Kirchhoff, G.R., Zur Theorie der Lichstrahlen, Annalen der Physik und Chemie, vol. 18, no. 8, pp. 663-695, 1883.

Kohonen, T. (1982), Self-organized formation of topologically correct feature maps. Biological Cybernetics, 43:59-69, 1982.

Kohonen, T (1990). The self-organising map. Proceedings of the IEEE, 78(9):1464-1480, 1990.

Kohonen, T. (1989), Self-Organisation and Associative Memory. Springer-Verlag, BerlinHeidelberg-New York-Tokio, 3 edition, 1989.

Kothari R., Pitts D. (1999), On finding the number of clusters, Pattern Recognition Letters, Vol 20, pp. , 405-416

Krishnapuram, R. (1993): Fuzzy Clustering Methods in Computer Vision. In: Proceedings von EUFIT'93, Vol. 2, pp. 720-730.

Krishnapuram, R., Keller, J. M. (1992): Fuzzy Set Theoretic Approach to Computer Vision: An Overview. In: IEEE International Conference on Fuzzy Systems, San Diego, pp. 135-142. 
Krishnapuram, R., Nasraoui, O., Frigui, H. (1992): The fuzzy C spherical shells algorithms: A new approach. In: IEEE Transactions on Neural Networks, Vol. 3, No. 5, pp. 663-671.

Kruse, R., E. Schwecke (1990), Fuzzy Reasoning in a Multidimensional Space of Hypotheses, Int. J. of Approximate Reasoning 1990, nr. 4, 47-68.

Kuncheva, L.I. Bezdek, J.C. (1998), An integrated framework for generalized nearest prototype classifier design, International Journal of Uncertainty, Fuzziness and Knowledge-based Systems 6 (1998) 437-457.

Lau, Th., 'Chinese Proverbs', Harperperennial Library, Boston, 1995.

Leblanc, B., Lutton, E., (1997), Bitwise Regularity Coefficients as a Tool for Deception Analysis of a Genetic Algorithm, INRIA Report RR-3274, October 1997

Legierse, P.E.J., Witgrefe, F. (1995), IntelPadPrint, the development of an intelligent padprinting system, project proposal Brite/Euram, European Union DG XII, grant BE3180

Legierse, P.E.J., (2000), IntelPadPrint, Final Project Report, Brite/Euram, European Union DG XII, grant BE3180

Leung Y., Zhang J.Sh., Xu Z.B. (2000), Clustering by Scale-Space Filtering, Vol 22, No 12, page 1396, IEEE Transactions on Pattern Analysis and Machine Intelligence

Lin, C.C., Chellappa R. (1987), Classification of partial 2-D shapes using Fourier descriptors, IEEE Transactions on Pattern Analysis and Machine Intelligence, volume 9 , number 5, pp. 686-690.

Lippert, R.A., Arias T.A., Edelman Alan (1998), Multiscale computation with interpolating wavelets, Journal of Computational Physics Vol. 140, pp. 278.

López-Rubio, E., Muñoz-Pérez, J., Gómez-Ruiz, J.A., (2001), Invariant pattern identification by self-organising networks, Vol. , 22, pp. 983-990

Luckiesh, M. (1965), Visual Illusions, Dover publications Inc., New York (USA), ISBN 0486-21530-X

Mallat S. G., Hwang, W.L., (1992), A Multiresolution Representation of Singular Functions, IEEE Trans. on Information Theory, Vol. 38, page 617.

Mallat, S.G. (1989), A Theory for Multiresolution Signal Decomposition: The Wavelet Representation, IEEE Transactions on Pattern Analysis and Machine Intelligence, July 1989

Mallet Y., Coomans D., Kautsky J., De Vel O. (1997), Classification using adaptive wavelets for feature extraction, Vol 19, No 10, page 1058, IEEE Transactions on Pattern Analysis and Machine Intelligence

Mandelbrot B. B. (1982), The Fractal Geometry of Nature, Freeman, San Francisco 1982.

Mandelbrot, B. B. (1975), Les Object Fractals, Flammarion, (Paris, 1975), in English as: Fractals: Form, Chance and Dimensions, Freeman, San Francisco 1977.

Mandelbrot, B. J. Fluid. Mech. 62, 331 (1974).

Mandelbrot, B., (1999), MultiFractals and l/F Noise : Wild Self-Affinity, Physics, pp. 19631976, Selecta Volume (1999), Springer Verlag; Berlin 
Marroquin J.L., Velasco F.A., Rivera M., Nakamura M. (2001), Gauss-Markov Measure Field Models for Low-Level Vision, Vol 23, No 4, page 337, IEEE Transactions on Pattern Analysis and Machine Intelligence

Mascle, S., Olivier, P., Vidal-Madjar, D., (1994), Identification of vegetation classes using multi-configuration polarimetric SAR data, Proceedings of Progress In Electromagnetics Reseach Symposium'94 [PIERS'94], in Noordwijk, the Netherlands, on July 1 1-15 1994.

Matsuyama T., Miura S., Nagao M. (1983), Structural analysis of natural textures by Fourier transformation, IEEE CVGIP, 24, 1983.

McCulloch, W.S., Pitts, W., (1943), A Logical Calculus of the Ideas Immanem in the Nervous Activity, Bulletin for Mathematical Biophysics, Vol. 7, pp. 115-133.

McCulloch, W.S., $(1965,1988)$, Embodiments of the Mind, $2^{\text {nd }}$ Eddition, Cambridge (USA), MIT Press.

Miller, W., Sutton, R., Werbos, P., (1990), Neural networks for control, Cambridge, MA, MIT Press, 1990.

Milnor, J., A Problem in Cartography, The American Mathematical Monthly, Vol. 76, 1969

Minsky, M.L., Papert, S.A., $(1969,1988)$, Perceptrons, $2^{\text {nd }}$ Eddition, Cambridge (USA), MIT Press.

Moghaddam B., Pentland A. (1997), Probabilistic Visual Learning for object representation, Vol 19, No 7, page 696, IEEE Transactions on Pattern Analysis and Machine Intelligence

Morlet, J, (1983), NATO ASI series, part FI (Springer Verlag, Berlin)

Mukherjee J., Das P.P., Aswata-Kumar M., Chatterji B.N. (2000), On approximating Euclidean metrics by digital distances in $2 D$ and $3 D$. Pattern Recognition Letters, Vol 21, pp. , 573-582

Narendra, K. Parthasarathy, (1990), Identification and control of dynamical systems using neural nenworks, K., IEEE Trans. Neural Networks, Vol. 1, No 1, pp 4-27, March 1990.

Nuygen D., Widrow B.,(1990), Neural networks for self-learning control systems, IEEE Control Systems Magazine, pp 18-23, April 1990.

Oh, G., Lee, S., Shin, S.Y. (1999), Fast determination of textural periodicity using distance matching functions, Pattern Recognition Letters 20 (1999), pp. 191-197.

Paglieroni, D.W. (1992a), Distance Transforms, Computer Vision, Graphics, and Image Processing, Vol. 54, pp. 56-74.

Paglieroni, D.W. (1992b), A Unified Distance Transform Algorithm and Architecture, Machine Vision and Applications, Vol. 5, pp. 47-55.

Pal, N.R., Bezdek, J.C. (1995), On cluster validity for the fuzzy c-means model, IEEE Trans. Fuzzy Sys., Vol. 3, Nr. 3.

Pal, N.R., Pal, K., Bezdek, J.C. (1997), A Mixed C-Means Clustering Model, Proc. 6th IEEE Int. Conf. on Fuzzy Systems, July 1-5, 1997, Barcelona (Spain) 
Pal, S. K. (1992): Fuzziness, Image Information and Scene Analysis. In: Yager, R., Zadeh, L.A., An Introduction to Fuzzy Logic Applications in Intelligent Systems, Kluwer Academic Publishers, pp. 147-184.

Pal, S. K. (1994): Fuzzy Sets in Image Processing and Recognition. In: Marks, R. J., Fuzzy Logic Technology and Applications, USA, pp.33-40.

Palmer, S., (1983), The Psychology of Perceptual Organization: A Transformational Approach, in: 'Human Vision', J. Beck, B. Hope, A. Rosenfeld, eds., Academic Press.

Paredis, J., Westra, R.L., (1997), Coevolutionary Computation for Path Planning, Proc. of the European Conference on Intelligent Techniques and Soft Computing, Aachen (Germany) 1997

Park H.J., Yang H.S. (2001), Invariant object detection based on evidence accumulation and Gabor features, Pattern Recognition Letters, Vol 22, pp. , 869-882

Pavlidis, T., (1992), Why Progress in Machine Vision Is So Slow. Pattern Recognition Letters, 13:221-225, 1992.

Peeters, R.L.M., Westra, R.L. (2000), Correlation of Variations in Base-Paper with Printability Defaults in End-Paper, Maastricht University Contract Research for SAPPI Nederland B.V.

Peitgen, H. O., Richter, Peter, The Beauty of Fractals (1986).

Pelah, A. (1997), The Vision of Natural and Complex Images, Vision Research, Volume 37, Number 23, 1997

Peña J.M., Lozano J.A., Larrañaga P. (1999), An emperical comparison of four initialisation methods for the K-means algorithm, Pattern Recognition Letters, Vol 20, pp. , 1027-1040

Pentland, A.P., (1984), Fractal-Based Description of Natural Scenes, IEEE Transactions on Pattern Analysis and Machine Intelligence, Vol. 6, No. 6, pp. 661-674, Nov 1984

Pentland, A.P., (1986), Perceptual Organization and the Representation of Natural Form, AI Journal, Vol. 28, No. 2, pp. 1-38.

Perlovsky L.I. (1998), The Conundrum of Combinatorial Complexity, Vol 20, No 6, page 666, IEEE Transactions on Pattern Analysis and Machine Intelligence

Peters, B.C., Walkers, H. (1978), An iterative procedure for obtaining maximum likelihood estimates of the parameters of a mixture of normal distributions, SIAM J. Appl. Math., Vol. 35, pp. 362-378.

Petrou, M., (2001), Learning in pattern recognition, Pattern Recognition Letters, Vol. , 22, pp. 3-13

Pfeifer, R., Schreter, Z., Fogelman-Soulié, Steels, L., Eds., (1989a), 'Connection in Perspecitve', North-Holland Publ., Amsterdam, 1989

Pfeifer, R., Schreter, Z., Fogelman-Soulié, Steels, L., Eds., (19896), 'Putting Connection in Perspecitve'in [Pfeifer et al, 1989a].

Pham, T. D., Yan, H. (1996): Color Image Segmentation- A Fuzzy-Integral MountainClustering Approach, In: Image Segmentation Workshop 1996, The Australian Pattern Recognition Society, Sydney, pp. 27-32. 
Philips P.J., Bowyer K.W. (1999), Introduction to the special section on empirical evaluation of computer vision algorithms, Vol 21, No 4, page 289, IEEE Transactions on Pattern Analysis and Machine Intelligence

Pietronero L., E. Tosatti (eds.), Fractals in Physics. North-Holland, Amsterdam 1986.

Pittner S., Kamarthi S.V. (1999), Feature Extraction from wavelet coefficients for pattern recognition tasks, IEEE Transactions on Pattern Analysis and Machine Intelligence, Vol 21, No 1, page 83

Postma, E.O., (1994), 'SCAN, a Neural Model of Covert Attention', PhD Thesis, Universiteit Maastricht, 1994, ISBN 90-9007320-5.

Price. K., (1986), Anything You Can Do, I Can Do Better (No You Can't). Computer Vision, Graphics, and Image Processing, 36:387-391, 1986.

Priestley, M.B. (1981), Spectral Analysis and Time Series, Academic Press, London (UK).

Ragnehalm, I. (1993), The Euclidean Distance Transform, Ph.D.Thesis, Linköping University, Linköping, Sweden.

Ratches J.A., Walters C.P., Buser R.G., Guenther B.D. (1997), Aided and Automatic Target Recognition based upon sensory inputs from image forming Systems, IEEE Transactions on Pattern Analysis and Machine Intelligence, Vol 19, No 9, page 1004

Raudys, S.J., Pikelis, V. (1980), On dimensionality, sample size, classification error, and complexity of classification algorithms in pattern recognition, Vol. 2, pp. 243251, IEEE Transactions on Pattern Analysis and Machine Intelligence.

Renyi, A. Probability Theory, North Holland, Amsterdam (1970)

Richardson, S., Green, P. (1997), On Bayesian Analysis of Mixtures with Unknown Number of Components, J. Royal Statistical Society (B), Vol. 59, pp. 731-792.

Rioul, O., (1993), Hölder regularity of subdivision schemes and wavelets, Proceedings of the 2nd International Conference on 'Curves and Surfaces', 1994, (Chamonix, France, 1993).

Roberts, L.G. (1965), Machine perception of three-dimensional solids, in: Tippit, J.T., et al. (Eds.), 'Optical and Electro Optical Information Processing. MIT Press, Cambridge (USA), pp. 159-197.

Roman, Jan, (1995), MultiFractals, Doctoral thesis, Chalmers University, 1995.

Rosenfeld, A., Chaudhuri, B. B. (1996): On a metric distance between fuzzy sets. In: Pattern Recognition Letters, Vol. 17, pp. 1157-1160.

Ruspini, E.H. (1969), A New Approach to Clustering, Information Control, Vol. 15, No. 1, pp 22-32, July 1969

Rutten, J.H.G.C. (1998), Polyhedral Clustering, Ph.D. Thesis, Universiteit Maastricht, 1998, ISBN 90-5278-236-9.

Sanna, A., Montrucchio, B., Sparavigna, A. (1999), A parallel algorithm of texture analysis for liquid crystal investigation. Pattern Recognition Letters 20 (1999), pp. 183190.

Schalkhoff, R. (1992), Pattern Recognition: Statistical, Structural, and Neural Approaches, Wiley Scientific Publishing, New York (USA), 1992. 
Sepulchre, J.A., Babloyants, A. (1991), Path Finding with Non-linear Waves, Phys. Rev. Lett. $66,1314$.

Serra, R., (1989), Dynamical Systems and Experts Systems, in Connnectionism in Perspective, ISBN: 0444880615 North Holland, (1989).

Shannon, C. E. (1948), A Mathematical Theory of Information, Bell System Technical Journal 27:379-423, 623-56

Sharman R., Tyler J.M., Piankyh O.S. (2000), A fast and accurate method to register medical images using Wavelet Modulus Maxima, Pattern Recognition Letters, Vol 21, pp. , 447-462

Shih, F.Y,C., Mitchell, O.R. (1992), A Mathematical Morphology approach to Euclidean distance transformation, IEEE Transactions on Image Processing, Vol 1, No 2, pp. 197-204.

Shippen, J.M., Westra, R.L., Freear, N.D., (1999), A Parallel Hardware Multiple Microprocessor Asynchronous Neural Network Architecture for Control Applications Proc. of the European Conference on Intelligent Techniques and Soft Computing, Aachen (Germany) September 1999

Sklansky, (1978), Image Segmentation and feature extraction, IEEE Trans Syst. Man cybern., $8,237-247,1978$

Stavrakakis, G.S., Pouliezos, A., Zervakis, M., Anagnostakis, E., Tselentis, G. (1997), Feature extraction and neuro-fuzzy classification of machine vibration signals for on-line quality control purposes, Proc. of the European Conference on Intelligent Techniques and Soft Computing, Aachen (Germany) September 1997, Aachen (Germany), pp. 1744-1748.

Steinberg, Y., Zeitouni, O. (1992), On tests for normality, IEEE Trans. Inform. Theory, Vol. 38., Nr. 9.

Subbarao M., Tyan J.Q. (1998), Selecting the optimal focus measure for autofocussing and depth-from-focus, Vol 20, No 8, page 864-867, IEEE Transactions on Pattern Analysis and Machine Intelligence

Sugeno, M. (1974): Theory of Fuzzy Integrals and its Applications, Ph.D.Thesis, Tokyo Institute of Technology, Japan.

Sugeno, M. (1977): Fuzzy Measures and Fuzzy Integrals: A Survey. In: Fuzzy Automata and Decision Processes, North Holland, Amsterdam, pp. 89-102.

Sziranyi, T., Hanis, A., (1999), Sub-pattern texture recognition using intelligent focal plane imaging sensor of small window-size. Pattern Recognition Letters 20 (1999), pp. 1132-1139.

Tagare H.D., Toyama K., Wang J.G. (2001), A maximum likelihood strategy for directing attention during visual search. IEEE Transactions on Pattern Analysis and Machine Intelligence, Vol. 23, No. 5, page 490.

Tao, Y., Lam. E.C.M., Tang, Y.Y., (2001), Feature extraction using wavelet and fractal. Pattern Recognition Letters, Vol. , 22, pp. 271-287

Theiler, J. (1990), Estimating fractal dimensions, Journal of the Optical Society of America, Vol A7, pp. 1055-1073. 
Titterington, D., Smith, A., Makov, U. (1985), Statistical Analysis of Finite Mixture Distributions. Chichester (U.K>), John Wiley \& Sons.

Tuma J.J., Numerical Calculations in Engineering, Mc Graw-Hill Publishing, New York, 1990.

Valkealahti K., Oja E. (1998), Rechced multidimensional co-occurrence histograms in texture classification, Vol 20, No 1, page 90-94, IEEE Transactions on Pattern Analysis and Machine Intelligence

Vanrell, M., Jvitria, Roca. X., (1997), A Multidimensional Scaling Approach to Explore the Behavior of a Texture Perception Algorithm. Machine Vision and Applications, 9(5):262-271, 1997.

Vogel, H., Jäger, U. (1997), Error detection on structured surfaces with digital image processing and neural networks, Proc. of the European Conference on Intelligent Techniques and Soft Computing, Aachen (Germany) September 1997, Aachen (Germany), pp. 1744-1748.

Vojak, R., Lévy-Véhel, J., Higher Order Multifractal Analysis, INRIA Report RR-2796, October 1997

Walker, E. L., Kang, H.-B. (1994): Fuzzy Measures of Uncertainy in Perceptual Grouping. In: Proceedings von 3th IEEE International Conference on Fuzzy Systems, Vol. 3, pp. 2020-2024.

Wang L., J. Liu (2000), Texture segmentation based on MRMRF modeling, Pattern Recognition Letters, Vol 21, pp., 189-200

Wang, L., Liu, J. (1999), Texture classification using multiresolution Markov random field models. Pattern Recognition Letters 20 (1999), pp. 171-182.

Watanabe, S. (1985), Pattern Recognition: Human and Mechanical, John Wiley \& Sons Pub., New York (USA).

Westra, R.L., (1982), On the non-integer Dimensions of non-linear dynamical Systems, Graduation paper, Utrecht State University

Westra, R.L., R.M.L. Frenken, H.E., Dijk (1997a), Classification of Power System States with Self-Organising Maps, Proc. of the European Conference on Intelligent Techniques and Soft Computing, Aachen (Germany) 1997

Westra, R.L., Paredis, J., (1997b), Coevolutionary Computation for Path Plamning, Proc. of the European Conference on Intelligent Techniques and Soft Computing, Aachen (Germany) 1997

Westra, R.L. (1998), Classification of Variations and Deviations in 2D-Images using a Using ID Fuzzy Correlation Approach, UM internal research report M98-09, 1998

Westra, R.L., (1999a), Printing Quality Control Using Template Independent NeuroFuzzy' Defect Classification, Proc. of the European Conference on Intelligent Techniques and Soft Computing, Aachen (Germany) September 1999. (Also available as UM internal research report M00-05).

Westra, R.L., (1999b), A Parallel Hardware Multiple Microprocessor Asynchronous Neural Nenwork Architecture for Control Applications see:. Shippen, J.M. et al., 1999.

Westra, R.L., (1999c), Applying Neural Networks to Fault Classification in Image Data, see: Freear, N.D. et al., 1999. 
Westra, R.L., (1999d), Geometry and Uniform Intensity Transformations for Images with Multiple Greyscale Structures, UM-report UM-Math M99-04, September 1999

Westra, R.L, (2000a), Adaptive Control Using CCD-Images: Towards a Template-Invariant Approach, Invited Lecture, Proceeding of the 7 th Conference on Intelligent Learning (CoIL), Chios (Greece) June 2000.

Westra, R.L. (2000b), Efficient On-line Classification based on Fuzzy Multivariate Class Membership Functions for Clustering and Optimal Parameter Selection, 7th UK conference on Fuzzy Systems, Sheffield, October 2000

Westra, R.L (2000c)., Final Technical Report of IntelPadPrint, EU DGXII, BRPR-CT960296, January 2000

Whitley, D. (1989), The Genitor Algorithm and Selection Pressure. Proc. 3rd ICGA.

Wieland, A. P. (1991), Evolving Controls for Unstable Systems, Connectionist Models: Proc. 1990 Summer School.

Wilson R.C., Hancock E.R. (1999), Graph matching with hierarchical discrete relaxation. Pattern Recognition Letters, Vol 20, pp. , 1041-1052

White, R., Engelen, G. (1994), Urban System Dynamics and Cellular Automata: Fractal Structures between Order and Chaos. In: Chaos, Solitons, and Fractals (Special Issue), Vol. 4 (1994), pp. 563-583.

Windham, M.P. (1981), Cluster validity for firzy clustering algorithms, Fuzzy Sets and Systems, Vol. 5., pp. 177-185.

Windham, M.P. (1982), Cluster validity for fuzzy C-means clustering, IEEE Pattern Analysis and Machine Intelligence, Vol. 4, pp. 357-363.

Yager, R., Filev, D. (1994), Generation of Fuzzy Rules by Moumtain Clustering, Journal of Intelligent and Fuzzy Systems, Vol. 2, No. 3, pp. 209-219, 1994

Zadeh, L.A.(1965), Fuzzy Sets and Systems, in: Systems Theory, ed. J. Fox, pp.29-37, Polytechnic Press, Brooklyn NY, 1965

Zadeh, L.A., (1978), Fuzzy Sets as a Basis for a Theory of Possibility, Fuzzy Sets and Systems, No 1, 1978, pp. 3-28.

Zadeh, L.A.(1979), A theory of approximate reasoning, In: 'Machine Intelligence', Vol. 9 (Hayes J.E., Michie D. and Mikulich L.I., Eds.). New York: Elsevier, 1979, pp. 149-194.

Zahid, N., Abouelala, O., Limouri, M., Essaid, A., (1999), Unsupervised fuzzy Chustering. Pattern Recognition Letters 20 (1999), pp. 123-129. 



\section{Author Index}

A

$\begin{array}{ll}\text { Altmann, M. } & 77 \\ \text { Amitrano, C. } & 107 \\ \text { Argoul, F. } & 123 \\ \text { Arneodo, A. } & 73,74,106,111- \\ & 115,123-125 \\ \text { Asvestas, G. } & 104,107 \\ \text { Azencott, R. } & 73,75,79\end{array}$

Bellman, R.E. $\quad 28$

Bezdek, J.C. $\quad 135,136,138$, 140-142, 144, 146,155

Biernacki, C. $\quad 142$

Bishop, C.M. 28

Bisoi, A.K. $\quad 104$

Bock, H.H. $\quad 157$

Bow, S-Tz 77

Bowyer, K.W. 4

Breu, $\mathrm{H}$. $\quad 30$

Buchanan, B. 2

C

Carlin, $\mathrm{M}$.

107

Chan, J.

7

Chaudhuri, B.B. 124

Chiu, S.

Christensen

Chui, Ch. K.

Courtney, P.

D

Darwin, Ch.

Daubechies, I.

Diamond, $\mathrm{P}$.

Drolon, $\mathrm{H}$.

Duda, R.O.

142,147

4

86,88

4

Dunn, J.C.

90,91

85

204

88

$135,141,142$,

145

$142,146,155$,

161

\section{E}

Elman, $\mathbf{J}$,

207

Engelen, G. $\quad 73$

Erlebacher, G. $\quad 74$
F

Farmer, J. D. $\quad 104,107$

Figueredo, M. 143

Firschein, O. 4

Fisher,R.A. $\quad 169,170$

Freear, N.D. $\quad 148$

Fukuyama, Y. 141

G

$141,144,160$

Gath, I.

$141,143,161$

Geva, A.B.

79,125

Goldberg, D.E. $\quad 90$

Gonzalez, R.C. $\quad 73,77$

Gould, S.J. $\quad 90$

Grabisch, M 201, 202

Guan, W. $\quad 30$

Gustafson, D.E. $\quad 157$

Guzman, A. 1

\section{$\mathbf{H}$}

Halsey, T. C. $\quad 106$

Hancock, E. $\quad 144$

Haralick, R.M. 2, 4

Hati, S. 7

Hausdorff, F. $\quad 103$

Hecht, E. $\quad 40$

Hentschel, H. G. 105

Hildreth, E.C. 6

Holland, J.H. $\quad 90$

Hsu, T. $\quad 75$

Huygens, Ch. $\quad 35,39$

Hwang, W.J. 145, 156

Jahne, B.

73

Jain, A.K.

$5,6,28,29$,

$141,143,144$,

170,203

K

Kaplan, L.M. $\quad 124$

Kaplanova, M. 7

Keller, J. M. $\quad 142$

Khalil, M.I. $\quad 74,88,111$, 125 
Kirchhoff, G.R. $\quad 43$

Kohonen, T. $\quad 2,76,77,138$,

Ratches, J.A. $\quad 4$

Raudys, S.J. $\quad 28$

174

Kothari, R. $\quad 144,146$

Richardson, S. $\quad 143$

Roberts, L.G. 1

Roman, J. $\quad 105$

L

Lau, Th. 1

Leblanc, B. 73

Legierse, P.E.J. $\quad 299,302,303$

Leung, $Y$. 7, 144, 146

Lin, C.C. $\quad 75$

López-Rubio, E. 74

$M$

Mallat, S. G. $\quad 74,86,113$, 114 ,

Mallet, Y. 88

Mandelbrot, B. B. 73, 103

Marroquin, J.L. 203

Mascle, S. $\quad 73$

Matsuyama, T. $\quad 75$

McCulloch, W.S. 2

Minsky, M.L. 141

Moghaddam, B. 6,7

Morlet, J $\quad 85$

Mukherjee, J. $\quad 30$

\section{O}

Oh, G.

79

$P$

Paglieroni, D.W. 30

Pal, N.R. $\quad 141$

Palmer, S. $\quad 6$

Paredis, J. $\quad 91$

Park, H.J. $\quad 74$

Pavlidis, T. 4

Peeters, R.L.M. 79

Pelah, A. 7

Peña, J.M. $\quad 142,157$

Pentland, A.P. $\quad 6,7,73,107$

Perlovsky, L.I. 2

Peters, B.C. $\quad 144$

Petrou, M. I

Pfeifer, R. 2

Philips, P.J. 4

Pittner, S. $\quad 88$

Postma, E.O. $\quad 6,7$

Price, K. 4

Priestley, M.B. 74

$\mathbf{R}$

Ragnehalm, I. $\quad 30$
Ruspini, E.H. 161

Rutten, J.H. 138

\section{$\mathrm{S}$}

Sanna, A. $\quad 79$

Schalkhoff, R. $\quad 145$

Shannon, C. E. $\quad 107$

Sharman R. $\quad 125$

Shih, F.Y,C. $\quad 30$

Shippen, J.M. 7

Sklansky $\quad 73$

Steinberg, Y. 141

Subbarao, M. 7

Sugeno, M. $\quad 19,141,201$, 202

Sziranyi, T. $\quad 79$

\begin{tabular}{ll}
\multicolumn{1}{c}{ T } & \\
Tagare, H.D. & 6,7 \\
Tao, Y. & 88,111 \\
Theiler, J. & 104 \\
Titterington, D. & [43
\end{tabular}

V

Valkealahti, K. $\quad 79$

Vogel, H. 183

W

Wang, L.J. 125

Watanabe, S. $\quad 28$

Westra, R.L. $\quad 11,13,44,79$, 91, 104, 125, $143,148,149$, $157,159,187$,

324

White, R., $\quad 73$

Whitley, D. 91

Wilson, R.C. $\quad 144$

Windham, M.P. $\quad 141,145$

$Y$

Yager, R.

146,147

Z

Zadeh, L.A.

138,140

Zahid, N.

$141,143,144$, 160 


\section{Subject Index}

1D-parameterisation
2DI-Map
2DI-Map, applications
2D-parameterisation

$23,35,38,53,54$

$52,55,56,60,62$

69

$52 \mathrm{ff}, 55$

A

B

Box covering

Box dimension

C

\begin{tabular}{|c|c|c|c|}
\hline absolute difference & see: $\mathrm{Z}$ & Clusters, validity & $141,146,159,161$ \\
\hline Alpha-exponent & 194,196 & criteria & \\
\hline Alpha-exponent, & 257,279 & Colours, greyscales & 17 \\
\hline evolution & & Colours, RGB & 17 \\
\hline $\begin{array}{l}\text { Artificial Neural } \\
\text { Networks }\end{array}$ & see: NN & $\begin{array}{l}\text { Combinatorial } \\
\text { complexity }\end{array}$ & 2 \\
\hline Autonomous sensing & 7 & Computational vision & $1,4,6$ \\
\hline B & & $\begin{array}{l}\text { Computational vision } \\
\text { control }\end{array}$ & 4,14 \\
\hline Box covering & 103,114 & Computer vision & $1,2,4,5,6$ \\
\hline $\begin{array}{r}\text { Box dimension } \\
\text { C }\end{array}$ & 111 & $\begin{array}{l}\text { Contraction } \\
\text { Cumulative Intensity } \\
\text { Distribution }\end{array}$ & $\begin{array}{l}222,223,226 \\
\text { see: CID }\end{array}$ \\
\hline Centroid & 140 & Curse of dimensionality & 3,28 \\
\hline CID & 44 & & \\
\hline $\begin{array}{l}\text { CID-representation, } \\
\text { planar }\end{array}$ & 50 & $\begin{array}{c}\text { D } \\
\text { Defect identification }\end{array}$ & $7,8,12,16,72$ \\
\hline $\begin{array}{l}\text { CID-representation, } \\
\text { tetrahedral }\end{array}$ & 48 & Distance measures, & $\begin{array}{l}116 \\
187,188\end{array}$ \\
\hline Classes, diffuse & 241 & normalised metric & \\
\hline Classes, overlap & $\begin{array}{l}182,187,189,191, \\
237,241,245,262, \\
263,268\end{array}$ & $\begin{array}{l}\text { Distancemap } \\
\text { Distancemap, } \\
\text { continuous }\end{array}$ & $\begin{array}{l}29,30,31 \\
35,39,42\end{array}$ \\
\hline Classification & 6,181 & Distancemap, & 33 \\
\hline $\begin{array}{l}\text { Cluster analysis, } \\
\text { iterative }\end{array}$ & 169 & $\begin{array}{l}\text { disconnected parts } \\
\text { Distancemap, discrete }\end{array}$ & 32 \\
\hline Cluster separation & $161,164,169,248$ & Distancemap, joining & 33 \\
\hline Clustering, automatic & 159 & Dynamicallity exponent & see: alpha-exponent \\
\hline Clustering, hierarchical & 144 & & \\
\hline Clustering, mountain & $138,144,146,172$ & $\mathbf{E}$ & \\
\hline $\begin{array}{l}\text { Clustering, non- } \\
\text { parametric }\end{array}$ & 141,142 & $\begin{array}{l}\text { Energy Functionals } \\
\text { Entire Measurement, }\end{array}$ & $\begin{array}{l}155,157 \\
244\end{array}$ \\
\hline Clustering, parametric & 141,142 & estimate & \\
\hline $\begin{array}{l}\text { Clustering, partitional } \\
\text { Clustering, scale-space }\end{array}$ & $\begin{array}{l}143,144,155 \\
145,172\end{array}$ & $\begin{array}{l}\text { Equilibrium Points, } \\
\text { mixed }\end{array}$ & 241,260 \\
\hline Clustering, subtractive & 147,172 & Euclidean Distance & 29 \\
\hline Clustering, supervised & 141,148 & Transform & \\
\hline
\end{tabular}

\begin{tabular}{|c|c|c|c|}
\hline Alpha-exponent & 194,196 & criteria & \\
\hline Alpha-exponent, & 257,279 & Colours, greyscales & 17 \\
\hline evolution & & Colours, RGB & 17 \\
\hline $\begin{array}{l}\text { Artificial Neural } \\
\text { Networks }\end{array}$ & see: NN & $\begin{array}{l}\text { Combinatorial } \\
\text { complexity }\end{array}$ & 2 \\
\hline Autonomous sensing & 7 & Computational vision & $1,4,6$ \\
\hline B & & $\begin{array}{l}\text { Computational vision } \\
\text { control }\end{array}$ & 4,14 \\
\hline Box covering & 103,114 & Computer vision & $1,2,4,5,6$ \\
\hline Box dimension & 111 & $\begin{array}{l}\text { Contraction } \\
\text { Cumulative Intensity }\end{array}$ & $\begin{array}{l}222,223,226 \\
\text { see: } \mathrm{CID}\end{array}$ \\
\hline C & & Distribution & \\
\hline Centroid & 140 & Curse of dimensionality & 3,28 \\
\hline CID & 44 & & \\
\hline $\begin{array}{l}\text { CID-representation, } \\
\text { planar }\end{array}$ & 50 & $\begin{array}{c}\text { D } \\
\text { Defect identification }\end{array}$ & $7,8,12,16,72$ \\
\hline $\begin{array}{l}\text { CID-representation, } \\
\text { tetrahedral }\end{array}$ & 48 & Distance measures, & $\begin{array}{l}116, \\
187,188\end{array}$ \\
\hline Classes, diffuse & 241 & normalised metric & \\
\hline Classes, overlap & $\begin{array}{l}182,187,189,191, \\
237,241,245,262, \\
263,268\end{array}$ & $\begin{array}{l}\text { Distancemap } \\
\text { Distancemap, } \\
\text { continuous }\end{array}$ & $\begin{array}{l}29,30,31 \\
35,39,42\end{array}$ \\
\hline Classification & 6,181 & Distancemap, & 33 \\
\hline $\begin{array}{l}\text { Cluster analysis, } \\
\text { iterative }\end{array}$ & 169 & $\begin{array}{l}\text { disconnected parts } \\
\text { Distancemap, discrete }\end{array}$ & 32 \\
\hline Cluster separation & $161,164,169,248$ & Distancemap, joining & 33 \\
\hline Clustering, automatic & 159 & Dynamicallity exponent & see: alpha-exponent \\
\hline Clustering, hierarchical & 144 & & \\
\hline Clustering, mountain & $138,144,146,172$ & $\mathbf{E}$ & \\
\hline $\begin{array}{l}\text { Clustering, non- } \\
\text { parametric }\end{array}$ & 141,142 & Energy Functionals & 155,157 \\
\hline $\begin{array}{l}\text { parametric } \\
\text { Clustering, parametric }\end{array}$ & 141,142 & $\begin{array}{l}\text { Entire Measurement, } \\
\text { estimate }\end{array}$ & 244 \\
\hline Clustering, partitional & $143,144,155$ & Equilibrium Points, & 241,260 \\
\hline Clustering, scale-space & 145,172 & mixed & \\
\hline Clustering, subtractive & 147,172 & Euclidean Distance & 29 \\
\hline Clustering, supervised & 141,148 & Transform & \\
\hline
\end{tabular}

Clustering,

unsupervised

Clusters, energy

Clusters, number of

Clusters, type

Clusters, validity

criteria

Colours, greyscales $\quad 17$

Colours, RGB 17

Combinatorial 2

complexity

Computational vision $\quad 1,4,6$

Computational vision 4,14

control

Computer vision

Contraction

Cumulative Intensity

Distribution

Curse of dimensionality 3,28

D

Defect identification

Distance measures,

see: CID

$7,8,12,16,72$,

116 ,

187, 188

$43,155 \mathrm{ff}, 159$,

$42,145,159,161$
$1,2,4,5,6$
$222,223,226$
17

normalised metric

\begin{tabular}{|c|c|c|c|}
\hline Alpha-exponent & 194,196 & criteria & \\
\hline Alpha-exponent, & 257,279 & Colours, greyscales & 17 \\
\hline evolution & & Colours, RGB & 17 \\
\hline $\begin{array}{l}\text { Artificial Neural } \\
\text { Networks }\end{array}$ & see: NN & $\begin{array}{l}\text { Combinatorial } \\
\text { complexity }\end{array}$ & 2 \\
\hline Autonomous sensing & 7 & Computational vision & $1,4,6$ \\
\hline B & & $\begin{array}{l}\text { Computational vision } \\
\text { control }\end{array}$ & 4,14 \\
\hline Box covering & 103,114 & Computer vision & $1,2,4,5,6$ \\
\hline Box dimension & 111 & $\begin{array}{l}\text { Contraction } \\
\text { Cumulative Intensity }\end{array}$ & $\begin{array}{l}222,223,226 \\
\text { see: } \mathrm{CID}\end{array}$ \\
\hline C & & Distribution & \\
\hline Centroid & 140 & Curse of dimensionality & 3,28 \\
\hline CID & 44 & & \\
\hline $\begin{array}{l}\text { CID-representation, } \\
\text { planar }\end{array}$ & 50 & $\begin{array}{c}\text { D } \\
\text { Defect identification }\end{array}$ & $7,8,12,16,72$ \\
\hline $\begin{array}{l}\text { CID-representation, } \\
\text { tetrahedral }\end{array}$ & 48 & Distance measures, & $\begin{array}{l}116, \\
187,188\end{array}$ \\
\hline Classes, diffuse & 241 & normalised metric & \\
\hline Classes, overlap & $\begin{array}{l}182,187,189,191, \\
237,241,245,262, \\
263,268\end{array}$ & $\begin{array}{l}\text { Distancemap } \\
\text { Distancemap, } \\
\text { continuous }\end{array}$ & $\begin{array}{l}29,30,31 \\
35,39,42\end{array}$ \\
\hline Classification & 6,181 & Distancemap, & 33 \\
\hline $\begin{array}{l}\text { Cluster analysis, } \\
\text { iterative }\end{array}$ & 169 & $\begin{array}{l}\text { disconnected parts } \\
\text { Distancemap, discrete }\end{array}$ & 32 \\
\hline Cluster separation & $161,164,169,248$ & Distancemap, joining & 33 \\
\hline Clustering, automatic & 159 & Dynamicallity exponent & see: alpha-exponent \\
\hline Clustering, hierarchical & 144 & & \\
\hline Clustering, mountain & $138,144,146,172$ & $\mathbf{E}$ & \\
\hline $\begin{array}{l}\text { Clustering, non- } \\
\text { parametric }\end{array}$ & 141,142 & Energy Functionals & 155,157 \\
\hline $\begin{array}{l}\text { parametric } \\
\text { Clustering, parametric }\end{array}$ & 141,142 & $\begin{array}{l}\text { Entire Measurement, } \\
\text { estimate }\end{array}$ & 244 \\
\hline Clustering, partitional & $143,144,155$ & Equilibrium Points, & 241,260 \\
\hline Clustering, scale-space & 145,172 & mixed & \\
\hline Clustering, subtractive & 147,172 & Euclidean Distance & 29 \\
\hline Clustering, supervised & 141,148 & Transform & \\
\hline
\end{tabular}

continuous

Distancemap, $\quad 33$

disconnected parts

Distancemap, discrete 32

Distancemap, joining 33

Dynamicallity exponent see: alpha-exponent

\section{E}

Energy Functionals $\quad 155,157$

Entire Measurement, 244

estimate

Equilibrium Points, $\quad 241,260$

mixed

Euclidean Distance 29

Transform

3

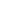
:

$138,141,142,144$,

$141,146,159,161$

\begin{tabular}{|c|c|c|c|}
\hline Alpha-exponent & 194,196 & criteria & \\
\hline Alpha-exponent, & 257,279 & Colours, greyscales & 17 \\
\hline evolution & & Colours, RGB & 17 \\
\hline $\begin{array}{l}\text { Artificial Neural } \\
\text { Networks }\end{array}$ & see: NN & $\begin{array}{l}\text { Combinatorial } \\
\text { complexity }\end{array}$ & 2 \\
\hline Autonomous sensing & 7 & Computational vision & $1,4,6$ \\
\hline B & & $\begin{array}{l}\text { Computational vision } \\
\text { control }\end{array}$ & 4,14 \\
\hline Box covering & 103,114 & Computer vision & $1,2,4,5,6$ \\
\hline Box dimension & 111 & $\begin{array}{l}\text { Contraction } \\
\text { Cumulative Intensity }\end{array}$ & $\begin{array}{l}222,223,226 \\
\text { see: } \mathrm{CID}\end{array}$ \\
\hline C & & Distribution & \\
\hline Centroid & 140 & Curse of dimensionality & 3,28 \\
\hline CID & 44 & & \\
\hline $\begin{array}{l}\text { CID-representation, } \\
\text { planar }\end{array}$ & 50 & $\begin{array}{c}\text { D } \\
\text { Defect identification }\end{array}$ & $7,8,12,16,72$ \\
\hline $\begin{array}{l}\text { CID-representation, } \\
\text { tetrahedral }\end{array}$ & 48 & Distance measures, & $\begin{array}{l}116, \\
187,188\end{array}$ \\
\hline Classes, diffuse & 241 & normalised metric & \\
\hline Classes, overlap & $\begin{array}{l}182,187,189,191, \\
237,241,245,262, \\
263,268\end{array}$ & $\begin{array}{l}\text { Distancemap } \\
\text { Distancemap, } \\
\text { continuous }\end{array}$ & $\begin{array}{l}29,30,31 \\
35,39,42\end{array}$ \\
\hline Classification & 6,181 & Distancemap, & 33 \\
\hline $\begin{array}{l}\text { Cluster analysis, } \\
\text { iterative }\end{array}$ & 169 & $\begin{array}{l}\text { disconnected parts } \\
\text { Distancemap, discrete }\end{array}$ & 32 \\
\hline Cluster separation & $161,164,169,248$ & Distancemap, joining & 33 \\
\hline Clustering, automatic & 159 & Dynamicallity exponent & see: alpha-exponent \\
\hline Clustering, hierarchical & 144 & & \\
\hline Clustering, mountain & $138,144,146,172$ & $\mathbf{E}$ & \\
\hline $\begin{array}{l}\text { Clustering, non- } \\
\text { parametric }\end{array}$ & 141,142 & Energy Functionals & 155,157 \\
\hline $\begin{array}{l}\text { parametric } \\
\text { Clustering, parametric }\end{array}$ & 141,142 & $\begin{array}{l}\text { Entire Measurement, } \\
\text { estimate }\end{array}$ & 244 \\
\hline Clustering, partitional & $143,144,155$ & Equilibrium Points, & 241,260 \\
\hline Clustering, scale-space & 145,172 & mixed & \\
\hline Clustering, subtractive & 147,172 & Euclidean Distance & 29 \\
\hline Clustering, supervised & 141,148 & Transform & \\
\hline
\end{tabular}




\section{F}

Feature extraction

Feature parameters

Feature parameters,

number of

Feature vector

FindAreas algorithm

FMV

FMV, performance of

Focus of attention

(FOA)

Fourier analysis

Fourier analysis,

ScanProfiles

Fourier analysis,

Textures

Fourier descriptors

Fourier, power spectral density

FPS

FPS, dynamic

FPS, functional fusion

FPS, functionals

FPS, global constraints

FPS, multi criteria

FPS, performance

FPS, static

Fractal

Fractal analysis

Fractal dimensions

Fractal functions

Fractal singularity

spectrum

Fractal wavelet analysis

Fractal wavelet

analysis, 1D-scans

Fractal wavelet

analysis, textures

Functional Parameter

Selection

Fuzzy C-Means (FCM)

Fuzzy correlations

Fuzzy integrals

Fuzzy membership

function

Fuzzy Multivariate

Clustering
27,29

$28,71,181,260$

28, 206,

$111,153,183$

$22,311,327$

$139,143,157,161$

158

6

74

76

79

77

79,133

$182,191 \mathrm{ff}$

194

196

192,195

197

$195 \mathrm{ff}, 281 \mathrm{ff}$

267

194

73,103

111,134

$73,103,107,111$,

$114,124,130,134$

111,114

123

$71,116,132$

111

124,125

see: FPS

$138,142,144,155$,

173,179

see: IPP-PT1

201, 202

$27,138,204$

see: FMV
G

Gaussian mixtures

$141,145,156,161$, 203, 212, 222,

Gaussian mixtures, see: NM normalised

Gaussian mixtures, see: UM

uncoupled

Genetic Algorithms

$2,7,76,90,138$, 141,207

Global Wavelet

89

Decomposition

(GWDA)

Greyscale

transformations

Greyscale

transformations, 1-D

Greyscale

transformations, 2-D

\section{H}

Helmholtz equation $\quad 29,41,42$

Hölder exponent $\quad 73,106,111$

Human vision $\quad 1,6,8,13$

Hypothesis, overturn of 247

I

IASC algorithm, as

209

iteration

IASC algorithm, as

transformation

IASC algorithm,

performance

IASC computation time 215

IASC estimate $\quad 244$

IASC explored

$192,216,228,249$,

parameters

257,259

IASC number of

$206,215,257,264$,

267,281

iterations

$215,219,241,253$,

$254,260,264,292$

IASC, control

265

parameters

IASC, convergence of $215 \mathrm{ff}$

IASC, descriptors $262 \mathrm{ff}$

IASC, dynamic $\quad 239 \mathrm{ff}$

properties of

IASC, information $\quad 203,212 \mathrm{ff}$

fusion

IASC, input parameters $262 \mathrm{ff}, 272$

IASC, Jacobian 


IASC, numerical
simulations
IASC, output
parameters
IASC, stability
IASC, trajectory
IASC: InterActive
Sensing and
Classification
IDC
Image analysis
Image processing
Images, continuous
Images, discrete/digital
Images, printed
Information fusion
Innovative aspects
Intelligent Binary
Search (IBS)
IntelPadPrint
Interactive analysis
Interactive
classification
Interactive sensing
InterActive Sensing and
Classification
Algorithm,
Interactive vision

35,52

22

$19,30,47,48$

$17 \mathrm{ff}$

$15,17 \mathrm{ff}$

$15 \mathrm{ff}$

$201 \mathrm{ff}$

8

$22 \mathrm{ff}, 311 \mathrm{ff}$

$3,14,295 \mathrm{ff}$

206, 207

$181 \mathrm{ff}, 184 \mathrm{ff}, 187$, $215,260 \mathrm{ff}$

$9,13,181,209 \mathrm{ff}$

see: IASC

Algorithm

$6,7,8 \mathrm{ff}, 13,317$,

$327 \mathrm{ff}$

IPP-prototypes

IPP-PT 1

IPP-PT2I

$308 \mathrm{ff}, 314 \mathrm{ff}$

$308 \mathrm{ff}$

$314 \mathrm{ff}$

see: IDC

Isodistance contour

Iterative cluster analysis 16

Iterative cluster

decomposition

Iterative cluster fusion

Iterative cluster tree

expansion

Iterative FMV

\section{K}

$161 \mathrm{ff}$

$164 \mathrm{ff}$

$161 \mathrm{ff}$

$168 \mathrm{ff}$

$\mathrm{K}$-nearest neighbour $\quad 142 \mathrm{ff}$

(K-NN)

L

Lipschitz, continuity $\quad 73,111,112,113$

\section{M}

$\begin{array}{ll}\text { Machine Vision } & 1,2 \\ \text { Mahalanobis distance } & 182,188,189 \\ \text { Many-parameter classes } & 181 \mathrm{ff} \\ \text { Mean, clustering } & 140 \\ \text { Mixture decomposition } & 143,144,145 \\ \text { MRA } & 72,86-90,125 \\ \text { Multi Resolution } & \text { see: MRA } \\ \text { Analysis } & \\ \text { Multi Resolution } & \text { see: MRA } \\ \text { Approach } & \\ \text { Multi-criteria } & 195\end{array}$

optimisation

Multifractal

Multivariate Gaussian

mixtures

\section{N}

$\begin{array}{ll}\begin{array}{l}\text { Nearest prototype } \\ \text { selection }\end{array} & 192,196 \\ \text { Neural Network } & \text { see: } \mathrm{NN} \\ \text { NM } & 203 \mathrm{ff} \\ \text { NN } & 2,75,76,148 \mathrm{ff} \\ \text { Noise, absolute } & 263 \\ \text { Noise, relative image } & 324 \\ \text { Noise, relative sensor } & 263 \\ \text { Noise, relative squared } & 253\end{array}$

$71,103,104,105 \mathrm{ff}$ $203 \mathrm{ff}, 212 \mathrm{ff}$

\section{O}

On-Line, classification

On-line, control

296,304

$295 \mathrm{ff}, 303$

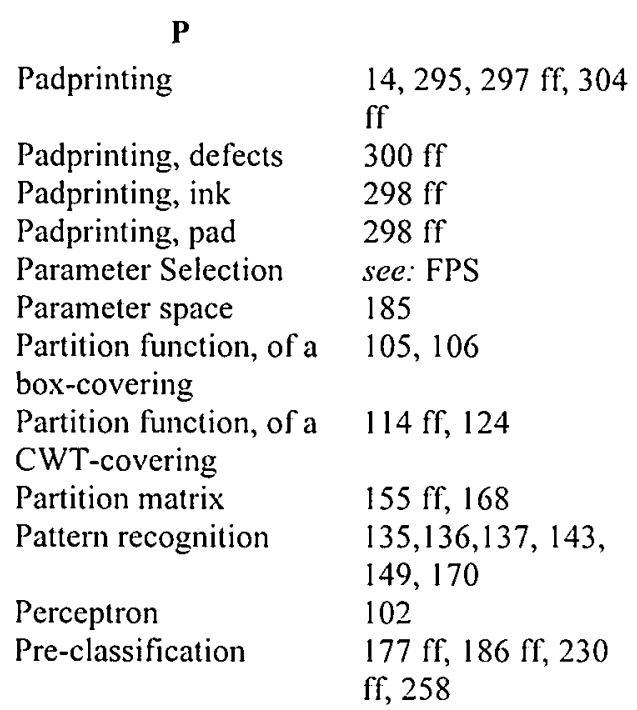


Principal template $\quad 17,19,20,69$

gauge (PTG)

Print border

PrintContext

18

18

$15,27 \mathrm{ff}$

Printing defects,

classification

Printing defects, defect $16,21 \mathrm{ff}$

classes

Printing defects,

$174 \mathrm{ff}$

parameterised

Probability fusion

$201 \mathrm{ff}$

Prototype, clustering

140

Prototype, IntelPadPrint see: PT1, PT2

\section{Q}

Quality control

Quality, classification

Quality, conclusion

Quality, data

Quality, image

Quality, print(ing)

\section{$\mathbf{R}$}

Ray

Real-time

Reasoning level,

general

Reasoning level,

interactive

Reasoning process

Reasoning strategy

relevance, parameter

Representation space

S

Scaling laws

Scaling laws, local

Scan curve

Scan curve, topology

Scan profiles

Scanning

Scanning, multi scan

line

Search box

Search box, energy

Search map
$1 \mathrm{ff}, 295$

$182,264,268,274$,

275

$186 \mathrm{ff}$

263

324

$7,8,12,299,302$, 328

35

$7,8,9$

$206 \mathrm{ff}$

$207 \mathrm{ff}$

$206 \mathrm{ff}$

$183 \mathrm{ff}$

$190 \mathrm{ff}$

$186,217,223,226$, 257

$73,75,103,104$, $112,115,116,119$

$123 \mathrm{ff}, 126$

$16,23 \mathrm{ff}$

24

$16,22,23$

16

$26 \mathrm{ff}$

$22 \mathrm{ff}$

22

22
Separation, class

189

Shape analysis

$77 \mathrm{ff}$

SOM map

174

State function

218

State function, general 232

State function, lower 254

State function, true $\quad 239 \mathrm{ff}$

State function, upper $\quad 254$

Supervised clustering $\quad 139,141 \mathrm{ff}, 148 \mathrm{ff}$

T

Template

15,17

Template-free

16

Template-free, strong $\quad 16$

Template-free, weak 16

Termination criteria

$206 \mathrm{ff}, 248 \mathrm{ff}, 260$,

$283 \mathrm{ff}$

Termination criteria, $\quad 283 \mathrm{ff}$

convergence-based

Termination criteria, $\quad 207 \mathrm{ff}, 283 \mathrm{ff}$

dynamic

Termination criteria, $283 \mathrm{ff}$

quality-based

Termination criteria, $207 \mathrm{ff}, 283 \mathrm{ff}$

static

Termination criteria, $\quad 206,207,229,230$, threshold

$250,251 \mathrm{ff}, 283$,

$286 \mathrm{ff}$

Texture

$71,72,73$

Texture analysis, fractal $72 \mathrm{ff}$

Texture classification $\quad 73,79$

\section{$\mathbf{U}$}

UM

Unifractal

$204 \mathrm{ff}$

105

V

Vision, active

$6 \mathrm{ff}, 13$,

Vision, human

$1,6 \mathrm{ff}, 8,13,302$,

317

Vision, interactive

$6,7,8 \mathrm{ff}, 13,317$, $327 \mathrm{ff}$

Visual attention $\quad 6,8$

W

Wavelet

$85 \mathrm{ff}, 85$

Wavelet analysis

$71,86,94,111 \mathrm{ff}$, 114,116

Wavelet analysis, N- $\quad 123 \mathrm{ff}$

dimensional 
Wavelet packages

Wavelet Transform

Modulus Maxima

Method

Wavelet transform, continuous

Wavelet transform, discrete

Wavelet, Coiflet

Wavelet, Daubechies

Wavelets, MRA
$87 \mathrm{ff}$

see: WTMM

$86 \mathrm{ff}$

$86 \mathrm{ff}$

85

85

see: MRA
Weakly separated WTMM

$139 \mathrm{ff}, 181,182$

$73,115 \mathrm{ff}, 119$,

$122,124,125,128$,

130

$\mathbf{Z}$

Z

see: Z-

representation

Z-representation

19 



\section{Summary}

\section{Chapter 1 INTRODUCTION}

The subject of this study is the automatic control of printing processes by means of images of the prints. This places our study in the field of 'Computer Vision'. This is a rapid developing area, caused by scientific and technological innovations. This development is driven by a steady decrease of price, combined with a ditto increase of performance, both of hardware and software. Due to these developments, there is much interest from industry, and the number of practical applications grows continually.

Certain characteristics of human vision are relevant for our subject. Especially, the active way in which humans visually explore an unknown object is very inspiring for our approach. This 'active vision' is a close cooperation between eye and brain, in which the brain analyses an interprets incoming images. From this interpretation of the image grows a certain hypothesis about the image, and from this certain expectations. Based on these assumptions, the brain selects another relevant part of the image. Thus, the eye is aimed at this area, and the image is analysed conditional to the actual hypothesis. In practice, this mechanism converges to the right conclusion within a 'blink of the eye'. I case of visual illusions the system is not able to converge to a solution, and we can as it were catch this mechanism in action. Based on this biological interpretation, we model the automatic analysis of prints in a similar way; as an interaction between a reasoning process that generates hypotheses and expectations regarding the image, and image-analysis techniques that perform and process requested sensing operations at certain identified locations of the image. The reasoning process thereby governs the position and the nature of the measurements. This process causes a 'real-time' diagnosis of the observed object, while only a fraction of all possible measurements is performed. This mechanism forms the central part of this thesis, and is treated in the Chapters 6, 6, and 7 .

Another inspiration from human vision concerns its ability to recognise textures. For humans, a small fragment of a texture, like textile, wood, stone, or paper suffices for an instantaneous recognition. Equipped with this capability, humans can promptly identify defects or imperfections in prints. We shall model this ability with the use of the new field of 'fractal wavelet-analysis'. This is described in Chapter 3.

Innovative subjects in this study include:

1. interactive classification based on the mechanism of active vision,

2. fractal wavelet analysis of print-pattern independent defect recognition,

3. geometrical transformations that represent images one-to-one on an interval or rectangle,

4. an automatic clustering method that is suitable for fast, 'unsupervised' partitioning of many-parameter data with not well-separated classes. This is relevant for our context of computer vision. 


\section{Chapter 2 THE ANALYSIS OF PRINTED IMAGES}

Main objective of this Chapter is to transform images (in our case CCD-images) of prints into one general representation. This frees us from all details of the print-pattern, called the template, that may vary in printing processes.

First we describe the structure and characteristics of prints. We define images of prints as continuous patterns of grey-scales. The print-border is the curve that separates the printed from the not-printed area. Most printing defects occur in specific areas relative to the printborder; near or far from the print-border, inside or outside printed areas. In this way four different types of area types can be distinguished, called print-contexts. The template is the print-pattern. Our ultimate objective is iemplate-free defect-recognition and print-quality control. By subtracting the template from the image, we obtain a direct impression from the printing defect, both its characteristic shape as its distribution over the print-contexts.

A printing-defect is characterised by its shape, texture, and greyscale-distribution. These parameters can be linked to the features of the printing-defects. These features are expressed in the so-called feature-parameters. These quantify the degree in which the feature is present. In this way, a printing-defect can be represented as a point in a feature-space. The position of this point in space determines to which defect-class it belongs, independent of the applied template.

The central subject of this Chapter concerns the transformation of printed images into one general representation. This representation makes the image independent of the template, and thereby enables template-free defect-classification. Starting point of this transformation is the Euclidean Distance Transform (EDT), that gives the distance from a point to a given geometric set. An addition to the EDT concerns the location of the point inside or outside the printed area. We define the primt-depth of a point in an image as the minimal Euclidean distance of that point to the print-border. The sign of the print-depth is positive if the point is outside, and negative if the point is inside the print. Using the print-depth, we can define a one-dimensional representation of printed images. This representation gives the normalised, cumulative intensity of all faults at a certain print-depth. Therefore, this transformation is called the 'Cumulative Intensity Distribution', short CID. This template-independent transformation enables a number of analyses, such as generalised moments, filters, deconvolution, or spectral analysis. Two examples are given of representations based on the CID that are able to separate specific clusters of defect classes. These representations are therefore suitable for pre-classification. Next, we try to construct a two-dimensional analogon of the CID. However, it appears to be impossible to construct continuous two-dimensional representations. The problems are analogous to map-projections: it is not possible to construct continuous flat representations of a globe. Locally, continuous representations are possible, or, consequently, as a collection of local maps. We define a transformation, called twodimensional Intensity Map (2DI-map), that is locally not-continuous, but does posses attractive local congruency characteristics. In this way, defects can sustain their characteristic form. With the 2DI-map arbitrary prints are represented in a rectangle, with above the printed, and below the non-printed area. More serious than all mathematical complications of the 2DImap is the discrete nature of the CCD-images. This causes Moiret-like segmentation, that seriously inhibit the practical use of the 2DI-map.

As a consequence of the representations discussed in this chapter, especially the feature-space representation and the CID-transform, we can now represent all kinds of printings as points in high-dimensional parameter-spaces. This allows us in later chapters to concentrate on clustering and classification techniques that are suitable for these kinds of spaces, without being concerned with the details of the template employed.

A separate appendix describes the determination of feature parameters fro printing defects. 


\section{Chapter 3 ANALYSIS AND CLASSIFICATION USING FRACTAL WAVELET ANALYSE}

In recent decennia much attention has been devoted to objects that reveal new details at each scale of magnification. These, so-called 'fractal' sets, appear to be relevant for the description of natural objects and physical processes. The main motivation in this Chapter is to investigate whether the characteristic features of printing defects can be expressed in a fractal representation. To that end we employ the 'multi-fractal' formalism, that provides a more appropriate, but also more complex description. In the multi-fractal formalism a set is represented by a mass or probability distribution. We can interpret the greyscales of an image as such a distribution. With methods from the macro canonical ensemble theory, we are able to calculate an entire spectrum of fractal characteristics of the set, including the fractal dimension, the entropy, and the auto-correlation. This spectrum of fractal properties is called the Generalised Fractal Dimension (GFD).

A practical problem concerns the determination of the GFD. Conventionally, a set is covered with a collection of hyper-boxes, and certain scaling properties of this covering is studied as the side of these hypercubes approaches to zero. Not only it is difficult to construct such a covering of most sets, it has also been demonstrated that many disadvantages are connected to this method. For instance, it can been shown that this method systematically underestimates the fractal dimension for dimensions larger than 2.6.

In this respect another and also recent formalism is helpful: wavelets and Multi Resolution Analysis (MRA). Wavelets are, like the name implies, small waves: they have integral zero (hence 'wave'), and a small compact support (hence wave-let). Similar to Fourier-analysis it is possible to represent images as (infinite) summations of wavelets. MRA offers an elegant method for this decomposition. The price paid for this elegancy is that besides the spectral components also the spatial components must be determined, causing a doubling of the number of coefficients. MRA concentrates at each scale of magnification on those parts of the set where this scale is especially relevant. But this characteristic is exactly what is needed to cover fractal sets with hypercubes. Therefore we can see wavelets as a kind of generalised hypercubes, and therefore MRA as a generalised method for covering fractal sets. For this reason, wavelets are especially suited for the analysis of fractal sets. In recent years this approach has been refined, especially by Mallat and Hwang. This has led to the development of the 'Wavelet-Transform Modulus Maxima' (WTMM) method. With the WTMM-method the GFD of an object can be computed efficiently. This method has been recently applied successfully for the analysis and classification of different types of textures.

In Chapter 3 all these formalisms are briefly discussed, and where necessary explained with an example. First, 'traditional' Fourier-analysis is applied. From this analysis global powerlaws can be computed, but no fractal dimensions. Therefore, the multifractal formalism is applied to the analysis of scan-profiles and images of printing defects. With this approach is it is possible to transform a printing-defect into a collection of numbers, the GFD. This collection can be seen as a specific kind of 'feature-vector'. In this representation, it is possible to significantly separate many printing defect classes. There are, however, many practical problems to this method, including the excessively long computation times, and the digital nature of the images, which allow only a limited degree of magnification.

The attractive side of this representation is that it 'strong' template-free, i.e. in no way assumes any knowledge of the applied template - where we assume that the template itself is no fractal. Moreover, it is - in principle - possible to relate the GFD of the image to the underlying physical-chemical printing process. the printing-defects are caused by a combination of rheological, mechanical, electro-dynamical, and chemical processes, which therefore also determine the GD of the resulting pattern. Knowledge of this mechanism would 
enable to estimate the relevant process-parameters from the GFD of the CCD-image, and thus to correct the process. At the moment, however, such an idea is totally infeasible.

We conclude that the idea of multifractal wavelet-analysis for classification of printingdefects has a potential for future use, but at the moment is not realistic.

In the cause of this chapter we also introduce an original generalisation of MRA, called the Global Wavelet Decomposition.

\section{Chapter 4 CLUSTERING METHODS FOR WEAKLY- SEPARATED MANY-PARAMETER CLASSES}

As a result of the definitions provided in Chapters 2 and 3, we can now represent an image from a printing-defect as a single point in a high-dimensional 'feature-space'. In this way collections of points group together in classes, the printing-defect classes. Many of these classes are found to be weakly separated, as they overlap significantly. Moreover, it seems that there is a large number of clusters in the data. This rises the question, which would be the best 'clustering' of the data in meaningful clusters. Perhaps a more significant taxonomy of defects is possible than provided by human experts. Another major motivation is the interactive classification-algorithm, discussed in Chapter 5, that requires only a small part of the feature-space.

In this Chapter several clustering paradigms are tested on their suitability for our featurespace. Direct clustering and classification of images using artificial neural networks appears to be computationally complex. The neural nets necessary for this application require an exceedingly high number of parameters. This would require an unrealistic number of empirical data, in order to train these parameters.

Accordingly, we study 'fuzzy clustering'. Techniques like Fuzzy C-Means (FCM) are 'unsupervised' (i.e. the training set is unlabeled) clustering techniques. We propose an addition to FCM, by replacing the spheroidal FCM-memberships-functions by ellipsoidal functions. This represents a better approximation of the local class-conditional metric. We call this method Fuzzy Multi-Variate clustering (FMV). This results in a clustering that provides equivalent partitions, but converges faster to the final result. Therefore, FMV is also more appropriate for high-dimensional spaces than FCM.

A large disadvantage of many clustering-methods, including FCM and FMV, is that they require some ad-hoc assumptions on the number of distribution of the clusters in featurespace. Therefore, we propose an automatic clustering algorithm, called iterative FMV cluster analysis, based on FMV, that can autonomously determine the number of significant classes in the data, by means of successive fusion and decomposition.

Finally, we apply clustering techniques to two representations that are based on the CIDtransformation from Chapter 2. In the first case we show the SOM-clustering technique can be used to estimate the intensity of a defect from a simple model for two kinds of printing defects. In the second case we show how iterative FMV cluster analysis can be used for partitioning a CID-based representation, which therefore is valuable for pre-classification. 


\section{Chapter 5 INTERACTIVE CLASSIFICATION OF NUMEROUS WEAKLY SEPARATED MANY-PARAMETER PROTOTYPES WITH NOISE}

Now we have reduced printing-defect analysis to the classification of an actual measurement expressed as a set of feature-parameters to one of a set of previous defined defect-classes. This could imply that first all possible measurements should be performed, which afterwards is straightforwardly assigned to the defect-class that corresponds to the identified part of feature-space. This would make classification very time-consuming and computationally demanding. Moreover, it is mostly unnecessary to perform all measurements, because during the process it has already become clear which defect-class occurred. Inspired by the mechanism of 'active vision' by humans, we opt for a different approach. In this Chapter, we propose an interactive method for simultaneous sensing and classification. We call this 'Interactive Classification'. The most vital problem here, is to - based on the present set of measurements and resulting hypotheses - select the 'best' possible candidate for the next measurement. The approach that we adopt is based on functional optimisation. We define a number of functionals, based on class-separation. First, however, we must define a useful metric in feature-space. The most frequent applied distance-measure is the so-called 'Mahalanobis-distance' (MD). However, the MD is non-symmetric for interclass-distances: in general: distance(klasse l,klasse2) is not equal to: distance(klasse2,klassel). therefore, we come with other propositions, like the 'geometric' distance. In the same sense, we define the 'relevance'; of a certain feature-parameter for a certain defect-class. With the use of these distance-measures, we can now construct functionals. Optimisation of these functionals leads to the feature-parameter that causes the largest separation of the classes, and thus the most useful measurement. By including the actual hypothesis - represented by the probabilitydistribution over the defect-classes - we make this process 'dynamic'. Thus, we select the feature-parameter for measurement that, given the current set of hypotheses, offers the best perspective to choose between competing hypotheses. In this respect it is important that during the reasoning process the weight of the actual hypotheses increases, for initially a hypothesis is more prawn to error. This increase is controlled by the so-called 'dynamicallityexponent'.

Next, the problem of integrating large amounts of heterogeneous information is considered, such as qualitative and quantitative information. Our choice is to represent qualitative information as 'fuzzy' parameters. Therefore, we only have quantitative information. Defectclasses in our representation are modelled by 'Gaussian mixtures', i.e. as multivariate normal distributions. Much emphasis is devoted to the control of the reasoning process. Some 'static' and 'dynamic' termination criteria are defined. 'Dynamic' here means that the history of the iteration is involved in the decision to terminate. We find that in case of a terminationcriterion with a constant threshold, an optimal value can be computed in the sense of a maximal success-rate with a minimal iteration-length.

All these subjects together form the ingredients for an integrated approach for simultaneous sensing and classifying, called: InterActive Sensing and Classification (IASC). Objective of $I A S C$ is the on-line classification of an object, based on selected sensor-readings. This method can be defined as an iterative algorithm, or as an iterated transformation in the space of hypotheses, where a sensing concerns 'just' one feature-parameter. For our assumption of a Gaussian mixture, this transformation can be further specified.

Of vital importance is the question of convergence and stability of the $I A S C$-algorithm. For this reason, we first define some measures for its performance: the quality ' $q$ ' of a conclusion 
( 1 for explicit, and 0 for indefinite conclusions), the success-rate ' $s$ ' of the algorithm ( 1 if the correct defect-class is identified, else 0 ), and the relative iteration-length ' $T$ ' (the number of performed measurements relative to the total number, so also in-between 0 and 1 ). As mentioned before, the actual hypothesis can be represented as a probability-distribution over the $K$ possible defect-classes. The actual hypothesis can therefore be represented as a normalised vector $\mathbf{p}$ in $\mathrm{IR}^{K}$, and, in the scenario where the probability of the individual classes lies in the interval $[0,1]$, more specific as a point in a $K$-dimensional hypercube: $[0,1]^{K}$. The edges of this hypercube correspond with the individual defect-classes. Therefore, the whole $I A S C$-iteration corresponds with a trajectory in this hypercube. The $I A S C$-iteration is said to converge if this trajectory end in an edge. The actual measurement, however, is a single vector in feature-space. During the iteration the components of this - initially unknown vector become known. The feature-space is partitioned in $K$ cells, which corresponds with the $K$ identified defect-classes.

The central conjecture in our approach is that if a measurement lies in a certain cell in featurespace, the $I A S C$-iteration converges with a quality $q$ to the defect-class associated to the cell, such that $q$ is determined by the distance between the measurement and the centre of the cell. This distance is also a measure for the relative noise in the measurement.

Several approaches are pursued in order to proof this conjecture. In Section 5.6.5 it is demonstrated that the $I A S C$-iteration is a contraction, because the Jacobian monotonously decreases to zero. All converging iterations are contractions. However, not all contractions correspond to converging iterations. For example, iterations with multiple or 'strange' attractors. In Section 5.6.6, three scenarios for the convergence are studied in more detail. (i) If the measurement coincides with the centre of a cell then the $I A S C$-iteration converges to the correct conclusion. (ii) If the measurement lies very close to the centre of the cell, the $I A S C$-iteration also converges. Here, close means: closer than the square root of the smallest eigen-value of the covariance-matrix of the associated defect-class. (iii) The measurement does not lie 'near' to a cell-centre. If the Euclidean distance to the cell-centre increases, there will be more feature-parameters for which this particular measurement lies closer to the centre of another cell-centre. These feature-parameters are therefore weakly separated, relative to the others, and have a low a-priori probability for selection in the functional parameter-selection that lies at the heart of the IASC-algorithm. Nevertheless, if the fraction of these 'wrong' parameters increases, the probability of a wrong conclusion increases also.

In Section 5.7, the dynamic properties of the IASC-iteration are studied in more detail. First, the evolution of the individual class- or state-probabilities are studied. Two simple observables are defined: the final value, and the average increase of the state-probabilities. By automatically clustering (NB: with iterated FMV from Chapter 4) a large number of observations, four primitive types of evolution are identified. These four types can be connected to the relative position of the clusters. Near clusters cause some 'confusion' in the evolution of state-probabilities during the iteration, and allow for a relatively late moment of changing the 'balance' of the hypothesis from one state to another. Some state-probabilities converge to a value somewhere in-between zero and one. This finding leads to a discussion of the role of diffuse classes and mixed equilibria. Mixed equilibria are points in feature-space that are 'undecidable'. Undecidable here means that the $I A S C$-iteration does not converge to an explicit conclusion. In case of an infinite iteration, these are the points on the separatrix between two classes. Because of the finite nature of the $I A S C$-iteration, points near to such separatrices will also be undecidable, especially for diffuse classes. Thus, the set of undecidable points are the vertices of a fuzified Voronoi-partitioning based on the cellcentres. 
The following sub-sections focus on the estimate and confidence-interval of the entire measurement-vector during the IASC-iteration. This allows for the calculation of the probability to reject the actual hypothesis based on a new measurement. It is shown that this probability decreases quasi-exponentially during the iteration.

Of major importance on the performance of the $I A S C$-algorithm are the termination-criteria. These are studied in Section 5.7.5. With a mean-field approach, a theoretic model is obtained that predicts the relation between the average success-rate $s$ and the relative iteration-time $T$. Finally, in Section 5.7.6 a number of models are proposed for the evolution of the dynamicallity-exponent. These determine the influence of the actual hypothesis on the functional feature-parameter selection.

We conclude that the $I A S C$-algorithm provides a framework for interactive classification, that under certain circumstances converges to the correct conclusion, while only a small fraction of all measurements is performed. This framework is not only applicable to Computational Vision, but in all cases where diagnosis and measurement concur, like in chemical analysis, medical diagnosis, and autonomous robots.

\section{Chapter 6 NUMERICAL EXPERIMENTS WITH IASC}

In this Chapter the $I A S C$-algorithm is studied by means of a number of numerical experiments. This will increase our understanding, and allows us to compare it with other methods. Moreover, some theoretical predictions from Chapter 5 can be tested. In order to quantify the performance, we measure the success-rate $s$, quality $q$, and the relative iterationtime $T$. Indicators for the complexity of the classification-problem are the average normalised intra-class geometric distance $\langle\vec{b}\rangle$, and the relative noise $v$ in the measurement - which is a measure for the normalised distance between the measurement and the defect-class cellcentre.

Control parameters for the $I A S C$-algorithm are the applied optimisation-functional, the representation of the state-probabilities (in our case Gaussian), the development of the dynamicallity-exponent, and the applied termination-criterion. Ads a measure for comparison, we regard two extremes: (i) exhaustively performing all measurements, and: (ii) random selection of feature-parameters. The conclusion of the analysis is that for overlapping classes $(<C<<1)$ or for small to moderate noise $(v<0.4)$ the $I A S C$-algorithm performs better than exhaustive or random parameter-selection. Also, some experiment is described of multicriteria functionals.

Next, some models for the evolution of the dynamicallity-exponent are tested. This reveals large differences between the scenario where the exponent is constant zero, and all others, but remarkably enough almost no difference in-between the other evolution-models.

Finally, several static and dynamic termination-criteria are compared. In Section 6.6 .4 the theoretically predicted relation between success-rate and iteration-length for a constant threshold is verified, as well as the theoretically predicted optimal value for this threshold.

The general conclusion for interactive classification with $I A S C$ can be summarised concisely in terms of class-overlap and relative noise:

- medium overlap and medium noise: all methods work

- medium overlap and high noise: $I A S C$ is better than random / exhaustive selection

- severe overlap and medium noise: $I A S C$ is better then random /exhaustive selection

- severe overlap - high noise: nothing works. 


\section{Chapter 7 ON-LINE CONTROL OF PRINTING QUALITY USING COMPUTATIONAL VISION.}

In this Chapter we return to our main topic; the quality-control of printing-processes. In this Chapter all ingredients of the previous Chapters are combined. This mainly concerns the results of the European research-project IntelPadPrimt, in which framework this study is largely performed. After a short description of the objectives, composition, and eventually obtained results of this project, a description is given of the area of application: industrial padprinting processes for mass-production. In a separate Section the relevant featureparameters for padprinting defects are discussed. Next, the two realised prototypes are considered.

The first prototype, called PTl, was based on scanning-analysis and a model for linear correlation between feature-parameters and defect-classes. A simple model controlled the spatial exploration of the CCD-image of a printed element for failures, and the eventual integration of all hypotheses. This iteration mostly converged within some hundred steps to a result. The average performance of this algorithm was $79 \pm 22 \%$. In fact, this was a primitive predecessor of the $I A S C$-algorithm.

The second prototype, PT2, was equipped with the IASC-algorithm (Chapter 5). It also employed the pre-classification method based on a CID-transformation of the image (Chapter 2). Some of the feature-parameters were computed using fractal wavelet analysis (Chapter 3 ). Moreover, the partition of feature-space was performed with itcrated FMV (Chapter 4). An intelligent camera system (developed by the University of Birmingham) produced CCDimages of predominantly good quality. The evaluation of this system involved three levels: (i) the identification that a printing-defect has occurred, (ii) the mentioned pre-classification of the template-subtracted and upgraded CCD-image, and: (iii) the result of the IASC-algorithm. For images of sufficient quality, about $77 \%$ of the total, the performance amounted to: for (i) $99 \%$, for (ii) (pre-classification): $97 \%$, and for (iii) (IASC): $95 \%$. The latter was determined largely by the efficiency of the feature-extraction.

The main conclusion regarding prototype PT2 is that IASC functions well, but that it is very sensitive to the image-quality. Therefore, in a practical application of this prototype the image-quality must be improved significantly.

The main conclusion of our thesis is that an approach based on interactive classification and fractal wavelet analysis provides a good framework for on-line print-quality control, but that more research is required to bring such a concept into industrial practice. 


\section{Samenvatting}

\section{Hoofdstuk 1 INLEIDING}

Het onderwerp van deze studie is de automatische controle van drukprocessen middels afbeeldingen van de bedrukkingen. Dit plaatst deze studie in het gebied dat 'Computer Vision' heet. Dit vakgebied maakt een stormachtige ontwikkeling door, zowel vakinhoudelijk alsmede door de gestadig toenemende prestatie en dalende prijzen van hardware, en meer veelzijdige software. Door deze ontwikkelingen neemt het aantal toepassingen eveneens stormachtig toe, en is er sprake van grote interesse vanuit industrie en bedrijfsleven.

Voor ons onderwerp zijn een aantal eigenschappen van het menselijke gezichtsvermogen uitermate belangwekkend. Met name de actieve manier waarop mensen een object visueel verkennen is zeer inspirerend voor onze aanpak. Dit 'actieve zien' is een nauwe samenwerking tussen brein en oog, waarin het brein binnenkomende beelden analyseert en interpreteert. Uit deze interpretatie ontstaat een hypothese omtrent het beeld, en daaruit bepaalde verwachtingen. Gebaseerd op deze veronderstellingen selecteert het brein een ander relevant onderdeel van het beeld voor verdere analyse, waarop het oog op dit gebied gericht wordt, en het beeld vanuit de actuele hypothese wordt geanalyseerd. In de praktijk convergeert dit mechanisme binnen een 'oogwenk' naar de juiste conclusie. In geval van visuele illusies lukt het het systeem niet om te convergeren, en kunnen we dit mechanisme als het ware betrappen. Vanuit deze biologische inspiratie modelleren we de automatische analyse van bedrukkingen op een analoge wijze; als een interactie tussen een redeneer proces dat hypothesen en verwachtingen omtrent een beeld genereert, en beeldverwerkingstechnieken die bepaalde locaties onderzoeken en daar enkele gevraagde metingen verrichten. Hierbij stuurt het redeneerproces de plaats en de aard van de metingen. Dit proces veroorzaakt een 'real-time' diagnose van het betrachte object, waarbij slechts een fractie van alle mogelijke metingen verricht wordt. Dit mechanisme vormt een centraal onderwerp van het proefschrift, en wordt behandeld in de hoofdstukken 5,6 en 7 .

Een andere inspiratie vanuit het visueel-cognitieve apparaat betreft het vermogen om 'texturen' te herkennen. Een afbeelding van een textuurfragment, b.v. hout, textiel, steen, huid, of papier, volstaat voor een instantane herkenning. Vanuit dezelfde vaardigheid kunnen mensen fouten of onregelmatigheden herkennen in b.v. bedrukkingen. We zullen deze vaardigheid trachten na te bootsen middels 'fractale wavelet-analyse', een recent opgekomen formalisme. Dit staat beschreven in hoofdstuk 3.

Vernieuwende onderwerpen in deze studie zijn onder meer:

1. interactieve classificatie gebaseerd op het mechanisme van 'actief zien',

2. fractale wavelet analyse van bedrukkingen voor bedrukkingsmotief-onafhankelijke foutherkenning,

3. transformaties die bedrukkingen op een eenduidige wijze afbeelden op een interval of rechthoek,

4. een automatische clusteringmethode, die geschikt is voor snelle, 'unsupervised' partitionering van veel-parameterdata met veel, slecht-gescheiden klassen. Dit is relevant in onze context, omdat wij precies met zulke parameterruimten te maken krijgen. 


\section{Hoofdstuk 2 DE ANALYSE VAN BEDRUKKINGSBEELDEN}

Doelstelling van dit hoofdstuk is om beelden (in ons geval CCD-foto's) van bedrukkingen in één algemene vorm af te beelden, zodat we niet meer te maken hebben met allerlei details die per bedrukking kan variëren.

Allereerst betrachten we de structuur en eigenschappen van bedrukkingen. We definiëren afbeeldingen van bedrukkingen als continue patronen van grijswaarden. De bedrukkingsrand is de curve die bedrukt van niet-bedrukt gebied scheidt. De meeste soorten bedrukkingsfouten treden alleen op in bepaalde soorten gebieden; nabij òf ver weg van de bedrukkingsrand, bimmen ò buiten het bedrukte gebied. Zodoende ontstaan vier gebiedstypen op een bedrukking, die we printcontexten noemen. De templaat is het patroon dat gedrukt moet worden. Onze uiteindelijke doelstelling is templaat-onafhankelijke foutherkenning en kwaliteitssturing. Door het beeld van de bedrukking over de templaat te leggen, en de twee beelden af te trekken, verkrijgen we een direct beeld van een eventuele bedrukkingsfout, zowel zijn karakteristieke vorm alsook zijn verdeling over de printcontexten.

Een bedrukkingsfout wordt gekarakteriseerd door zijn vorm, textuur, en grijsheidswaardenverdeling. Tal van eigenschappen van drukfouten kunnen hieraan worden gerelateerd. Deze eigenschappen worden weergegeven door de 'featureparameters'. De mate waarin de eigenschap voorkomt wordt hierbij weergegeven door een getalswaarde. Op deze wijze kan een drukfout als een punt in de 'feature-ruimte' worden weergegeven. De positie van een punt in deze ruimte bepaald tot welke drukfoutenklasse het behoord, ongeacht zijn templaat.

Het centrale onderwerp van dit hoofdstuk betreft de transformatie van bedrukkingen naar een algemene representatie. Ook deze representatie makt de afbeelding onafhankelijk van de templaat, en maakt zodoende templaat-onafhankelijke foutclassificatie mogelijk. Uitgangspunt voor de transformatie is de Euclidische afstandstransformatie (EDT), die de afstand van een punt tot een verzameling geeft. Een aanvulling op de EDT betreft de positie van een punt binnen ò buiten het bedrukte gebied. De afstand in een afbeelding van een bedrukking definiëren wij nu als de minimale Euclidische afstand tot een bedrukkingsrand, met positief teken buiten, en negatief teken binnen het bedrukte gebied. Deze afstandsmaat noemen we de 'bedrukkingsdiepte'. Middels de bedrukkingsdiepte kan nu een eendimensionale transformatie op beelden van bedrukkingen worden gedefinieerd. Deze afbeelding geeft de (genormeerde) som van alle fouten op een bepaalde bedrukkingsdiepte. We noemen deze afbeelding de 'Cumulatieve Intensiteit Distributie', kortweg CID. Deze templaat-onafhankelijke afbeelding staat een aantal analyses toe, zoals gegeneraliseerde momenten, filteren of deconvolutie, of spectrale analyse. Twee voorbeelden worden gegeven van representaties gebaseerd op de CID, die in staat zijn verscheidene clusters foutklassen te scheiden. Deze representaties zijn derhalve geschikt voor preclassificatie.

Vervolgens trachten we een twee-dimensionaal analogon van de CID te construeren. Het blijkt echter niet mogelijk om een continue twee-dimenionale representatie te construeren. De problemen zijn analoog aan kaart-projecties: het is niet mogelijk om een continue vlakke representatie van een globe te construeren. Dit kan wel lokaal, of via een collectie van lokale kaarten. Wị definiëren een afbeelding, 2DI-map geheten, die inderdaad lokaal niet-continu is, maar wel aantrekkelijke lokale congruentie-eigenschappen heeft. Zodoende behouden fouten veelal hun karakteristiek vorm. Met de 2DI-map worden willekeurige bedrukkingen weergegeven in een rechthoek, met boven de centrale horizontale as het binnengebied, en daaronder het buitengebied van de bedrukking. Een veel groter probleem dan alle mathematische complicaties blijkt het discrete karakter van de CCD-foto's te zijn. Hierdoor ontstaan Moiret-achtige coupes, die verder gebruik van de 2DI-map voor classificatiedoeleinden ernstig beperken. 
Door deze transformaties, met name de feature-representatie en de CID-transformatie, kunnen we nu alle soorten bedrukkingen weergeven als punten in parameterruimten. Hierdoor kunnen we ons in latere hoofdstukken concentreren op clustering- en classificatietechnieken in dit type parameterruimten, zonder ons te bekommeren om allerhande details van de templaat.

Een aparte en uitgebreide appendix is gewijd aan de bepaling van featureparameters voor bedrukkingsfouten. De reden hiervoor is dat in de praktijk snel heengelopen wordt over de problematiek om goede featureparameters te definiëren. Vaak is het niet goed mogelijk om deugdelijke algoritmen voor de kwantificatie van bepaalde kenmerkende eigenschappen te construeren. In dergelijke gevallen hebben alle verdere clustering- en classificatietechnieken ook weinig zin meer. In deze appendix wordt in detail een groot aantal featureparameters besproken, soms vergezeld van een evaluatie van de performantie.

\section{Hoofdstuk 3 ANALYSE EN CLASSIFICATIE MIDDELS FRACTALE WAVELET ANALYSE}

De laatste decennia is er veel aandacht voor objecten die op elke schaal van vergroting relevante details vertonen. Deze 'fractale' verzamelingen blijken van groot belang te zijn voor de beschrijving van fysieke objecten en fysische processen. Belangrijkste motivatie in dit hoofdstuk is om te onderzoeken of het karakteristieke uiterlijk van bedrukkingsfouten weergegeven kan worden middels een fractale beschrijving. Daarbij maken we gebruik van het 'multi-fractale' formalisme, dat een meer geschikte, maar ook meer gecompliceerde beschrijving verschaft. In het multifractaal formalisme wordt de verzameling gerepresenteerd middels een dichtheidsfuncie. Met behulp van methoden uit de macrocanonieke ensembletheorie wordt hieruit een heel spectrum van fractale eigenschappen berekend, waaronder de fractale dimensie, de entropie, en de autocorrelatie. Dat spectrum van fractale eigenschappen wordt de 'Gegeneraliseerde Fractale Dimensie' (GFD) genoemd.

Een groot praktisch probleem is de bepaling van de GFD. Conventioneel gebeurt dat door de verzameling te overdekken met een collectie hyperkubussen, en dan bepaalde schalingseigenschappen van de overdekking te bestuderen terwijl de zijde van de kubussen naar lengte nul daalt. Niet alleen is het moeilijk zo'n overdekking te construeren voor de meeste verzamelingen, het is bovendien aangetoond dat er verscheidene nadelen kleven aan deze methode. Zo kan worden aangetoond dat deze methode consequent een te lage schatting levert voor dimensies hoger dan 2.6 .

In dat verband komt een geheel andersoortig, en eveneens zeer recent, formalisme te hulp: 'wavelets' en 'multi resolutie analyse' (MRA). Wavelets zijn - zoals de naam al aangeeft kleine 'golfjes': ze hebben integraal nul (vandaar: golf), en een beperkte compacte support (vandaar 'golfje'). Geheel analoog aan Fourier-analyse is het mogelijk om afbeeldingen weer te geven als (oneindige) sommen van wavelets. MRA biedt een elegante methode voor een dergelijke decompositie. De prijs die daarvoor betaald moet worden is dat er nu behalve de spectrale componenten ook spatiële componenten zijn, zodat het aantal coëfficiënten feitelijk verdubbelt. MRA richt zich bij elke schaal van vergroting juist op die gebieden van het object waar deze schaal bijzonder relevant is. Maar dit is juist de eigenschap die we nodig hebben voor het overdekken van het fractale object met hyperkubussen. Het is mogelijk om wavelets te zien als een soort gegeneraliseerde hyperkubussen, en zodoende de MRA als een soort gegeneraliseerde overdekking van het object. Wavelets zijn derhalve bijzonder geschikt voor de analyse van fractale objecten. De laatste paar jaren is deze aanpak verder uitgewerkt, met name door Mallat en Hwang. Dit heeft geleid tot de 'Wavelet-Transform Modulus Maxima' (WTMM) methode. Met de WTMM-methode kan de GFD van een object efficiënt worden 
berekend. Deze methode is recentelijk met succes toegepast om texturen te analyseren en te classificeren.

In hoofdstuk 3 worden al deze formalismen beknopt besproken, en waar nodig toegelicht met een voorbeeld. Allereerst wordt 'traditionele' Fourier-analyse toegepast. Hieruit kunnen slechts 'power-laws' gedistilleerd worden, maar geen fractale dimensies. Uiteindelijk wordt het multifractale wavelet formalisme toegepast op de analyse van scanprofielen en beelden van drukfouten. Daardoor wordt een beeld van een drukfout afgebeeld in een collectie getallen, namelijk de GFD. De GFD kunnen we dus zien als een specifieke 'featurevector'. In deze representatie blijken veel drukfoutklassen goed gescheiden te worden. Er zijn echter veel praktische problemen aan deze methode, met name de excessief lange rekentijden en het digitale karakter van de gebruikte beelden, die maar een beperkte vergroting toestaat.

Het aantrekkelijke van deze representatie is dat ze 'sterk' templaat-onafhankelijk is, d.w.z. op geen enkele wijze de kennis van de gebruikte templaat veronderstelt (waarbij we er van uitgaan dat deze zelf geen fractaal is). Bovendien is het - in principe - mogelijk om de GFD van de afbeelding te relateren aan het onderliggende fysisch-chemische drukkingproces. De fouten ontstaan door een combinatie van rheologische, mechanische, en chemische processen die derhalve ook de GFD van het doordoor ontstane patroon bepalen. Kennis van dit mechanisme zou het mogelijk maken om, uitgaande van de schatting van de GFD van het CCD-beeld, de relevante procesparameters te schatten, en derhalve het proces bij te sturen. Momenteel is een dergelijk idee echter volstrekt onhaalbaar.

We concluderen dat het idee van multifractale wavelet-analyse voor classificatie van drukfouten veel potentie heeft, maar om genoemde praktische redenen nog niet bruikbaar is. En passant wordt in dit hoofdstuk ook een eigen generalisatie van MRA gepresenteerd, GWDA genaamd, en voorbeelden gegeven van de toepassing daarvan.

\section{Hoofdstuk 4 AUTOMATISCHE CLUSTERINGSMETHODEN VOOR HOOG-DIMENSIONALE FEATURE-RUIMTEN MET SLECHT GESCHEIDEN KLASSEN}

Dankzij met name hoofdstuk 2 kunnen we nu een drukfout als een punt in een hoogdimensionale 'feature-ruimte' weergeven. Het blijkt echter dat verscheidene foutklassen in deze ruimte slecht gescheiden zijn. Bovendien zijn er relatief veel foutklassen, en is het wellicht mogelijk om met behulp van deze representatie, een andere, meer relevante foutklassen-taxonomie te ontdekken. Een belangrijke motivatie is bovendien dat we in hoofdstuk 5 een interactief classificatiealgoritme gaan beschrijven, zodat slechts een deelverzameling van de feature-ruimte nodig is.

In dit hoofdstuk worden daarom verschillende clusteringparadigma's getest op hun bruikbaarheid voor deze ruimte. Directe clustering en classificatie van beelden met behulp van neurale netwerken blijkt vanwege de resulterende computationele complexiteit bezwaarlijk. De neurale netwerken die nodig zijn voor deze toepassing hebben bijzonder veel parameters. Daardoor zouden onrealistische hoeveelheden voorbeelden noodzakelijk zijn om deze parameters te trainen.

Onze aandacht gaat daardoor uit naar 'fuzzy clustering'. Dergelijke technieken, zoals Fuzzy C-Means (FCM) zijn sterke 'unsupervised' (dat wil zeggen dat de meetpunten niet gelabeld zijn) clusteringtechnieken. We stellen een aanvulling op FCM voor, door in plaats van bolvormige 'memberships-functies' ellipsoidale functies te gebruiken, die een betere benadering geven van de locale metriek van de klasse. Deze methoden noemen we Fuzzy Multi-Variate clustering (FMV). Dit resulteert in clustering die op zijn minst gelijkwaardige 
partities van de feature-ruimte geeft, maar aanzienlijk sneller een resultaat bereikt. Derhalve is FMV ook meer geschikt voor meer-dimensionale ruimten dan FCM.

Een groot nadeel van clusteringmethoden, inclusief FCM en FMV, is dat zijn enige veronderstellingen vereisen van het aantal of de verdeling van de klassen over de featureruimte. Daarom stellen we een automatisch clusteringalgoritme voor, genaamd iteratieve FMV cluster analyse, gebaseerd op FMV, die zelfstandig het aantal significante klassen in de data bepaalt middels successievelijke splitsing of samenvoeging van klassen.

Tenslotte passen we clusteringtechnieken toe op de twee representaties die gebaseerd zijn op de CID-transformatie uit hoofdstuk 2. In het eerste geval laten we zien hoe de SOMclusteringtechniek gebruikt kan worden bij een eenvoudig model van twee soorten drukfouten, en voor de schatting van de grootte van de verstoring. In het tweede geval laten we zien hoe iteratieve FMV cluster analyse gebruikt kan worden voor de partitionering van een CID-gebaseerde representatie, die daardoor zeer geschikt wordt om te dienen als preclassificatie.

\section{Hoofdstuk 5 INTERACTIEVE CLASSIFICATIE VOOR HOOG- DIMENSIONALE, SLECHT-GESCHEIDEN KLASSEN EN RUIS}

$\mathrm{Nu}$ hebben we drukfoutanalyse teruggebracht tot de classificatie van een actuele meting van feature-waarden in één van de - van te voren geïdentificeerde - foutklassen. Dit kan betekenen dat eerst alle mogelijke metingen worden verricht, waarna een simpele toewijzing in de feature-ruimte volgt. Hierdoor zou de classificatie zeer tijdrovend en rekenintensief zijn. Bovendien is het veelal onnodig om alle metingen te verrichten, omdat gedurende het proces reeds duidelijk is geworden wat de waargenomen foutcategorie is. Mede geïnspireerd door het 'actief zien' bij levende wezens kiezen we voor een andere aanpak. In dit hoofdstuk stellen we een interactieve methode voor om gelijktijdig te kunnen classificeren en meten. We noemen dit 'interactieve classificatie'. Belangrijkste probleem hierbij is om, gebaseerd op de huidige metingen en resulterende veronderstellingen, de 'beste' volgende meting te kiezen. De aanpak die we kiezen is gebaseerd op functionale optimalisatie. We definiëren een aantal functionalen die gebaseerd zijn op de scheiding van klassen. Daartoe moet een eerst zinvolle metriek in de feature-ruimte gedefinieerd worden. De meest-gebruikte afstandsmaat is de 'Mahalanobis-afstand', maar deze is niet-symmetrisch voor klassenafstanden: afstand(klassel,klasse2) is i.h.a. niet gelijk aan afstand(klasse2,klassel). Daarom komen we met andere voorstellen, zoals de 'geometrische' afstand. In dezelfde zin definiëren we de relevantie van een bepaalde featureparameter voor een bepaalde foutklasse. Met behulp van deze afstandsmaten definiëren we nu functionalen. Optimalisatie van deze functionalen leidt tot de featureparameter die de meeste scheiding van de klassen geeft, en in die zin de meest zinvolle meting is. Door de actuele hypothese - weergegeven door de kansverdeling over de foutklassen - te integreren in deze functionaal maken we dit proces dynamisch. Zodoende kiezen we de featureparameter die, gegeven de huidige hypothesen, het meeste perspectief biedt om tussen de concurrerende hypothesen te kiezen. Van belang is dat gedurende het redeneerproces aanvankelijk weinig gewicht wordt toegekend aan een hypothese, maar later geleidelijk aan steeds meer. Dit wordt tot uitdrukking gebracht in de z.g. 'dynamiciteitsexponent'. Vervolgens wordt de aandacht gevestigd op het probleem om ongelijkaardige informatie te integreren. Hierbij moet worden gedacht aan de grote hoeveelheid sensorinformatie die beschikbaar is, zoals betreffende viscositeit, temperatuur, en mechanische druk. Gekozen wordt voor een representatie als normale multivariate 
verdelingen, de zogenaamde Gausische mixtures. Veel nadruk krijgt de controle van het redeneerproces. Zo worden enkele statische en dynamische stopcriteria gedefinieerd. Hierbij slaat 'dynamisch' op het feit dat deze de geschiedenis van de iteratie betrekt bij de beslissing tot beëindiging van de iteratie. Hierbij blijkt dat in het geval van een stopcriterium met een constante 'threshhold' een optimale waarde berekend kan worden voor die threshhold in de zin van maximale slaagkans bij minimale iteratielengte.

Al deze onderwerpen tezamen vormen de ingrediënten voor een geïntegreerde aanpak voor simultaan meten en classificeren, genaamd: InterActive Sensing and Classification (IASC). Doel van $I A S C$ is de 'on-line' classificatie van een object, gebaseerd op geselecteerde sensormetingen. De methode kan gedefinieerd worden als een iteratief algoritme, of als een geïtereerde afbeelding op de ruimte van hypothesen, waarbii de meting 'slechts' een parameter is. Voor onze aanname van Gausisch verdeelde klassen kan deze afbeelding verder worden uitgewerkt.

Een centrale plaats neemt de convergentie en stabiliteit van de $I A S C$-methode in. Daartoe worden eerst enkele maatstaven voor de performantie gedefinieerd: de kwaliteit ' $q$ ' van de conclusie (die 1 is voor éénduidige conclusies, en 0 voor meerduidige conclusies), de slaagkans ' $s$ ' van het algoritme (die 1 is als de correcte foutklasse wordt gevonden en anders 0 ), en de relatieve lengle ' $T$ ' van de iteratie (dat is het aantal verrichte metingen t.o.v. het totaal aantal mogelijke metingen, dus eveneens tussen 0 en 1). Zoals reeds eerder vermeld, kan de actuele hypothese weergegeven worden als een kansverdeling over de $K$ mogelijke foutklassen. De actuele hypothese kan dus gerepresenteerd worden door een vector in $\mathrm{IR}^{K}$, en, daar de kansmaat voor iedere afzonderlijke klasse in het interval $[0,1]$ ligt, meer specifiek als een punt in een $K$-dimensionale hyperkubus $[0,1]^{K}$. De hoekpunten van deze hyperkubus corresponderen met concreet voorkomende foutklassen. De hele IASC-iteratie correspondeert zodoende met een baan in deze hyperkubus. De $I A S C$-iteratie convergeert derhalve als die baan eindigt in een hoekpunt. De actuele meting daarentegen is een vector in de featureruimte, waarvan gedurende de iteratie steeds meer componenten 'bekend' worden. Die feature-ruimte is opgedeeld in $K$ cellen, die corresponderen met de $K$ bekende foutklassen. Het centrale vermoeden is nu dat als de meting in een bepaalde cel ligt, de $I A S C$-iteratie naar de betreffende foutklasse convergeert met een kwaliteit $q$ die bepaald wordt door de afstand van de meting tot het centrum van de corresponderende cel. Deze afstand is tevens een maat voor de relatieve ruis in de meting.

Verscheidene wegen worden gevolgd om deze veronderstelling te bewijzen. In sectie 5.6.5 wordt aangetoond dat de $I A S C$-iteratie een contractie is, daar de Jacobiaan monotoon naar nul nadert. Alle convergerende iteraties zijn contracties. Echter, niet alle contracties corresponderen met convergerende iteraties, bijvoorbeeld bij meervoudige convergentiepunten of bij chaotische gedrag nabij 'strange attractors'. In sectie 5.6.6 worden drie scenario's nader onder de loep genomen. i. Als de meting identiek is aan het centrum van een cel dan convergeert de $I A S C$-iteratie naar de correcte conclusie. ii. Als de meting erg dicht bij het centrum van een cel ligt, dan convergeert de iteratie eveneens. Dichtbij blijkt hier te zijn: dichter bij dan de wortel uit de kleinste eigenwaarde van de covariantiematrix van de bijbehorende foutklasse. Tenslotte: iii. de meting ligt niet 'dicht' bij een cel-centrum. Naarmate de Euclidische afstand tot het centrum toeneemt, zullen er featureparameters zijn waarvoor de meting dichter bij een centrum van een andere cel ligt. Deze featureparameters zijn dan slecht gescheiden t.o.v. de anderen, en hebben dus een lage selectiekans bij de functionale parameterselectie die ten grondslag aan $I A S C$. Desalniettemin, als de fractie van 'foute' parameters toeneemt, neemt de kans op een verkeerde beslissing ook toe.

In sectie 5.7 worden de dynamische eigenschappen van de $I A S C$-iteratie nader bestudeerd. Eerst bestuderen we de evolutie van de klasse-waarschijnlijkheden gedurende de iteratie. We definiëren twee eenvoudige kengetallen: de einchraarde en de gemiddelde stijging van deze 
klasse-waarschijnlijkheden. Door middel van automatische clustering (NB: met iterated FMV uit hoofdstuk 4) van een groot aantal observaties worden vier typen evolutie onderscheiden. Deze vier typen kunnen gerelateerd worden aan de relatieve onderlinge ligging van de clusters. Nabije clusters zorgen voor enige 'verwarring' bij de evolutie van de klassewaarschijnlijkheidsfunctie gedurende de iteratie, en kunnen op een relatief laat moment de 'balans' nog laten omslaan. Sommige klassewaarschijnlijkheden convergeren naar een waarde ergens tussen nul en een in. Dit inzicht leidt tot een bespreking van de rol van diffuse klassen en gemengde evenwichtspunten. Gemengde evenwichtspunten zijn punten in de featureruimte die 'onbeslisbaar' zijn. Onbeslisbaar betekent hier dat de $I A S C$-iteratie niet naar een éénduidige conclusie convergeert. In het geval van een oneindig lange iteratie zijn dit de punten op de separatrix tussen twee klassen. Daar echter de $I A S C$-iteratie eindig lang is, zullen punten nabij de separatrix, met name bij diffuse klassen, echter ook onbeslisbaar zijn.

In de volgende subsecties wordt iets verteld over de schatting van de gehele meting, gedurende de $I A S C$-iteratie, alsmede over de betrouwbaarheid daarvan. Daarop gebaseerd wordt de kansmaat bepaald dat de actuele hypothese wordt verworpen op grond van een nieuwe meting, gedurende de IASC-iteratie. Aangetoond wordt dat deze kans quasiexponentieel afneemt gedurende de iteratie.

Van grote invloed zijn de stop-criteria op de prestatie van het $I A S C$-algoritme. Deze worden nader bestudeerd in sectie 5.7.5. Middels een mean-field approach wordt een theoretisch model verkregen dat de relatie tussen de gemiddelde slaagkans $s$ en de relatieve iteratielengte $T$ voorspelt.

Tenslotte wordt in sectie 5.7.6 een aantal modellen gepresenteerd voor de evolutie van de dynamiciteits-exponent. Deze bepalen de invloed van de actuele hypothese op de featureparameter selectie.

We concluderen dat het $I A S C$-algoritme een raamwerk biedt voor interactieve classificatie, die onder bepaalde omstandigheden tot de juiste conclusie leidt, terwijl maar een klein deel van alle mogelijke metingen is verricht. Dit raamwerk is niet alleen toepasbaar binnen computer vision, maar ook in alle gevallen waar diagnose en meting samengaan, zoals in chemische analyse, medische diagnose, en autonome robotten.

\section{Hoofdstuk 6 NUMERIEKE EXPERIMENTEN MET IASC}

In dit hoofdstuk worden tal van numerieke experimenten met het $I A S C$-algoritme uitgevoerd. Dit om inzicht te krijgen in de werking van $I A S C$ en ter vergelijking met andere methoden. Bovendien kunnen zo enkele theoretische voorspellingen uit hoofdstuk 5 worden getoetst. Om de performantic te kwantificeren meten we de reeds genoemde slaagkans $s$, kwaliteit $q$, en relatieve iteratielengte $T$. Grootheden die de complexiteit van het classificatieprobleem weergeven zijn de gemiddelde genormaliseerde afstand $\langle d\rangle$ tussen de klassen, en de relatieve ruis $v$ in de meting - die een maat is voor de afstand van de meting tot het centrum van de foutklasse in de featureruimte. Controle parameters in het IASC-algoritme zijn de gekozen optimalisatie-functionaal, de representatie van de klassewaarschijnlijkheden (in ons geval Gausisch), de ontwikkeling van de dynamiciteits-exponent, en het gebezigde stopcriterium. Als eerste worden verschillende optimalisatic-functionalen getest. Als maatstaf voor de vergelijking dienen twee uitersten: $i$. uitputtend alle metingen verrichten, en ii. random selectie van metingen. De conclusie is dat bij overlappende klassen $(<d><1)$ òf enige ruis $(v$ $<0.4$ ), IASC beter presteert dan random of uitputtende parameterselectie. Tevens wordt een enkel experiment met multi-criteria-functionalen beschreven.

Vervolgens worden enkele modellen voor de evolutie van de dynamiciteits-exponent getest. Daarbij blijkt dat er grote verschillen zijn tussen scenario's waar deze constante waarde nul 
heeft, maar dat verrassend genoeg er verder weinig verschil valt te meten tussen de specifieke evolutiemodellen. Tenslotte worden verscheidene statische en dynamische stopcriteria vergeleken. In sectie 6.6.4 wordt zodoende de theoretisch voorspelde relatie tussen slaagkans en iteratielengte voor constante threshhold geverifieerd, alsmede de theoretisch voorpelde optimale waarde voor de threshhold.

De algemene conclusie betreffende interactief classificeren met IASC kan bondig worden samengevat als:

- geringe klassenoverlap en weinig ruis: alle methoden werken

- sterke klassenoverlap òf veel ruis: IASC werkt beter dan random en uitputtend

- sterke klassenoverlap èn veel ruis: niets werkt meer

\section{Hoofdstuk 7 KWALITEITSGESTUURDE BEDRUKKINGS- PROCESSEN.}

In dit hoofdstuk keren we terug naar ons hoofdthema; de kwaliteitssturing van drukprocessen. In dit hoofdstuk komen alle ingrediënten terug die eerder besproken zijn. Dit hoofdstuk behandeld de resultaten van het Europees onderzoeksproject IntelPadPrint, waarbinnen deze studie grotendeels is verricht. Na een korte beschrijving van de doelstellingen, samenstelling en uiteindelijke behaalde resultaten van dit project wordt een beschrijving gegeven van het probleemgebied: industriële bedrukkingsprocessen voor massaproductie. In een aparte paragraaf worden de relevante featureparameters voor bedrukkingsfouten besproken. Vervolgens worden de twee gerealiseerde prototypen besproken.

Het eerste prototype, PTl genaamd, was gebaseerd op scananalyse en een model voor lineaire correlatie tussen featureparameters en foutklassen. Een eenvoudig model regelde de ruimtelijke exploratie van het beeld naar fouten en de integratie van de conclusies. Deze iteratie convergeerde meestal binnen zo'n honderd stappen naar een resultaat. De gemiddelde performantie van dit algoritme bedroeg zo'n $79 \pm 22 \%$. In feite was dit een primitieve voorganger van het $I A S C$-algoritme.

Het tweede prototype, PT2, was uitgerust met het $I A S C$-algoritme (hoofdstuk 5). Maar tevens werd gebruik gemaakt van een preclassificatie-methode van een representatic van het beeld gebaseerd op de CID-transformatie (hoofdstuk 2). Sommige van de featureparameters werden met behulp van fractale wavelet analyse berekend (hoofdstuk 3). Bovendien was de partitic van de feature-ruimte verricht middels iterated FMV (hoofdstuk 4). Een intelligent camera system (ontwikkeld door de Universiteit van Birmingham) leverde de CCD-beelden van overwegend redelijke kwaliteit. De evaluatie van dit systeem betreft drie gradaties: i. de identificatie dàt een drukfout optreed, ii. de genoemde preclassificatic van het beeld, iii. het resultaat van het IASC-algoritme. Voor bruikbare beelden, zo'n $77 \%$ van het totaal, was de performantie van i: $99 \%$, van ii (preclassificatie): $97 \%$ en van iii $(I A S C): 95 \%$. De laatste werd voornamelijk bepaald door de kwaliteit van de feature-extraction. De conclusies betreffende PT2 zijn dat IASC goed functioneert, maar erg gevoelig is voor de beeldkwaliteit. Daarom moet in een praktische uitwerking van dit prototype met name de beeldkwaliteit sterk verbeterd worden.

De belangrijkste conclusie van onze studie is dat onze aanpak, mede gebaseerd op interactieve classificatie en fractale wavelet analyse, een goed raamwerk biedt voor directe kwaliteitssturing met behulp van 'computationele visie', maar dat meer onderzoek noodzakelijk is om dit concept op een niveau te brengen die noodzakelijk is voor toepassing in de industriële praktijk. 


\section{Curriculum Vitae}

Ronald Westra was born on Friday, April 13 in Amsterdam. After completion of VWO in Roermond, the Netherlands, he studied at Utrecht University. There, he received a baccalaureate in Theoretical Astrophysics, and graduated in 1982 in Theoretical Physics on the fractal geometry of dynamical systems. He held various positions as scientific researcher in science, industry and ITC-engineering, including Nuclear and Radiation Physics, the University of Amsterdam, Pandata (CGS-group) research \& development. In 1990 he became group leader of the Control and Engineering Group of the Research Institute for Knowledge Systems (RIKS) in Maastricht, where he was responsible for the acquisition and management of industrial and scientific research projects. There, he instigated and defined several national and international research projects, including European research projects: BE-4489 'Camaro', and BE-7686 'Psycho'. In this position he also initiated close scientific collaboration with national and international universities. In 1997 he joined the department of Mathematics of the Universiteit Maastricht as research manager, where he coordinates applied research activities, especially aimed at industrial applications. Besides his work, he held several official positions, such as president of the section Theoretical Astronomy of the Dutch Society for Meteorology and Astronomy, president of Studium Generale Maastricht, and external examiner and reviewer. He participated in many national and international conferences, and was invited speaker at the 7 th Conference on Intelligent Learning (CoIL), Chios (Greece) June 2000, and the 7th UK Conference on Fuzzy Systems, Sheffield, October 2000.

$\mathrm{He}$ is married, has two daughters, and momentarily lives in the village of Bunde, near Maastricht. 